UNITED STATES DEPARTMENT OF THE INTERIOR GEOLOGICAL SURVEY

\title{
PRELIMINARY DETERMINATION OF EPICENTERS MONTHLY LISTING
}

$\underline{A P R I L}$ - JUNE 1989

NATIONAL EARTHQUAKE INFORMATION CENTER

Open File Report

$89-600-B$

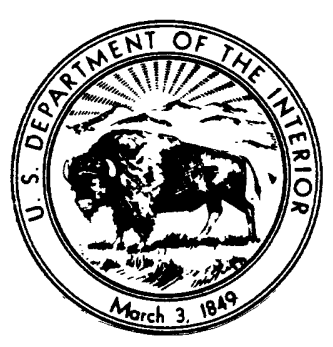

This report is preliminary and has not been reviewed for conformity with U.S. Geological Survey editorial standards. 


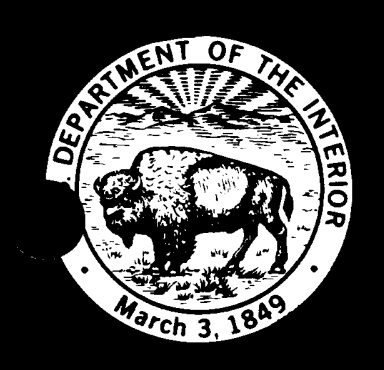

APR I L 1989

DEPTH MAGNITUDES SD NO $\begin{array}{cl}\text { GS } & \text { STA } \\ \text { MB MSZ } & \text { USED }\end{array}$

$\begin{array}{llr}K & \text { DAY ORIGIN TIME } \\ \text { E GEOGRAPHIC } \\ Y\end{array}$ HR MN SEC LAT LONG

$\begin{array}{llllll}01 & 27 & 55.6 & 42.771 \mathrm{~N} & 18.585 \mathrm{E}\end{array}$ $013126.8 \% \quad 30.954 \mathrm{~S} \quad 116.836 \mathrm{E}$ $034155.0860 .704 \mathrm{~N} \quad 151.134 \mathrm{~W}$ $043051.7 \quad 5.271 \mathrm{~N} \quad 126.514 \mathrm{E}$ 044557.0 ? 32.56 S $69.63 \mathrm{~W}$ $05 \quad 1737.9 * 19.290 \mathrm{~S} \quad 127.767 \mathrm{E}$ $08 \quad 22 \quad 21.9 ? \quad 37.61 \mathrm{~N}$ $08 \quad 27 \quad 43.8 \quad 35.497 \mathrm{~N}$ $\begin{array}{llllll}08 & 42 & 49.7 \% & 39.289 & N\end{array}$ $08 \quad 58 \quad 35.4 \% 41.278 \mathrm{~N}$ $091021.0 * 13.133 \mathrm{~s}$ $092543.7 * 63.561 \mathrm{~N}$ $095152.4 \% 39.133 \mathrm{~N}$ $\begin{array}{llll}10 & 05 & 02.6 \% & 39.163 \mathrm{~N}\end{array}$ $10 \quad 16 \quad 35.7 * 50.003 \mathrm{~N}$ $\begin{array}{llll}11 & 34 & 38.7 & 3.152 \mathrm{~S}\end{array}$ $\begin{array}{lllll}11 & 36 & 12.5 & 5.119 & \mathrm{~S}\end{array}$ $1143 \quad 25.7 \% \quad 41.209 \mathrm{~N}$ $\begin{array}{lllll}12 & 31 & 36.5 & 40.872 \mathrm{~N}\end{array}$ $\begin{array}{llll}13 & 19 & 45.7 & 39.214 \mathrm{~N}\end{array}$ $\begin{array}{lllll}15 & 06 & 37.9 \% & 37.937 \mathrm{~N}\end{array}$ $17 \quad 1009.3 ? 37.62 \mathrm{~N}$ $21 \quad 15 \quad 19.4$ ? $61.56 \mathrm{~N}$ $\begin{array}{llll}21 & 29 & 58.7 & 32.849\end{array}$

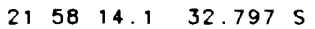

$222259 . \theta * 34.438 \mathrm{~N}$ $00 \quad 1420.3 \quad 44.623 \mathrm{~N}$ $003750.5 * 12.117 \mathrm{~S}$ $011019.9 * 19.7915$ $012411.5 \quad 36.212 \mathrm{~N}$ 015952.6 ? $66.01 \mathrm{~N}$ $020104.8 ? 41.26 \mathrm{~N}$ $024132.2 ? 13.60 \mathrm{~S}$ $\begin{array}{lllll}03 & 46 & 19.8 & 44.627 \mathrm{~N}\end{array}$ $05 \quad 1224.9 * 17.237 \mathrm{~N}$ $0528 \quad 19.9 * 8.098 \mathrm{~S}$ $06 \quad 4204.5 \quad 28.252 \mathrm{~N}$ $\begin{array}{llll}06 & 59 & 00.6 & 47.245 \mathrm{~N}\end{array}$

$072126.2 * 29.587 \mathrm{~s}$ $\begin{array}{llllllll}99 & 21 & 21 & 02 & 43.97 & \mathrm{~N}\end{array}$ $092521.4 \quad 8.846 \mathrm{~S}$ $092935.2 ? \quad 34.47 \mathrm{~N}$

$1030 \quad 13.9 * 35.297 \mathrm{~N}$ $103557.1 \quad 11.063 \mathrm{~N}$
$71.21 \mathrm{E}$

$24.035 \mathrm{E}$

$27.702 \mathrm{E}$

$19.989 E$

$34.089 \mathrm{E}$

164.270

$27.612 \mathrm{E}$

$27.583 \mathrm{E}$

127.791

$127.909 \mathrm{E}$

$151.477 \mathrm{E}$

$20.005 E$

$22.780 \mathrm{E}$

$23.664 \mathrm{E}$

$27.337 \mathrm{E}$

$20.55 \mathrm{E}$

$146.48 \mathrm{~W}$

$39.816 \mathrm{~W}$

$69.947 w$

$26.770 \mathrm{E}$

$7.049 \mathrm{E}$

166. $130 \mathrm{E}$

$175.487 \mathrm{~W}$

$28.036 \mathrm{E}$

$156.41 \mathrm{~W}$

$20.43 \mathrm{E}$

$167.77 \mathrm{E}$

$7.041 \mathrm{E}$

$62.005 W$

$107.716 \mathrm{E}$

$57.315 \mathrm{E}$

8. $991 \mathrm{E}$

$68.073 \mathrm{~W}$ $149.06 \mathrm{E}$ $127.134 \mathrm{E}$

$140.18 \mathrm{E}$

$46.507 \mathrm{E}$ $85.352 w$
$5 \mathrm{G}$

$10 \mathrm{G}$

52

63.

$10 \mathrm{C}$

$10 \mathrm{C}$

$33 \mathrm{~N} 3.8$

113

$10 \mathrm{G}$

10

$10 \mathrm{G} 4.3$

$33 \mathrm{~N}$

$10 \mathrm{G}$

10

10 G 4.2

$33 \mathrm{~N} 5.1$

$110 * 4.8$

$10 \mathrm{G}$

$10 \mathrm{G}$

$10 \mathrm{G}$

$10 \mathrm{G}$

$10 \mathrm{G}$

$33 N$

$10 \mathrm{G} \quad 4.4$

$1100 \quad 5.5$

$137 ?$

$10 \mathrm{G}$

332.5 .1

$10 \mathrm{G}$

$33 N$

$10 \mathrm{C}$

$33 \mathrm{~N}$

$10 \mathrm{C}$

$33 N$

$33 \mathrm{~N} 4.6$

$14 \mathrm{G}$

$150 *$

$33 \mathrm{~N} 3.9$

$33 \mathrm{~N} 4.9$

$10 \mathrm{G}$

5 G 4.3 $70 \quad 5.0$

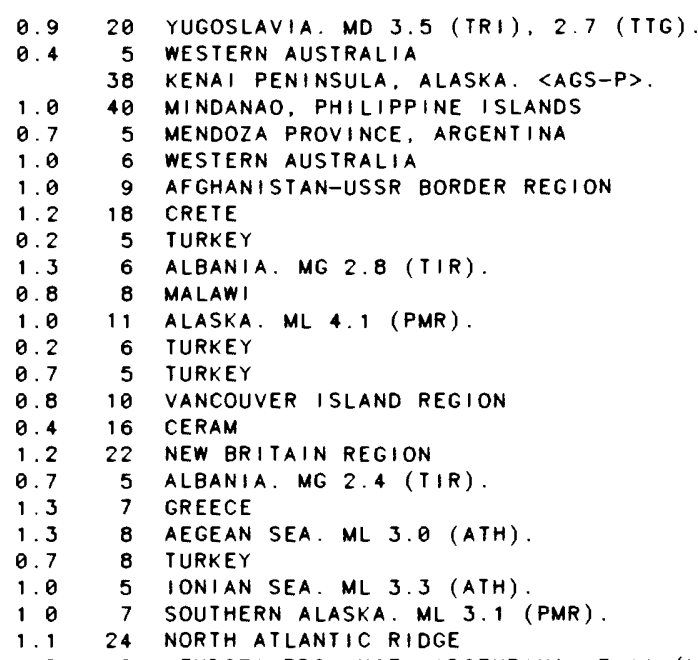

AFGHANISTAN-USSR BORDER REGION

CRETE

5 TURKEY

6 ALBANIA. MG 2.8 (TIR).

B MALAWI

ALASKA. ML 4.1 (PMR)

6 TURKEY

TURKEY

VANCOUVER ISLAND REgION

CERAM

NEW BRITAIN REGION

5 ALBANIA. MG 2.4 ( $T I R)$

GREECE

AEGEAN SEA. ML 3.0 (ATH)

TURKEY

IONIAN SEA. ML 3.3 (ATH)

SOUTHERN ALASKA. ML 3.1 (PMR)

NORTH ATLANTIC RIDGE

MENDOZA PROVINCE, ARGENTINA. Fell (V) at Sontiago,

Chile. Also felt in the Valporaiso, Chile area.

8 CRETE. MD 3.7 (ATH)

25 NORTHERN ITALY. ML 2.5 (GEN)

SANTA CRUZ ISLANDS

TONGA ISLANDS

DODECANESE ISLANDS. MD 3.2 (ATH).

6 ALASKA. ML 3.2 (PMR).

ALBANIA

VANUATU ISLANDS

NORTHERN I TALY. ML 2.3 (GEN), 2.3 (LDG)

LEEWARD ISLANDS. ML 3.6 (FDF)

JAVA

SOUTHERN IRAN

SWITZERLAND. ML 3.3 (GRF), 3.2 (LOG), 3.2 (KBA). MD 3.0 (STR)

9 SAN JUAN PROVINCE, ARGENTINA

10 KURIL ISLANDS REGION

TIMOR

NEAR EAST COAST OF HONSHU, JAPAN. MG 2.8 (JMA). FEIt ( JMA) ot Toteyomo.

IRAN-IRAO BORDER REGION

NICARAGUA. MD 5.2 (HDC). Felt (V) ot Ployo Hermoso and Playa del Coco; (IV) ot Nicoyo and Santo Cruz; (III) ot Puntarenos: ( 11 ) at Atenos and Las Chiles, Costa Rica. Felt at Son Jose and in southern Casto Rico. Felt (111) at Monoguo, Nicar aguo.

$\begin{array}{rrrlrrrrrrr}002 & 10 & 43 & 32.7 & 5.428 & \mathrm{~S} & 146.785 & \mathrm{E} & 251 & \mathrm{D} & 5.6 \\ 02 & 12 & 22 & 37.6 & 36.176 & \mathrm{~N} & 28.010 & \mathrm{E} & 10 & \mathrm{G} & \\ \theta 2 & 12 & 55 & 29.5 \% & 46.618 & \mathrm{~N} & 6.584 & \mathrm{E} & 10 & \mathrm{G} & \\ 02 & 14 & 11 & 04.4 * & 36.052 & \mathrm{~N} & 31.182 & \mathrm{E} & 10 & \mathrm{G} & \\ 02 & 14 & 43 & 34.47 & 39.084 & \mathrm{~N} & 29.136 & \mathrm{E} & 10 & \mathrm{G} & \\ 02 & 14 & 50 & 52.6 ? & 12.37 & \mathrm{~N} & 144.36 & \mathrm{E} & 33 & \mathrm{~N} & \\ 02 & 15 & 23 & 33.5 ? & 23.82 & \mathrm{~N} & 142.56 & \mathrm{E} & 33 & \mathrm{~N} & 4.6\end{array}$

0.9147 EAST PAPUA NEW GUINEA REGION

0.56 DODECANESE ISLANDS. MD 3.5 (ATH)

0.710 SWITZERLAND. ML 2.7 (LDG)

1.1 TURKEY

0.6 8 SOUTH OF MARIANA ISLANDS

0.99 VOLCANO ISLANDS REGION
1.45 TURKEY 
$\begin{array}{llllllllllll}02 & 16 & 12 & 20.8 ? & 22.65 & S & 66.75 & \text { W } & 326 & ?\end{array}$

02 $18 \quad 26 \quad 49.8 * 37.740 \mathrm{~N}$

$02 \quad 212202.5 ? 31.30 \mathrm{~S}$

$\begin{array}{lllll}0 & 02 & 21 & 36.9 & 32.621 \mathrm{~N}\end{array}$

$02 \quad 213030.7 * 6.602 \mathrm{~N}$

0300 05 58.8 ? $36.18 \mathrm{~N}$

$03 \quad 000905.4 \& \quad 60.639 \mathrm{~N}$

$\begin{array}{llllllll}03 & 00 & 26 & 22.3 ? & 10.75 \mathrm{~N}\end{array}$

$\begin{array}{lllllll}03 & 01 & 18 & 13.1 ? & 51.63 & \mathrm{~N} \\ 03 & 01 & 37 & 21.2 ? & 33.08 & \mathrm{~S}\end{array}$

$\begin{array}{llllllll}03 & 04 & 26 & 52.2 ? & 21.36 \quad \mathrm{~S}\end{array}$

$\begin{array}{llllll}03 & 05 & 16 & 06.9 \% & 39.667 & \mathrm{~N}\end{array}$

$\begin{array}{llllll}03 & 06 & 58 & 44.2 & 39.322 \mathrm{~N}\end{array}$

$03 \quad 08 \quad 36 \quad 23.9 * 38.174 \mathrm{~N}$

$\begin{array}{lllll}08 & 58 & 49.6 \% & 39.270 \mathrm{~N}\end{array}$

03

03

$03 \quad 09 \quad 33 \quad 05.6 \% \quad 39.589 \mathrm{~N}$ $03 \quad 0958 \quad 05.2 \quad 46.110 \mathrm{~N}$ $03 \quad 112932.3 * 17.897 \mathrm{~N}$ $03 \quad 131557.1 \% 60.006 \mathrm{~N}$ $\begin{array}{llllllll}03 & 14 & 19 & 39.7 ? & 44.24 \mathrm{~N}\end{array}$ $03 \quad 170853.4 * 39.254 \mathrm{~N}$ $03 \quad 1745 \quad 07.5 * 3.555 \mathrm{~N}$
36.755 E $10 \mathrm{G}$ 179.99 $47.782 \mathrm{E}$

73.416 W 27.23 $150.551 \mathrm{~W}$ 60.82 $170.31 w$ $72.31 \mathrm{w}$ $62.390 \mathrm{~W}$

$68.680 \mathrm{w}$ $173.75 \mathrm{~W}$ $29.360 \mathrm{E}$

72.08 W $25.516 \mathrm{E}$ 138.333 27.725 $14.138 \mathrm{E}$

$142.378 \mathrm{E}$

$30.515 \mathrm{E}$ 14. $106 \mathrm{E}$ $99.654 W$

$4.772 \mathrm{E}$

$7.53 \mathrm{E}$ $151.204 \mathrm{~W}$ $60.027 \mathrm{~W}$ $23.485 \mathrm{E}$ 124.562 $121.795 \mathrm{~W}$
02 $173727.1 \quad 35.194 \mathrm{~N} \quad 135.552 \mathrm{E} \quad 10 \mathrm{G}$

- $02 \quad 205202.6 \quad 30.941 \mathrm{~S}$

$03014550.7 * 10.715 \mathrm{~N}$

$03 \quad 031930.4 * 31.5915$

$03 \quad 05 \quad 1658.0 ? 33.90 \quad S$ $03163524.6862 .236 \mathrm{~N}$ $03 \quad 170418.2 * 15.695 \mathrm{~N}$ $03 \quad 1746 \quad 34.4 \& 37.422 \mathrm{~N}$ 68.37 W $116 ?$

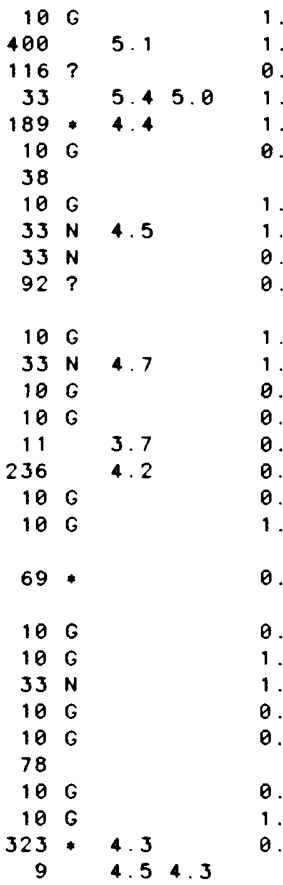

0.7

0.6

1.1

1. 0

0. 2 .

1. 0.339

1.5
0.8

1.2

1.5

0.6
0.7

1.0

1. 4

0.4

0.9

0.5

1.0

0.8

0.7

1.4

1. 2

0.1
7 JUJUY PROVINCE. ARGENTINA

8 SOUTHERN HONSHU, JAPAN. MG 3.6 (JMA). FeIt (I JMA) ot Kyoto.

7 TURKEY

59 KERMAOEC ISLANOS REGION

5 SAN JUAN PROVINCE, ARGENTINA

IRAN-IRAO BOROER REGION. FeIt ot II am and AhYOZ, I $\mathrm{r}$ an

1 NORTHERN COLOMBIA

4 DODECANESE ISLANDS. MO 3.2 (ATH)

40 KENAI PENINSULA. ALASKA. LAGS-P>. ML 3.1 (PMR)

6 TRINIOAO. MO 3.3 (TRN)

14 FOX ISLANOS. ALEUTIAN ISLANDS. ML 4.3 (PMR)

7 OFF COAST OF CENTRAL CHILE

22 NEAR COAST OF VENEZUELA. MO 3.9 (TRN). Felt (11) on Trinidod

6 SAN JUAN PROVINCE, ARGENTINA

13 TONGA ISLANOS

5 TURKEY

8 OFF COAST OF CENTRAL CHILE

3 AEGEAN SEA. ML 3.6 (ATH)

15 NEAR WEST COAST OF HONSHU, JAPAN

6 TURKEY

10 YUgOSLAVIA. ML 2.7 (KBA), 2.1 (LJU). MO 2.7 (LJU). Fell (IV) ot Poljone nod Skofjo Loko.

- NEAR EAST COAST OF HONSHU, JAPAN. MG 3.7 (JMA). Felt (II JMA) ot Miyoko.

5 TURKEY

7 YUgOSLAVIA. ML 2.1 (KBA), 1.9 (LJU). MO 1.7 (TRI).

6 GUERRERO, MEXICO

7 SOUTHERN NORWAY. MO 1.8 (BER).

4 NORTHERN ITALY. MO 1.5 (STR).

5 CENTRAL ALASKA. CAGS-P>.

11 LEEWARD ISLANDS. ML 3.6 (FDF).

6 AEGEAN SEA. ML 2.7 (ATH).

15 CELEBES SEA

69 CENTRAL CALIFORNIA. <BRK>. ML 4.7 (BRK). MO=2.2*10**16 $\mathrm{Nm}$ (BRK). Slight domage (VI) ot Son Jose. Felt (V) at Gilroy, Monte Serono, Sonta Claro, Sonta Cruz and Sunnyvale. Felt (IV) at Alviso, Brisbane, Braokdale. Burlingame, Campbell, Cayote. Felton. Fremant, Livermare, Los Altos, Los Gatas, Millibrae, Madesto. Monterey, Mountain View. New Almoden. Pleasantan. Redwaad Estates, San Anselmo, San Bruno, San Carlos. Son Francisco. Son Martin. San Mateo and Stockton. Felt from Monterey to Sonto Roso.

$\begin{array}{llllllrlrlll}03 & 18 & 01 & 41.6 & 45.983 & \mathrm{~N} & 14.099 & \mathrm{E} & 10 & \mathrm{G} & & \\ 03 & 18 & 06 & 10.6 ? & 14.93 & \mathrm{~S} & 167.99 & \mathrm{E} & 195 & ? & 4.9 & \\ 03 & 19 & 32 & 41.1 & 41.616 & \mathrm{~N} & 143.896 & \mathrm{E} & 43 & & 4.8 & 5.0 \\ 03 & 19 & 39 & 32.2 & 25.170 & \mathrm{~N} & 94.652 & \mathrm{E} & 67 & 0 & 5.2 & \\ 03 & 22 & 20 & 30.6 & 39.731 & \mathrm{~N} & 122.680 & \mathrm{~W} & 5 & \mathrm{G} & & \\ 03 & 23 & 15 & 17.5 \& & 36.770 & \mathrm{~N} & 121.258 & \mathrm{~W} & 7 & & & \end{array}$

1.58 YUGOSLAVIA. ML 1.8 (KBA), 1.6 (LJU).

1.28 VANUATU ISLANDS

1.046 HOKKAIOO, JAPAN REGION. FEII (I JMA) Ot UrokOWO.

1.0235 BURMA-INOIA BORDER REGION

1.077 NORTHERN CALIFORNIA. ML 2.7 (BRK).

15 CENTRAL CALIFORNIA. CBRK>. ML 2.5 (BRK). Felt ot Prunedole.

1.313 GREECE-ALBANIA BOROER REGION. MO 3.1 (ATH)

0.715 HOKKAIDO, JAPAN REGION

0.95 EASTERN IDAHO. ML 3.0 (BUT).

0.36 TURKEY

0.27 SOUTHERN NORWAY. MO $2 . \theta$ (BER)

0.827 SOUTH OF FIJI ISLANOS

0.2

0.0

0.9

1. 1

0.9

0.1

0. 7

1. 1

1. 2

1. 3

0.5

1. 2

1. 8

0. 5

7 SOUTHERN NORWAY. MO 1.8 (BER)

4 SOUTHERN NORWAY. MO 1.9 (BER)

50 SOUTH OF FIJI ISLANOS

4 YUGOSLAVIA

5 RED SEA

5 SOUTHERN NORWAY. MO 1.8 (BER).

6 SOUTHERN NORWAY. MO 1.7 (BER)

12 SOUTH OF FIJI ISLANDS

50 CENTRAL ALASKA. 〈AGS-P>.

14 FRANCE. ML 3.1 (LOG).

8 NEAR COAST OF CENTRAL CHILE

11 AEGEAN SEA. ML 2.8 (ATH).

7 BANDA SEA

6 FRANCE. ML 2.4 (GEN).

78 HAWAll. SHVO-P>. MO 4.4 (HVO). Felt (IV) ot Ninole ond $(111)$ at Poouhau. Also felt at Howai $i$ Volconoes National Park. Howai ion Ocean View Estates, Honakao, Kano, Pahala, Papaikou, Noalehu, Parodise Pork, Royal Gardens and waimeo.

04
0
0
0
0
0
05
0
05
05
05
05
05
05
05
05

$\begin{array}{llllllll}19 & 38 & 22.4 ? & 37.87 & \mathrm{~N} & 28.31 \quad \mathrm{E}\end{array}$ $201052.1 \quad 25.017 \mathrm{~N} \quad 123.359 \mathrm{E} \quad 141$ $\begin{array}{llllllll}21 & 20 & 06.9 ? & 37.19 & \mathrm{~N} & 17.89 & \mathrm{E}\end{array}$ $\begin{array}{llllllll}22 & 46 & 59.2 \% & 58.181 \mathrm{~N} & 151.546 \mathrm{~W}\end{array}$ $000427.7 \quad 3.473 \mathrm{~S} \quad 136.515 \mathrm{E}$ $\begin{array}{lllllll}01 & 30 & 19.9 & 46.887 \mathrm{~N} & 154.046 \mathrm{E}\end{array}$ 022536.8 ? $39.63 \mathrm{~N} 142.58 \mathrm{E}$

$\begin{array}{llllll}0436 & 01.1 & 46.442 \mathrm{~N} & 12.378 \mathrm{E}\end{array}$ $0800 \quad 58.8862 .394 \mathrm{~N} \quad 151.445 \mathrm{~W}$ $0923 \quad 32.37 \quad 39.148 \mathrm{~N} \quad 27.610 \mathrm{E}$ $105752.1 * 3.009 \mathrm{~N} \quad 127.900$ $105835.2 * 35.344 \mathrm{~N} \quad 24.531 \mathrm{E}$ $112748.0 \quad 20.790 \mathrm{~S} \quad 69.923 \mathrm{~W}$ $115403.0 \% 47.517 \mathrm{~N} \quad 6.652 \mathrm{E}$

$\begin{array}{rlll}10 & G & \\ 141 & & 5.0 \\ 10 & G & \\ 50 & & & \\ 81 & 0 & 5.1 & \\ 35 & D & 5.2 & 4.4 \\ 33 & N & & \\ & & \\ 10 & G & \\ 87 & & \\ 10 & G & \\ 33 & N & 4.3 \\ 10 & G & \\ 37 & 0 & 4.8 \\ 10 & G & \end{array}$

1.
1.
0
0
0
0.
1.
0.
0
0
1.
0
1.05 TURKEY

1.0106 NORTHEAST OF IAIWAN

$9.7 \quad 7$ IONIAN SEA

42 KODIAK ISLANO REGION. CAGS-P>. ML 4.0 (PMR)

0.818 WEST IRIAN

0.9102 KURIL ISLANOS REGION

8 NEAR EAST COAST OF HONSHU, JAPAN, MG 3.6 (JMA). Felt (1) JMA) of Miyoko and (I JMA) ot ofunoto.

1.06 NORTHERN ITALY. ML 2.0 (KBA). MD 2.5 (LJU)

26 CENTRAL ALASKA. CAGS-P>

5 TURKEY

7 TALAUD ISLANOS

5 CRETE. MO 3.8 (ATH)

42 NORTHERN CHILE

6 FRANCE. ML 2.5 (LOG). 


\begin{tabular}{|c|c|c|c|c|c|c|c|c|c|c|c|}
\hline 65 & 12 & 61 & 18.68 & 48.807 & $N$ & 122.070 & $w$ & 2 & & & \\
\hline 05 & 12 & 03 & $26.2 \%$ & 60.629 & $\mathbf{N}$ & 6.251 & $E$ & 10 & G & & \\
\hline 05 & 12 & 34 & $46.7 *$ & 18.976 & $N$ & 145.329 & $E$ & 232 & $*$ & 4.4 & \\
\hline 05 & 12 & 47 & 32.5 & 40.852 & $\mathbf{N}$ & 30.471 & $E$ & 10 & G & 3.8 & \\
\hline 85 & 15 & 09 & 06.5 & 34.667 & $\mathbf{N}$ & 24.146 & $E$ & 33 & & 4.8 & 4.2 \\
\hline 65 & 16 & 11 & $27.4 ?$ & 9.78 & $\mathbf{N}$ & 56.54 & $E$ & 10 & G & 4.9 & \\
\hline 05 & 18 & 86 & $04.2 ?$ & 23.02 & $N$ & 142.46 & $E$ & 33 & $\mathbf{N}$ & 4.5 & 3.9 \\
\hline 85 & 18 & 44 & 45.6 . & 18.122 & $S$ & 178.072 & $w$ & 620 & - & 4.9 & \\
\hline 05 & 21 & 43 & $33.3 *$ & 36.710 & $N$ & 71.568 & $E$ & 33 & $\mathbf{N}$ & 3.8 & \\
\hline 85 & 21 & 45 & $46.8 ?$ & 51.05 & $\mathbf{N}$ & 15.78 & $E$ & 10 & G & & \\
\hline 85 & 22 & 84 & $33.9 \%$ & 40.573 & $N$ & 27.988 & $\mathrm{E}$ & 10 & G & & \\
\hline 05 & 22 & 33 & $50.1 *$ & 11.384 & $\mathrm{~s}$ & 122.832 & $E$ & 151 & $*$ & 4.8 & \\
\hline 05 & 22 & 33 & 55.18 & 60.068 & $\mathbf{N}$ & 152.821 & $w$ & 90 & & & \\
\hline 05 & 22 & 36 & $35.9 *$ & 45.875 & $\mathbf{N}$ & 150.571 & $E$ & 33 & $N$ & 4.5 & \\
\hline 85 & 23 & 47 & 49.3 & 20.857 & $\mathrm{~s}$ & 69.028 & $w$ & 112 & $D$ & 5.7 & \\
\hline 86 & $\theta \theta$ & 34 & 12.6 & 40.578 & $\mathbf{N}$ & 19.704 & $\mathrm{E}$ & 5 & $\mathbf{G}$ & & \\
\hline 06 & 02 & 35 & 51.3 & 44.511 & $\mathbf{N}$ & 71.144 & $w$ & 5 & G & & \\
\hline
\end{tabular}

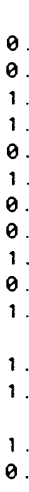

$\begin{array}{lr}0.8 & 19 \\ 0.5 & 15 \\ 1.0 & 25 \\ 1.2 & 15 \\ 0.4 & 1 \\ 1.3 & \\ 0.9 & 26 \\ 0.4 & \\ 1.2 & \\ 0.5 & \\ 1.3 & 1 \\ 1.3 & 3 \\ 1.1 & 19 \\ 1.1 & \\ 0.6 & 16 \\ & \end{array}$

19
7
15
25
155
11
7
26
8
5
8
14
27
32
199
9
9
16

WASHINGTON. ¿SEA>. CL 2.8 (SEA). Fell ol Deming

SOUTHERN NORWAY. MD 1.6 (BER).

MARIANA I SLANDS

TURKEY. FeI $t$ ot AdOPOZOT

CRETE. ML 4.4 (ATH)

CARLSBERG RIDGE

VOLCANO ISLANDS REGION

FIJI ISLANDS REGION

AFGHANISTAN-USSR BOROER REGION

POLAND. ML 3.0 (VKA), 2.7 (KBA).

TURKEY

SOUTH OF TIMOR

SOUTHERN ALASKA. 〈AGS-P>.

KURIL I SLANDS

NORTHERN CHILE. FeIt (IV) in narthern Chile and parts of southern peru.

9 ALBANIA

NORTHERN NEW ENGLAND. MOLg 3.4 (NEIS), 3.5 (WES). Felt

(IV) of Berlin. Gorhom and Lancoster. New Hampshire.

Also felt (IV) at Bethel, Maine; Guildhall and

Lunenburg. Vermant. Felt (III) at Bartlell, Groveton,

Jeffersan, Lisban. Littleton. Milon. Randoph,

Sonbornville and Woadsville. New Hampshire. Also felt

( 111 ) ot Bryont Pand. East Andover. Honover. Locke

Mills. Mexico, Rumford Center, Rumford Point, West

Bethel and West Paris, Maine.

$032439.6 * 11.019 \mathrm{~S}$

$167.047 \mathrm{E}$

$33 \mathrm{~N}$

$05 \quad 4752.6 .25 .120 \mathrm{~N}$

$\begin{array}{llllll}06 & 45 & 43.2 ? & 16.16 \quad \mathrm{~S}\end{array}$

$\begin{array}{llll}06 & 47 & 20.7 & 51.479 \mathrm{~N}\end{array}$

$71.071 \mathrm{~W}$

$95.257 \mathrm{E}$

$173.14 \mathrm{~W}$

$175.730 \mathrm{~W}$

$\begin{array}{lllllll}07 & 32 & 24.9 \& & 60.116 & \mathrm{~N} & 153.289 \mathrm{~W} \\ 08 & 05 & 57.1 & 19.306 & \mathrm{~S} & 169.002 & \mathrm{E}\end{array}$

$116 ?$

$33 \mathrm{~N} \quad 4.7$

$33 \mathrm{~N} \quad 4.7$

147

f 06

169.002 E 166 G 6.1

44.1
1
7
7
1

1.3

0.7

1. 5

1. 240

1. 3282

\section{SANTA CRUZ ISLANOS}

NEAR COAST OF CENTRAL CHILE

10 BURMA-INDIA BORDER REGION

TONGA ISLANDS

andREANOF ISLANDS. ALEUTIAN IS. Felt on adok.

SOUTHERN ALASKA. 〈AGS-P>.

VANUATU ISLANDS. mb 6.3 (BRK). Felt at Chepenehe, $\begin{array}{lllll}08 & 45 & 54.1 & 33.480 \mathrm{~N}\end{array}$ $0902 \quad 15.8 \& 60.288 \mathrm{~N}$ $\begin{array}{lllll}09 & 27 & 09.0 & 44.582 \mathrm{~N}\end{array}$ $103251.3 ? 61.15 \mathrm{~N}$ $104424.5 ? 60.91 \mathrm{~N}$ $\begin{array}{lllll}10 & 46 & 30.0 \% & 41.154 \mathrm{~N}\end{array}$ $112600.2739 .311 \mathrm{~N}$ $\begin{array}{lllll}11 & 41 & 48.9 & 40.015 \mathrm{~N}\end{array}$ $1230 \quad 37.4 \% 60.722 \mathrm{~N}$

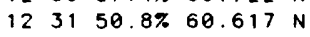
$1305 \quad 15.1 \quad 44.716 \mathrm{~N}$ $131258.7 ? 58.10 \mathrm{~N}$ $\begin{array}{llll}13 & 48 & 44.3 & 43.925 \mathrm{~N}\end{array}$

$160431.3860 .720 \mathrm{~N}$ $\begin{array}{llll}16 & 10 & 04.0 \quad 36.046 \mathrm{~N}\end{array}$

$\begin{array}{llll}16 & 54 & 29.9 & 19.072 \mathrm{~s}\end{array}$ $\begin{array}{lllllll}17 & 33 & 24.3 ? & 29.74 \quad \mathrm{~S}\end{array}$ $\begin{array}{lllll}18 & 34 & 35.7 & 39.268 \mathrm{~N}\end{array}$ $\begin{array}{llll}18 & 35 & 03.8 & 46.235 \mathrm{~N}\end{array}$ $\begin{array}{lllll}18 & 50 & 44.4 & 40.321 \mathrm{~S}\end{array}$ $1855 \quad 16.5 * 22.503 \mathrm{~S}$ $\begin{array}{lllll}18 & 58 & 33.8 ? & 39.08 \quad \mathrm{~N}\end{array}$ $\begin{array}{lllll}18 & 59 & 18.5 ? \quad 4.85 & 5\end{array}$ $191505.2 * 11.020 \mathrm{~S}$ $221021.0 .45 .967 \mathrm{~N}$

$\begin{array}{llll}22 & 16 & 16.8 \% & 40.094 \mathrm{~N}\end{array}$ $\begin{array}{lllll}22 & 19 & 56.7 \% & 40.095 \mathrm{~N}\end{array}$ $223038.1 .33 .640 \mathrm{~N}$ $\begin{array}{llllll}22 & 33 & 41.0 & 19.652 \mathrm{~S}\end{array}$ $\begin{array}{llll}23 & 36 & 49.5 & 34.022 \mathrm{~N}\end{array}$ $\begin{array}{lllll}23 & 49 & 47.6 & 4.255 \mathrm{~S}\end{array}$ $00 \quad 4146.0 .33 .462 \mathrm{~N}$ $032511.8 ? \quad 3.21 \mathrm{~S}$ $032700.5 \cdot 36.106 \mathrm{~N}$ 043234.8 ? $34.41 \mathrm{~N}$ $\begin{array}{llll}04 & 33 & 01.2 & 43.393 \mathrm{~N}\end{array}$ $05 \quad 4322.7 .33 .720 \mathrm{~N}$ $06 \quad 3536.9 ? \quad 24.53 \mathrm{~S}$ $\begin{array}{llll}06 & 36 & 13.5 & 48.090 \mathrm{~N}\end{array}$ $\begin{array}{llllll}06 & 45 & 29.0 ? & 15.07 \quad \mathrm{~S}\end{array}$ $\begin{array}{llll}08 & 26 & 48.9 & 47.716 \mathrm{~N}\end{array}$ $085148.9 .36 .702 \mathrm{~N}$ $092458.0 \% 39.150 \mathrm{~N}$ $\begin{array}{llllll}09 & 55 & 15.27 & 30.76 \quad \mathrm{~N}\end{array}$ $\begin{array}{llllll}10 & 33 & 34.4 & 42.941 \mathrm{~N}\end{array}$ $123141.8 \& 60.130 \mathrm{~N}$ $125441.6 \quad 6.809 \mathrm{~N}$

$13 \quad 32,11.6 \quad 51.341 \mathrm{~N}$ $\begin{array}{llll}13 & 49 & 48.3 & 45.729 \mathrm{~N}\end{array}$ $\begin{array}{llllll}15 & 27 & 57.5 ? & 59.51 \mathrm{~S}\end{array}$ $\begin{array}{lllllll}15 & 28 & 19.0 \% & 60.634 \mathrm{~N}\end{array}$ $\begin{array}{lllll}16 & 04 & 04.7 ? & 2.96 \quad \mathrm{~N}\end{array}$
$132.429 \mathrm{E} \quad 13$

$153.699 \mathrm{~W} 192$

$7.225 \mathrm{E} \quad 10 \mathrm{G}$

2. 28 E $10 \mathrm{G}$

4.86 E $10 \mathrm{G}$

28.892 E $10 \mathrm{G}$

$27.723 \mathrm{E} \quad 10 \mathrm{G}$

$19.750 \mathrm{E} \quad 10 \mathrm{G}$

$5.536 \mathrm{E} \quad 10 \mathrm{G}$

5.492 E $10 \mathrm{G}$

$3.585 W \quad 10 \mathrm{G}$

$6.35 \mathrm{E}$

146.686

$150.771 w 50$

$5 \mathrm{G}$

$67.130 \mathrm{~W}$

$177.86 W$

$23496 \mathrm{E}$

$13.505 \mathrm{E}$

$75.036 \mathrm{~W}$

$179.223 \mathrm{E}$

$29.53 E$

$136.13 \mathrm{E}$

$166.739 \mathrm{E}$

$15.493 \mathrm{E}$

29. $180 \mathrm{E}$

$29.156 \mathrm{E}$

$24.568 \mathrm{E}$

$64.083 \mathrm{~W}$

$24.804 E$

$143.406 \mathrm{E}$

$24.470 \mathrm{E}$

$104.11 \mathrm{E}$

$27.066 \mathrm{E}$

$17.58 E$

$5.429 \mathrm{E}$

$73.078 \mathrm{E}$

$178.46 \mathrm{E}$

174.85

$105.602 \mathrm{~W}$

$71.249 \mathrm{E}$

$27.582 \mathrm{E}$

$51.65 \mathrm{E}$

$18.403 \mathrm{E}$

$153.562 \mathrm{~W}$

73.036 W 151

$29.981 \mathrm{~W}$

$14.337 \mathrm{E}$

$29.70 \mathrm{~W}$

$6.239 \mathrm{E}$

$126.99 \mathrm{E}$
24248

$56 ? 5.0$

$10 \mathrm{G}$

$10 \mathrm{G}$

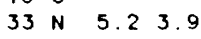

$633 * 4.5$

$10 \mathrm{G}$

$33 \mathrm{~N} 4.4$

$\begin{array}{llll}33 \mathrm{~N} & 4.6 & 4.3\end{array}$

$10 \mathrm{G}$

$10 \mathrm{G}$

$10 \mathrm{G}$

$27 * 3.5$

24.5 .0

$45 \quad 4.5$

149.5 .0

$33 \mathrm{~N} 4.1$

$341 * 4.7$

$10 \mathrm{G}$

$10 \mathrm{G}$

10

$33 N 4.2$

$646 ? 4.8$

$10 \mathrm{G}$

$33 \mathrm{~N}$

5

$203 ? 4.3$

$10 \mathrm{G}$

$33 \mathrm{~N}$

$10 \mathrm{G}$

55

$10 \mathrm{G} \quad 5.34 .8$

$10 \mathrm{G}$

$10 \mathrm{G}$

$10 \mathrm{G}$

89 ? 4.5 Loyalty islands and Noumeo, New Coledonio. Two events about 4 secands apart. Depth from broodbond displocement seismograms, bosed on second, larger event.

0.7

0 .

0.5

0.5

0. 8

1. 2

0. 5

0.3

1.0
0.5
1.0

0.6

1. 1

1.1
1.2
0.8
0.6

0.6

1. 1

1.1
1.1
1.6

1. 2

1. 2

1. 2

1.2
1.1

1. 1

1. 1

1.4
0.9

0.8

1.4

1.0

0.7

1. 3

0.6

1.4

1. 2

0.7

0.9
0.9

๑. 8

0.2

0.6

1.3

1.1

1.0
1.0

1.0

4.3

SHIKOKU JAPAN MG 39 (JMA). FeIt ( I I JMA) ot Uwojima.

52 SOUTHERN ALASKA. LAGS-P>.

24 NORTHERN ITALY. ML 2.9 (LOG), 2.5 (GEN)

NORWEGIAN SEA. MD 2.1 (BER).

SOUTHERN NORWAY. MD 1.7 (BER)

6 TURKEY

TURKEY

2 ALBANIA. MG 2.8 (TIR)

SOUTHERN NORWAY. MO 1.6 (BER)

6 SOUTHERN NORWAY. MO 1.6 (BER).

39 BAY OF BISCAY. ML 4.2 (LDG).

5 SOUTHERN NORWAY. MD 2.2 (BER). Proboble explasion.

83 KURIL ISLANDS. FeIt (I I JMA) ot Nemuro and ( I JMA) ot Kushiro, Hokkaido.

41 KENAI PENINSULA, ALASKA. 〈AGS-P>

17 SOUTHERN NEVADA. ML 2.7 (NEIS). Felt in the Boulder $C i t y$ orea and ot Hoover Dom.

22 SOUTHERN BOLIVIA

20 KERMADEC ISLANDS. FeIt (IV) On ROOUl Is land.

AEGEAN SEA. ML 3.1 (ATH)

AUSTRIA. ML 1.9 (LJU), 1.9 (KBA). MD 2.1 (TRI)

53 OFF COAST OF SOUTHERN CHILE

28 SOUTH OF FIJI ISLANDS

5 TURKEY

6 WEST IRIAN REGION

19 SANTA CRUZ ISLANDS

YUGOSLAVIA. MD 2.8 (LJU). ML 2.2 (KBA). FeIt (V) ot Brestanica. 


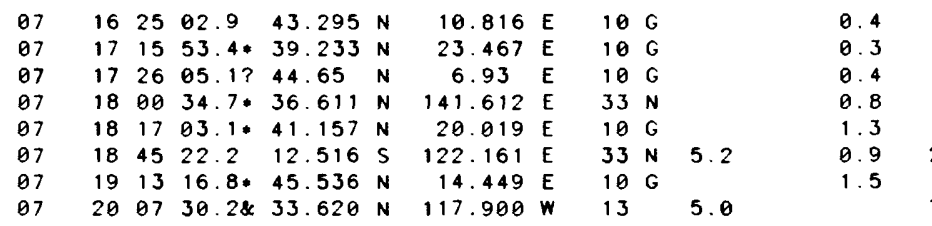

$\begin{array}{lllllll}07 & 20 & 46 & 20.4 * 34.103 & \mathrm{~N} \\ 07 & 20 & 51 & 48.4 & 2.428 & \mathrm{~S} \\ 07 & 20 & 56 & 49.0 ? & 34.11 & \mathrm{~N} \\ 07 & 21 & 06 & 21.5 & 58.685 & \mathrm{~S} \\ 07 & 21 & 39 & 39.1 & 20.434 & \mathrm{~N} \\ 07 & 21 & 40 & 43.9 & 54.085 & \mathrm{~N} \\ 07 & 21 & 45 & 18.6 & 20.576 & \mathrm{~N} \\ 07 & 22 & 12 & 42.1 & 39.284 & \mathrm{~N} \\ 07 & 22 & 35 & 25.7 * & 28.194 & \mathrm{~N} \\ 07 & 22 & 43 & 22.5 * & 19.067 & \mathrm{~S} \\ 07 & 23 & 17 & 59.9 \& & 57.346 & \mathrm{~N} \\ 07 & 23 & 24 & 39.9 & 44.509 & \mathrm{~N} \\ 07 & 23 & 56 & 07.3 * & 36.023 & \mathrm{~N} \\ 08 & 00 & 04 & 44.2 & 12.938 & \mathrm{~N} \\ 08 & 00 & 07 & 00.8 * & 0.374 & \mathrm{~S} \\ 08 & 00 & 09 & 04.5 & 12.976 & \mathrm{~N} \\ 08 & 00 & 24 & 32.5 & 32.012 & \mathrm{~S} \\ 08 & 00 & 57 & 43.6 & 33.999 & \mathrm{~N} \\ 08 & 01 & 15 & 13.6 \% & 36.813 & \mathrm{~N} \\ 08 & 01 & 16 & 08.08 & 57.093 & \mathrm{~N} \\ 08 & 01 & 22 & 23.2 \& & 57.064 & \mathrm{~N} \\ 08 & 01 & 24 & 43.8 * 57.062 & \mathrm{~N}\end{array}$

\begin{abstract}
$135.214 \mathrm{E}$
\end{abstract}
$139.434 \mathrm{E}$

$148.750 \mathrm{E}$

$100.705 \mathrm{E}$

$162.711 \mathrm{~W}$

$100.586 \mathrm{E}$

$23.603 \mathrm{E}$

$96.493 \mathrm{E}$

$167.656 \mathrm{E}$

$143.075 \mathrm{~W}$

$6.867 \mathrm{E}$

25. $922 \mathrm{E}$

44.938 W

$126.240 \mathrm{E}$

$44.780 \mathrm{~W}$

$71.545 W$

24. $582 \mathrm{E}$

$30.139 \mathrm{E}$

$143.388 W$

$143.490 W$

$143.429 w$

$50.071 \mathrm{E}$

$21.191 \mathrm{E}$

$173.001 \mathrm{~W}$

$8.913 w$

153. $231 \mathrm{~W}$

$18.733 \mathrm{E}$

$18.755 \mathrm{E}$

$2.412 w$

$141.717 \mathrm{E}$

$8.91 \mathrm{~W}$

23. $599 \mathrm{E}$

$27.730 \mathrm{E}$

$24.354 \mathrm{~W}$

$27.654 \mathrm{E}$

2. $56 \mathrm{E}$

$96.721 \mathrm{E}$

$164.601 \mathrm{E}$

$123.508 \mathrm{E}$

153.242 E

$75.326 \mathrm{~W}$

$179.31 \mathrm{~W}$

$174.07 \mathrm{E}$

$17.477 \mathrm{E}$

$101.275 w$

$7.245 \mathrm{E}$

$69.452 \mathrm{E}$

$20.781 \mathrm{E}$

$69.436 \mathrm{~W}$

$120.800 \mathrm{~W}$

$90.077 \mathrm{E}$

129.00

$42.211 \mathrm{E}$

$145.085 \mathrm{E}$

$21.655 \mathrm{E}$

$1.197 \mathrm{~W}$

$178.386 w$

$177.999 w$ $117.100 \mathrm{~W}$ $26.35 \mathrm{E}$ $151.827 \mathrm{~W}$ $151.790 \mathrm{~W}$ $172.845 \mathrm{~W}$
$10 \mathrm{G}$

$33 \mathrm{~N} \quad 4.65 .0$

$10 \mathrm{G}$

10 G 5.25 .1

$10 \mathrm{G} 4.2$

$33 \mathrm{~N}$

$10 \mathrm{G} \quad 4.6$

$10 \mathrm{G}$

$33 \mathrm{~N}$

$10 \mathrm{G} \quad 3.9$

$10 \mathrm{G}$

$10 \mathrm{G}$

$33 \mathrm{~N}$

$10 \mathrm{G} \quad 4.7 \quad 4.5$

$76 * 4.9$

10 G 4.44 .8

$72 * 5.5$

$33 \mathrm{~N} 4.0$

$5 \mathrm{G}$

$10 \mathrm{G}$

$10 \mathrm{G}$

$10 \mathrm{G} \quad 5.04 .9$

$10 \mathrm{G}$

$\begin{array}{llll}33 & D & 5.4 & 5.7\end{array}$

128

$40 \cdot 3.7$

23

$10 \mathrm{G}$

$33 \mathrm{~N} 4.5$

$5 \mathrm{G}$

10

$10 \mathrm{G} \quad 4.94 .4$

$10 \mathrm{G}$

$10 \mathrm{G}$

$33 \mathrm{~N} 4.4$

10 G 4.9

$75 * 4.7$

56 D 4.9

87 *

$33 \mathrm{~N} 4.0$

$33 \mathrm{~N}$

4.5

37

$10 \mathrm{G} 4.8$

$10 \mathrm{G}$

$126 ?$

$19 \mathrm{G} \quad 5.2 \quad 4.7$

$209 ? 4.4$

$33 \mathrm{~N} \quad 4.6$

$33 \mathrm{~N} \quad 4.9$

$10 \mathrm{G}$

$10 \mathrm{G}$

$\begin{array}{llll}33 \mathrm{~N} & 5.2 & 4.7\end{array}$

$33 N \quad 4.8$

$33 \mathrm{~N}$

0

$\begin{array}{lllll}0 & & & & \\ 25 & 5.1 & 4.9\end{array}$
$10 \mathrm{G} \quad 4.9 \quad 4.7$

$\begin{array}{rrrr}25 & D & 5.1 & 4.9\end{array}$
0. 9

1. 1

0. 3

1.0

1.4
1.4

1.4
1.2

1. 2

0. 7

1. 1

0. 4

1. 3

1. 1

1. 1

1.8

1.0
1.0

1.4

1.0

1.0

1.4
1.1

1. 3198

12
$1.4 \quad 118$

$1.2 \quad 48$

1. 09

D. 9

0. 9

1. 1

0.7
0.4

(9. 9

D. 4

1. 1

1.2

0. 8

1. 2

0.8

1. 2

0.4

0.4
1.2

1. 5

0. 3

1.0

1.0

1.3

1.0

0.7

1.2

1. 1

1. 5

1.3

อ. 8

1. 3

0.5

4 SOUTHERN CALIFORNIA 〈PAS-P>. ML 3.2 (PAS).

MD 3.1 (ATH)

39 SOUTHERN ALASKA. $\angle A G S-P\rangle$. ML 3.1 (PMR).

36 SOUTHERN ALASKA. 〈AGS-P>.

$\begin{array}{lrl}1.2 & 76 & \text { SAMOA ISLANDS REGION } \\ 0.8 & 140 & \text { FIJI ISLANDS REGION }\end{array}$

(I) JMA) at Wakayama. JMA) at Wakayama.

SOUTHEAST ASIA ISLAND

ALASKA PENINSULA

AEGEAN SEA. MD 3.4 (ATH)

INDIA-CHINA BORDER REGION

GULF OF ALASKA LAGS-P>

FRANCE. ML 1.8 (GEN)

DODECANESE ISLANDS. ML 3.9 (ATH).

MOLUCCA SEA

7 MEDITERRANEAN SEA

TURKEY

GULF OF ALASKA. $\angle A G S-P>$ coda of previaus event.

ATLANTIC-INDIAN RISE

SOUTHERN ALASKA. 〈AGS-P>

i tagrad, and Dubravnik.

PORTUGAL. MG 3.0 (MDD)

5 TURKEY (III) on southeostern Soo Miguel.

5 NORTH SEA. MD 1.8 (BER).

TEMASSA PENINSULA

NORTHERN COLOMEIA

5 VANUATU ISLANDS REGION

8 NORTHERN ITALY. ML 2.1 (GEN).

MID-INDIAN RISE

6 NORTHERN CHILE

CENTRAL CALIFORNIA. $\angle B R K\rangle$. ML 2.7 (BRK).

7 TIMOR SEA

IRAQ

(TIG).

SPAIN. MD 3.1 (MDD)
AEGEAN SEA. ML 2.9 (ATH)

FRANCE. ML 2.0 (GEN)

NEAR EAST COAST OF HONSHU, JAPAN

ALBANIA. ML 2.7 (SKO)

YUGOSLAVIA MD 2.5 (LJU), 2.1 (TRI). (VI) at Corona del Mor. Costo Mesa and (v) artesia. Anaheim, Bueno Grove, Huntington Beach, Irvine. Loguna Beoch, Long Beach, Orange, Santa Ana, Surfside and Tustin. Felt , Avalon, Bower, Ball. Downey, East Angeles. Las Alamitos. Norwalk. Oceanside, Pico Rivera. acentia, Son Clemente. San Luis Rey, South Gate.

Punset Beoch and Yorbo Linda. Felt throughout much of southern California.

NEAR S. COAST OF SOUTHERN HONSHU. MG 2.7 (JMA). FeIt (I

TONGA ISLANDS, MO=1.3*10**18 $\mathrm{Nm}$ (PPT). MS 5.7 (BRK).

PRTGAL. Felt (V) in the Amiaes de Baixo area and (1I)

(

AZORES ISLANDS REgION. Fell (IV) an Santa Maria and

7 YUGOSLAVIA. ML 3.6 (ZAG), 3.4 (VKA), 3.2 (KBA), 2.7 (LJU). MD 3.6 (TRI). Felt (IV) ot Banja Luka. 





$11 \quad 1126 \quad 53.2 * 18.211 \mathrm{~S} \quad 167.316 \mathrm{E}$

$11 \quad 1233127.1040 .474 \mathrm{~N} \quad 23.406 \mathrm{E}$

$\begin{array}{llllll}13 & 06 & 16.1 & 63.651 \mathrm{~N} & 148.378 \mathrm{~W}\end{array}$ $\begin{array}{lllll}13 & 49 & 15.2 & 34.895 & \mathrm{~N}\end{array}$

135008.5 ? $35.86 \mathrm{~N}$

$135322.4 \quad 1.040 \mathrm{~S}$

$\begin{array}{lllll}14 & 02 & 02.4 & 63.425 \mathrm{~N}\end{array}$

$144039.9 * 6.867 \mathrm{~S}$

$\begin{array}{lllll}14 & 45 & 27.0 & 40.294 \mathrm{~N}\end{array}$

$153024.1 * 38.486 \mathrm{~S}$

$\begin{array}{llllll}16 & 18 & 54.3 & 44.152 \mathrm{~N}\end{array}$

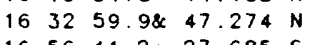

$165641.2 * 27.685 \mathrm{~S}$

$180425.5 * 24.056 \mathrm{~N}$

$190120.2 * 11.736 \mathrm{~N}$

$\begin{array}{lllll}19 & 40 & 59.8 & 35.991 \mathrm{~N}\end{array}$

$2008 \quad 41.6 ? \quad 5.53 \quad \mathrm{~S}$ $\begin{array}{llll}20 & 26 & 21.6 \% & 60.257 \mathrm{~N}\end{array}$ $\begin{array}{lllll}20 & 40 & 34.8 & 44.624 \mathrm{~N}\end{array}$ $21 \quad 26 \quad 12.6 * 41.956 \mathrm{~N}$ $2202 \quad 26.7 * 40.727 \mathrm{~N}$ $225753.2860 .137 \mathrm{~N}$

$230802.8 * 18.261 \mathrm{~N}$

$000119.6 * 31.160 \mathrm{~N}$ $\begin{array}{lllllll}01 & 28 & 14.8 ? & 0.53 \quad \mathrm{~N}\end{array}$ $03 \quad 3732.7 * 39.265 \mathrm{~N}$ $\begin{array}{llll}04 & 26 & 07.4 & 44.245 \mathrm{~N}\end{array}$ $\begin{array}{llll}04 & 46 & 59.9 & 44.299 \mathrm{~N}\end{array}$

$\begin{array}{llll}05 & 05 & 15.9 & 13.308 \mathrm{~N}\end{array}$ $06 \quad 3938.4837 .805 \mathrm{~N}$

$06 \quad 4731.0 * 11.281 \mathrm{~S}$ $\begin{array}{llllll}07 & 26 & 27.9 & 41.440 \mathrm{~N} & 142.227 \mathrm{E}\end{array}$ $08 \quad 4038.7831 .700 \mathrm{~N} \quad 115.760 \mathrm{~W}$ $\begin{array}{lllll}10 & 05 & 43.2 & 38.098 & \mathrm{~N}\end{array}$

$\begin{array}{lllll}10 & 46 & 02.6 & 39.246 \quad \mathrm{~N}\end{array}$

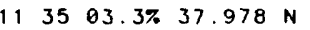
$12 \quad 1245.1848 .419 \mathrm{~N}$ $\begin{array}{lllllll}12 & 45 & 29.8 & 7.56 \quad \mathrm{~S}\end{array}$ $\begin{array}{llll}13 & 53 & 29.7 & 38.090 \mathrm{~N}\end{array}$ $\begin{array}{lllll}15 & 48 & 52.6 \% & 39.841 \mathrm{~N}\end{array}$ $\begin{array}{lllll}16 & 3706.48 & 40.797 \mathrm{~N} & \\ 17 & 02 & 11.7 & 35.285 \mathrm{~N} & \end{array}$ $\begin{array}{lllll}17 & 02 & 11.7 & 35.285 \mathrm{~N}\end{array}$ $17 \quad 1029.5 \& 40.818 \mathrm{~N}$ $172659.7 * 51.103 \mathrm{~N}$ $\begin{array}{lllll}18 & 22 & 05.8 & 40 & 342 \mathrm{~N}\end{array}$ $\begin{array}{llllll}18 & 30 & 18.6 ? & 25.65 \quad \mathrm{~N}\end{array}$ $\begin{array}{lllll}19 & 09 & 37.9 & 43.683 \mathrm{~N}\end{array}$ $\begin{array}{llllll}19 & 24 & 02.7 & 38.104 & N\end{array}$ $20 \quad 1053.6 * 44.600 \mathrm{~N}$ $201706.9838 .800 \mathrm{~N}$ $21 \quad 1149.0 \% 31.655 \mathrm{~N}$ $\begin{array}{lllll}21 & 43 & 50.4 & 39.273 \mathrm{~N}\end{array}$ $22 \quad 2338.4 * 59.958 \mathrm{~S}$ $\begin{array}{lllll}23 & 31 & 59.9 & 39.278 \mathrm{~N}\end{array}$ $\begin{array}{lllll}23 & 49 & 25.67 & 39.576 \mathrm{~N}\end{array}$ $\begin{array}{lllllll}23 & 57 & 05.13 & 35.78 \quad \mathrm{~N}\end{array}$ $0008 \quad 34.6 \quad 38.604 \mathrm{~N}$ $0042 \quad 15.9$ ? $10.69 \mathrm{~N}$ $\begin{array}{lllll}00 & 43 & 11.2 & 39.533 & \mathrm{~S}\end{array}$ $\begin{array}{llllll}00 & 50 & 29.9 \% & 40.106 \quad \mathrm{~N}\end{array}$ $\begin{array}{lllllllll}01 & 15 & 19 & 13 & 4 & 1.70 & N\end{array}$ $013051.5 \quad 16.429 \mathrm{~N}$ $\begin{array}{lllll}02 & 07 & 35.9 & 34.183 & \mathrm{~N}\end{array}$ $02 \quad 1001.0 \quad 38.919 \mathrm{~N}$ $921756.3 * 39.402 \mathrm{~N}$ $02 \quad 3950.3 \quad 45.413 \mathrm{~N}$ $\begin{array}{lllll}02 & 42 & 45.2 \pi & 38.599 \mathrm{~N}\end{array}$ $\begin{array}{lllll}02 & 46 & 23.3 \% & 38.603 \mathrm{~N}\end{array}$ $\begin{array}{lllllll}03 & 26 & 27.83 & 38.07 & \end{array}$ $\begin{array}{llll}03 & 33 & 28.3 & 38.640 \mathrm{~N}\end{array}$ $03 \quad 4943.7 * 27.287 \mathrm{~S}$ $040430.1 * 53.482 \mathrm{~S}$ $0502 \quad 35.6 \% 37.420 \mathrm{~N}$

$\begin{array}{llllll}05 & 26 & 27.13 & 17.82 \quad \mathrm{~N}\end{array}$ $\begin{array}{llllll}05 & 45 & 23.9 ? & 17.82 & \mathrm{~N}\end{array}$ $06 \quad 3351.0738 .610 \mathrm{~N}$ 071640.4 ? $35.70 \mathrm{~N}$

$07 \quad 25 \quad 36.7 \quad 24.472 \mathrm{~N}$

$9.142 \mathrm{E}$ $81.23 \mathrm{E}$

$126.558 \mathrm{E}$ $47.630 \mathrm{~W}$ $147.742 \mathrm{E}$ $29.459 \mathrm{E}$

$4.718 \mathrm{~W}$ 8. $197 \mathrm{E}$ $122.907 \mathrm{~W}$ $26.363 \mathrm{E}$ $20.846 \mathrm{E}$ $59.843 \mathrm{~W}$ $139.951 \mathrm{E}$

$29.57 \mathrm{E}$ $5.017 \mathrm{E}$ $7.043 \mathrm{E}$

$19.212 \mathrm{E}$ $29.739 \mathrm{E}$

$50.539 \mathrm{E}$ $132.57 \mathrm{E}$

$23.560 \mathrm{E}$

$6.753 \mathrm{E}$

$10.798 \mathrm{E}$

$39.984 \mathrm{E}$

$22.029 \mathrm{E}$

$23.560 \mathrm{E}$

$21.847 \mathrm{E}$

$22.230 \mathrm{~W}$

$131.07 \mathrm{E}$

$22.080 \mathrm{E}$

$27.629 \mathrm{E}$

$124.668 \mathrm{~W}$

$135.445 \mathrm{E}$

$124.682 \mathrm{~W}$

$21.842 \mathrm{E}$

$144.07 \mathrm{E}$

8. $414 \mathrm{E}$

$21.972 \mathrm{E}$

$6.620 \mathrm{E}$

$122.800 \mathrm{~W}$

$35.671 \mathrm{E}$

23. $592 \mathrm{E}$

53.619 W

$23.498 \mathrm{E}$

$28.856 \mathrm{E}$

$140.65 \mathrm{E}$

$27.291 \mathrm{E}$

86.46 w

$75.002 \mathrm{~W}$

$29.325 \mathrm{E}$

$12.69 \mathrm{E}$

$119.916 \mathrm{E}$

$96.713 \mathrm{E}$

$24.961 \mathrm{E}$

$23.537 \mathrm{E}$

$26.138 \mathrm{E}$

$27.145 \mathrm{E}$

$27.153 \mathrm{E}$

73.56 W

27. $241 \mathrm{E}$

$177.143 \mathrm{~W}$

$140.176 \mathrm{E}$

$121.813 \mathrm{~W}$

$65.63 \mathrm{w}$

$65.66 \mathrm{~W}$

$27.192 \mathrm{E}$ 153.036 W 120

$65.339 \mathrm{~W} 10 \mathrm{G}$

$\begin{array}{rlll}33 & N & 4.9 & 4.4 \\ 10 & G & & \\ 6 & G & & \\ 33 & N & & \\ 38 & * & 4.8 & \\ 33 & N & 4.1 & \\ 33 & N & 5.0 & 4.6 \\ 33 & N & & \\ 31 & & & \\ 10 & G & & \\ 10 & G & 4.8 & \\ 10 & G & & \\ 47 & & & \\ 5 & G & & \\ 33 & N & & \\ 10 & G & & \\ 64 & & 4.7 & \end{array}$
$121.732 \mathrm{~W}$

\begin{tabular}{|c|c|c|}
\hline $33 \mathrm{~N}$ & & 1.3 \\
\hline $33 \mathrm{~N}$ & 4.93 .9 & 1.6 \\
\hline 12 & & 1.4 \\
\hline $10 \mathrm{G}$ & & 0.4 \\
\hline $10 \mathrm{G}$ & & 1.1 \\
\hline
\end{tabular}

$10 \mathrm{G}$

$10 \mathrm{G}$

$10 \mathrm{G}$

$10 \mathrm{G}$

$10 \mathrm{C}$

$141.39 \mathrm{E}$

$33 \mathrm{~N} \quad 4.9$

12

0.8

$\begin{array}{rrrrrr}42 & ? & 4.8 & & 1.2 & 14 \\ 72 & & 4.1 & & 1.1 & 20 \\ 6 & G & & & & 24 \\ 10 & G & 4.4 & 3.7 & 1.2 & 90\end{array}$

$10 \mathrm{G} \quad 4.4 \quad 3.7$

$10 G$

$10 \mathrm{G}$

$33 \times 4.3$

$14 \quad 4.2$

$10 \mathrm{G}$

$\begin{array}{ll}15 \\ 371 & 4 \\ 14 & 2\end{array}$

$\begin{array}{rr}371 & 4.2\end{array}$

$10 \mathrm{G}$

$10 \mathrm{G}$

$33 \mathrm{~N} \quad 4.5$

$10 \mathrm{G}$

$28 \quad 4.2$

$10 \mathrm{G}$

10
10

$11 \quad 4.0$

$10 G \quad 5.0$

$10 \mathrm{G}$

$10 \mathrm{G}$

$70 \cdot 4.2$

14

$33 \mathrm{~N}$

$33 \mathrm{~N} \quad 5.8 \quad 5.5$

$10 \mathrm{G}$

1100.9

$\begin{array}{llll}54 & 4.9 & 4.0 & 1.0\end{array}$

$33 \mathrm{~N} \quad 4.4 \quad 1.3$

$10 \mathrm{G}$

$10 \mathrm{G}$

$10 \mathrm{G}$

$10 \mathrm{G}$

$10 \mathrm{C}$

$33 \mathrm{~N}$

$10 \mathrm{G}$

33 N 4.8

10 G 5.1

5

$10 G$

$10 \mathrm{G}$

$10 \mathrm{G}$

31

92.505 E $33 \mathrm{~N} \quad 5.15 .0$

$1.5 \quad 12$

1.

1. 2

1.0225

0.610

220

7

$\cdot 4$

1. 0

1.3

0.3

1.3

42

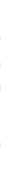

0. 6

8
1. 329

1.58

27 ETHIOPIA. ML 5.O (ARO)

CENTRAL CALIFORNIA. CBRK>. ML 3.6 (BRK). MO=5.1*10*14 $\mathrm{Nm}$ (BRK). Felt (IV) ot Dublin and Pleosonton; (111) ot Son Lorenzo. Also felt ot Livermore.

SANTA CRUZ ISLANDS

HOKKAIDO, JAPAN REgION. FeIt (II JMA) ot Hochinohe.

BAJA CALIFORNIA. SPAS-P>. ML 3.4 (PAS).

GREECE. ML 4.3 (ATH). Minor domage ot Leondion. Felt throughaut Akhoia.

1.114 AEGEAN SEA. ML 3.5 (ATH).

0.77 SOUTHERN GREECE. MG 3.6 (TRN)

9 WASHINGTON. 〈SEA>, CL 1.5 (SEA). Felt at Mount vernan.

TANIMBAR ISLANDS REGION

1.261 GREECE. ML 4.0 (ATH).

1.27 TURKEY

7 NEAR COAST OF NORTHERN CALIF. ¿BRK>. ML 3.4 (BRK).

48 SOUTHERN HONSHU, JAPAN

7 NEAR COAST OF NORTHERN CALIF. CBRK>. ML 3.3 (BRK)

5 POLAND

0 GREECE. MG 3.2 (TIR)

9 VOLCANO ISLANDS REGION

23 CORSICA. ML 2.7 (GEN), 2.7 (LDG).

69 GREECE. ML 3.9 (ATH)

FRANCE. ML 2.5 (GEN)

8 NORTHERN CALIFORNIA. LBRK>. ML 2.6 (BRK)

6 DEAD SEA REGION

59 AEGEAN SEA. ML 3.6 (ATH)

29 SCOTIA SEA

19 AEGEAN SEA. ML 3.1 (ATH).

5 TURKEY

5 NEAR EAST COAST OF HONSHU, JAPAN

1 TURKEY

3 OFF COAST OF COSTA RICA. MD 4.2 (HDC).

OFF COAST OF CENTRAL CHILE

TURKEY

SOUTHERN ITALY

LUZON, PHILIPPINE ISLANDS

OINGHAI PROVINCE, CHINA

AEGEAN SEA. ML 3.0 (ATH).

AEGEAN SEA. ML 3.2 (ATH)

ROMANIA

TURKEY

TURKEY

NEAR COAST OF CENTRAL CHILE

TURKEY

KERMADEC ISLANDS REGION

30 WEST OF MACOUARIE ISLAND

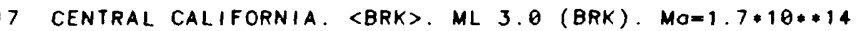
$\mathrm{Nm}$ (BRK). Felt ot San Jose.

0.36 PUERTO RICO REGION. Felt at Caguas.

0.2 5 PUERTO RICO REGION

1.15 TURKEY

NEAR EAST COAST OF HONSHU. JAPAN. MG 3.6 (JMA). Felt (I JMA) ot Mito.

1.2140 INDIA-BANGLADESH BORDER REGION. Felt in the

Dhaka-Chittagong area, Bangladesh. Also felt in the

Agartala-Silchor areo. India. 


\begin{tabular}{|c|c|c|c|c|c|c|c|c|c|}
\hline 13 & 07 & 45 & $13.9 \%$ & 40.829 & $\mathbf{N}$ & 27.913 & E & 10 & G \\
\hline 3 & 07 & 49 & 203 & 41.764 & $\mathbf{N}$ & 45.855 & E & 33 & $N$ \\
\hline 3 & 07 & 55 & 11.3 & 13.263 & $\mathbf{N}$ & 39.925 & E & 33 & $\mathbf{N}$ \\
\hline 13 & 08 & 04 & $41.2 *$ & 38.579 & $\mathbf{N}$ & 27.191 & $\mathbf{E}$ & 10 & G \\
\hline 13 & 08 & 22 & $49.6 \%$ & 38.615 & $\mathbf{N}$ & 27.180 & E & 10 & G \\
\hline 13 & 08 & 44 & $56.0 *$ & 38.636 & $\mathbf{N}$ & 27.285 & $E$ & 10 & G \\
\hline 13 & 09 & 03 & 39.5 & 27.972 & $\mathbf{S}$ & 66.491 & $w$ & 173 & \\
\hline 13 & 09 & 52 & $03.0 \%$ & 60.693 & $\mathbf{N}$ & 6.009 & E & 10 & G \\
\hline 13 & 10 & 47 & $44.9 *$ & 27.353 & $\mathbf{N}$ & 142.566 & $E$ & 33 & $\mathbf{N}$ \\
\hline 13 & 12 & 00 & $57.0 *$ & 22.045 & $\mathrm{~S}$ & 68.314 & $w$ & 116 & 0 \\
\hline 13 & 12 & 17 & 31.3 & 13.290 & $\mathbf{N}$ & 39.980 & $E$ & 33 & $N$ \\
\hline 13 & 12 & 18 & 23.8 & 38.652 & $\mathbf{N}$ & 27.192 & $E$ & 10 & G \\
\hline 13 & 12 & 35 & $043 *$ & 13.336 & $\mathbf{N}$ & 39.963 & $\mathbf{E}$ & 33 & $\mathbf{N}$ \\
\hline 13 & 12 & 55 & 2018 & 61.710 & $\mathbf{N}$ & 149.657 & $w$ & 30 & \\
\hline 13 & 13 & 00 & 48.7 & 13.351 & $\mathbf{N}$ & 40.011 & $E$ & 33 & $\mathbf{N}$ \\
\hline 13 & 13 & 04 & 04.1 & 38.626 & $\mathbf{N}$ & 27.275 & $\mathbf{E}$ & 10 & G \\
\hline 13 & 13 & 55 & $49.5 *$ & 5.911 & $s$ & 130.544 & $E$ & 33 & $\mathbf{N}$ \\
\hline 13 & 14 & 14 & $31.7 \%$ & 11.375 & $\mathbf{N}$ & 61.799 & $w$ & 10 & G \\
\hline 13 & 14 & 34 & 37.5 & 45.767 & $\mathbf{N}$ & 26.691 & $\mathbf{E}$ & 120 & \\
\hline 13 & 14 & 38 & $28.6 ?$ & 4. 11 & $N$ & 32.51 & $w$ & 10 & G \\
\hline 13 & 15 & 52 & 46.9 * & 5.727 & $S$ & 131.027 & $E$ & 111 & $*$ \\
\hline 13 & 16 & 08 & 40.2 & 38.623 & $\mathbf{N}$ & 27.188 & $\mathbf{E}$ & 10 & G \\
\hline 13 & 16 & 17 & $29.1 \%$ & 38.665 & $N$ & 27.234 & $\mathbf{E}$ & 10 & G \\
\hline 13 & 16 & 19 & $49.7 *$ & 33.563 & $\mathbf{N}$ & 24.709 & $\mathbf{E}$ & 10 & G \\
\hline 13 & 16 & 58 & $50.8 *$ & 2.103 & $\mathbf{N}$ & 126.162 & $E$ & 33 & $\mathbf{N}$ \\
\hline 13 & 17 & 10 & 15.97 & 38.559 & $\mathbf{N}$ & 26.960 & $\mathbf{E}$ & 10 & G \\
\hline 13 & 18 & 49 & $24.6 ?$ & 55.88 & $N$ & 160.90 & $w$ & 168 & $*$ \\
\hline 13 & 19 & 36 & $11.5 *$ & 51.204 & $\mathbf{N}$ & 15.552 & $\mathbf{E}$ & 10 & G \\
\hline 13 & 20 & 04 & 19.6 & 36.514 & $\mathbf{N}$ & 24.025 & $\mathbf{E}$ & 66 & $*$ \\
\hline 13 & 21 & 34 & $43.5 *$ & 50.730 & $\mathbf{N}$ & 14.244 & $\mathbf{E}$ & 10 & G \\
\hline 13 & 21 & 39 & 29.0 & 39.549 & $\mathbf{N}$ & 29.157 & $\mathbf{E}$ & 10 & G \\
\hline 13 & 22 & 39 & 10.3 & 40.384 & $\mathbf{N}$ & 25.953 & $\mathbf{E}$ & 10 & G \\
\hline 13 & 23 & 45 & $12.9 ?$ & 70.11 & $N$ & 8.34 & $\mathbf{E}$ & 0 & G \\
\hline 14 & 01 & 15 & 44.5 & 14.879 & $S$ & 167.211 & $\mathbf{E}$ & 158 & $*$ \\
\hline 14 & 01 & 57 & $08.6 ?$ & 42.14 & $\mathbf{N}$ & 145.97 & E & 33 & $N$ \\
\hline 14 & 02 & 34 & $00.0 *$ & 9.342 & $S$ & 118.658 & $\mathbf{E}$ & 33 & $N$ \\
\hline 14 & 04 & 17 & $48.0 ?$ & 39.05 & $\mathbf{N}$ & 23.67 & $\mathbf{E}$ & 10 & G \\
\hline 14 & 05 & 55 & 14.18 & 59.928 & $\mathbf{N}$ & 153.222 & $w$ & 126 & \\
\hline 14 & 05 & 57 & 06.5 & 37.586 & $\mathbf{N}$ & 20.873 & $\mathbf{E}$ & 54 & $*$ \\
\hline 14 & 96 & 39 & $13.8 ?$ & 49.37 & $\mathbf{N}$ & 155.97 & $\mathbf{E}$ & 33 & $\mathbf{N}$ \\
\hline 14 & 06 & 45 & 55.08 & 36.563 & $\mathbf{N}$ & 121.202 & $w$ & 8 & \\
\hline 14 & 07 & 00 & 30.98 & 58.422 & $\mathbf{N}$ & 142.716 & $w$ & 10 & G \\
\hline 14 & 07 & 17 & $43.6 *$ & 11.008 & $\mathrm{~S}$ & 164.165 & $\mathbf{E}$ & 50 & $?$ \\
\hline 14 & 07 & 56 & 45. 1* & 6.954 & $N$ & 72.998 & $w$ & 150 & * \\
\hline 14 & 08 & 56 & 06.38 & 59.809 & $\mathbf{N}$ & 153.404 & $w$ & 120 & \\
\hline 14 & 10 & 03 & 27.7 & 19.116 & $\mathbf{N}$ & 69.598 & $w$ & 94 & \\
\hline 14 & 10 & 28 & $59.8 *$ & 37.661 & $\mathbf{N}$ & 24.912 & $E$ & 10 & G \\
\hline 14 & 10 & 55 & 27.3 & 15.031 & $\mathbf{N}$ & 59.602 & $w$ & 22 & \\
\hline 14 & 11 & 07 & $41.9 *$ & 38.629 & $\mathbf{N}$ & 27.168 & $E$ & 10 & G \\
\hline 14 & 11 & 11 & 48.38 & 47.655 & $\mathbf{N}$ & 122.200 & $w$ & $\theta$ & \\
\hline 14 & 11 & 17 & $44.7 *$ & 37.879 & $\mathbf{N}$ & 72.415 & E & 33 & $\mathbf{N}$ \\
\hline 14 & 11 & 34 & 16.1 & 40.253 & $\mathbf{N}$ & 19.872 & $E$ & 10 & G \\
\hline 14 & 11 & 44 & 39.6 . & 53.122 & $\mathbf{N}$ & 171.401 & $E$ & 33 & $N$ \\
\hline 14 & 11 & 54 & $49.0 ?$ & 44.60 & $N$ & 27.48 & $\mathbf{E}$ & 10 & G \\
\hline 14 & 12 & 33 & $43.8 *$ & 40.663 & $\mathbf{N}$ & 29.990 & $\mathbf{E}$ & 10 & $G$ \\
\hline 14 & 12 & 52 & 26.7 & 19.201 & $\mathbf{N}$ & 145.522 & $\mathbf{E}$ & 168 & $*$ \\
\hline 14 & 13 & 02 & 50.2 * & 38.182 & $N$ & 20.705 & $\mathbf{E}$ & 10 & G \\
\hline 14 & 13 & 82 & 52.6 & 18.172 & $S$ & 178.393 & $w$ & 636 & \\
\hline 14 & 13 & 03 & 44. 3? & 25.27 & $\mathrm{~S}$ & 174.66 & $w$ & 33 & $\mathbf{N}$ \\
\hline 14 & 13 & 04 & 42.2 & 18.180 & $S$ & 178.475 & $w$ & 635 & $*$ \\
\hline 14 & 14 & 53 & 57.7 & 42.303 & $N$ & 19.851 & $\mathbf{E}$ & 10 & G \\
\hline 14 & 16 & 19 & 01.9 & 17.794 & $N$ & 65.616 & $w$ & 10 & G \\
\hline 14 & 16 & 31 & $35.6 *$ & 14.085 & $\mathrm{~S}$ & 34.143 & $\mathbf{E}$ & 10 & G \\
\hline 14 & 17 & 05 & $42.9 \%$ & 16.863 & $N$ & 61.783 & $w$ & 10 & G \\
\hline 14 & 17 & 50 & 51.0 & 49.100 & $N$ & 6.889 & $\mathbf{E}$ & 10 & G \\
\hline 14 & 18 & 19 & $14.8 ?$ & 19.53 & $\mathrm{~S}$ & 177.58 & $w$ & 437 & $\bullet$ \\
\hline 14 & 19 & 45 & 08.0 & 67.561 & $N$ & 142.655 & $w$ & 33 & $\mathbf{N}$ \\
\hline 14 & 20 & 08 & 00.3 & 44.381 & $\mathbf{N}$ & 7.171 & $\mathbf{E}$ & 10 & G \\
\hline 14 & 20 & 23 & 57.2 & 42.352 & $N$ & 19.913 & $\mathbf{E}$ & 14 & $\bullet$ \\
\hline 14 & 20 & 36 & $12.0 ?$ & 17.81 & $\mathbf{N}$ & 65.65 & $w$ & 10 & G \\
\hline 14 & 22 & 51 & 52.4 & 23.322 & $N$ & 99.308 & $\mathbf{E}$ & 33 & $N$ \\
\hline 14 & 22 & 57 & 59.6 & 41.132 & $N$ & 74.525 & $\mathbf{E}$ & 33 & $\mathbf{N}$ \\
\hline 14 & 23 & 24 & 19.3 & 17.845 & $N$ & 65.624 & $w$ & 22 & \\
\hline 14 & 23 & 30 & 39.8 & 32.632 & $N$ & 47.743 & $\mathbf{E}$ & 33 & $\mathbf{N}$ \\
\hline 14 & 23 & 353 & 30.1 & 33.642 & $S$ & 71.633 & $w$ & 15 & $\bullet$ \\
\hline 15 & 00 & 272 & $28.2 \%$ & 44.002 & $N$ & 7.436 & $\mathbf{E}$ & 10 & G \\
\hline 15 & 02 & 24 & 00.6 & 7.193 & $S$ & 126.935 & E & 325 & $*$ \\
\hline 15 & 03 & 02 & $17.6 \%$ & 40.803 & $N$ & 27.955 & $E$ & 10 & G \\
\hline 15 & 05 & 42 & 13.4 & 44.227 & $N$ & 6.329 & $\mathbf{E}$ & 2 & \\
\hline 15 & 06 & 35 & $03.2 ?$ & 50.34 & $\mathbf{N}$ & 18.37 & E & 10 & G \\
\hline 15 & 06 & 40 & 24.9 & 87.118 & $N$ & 47.405 & E & 10 & G \\
\hline 15 & 07 & 23 & 19.6 . & 38.060 & $N$ & 21.915 & $E$ & 10 & G \\
\hline 15 & 07 & 511 & $10.1 ?$ & 17.85 & $N$ & 65.72 & $w$ & 10 & G \\
\hline 15 & 07 & 56 & 53.3 & 42.284 & $\mathbf{N}$ & 19.877 & E & 10 & G \\
\hline 15 & 08 & 048 & $83.4 *$ & 3.779 & $N$ & 82.721 & $w$ & 33 & $\mathbf{N}$ \\
\hline 15 & 08 & 06 & $13.3 *$ & 35.443 & $N$ & 141.112 & $E$ & 33 & $\mathbf{N}$ \\
\hline 15 & 88 & 582 & $24.5 \%$ & 39.489 & $N$ & 29.067 & E & 10 & G \\
\hline 15 & 10 & 150 & 08.0 & 46.042 & $N$ & 14.142 & $E$ & 10 & G \\
\hline & $\begin{array}{l}12 \\
12\end{array}$ & $\begin{array}{l}10 \\
30\end{array}$ & $\begin{array}{l}45.2 * \\
59.5 *\end{array}$ & $\begin{array}{r}41.845 \\
3.128\end{array}$ & $\begin{array}{l}N \\
S\end{array}$ & $\begin{array}{r}22.759 \\
47335\end{array}$ & $E$ & $\begin{array}{l}10 \\
33\end{array}$ & G \\
\hline
\end{tabular}

6 TURKEY

91 EASTERN CAUCASUS. Felt (V) at Tbilisi and Stepanavan: (IV) ot Ki rovakan. Tumanyon and Krasnaselsk; (III) a Bakuriani and Makhachkalo.

2 ETHIOPIA. ML 5.2 (ARO).

1 TURKEY

5 TURKEY

8 TURKEY

35 CATAMARCA PROVINCE, ARGENTINA

5 SOUTHERN NORWAY. MD 1.9 (BER).

24 BONIN ISLANDS REGION. FeIt (I JMA) an Chichi-shima.

18 NORTHERN CHILE

91 ETHIOPIA

14 TURKEY Felt ot I $2 \mathrm{mir}$

9 ETHIOPIA. ML 4.3 (ARO).

36 SOUTHERN ALASKA LAGS-P>

43 ETHIOPIA. ML 4.8 (ARO).

14 TURKEY

8 BANDA SEA

7 WINDWARD ISLANDS. MG 3.6 (TRN)

47 ROMANIA

7 CENTRAL MID-ATLANTIC RIDGE

15 BANDA SEA

26 TURKEY

8 TURKEY

5 MEDITERRANEAN SEA. MO 3.8 (ATH).

17 MOLUCCA PASSAGE

7 AEGEAN SEA

11 ALASKA PENINSULA

10 POLAND. ML 3.7 (VKA), 3.4 (KBA)

21 SOUTHERN GREECE. MD 3.7 (ATH).

5 CZECHOSLOVAKIA. ML 3.4 (VKA).

18 TURKEY

6 AEGEAN SEA. MD 2.9 (ATH).

5 NORWEGIAN SEA. MD 3.1 (BER)

91 VANUATU ISLANDS

13 HOKKAIDO, JAPAN REGION

5 SUMBAWA ISLAND REGION

AEGEAN SEA

20 SOUTHERN ALASKA. LAGS-P>.

33 IONIAN SEA. MD 3.6 (ATH).

10 KURIL ISLANDS

21 CENTRAL CALIFORNIA. $\angle B R K\rangle$, ML 3.3 (BRK).

23 GULF OF ALASKA. LAGS-P>.

14 SANTA CRUZ ISLANDS REGION

16 NORTHERN COLOMBIA

II SOUTHERN ALASKA. $\angle A G S-P>$

82 DOMINICAN REPUBLIC REGION

5 SOUTHERN GREECE. ML 2.7 (ATH)

22 LEEWARD ISLANOS. ML 3.9 (FDF).

6 TURKEY

15 WASHINGTON. LSEA>. CL 1.5 (SEA). Felt in the Juonito Creek orea

11 TAJIK SSR

12 ALBANIA MD 3.2 (ATH).

23 NEAR ISLANDS, ALEUTIAN ISLANDS

8 ROMANIA

9 TURKEY

46 MARIANA ISLANDS

6 GREECE. MD 3.4 (ATH)

23 FIJI ISLANDS REGION

6 SOUTH OF TONGA ISLANDS

92 FIJI ISLANDS REGION

62 YUGOSLAVIA. MD 3.8 (ATH), 3.5 (TTG).

12 PUERTO RICO REGION

5 MALAWI. MG 3.7 (LSZ)

5 LEEWARD ISLANDS, ML 3.1 (FDF).

GERMANY. MD 2.6 (STR).

4 FIJI ISLANDS REGION

ALASKA

13 NORTHERN ITALY. ML 3.6 (LDG)

13 YUGOSLAVIA. MD 2.2 (TTG).

5 PUERTO RICO REGION. FeIt in eostern Puerto Rico.

34 BURMA-CHINA BORDER REGION. ML 4.7 (BJI).

12 KIRGHIZ SSR

13 PUERTO RICO REgION. Felt in eostern Puerto Rico.

83 IRAN-IRAQ BORDER REGION

11 NEAR COAST OF CENTRAL CHILE

5 NORTHERN ITALY. MD 1.0 (STR).

21 BANDA SEA

7 TURKEY

14 FRANCE. ML 2.5 (LDG)

5 POLAND

36 NORTH OF FRANZ JOSEF LAND

6 GREECE. ML 3.0 (ATH)

PUERTO RICO REGION

46 YUGOSLAVIA. MD 3.4 (ATH), 3.2 (TTG).

15 SOUTH OF PANAMA

10 NEAR EAST COAST OF HONSHU, JAPAN

TURKEY

YUGOSLAVIA. ML 1.9 (KBA)

6 YUGOSLAVIA. ML 2.7 (SKO).
19 BISMARCK SEA 
$\begin{array}{llllllllll}15 & 14 & 22 & 34.2 & 41.837 \mathrm{~N} & 23.692 \mathrm{E}\end{array}$ $\begin{array}{llllll}0 & 15 & 142641.2 & 8.453 \mathrm{~N} & 61.039 \mathrm{~W}\end{array}$ $\begin{array}{llllllll}15 & 04 & 08.7 ? & 13.59 & \mathrm{~N} & 120.38 \mathrm{E}\end{array}$ $\begin{array}{lllllll}16 & 03 & 04.5 & 40.542 \mathrm{~N} & 139.396 \mathrm{E}\end{array}$ $\begin{array}{llllll}16 & 3951.1 & 36.557 \mathrm{~N} & 89.711 \mathrm{~W}\end{array}$ $\begin{array}{llllll}17 & 22 & 02.4 & 39.853 \mathrm{~N} & 19.602 \mathrm{E}\end{array}$ $\begin{array}{llllll}1740 & 01.9 ? & 5.72 & \mathrm{~S} & 130.14 \mathrm{E}\end{array}$ $\begin{array}{llllllll}18 & 15 & 56.3 ? & 38.28 \mathrm{~N} & 20.23 \mathrm{E}\end{array}$ $\begin{array}{lllrllll}18 & 43 & 17.4 & 5.341 \mathrm{~S} & 145.516 \mathrm{E}\end{array}$ f $15203408.929 .987 \mathrm{~N} 99.195 \mathrm{E}$ $\begin{array}{llllllll}15 & 21 & 05 & 27.6 ? & 29.92 \mathrm{~N}\end{array}$

$\begin{array}{lllllll}15 & 21 & 16 & 43.6 ? & 30.52 \mathrm{~N}\end{array}$

$\begin{array}{llllll}15 & 22 & 03 & 27.5 & 42.035 & \mathrm{~N}\end{array}$
$99.38 \quad E$

$98.45 \mathrm{E}$

$142.612 \mathrm{E}$
$33 \mathrm{~N} \quad 4.6$

$33 \mathrm{~N} 4.1$

$72 \quad 4.8$

11804.5

$\begin{array}{lllll}22 & 33 & 32.5 & 24.828 \mathrm{~N}\end{array}$ $\begin{array}{lllll}23 & 48 & 36.8 & 13.723 & \mathrm{~S}\end{array}$ $00 \quad 20 \quad 37.0 \% 39.374 \mathrm{~N}$ $00 \quad 26 \quad 44.6 ? \quad 10.86 \quad S$ $00 \quad 58 \quad 07.3 \% \quad 39.405 \mathrm{~N}$ 01 1006.1 ? $51.99 \mathrm{~N}$ $0348 \quad 39.5 ? \quad 17.99 \mathrm{~N}$ $043758.2 \% 40.841 \mathrm{~N}$ $\begin{array}{llll}04 & 46 & 29.6 & 47.572 \mathrm{~N}\end{array}$ $\begin{array}{lllll}05 & 23 & 04.2 \% & 37.903 & N\end{array}$ $\begin{array}{lllll}05 & 24 & 23.3 & 26.533 \mathrm{~N}\end{array}$

$062054.9 * 13.855 \mathrm{~N}$ $065024.4 \quad 1.012 \mathrm{~N}$ 07 $1539.7 * 24.324 \mathrm{~S}$ 07 $28 \quad 14.6 * 39.513 \mathrm{~N}$ $080429.5 * 4.612 \mathrm{~N}$ $\begin{array}{llllll}08 & 07 & 11.4 ? & 38.71 \mathrm{~N}\end{array}$ $083256.0 \quad 38.640 \mathrm{~N}$ $\begin{array}{lllll}08 & 35 & 03.2 & 4.462 \mathrm{~N}\end{array}$ $\begin{array}{llll}08 & 43 & 04.1 & 22.000 \mathrm{~S}\end{array}$ $\begin{array}{llllll}08 & 51 & 11.7 & 39.199 \mathrm{~N}\end{array}$ $09 \quad 1926.1 * 23.856 \mathrm{~S}$ $\begin{array}{lllll}09 & 29 & 16.7 ? \quad 4.94 \mathrm{~N}\end{array}$ $\begin{array}{llll}09 & 46 & 01.8 & 39.105 \mathrm{~N}\end{array}$ $\begin{array}{lllll}10 & 33 & 05.5 & 34.229 \mathrm{~N}\end{array}$ $\begin{array}{llll}10 & 51 & 16.5 & 4.630 \mathrm{~N}\end{array}$ $110529.3 * 31.582 \mathrm{~s}$ $\begin{array}{llll}11 & 49 & 38.6 & 25.137 \mathrm{~N}\end{array}$ $\begin{array}{llllll}12 & 28 & 41.9 ? & 46.33 \quad \mathrm{~N}\end{array}$ $\begin{array}{llllll}13 & 13 & 49.6 ? & 5.93 \quad 5\end{array}$ $\begin{array}{llllll}13 & 15 & 32.8 \% & 42.585\end{array}$ $\begin{array}{llll}13 & 22 & 37.5 & 42.639 \mathrm{~N}\end{array}$ $\begin{array}{lllll}14 & 38 & 22.0 \% & 15.503 \mathrm{~N}\end{array}$ $145303.5 * 34.991 \mathrm{~N}$

$1457 \quad 15.5 * 0.757 \mathrm{~N}$ $1508 \quad 12.8 * 4.140 \mathrm{~S}$ $153555.1848 .421 \mathrm{~N}$ $1548 \quad 08.3860 .091 \mathrm{~N}$ $\begin{array}{llllll}16 & 18 & 57.5 ? & 23.23 & S\end{array}$ $\begin{array}{llll}18 & 25 & 52.2 & 29.872 \mathrm{~N}\end{array}$ $\begin{array}{llllll}18 & 33 & 35 & 0 \% & 42.300 \quad \mathrm{~N}\end{array}$ $\begin{array}{lllll}19 & 46 & 11.5 \% & 39.360 \mathrm{~N}\end{array}$ $\begin{array}{lllll}19 & 48 & 14.6 & 21.039 & 5\end{array}$ $\begin{array}{llllll}19 & 51 & 01.0 & 29.801 \mathrm{~N}\end{array}$ $22 \quad 17 \quad 12.9 * 32.515 \mathrm{~N}$ $233337.6 * 41.201 \mathrm{~N}$ 01 $5952.3 \quad 39.316 \mathrm{~N}$ $02 \quad 5907.9 \quad 29.865 \mathrm{~N}$ $0349 \quad 37.5 * 28.764 \mathrm{~S}$ $042936.6 \quad 35.582 \mathrm{~N}$

042943.8 ? $18.00 \quad N$ $045526.7 * 30.644$ 050038.2 ? $31.03 \mathrm{~S}$ $\begin{array}{lllll}05 & 47 & 26.6 & 45.252 \mathrm{~N}\end{array}$ $062953.9 * 44.390 \mathrm{~N}$ $\begin{array}{llllll}06 & 33 & 16.2 ? & 6.24\end{array}$ $06 \quad 33 \quad 47.7 * 36.343 \mathrm{~N}$ $\begin{array}{lllll}08 & 19 & 54.3 \& & 59.806 \mathrm{~N}\end{array}$ $08 \quad 56 \quad 17.0 * 39.089 \mathrm{~N}$ $\begin{array}{lllllll}09 & 11 & 36.6 \% & 37.397 & N\end{array}$ 092653.3 ? $32.94 \mathrm{~S}$ $093431.5 * 11.3495$ $\begin{array}{lllll}09 & 52 & 23.1 & 32.345\end{array}$ $\begin{array}{llll}11 & 15 & 52.4 & 6.932\end{array}$ $\begin{array}{lllll}12 & 17 & 02.0 \% & 39.303 \mathrm{~N}\end{array}$ $\begin{array}{llll}12 & 57 & 13.0 \% & 11.459 \mathrm{~N}\end{array}$ $\begin{array}{llll}13 & 05 & 14.9 & 37.995\end{array}$

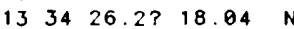
$135916.7 * 32.818 \mathrm{~N}$ $\begin{array}{lllll}1420 & 31.1 & 8.179 & 5\end{array}$

\begin{tabular}{|c|c|c|c|c|c|}
\hline 122.440 & $E$ & 118 & & 4.5 & \\
\hline 171.926 & E & 602 & & 5.2 & \\
\hline 27.730 & $\mathbf{E}$ & 10 & G & & \\
\hline 165.49 & $\mathbf{E}$ & 33 & $\mathbf{N}$ & 4.9 & 3.8 \\
\hline 27.697 & $\mathbf{E}$ & 10 & $G$ & & \\
\hline 176.25 & $E$ & 33 & $N$ & 4. 1 & \\
\hline 65.70 & $w$ & 33 & $\mathbf{N}$ & & \\
\hline 27.779 & $\mathbf{E}$ & 10 & G & & \\
\hline 12.183 & $\mathbf{E}$ & 10 & G & & \\
\hline 27.356 & $\mathbf{E}$ & 10 & G & & \\
\hline 126.388 & $\mathbf{E}$ & 133 & & 5.3 & \\
\hline 43.113 & $\mathbf{E}$ & 10 & G & & \\
\hline 120.061 & $\mathbf{E}$ & 27 & $D$ & 5.3 & 4.9 \\
\hline 67.216 & $w$ & 200 & $*$ & & \\
\hline 74.191 & $\mathbf{E}$ & 33 & $N$ & 4.1 & \\
\hline 32.651 & $w$ & 10 & G & 4.9 & 4.7 \\
\hline 19.73 & $\mathbf{E}$ & 10 & $G$ & & \\
\hline 102.524 & $\mathbf{E}$ & 66 & $\bullet$ & 4.3 & \\
\hline 32.573 & $w$ & 10 & $G$ & 5.4 & 4.9 \\
\hline 169.914 & $\mathbf{E}$ & 33 & $\mathbf{N}$ & 5.2 & 5.1 \\
\hline 23.687 & $\mathbf{E}$ & 10 & $G$ & & \\
\hline 114.850 & $w$ & 10 & $G$ & & 4.6 \\
\hline 32.41 & $w$ & 10 & $G$ & 4.9 & \\
\hline 27.619 & $E$ & 10 & $G$ & & \\
\hline 33.798 & $E$ & 29 & $\bullet$ & & \\
\hline 32.664 & $w$ & 10 & $G$ & 5.4 & 5.4 \\
\hline 177.735 & $w$ & 33 & $N$ & 4.8 & 4.8 \\
\hline 95.846 & $E$ & 33 & $N$ & 4.7 & 4.2 \\
\hline 12.66 & $E$ & 10 & $G$ & & \\
\hline 107.81 & $E$ & 33 & $N$ & 4. 8 & \\
\hline 18.992 & $E$ & 10 & $G$ & & \\
\hline 18.965 & $E$ & 10 & G & & \\
\hline 60.885 & $w$ & 33 & $\mathbf{N}$ & & \\
\hline 140.709 & $E$ & 33 & $N$ & 4.7 & \\
\hline 126.994 & $E$ & 33 & $N$ & 4.8 & \\
\hline 142.516 & $E$ & 66 & * & 4.5 & \\
\hline 122.225 & $w$ & $\theta$ & & & \\
\hline 153.685 & $w$ & 159 & & & \\
\hline 178.12 & $w$ & 348 & $?$ & 4.5 & \\
\hline 99.270 & E & 35 & $\bullet$ & 5.1 & \\
\hline 19.877 & E & 10 & $G$ & & \\
\hline 16.977 & E & 10 & G & & \\
\hline 178.942 & $w$ & 610 & $D$ & 5.7 & \\
\hline 99.196 & E & 43 & $\star$ & 4.9 & \\
\hline 47.787 & E & 33 & $\mathbf{N}$ & 4.6 & \\
\hline 20.048 & E & 10 & G & & \\
\hline 23.460 & E & 10 & G & & \\
\hline 99.049 & E & 25 & $\bullet$ & 4.9 & \\
\hline 123.728 & E & 10 & G & & \\
\hline 140.249 & $E$ & 78 & * & & \\
\hline
\end{tabular}

65.71 $71.683 \mathrm{~W}$ $71.51 \mathrm{~W}$ $7.511 \mathrm{E}$ $141.467 \mathrm{E}$ $151.06 \mathrm{E}$ $27.684 \mathrm{E}$ $153.724 \mathrm{~W}$

$27.612 \mathrm{E}$ $2.297 \mathrm{~W}$ $72.03 \mathrm{~W}$ $119.208 \mathrm{E}$ $70.081 \mathrm{~W}$ $129.426 E$ $16.891 \mathrm{E}$ $61.479 \mathrm{~W}$

$65.69 \mathrm{~W} 33 \mathrm{~N}$

$130.639 \mathrm{E} \quad 10 \mathrm{G}$ 112.924 E $113 ? 5.1$

99 ?

141

$\begin{array}{ll}10 & G \\ 10 & G\end{array}$

$33 \mathrm{~N}$

$33 \mathrm{~N} 4.2$

$33 \mathrm{~N}$

$10 \mathrm{G}$

$10 \mathrm{G}$

5.0

5. 1
$10 \mathrm{G}$

$23 \mathrm{G} \quad 5.85 .1$

$33 \mathrm{~N} 4.6$

41

$5 \mathrm{G}$
$10 \mathrm{G}$

$33 \mathrm{~N} 4.6$

$10 \mathrm{G}$

$13 \mathrm{G} 6.26 .2$
$95 * 4.7$
0.7

0.9358

0.49

1.044

0. $6 \quad 9$

1. 0

1.2406
GREECE-BULGARIA BORDER REGION, ML 2.4 (SKO)

VENEZUELA. Felt on southern Trinidod. Depth from broodbond displacement seismogroms.

MINDORO, PHILIPPINE ISLANDS

NEAR WEST COAST OF HONSHU, JAPAN

NEW MADRID, MISSOURI REGION. MBLg 3.0 (NEIS)

GREECE-ALBAT

GREECE. MD 3.4 (ATH)

EAST PAPUA NEW GUINEA REGION

SICHUAN PROVINCE, CHINA. Four people killed, 5 injured

and considerable domage in the Botang oreo. Seven

people killed, ot least 37 injured and odditianal

damage in the oreo due to a number of oftershocks.

Depth from broodband displacement sei smograms.

SICHUAN PROVINCE, CHINA

1.07 TIBET

$\begin{array}{rr}1.0 & 7 \\ 0.9 & 56\end{array}$

HOKKAIDO. JAPAN REGION. FeIt (I JMA) at Hiroo,

Tomakamai, Urokawa and Obihiro. Also felt (I JMA) at

Hachinahe, Honshu.

0.8 17 TAIWAN REGION

1.2173 VANUATU ISLANDS REGION

0.25 TURKEY

10 SANTA CRUZ ISLANDS

6 TURKEY

9 RAT ISLANDS, ALEUTIAN ISLANDS

PUERTO RICO REGION

7 TURKEY

9 AUSTRIA. ML 3.0 (VKA), 2.3 (KBA). Felt (V) ot Woergl.

5 TURKEY

199 RYUKYU ISLANDS. Felt (II JMA) on Kume-shimo. Also felt

(I I JMA) at Naho and (I JMA) at Nago, Okinowa.

0.1 7 WESTERN ARABIAN PENINSULA. ML 4.1 (ARO).

1.198 MINAHASSA PENINSULA

9 CHILE-ARGENTINA BORDER REGION

8 SOUTHERN XINJIANG, CHINA

34 CENTRAL MID-ATLANTIC RIDGE

5 IONIAN SEA. MD 3.2 (ATH)

17 GANSU PROVINCE, CHINA

93 CENTRAL MID-ATLANTIC RIDGE

71 LOYALTY ISLANDS REGION

9 AEGEAN SEA. ML 2.6 (THE)

17 EASTER ISLAND REGION

6 CENTRAL MID-ATLANTIC RIDGE

6 TURKEY

8 CYPRUS

214 CENTRAL MID-ATLANTIC RIDGE. MS 5.6 (BRK)

17 KERMADEC ISLANDS REGION

53 BURMA-INDIA BORDER REGION

5 NORTHERN ITALY. ML 1.7 (KBA)

19 JAVA

8 YUGOSLAVIA. MD 2.0 (TTG)

23 YUGOSLAVIA. MD 3.0 (TTG)

9 LEEWARD ISLANDS. ML 2.8 (FDF).

1.312 NEAR EAST COAST OF HONSHU, JAPAN FeIt (I JMA) ot UT SUNOMIYO

0.514 MINAHASSA PENINSUla

0.920 PAPUA NEW GUINEA

21 WASHINGTON. SSEA) CL 2.7 (SEA).

13 SOUTHERN ALASKA. $\angle A G S-P\rangle$

8 SOUTH OF FIJI ISLANDS

1.332 SICHUAN PROVINCE, CHINA

0.46 YUGOSLAVIA. MD 2.5 (TTG)

6 SOUTHERN ITALY

338 FIJI ISLANDS REGION. MO 5.4 (BRK).

24 SICHUAN PROVINCE, CHINA

14 IRAN-IRAO BORDER REGION

5 ALBANIA. ML 2.7 (SKO).

12 AEGEAN SEA

37 SICHUAN PROVINCE, CHINA

6 WESTERN AUSTRALIA

11 NEAR EAST COAST OF HONSHU, JAPAN. FEIt ( I JMA) ot Yokohoma and Ajiro.

$33 \mathrm{~N} \quad 1.1 \quad 5$ PUERTO RICO REGION

$44 * 4.94 .8 \quad 1.4$ 48 NEAR COAST OF CENTRAL CHILE

$33 \mathrm{~N} \quad 1.3 \quad 8$ NEAR COAST OF CENTRAL CHILE

1.057 NORTHERN ITALY. ML 3.5 (LDG). MD 3.1 (STR).

$33 \mathrm{~N} 4.7 \quad 0.6 \quad 10$ HOKKAIDO, JAPAN REGION

$33 \mathrm{~N} 4.4 \quad 1.6 \quad 9$ NEW BRITAIN REGION

$78 \quad 5.0$
DODECANESE ISLANDS

SOUTHERN ALASKA. 〈AGS-P>

0.25 TURKEY

0. 16 SPAIN. MG 2.9 (MDD)

0.58 OFF COAST OF CENTRAL CHILE

1.414 SOUTH OF SUMBA ISLAND

1.112 CHILE-ARGENTINA BORDER REGION

1.248 BANDA SEA

1.16 SOUTHERN ITALY

1.46 WINDWARD ISLANDS. MD 3.3 (TRN)

1.446 NORTH ISLAND, NEW ZEALAND

1.36 PUERTO RICO REGION

0.75 KYUSHU. JAPAN. MG 2.9 (JMA). Felt (I JMA) at Kumamata.

1.164 JAVA 


\begin{tabular}{|c|c|c|c|c|c|c|c|c|c|c|c|}
\hline 17 & 14 & 24 & $03.6 ?$ & 31.90 & $S$ & 178.00 & $w$ & 33 & $N$ & & 1.4 \\
\hline 17 & 15 & 36 & 48.2 & 40.517 & $N$ & 29.363 & $E$ & 10 & $G$ & & 0.4 \\
\hline 17 & 15 & 39 & 46.42 & 60.055 & $\mathbf{N}$ & 152.836 & $w$ & 104 & & 4.6 & \\
\hline 17 & 16 & 24 & 33.28 & 38.778 & $N$ & 122.775 & $w$ & 5 & & & \\
\hline 17 & 16 & 30 & $54.3 ?$ & 23.69 & $N$ & 122.36 & $\mathbf{E}$ & 33 & $N$ & 4.3 & 1.5 \\
\hline 17 & 17 & 08 & 37.2 & 3.768 & $S$ & 138.071 & $E$ & 86 & $*$ & 4.7 & 0.9 \\
\hline 17 & 17 & 32 & 16.3 & 2.881 & $\$$ & 138.924 & $E$ & 59 & $?$ & 5.1 & 1.3 \\
\hline 17 & 19 & 28 & $53.1 *$ & 9.614 & $S$ & 122.629 & $E$ & 33 & $N$ & 4.5 & 1.4 \\
\hline 17 & 19 & 52 & 59.98 & 35.200 & $\mathrm{~N}$ & 120.900 & $w$ & 5 & & & \\
\hline 17 & 19 & 53 & 54.6 & 23.417 & $N$ & 123.407 & $E$ & 24 & * & 4.5 & 1.0 \\
\hline 17 & 20 & 14 & $57.4 ?$ & 18.01 & $N$ & 65.71 & $w$ & 33 & $N$ & & 1.0 \\
\hline 17 & 21 & 01 & $55.5 \%$ & 40.267 & $N$ & 29.215 & $E$ & 10 & G & & 0.9 \\
\hline 17 & 21 & 28 & 15.5 & 18.254 & $N$ & 119.244 & $E$ & 52 & $\bullet$ & 4.7 & 0.6 \\
\hline 17 & 23 & 05 & 12.2 & 29.779 & $N$ & 99.113 & $E$ & 33 & $\mathbf{N}$ & 4.5 & 0.9 \\
\hline 18 & 00 & 01 & 36.3 & 33.718 & $N$ & 133.462 & $E$ & 10 & G & & 1.4 \\
\hline 18 & 00 & 47 & 58.1 & 39.296 & $N$ & 23.600 & $\bar{E}$ & 10 & G & 3.9 & 1.3 \\
\hline 18 & 00 & 50 & $59.3 *$ & 39.261 & $N$ & 23.532 & $E$ & 10 & G & & 1.5 \\
\hline 18 & 01 & 20 & $56.3 *$ & 6.336 & $S$ & 154.072 & $E$ & 54 & $?$ & & 1.6 \\
\hline 18 & 01 & 25 & 11.12 & 60.066 & $N$ & 152.774 & $w$ & 98 & & & \\
\hline 18 & 01 & 35 & $17.7 ?$ & 30.67 & $s$ & 71.92 & $w$ & 33 & $N$ & & 0.6 \\
\hline 18 & 02 & 01 & 27.1 & 21.538 & $S$ & 178.955 & $w$ & 580 & & 5.2 & 0.9 \\
\hline 18 & 02 & 41 & $59.4 *$ & 52.607 & $N$ & 169.152 & $w$ & 33 & $N$ & 4.6 & 0.7 \\
\hline 18 & 03 & 04 & 20.4 & 28.914 & $N$ & 95.764 & $E$ & 33 & $N$ & 4.5 & 0.8 \\
\hline 18 & 03 & 53 & 23.9 & 39.305 & $N$ & 23.608 & $E$ & 10 & G & & 1.1 \\
\hline 18 & 04 & 21 & $58.4 *$ & 21.848 & $s$ & 70.492 & $w$ & 52 & $*$ & 4.4 & 1.1 \\
\hline 18 & 06 & 59 & 50.28 & 38.793 & $N$ & 122.772 & $w$ & 4 & & & \\
\hline 18 & 07 & 58 & $11.0 *$ & 9.788 & $S$ & 127.882 & $E$ & 250 & $*$ & 4.6 & 0.9 \\
\hline 18 & 08 & 20 & 16.4 & 31.635 & $N$ & 104.118 & $E$ & 33 & $N$ & 4.3 & 0.9 \\
\hline 18 & 08 & 33 & $51.7 \%$ & 60.717 & $N$ & 5.544 & $E$ & 10 & $G$ & & 0.7 \\
\hline 18 & 08 & 48 & 46.6 & 50.664 & $N$ & 114.160 & $w$ & 5 & $G$ & & 0.5 \\
\hline 18 & 09 & 14 & $34.3 *$ & 34.245 & $N$ & 26.617 & $E$ & 33 & $N$ & & 0.8 \\
\hline 18 & 09 & 54 & 51.9 & 30.648 & $S$ & 71.990 & $w$ & 41 & $*$ & 4.6 & 1.2 \\
\hline 18 & 10 & 45 & 47.6 & 34.669 & $N$ & 110.925 & $w$ & 5 & G & & 0.8 \\
\hline 18 & 11 & 08 & $22.6 *$ & 30.666 & $S$ & 71.520 & $w$ & 33 & $N$ & & 1.4 \\
\hline 18 & 11 & 33 & $30.4 \%$ & 60.901 & $N$ & 4.844 & $E$ & 10 & G & & 0.6 \\
\hline 18 & 12 & 33 & 52.1 & 23.834 & $s$ & 179.944 & $E$ & 524 & $G$ & 5.8 & 0.9 \\
\hline 18 & 12 & 56 & $45.8 ?$ & 37.24 & $N$ & 52.72 & $E$ & 33 & $N$ & & 1.5 \\
\hline 18 & 14 & 45 & 11.1 & 39.266 & $N$ & 23.599 & $E$ & 10 & G & & 1.1 \\
\hline 18 & 15 & 33 & $21.0 \%$ & 42.331 & $\mathrm{~N}$ & 19.852 & $E$ & 10 & G & & 0.4 \\
\hline 18 & 17 & 02 & 20.88 & 10.599 & $N$ & 85.032 & $w$ & 165 & & & \\
\hline 18 & 17 & 13 & $47.1 *$ & 52.119 & $\mathrm{~N}$ & 175.560 & $E$ & 69 & - & 4.0 & 0.8 \\
\hline $\begin{array}{l}18 \\
18\end{array}$ & $\begin{array}{l}17 \\
18\end{array}$ & $\begin{array}{l}14 \\
15\end{array}$ & $\begin{array}{l}12.1 \\
35.78\end{array}$ & $\begin{array}{l}39.293 \\
33.690\end{array}$ & $\begin{array}{l}\mathrm{N} \\
\mathrm{N}\end{array}$ & $\begin{array}{r}23.545 \\
120.080\end{array}$ & $\begin{array}{l}E \\
W\end{array}$ & $\begin{array}{r}10 \\
6\end{array}$ & G & & 0.6 \\
\hline $\begin{array}{l}18 \\
18\end{array}$ & 18 & 29 & $01.8 ?$ & 18.33 & $\mathrm{~N}$ & 62.03 & $w$ & 10 & $G$ & & 0.4 \\
\hline 18 & 19 & 00 & 22.7 & 63.493 & $\mathrm{~N}$ & 148.660 & $w$ & 33 & $\mathrm{~N}$ & & 1.0 \\
\hline 18 & 19 & 10 & $14.3 ?$ & 29.52 & $S$ & 178.81 & $w$ & 227 & $?$ & 4.7 & 1.4 \\
\hline 18 & 19 & 28 & 25.8 & 58.294 & $N$ & 6.769 & $E$ & 10 & G & & 0.9 \\
\hline 18 & 19 & 28 & $46.8 *$ & 43.454 & $\mathbf{N}$ & 12.640 & $E$ & 10 & G & & 0.8 \\
\hline 18 & 19 & 43 & $45.3 ?$ & 9.55 & $S$ & 129.80 & $E$ & 284 & $?$ & 4.5 & 0.6 \\
\hline 18 & 21 & 28 & 15.32 & 57.778 & $\mathbf{N}$ & 143.129 & $w$ & 10 & G & & \\
\hline 18 & 21 & 46 & 30.28 & 58.092 & $N$ & 151.656 & $w$ & 53 & G & & \\
\hline 18 & 21 & 54 & 57.42 & 58.095 & $N$ & 151.657 & $w$ & 53 & G & & \\
\hline 18 & 22 & 02 & $11.9 \%$ & 39.310 & $N$ & 28.977 & $E$ & 10 & G & & 10 \\
\hline 18 & 22 & 123 & $38.3 \%$ & 18.183 & $N$ & 66.080 & $w$ & 31 & $*$ & & 0.2 \\
\hline 18 & 22 & 332 & 26.8 & 51.552 & $N$ & 16.042 & $\mathbf{E}$ & 10 & G & & 0.9 \\
\hline 18 & 23 & 01 & 45.7 & 50.476 & $N$ & 18.868 & $\mathbf{E}$ & 10 & G & & 1.1 \\
\hline 18 & 23 & 18 & 04.72 & 62.020 & $N$ & 151.596 & $w$ & 95 & & & \\
\hline 19 & 00 & 08 & 19.6 & 31.310 & $S$ & 177.815 & $w$ & 11 & G & 5.65 .9 & 11 \\
\hline 19 & 03 & 125 & $55.0 ?$ & 16.71 & $N$ & 59.68 & $w$ & 22 & & & 09 \\
\hline 19 & 03 & 32 & 45.6 & 39.648 & $N$ & 28.958 & E & 10 & $G$ & & 1.5 \\
\hline 19 & 03 & 46 & 08.4. & 36.247 & $N$ & 27.090 & E & 10 & G & & 0.8 \\
\hline 19 & 03 & 59 & $16.3 \%$ & 39.100 & $N$ & 27.842 & E & 10 & $G$ & & 0.4 \\
\hline 19 & 05 & 55 & $20.3 ?$ & 61.50 & $N$ & 3.93 & $\mathbf{E}$ & 10 & $G$ & & 0.8 \\
\hline 19 & 06 & 015 & $58.1 ?$ & 38.18 & $N$ & 3.88 & $w$ & 10 & G & & 0.9 \\
\hline 19 & 07 & 035 & $59.3 *$ & 31.521 & $S$ & 177.763 & $w$ & 61 & D & 5.2 & 1.2 \\
\hline 19 & 07 & 30 & $46.9 \%$ & 40.273 & $N$ & 29.557 & $\mathbf{E}$ & 10 & G & & 0.3 \\
\hline 19 & 07 & 32 & 23.1 & 36.469 & $N$ & 73.415 & $\mathbf{E}$ & 45 & $*$ & 4.9 & 1.1 \\
\hline 19 & 08 & 59 & 49.6 & 16.355 & $N$ & 98.682 & $w$ & 10 & $G$ & 4.9 & 1.1 \\
\hline 19 & $11-$ & 13 & $41.4 *$ & 42.702 & $\mathbf{S}$ & 83.394 & $w$ & 10 & $G$ & 4.9 & 0.7 \\
\hline 19 & 11 & 55 & $47.7 \%$ & 39.117 & $N$ & 27.614 & $\mathbf{E}$ & 10 & $G$ & & 0.5 \\
\hline 19 & 12 & 26 & 41.8 & 42.551 & $N$ & 24.172 & $E$ & 10 & G & & 1.5 \\
\hline 19 & 12 & 43 & $33.0 ?$ & 13.46 & $\mathrm{~N}$ & 90.74 & $w$ & 48 & $?$ & 4.7 & 1.5 \\
\hline 19 & 13 & 09 & $25.2 ?$ & 27.92 & $S$ & 178.54 & $w$ & 268 & $?$ & 4. 1 & 1.0 \\
\hline 19 & 13 & 25 & 36.2 * & 5.451 & $S$ & 129.856 & $E$ & 197 & $*$ & 4.5 & 0.6 \\
\hline 19 & 13 & 27 & 13.98 & 48.696 & $N$ & 123.343 & $w$ & 20 & & & \\
\hline 19 & 13 & 35 & $07.6 ?$ & 43.96 & $N$ & 7.31 & $E$ & 10 & G & & 0.2 \\
\hline 19 & 13 & 41 & 37.3 & 36.414 & $\mathrm{~N}$ & 5.550 & $w$ & 10 & $G$ & & 1.1 \\
\hline 19 & 14 & 48 & 56.7 & 17.858 & $N$ & 105.228 & $w$ & 10 & G & 5.15 .4 & 1.2 \\
\hline 19 & 15 & 00 & $33.5 *$ & 17.643 & $N$ & 105.077 & $w$ & 10 & G & & 1.3 \\
\hline 19 & 16 & 15 & $28.8 *$ & 17.659 & $N$ & 105.270 & $w$ & 10 & G & 4.2 & 1.0 \\
\hline 19 & 16 & 23 & 52.4 & 6.947 & $S$ & 129.905 & $E$ & 69 & * & 5.2 & 1.0 \\
\hline 19 & 16 & 38 & $22.5 \&$ & 60.737 & $N$ & 151.901 & $\boldsymbol{w}$ & 76 & & & \\
\hline 19 & 16 & 40 & 28.3 & 30.133 & $N$ & 90.046 & $E$ & 33 & $N$ & 4.5 & 1.1 \\
\hline 19 & 17 & 30 & 56.98 & 61.226 & $N$ & 151.858 & $w$ & 82 & & & \\
\hline 19 & 19 & 06 & $15.0 \%$ & 37.651 & $N$ & 30.013 & $E$ & 10 & G & & 1.1 \\
\hline 19 & 19 & 30 & $36.8 \%$ & 40.137 & $N$ & 29.345 & $E$ & 10 & G & & 1.0 \\
\hline 19 & 19 & 49 & $34.6 *$ & 37.711 & $N$ & 14.693 & $E$ & 10 & G & & 0.6 \\
\hline 19 & 20 & 08 & 41.08 & 59.692 & $N$ & 153.084 & $w$ & 99 & & & \\
\hline
\end{tabular}




\begin{tabular}{|c|c|c|c|c|c|c|c|c|c|c|c|}
\hline 19 & 20 & 25 & 30.1 & 37.849 & $\mathbf{N}$ & 14.785 & $E$ & 10 & $G$ & & \\
\hline 19 & 21 & 34 & 44.4 & 37.818 & $\mathbf{N}$ & 14.744 & $E$ & 10 & $G$ & & \\
\hline 19 & 22 & 05 & $57.8 *$ & 44.707 & $\mathbf{N}$ & 147.685 & $E$ & 33 & $N$ & 4.6 & \\
\hline 19 & 22 & 39 & 28.7 & 37.257 & $N$ & 115.896 & $w$ & 3 & & & \\
\hline 19 & 23 & 40 & 33.0 & 42.602 & $\mathbf{N}$ & 18.959 & $E$ & 10 & $G$ & & \\
\hline 19 & 23 & 54 & $57.2 ?$ & 7.18 & $N$ & 73.81 & $w$ & 129 & $?$ & & \\
\hline 20 & 00 & 10 & $37.6 *$ & 46.862 & $N$ & 152.900 & $E$ & 33 & $N$ & 4.8 & 4.3 \\
\hline 20 & 00 & 37 & 24.1 & 42.350 & $\mathbf{N}$ & 19.999 & $E$ & 10 & G & & \\
\hline 20 & 00 & 56 & $13.2 ?$ & 32.36 & S & 72.05 & $w$ & 33 & $\mathbf{N}$ & & \\
\hline 20 & 01 & 07 & $02.5 \&$ & 59.892 & $\mathbf{N}$ & 150.464 & $w$ & 50 & & & \\
\hline 20 & 02 & 16 & $05.9 *$ & 55.494 & $\mathbf{N}$ & 4.826 & $w$ & 10 & $G$ & & \\
\hline 20 & 02 & 45 & $45.5 ?$ & 44.19 & $\mathbf{N}$ & 150.01 & $E$ & 33 & $N$ & 4.2 & \\
\hline 20 & 03 & 14 & $02.5 *$ & 22.708 & $\mathbf{N}$ & 121.691 & $E$ & 33 & $N$ & 4.0 & \\
\hline 20 & 04 & 36 & $04.0 \%$ & 15.355 & $\mathbf{N}$ & 60.777 & $w$ & 10 & G & & \\
\hline 20 & 05 & 31 & $29.9 ?$ & 44.32 & $\mathbf{N}$ & 149.84 & $E$ & 33 & $\mathbf{N}$ & 4.2 & \\
\hline 20 & 06 & 13 & 53.2 & 13.328 & $\mathbf{N}$ & 90.757 & $w$ & 65 & & 4.8 & \\
\hline 20 & 06 & 35 & $55.3 \&$ & 58.946 & $\mathbf{N}$ & 152.128 & $w$ & 66 & & & \\
\hline 20 & 06 & 53 & $26.3 ?$ & 35.95 & $\mathbf{N}$ & 70.32 & $E$ & 33 & $\mathbf{N}$ & 3.8 & \\
\hline 20 & 06 & 53 & 33.0 & 2. 968 & $\mathbf{N}$ & 126.400 & $E$ & 94 & $*$ & 4.9 & \\
\hline 20 & 07 & 54 & 03.1 & 7.775 & $s$ & 107.922 & $E$ & 33 & $N$ & 4.7 & \\
\hline 20 & 08 & 08 & 51.0 & 9.259 & $s$ & 79.033 & $w$ & 63 & $G$ & 5.8 & \\
\hline 20 & 08 & 12 & $32.4 *$ & 39.899 & $N$ & 29.817 & $w$ & 10 & $G$ & 4.5 & \\
\hline 20 & 08 & 42 & 03.4 & 40.001 & $N$ & 29.869 & $w$ & 10 & $G$ & 4.9 & 4.7 \\
\hline 20 & 09 & 37 & $37.3 ?$ & 44.48 & $N$ & 128.74 & $w$ & 10 & $G$ & 4.0 & \\
\hline 20 & 09 & 48 & 21.3 & 44.281 & $N$ & 7.492 & $E$ & 10 & $G$ & & \\
\hline 20 & 10 & 40 & 33.4 & 34.784 & $N$ & 140.866 & $E$ & 62 & $\bullet$ & 4.7 & \\
\hline 20 & 11 & 14 & $49.9 \%$ & 39.300 & $N$ & 28.924 & $E$ & 10 & $G$ & & \\
\hline 20 & 11 & 16 & 50.77 & 39.267 & $\mathbf{N}$ & 28.932 & $E$ & 10 & $G$ & & \\
\hline 20 & 11 & 28 & 09.1 & 28.858 & $\mathbf{N}$ & 139.185 & $E$ & 470 & - & 4.5 & \\
\hline 20 & 11 & 46 & 09.3 & 36.281 & $\mathbf{N}$ & 5.543 & $w$ & 10 & $G$ & & \\
\hline 20 & 12 & 11 & $39.7 *$ & 38.845 & $\mathbf{N}$ & 71.721 & $E$ & 33 & $\mathbf{N}$ & 4.5 & \\
\hline 20 & 12 & 13 & $59.6 ?$ & 7.00 & $N$ & 76.21 & $w$ & 34 & $?$ & 4. 2 & \\
\hline 20 & 12 & 45 & 52.7 & 38.494 & $\mathbf{N}$ & 117.806 & $w$ & 4 & & 4.3 & \\
\hline 20 & 13 & 06 & $00.3 ?$ & 46.22 & $s$ & 75.63 & $w$ & 33 & $\mathbf{N}$ & 4.9 & 4.9 \\
\hline 20 & 13 & 59 & 39.8 & 37.793 & $\mathbf{N}$ & 2.557 & $w$ & 10 & $G$ & & \\
\hline 20 & 15 & 02 & $16.5 *$ & 45.712 & $\mathrm{~s}$ & 75.560 & $w$ & 10 & $G$ & 5.5 & 5.3 \\
\hline 20 & 16 & 14 & $14.2 ?$ & 20.07 & $\mathbf{N}$ & 144.42 & $E$ & 33 & $\mathbf{N}$ & 4.6 & \\
\hline 20 & 16 & 14 & $39.9 ?$ & 9.93 & $\mathrm{~s}$ & 124.04 & $E$ & 33 & $\mathbf{N}$ & 4.5 & \\
\hline 20 & 16 & 29 & $07.3 \&$ & 59.776 & $\mathbf{N}$ & 153.180 & $w$ & 104 & & & \\
\hline 20 & 17 & 26 & 51.9 & 49.124 & $N$ & 6.820 & $E$ & 10 & $G$ & & \\
\hline 20 & 18 & 47 & 40.2 & 35.769 & $\mathbf{N}$ & 21.757 & $E$ & 10 & G & 4.4 & \\
\hline 20 & 19 & 57 & $12.3 \&$ & 58.490 & $\mathbf{N}$ & 142.803 & $w$ & 10 & G & & \\
\hline 20 & 20 & 31 & 00.4 & 11.974 & $\mathbf{N}$ & 142.942 & $E$ & 33 & $\mathbf{N}$ & 5.1 & 4.2 \\
\hline 20 & 22 & 59 & 54.0 & 57.166 & $N$ & 121.976 & $E$ & 26 & G & 6.1 & 6.5 \\
\hline
\end{tabular}

20
20
21
21
21
21
21
21
21
21
21
21
21
21
21
21
21
21
21
21
21
21
21
21
21
21
21
21
21
21
21
22
22
22
22
22
22
22
22
22
$234433.2 \& 37.258 \mathrm{~N}$

$00 \quad 2417.2 * 41.265 \mathrm{~N}$ $0106 \quad 13.6 ? 21.04 \mathrm{~S}$ 01 $3346.5 \cdot 11.135 \mathrm{~N}$ 030148.1 ? $7.42 \mathrm{~S}$ 041705.1 ? $0.13 \mathrm{~N}$ $053533.4 * 10.189 \mathrm{~S}$ $07 \quad 2936.4 ? \quad 41.29$ $08 \quad 16 \quad 06.5 \quad 36.495 \mathrm{~N}$ बB $38 \quad 20.5 * 27.949 \mathrm{~N}$ $\begin{array}{llllll}09 & 40 & 43.7 \& & 47.392 \mathrm{~N}\end{array}$ $09 \quad 55 \quad 32.3 \% 38.613 \mathrm{~N}$ $\begin{array}{lllll}12 & 10 & 44.5 & 38.389 & \mathrm{~N}\end{array}$ $\begin{array}{lllll}12 & 17 & 35.5 & 42.752 \mathrm{~N}\end{array}$ $124629.7 * 21.652 \mathrm{~N}$ $13 \quad 34 \quad 35.3 \% 59.046 \mathrm{~N}$

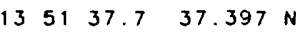
$\begin{array}{llll}1406 & 27.0 & 39.386 \mathrm{~N}\end{array}$ $\begin{array}{lllll}15 & 10 & 06.1 & 42.341 \mathrm{~N}\end{array}$ $16 \quad 1504.7 * 42.931 \mathrm{~N}$ $183911.3 * 18.311 \mathrm{~N}$ $1908 \quad 41.0 \quad 57.168 \mathrm{~N}$ $\begin{array}{llll}19 & 28 & 51.6 & 40.519\end{array}$ $194359.8 \% 40.524 \mathrm{~N}$ $\begin{array}{lllll}20 & 34 & 28.3 & 16.366 \mathrm{~N}\end{array}$ $\begin{array}{lllll}20 & 36 & 04.2 & 13.302 \mathrm{~N}\end{array}$ $\begin{array}{llll}21 & 02 & 46.9 \% & 37.762 \mathrm{~N}\end{array}$ $\begin{array}{llll}22 & 06 & 27.7 \% & 40.524 \mathrm{~N}\end{array}$ $22 \quad 2021.0 * 37.537 \mathrm{~N}$ $231630.4 * 36.439 \mathrm{~N}$ $005110.0 * 51.079 \mathrm{~N}$ $040639.4 ? 44.56 \mathrm{~N}$ $06 \quad 15 \quad 17.3 * 0.997 \mathrm{~S}$ $\begin{array}{lllll}06 & 19 & 37.1 & 8.966\end{array}$ $071556.9 ? \quad 17.83 \mathrm{~N}$ $074441.6 ? 24.94 \mathrm{~N}$ $\begin{array}{llll}10 & 58 & 19.6 & 40.945 \mathrm{~N}\end{array}$ $044830.8 * 35.576 \mathrm{~S}$ $\begin{array}{lllll}08 & 03 & 25.1 & 40.513 \mathrm{~N}\end{array}$

$22.760 \mathrm{E} \quad 10 \mathrm{G}$

$68.45 W 133$ ?

$62.132 \mathrm{~W} 33 \mathrm{~N}$

128.49 E $146 ? 4.9$

161.377 E $101 * 4.7$

22.77 E $10 \mathrm{G}$

26.755 E 157 .

$64.271 \mathrm{E} \quad 33 \mathrm{~N} 4.4$

$122.856 \mathrm{~W}$

$27.947 \mathrm{E}$

$22.129 E \quad 10 G \quad 4.2$

$13.334 E \quad 10$

$95.494 E \quad 59 ? 4.7$

5.966 E $10 \mathrm{G}$

$23.554 E$ 21.

$26.417 \mathrm{E} \quad 10 \mathrm{G}$

$19.870 \mathrm{E} 7$

$19.454 \mathrm{E} \quad 10 \mathrm{G}$

$100.829 \mathrm{~W}$

$123.768 \mathrm{E}$

$15.789 \mathrm{E}$

$15.880 \mathrm{E}$

$122.198 \mathrm{E}$

$144.381 \mathrm{E}$

29.172 E

$15.856 \mathrm{E}$

$20.621 \mathrm{E}$

$71.253 \mathrm{E}$

$178.578 \mathrm{~W}$

$7.28 \mathrm{E}$

$102.935 \mathrm{~W}$

$123.202 \mathrm{E}$

$115.512 \mathrm{E}$

65.67

$20.551 \mathrm{E} 10 \mathrm{G}$

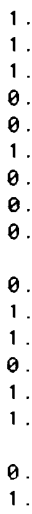

1.4 12 SICILY

$1.5 \quad 13$ SICILY

KURIL ISLANDS

35 SOUTHERN NEVADA. ML 3.5 (NEIS)

0.96 YUGOSLAVIA. MO 2.0 (TTG)

1.27 NORTHERN COLOMBIA

0.931 KURIL ISLANDS

510 YUgoslavia. MO 2.4 (TTG)

0.68 OFF COAST OF CENTRAL CHILE

62 KENAI PENINSULA. ALASKA. 〈AGS-P>

0.27 UNITED KINGDOM

1.56 KURIL ISLANDS REGION

1.4 11 TAIWAN REGION

0.66 LEEWARD ISLANDS. ML 2.5 (FDF)

6 KURIL ISLANDS

1.274 NEAR COAST OF GUATEMALA

39 KODIAK ISLANO REGION. LAGS-P>

8 HINDU KUSH REGION

1.276 MOLUCCA PASSAGE

0.826 JAVA

1.0299 OFF COAST OF NORTHERN PERU. Felt ot Limo, Chimbote and Trujilla. Depth fram braadband displacement se i smagrams.

0.930 AZORES ISLANDS

1.296 AZORES ISLANDS REGION

0.920 OFF COAST OF OREGON

9. 510 NORTHERN ITALY

0.722 NEAR EAST COAST OF HONSHU, JAPAN. FEIt (I JMA) on OShIMO.

1.19 TURKEY

0. 59 TURKEY

0.936 BONIN ISLANDS REGION

1.27 STRAIT OF GIBRALTAR

1.114 AFGHANISTAN-USSR BORDER REGION

1.513 NORTHERN COLOMBIA

0.779 NEVADA. ML 4.2 (BRK). Felt (IV) ot Mino. (111) ot Luning and Manhotton.

1.615 NEAR COAST OF SOUTHERN CHILE

1.211 SPAIN. MG 3.4 (MDD). Felt (111) in the Golero oreo.

1.656 OFF COAST OF SOUTHERN CHILE

0.812 MARIANA ISLANOS

1.57 TIMOR

30 SOUTHERN ALASKA. $\angle A G S-P\rangle$.

0.77 GERMANY. MD 1.5 (STR).

1.372 MEDITERRANEAN SEA. ML 4.2 (ATH).

14 GULF OF ALASKA. 〈AGS-P>.

0. 884 SOUTH OF MARIANA ISLANDS

0.9545 EASTERN USSR. MS 6.3 (BRK), 5.9 (PAS). FeIt (VI) ot Khoni, Olekso and Yuktali; (V) ot lkabyo, Kuvykto. Khatymi. Neryungri and Berkakit: (IV) of Yokutsk, Chito and Blagoveshchensk. Also felt strangly at Ust-Nyukzha. Depth from broodband displacement seismagrams.

1.58 CENTRAL CHILE

17 CENTRAL CALIFORNIA. <BRK>. ML 3.2 (BRK). MO $=1.4 * 10 * 14$

123.44 E $198 ? 4.7$

23.94 E 109 ?

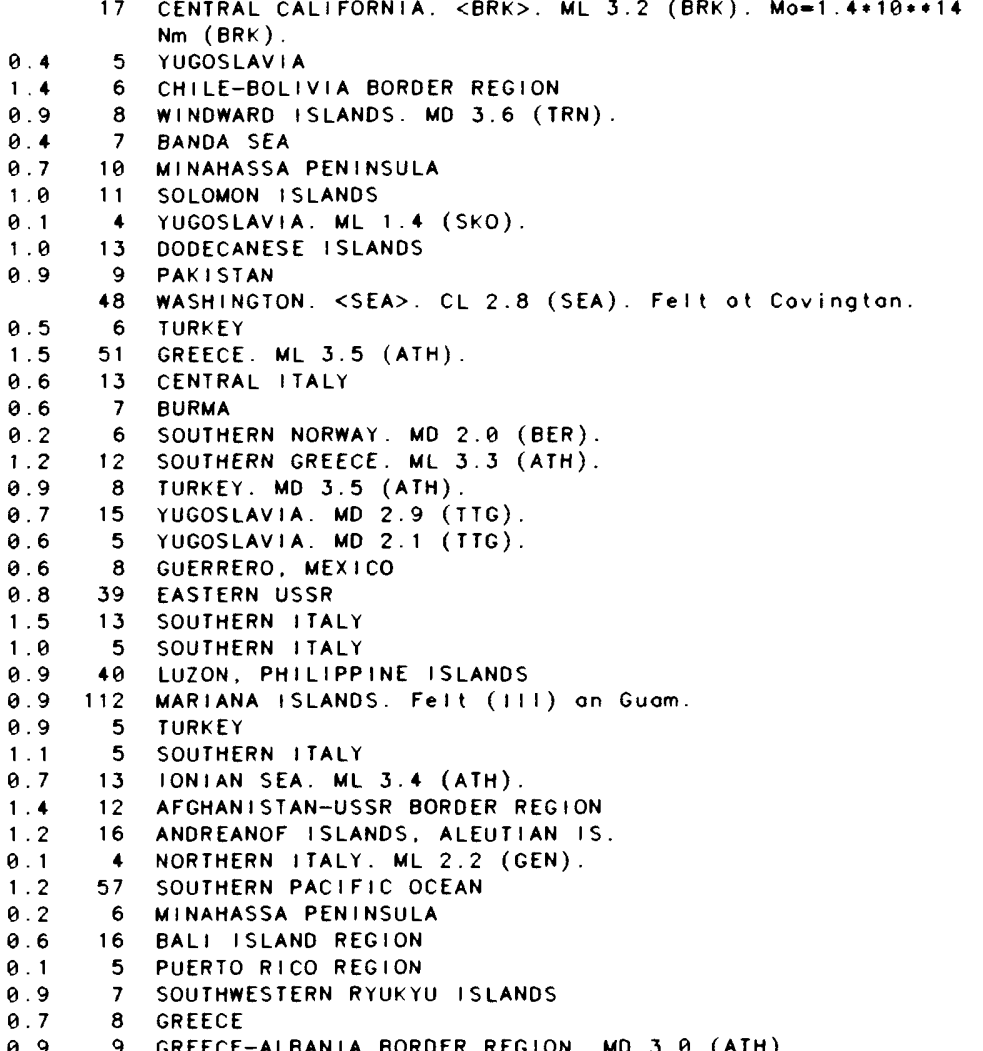
$\mathrm{Nm}$ (BRK) 


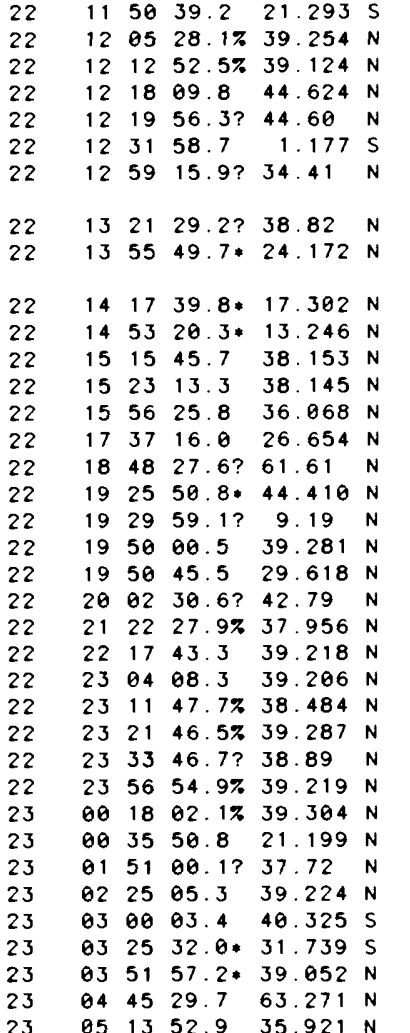

$054641.0 ? 16.66 \mathrm{~S}$ $\begin{array}{lllll}06 & 16 & 25.3 & 39.327 \mathrm{~N}\end{array}$ $064555.1 * 37.545 \mathrm{~S}$ $064727.6 .39 .613 \mathrm{~N}$ 070822.0 ? $23.81 \mathrm{~N}$ $074406.2 ? \quad 42.16 \mathrm{~N}$ $\begin{array}{lllll}07 & 50 & 30.4 & 14.826 \mathrm{~S}\end{array}$ $\begin{array}{llllll}07 & 55 & 02.4 \% & 36.081 \mathrm{~N}\end{array}$ $082749.4 \cdot 58.736 \mathrm{~s}$ $084528.7 ? \quad 17.35 \mathrm{~N}$ $084706.5 .5 .611 \mathrm{~S}$ $085034.0 .44 .197 \mathrm{~N}$ 105151.0 ? $31.98 \mathrm{~S}$ $\begin{array}{lllll}11 & 49 & 14.3 \% & 16.944 \mathrm{~N}\end{array}$ $12 \quad 1805.1 \& 59.740 \mathrm{~N}$ $1254 \quad 05.5 ? \quad 17.92$ $\begin{array}{llll}13 & 03 & 10.9 & 36.183 \mathrm{~N}\end{array}$ $\begin{array}{llll}13 & 47 & 21.9 * 16.570 \mathrm{~S}\end{array}$ $\begin{array}{lllll}13 & 55 & 12.5 & 8.914 \mathrm{~S}\end{array}$ $1356 \quad 53.4 \% 38.964 \mathrm{~N}$ $\begin{array}{lllll}14 & 17 & 04.8 \text { ? } & 18.26 \mathrm{~N}\end{array}$ $\begin{array}{llllll}14 & 23 & 49.7 \% & 39.106 \mathrm{~N}\end{array}$ $\begin{array}{llllll}15 & 17 & 15.9 & 6.10 \mathrm{~S}\end{array}$ $\begin{array}{lllll}15 & 22 & 34.1 & 44.958 \mathrm{~N}\end{array}$ $\begin{array}{llll}15 & 41 & 45.0 * 44.068 \mathrm{~N}\end{array}$ $\begin{array}{llll}16 & 17 & 22.3 * 61.256 \mathrm{~N}\end{array}$ $\begin{array}{lllll}16 & 35 & 27.6 * 3.3735\end{array}$ $\begin{array}{llll}17 & 18 & 04.3 & 2.690\end{array}$ $1756 \quad 14.8 * 14.012 \mathrm{~N}$ $181132.8 * 40.515 \mathrm{~N}$ $1911127.3 * 66.946 \mathrm{~N}$ $1920 \quad 35.5 ? \quad 66.89 \mathrm{~N}$ $192106.466 .960 \mathrm{~N}$

$\begin{array}{llll}19 & 51 & 24.3 & 39.230 \mathrm{~N}\end{array}$ $201328.5 * 66.980 \mathrm{~N}$ $\begin{array}{lllll}21 & 31 & 19.4 & 66.946 & \mathrm{~N}\end{array}$ $\begin{array}{llllll}21 & 35 & 34.2 & 6.538\end{array}$ $\begin{array}{llllll}22 & 14 & 47.6 \% & 39.305 & \mathrm{~N}\end{array}$ $\begin{array}{lllll}22 & 17 & 50.0 & 39.319 \mathrm{~N}\end{array}$ $\begin{array}{lll}22 & 19 & 12.3 * 66.900 \mathrm{~N}\end{array}$ $\begin{array}{llllll}22 & 31 & 24.1 & 33.46 & \end{array}$ $\begin{array}{lllll}23 & 32 & 20.6 & 41.764 \mathrm{~N}\end{array}$ $234436.6 * 2.101 \mathrm{~S}$ $\begin{array}{lllll}23 & 47 & 27.3 & 66.970 \mathrm{~N}\end{array}$ $\begin{array}{lllll}23 & 56 & 19.4 & 41.966 \mathrm{~N}\end{array}$ $005040.9 * 66.989 \mathrm{~N}$ $\begin{array}{lllll}01 & 09 & 45.2 & 66.905 \mathrm{~N}\end{array}$ $013041.9 \quad 28.795 \mathrm{~N}$ $013401.2 \quad 57.317 \mathrm{~N}$ $013531.1 \& 59.528 \mathrm{~N}$

$\begin{array}{cc}179.193 & W \\ 27.733 & E \\ 27.578 & E \\ 7.039 & E \\ 7.00 & E \\ 136.925 & E \\ 134.82 & E\end{array}$

23.74 E $10 \mathrm{G}$ $125.239 \mathrm{E}$

$94.925 E$ $144.738 \mathrm{E}$ $117.811 \mathrm{~W}$ $117.818 \mathrm{~W}$ $1.582 W$

$96.827 \mathrm{E}$

$3.34 \mathrm{E}$

6. $469 \mathrm{E}$

$125.55 \mathrm{E}$

$29.006 \mathrm{E}$

$141.357 \mathrm{E}$

$18.53 \mathrm{E}$

$14.135 E$

$23.583 \mathrm{E}$

$23.589 \mathrm{E}$

$15.387 \mathrm{E}$

$28.970 \mathrm{E}$

$18.12 \mathrm{E}$

$28.845 E$

$29.024 \mathrm{E}$

$119.558 \mathrm{E}$

$15.01 \mathrm{E}$

$23.648 \mathrm{E}$ $175.997 \mathrm{E}$

$67.629 \mathrm{~W}$

$72.655 \mathrm{E}$

$150.655 \mathrm{~W}$

$140.250 \mathrm{E}$
$173.79 \mathrm{~W}$

$73.214 \mathrm{E}$ $176.255 E$ $22.792 \mathrm{E}$ $93.70 \mathrm{E}$ $22.69 \mathrm{E}$ $166.692 \mathrm{E}$ $1.657 \mathrm{~W}$ $25.261 w$ $61.10 \mathrm{~W}$ $151.149 \mathrm{E}$ $17.772 \mathrm{E}$ $71.71 \mathrm{~W}$ $152.829 w$ 65.75 $139.132 \mathrm{E}$ $172.250 \mathrm{~W}$ $117.970 \mathrm{E}$ $27.172 \mathrm{E}$ 61.26 $27.572 \mathrm{E}$ $135.24 E$ 150. $238 E$ $7.316 \mathrm{E}$ $151.839 \mathrm{~W}$ $139.769 \mathrm{E}$ $141.482 \mathrm{E}$ $93.220 \mathrm{E}$ $24.293 \mathrm{E}$ 156.186 W 156.28 156.289

$23.676 \mathrm{E}$ $156.236 \mathrm{~W}$ $156.296 \mathrm{~W}$ $105.826 \mathrm{E}$ $29.013 \mathrm{E}$ $29.022 \mathrm{E}$ $156.293 \mathrm{~W}$ 70.90 W $12.684 \mathrm{E}$ $128.465 \mathrm{E}$ $156.350 \mathrm{~W}$ $23.509 \mathrm{E}$ $156.390 \mathrm{~W}$ $156.331 \mathrm{~W}$ $129.569 \mathrm{E}$ $121.904 \mathrm{E}$ $152.804 \mathrm{~W}$ $61.637 w$

$33 \mathrm{~N}$
614

$10 \mathrm{G}$

$10 \mathrm{G}$

$10 \mathrm{G}$

$33 \mathrm{~N}$

4. 9

$3 \mathrm{~N} 4.2$

$56 \cdot 4.9$

10

$33 \mathrm{~N} 4.4$

$10 \mathrm{G}$

$10 \mathrm{G}$

$41 ?$

$10 \mathrm{G}$

$33 \mathrm{~N} \quad 4.5$

$10 \mathrm{G}$

$10 \mathrm{G}$

10 11

150 ?

$10 \mathrm{G}$

$10 \mathrm{G}$

$10 \mathrm{G}$

$10 \mathrm{G}$

$33 \mathrm{~N} 4.1$

$10 \mathrm{G}$

10 G 4.1

425.1

$10 \mathrm{G}$

$33 \mathrm{~N} 4.5$

$33 \mathrm{~N}$

$33 \mathrm{~N} 4.6$

$33 \mathrm{~N} 4.6$
277

10

$\begin{array}{ll}10 & \mathrm{G} \\ 33 & \mathrm{~N}\end{array}$

106

$61 * 5.8$

$10 \mathrm{G}$

$33 \mathrm{~N} 5.2$

$10 \mathrm{G}$

$10 \mathrm{G} 4.0$

10

$33 \mathrm{~N}$

$33 \mathrm{~N}$

90

100

$73 *$

3305.05 .0

$118 * 4.2$

$10 \mathrm{G}$

$10 \mathrm{G}$

$10 \mathrm{G}$

$33 \mathrm{~N} 4.3$

$\begin{array}{lll}89 & D & 4.9\end{array}$

$10 \mathrm{G}$

86

97.4 .4

$18 * 4.2$

$33 \mathrm{~N} \quad 4.2$

$10 \mathrm{G}$

56

$5 G$

$\begin{array}{llll}6 & G & 5.7 & 5.1\end{array}$

$\begin{array}{rllll}10 & & 3.7 & & 1.1 \\ 5 & G & & & 1.2 \\ 5 & G & & & 0.5 \\ 75 & . & 5.1 & & 1.1 \\ 10 & G & & 0.4 \\ 9 & & & & 1.1 \\ 5 & G & & & 0.9 \\ 33 & N & & & 1.0 \\ 17 & & & & 1.1 \\ 61 & P & 4.3 & & 0.6 \\ 5 & G & & & 0.7 \\ 7 & & & & 0.8 \\ 5 & G & & & 1.3 \\ 5 & G & & & 0.3 \\ 33 & & 4.9 & & 0.8 \\ 33 & N & 4.8 & 4.4 & 0.9 \\ 85 & & 4.6 & & \end{array}$

0.979 FIJI ISLANDS REGION

5 TURKEY

0.55 TURKEY

0.522 NORTHERN ITALY. ML 2.6 (LDG), 2.4 (GEN)

0.34 FRANCE. ML 2.0 (GEN).

22 WEST IRIAN REGION

4 NEAR S. COAST OF SOUTHERN HONSHU. MG 3.4 (JMA). FEIt (I JMA) at Sumoto

0.411 GREECE. MD 3.3 (ATH)

0.25 SOUTHWESTERN RYUKYU ISLANDS. FeIt ( 1 JMA) On Mi yako-jimo

\section{BURMA}

18 MARIANA ISLANDS FeIt (111) on Guam.

34 NEVADA. ML 3.2 (NEIS).

34 NEVADA. ML 2.9 (NEIS)

21 WESTERN MEDITERRANEAN SEA. MG 3.5 (MOD)

23 BURMA

11 NORWEGIAN SEA. MD 2.6 (BER)

10 FRANCE

7 MINDANAO, PHILIPPINE ISLANDS

16 TURKEY

29 SOUTH OF HONSHU, JAPAN

4 YUGOSLAVIA. MD 2.0 (TTG).

9 SICILY

18 AEGEAN SEA. ML 3.0 (ATH)

31 AEGEAN SEA. ML 3.2 (ATH).

16 SICILY

TURKEY

6 IONIAN SEA. ML 3.1 (ATH).

5 TURKEY

11 TURKEY

24 TAIWAN REGION

4 SICILY

73 AEGEAN SEA. ML 4.1 (ATH)

29 NORTH ISLAND, NEW ZEALAND

6 SAN JUAN PROVINCE, ARGENTINA

14 KIRGHIZ SSR

$\begin{array}{lrl}0.8 & 8 & \text { CENTRAL ALASKA. ML } 3.3 \text { (PMR). } \\ 0.8 & 18 & \text { NEAR EAST COAST OF HONSHU, JAPAN. FeIt (I JMA) ot Mito, }\end{array}$ Tokyo and Utsunomiyo.

1.18 TONGA ISLANDS

1.226 TAJIK-XINJIANG BORDER REGION

1.120 NORTH ISLAND, NEW ZEALAND

8 GREECE

0.57 BURMA-INDIA BORDER REGION

0.76 BULGARIA. ML 1.8 (SKO).

98 VANUATU ISLANDS

6 WESTERN MEDITERRANEAN SEA. MG 3.1 (MDD)

17 SOUTH SANDWICH ISLANOS REGION

5 LEEWARD ISLANDS. ML 3.1 (FDF) 


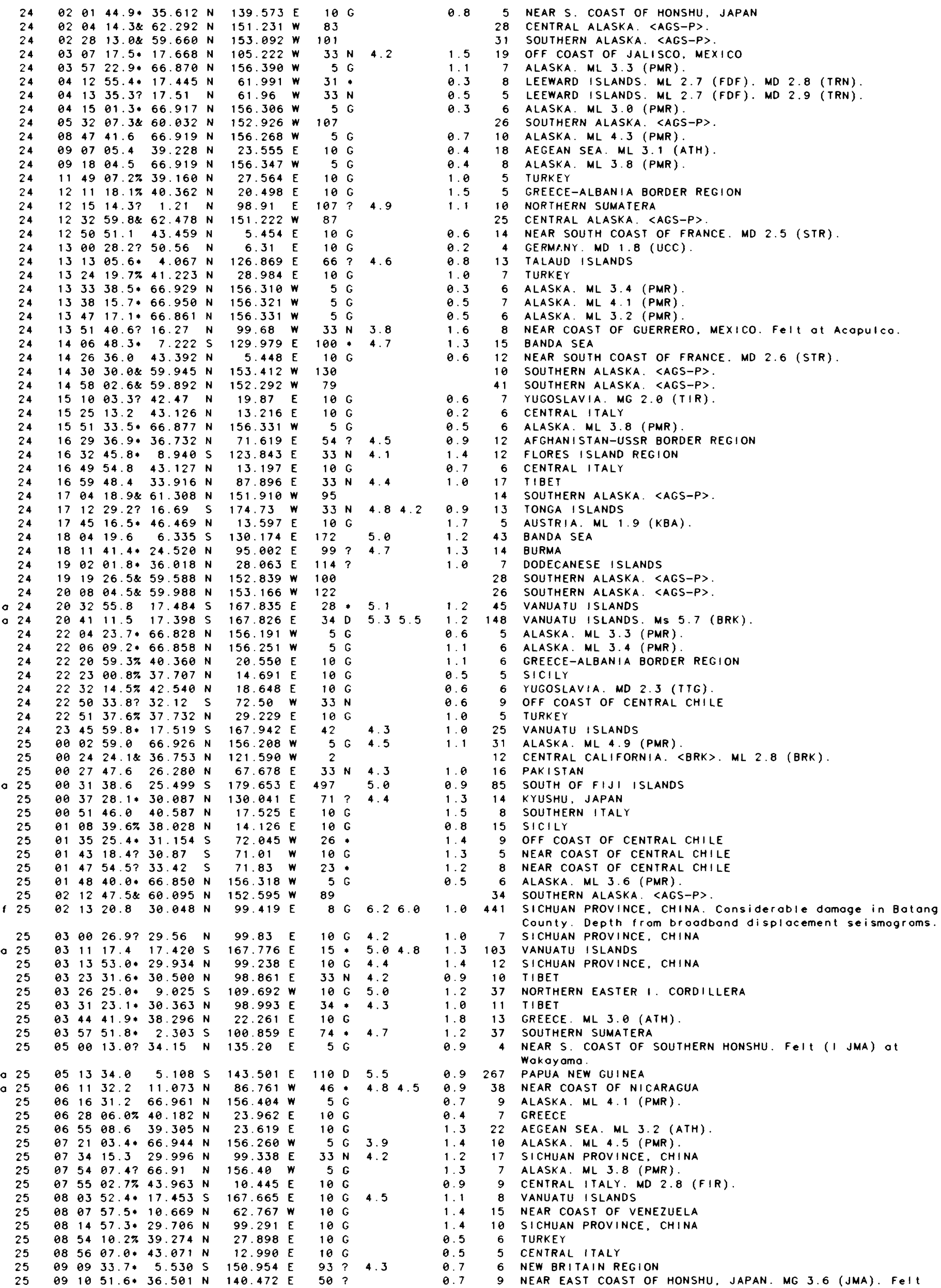




\begin{tabular}{|c|c|c|c|c|c|c|c|}
\hline 25 & 10 & 27 & 44.4 & 29.890 & $\mathbf{N}$ & 99.296 & E \\
\hline 25 & 10 & 33 & $02.5 *$ & 66.900 & $\mathbf{N}$ & 156.337 & $w$ \\
\hline 25 & 11 & 16 & $17.4 \%$ & 33.626 & $S$ & 71.741 & $w$ \\
\hline 25 & 11 & 31 & 57.4 & 42.332 & $\mathrm{~N}$ & 19.958 & E \\
\hline 25 & 11 & 44 & 17.5 & 42.986 & $\mathrm{~N}$ & 13.701 & E \\
\hline 25 & 13 & 42 & 59.9 & 66.902 & $\mathbf{N}$ & 156.331 & $w$ \\
\hline 25 & 13 & 43 & $12.3 ?$ & 51.38 & $\mathrm{~N}$ & 179.51 & $w$ \\
\hline 25 & 13 & 52 & 36.5 & 38.916 & $N$ & 21.090 & E \\
\hline 25 & 13 & 55 & $02.9 ?$ & 39.08 & $N$ & 27.54 & E \\
\hline 25 & 14 & 16 & $04.1 ?$ & 42.39 & $N$ & 0.38 & $w$ \\
\hline 25 & 14 & 18 & $12.3 \%$ & 60.589 & $N$ & 5.885 & $E$ \\
\hline 25 & 14 & 29 & 00.5 & 16.773 & $\mathrm{~N}$ & 99.328 & $w$ \\
\hline
\end{tabular}

$10 \mathrm{G}$

$5 \mathrm{G}$

18.

$10 \mathrm{G}$

$10 \mathrm{G}$

$33 \mathrm{~N} 4$

$10 \mathrm{G}$

$10 \mathrm{G}$

$10 \mathrm{G}$

$10 \mathrm{G}$
19 G 6.26 .8

( I I JMA) ot Mi to

1.112 SICHUAN PROVINCE, CHINA

0.45 ALASKA ML 3.5 (PMR)

Q.4 8 NEAR COAST OF CENTRAL CHILE

0.512 YUGOSLAVIA. MD 2.4 (TTG)

$0.7 \quad 7$ CENTRAL ITALY

0.25 ALASKA. MD 2.4 (TTG)

5 ANDREANOF ISLANDS, ALEUTIAN IS.

5 GREECE. MD 2.9 (ATH).

4 TURKEY

0.96 PYRENEES. ML 3.0 (LDG).

0.79 SOUTHERN NORWAY. MD 1.9 (BER).

380 NEAR COAST OF GUERRERO, MEXICO. MS 7.1 (BRK), 6.5 (PAS). Mo=1.4*10*19 Nm (PPT). Three people killed, o few injured and some damage of Mexico City. Minar damage reported in the Acopulco orea. Felt strongly in much of southern Mexica ond as far away as Guadalajara. Depth from broadband displacement sei smograms.$$
25
$$

$\begin{array}{llll}1456 & 25.0 & 40.715 \mathrm{~N}\end{array}$ $\begin{array}{llll}15 & 12 & 48.4 & 29.803 \mathrm{~N}\end{array}$ $155916.9 * 66.908 \mathrm{~N}$ $\begin{array}{lllll}16 & 26 & 43.9 & 16.411 \mathrm{~N}\end{array}$

$23.387 \mathrm{E}$ $99.171 \mathrm{E}$ $156.180 \mathrm{~W}$

$10 \mathrm{G}$

$\begin{array}{rrr}10 & G & 4.5\end{array}$

$5 \mathrm{G}$

22 D 4.8

$\begin{array}{llllll}16 & 42 & 19.6 ? & 39.29 \quad \mathrm{~N}\end{array}$

$\begin{array}{llll}16 & 42 & 58.6 & 30.053 \mathrm{~N}\end{array}$

$\begin{array}{lllll}16 & 44 & 33.1 & 66.929 \mathrm{~N}\end{array}$

$164956.3 \% 44.619 \mathrm{~N}$

$171838.0 \quad 35.842 \mathrm{~N}$

30 .

$99.461 \mathrm{E} \quad 10 \mathrm{G} 4.7$

$156.273 w$

$7.045 \mathrm{E}$

$140.361 \mathrm{E}$

$5 \mathrm{G}$

106

$71 \quad 5.5$

$1753 \quad 17.1 ? 66.94 \mathrm{~N}$

156.28

$99.306 \mathrm{E}$

156.34

$151.319 \mathrm{~W}$

$\begin{array}{llllll}19 & 02 & 39.3 ? & 66.93 \mathrm{~N}\end{array}$

$\begin{array}{llll}19 & 19 & 23.28 & 58.010 \mathrm{~N}\end{array}$

$\begin{array}{lllll}19 & 37 & 07.6 & 45.957 & \mathrm{~N} \\ 20 & 45 & 16.8 & 36.884 & \mathrm{~N}\end{array}$

$14.700 \mathrm{E}$ 28.45 .16 .8 .36 .8

$\begin{array}{llll}22 & 12 & 37.0 & 66.941 \mathrm{~N}\end{array}$ $\begin{array}{llll}22 & 22 & 32.1 & 42.258 \mathrm{~N}\end{array}$ $\begin{array}{lllll}23 & 10 & 12.5 ? & 15.82 \mathrm{~N}\end{array}$ $\begin{array}{llll}23 & 17 & 50.1 \% 33.472 \mathrm{~S}\end{array}$ $012257.7 .66 .898 \mathrm{~N}$ $03 \quad 30 \quad 27.1$ ? $9.48 \quad \mathrm{~S}$ $033121.6 \% 45.998 \mathrm{~N}$ $05 \quad 22 \quad 18.9 \& \quad 37.263 \mathrm{~N}$ $\begin{array}{llll}06 & 12 & 07.9 & 39.290 \mathrm{~N}\end{array}$ $\begin{array}{llllll}06 & 47 & 19.5 ? & 12.38 \quad \mathrm{~S}\end{array}$ $\begin{array}{lllll}08 & 23 & 18.3 & 42.288 \mathrm{~N}\end{array}$ $08 \quad 4658.1 \% 39.093 \mathrm{~N}$ $\begin{array}{llllll}09 & 06 & 49.47 & 39.124 \mathrm{~N}\end{array}$ $09 \quad 1644.7 * 23.102 \mathrm{~N}$ $\begin{array}{lllllll}10 & 08 & 35.7 ? & 39.17 & \mathrm{~N}\end{array}$ $102958.2 .37 .675 \mathrm{~N}$ $1109 \quad 04.8 \% \quad 39.249 \mathrm{~N}$ $\begin{array}{llllll}11 & 09 & 13.2 ? & 14.67 \mathrm{~N}\end{array}$ $\begin{array}{lllll}12 & 19 & 41.0 & 44.622 \mathrm{~N}\end{array}$ $\begin{array}{lllll}12 & 28 & 14.1 & 41.236 \mathrm{~N}\end{array}$

$134035.1 * 27.850 \mathrm{~N}$ $\begin{array}{llll}13 & 44 & 29.0 & 0.200 \mathrm{~N}\end{array}$ $1356 \quad 49.7 \% 60.635 \mathrm{~N}$ $1427 \quad 12.4 * 66.969 \mathrm{~N}$ $144410.4 \% 59.870 \mathrm{~N}$ $\begin{array}{llll}14 & 45 & 59.8 & 44.593 \mathrm{~N}\end{array}$ $\begin{array}{lllll}14 & 47 & 12.48 & 34.510 \mathrm{~N}\end{array}$ $\begin{array}{lllll}15 & 37 & 17.0 & 44.588 & N\end{array}$ $\begin{array}{llll}16 & 1206.9 * 52.373 \mathrm{~N}\end{array}$ $\begin{array}{llll}16 & 29 & 28.7 & 44.583 \mathrm{~N}\end{array}$ $\begin{array}{lllll}16 & 33 & 09.3 & 44.586 & N\end{array}$ $164730.8 * 36.273 \mathrm{~N}$

$\begin{array}{lllllll}16 & 50 & 39.5 ? & 31.01 \mathrm{~N}\end{array}$ $\begin{array}{llll}18 & 46 & 03.3 & 39.318 \mathrm{~N}\end{array}$ $\begin{array}{llll}18 & 51 & 22.1 & 39.278 \mathrm{~N}\end{array}$ $1900 \quad 40.6 \% 39.305 \mathrm{~N}$

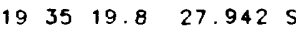
$\begin{array}{llllll}19 & 45 & 37.4 \% & 44.555 \mathrm{~N}\end{array}$ $\begin{array}{lllll}20 & 43 & 16.3 & 38.424 \mathrm{~N}\end{array}$ $2108 \quad 17.5 \cdot 36.608 \mathrm{~N}$ $\begin{array}{llllll}21 & 28 & 58.2 ? & 31.56 \quad \mathrm{~N}\end{array}$ $221008.3 * 46.443 \mathrm{~N}$ $221031.8 \& 33.930 \mathrm{~N}$ $18.721 \mathrm{E}$ $99.88 \mathrm{~W}$ $70.470 \mathrm{~W}$ $156.363 \mathrm{~W}$ $123.81 \mathrm{E}$ $27.737 \mathrm{E}$ $121.682 W$

$23.311 \mathrm{E}$ $117.74 \mathrm{E}$ $12.821 \mathrm{E}$ $27.578 \mathrm{E}$ $27.630 \mathrm{E}$ $98.959 \mathrm{E}$ $23.57 \mathrm{E}$

$20.647 \mathrm{E}$

$27.680 E$

93.32

$7.047 \mathrm{E}$

$20.892 \mathrm{E}$

$5 G$

$10 \mathrm{G} \quad 4.5$

56

57

$10 \mathrm{G}$ 1024.6

85. 229 122.119

$6.244 \mathrm{E}$

$156.510 \mathrm{~W}$

$5.089 \mathrm{E}$

$7.067 \mathrm{E}$

$120.690 \mathrm{~W}$

$7.065 \mathrm{E}$

176.306

$7.022 \mathrm{E}$

$7.070 \mathrm{E}$

$141.350 \mathrm{E}$

$\begin{array}{lll}5 & \mathrm{G} & 4.2\end{array}$

14

$33 \mathrm{~N} 4.5$

$83 ?$

5 G

$33 \mathrm{~N} 4.2$

$33 \mathrm{~N}$

$33 \mathrm{~N} 4.1$

10

$10 \mathrm{G}$

$10 \mathrm{~B}$

$33 \mathrm{~N}$

$10 \mathrm{G}$

$10 \mathrm{G}$

$10 \mathrm{G}$

$49 \cdot 4.5$

$10 \mathrm{G}$

113.9

33 .

211

5.0

5

$\begin{array}{rr}5 & G \\ 10 & G\end{array}$

$10 \mathrm{G}$

1

$10 \mathrm{G}$

$33 \mathrm{~N}$

$10 \mathrm{G}$

$10 \mathrm{G}$

$16 * 4.3$

$131.64 \mathrm{E}$

$23.457 \mathrm{E}$

$23.564 \mathrm{E}$

$28.996 \mathrm{E}$

66.846

$6.977 \mathrm{E}$

119.161

$30.150 \mathrm{E}$

$35.57 \quad E$

$9.526 \mathrm{E}$

$118.580 \mathrm{~W}$

$55:$

$10 \mathrm{G}$

$10 \mathrm{G}$

$92 * 4.6$

$10 \mathrm{G}$

$5 \mathrm{G}$

$59 ?$

10

11

$2230 \quad 13.9 ? \quad 31.40 \quad 5$

177.76

$126 ? 4.7$

$\begin{array}{llll}23 & 40 & 46.2 \quad 5.165 \mathrm{~S}\end{array}$ $00 \quad 0857.1 * 29.954 \mathrm{~N}$ $\begin{array}{llllll}01 & 08 & 37 & 3 \& & 60 & 138\end{array}$ $020222.9860 .032 \mathrm{~N}$

$43.491 E 111 * 5.0$

99.288 E $33 \mathrm{~N} 4.1$

$153.136 \mathrm{~W} 121$

$153.199 W 127$

$\begin{array}{llllllllll}02 & 20 & 04.7 & 30.601 & \mathrm{~N} & 140.589 \mathrm{E} & 85 & \mathrm{G} & 6.1\end{array}$
GREECE. ML 2.4 (SKO)

$1.0 \quad 10$ GREECE. ML 15 SICHUAN PROVINCE, CHINA

0.97 ALASKA. ML 3.8 (PMR)

1.244 NEAR COAST OF GUERRERO, MEXICO. FeIt in the

ACOPUICO-MEXico City area.

0.69 AEGEAN SEA

1.331 SICHUAN PROVINCE, CHINA

5 10 ALASKA. ML 3.9 (PMR)

25 NORTHERN ITALY. ML 2.2 (GEN)

0.9253 NEAR EAST COAST OF HONSHU, JAPAN. FeIt $(111$ JMA) at

Chibo, (II JMA) ot Tokyo and (I JMA) at Shizuoka.

0.75 ALASKA. ML 3.1 (PMR)

1.215 SICHUAN PROVINCE, CHINA

28 KODIAK ISLAND REGION. LAGS-P>

0.99 YUGOSLAVIA. MD 3.4 (LJU), 2.8 (TRI)

1.059 NEAR EAST COAST OF HONSHU, JAPAN. FeIt (1।1 JMA) at Onahama and Mito. ( 1 I JMA) at Utsunomiya and ( 1 JMA) at Shirakawa

0.811 ALASKA. ML 4.2 (PMR)

1.211 YUGOSLAVIA. MD 2.5 (TTG)

1.66 OFF COAST OF GUERRERO, MEXICO

9 CHILE-ARGENTINA BORDER REGION

5 ALASKA. ML 3.2 (PMR)

5 TIMOR

5 ROMANIA

11 CENTRAL CALIFORNIA. 〈BRK>. ML 2.6 (BRK).

1.231 AEGEAN SEA. ML 3.5 (ATH).

0.75 SOUTH OF SUMBAWA ISLAND

1.010 CENTRAL ITALY

0.4 TURKEY

0. 55 TURKEY

0. 25 BURMA-CHINA BORDER REGION

0. 47 AEGEAN SEA

0.75 IONIAN SEA. MD 3.3 (ATH)

0.76 TURKEY

1.1 20 NEAR COAST OF CHIAPAS. MEXICO

0.3 21 NORTHERN ITALY. ML 2.8 (LDG), 2.3 (GEN).

55 ALBANIA. ML 3.7 (TTG) 3.7 (ATH). Felt (V) at Kicevo and Resen, Yugosiavia.

1.58 NEPAL

1.0 80 MINAHASSA PENINSULA

0.86 SOUTHERN NORWAY. MD 1.8 (BER)

0.78 ALASKA. ML 3.4 (PMR)

0.6 6 SOUTHERN NORWAY. MD 1.9 (BER)

0.618 NORTHERN ITALY. ML 2.4 (GEN), 2.3 (LDG)

11 SOUTHERN CALIFORNIA, $\angle P A S-P\rangle$. ML 3.1 (PAS)

0.6 20 NORTHERN ITALY. ML 2.4 (LDG), 2.3 (GEN)

1.25 ANDREANOF ISLANDS. ALEUTIAN IS. FeIt on Adak.

0. 314 NORTHERN ITALY. ML 2.0 (GEN), 2.0 (LDG).

0.844 NORTHERN ITALY. ML 3.1 (LDG), 3.0 (GEN)

1.213 NEAR EAST COAST OF HONSHU, JAPAN. FeIt (II JMA) ot Mito and ( I JMA) at Onahama

0.9 10 KYUSHU, JAPAN

0.814 AEGEAN SEA. ML 3.0 (ATH)

0.924 AEGEAN SEA. ML 3.3 (ATH).

1.37 TURKEY

0.920 CATAMARCA PROVINCE, ARGENTINA

0.27 FRANCE. ML 2.0 (GEN)

1.211 CALIFORNIA-NEVADA BORDER REGION. ML 2.7 (BRK).

0.67 TURKEY

0.5 DEAD SEA REGION

0.45 SWITZERLAND. ML 2.5 (LDG)

16 SOUTHERN CALIFORNIA. 〈PAS-P>. ML 3.4 (PAS). Felt in the Sonta Monica-Tar rance area.

1.39 KERMADEC ISLANDS REGION

19 NORTHERN CALIFORNIA. <BRK>. ML 3.4 (BRK). FeIt (IV) at COBD.

1.116 PAPUA NEW GUINEA

0.78 SICHUAN PROVINCE, CHINA

16 SOUTHERN ALASKA. 〈AGS-P>.

18 SOUTHERN ALASKA. LAGS-P>.

1.0454 SOUTH OF HONSHU, JAPAN, mb 6.6 (BRK). MO=6.0*10*17 $\mathrm{Nm}$ (PPT). Felt (II JMA) at Takya, Tateyama and on

Chichi-shima: (I JMA) at Choshi. Hiroo, Onohama ond on
0.35 ALASKA. ML 3.6 (PMR) 


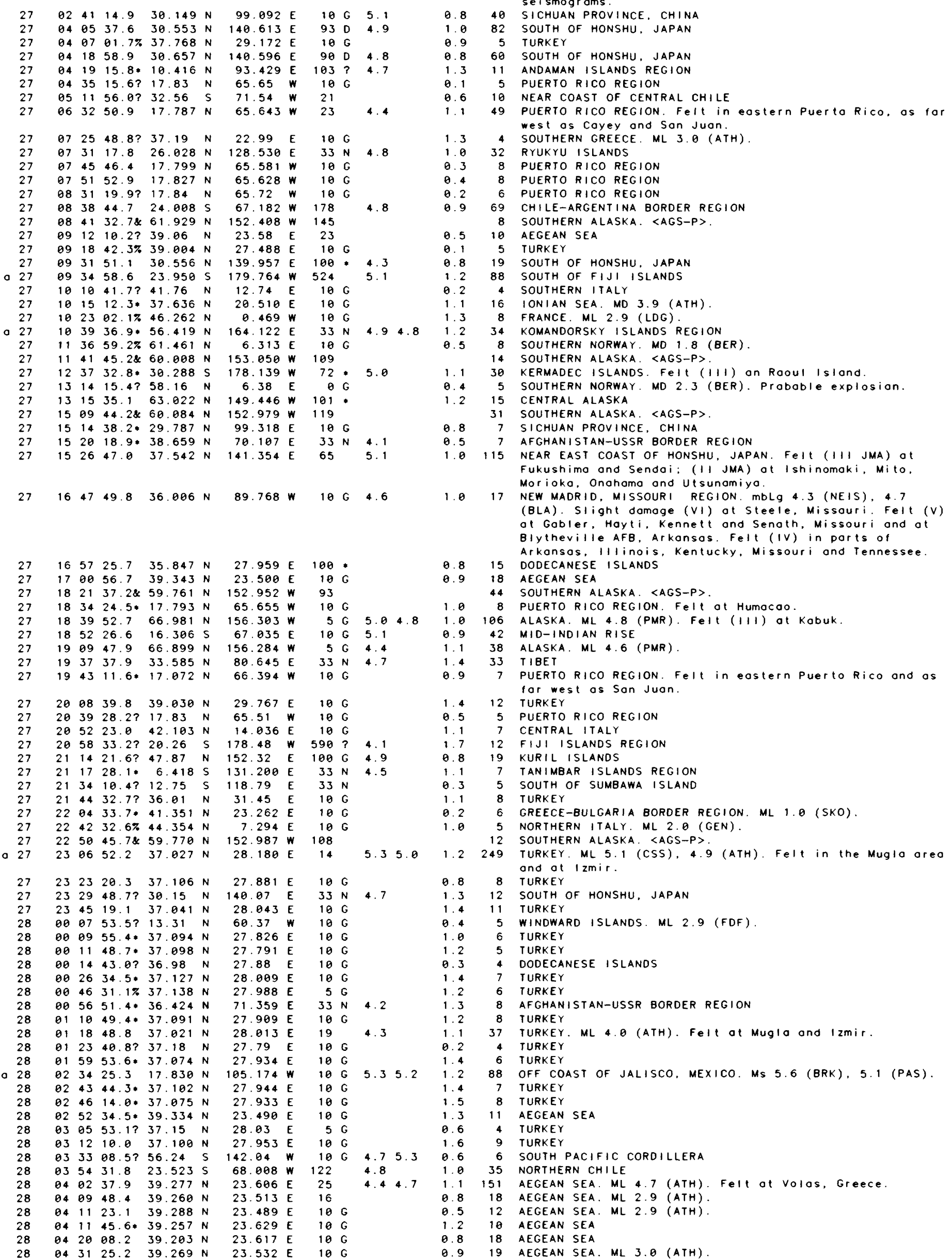

Hachijo-jima. Depth from broadband displacement se ismograms.

840 SichUAN PROVINCE, CHINA

1. 82 SOUTH OF HONSHU, JAPAN

TURKEY

1.311 ANDAMAN ISLANDS REgION

O NEAR COAST OF CENTRAL CHILE

PUERTO RICO REGION Felt in eostern puerto Rico, as far

PUERTO RICO REGION

PUERTO RICO REGION

6 PUERTO RICO REGION

KOMANDORSKY ISLANDS REGION

explosian. (BLA). Slight damage (VI) at Steele. Missauri. Felt (V) SE ISLANDS

4 SOUTHERN ALASKA <AGS-P

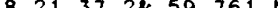
$83424.5 * 17.793 \mathrm{~N}$ $\begin{array}{llll}18 & 52 & 26.6 & 16.306\end{array}$

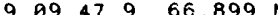
$\begin{array}{lllll}9 & 37 & 37.9 & 33.585\end{array}$

$\begin{array}{llllll}28 & 08 & 39.8 & 39.030 \mathrm{~N}\end{array}$ $211728.1 * 6.4185$ $\begin{array}{llllll}21 & 34 & 10.4 ? & 12.75\end{array}$ $\begin{array}{lllll}22 & 42 & 32.67 & 44.354 \mathrm{~N}\end{array}$ $2250 \quad 45.7 \& 59.770 \mathrm{~N}$ $\begin{array}{lllll}23 & 23 & 20.3 & 37.106 \mathrm{~N}\end{array}$

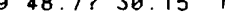
$\begin{array}{lllllll}00 & 07 & 53.5 ? & 13.31 \mathrm{~N}\end{array}$ $000955.4 * 37.094 \mathrm{~N}$ $90 \quad 26 \quad 34.5 * 37.127 \mathrm{~N}$ $\begin{array}{lllllll}90 & 46 & 31.1 \% & 37.138 \mathrm{~N}\end{array}$ $\begin{array}{llllllllll}03 & 95 & 53 & 1 & 37 & 15 & \mathrm{~N}\end{array}$ $04 \quad 02 \quad 37.9 \quad 39.277 \mathrm{~N}$ $04 \quad 3125.2 \quad 39.269 \mathrm{~N}$
$10 \mathrm{G}$

$5 G \quad 5.04 .8$

5.

G 4 . 4

$10 \mathrm{G}$

$10 \mathrm{G}$

$10 \mathrm{G}$

? 4.

$33 \mathrm{~N} 4.5$

$33 \mathrm{~N}$

$16 \mathrm{G}$

33

10

$10 G$

19

(日)

10

$10 \mathrm{G}$

$10 \mathrm{G}$

4.75 .3

4.8

$23.606 \mathrm{E}$

$23.513 \mathrm{E}$

23. $532 \mathrm{E}$ 


\begin{tabular}{|c|c|c|c|c|c|c|c|c|c|c|c|c|}
\hline & 28 & 04 & 39 & 49.3 & 39.283 & N & 23.718 & E & 10 & G & & 1.2 \\
\hline & 28 & 64 & 54 & 57.6 & 39.244 & $\mathbf{N}$ & 23.609 & $E$ & 10 & G & & 0.8 \\
\hline & 28 & 05 & 05 & $48.6 ?$ & 37.26 & $N$ & 27.97 & $E$ & 10 & G & & 1.4 \\
\hline & 28 & 05 & 15 & 07.7 . & 39.054 & $N$ & 23.623 & $E$ & 10 & G & & 0.5 \\
\hline & 28 & 05 & 29 & $55.2 *$ & 37.059 & $\mathbf{N}$ & 27.992 & $E$ & 10 & G & & 1.2 \\
\hline & 28 & 05 & 30 & 03.0 & 39.252 & $\mathbf{N}$ & 23.648 & $E$ & 10 & G & & 0.9 \\
\hline & 28 & 05 & 40 & $22.9 ?$ & 38.85 & $\mathbf{N}$ & 23.65 & $E$ & 12 & & & 0.6 \\
\hline & 28 & 66 & 37 & $32.5 ?$ & 37.18 & $\mathbf{N}$ & 28.11 & $E$ & 10 & G & & 0.4 \\
\hline & 28 & 06 & 48 & 47.5 & 37.092 & $N$ & 27.912 & $E$ & 5 & G & & 1.4 \\
\hline & 28 & 07 & 02 & 45.6 & 39.265 & $N$ & 23.497 & $E$ & 10 & G & & 0.8 \\
\hline & 28 & 67 & 24 & $27.5 \%$ & 37.207 & $\mathbf{N}$ & 27.997 & $E$ & 10 & G & & 1.2 \\
\hline o & 28 & 07 & 48 & 19.6 & 13.245 & $\mathbf{N}$ & 89.675 & $w$ & 59 & & 5.2 & 1.2 \\
\hline & 28 & 08 & 09 & $54.6 *$ & 37.077 & $N$ & 27.925 & $E$ & 10 & G & & 1.3 \\
\hline & 28 & 08 & 12 & $13.9 ?$ & 17.83 & $N$ & 65.65 & $w$ & 10 & G & & 0.1 \\
\hline & 28 & 08 & 16 & $05.1 *$ & 37.076 & $\mathbf{N}$ & 27.999 & $E$ & 10 & G & & 1.1 \\
\hline & 28 & 08 & 22 & $43.38 c$ & 60.126 & $\mathbf{N}$ & 153.249 & $w$ & 134 & & & \\
\hline & 28 & 08 & 58 & $12.9 \%$ & 39.032 & $\mathbf{N}$ & 27.650 & $E$ & 10 & G & & 1.4 \\
\hline o & 28 & 09 & 13 & 46.2 & 20.163 & $\mathrm{~S}$ & 67.478 & $\mathbf{E}$ & 10 & G & 5.24 .9 & 1.0 \\
\hline & 28 & 09 & 22 & $06.9 *$ & 29.901 & $\mathbf{N}$ & 99.359 & $E$ & 10 & G & 4.8 & 1.2 \\
\hline o & 28 & 09 & 23 & 07.3 & 16.606 & $\mathrm{~S}$ & 173.645 & $w$ & 93 & D & 5.4 & 1.0 \\
\hline & 28 & 10 & 04 & $00.8 ?$ & 38.85 & $\mathbf{N}$ & 23.63 & $E$ & 10 & G & & 0.4 \\
\hline & 28 & 10 & 10 & $02.8 ?$ & 37.20 & $N$ & 27.90 & $E$ & 10 & G & & 0.8 \\
\hline & 28 & 11 & 12 & 53.7 & 30.588 & $\mathbf{N}$ & 140.556 & $E$ & 117 & * & 4.6 & 1.3 \\
\hline & 28 & 11 & 21 & $22.3 ?$ & 36.95 & $N$ & 27.81 & $E$ & 10 & G & & 1.4 \\
\hline & 28 & 11 & 39 & 46.4 & 39.256 & $N$ & 23.570 & $E$ & 10 & & & 0.7 \\
\hline & 28 & 11 & 51 & $16.3 \%$ & 31.715 & $\mathrm{~S}$ & 117.064 & $E$ & 10 & G & & 0.3 \\
\hline & 28 & 12 & 31 & 19.0 & 24.840 & $N$ & 122.263 & E & 100 & $\bullet$ & 3.9 & 1.4 \\
\hline & 28 & 12 & 40 & $10.8 *$ & 37.103 & $N$ & 27.998 & $E$ & 10 & G & & 1.4 \\
\hline & 28 & 13 & 14 & $58.5 *$ & 29.888 & $N$ & 99.083 & $\mathbf{E}$ & 10 & G & 4.6 & 1.5 \\
\hline & 28 & 13 & 19 & $59.6 ?$ & 41.98 & $N$ & 23.08 & $E$ & 10 & G & & 0.7 \\
\hline o & 28 & 13 & 30 & 19.2 & 37.004 & $N$ & 28.139 & $E$ & 17 & & 5.15 .1 & 1.0 \\
\hline & 28 & 13 & 39 & $12.5 *$ & 37.086 & $N$ & 27.881 & E & 10 & G & & 1.5 \\
\hline & 28 & 13 & 43 & $34.4 ?$ & 16.57 & $\mathrm{~S}$ & 174.51 & $w$ & 173 & * & 4.6 & 0.3 \\
\hline & 28 & 13 & 44 & 05.1 & 36.982 & $\mathrm{~N}$ & 28.006 & $E$ & 20 & & 4.0 & 1.2 \\
\hline & 28 & 14 & 04 & $00.0 ?$ & 37.12 & $N$ & 28.05 & $E$ & 10 & G & & 0.4 \\
\hline & 28 & 14 & 05 & 35.2 * & 37.122 & $N$ & 27.867 & $E$ & 10 & G & & 1.3 \\
\hline & 28 & 14 & 18 & $33.4 *$ & 37.123 & $\mathrm{~N}$ & 27.847 & $E$ & 10 & G & & 1.3 \\
\hline & 28 & 14 & 33 & 59.7 & $\begin{array}{l}36.537 \\
37\end{array}$ & $\mathrm{~N}$ & $\begin{array}{r}70.890 \\
27.860\end{array}$ & $E$ & $\begin{array}{r}174 \\
19\end{array}$ & 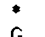 & 3.9 & 0.7 \\
\hline & $\begin{array}{l}28 \\
28\end{array}$ & $\begin{array}{l}14 \\
14\end{array}$ & $\begin{array}{l}50 \\
52\end{array}$ & 56.4 & $\begin{array}{l}37.089 \\
37.063\end{array}$ & $\begin{array}{l}N \\
N\end{array}$ & $\begin{array}{l}27.860 \\
27.962\end{array}$ & $\begin{array}{l}E \\
E\end{array}$ & $\begin{array}{r}10 \\
7\end{array}$ & 6 & 4.2 & $\begin{array}{l}1.3 \\
1.5\end{array}$ \\
\hline & 28 & 15 & 13 & 54.7 & 39.226 & $N$ & 23.632 & $E$ & 10 & G & & 0.7 \\
\hline & 28 & 15 & 29 & 37.1 & 39.241 & $N$ & 23.601 & $E$ & 22 & & 3.6 & 0.9 \\
\hline & 28 & 15 & 49 & 20.5 & 43.268 & $N$ & 0.407 & $w$ & 10 & G & & 1.4 \\
\hline & 28 & 15 & 54 & 42.0 & 39.310 & $N$ & 23.502 & $E$ & 10 & G & & 0.6 \\
\hline & 28 & 16 & 01 & $50.5 *$ & 37.093 & $\mathbf{N}$ & 27.952 & $E$ & 10 & G & & 1.5 \\
\hline & 28 & 16 & 11 & 21.6 & 37.089 & $N$ & 28.005 & $E$ & 10 & G & & 0.4 \\
\hline & 28 & 16 & 48 & $16.7 *$ & 37.111 & $\mathrm{~N}$ & 27.967 & E & 10 & G & & 1.5 \\
\hline & 28 & 17 & 13 & 22.9. & 37.095 & $\mathbf{N}$ & 27.909 & $E$ & 10 & G & & 1.3 \\
\hline o & $\begin{array}{l}28 \\
28\end{array}$ & 17 & $\begin{array}{l}24 \\
38\end{array}$ & $\begin{array}{l}33.6 \\
26.4\end{array}$ & $\begin{array}{r}37.115 \\
4.354\end{array}$ & $\begin{array}{l}N \\
S\end{array}$ & $\begin{array}{r}27.940 \\
101.413\end{array}$ & $E$ & 10 & G & & 1. 2 \\
\hline 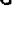 & $\begin{array}{l}20 \\
28\end{array}$ & 17 & 40 & 13.4 . & 37.107 & $\mathrm{~N}$ & 27.930 & $E$ & $\begin{array}{l}27 \\
10\end{array}$ & G & 5.45 .8 & $\begin{array}{l}1.0 \\
1.5\end{array}$ \\
\hline & 28 & 18 & 33 & 12.8 & 37.100 & $\mathbf{N}$ & 27.950 & $E$ & 10 & G & & 1.0 \\
\hline & 28 & 19 & 26 & $15.2 ?$ & 41.93 & $\mathbf{N}$ & 128.24 & $w$ & 10 & G & 4.5 & 1.7 \\
\hline & 28 & 19 & 32 & 55.5 & 37.084 & $N$ & 27.814 & $E$ & 10 & G & & 0.6 \\
\hline $\mathbf{0}$ & 28 & 20 & 08 & 51.5 & 4.294 & $\mathrm{~S}$ & 101.363 & $E$ & 30 & D & 5.25 .6 & 1.1 \\
\hline $\boldsymbol{a}$ & 28 & 20 & 26 & 17.9 & 59.515 & S & 29.397 & $w$ & 22 & D & $5.5 \quad 5.7$ & 1.4 \\
\hline & 28 & 20 & 43 & $24.7 ?$ & 37.17 & $N$ & 27.99 & $\mathbf{E}$ & 10 & G & & 1.5 \\
\hline & 28 & 20 & 53 & 50.2 & 43.344 & $\mathbf{N}$ & 5.679 & $E$ & 10 & G & & 0.4 \\
\hline & 28 & 21 & 08 & $56.1 *$ & 37.083 & $N$ & 27.916 & $E$ & 10 & G & & 0.3 \\
\hline a & 28 & 21 & 09 & 57.6 & 4.210 & $\mathrm{~S}$ & 101.370 & $E$ & 32 & D & 5.45 .7 & 1.0 \\
\hline & 28 & 21 & 11 & 42.6 & 37.081 & $N$ & 27.915 & $E$ & 10 & G & & 1.1 \\
\hline & 28 & 22 & 00 & $41.7 \%$ & 37.162 & $\mathrm{~N}$ & 27.938 & $E$ & 10 & G & & 0.8 \\
\hline & 28 & 22 & 13 & $03.5 *$ & 4. 187 & $\mathrm{~S}$ & 101.503 & $E$ & 33 & $\mathbf{N}$ & 5.0 & 0.7 \\
\hline & 28 & 22 & $\begin{array}{l}18 \\
59\end{array}$ & 23.1 & 39.942 & $N_{N}^{N}$ & 23.893 & E & 10 & G & & 0.9 \\
\hline & 29 & 00 & 10 & 40.5 & 39.800 & $\mathrm{~N}$ & 30.503 & E & 10 & $G$ & 3.5 & 1.0 \\
\hline o & 29 & 01 & 03 & 06.8 & 5.494 & $s$ & 102.976 & $E$ & 38 & $D$ & 5.45 .1 & 1.1 \\
\hline & 29 & 01 & 06 & $55.1 ?$ & 37.74 & $\mathbf{N}$ & 23.61 & $E$ & 10 & G & & 1.0 \\
\hline & 29 & 01 & 38 & $33.6 ?$ & 34.03 & $S$ & 71.92 & $w$ & 33 & $\mathbf{N}$ & & 0.2 \\
\hline & 29 & 01 & 51 & $57.2 ?$ & 39.22 & $\mathbf{N}$ & 23.28 & $E$ & 10 & G & & 0.6 \\
\hline & 29 & 02 & 14 & $20.3 *$ & 39.371 & $\mathbf{N}$ & 16.965 & $E$ & 10 & G & & 0.4 \\
\hline & 29 & 02 & 22 & $56.6 \%$ & 37.156 & $N$ & 27.968 & $E$ & 5 & G & & 0.9 \\
\hline & 29 & 02 & 23 & 57.4 & 39.327 & $N$ & 16.774 & $E$ & 10 & G & & 0.6 \\
\hline & 29 & 02 & 25 & $20.2 \%$ & 39.370 & $\mathbf{N}$ & 16.799 & $E$ & 10 & G & & 0.7 \\
\hline & 29 & 02 & 26 & $37.2 \%$ & 39.329 & $\mathbf{N}$ & 16.797 & $E$ & 10 & G & & 0.7 \\
\hline & 29 & 02 & 55 & 51.0 & 37.787 & $\mathrm{~N}$ & 142.446 & E & 33 & & 4.6 & 1.1 \\
\hline & 29 & 03 & 10 & $40.8 *$ & 66.938 & $N$ & 156.412 & $w$ & 5 & G & & 0.4 \\
\hline & 29 & 03 & 16 & 32.4 & 66.940 & $\mathbf{N}$ & 156.339 & $w$ & 5 & G & & 0.2 \\
\hline & 29 & 04 & 04 & $03.3 *$ & 37.022 & $N$ & 27.966 & $E$ & 10 & G & & 1.4 \\
\hline & 29 & 04 & 11 & $12.1 *$ & 17.356 & $S$ & 174.288 & $w$ & 149 & D & 4.6 & 1.0 \\
\hline & 29 & 04 & 11 & $12.8 *$ & 37.080 & $\mathbf{N}$ & 27.973 & E & 10 & G & & 1.6 \\
\hline & 29 & 04 & 23 & 39.8 & 37.119 & $N$ & 27.939 & $E$ & 10 & G & & 0.9 \\
\hline & 29 & 04 & 44 & $47.0 \%$ & 37.204 & $N$ & 27.921 & $E$ & 10 & G & & 1.4 \\
\hline & 29 & 05 & 41 & 33.3 & 37.105 & $N$ & 27.886 & $E$ & 10 & G & & 1.4 \\
\hline & 29 & 06 & 15 & $15.7 \%$ & 39.429 & $N$ & 28.094 & $E$ & 10 & G & & 1.3 \\
\hline & 29 & 06 & 25 & 39.1 & 57.190 & $\mathbf{N}$ & 122.069 & E & 30 & D & 5.35 .1 & 0.9 \\
\hline & 29 & 06 & 26 & 52.8 & 46.167 & $\mathrm{~N}$ & 11.919 & $E$ & 10 & G & & 0.8 \\
\hline & 29 & 06 & 40 & $49.7 *$ & 37.074 & $\mathbf{N}$ & 27.909 & $E$ & 10 & G & & 1.5 \\
\hline & $\begin{array}{l}29 \\
29\end{array}$ & $\begin{array}{l}06 \\
07\end{array}$ & $\begin{array}{l}47 \\
29\end{array}$ & $\begin{array}{l}18.7 \\
27.2 .\end{array}$ & $\begin{array}{l}39.262 \\
37.143\end{array}$ & $\begin{array}{l}\mathrm{N} \\
\mathrm{N}\end{array}$ & $\begin{array}{l}23.424 \\
27.959\end{array}$ & $\begin{array}{l}E \\
F\end{array}$ & $\begin{array}{l}10 \\
10\end{array}$ & G & & $\begin{array}{l}1.2 \\
1.5\end{array}$ \\
\hline
\end{tabular}




\begin{tabular}{|c|c|c|c|c|c|c|c|c|c|c|c|c|c|c|}
\hline 29 & 07 & 40 & $52 \cdot 4 *$ & 25.610 & $\mathrm{~s}$ & 179.378 & $w$ & 457 & $?$ & 4.6 & & 1.1 & 18 & SOUTH OF FIJI ISLANDS \\
\hline 29 & 08 & 08 & $08.0 ?$ & 37.02 & $N$ & 28.05 & $\mathbf{E}$ & 5 & G & & & 0.9 & 4 & TURKEY \\
\hline 29 & 08 & 26 & $39.5 *$ & 44.292 & $N$ & 149.107 & $E$ & 33 & $N$ & 4.7 & & 1.4 & 12 & KURIL ISLANDS \\
\hline 29 & 08 & 43 & 58.5 & 40.565 & $N$ & 24.180 & $\mathbf{E}$ & 10 & G & & & 0.8 & 7 & AEGEAN SEA \\
\hline 29 & 08 & 58 & 04.7 & 44.291 & N & 7.487 & $\mathbf{E}$ & 10 & G & & & 0.5 & 16 & NORTHERN ITALY. ML 2.2 (GEN) \\
\hline 29 & 09 & 09 & $42.0 \%$ & 39.111 & $N$ & 27.556 & $\mathbf{E}$ & 10 & G & & & 0.7 & 5 & TURKEY \\
\hline 29 & 09 & 35 & 39.0. & 66.876 & $N$ & 156.230 & $w$ & 5 & G & & & 0.6 & 6 & ALASKA. ML 2.8 (PMR) \\
\hline 29 & 10 & 14 & 33.0 & 7.320 & $S$ & 120.692 & E & 490 & & 4. 6 & & 1.0 & 58 & FLORES SEA \\
\hline 29 & 10 & 37 & $26.1 ?$ & 37.12 & $N$ & 27.91 & $\mathbf{E}$ & 10 & G & & & 1.6 & 4 & TURKEY \\
\hline 29 & 11 & 37 & $54.0 ?$ & 37.02 & $N$ & 27.97 & $E$ & 10 & G & & & 0.8 & 4 & TURKEY \\
\hline 29 & 12 & 10 & $33.2 ?$ & 37.12 & $N$ & 28.02 & $E$ & 10 & G & & & 1.3 & 5 & TURKEY \\
\hline 29 & 12 & 35 & $53.1 ?$ & 34.28 & $N$ & 139.87 & $E$ & 33 & $N$ & 48 & & 0.9 & 6 & NEAR S. COAST OF HONSHU, JAPAN \\
\hline 29 & 12 & 55 & $14.5 ?$ & 25.25 & $N$ & 91.60 & $\mathbf{E}$ & 33 & N & 4.3 & & 0.4 & 7 & INDIA-BANGLADESH BORDER REGION \\
\hline 29 & 13 & 50 & 12.0 & 36.599 & N & 141.038 & $\mathbf{E}$ & 58 & & 4.3 & & 0.8 & 26 & $\begin{array}{l}\text { NEAR EAST COAST OF HONSHU, JAPAN. Felt ( I JMA) ot Mito } \\
\text { and Onohomo; ( } 1 \text { JMA) of Utsunomiyo. }\end{array}$ \\
\hline 29 & 15 & 04 & $25.0 ?$ & 21.72 & $s$ & 177.22 & $\mathbf{w}$ & 400 & G & 4.6 & & 0.8 & 12 & FIJI ISLANDS REGION \\
\hline 29 & 15 & 12 & 02.4 & 51.786 & N & 175.226 & $w$ & 33 & N & 5.1 & 4.3 & 0.8 & 132 & $\begin{array}{l}\text { ANDREANOF ISLANDS, ALEUTIAN IS. ML } 4.7 \text { (PMR). FeIt (IV) } \\
\text { ON AdOK. }\end{array}$ \\
\hline 29 & 15 & 52 & 53.2 & 66.922 & N & 156.321 & $w$ & 5 & G & 4.1 & & 0.7 & 22 & ALASKA. ML 4.2 (PMR). Felt ot Kobuk. \\
\hline 29 & 16 & 40 & $07.7 \%$ & 41.681 & N & 27.563 & $\mathbf{E}$ & 10 & G & & & 1.1 & 5 & TURKEY \\
\hline 29 & 18 & 10 & $00.0 ?$ & 2.03 & N & 126.53 & $\mathbf{E}$ & 113 & $?$ & 4.0 & & 0.4 & 7 & MOLUCCA PASSAGE \\
\hline 29 & 18 & 51 & $22.2 *$ & 66.900 & N & 156.327 & $w$ & 5 & $\mathbf{G}$ & & & 0.2 & 5 & ALASKA. ML 3.6 (PMR). \\
\hline 29 & 19 & 26 & 48.0 & 37.139 & N & 27.932 & $\mathbf{E}$ & 10 & G & & & 0.5 & 7 & TURKEY \\
\hline 29 & 19 & 34 & 13.7 & 37.099 & $N$ & 27.908 & $\mathbf{E}$ & 10 & G & & & 1.1 & 12 & TURKEY \\
\hline 29 & 20 & 18 & $39.4 *$ & 34.166 & $S$ & 71.130 & $w$ & 88 & $\bullet$ & & & 0.2 & 12 & NEAR COAST OF CENTRAL CHILE \\
\hline 29 & 20 & 32 & $25 \cdot 4 ?$ & 16.04 & $\mathrm{~S}$ & 173.63 & $w$ & 92 & D & 4.6 & & 0.9 & 12 & TONGA ISLANDS \\
\hline 29 & 20 & 58 & $08.2 *$ & 37.068 & $N$ & 27.876 & $\mathbf{E}$ & 10 & G & & & 1.3 & 7 & TURKEY \\
\hline 29 & 21 & 42 & $52 \cdot 2 ?$ & 45.01 & $N$ & 3.57 & $\mathbf{E}$ & 10 & G & & & 1.1 & 9 & FRANCE. ML 2.9 (LDG). \\
\hline 29 & 21 & 57 & $48.0 *$ & 0.867 & $\mathrm{~S}$ & 98.064 & $\mathbf{E}$ & 42 & $?$ & $4 \cdot 4$ & & 1.2 & 23 & SOUTHERN SUMATERA \\
\hline 29 & 21 & 59 & 28.18 & 60.391 & $N$ & 152.220 & $w$ & 99 & & & & & 39 & SOUTHERN ALASKA. 〈AGS-P>. \\
\hline 29 & 22 & 01 & $45.7 ?$ & 0.90 & $S$ & 80.41 & $w$ & 33 & N & 4.6 & & 1.3 & 13 & NEAR COAST OF ECUADOR \\
\hline 29 & 22 & 19 & 54.7 & 37.099 & $N$ & 27.882 & $\mathbf{E}$ & 10 & G & & & 1.1 & 14 & TURKEY \\
\hline 29 & 22 & 26 & $25.1 \%$ & 18.256 & $N$ & 66.310 & $w$ & 10 & G & & & 0.3 & 6 & PUERTO RICO REGION \\
\hline 29 & 22 & 28 & 34.1 & 39.282 & $N$ & 23.591 & $E$ & 11 & & & & 1.0 & 38 & AEGEAN SEA. ML 3.3 (ATH) \\
\hline 29 & 22 & 31 & 06.0 & 37.098 & $N$ & 27.904 & $\mathbf{E}$ & 10 & G & & & 1.3 & 9 & TURKEY \\
\hline 29 & 23 & 52 & $26.7 \%$ & 10.942 & N & 62.120 & $w$ & 33 & $N$ & & & 0.7 & 8 & NEAR COAST OF VENEZUELA. MD 3.7 (TRN) \\
\hline 30 & 00 & 07 & $57.7 *$ & 0.868 & S & 98.062 & E & 33 & N & 4.3 & & 1.2 & 19 & SOUTHERN SUMATERA \\
\hline 30 & 00 & 10 & $58.0 ?$ & 41.79 & N & 12.74 & $\mathbf{E}$ & 10 & G & & & 0.1 & 4 & SOUTHERN ITALY \\
\hline 30 & 00 & 29 & 44.9 & 11.297 & N & 68.071 & $w$ & 10 & G & 4.9 & 4. 2 & 1.3 & 50 & NEAR COAST OF VENEZUELA \\
\hline 30 & 00 & 37 & $20.1 *$ & 66.920 & N & 156.230 & w & 5 & G & & & 0.4 & 6 & ALASKA. ML 3.1 (PMR). \\
\hline 30 & 00 & 48 & 21.2 & 66.978 & $N$ & 156.159 & $w$ & 5 & G & 4.5 & & 1.1 & 34 & ALASKA. ML 4.8 (PMR). \\
\hline 30 & 02 & 18 & $27.2 ?$ & 41.58 & N & 12.45 & $E$ & 10 & G & & & 0.2 & 4 & SOUTHERN ITALY \\
\hline 30 & 02 & 38 & $29.2 *$ & 37.082 & N & 27.834 & $\mathbf{E}$ & 10 & G & & & 0.7 & 6 & TURKEY \\
\hline 30 & 02 & 38 & $38.8 \&$ & 58.048 & N & 154,134 & $w$ & 73 & & & & & 10 & ALASKA PENINSULA. $\angle A G S-P\rangle$. \\
\hline 30 & 03 & 04 & $04.6 ?$ & 17.88 & N & 65.75 & $w$ & 10 & G & & & 0.2 & 5 & PUERTO RICO REGION \\
\hline 30 & 03 & 07 & $54.3 ?$ & 18.46 & N & 65.91 & $w$ & 10 & G & & & 1.1 & 4 & PUERTO RICO REGION \\
\hline 30 & 03 & 15 & $21.4 ?$ & 20.29 & $S$ & 176.41 & $w$ & 278 & $?$ & 4. 4 & & 0.6 & 11 & FIJI ISLANDS REGION \\
\hline 30 & 03 & 36 & $20.0 *$ & 34.925 & N & 137.072 & $\mathbf{E}$ & 33 & $N$ & & & 0.4 & 7 & $\begin{array}{l}\text { NEAR S. COAST OF HONSHU, JAPAN. MG } 3.6 \text { (JMA). Felt ( } \\
\text { JMA) at NagOYO and Gifu }\end{array}$ \\
\hline 30 & 03 & 38 & 00.6 & 47.298 & N & 6.605 & $E$ & 21 & & & & 0.8 & 51 & FRANCE. ML 3.8 (LDG). \\
\hline 30 & 03 & 50 & 15.5 & 34.926 & N & 137.028 & $\mathbf{E}$ & 33 & $N$ & & & 0.6 & 9 & $\begin{array}{l}\text { NEAR S. COAST OF HONSHU, JAPAN. MG } 3.4 \text { (JMA). FEIt ( } \\
\text { JMA) at NOgOYO. }\end{array}$ \\
\hline 30 & 05 & 04 & $42.5 *$ & 32.638 & $s$ & 69.904 & $w$ & 33 & $N$ & & & 1.5 & 7 & MENDOZA PROVINCE, ARGENTINA \\
\hline 30 & 05 & 11 & 00.5 & 39.277 & N & 23.580 & $\boldsymbol{E}$ & 20 & & 4.0 & & 1.0 & 70 & AEGEAN SEA. ML 4.1 (ATH). \\
\hline 30 & 05 & 15 & 13.3 & 39.261 & N & 23.526 & $E$ & 10 & G & & & 0.4 & 13 & AEGEAN SEA. ML 3.0 (ATH) \\
\hline 30 & 06 & 49 & $48 \cdot 3$ & 42.019 & N & 19.206 & $\mathbf{E}$ & 10 & G & & & 1.1 & 11 & YUGOSLAVIA. MD 2.9 (TTG) \\
\hline 30 & 07 & 44 & $05.0 \%$ & 45.640 & N & 3.569 & $\mathbf{E}$ & 10 & G & & & 0.8 & 7 & FRANCE. ML 2.5 (LDG). \\
\hline 30 & 07 & 44 & $58.5 \&$ & 60.321 & $N$ & 150.862 & $w$ & 57 & & & & & 34 & KENAI PENINSULA, ALASKA. CAGS-P> \\
\hline 30 & 08 & 22 & 54.0 & 10.960 & N & $68 \cdot 325$ & $w$ & 20 & G & 5.9 & 6.0 & 1.0 & 345 & $\begin{array}{l}\text { NEAR COAST OF VENEZUELA. MS } 6.4 \text { (BRK), } 6.0 \text { (PAS). } \\
\text { SI ight damage in the Valencio orea. Felt ot Caracas and } \\
\text { in Aragua, Carabobo. Falcon ond Yaracuy states. Depth } \\
\text { from braodband displacement se ismagrams. }\end{array}$ \\
\hline 30 & 09 & 10 & 56.68 & 61.351 & N & 149.695 & $w$ & 29 & & 4. 4 & & & 65 & $\begin{array}{l}\text { SOUTHERN ALASKA. CAGS-P>. ML } 44 \text { (PMR). Felt (V) at } \\
\text { Chugiok ond Eagle River: (IV) ot Anchoroge and Palmer. }\end{array}$ \\
\hline 30 & 09 & 30 & $06.4 *$ & 11.758 & N & 68.416 & $w$ & 10 & G & 4.6 & & 1.2 & 12 & NEAR COAST OF VENEZUELA \\
\hline 30 & 10 & 15 & $29.7 ?$ & 21.84 & $\mathrm{~S}$ & 67.10 & $w$ & 213 & $?$ & & & 1.6 & 6 & CHILE-BOLIVIA BORDER REGION \\
\hline 30 & 10 & 42 & $06.4 ?$ & 39.24 & $N$ & 23.54 & $E$ & 10 & G & & & 0.5 & 9 & AEGEAN SEA \\
\hline 30 & 11 & 03 & $36.4 \%$ & 39.218 & $N$ & 28.846 & $\mathbf{E}$ & 10 & G & & & 0.5 & 6 & TURKEY \\
\hline 30 & 11 & 04 & 26.3 & 30.110 & $\mathbf{N}$ & 99.502 & $\mathbf{E}$ & 44 & $*$ & 4.8 & & 1.0 & 56 & SICHUAN PROVINCE, CHINA \\
\hline 30 & 11 & 12 & $18.3 \%$ & 60.600 & $N$ & 5.893 & E & 10 & G & & & 1.0 & 10 & SOUTHERN NORWAY. MD 1.8 (BER) \\
\hline 30 & 11 & 49 & $25 \cdot 9 *$ & 30.743 & $\mathbf{S}$ & 72.066 & $w$ & 62 & $?$ & 4.8 & & 1.4 & 16 & OFF COAST OF CENTRAL CHILE \\
\hline 30 & 11 & 54 & $32.9 *$ & 66.888 & N & 156.340 & $w$ & 5 & G & & & 0.3 & 6 & ALASKA. ML 3.4 (PMR). \\
\hline 30 & 11 & 58 & $57.7 *$ & 32.677 & $S$ & 69.761 & $w$ & 134 & $?$ & & & 0.3 & 9 & MENDOZA PROVINCE, ARGENTINA \\
\hline 30 & 12 & 52 & $42 \cdot 2 *$ & 29.913 & $\mathrm{~N}$ & 99.268 & $E$ & 33 & $N$ & 4.2 & & 1.0 & 9 & SICHUAN PROVINCE, CHINA \\
\hline 30 & 13 & 12 & 13.4 & 45.673 & $N$ & 3.435 & $\mathbf{E}$ & 11 & & & & 0.7 & 18 & FRANCE. ML 3.5 (LDG). \\
\hline 30 & 13 & 22 & $08.4 \%$ & 45.708 & $N$ & 3.492 & $\mathbf{E}$ & 10 & G & & & 1.1 & 14 & FRANCE. ML 3.0 (LDG) \\
\hline 30 & 14 & 41 & $36.8 *$ & 66.879 & $\mathbf{N}$ & 156.300 & $w$ & 5 & G & & & 0.3 & 5 & ALASKA. ML 3.4 (PMR) \\
\hline 30 & 15 & 32 & $57.9 ?$ & 17.45 & $N$ & 62.12 & $w$ & 33 & N & & & 1.4 & 8 & LEEWARD ISLANDS. ML 2.8 (FDF) \\
\hline 30 & 15 & 33 & 52.7 & 17.912 & $\mathbf{S}$ & 178.665 & $\mathbf{w}$ & 570 & D & 5.4 & & 1.0 & 331 & FIJI ISLANDS REGION. mb 5.6 (BRK) \\
\hline 30 & 16 & 11 & $23.9 *$ & 35.596 & $N$ & 30.951 & $\mathbf{E}$ & 33 & $N$ & & & 1.0 & 9 & EASTERN MEDITERRANEAN SEA \\
\hline 30 & 16 & 18 & $29.8 *$ & 35.640 & $N$ & 30.742 & E & 33 & N & & & 0.4 & 5 & EASTERN MEDITERRANEAN SEA \\
\hline 30 & 16 & 26 & 43.9. & 43.163 & $N$ & 136.072 & $\mathbf{E}$ & 356 & $*$ & 4.2 & & 0.9 & 24 & EASTERN SEA OF JAPAN \\
\hline
\end{tabular}




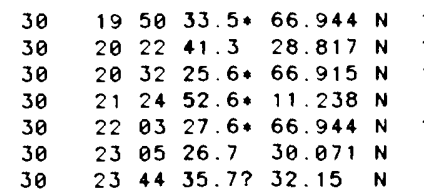

$5 G$

$33 \mathrm{~N} \quad 4.3$

5 G

$10 \mathrm{G} 4.6$

$5 \mathrm{G}$

15 D 5.14 .8
1.36 ALASKA. ML 3.5 (PMR)

1.330 BONIN ISLANDS REgION

0.86 ALASKA. ML 3.6 (PMR)

1.314 NEAR COAST OF VENEZUELA

0.16 ALASKA. ML 3.8 (PMR)

0.9142 SICHUAN PROVINCE, CHINA

1.211 OINGHAI PROVINCE, CHINA

A D D I T I ONAL SOURCE PARAMETERS

01 $2158 \quad 14.19 \quad 32.797 \mathrm{~S} \quad 69.947 w 110 \mathrm{~km}$ $5.5 \mathrm{mb}$ ( 22 abs.)

MENDOZA PROVINCE, ARGENTINA

FAULT PLANE SOLUTION: P-WoVES NP 1: Strike $=25$ Dip=75 SI ip $=-60$ $\begin{array}{llll}\text { NP2 : } & 139 & 33 & -152\end{array}$ Principol Axes:

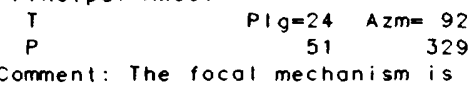
poorly cantrolled and

corresponds to normal faulting with a maderate strike-slip component. The preferred fout Plane is NP1.

CENTROID, MOMENT TENSOR (HRV)

Data Used: GDSN

L.P.B.: 16S, 31C

Centroid Lacotion

Origin Time $\quad 21: 58: 18.40 .4$

Lat $32.86 \mathrm{~S} 0.04$ Lon $70.19 \mathrm{~W} 0.08$

Dep 119.03 .2 Half-duration 1.6 Principal Axes:

Scale $10 * * 16 \mathrm{Nm}$

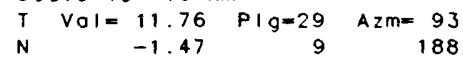

$\begin{array}{lrrr}N & -10.47 & 9 & 294\end{array}$

Best Double Couple: $M O=1.1 * 10 * 17$ NP1: Strike $=158$ Dip $=18$ Slip $=-122$ NP2: $\quad 10 \quad 75 \quad-80$

$\begin{array}{lllllllll}02 & 06 & 42 & 04.57 & 28.252 \mathrm{~N} & 57.315 \mathrm{E} & 44 \mathrm{~km}\end{array}$ $5.3 \mathrm{mb}$ ( 55 ODS.) $4.8 \mathrm{Msz}$ ( 10 Obs.) SOUTHERN IRAN

CENTROID, MOMENT TENSOR (HRV)

Doto Used: GOSN

L.P.B.: $14 \mathrm{~S}, 24 \mathrm{C}$

Centroid Location:

Origin Time $\quad 06: 42: 9.40 .5$

Lat $27.91 \mathrm{~N} 0.06$ Lon $57.25 \mathrm{E} 0.06$

Dep 42.8 BDY Half-duration 1.7

Principol Axes:

Scole $10 * * 16 \mathrm{Nm}$

$T \quad V_{0}=11.62 \quad$ PIg $=69 \quad$ Azm $=350$

$\begin{array}{rrrr}N & 1.21 & 4 & 251 \\ P & -12.83 & 21 & 159\end{array}$

Best Double Couple:Mo=1.2*10**17 NP1: Strike $=242$ Dip=24 Slip= 81 $\begin{array}{rrr}\text { NP2: } & 72 & 66\end{array}$

$02 \quad 10 \quad 35 \quad 57.15 \quad 11.063 \mathrm{~N} \quad 85.352 \mathrm{~W} \quad 70 \mathrm{~km}$ $5.0 \mathrm{mb}$ ( 26 obs.)

NI CARAGUA

CENTROID, MOMENT TENSOR (HRV)

Data Used: GDSN

L.P.B.: 16S, $29 \mathrm{C}$

Centroid Location:

Origin Time 10:36: 1.80 .6

Lat 10.98N 0.07 Lan 85.26w 0.08

Dep 80.59 .3 Half-duration 1.8

Principal Axes:

Scale $10 * * 16 \mathrm{Nm}$

$T \quad V O I=14.02 \quad P I g=39 \quad A z m=41$

$\begin{array}{rrrr}N & -2.90 & 2 & 309\end{array}$

$\begin{array}{ccrr}P & -11.11 & 51 & 216 \\ \text { Best Double Couple: } M O=1 & 3 * 10 * 17\end{array}$

NP1: Strike=150 Dip=6 Slip= -69

$\begin{array}{llll}\text { NP2: } & 309 & 84 & -92\end{array}$

$\begin{array}{lllllll}02 & 10 & 43 & 32.79 & 5.428 \mathrm{~S} & 146.785 \mathrm{E} \quad 251 \mathrm{~km}\end{array}$ $5.6 \mathrm{mb}(41$ Obs.)

EAST PAPUA NEW GUINEA REgION

FAULT PLANE SOLUTION: P-WOVES

NP1: Strike $=300$ Dip $=72$ SI $i p=-90$

NP2: $\quad 120 \quad 18 \quad-90$

Principol Axes:

$$
\begin{array}{rrr}
T & P I g=27 & A z m=30 \\
P & 63 & 210
\end{array}
$$

Comment: The focal mechanism is

moderotely well cantrolled and

carresponds ta narmal

foulting. The preferred foult
Plane is NPI.

MOMENT TENSOR SOLUTION

Dep 238 Principol Axes:

Scale $10 * 17 \mathrm{Nm}$

$\mathrm{T} \quad \mathrm{Val}=2.86 \quad \mathrm{PIg}=34 \quad$ Azm $=33$

$\begin{array}{rrrr}\mathrm{N} & 0.06 & 5 & 126 \\ \mathrm{P} & -2.92 & 55 & 224\end{array}$

Best Double Couple: $M O=2.9 * 10 * 17$

NP1: Strike=101 $\quad$ i $p=12$ SI i $p=-116$ NP2 : 307

-116
-85

CENTROID, MOMENT TENSOR (HRV)

Data Used: GDSN

L.P.B.: 13S, $25 \mathrm{C}$

Centroid Locotion

Origin Time $\quad 10: 43: 39.00 .6$

Lat 5.2350 .05 Lan $146.85 \mathrm{E} 0.04$

Dep 227.82 .3 Half-duration 2.5 Principol Axes:

Scale $10 * * 17 \mathrm{Nm}$

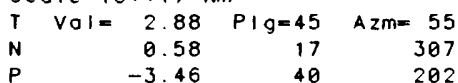

Best Double Couple:Mo=3.2*10**17

NP1: Strike $=225$ Dip $=17$ Si i $p=8$

NP2: $\quad 128 \quad 88 \quad 107$

$02 \quad 2052 \quad 02.67 \quad 30.941 \mathrm{~S} \quad 179.998 E 400 \mathrm{~km}$

5. $1 \mathrm{mb}$ ( 32 ODS.)

KERMADEC ISLANDS REGION

CENTROID, MOMENT TENSOR

(HRV)

Doto Used: GDSN

L.P.B.: 14S, $27 \mathrm{C}$

Centroid Location:

Origin Time $\quad 20: 52: 6.10 .8$

Lot $31.10 \mathrm{~S} 0.09$ Lon 179.91W 0.06

Dep 428.2 3.1 Half-duration 1.8 Principal Axes:

Scale $10 * * 17 \mathrm{Nm}$

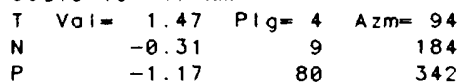

$\begin{array}{rrr}-1.17 & 80 & 342 \\ \text { Best Double Couple } & -0.31 & 3.10 .017\end{array}$

NP1: Strike=174 Dip=42 Si ip $=-104$

$\begin{array}{rrr}\text { NP2: } & 12 & 50\end{array}$

$022124 \quad 36.96 \quad 32.621 \mathrm{~N} \quad 47.782 \mathrm{E} \quad 33 \mathrm{~km}$ $5.4 \mathrm{mb}$ ( 71 ODS.) 5.OMSZ ( 7 ODS.)

IRAN-IRAO BORDER REGION

CENTROID. MOMENT TENSOR

Doto Used: GOSN

L.P.B.: 15S, 30C

Centraid Location

Origin Time $\quad 21: 24: 40.10 .9$

Lat $32.89 \mathrm{~N} 0.09$ Lon $47.62 \mathrm{E} 0.09$

Dep 15.0 FIX Half-duration 1.8

Principol Axes:

Scole $10 * 17 \mathrm{Nm}$

T $\quad \mathrm{OOI}=1.12 \quad P \mid \mathrm{g}=29 \quad$ AZm $=112$

$\begin{array}{rrrr}N & 0.60 & 40 & 355 \\ P & -1.72 & 37 & 227\end{array}$

Best Double Couple: $M O=1.4 * 10 * * 17$

NP1: Strike $=256$ Dip $=40$ SI ip $=-7$

$\begin{array}{llll}\text { NP 2: } & 351 & 85 & -130\end{array}$

$\begin{array}{llllllll}03 & 19 & 39 & 32.28 & 25.170 \mathrm{~N} & 94.652 \mathrm{E} & 67 \mathrm{~km}\end{array}$

5. $2 \mathrm{mb}$ ( 70 Obs.)

BURMA-INDIA BORDER REGION

CENTROID, MOMENT TENSOR

(HRV)

Data Used: GDSN

L.P.: $125,24 C$

Centroid Lacation

$19: 39: 37.8 \quad 0.4$

Lat $25.15 \mathrm{~N} 0.06$ Lon $94.81 \mathrm{E} 0.06$

Dep 85.33 .2 Half-duration $2 . \theta$

Principal Axes

Scole $10 * * 17 \mathrm{Nm}$

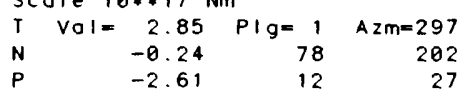

Best Dauble Cauple:Ma=2.7*10*17

NP1: Strike= 71 Dip=81 SIip= -8
NP2 :

163

$0420 \quad 10 \quad 52.15 \quad 25.017 \mathrm{~N} \quad 123.359 \mathrm{E} \quad 141 \mathrm{~km}$ $5.0 \mathrm{mb}$ ( 27 ODS.)

NORTHEAST OF TAIWAN

CENTROID, MOMENT TENSOR

Doto Used: GOSN

L.P.B.: 10S $18 \mathrm{C}$

Centraid Lacatian:

Origin Time

20: $10: 50.0 \quad 0.6$

Lat $24.62 \mathrm{~N} 0.08$ Lon $122.96 \mathrm{E} 0.11$

Dep 121.1 5.2 Half-duration 1.7 Principal Axes:

Scole $10 * * 16 \mathrm{Nm}$

$\begin{array}{rrrr}T & V a l=12.08 & P \mid g=55 & \text { A }=192=192 \\ N & -1.18 & 19 & 73 \\ P & -10.89 & 28 & 332\end{array}$

$\begin{array}{cccr}P & -10.89 & 28 & 332 \\ \text { Best Double Couple:Mo=1.1*10*17}\end{array}$ NP1:Strike $=22$ Dip=24 SIip= 37

NP2: $\quad 258 \quad 76 \quad 110$

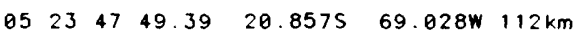
$5.7 \mathrm{mb}$ ( 46 abs.)

NORTHERN CHILE

CENTROID, MOMENT TENSOR

(HRV)

Data Used: GOSN

Centroid $215,55 C$

$23: 47: 54.8 \quad 0.2$

Lot 21.1350 .02 Lon 69.09 W 0.03

Dep 122.31 .3 Half-duration 4.6 Principal Axes:

Scale $10 * 18 \mathrm{Nm}$

$T \quad V a I=2.69 \quad P I g=16 \quad$ A $=2 m=78$

$\begin{array}{llll}N & -0.86 & 11 & 345\end{array}$

Best Double Couple:Mo=2.3*10*18 NP 1: Strike $=185$ D i $p=30$ SI i $p=-67$

NP2 : $\quad 339 \quad 62 \quad-103$

$06 \quad 08 \quad 05 \quad 57.10 \quad 19.306 \mathrm{~S} \quad 169.002 \mathrm{E} \quad 166 \mathrm{~km}$ $6.1 \mathrm{mb}$ ( 30 Obs.)

VANUATU ISLANDS

FAULT PLANE SOLUTION: P-WOVES

NP1: Strike $=158$ Dip $=75$ Slip= 90

NP2: $\quad 338 \quad 15 \quad 90$

Principol Axes:

$\begin{array}{rrr}T & P I g=60 & A z m=68 \\ P & 30 & 248\end{array}$

Comment: The focal mechanism is

moderately well cantralled and

corresponds to reverse

foulting. The preferred foult Plane is NP2.

RADIATED ENERGY

Na. of sta: 10 Focal mech. F Energy $0.3 \pm 0.1 * 10 * * 15 \mathrm{Nm}$ MOMENT TENSOR SOLUTION

DeP 151 Na. of sta: 12 Principol Axes:

Scale $10 *+19 \mathrm{Nm}$

$T \quad V a l=2.69 \quad P \mid g=61 \quad A z m=78$

$\begin{array}{rrrr}N & -0.26 & 1 & 346 \\ P & -2.43 & 29 & 255\end{array}$

Best Dauble Cauple: $M a=2.6 * 10 * * 19$

NP 1: Strike=343 Dip=16 Slip= 87

NP2: $\quad 166 \quad 74 \quad 91$

CENTROID, MOMENT TENSOR (HRV)

Data Used: GDSN

L.P.B.: 23S, 62C M.W.: $18 \mathrm{~S}, 47 \mathrm{C}$ Centroid Lacation:

Origin Time

$08: 06: 7.60 .1$

Lat 19.48S 0.01 Lon 168.94E 0.01

Dep $164.60 .5 \mathrm{Holf}$-duration 11.0

Principol Axes:

Scole $10 * 19 \mathrm{Nm}$

$T$ Vol $=2.73 \quad \mathrm{Plg}=59 \quad$ Azm $=90$

$\begin{array}{rrrr}N & 0.04 & 8 & 346 \\ P & -2.77 & 29 & 252\end{array}$

Best Double Couple: $M O=2.8 * 10 * 19$ NP1: Strike=318 Dip=17 SIip= 61

NP2: $\quad 169 \quad 75 \quad 99$ 
$072106 \quad 21.54 \quad 58.685 \mathrm{~S} \quad 148.750 \mathrm{E} \quad 10 \mathrm{~km}$ $5.2 \mathrm{mb}(6$ obs.) 5.1Msz ( 2 obs.) WEST OF MACOUARIE ISLAND

CENTROID, MOMENT TENSOR

(HRV)

Data Used: GOSN

L.P.日.: 17S, $30 \mathrm{C}$

Centroid Location

Origin Time

$21: 06: 26.70 .5$

Lot 59.025 S. 05 Lon $148.35 \mathrm{E} \theta .10$

Dep 15.0 FIX Half-duration 2.3

Principal Axes:

Scale $10 \cdots 17 \mathrm{Nm}$

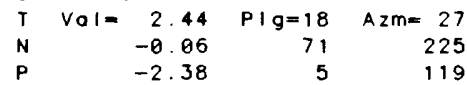

Best Double Couple: Mo=2,4*10*17

NP1: Strike $=165$ D i $p=74$ Si i $=9$

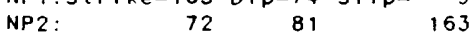

$080122 \quad 23.26 \quad 57.064 \mathrm{~N} \quad 143.490 \mathrm{~W} \quad 10 \mathrm{~km}$ $4.9 \mathrm{mb}$ ( 46 obs.) 4.7Msz ( 3 obs.) GULF OF ALASKA

CENTROID, MOMENT TENSOR (HRV)

Dato Used: GOSN

L.P.B.: 15S, $24 \mathrm{C}$

Centroid Location:

Origin Time $\quad 01: 22: 23.91 .5$

Lat $57.27 \mathrm{~N} \theta .15$ Lon $144.01 \mathrm{~W} \theta 17$

Dep $15.0 \mathrm{FIX}$ Half-duration 1.5

Principol Axes:

Scole $10 * * 16 \mathrm{Nm}$

T $\quad V a I=5.22 \quad P I g=\theta \quad A z m=132$

$\begin{array}{rrrr}N & 0.09 & 90 & 180 \\ P & -5.30 & 0 & 42\end{array}$

Best Double Couple:Mo:5.3*10**16

NP1:Strike $=177$ Di $=90$ SIip $=180$

$$
\text { NP2: } \quad 267 \quad 90 \quad 0
$$

$08 \quad 01 \quad 32 \quad 48.56 \quad 37.731 \mathrm{~s} \quad 50.071 \mathrm{E} \quad 10 \mathrm{~km}$ $5.0 \mathrm{mb}$ ( 15 obs.) 4.9Msz ( 1 obs.) ATLANTIC-INDIAN RISE

CENTROID, MOMENT TENSOR

(HRV)

L.P.B.: 19S, 39C

Centroid Locotion:

Origin Time $\quad 01: 32: 56.20 .7$

Lot 37.5750 .08 Lon $50.61 \mathrm{E} 0.09$

Dep $15.0 \mathrm{FIX} \mathrm{Holf-durotion} 1.9$

Principol Axes:

Scale $10 * 17 \mathrm{Nm}$

$T \quad V O l=1.48 \quad P \mid g=14 \quad A z m=2$

$\begin{array}{rrrr}N & -0.18 & 2 & 271 \\ P & -1.38 & 76 & 172\end{array}$

Best Double Couple:Mo=1.4*10*17

NPI: Strike $=95$ Dip=31 Slip= $=-85$

NP2: $\quad 270 \quad 59 \quad-93$

$88 \quad 0306 \quad 01.59 \quad 15.7375 \quad 173.001 \mathrm{~W} \quad 33 \mathrm{~km}$ $5.4 \mathrm{mb}(28$ obs.) $5.7 \mathrm{Msz}(24$ obs.) TONGA ISLANDS

CENTROID, MOMENT TENSOR (HRV)

Doto Used: GOSN

L.P. B. 17S, 410

Centroid Locotion

Origin Time 03:06:8.7 0.2

Lot 15.90S 0.03 Lon 172.91w 0.03

Dep 15.0 BDY Half-durotion 3.7

Principal Axes:

Scale $10 * * 17 \mathrm{Nm}$

$T \quad V O I=10.66 \quad P / g=66 \quad$ Azm $=257$

$$
\begin{array}{rrrr}
N & 0.88 & 3 & 354 \\
P & -11.54 & 24 & 86
\end{array}
$$

Best Double Couple: Mo=1.1*10**18

NP 1: Strike $=183$ Dip $=21$ SIip $=99$

NP2 : $\quad 353 \quad 69 \quad 86$

$0902 \quad 3136.17 \quad 29.177 \mathrm{~N} \quad 90.077 \mathrm{E} \quad 10 \mathrm{~km}$ $5.2 \mathrm{mb}$ ( 52 obs.) $4.7 \mathrm{Msz}$ ( 6 obs.) T I BET

CENTROIO, MOMENT TENSOR (HRV)

Dota Used: GOSN

L.P.B. : $15 \mathrm{~S}, 23 \mathrm{C}$

Centroid Location:

$02: 31: 42.7 \quad 0.7$

Lat $28.74 \mathrm{~N} 0.13$ Lon $89.94 \mathrm{E} 0.18$

DeP $15.0 \mathrm{FIX} \mathrm{Half-duration} 1.5$

Principol Axes:

Scale $10 * 16 \mathrm{Nm}$

$T \quad \mathrm{Val}=6.66 \quad \mathrm{Plg}=5 \quad$ Azm $=260$

$\begin{array}{llll}N & -0.49 & 19 & 352 \\ P & -6.17 & 70 & 155\end{array}$

Best Double Couple:Mo=6.4*10*16

NP 1: Strike=330 Dip=43 SI ip $=-119$
NP2 :

187

53

$090507 \quad 50.60 \quad 51.510 \mathrm{~N} \quad 178.386 \mathrm{w} \quad 33 \mathrm{~km}$ $5.2 \mathrm{mb}$ ( 59 abs.) 4.7Msz ( 3 obs.) ANOREANOF ISLANDS, ALEUTIAN IS.

CENTROIO, MOMENT TENSOR (HRV)

Doto Used: GDSN

L.P.B.: 19S, 35C

Centroid Location:

Origin Time

$05: 07: 56.0 \quad 0.8$

Lot $51.92 \mathrm{~N} 0.07$ Lon $178.13 \mathrm{~W} 0.10$

Dep $32.0 \mathrm{FIX} \mathrm{Holf-durotion} 1.5$

Principol Axes:

Scole $10+16 \mathrm{Nm}$

I $\quad$ O I $=8.45 \quad \mathrm{PIg}=71 \quad$ Azm $=334$

$\begin{array}{rrrr}N & 1.18 & 2 & 239 \\ P & -9.63 & 19 & 148\end{array}$

Best Double Couple: Mo=9.0*10**16

NP 1: Strike $=235$ Dip=26 SIip= 86

NP2: $\quad 60 \quad 64 \quad 92$

$\begin{array}{llllllll}09 & 09 & 14 & 34.17 & 16.265 \mathrm{~S} & 172.845 \mathrm{~W} & 25 \mathrm{~km}\end{array}$ 5.1mb ( 15 obs.) 4.9Msz ( 2 obs.)

SAMOA ISLANDS REGION

CENTROID, MOMENT TENSOR (HRV)

Doto Used: GOSN

L.P.B.: 15S, 31C

Centroid Locotion :

Origin Time

$09: 14: 42.9 \quad 0.6$

Lot $16.24 \mathrm{~S} 0.06$ Lon $172.79 \mathrm{~W} 0.07$

Dep $15.0 \mathrm{FIX} \mathrm{Half-duration} 1.8$

Principal Axes:

Scale $10 * * 17 \mathrm{Nm}$

T $\quad V_{0} l=1.28 \quad P / g=69 \quad A z m=297$

P 0.16

20

99

Best Double Couple:Mo=1,4*10**17

NP 1 : Strike=178 Dip=26 SIip= 76

NP2 :

$13 \quad 65$

$090922 \quad 34.81 \quad 20.791 \mathrm{~S} \quad 178.703 w 593 \mathrm{~km}$ $5.1 \mathrm{mb}$ ( 30 Obs.)

FIJ I ISLANDS REGION

CENTROID MOMENT TENSOR

(HRV)

Doto Used: GOSN

L.P.B.: 15S, $25 \mathrm{C}$

Centroid Location

Origin Time

$09: 22: 41.6 \quad 0.8$

Lat 20.4250 .08 Lon $179.23 w 0.08$

Dep 604.1 5.4 Holf-durotion $2 . \theta$

Principal Axes:

Scale $10 *+17 \mathrm{Nm}$

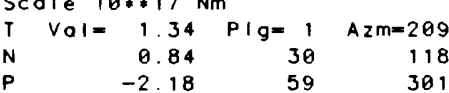

Best Double Couple: Mo $=1.8 * 10 * 17$

NP 1 : Strike=326 Dip=51 S1 ip $=-50$

NP2: $\quad 92 \quad 53 \quad-129$

$\begin{array}{lllllll}09 & 16 \quad 57 & 06.52 \quad 20.5275 \quad 174.378 E \quad 33 k m\end{array}$ $5.1 \mathrm{mb}(12$ obs.) 5.1Msz ( 2 obs.) VANUATU ISLANOS REGION

CENTROID MOMENT TENSOR

(HRV)

Dota Used: GOSN

L.P.B. 16S, $31 \mathrm{C}$

Centroid Location:

Origin Time $\quad 16: 57: 11.80 .5$

Lat $20.34 \mathrm{~S} 0.06$ Lan $173.91 \mathrm{E} 0.04$

Dep 39.64 .9 Half-durotion 1.9

Principol Axes:

Scale $10 * 17 \mathrm{Nm}$

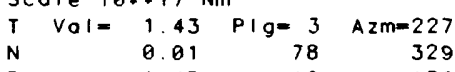

$\begin{array}{llll}\mathrm{N} & -145 & 42 & 136\end{array}$

Best Double Couple: $M 0=1.4 * 10 * * 17$

NP 1 : Strike $=272$ D i $p=80$ SI i $=-174$

NP2 :

181

84

$\begin{array}{lllll}03 & 56 & 36.91 & 49.488 \mathrm{~N} & 159.185 \mathrm{E} \quad 16 \mathrm{~km}\end{array}$ $6.3 \mathrm{mb}$ ( 80 obs.) $6.6 \mathrm{Msz}$ ( 23 obs.) KURIL ISLANDS REGION

FAULT PLANE SOLUTION: P-WOVES

NP1: Strike $=230$ Dip=68 SI i $=-90$

NP2: $\quad 50 \quad 22 \quad-90$

Principol Axes:

T PIg=23 Azm=320

Comment: The focol mechonism is poorly controlled and

corresponds to normal

foulting. The preferred foult

Plane is NP1.

RADIATED ENERGY
No of sta: 13 Focal mech. F Energy $3.0 \pm 0.6 * 10 * 14 \mathrm{Nm}$ MOMENT TENSOR SOLUTION

Dep 26 No. of sta: 22

Principal Axes:

Scale $10 * * 19 \mathrm{Nm}$

$T \quad V a I=1.10 \quad P I^{\prime} g=20 \quad$ Azm $=332$

$\begin{array}{llll}N & -0.03 & 13 & 237 \\ P & -1.07 & 66 & 116\end{array}$

Best Double Couple:Ma=1.1*10**19

NP1: Strike $=83$ Dip=28 Slip= -62

NP2: $\quad 231 \quad 66 \quad-104$

CENTROID, MOMENT TENSOR (HRV)

Doto Used: GOSN

L.P.B.: 18S, 51C M.W.: 15S, $31 \mathrm{C}$

Centroid Locotion:

Origin Time $03: 56: 42.8 \quad 0.1$

Lot $49.64 \mathrm{~N} 0.01$ Lon 159.41E 0.02

Dep 15.0 FIX Half-duration 9.0

Principol Axes:

Scole $10 * 19 \mathrm{Nm}$

$T$ Vol $=1.55 \quad P \mid g=11 \quad A 2 m=326$

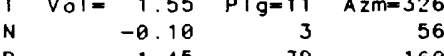

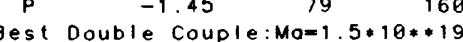

NP 1: Strike $=52$ Dip $=35$ Slip $=-95$

NP2: $\quad 238 \quad 56 \quad-87$

GEOSCOPE MOMENT TENSOR (PAR)

Dep 15.0 Half-duration 8.0

Best Double Couple:Mo=2.0*10**19

NP1: Strike $=59$ Dip=29 SIip= -71

NP2: $\quad 218 \quad 63 \quad 260$

$1300 \quad 43 \quad 11.28 \quad 39.5335 \quad 75.002 \mathrm{~W} \quad 33 \mathrm{~km}$

$5.8 \mathrm{mb}$ ( 27 obs.) 5.5Msz ( 6 obs.)

OFF COAST OF CENTRAL CHILE

FAULT PLANE SOLUTION: P-WOVES

NP1: Strike $=5$ Dip=75 Slip= -90

NP2 : 185

Principal Axes:

$\begin{array}{lrr}T & P I g=30 & \text { Azm }= \\ P & 60 & 95 \\ & 275\end{array}$

Comment: The focol mechonism is

poorly controlled and

corresponds to normol

foulting. The preferred foult

Plone is NPI.

MOMENT TENSOR SOLUTION

DeP 8 No. of sta: 5

Principal Axes:

Scale $10 * 17 \mathrm{Nm}$

$T \quad V O I=5.50 \quad \mathrm{Plg}=27 \quad$ Azm $=98$

$\begin{array}{rrrr}\mathrm{N} & 0.18 & 7 & 4 \\ \mathrm{P} & -5.68 & 62 & 262\end{array}$

Best Double Couple:Mo=5.6*10**17 NP1 : Strike $=204$ Dip=19 S1 ip $=-69$

$\begin{array}{cccr}\text { NP2: } & 2 & 72 & -97 \\ \text { CENTROID } & 2 & \\ \end{array}$

Doto Used: GOSN

L.P.B.: 19S, $44 \mathrm{C}$

Centraid Location:

Origin Time $\quad 00: 43: 10.80 .2$

Lat 39.9650 .03 Lon $75.07 w 0.04$

Dep 15.0 BOr Holf-duration 2.5

Principal Axes:

Scole $10 * 17 \mathrm{Nm}$

T $\quad \mathrm{Val}=5.58 \quad \mathrm{Plg}=16 \quad$ Azm $=86$

$\begin{array}{rrrr}N & 0.30 & 21 & 350 \\ P & -5.88 & 63 & 211\end{array}$

Best Double Couple:Mo=5.7*10*17 NP 1: Strike $=204$ D ip $=34$ SI ip $=-51$

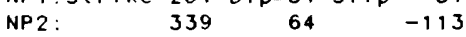

$\begin{array}{llllllll}13 & 07 & 25 & 36.70 & 24.472 \mathrm{~N} & 92.505 \mathrm{E} & 33 \mathrm{~km}\end{array}$ $5.1 \mathrm{mb}(44$ Obs.) $5.0 \mathrm{Msz}$ ( 9 obs.) INOIA-BANGLADESH BOROER REGION CENTROID, MOMENT TENSOR (HRV)

Dat o Used: GOSN

L.P. B.: 12S, $23 \mathrm{C}$

Centroid Locotion:

Origin $T$ ime

$07: 25: 37.9 \quad 0.8$

Lat $2425 \mathrm{~N} 0.09$ Lan $91.71 \mathrm{E} 0.16$

DeP $33.0 \mathrm{FI}$ ( Half-duration 1.9

Principal Axes:

Scole $10 * * 17 \mathrm{Nm}$

T $\mathrm{Val}=2.01 \quad \mathrm{PIg}=47 \quad \mathrm{AZm}=97$

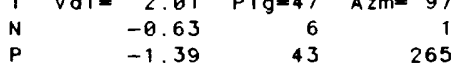

Best Double Couple:Mo=1.7*10**17

NP 1: Strike=291 Dip=6 Slip= 20

$\begin{array}{llll}\text { NP2: } & 181 & 88 & 96\end{array}$

$\begin{array}{llllllll}13 & 12 & 17 & 31.30 & 13.290 \mathrm{~N} & 39.980 \mathrm{E} & 33 \mathrm{~km}\end{array}$ 
4. $9 \mathrm{mb}$ ( 23 obs.) 4.9Msz ( 1 obs.) ETHIOPIA

CENTROID, MOMENT TENSOR (HRV)

Doto Used: GDSN

L.P.B.: 16S, 35C

Centroid Locotion:

Origin Time 12:17:25.7 0.9

Lot $12.94 \mathrm{~N} 0.06$ Lon $39.48 \mathrm{E} \quad 0.06$

Dep 15.0 FIX Half-duration 2.0 Principal Axes:

Scole $10 *+17 \mathrm{Nm}$

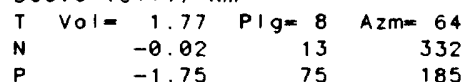

Best Double Couple:Mo=1.8*10**17 NP 1 : Strike=168 Dip=39 SI ip $=-70$ $\begin{array}{llll}\text { NP2 : } & 323 & 54 & -106\end{array}$

$\begin{array}{lllllllll}14 & 01 & 15 & 44.55 & 14.879 S & 167.211 \mathrm{E} & 158 \mathrm{~km}\end{array}$ $5.2 \mathrm{mb}(17$ ODS.)

VANUATU ISLANDS

CENTROID, MOMENT TENSOR

(HRV)

Doto Used: GDSN

L.P.B.: 9S, $17 \mathrm{C}$

Centroid Locotion

Origin Time $\quad 01: 15: 48.21 .7$

Lot $15.44 \mathrm{~S} 0.18$ Lon $166.97 \mathrm{E} \quad 0.17$

Dep 133.46 .0 Half-duration 1.5 Principol Axes:

Scale $10 * 16 \mathrm{Nm}$

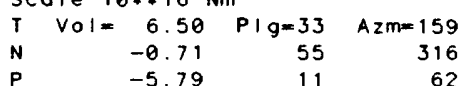

Best Double Couple:Mo=6.1*10**16

NP1: Strike=196 Dip*59 Si ip $=163$

$\begin{array}{llll}\text { NP2: } & 295 & 76 & 32\end{array}$

$\begin{array}{lllllllll}14 & 12 & 52 & 26.76 & 19.201 \mathrm{~N} & 145.522 \mathrm{E} & 168 \mathrm{~km}\end{array}$ $5.4 \mathrm{mb}$ ( 36 ODS.)

MARIANA ISLANOS

CENTROID, MOMENT TENSOR (HRV)

Doto Used: GDSN

L.P.B.: 14S, $25 \mathrm{C}$

Centroid Locotion:

Origin Time 12:52:28.10.9

Lot $19.46 \mathrm{~N} 0.07$ Lon $145.08 \mathrm{E} 0.10$

Dep 156.13 .8 Holf-durotion 1.5 Principol Axes:

Scole $10 *+16 \mathrm{Nm}$

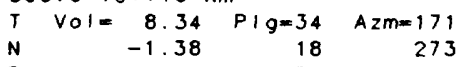

$\begin{array}{rrrr}N & -1.38 & 18 & 273 \\ P & -6.96 & 50 & 27\end{array}$

Best Double Couple:Mo: $=7.6 * 10 * 16$ NP1: Strike=211 Dip=20 SI ip=-154

NP2: $\quad 96 \quad 81 \quad-71$

$\begin{array}{llllll}14 & 1302 & 52.66 & 18.1725 & 178.393 w \quad 636 \mathrm{~km}\end{array}$

$5.4 \mathrm{mb}(37$ obs.

FIJI ISLANDS REGION

CENTROID. MOMENT TENSOR

(HRV)

Doto Used: GDSN

L.P.8.: 19S, 44C

Centroid Locotion

Origin Time 13:03:0.50.7

Lot 17.8750 .05 Lon $178.74 w 0.05$

Dep 648.0 2.4 Half-durotion 2.8

Principol Axes:

Scale $10 * * 17 \mathrm{Nm}$

$T \quad V a l=4.50 \quad P / g=8 \quad A z m=328$

$\begin{array}{rrrr}N & 0.43 & 31 & 63 \\ P & -4.93 & 57 & 225\end{array}$

Best Double Couple:Mo=4.7*10*17

NP 1: Strike $=27$ Dip $=46$ s l i $p=-136$

NP2: $\quad 263 \quad 60 \quad-53$

$\begin{array}{lllllll}15 & 14 & 26 & 41.25 & 8.453 \mathrm{~N} & 61.039 \mathrm{~W} & 23 \mathrm{~km}\end{array}$ $5.8 \mathrm{mb}$ ( 64 abs.) 5.1Msz ( 3 abs.) VENEZUELA

FAULT PLANE SOLUTION: P-Woves

NP1: Strike $=95$ Di $p=75$ Slip= -50

NP2 :

202

$42-157$

Principol Axes

$\begin{array}{lll}T & P I g=20 & A z m=156\end{array}$

Comment: The focol mechanism is

poorly controlled ond

corresponds to strike-slip

foulting with o moderote

normal component. The

preferred fault plone is not

determined.

RADIATED ENERGY

No. of sto: 5 focol mech.
Energy

$8 \cdot 4 \pm 3 \cdot 5 * 10 * 12 \mathrm{Nm}$

Dep 13

Principal Axes:

Scale $10 * * 17 \mathrm{Nm}$

$T \quad V_{0} I=1.56 \quad P I g=9 \quad A 2 m=151$

$\begin{array}{rrrr}N & -0.01 & 44 & 249 \\ P & -1.55 & 45 & 52\end{array}$

Best Double Couple:Mo=1.6*10**17

NP1: Strike=202 Dip=53 SI ip=-151

NP2 :

CENTROIO, MOMENT TENSOR (HRV)

Do to Used: GDSN

L.P.B.: 20S, $42 \mathrm{C}$

Centroid Locotion:

Origin Time

$14: 26: 41.40 .6$

Lot $8.50 \mathrm{~N} 0.06$ Lon $60.32 \mathrm{~W} 0.06$

Dep $18.54 .6 \mathrm{Holf}$-durotion 1.9 Principol Axes:

Scole $10 * * 17 \mathrm{Nm}$

$T \quad V_{0} l=1.60 \quad P \mid g=28 \quad$ Azm $=149$

$\begin{array}{lllr}N & -0.36 & 26 & 254 \\ P & -1.24 & 51 & 28\end{array}$

Best Double Couple:Mo=1.4*10**17

NP1: Strike=194 D i $p=29$ SI i $p=-153$

NP2: $\quad 80 \quad 77 \quad-63$

$\begin{array}{lllllll}15 & 20 & 34 & 08.93 & 29.987 \mathrm{~N} & 99.195 \mathrm{E} \quad 13 \mathrm{~km}\end{array}$ $6.2 \mathrm{mb}$ ( 68 obs.) $6.2 \mathrm{Msz}$ ( 18 obs.) SICHUAN PROVINCE CHINA

FAULT PLANE SOLUTION: P-WoveS

NP1: Strike $=85$ Dip $=40$ SIip $=-90$

NP2: $\quad 265 \quad 50 \quad-90$

Principol Axes:

$$
\begin{array}{rrr}
T & P I g=5 & \text { Azm }=355 \\
P & 85 & 175
\end{array}
$$

Camment: The focol mechanism is

poorly controlled ond

carresponds to normo

foulting. The preferred foult

plane is not determined.

RADIATED ENERGY

No. of sto: 9 Focol mech. Energy $\quad 1 . \theta \pm 0.2 * 10 * * 14 \mathrm{Nm}$ MOMENT TENSOR SOLUTION

DeP 7 No of sto: 14 Principol Axes:

Scole $10 * 18 \mathrm{Nm}$

$\begin{array}{rrrrr}T & V 01= & 3.70 & P \mid g=3 & A 2 m= \\ N & -0.33 & 15 & 89 \\ P & -3.37 & 75 & 266\end{array}$

Best Double Couple:Mo=3.5*10**18

NP1: Strike $=83$ Dip=44 SI ip=-111

NP2 : $291 \quad 50 \quad-7$

CENTROID, MOMENT TENSOR (HRV) DOto Used: GOSN

L.P.: 15S, 38C M.W.: 19S, 39C Centroid Locotion: $20: 34: 21.20 .1$

Lot $29.92 \mathrm{~N} 0.01$ Lon $99.37 \mathrm{E} 0.02$ Dep 15 . BDY Holf-durotion 6.3 Principol Axes:

Scole $10 * * 18 \mathrm{Nm}$

$$
\begin{aligned}
& T \quad V O I=5.38 \quad P I g=16 \quad A z m=171 \\
& \begin{array}{rrrr}
N & -0.11 & 8 & 78 \\
P & -5.27 & 72 & 323
\end{array}
\end{aligned}
$$

Best Double Couple:Mo=5.3*10*18

NP1: Strike $=273$ Dip $=29$ Stip= $=73$

$\begin{array}{llll}\text { NP2: } & 74 & 62 & -99\end{array}$

$\begin{array}{lllllll}15 & 23 & 48 & 36.81 & 13.723 S & 171.926 \mathrm{E} & 602 \mathrm{~km}\end{array}$

$5.2 \mathrm{mb}$ ( 36 obs.)

VANUATU ISLANDS REGION

CENTROID, MOMENT TENSOR

(HRV)

Doto Used: GOSN

P.B.: 9S, 130

Centroid Locotion:

$23: 48: 42.9 \quad 1.6$

Lot 13.69S FIX; Lon 171.89E FIX

Dep 575.010.8 Holf-durotion 2.0

Principol Axes

Scale $10 * * 17 \mathrm{Nm}$

$T \quad V O I=2.73 \quad P \mid g=41 \quad$ Azm $=55$

$\begin{array}{llrr}\mathrm{N} & 0.02 & 36 & 185\end{array}$

Best Double Couple: $M_{0}=2.7 * 10 * 17$

NP1:Strike $=80$ Dip=37 Siip= 168

NP2: $\quad 179 \quad 83$

$\begin{array}{lllllll}16 & 05 \quad 24 & 23.34 & 26.533 \mathrm{~N} & 126.388 \mathrm{E} \quad 133 \mathrm{~km}\end{array}$ $5.3 \mathrm{mb}(54$ Obs.)

RYUKYU ISLANDS

CENTROID, MOMENT TENSOR
Doto Used: GDSN

L.P.B.: 12S, 24C

Centroid Locotion:

Origin Time $05: 24: 25.30 .5$

Lot $26.33 \mathrm{~N} 0.04$ Lon 125.96E 0.08 Dep 125.92 .2 Holf-durotion $2 . \theta$

Principol Axes:

Scole $10 * * 17 \mathrm{Nm}$

$T$ Vol $=1.57 \quad$ PIg $=23 \quad$ Azm $=227$

$\begin{array}{llll}N & 0.39 & 22 & 327\end{array}$

Best Double Couple:Mo*1.8*10**17

NP1: Strike $=281$ Di $p=29$ SIi $p=-14 \theta$

$\begin{array}{llll}\text { NP2: } & 155 & 72 & -67\end{array}$

$\begin{array}{llllll}16 \quad 06 & 50 & 24.46 & 1.012 \mathrm{~N} & 120.061 \mathrm{E} & 27 \mathrm{~km}\end{array}$ 5. $3 \mathrm{mb}$ ( 27 obs.) 4.9Msz ( 15 obs.) MINAHASSA PENINSULA

CENTROID, MOMENT TENSOR (HRV)

Doto Used: GDSN

L.P.B.: 17S, 39C

Centroid Locotion:

Origin Time $\quad 06: 50: 29.60 .4$

Lot $1.52 \mathrm{~N} 0.05$ Lon $120.28 \mathrm{E} 0.07$ Dep 35.14 .3 Half-durotion 2.3 Principol Axes:

Scale $10 * * 17 \mathrm{Nm}$

T Val $=2.38 \quad$ Plg $=59 \quad$ Azm $=204$

$\begin{array}{rrrr}N & -0.03 & 23 & 71\end{array}$

Best Double Couple:Ma=2.4*10*17

NP1: Strike $=28$ Dip=31 Slip= 43

NP2: $\quad 260 \quad 69 \quad 114$

$\begin{array}{llllllll}16 & 08 & 04 & 29.57 & 4.612 \mathrm{~N} & 32.651 \mathrm{~W} & 10 \mathrm{~km}\end{array}$ $4.9 \mathrm{mb}$ ( 11 ObS.) 4.7MSZ ( 2 obs.) CENTRAL MID-ATLANTIC RIDGE

CENTROID, MOMENT TENSOR

(HRV)

Doto Used: GOSN

L.P.B.: $11 \mathrm{~S}, 18 \mathrm{C}$

Centraid Location:

Origin Time $08: 04: 34.21 .2$

Lot 4.74N FIX; Lon $32.62 \mathrm{~W}$ FIX Dep 15.0 FIX Half-duration 1.6 Principol Axes:

Scole $10 * * 16 \mathrm{Nm}$

T $\quad$ O I $=3.57 \quad \mathrm{PIg}=0 \quad$ Azm $=264$

$\begin{array}{rrrr}N & -0.64 & 0 & 174 \\ P & -2.93 & g & 180\end{array}$

Best Double Couple:Mo=3.3*10**16 NP 1 : Strike $=354$ Dip=45 Si i $p=-90$

NP2: $\quad 174 \quad 45 \quad-90$

$\begin{array}{llllllll}16 & 08 & 35 & 03.25 & 4.462 \mathrm{~N} & 32.573 \mathrm{~W} & 10 \mathrm{~km}\end{array}$ $5.4 \mathrm{mb}(18$ Obs.) 4.9Msz ( 3 obs.) CENTRAL MID-ATLANTIC RIOGE

CENTROID, MOMENT TENSOR

(HRV)

Dato Used: GOSN

L.P.B.: $14 \mathrm{~S}, 24 \mathrm{C}$

Centroid Locotian:

Origin Time $\quad 08: 35: 9.80 .8$

Lot $4.73 \mathrm{~N} 0.08$ Lon $32.41 \mathrm{~W} 0.06$

Dep 15.0 FIX Half-duration 1.6

Principol Axes:

Scole $10 * 16 \mathrm{Nm}$

$T$ Vol $=6.93 \quad$ Plg $=0 \quad$ Azm $=264$

$\begin{array}{rrrr}N & 1.06 & 0 & 174 \\ P & -7.98 & 90 & 180\end{array}$

Best Double Couple:Mo=7.5*10*16

NP1: Strike $=354$ Dip=45 SI i p $=-90$

$\begin{array}{llll}\text { NP2: } & 174 & 45 & -90\end{array}$

$\begin{array}{lllllll}16 & 10 & 51 & 16.52 & 4.630 \mathrm{~N} & 32.664 \mathrm{~W} & 10 \mathrm{~km}\end{array}$ 5. $4 \mathrm{mb}$ ( 50 obs.) 5.4Msz ( 12 obs.) CENTRAL MID-ATLANTIC RIDGE

CENTROID, MOMENT TENSOR

(HRV)

Dato Used: GDSN

L.P.B.: 19S, $45 \mathrm{C}$

Centroid Location:

Origin Time $\quad 10: 51: 26.80 .3$

Lat $4.89 \mathrm{~N} 0.04$ Lon $32.31 \mathrm{~W} 0.03$

Dep 15.0 FIX Half-durotion 2.6 Principol Axes:

Scole $10 * 17 \mathrm{Nm}$

$T$ VOI= $3.37 \mathrm{Plg}=6 \quad A=\mathrm{Nm}=267$

\begin{tabular}{rrrr}
$\mathrm{N}$ & 3.37 & $\mathrm{Plg}=6$ & $\mathrm{Azm}=267$ \\
$\mathrm{P}$ & -3.51 & 2 & 177 \\
\hline
\end{tabular}

Best Double Couple:Mo=3.4*10**17

NP1: Strike $=0$ Dip $=39$ S1 i $=-87$

NP2: $\quad 176 \quad 51 \quad-93$

$\begin{array}{lllllll}16 & 19 & 48 & 14.69 & 21.039 \mathrm{~S} & 178.942 \mathrm{~W} & 610 \mathrm{~km}\end{array}$ $5.7 \mathrm{mb}$ ( 57 obs.) 
FIJI ISLANDS REGION

CENTROID. MOMENT TENSOR

(HRV)

Data Used: GOSN

L.P.B.: 18S, $48 \mathrm{C}$

Centroid Lacatian:

Origin Time 19:48:22.9 0.2

Lat 20.7950 .02 Lon $179.18 \mathrm{~W} 0.02$

Dep $600.81 .3 \mathrm{Half}$-duration 5.1

Principal Axes:

Scale $10 * 18 \mathrm{Nm}$

$T \quad V a l=2.43 \quad P \mid g=21 \quad A Z m=78$

$\begin{array}{rrrr}N & 0.98 & 30 & 182 \\ P & -3.41 & 51 & 319\end{array}$

Best Double Couple: $M_{0}=2.9 * 10 * 18$

NP1: Strike $=127$ Di $p=36 \quad$ SI i $p=-150$

NP2: $\quad 12 \quad 73 \quad-58$

$\begin{array}{llllll}17 & 94 & 55 & 26.77 & 30.644 S \quad 71.683 W \quad 44 k m\end{array}$

4.9mb ( 14 obs.) 4.8MSZ (1 obs.) NEAR COAST OF CENTRAL CHILE

CENTROID, MOMENT TENSOR (HRV)

Data Used: GOSN

L.P.B.: 14S, $20 \mathrm{C}$

Centroid Location

Origin

$04: 55: 28.8 \quad 0.8$

Lat 30.9250 .12 Lon $71.85 W 0.13$

Dep $15.0 \mathrm{Fix} \mathrm{Half-duration} 1.5$

Principal Axes

Scale $10 * * 16 \mathrm{Nm}$

$T \quad V a l=6.44 \quad$ PIg $=68 \quad$ Azm $=90$

$\begin{array}{rrrr}N & 1.73 & 2 & 355\end{array}$

$\begin{array}{ccrr}P & -8.17 & 22 & 265 \\ \text { Best Double Couple:Mo=7.3*10*16 }\end{array}$

NP 1: Strike $=351$ Dip $=23$ SIip= 85

NP2: $\quad 176 \quad 67 \quad 92$

$\begin{array}{lllll}1802 \quad 01 & 27.13 \quad 21.538 \mathrm{~S} \quad 178.955 \mathrm{~W} 580 \mathrm{~km}\end{array}$ $5.2 \mathrm{mb}$ ( 36 obs.)

FIJI ISLANDS REGION

CENTROID, MOMENT TENSOR (HRV)

Data Used: GDSN

L.P.B.: 20S, $31 \mathrm{C}$

Centroid Location

Origin Time

$02: 01: 34.9 \quad 0.6$

Lat 21.235 0.05 Lon 179.40 W 0.05

Dep $603.53 .0 \mathrm{Half}$-duration 1.9

Principal Axes

$$
\begin{array}{llrr}
\text { Scale } 10 * 17 & \mathrm{Nm} & & \\
\mathrm{T} \text { Val } & 1.48 & \text { PIg }=22 & \text { Azm }=123 \\
\mathrm{~N} & 0.33 & 16 & 220
\end{array}
$$

\begin{tabular}{cccr}
$P$ & -1.81 & 62 & 344 \\
Best Double Couple: & \multicolumn{1}{c}{ Co=1 } & $6 * 10 * 117$
\end{tabular}

NP1: Strike $=185$ Dip $=27$ SI i $p=-128$

$\begin{array}{llll}\text { NP2: } & 47 & 69 & -73\end{array}$

$\begin{array}{lllllll}18 & 12 & 33 & 52.16 & 23.834 \mathrm{~S} & 179.944 \mathrm{E} & 524 \mathrm{~km}\end{array}$ $5.8 \mathrm{mb}$ ( 50 obs.)

SOUTH OF FIJI ISLANDS

FAULT PLANE SOLUTION: P-WaVES

NP1:Strike $=190$ Dip=82 Slip $=90$

NP2: $10 \quad 8 \quad 90$

Principal Axes:

$$
\begin{array}{lrr}
T & P I g=53 & A z m=100 \\
P & 37 & 280
\end{array}
$$

Camment: The facal mechanism is

poorly controlled and

corresponds to reverse

tauling. The preferred faull

plane is NP2.

RADIATED ENERGY

No. of sta: 7 Focol mech. M Energy $\quad 5.2 \pm 1.5 * 10 * * 12 \mathrm{Nm}$ MOMENT TENSOR SOLUTION

Dep $548 \quad \mathrm{Na}$. of sta: 13 Principal Axes:

Scale $10 * 18 \mathrm{Nm}$

$T \quad \mathrm{Val}=1.60 \quad \mathrm{PIg}=50 \quad$ Azm $=75$

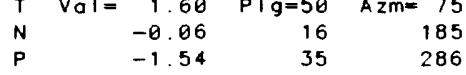

Best Double Couple: $M O=1.6 * 10 * 18$

NP 1:Strike $=67$ Dip=18 SIip= 154

NP2 :

$182 \quad 82$

(HRV)

CENTROID. MOMENT TENSOR

Data Used: GOSN

L.P.B.: $12 \mathrm{~S}, 30 \mathrm{C}$

Centroid Locatian:

Origin Time 12:34: 1.20 .3

Lat $23.87 \mathrm{~S} 0.03$ Lon 179.79E 0.02

Dep $547.91 .3 \mathrm{Half}$-duration 3.8

Principal Axes:

Scale $10 * 17 \mathrm{Nm}$

T $\quad$ Val $=9.72 \quad$ PIg $=48 \quad$ AZm $=85$
$\begin{array}{llll}P & -11.45 & 36 & 300\end{array}$

Best Double Couple: $M O=1,1 * 10 * 18$ NP1: Strike $=85$ Dip $=19$ Slip $=160$ NP2: $\quad 194 \quad 84 \quad 72$

$1900 \quad 08 \quad 19.65 \quad 31.3105 \quad 177.815 \mathrm{~W} \quad 11 \mathrm{~km}$ $5.6 \mathrm{mb}$ ( 17 obs.) $5.9 \mathrm{Msz}$ ( 27 obs.) KERMADEC ISLANDS REGION

FAULT PLANE SOLUTION: P-Waves

NP 1: Strike $=15$ Dip=70 SIip $=90$

$\begin{array}{llll}\text { NP2: } & 195 & 20 & 90\end{array}$

$\begin{array}{lrr}\text { Principal Axes: } & \\ T & P \mid g=65 & \text { Azm }=285 \\ P & 25 & 105\end{array}$

Comment: The focal mechanism is

poorly controlled and

corresponds to reverse

fauting. The preferred fault

plane is NP2

MOMENT TENSOR SOLUTION

Dep 10 No. of sta: 14

Principal Axes

Scale $10 * * 17 \mathrm{Nm}$

T $\quad V_{a l}=6.27 \quad P I g=59 \quad$ Azm $=254$

$\begin{array}{rrrr}\mathrm{N} & -0.26 & 20 & 20 \\ \mathrm{P} & -6.01 & 23 & 119\end{array}$

Best Double Couple:Mo=6.1*10**17 NP 1: Strike $=242$ Dip=28 Slip=135 NP

CENTROID, MOMENT TENSOR (HRV)

Data Used: GOSN

L.P.B.: $18 \mathrm{~S}, 39 \mathrm{C}$

Centroid Lacation

Origin Time $\quad 00: 08: 27.40 .4$

Lat $31.36 \mathrm{~S} 0.03$ Lan 177.54W 0.04

Dep 19.71 .6 Halt-duration 3.2

Principal Axes:

Scale $10 \cdots 17 \mathrm{Nm}$

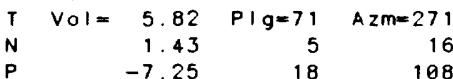

Best Double Couple.Ma=6.5*10*17

NP1 : Strike $=206$ Dip $=28$ SIip $=101$

$\begin{array}{llll}\text { NP2 : } & 14 & 63 & 84\end{array}$

$1907 \quad 03 \quad 59.31 \quad 31.521 \mathrm{~s} \quad 177.763 w \quad 61 \mathrm{~km}$

$5.2 \mathrm{mb}(8$ Obs.)

KERMADEC ISLANDS REGION

CENTROID, MOMENT TENSOR

Data Used: GDSN

L.P.B.: 145,220

Centroid Location

07:04: $1.5 \quad 1.9$

Lat $31,15 \mathrm{~S}$ 0. 12 Lon $177.66 \mathrm{~W}$ 0.14

Dep 40.97 .2 Half-duration 1.4

Principal Axes

Scale $10 * 16 \mathrm{Nm}$

$T \quad V a l=4.48 \quad P I g=62 \quad A z m=228$

$\begin{array}{rrrr}N & 0.95 & 26 & 17 \\ P & -5.43 & 10 & 112\end{array}$

Best Double Couple:Mo=4.9*10*16 NP 1: Strike $=230$ Dip $=42$ Slip $=131$

NP2 : 60 59

$19 \quad 14 \quad 48 \quad 56.70 \quad 17.858 \mathrm{~N} \quad 105.228 \mathrm{~W} \quad 10 \mathrm{~km}$ $5.1 \mathrm{mb}$ ( 10 obs.) 5.4Msz ( 7 obs.) OFF COAST OF JALISCO, MEXICO

CENTROID, MOMENT TENSOR

(HRV)

Data Used: GDSN

P. B : 16S, 39C

Centroid Location:

Origin Time 14:49:2.1 0.4

Lat $17.99 \mathrm{~N} 0.04$ Lon $105.47 \mathrm{~W} 0.04$

Dep $15.0 \mathrm{FIX}$ Half-duration 2.9

Principal Axes

Scale $10 * 17 \mathrm{Nm}$

T $\quad \mathrm{Val}=5.40 \quad \mathrm{PIg}=6 \quad \mathrm{Azm}=82$

$\begin{array}{rrrr}N & -0.47 & 84 & 254 \\ P & -4.93 & 1 & 352\end{array}$

Best Double Couple: $M_{0}=5.2 * 10 * * 17$

NP 1: Strike $=127$ Dip $=85$ SIip $=176$

NP2: $\quad 217 \quad 86$

$20 \quad 06 \quad 53 \quad 33.06 \quad 2.968 \mathrm{~N} \quad 126.400 \mathrm{E} \quad 94 \mathrm{~km}$ 4. $9 \mathrm{mb}(15$ obs.)

MOLUCCA PASSAGE

CENTROID. MOMENT TENSOR

(HRV)

Data Used: GDSN

L.P.B.: $17 \mathrm{~S}, 35 \mathrm{C}$

Centroid Lacation:

$06: 53: 32.5 \quad 0.4$

Lat $3.07 \mathrm{~N} 0.05$ Lon $126.43 \mathrm{E} 0.06$

Dep 33.33 .2 Half-durotion 2.0
Principal Axes:

Scale $10 * * 17 \mathrm{Nm}$

T $\quad \mathrm{O} O \mathrm{l}=1.58 \quad \mathrm{PIg}=61 \quad$ Azm $=308$

$\begin{array}{llll}\mathrm{N} & 0.24 & 12 & 195\end{array}$

Best Dauble Couple:Ma=1.7*10**17

NP1: Strike $=163$ Dip $=22$ Slip= 56

NP2: $\quad 19 \quad 72 \quad 103$

$2008 \quad 08 \quad 51.09 \quad 9.259 \mathrm{~S} \quad 79.033 w \quad 63 \mathrm{~km}$

$5.8 \mathrm{mb}(55 \mathrm{abs}$.)

OFF COAST OF NORTHERN PERU

RADIATED ENERGY

$\mathrm{Na}$. of sta: 4 Focal mech. C

Energy $1.2 \pm 0.6 * 10 * 13 \mathrm{Nm}$

CENTROID, MOMENT TENSOR (HRV)

Data Used: GDSN

L.P.B.: 19S, $42 C$

Centroid Lacatian:

Origin Time $08: 08: 56.40 .3$

Lat 9.4350 .03 Lon $79.16 \mathrm{~W} 0.04$

Dep 63.12 .3 Half-duration 2.4

Principal Axes:

Scale $10 * * 17 \mathrm{Nm}$

$T \quad \mathrm{Val}=3.14 \quad \mathrm{PIg}=5 \quad$ Azm $=240$

$\begin{array}{rrrr}N & -0.07 & 4 & 150 \\ P & -3.07 & 83 & 24\end{array}$

Best Double Couple:Ma=3.1*10**17

NP 1 : Strike $=335$ Dip=40 SI ip= -84

NP2: $\quad 147 \quad 51 \quad-95$

$20 \quad 22 \quad 5954.07 \quad 57.166 \mathrm{~N} 121.976 \mathrm{E} \quad 26 \mathrm{~km}$ $6.1 \mathrm{mb}$ ( 65 obs.) 6.5MSZ ( 21 abs.) EASTERN USSR

FAULT PLANE SOLUTION: P-Waves

NP1: Strike $=129$ Dip=75 SIip 90 NP2 :

Principal Axes:

$$
\begin{array}{rrr}
T & P I g=60 & A z m= \\
P & 30 & 219
\end{array}
$$

Camment: The focal mechanism is

paorly cantrolled and

corresponds ta reverse

faulting. The preferred fault plane is NP2

RADIATED ENERGY

No. of sta: 7 focal mech. M Energy $\quad 0.6 \pm 0.2 * 10 * * 14 \mathrm{Nm}$ MOMENT TENSOR SOLUTION

Dep 22 No. af sta: 18 Principal Axes:

Scale $10 * 18 \mathrm{Nm}$

$T \quad V a l=2.74 \quad P \mid g=53 \quad$ AZm $=22$

$\begin{array}{lllr}N & 0.34 & 16 & 134\end{array}$

Best Double Couple:Mo=2.9*10**18 NP1:Strike $=7$ Dip $=19$ SI ip $=145$ NP2: $\quad 131 \quad 79 \quad 74$

CENTROID, MOMENT TENSOR (HRV)

Data Used: GDSN

L.P.B.: 16S, 44C M.W.: 13S, $23 \mathrm{C}$

Centraid Lacatian:

Lat $57.03 \mathrm{~N} 0.01$ Lon $121.23 \mathrm{E} 0.04$

Principal Axes:

Scale $10 * * 18 \mathrm{Nm}$

$T$ Val $=3.25 \quad$ PIg $=30 \quad$ Azm $=329$

$\begin{array}{rrrr}N & -0.32 & 54 & 113\end{array}$

$\begin{array}{cccr}P & -2.93 & 17 & 229 \\ \text { Best Double Couple:Mo=3.1*10**18}\end{array}$

NP1:Strike $=6$ Dip=56 SIip= 170

NP2: $101 \quad 82 \quad 35$

GEOSCOPE MOMENT TENSOR (PAR)

Dep 25.0 Half-duratian 7.0

Best Double Couple:Ma=5.0*10**18

NP 1:Strike=115 Dip=86 SIip= 68

NP2: $\quad 14 \quad 23 \quad 168$

$\begin{array}{llllllll}21 & 20 & 36 & 04.23 & 13.302 \mathrm{~N} & 144.381 \mathrm{E} & 115 \mathrm{~km}\end{array}$

5. $2 \mathrm{mb}(25$ obs.)

MARIANA I SLANDS

CENTROID, MOMENT TENSOR

Data Used: GDSN

L.P.B.: 16S, 26C

Centraid Lacation:

Origin Time

$20: 36: 8.60 .4$

Lat 13.19N 0.04 Lan 144.04E 0.06

Dep $107.23 .1 \mathrm{Half}$-duration 1.8

Principal Axes:

Scale $10 * 16 \mathrm{Nm}$

$T \quad V a l=12.32 \quad P I g=1 \quad A z m=210$

$N \quad 1.48 \quad 50 \quad A 2 m=210$ 
$\begin{array}{llll}P & -13.80 & 39 & 119\end{array}$

Best Dauble Cauple: $M_{0}=1.3 \cdot 10 \cdot 117$ NP 1: Strike=262 Dip=62 S1 ip =-150 $\begin{array}{llll}\text { NP2 : } & 157 & 64 & -31\end{array}$

$\begin{array}{lllllll}22 & 04 & 48 & 30.85 & 35.576 \mathrm{~s} & 102.935 \mathrm{w} & 10 \mathrm{~km}\end{array}$ $5.0 \mathrm{mb}(10$ abs.) 4.8Msz ( 2 obs.) SOUTHERN PACIFIC OCEAN

CENTROID, MOMENT TENSOR

(HRV)

Data Used: GDSN

L.P.B.: 19S, $42 \mathrm{C}$

Centroid Lacation:

Origin Time $04: 48: 35.50 .3$

Lat 36.0550 .04 Lon $102.82 W 0.04$

Dep $15.0 \mathrm{FIX}$ Holf-duration 2.0 Principal Axes:

Scale $10 * * 17 \mathrm{Nm}$

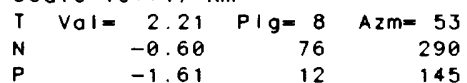

Best Double Couple: $M 0=1.9 * 10 * 17$ NP1 : Strike=189 Dip=76 SIip= -3

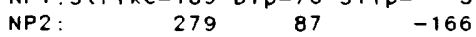

$\begin{array}{llllllll}23 & 15 & 22 & 34.17 & 44.958 \mathrm{~N} & 150.238 \mathrm{E} & 89 \mathrm{~km}\end{array}$

4. $9 \mathrm{mb}$ ( 43 ODS.)

KURIL ISLANDS REGION

CENTROID. MOMENT TENSOR

(HRV)

Dota Used: GDSN

L.P.B.: 17S, $29 \mathrm{C}$

Centraid Lacatian:

Origin Time $\quad 15: 22: 32.50 .7$

Lat $45.37 \mathrm{~N} 0.05$ Lon 150.65E 0.09

Dep 44.24 .7 Holf-duration 1.5 Principal Axes

Scale $10 * 16 \mathrm{Nm}$

T $\quad V O I=6.10 \quad P \mid g=72 \quad$ Azm $=257$

$\begin{array}{rrrr}N & 0.36 & 13 & 35 \\ P & -6.46 & 12 & 128\end{array}$

Best Double Couple Mo=6.3*10*16

NPI: Strike=235 Dip=35 SI ip= 114

NP2: $\quad 27 \quad 58 \quad 74$

$\begin{array}{llllllll}23 & 192106.47 & 66.960 \mathrm{~N} & 156.289 \mathrm{w} & 6 \mathrm{~km}\end{array}$ $5.7 \mathrm{mb}(65$ obs.) 5.1Msz ( 16 obs) ALASKA

CENTROID, MOMENT TENSOR

(HRV)

Data Used: GDSN

L.P.B.: 19S, $37 \mathrm{C}$

Centraid Lacatian

Origin Time

19. $21: 10.4 \quad 0.3$

Lat $67.30 \mathrm{~N} 0.05$ Lon 157.02W 0.08

Dep 15.0 BoY Half-duration 2.1 Principal Axes

Scole $10 * 17 \mathrm{Nm}$

$T \quad V 0 l=1.96 \quad P / g=6 \quad$ Azm $=258$

$\begin{array}{rrrr}\mathrm{N} & -0.29 & 3 & 348 \\ \mathrm{P} & -1.67 & 83 & 104\end{array}$

Best Double Couple. Mo=1.8*10*17

NP 1: Strike=345 Dip=39 SI ip $=-95$

NP2: $\quad 171 \quad 51 \quad-86$

$\begin{array}{llllllll}24 & 20 & 32 & 55.84 & 17.484 \mathrm{~S} & 167.835 \mathrm{E} \quad 28 \mathrm{~km}\end{array}$ $5.1 \mathrm{mb}$ ( 3 obs.)

VANUATU ISLANDS

CENTROID, MOMENT TENSOR (HRV)

Doto Used: GDSN

L.P.B. 10S, $17 \mathrm{C}$

Centroid Locotion

Origin Time 20:32:59.7 1.4

Lot 17.49S 0.14 Lon 167.75E 0.14

Dep 37.39 .4 Holf-durotion 1.5

Principol Axes

Scole $10 * 16 \mathrm{Nm}$

T $\quad \mathrm{OOI}=6.12 \quad \mathrm{Plg}=74 \quad \mathrm{Azm}=48$

$\begin{array}{rrrr}N & -0.86 & 7 & 157 \\ P & -5.26 & 14 & 249\end{array}$

Best Double Couple:Mo=5.7*10*16

NP1: Strike=350 Dip=32 SI ip= 104

NP2: $\quad 153 \quad 60 \quad 81$

$\begin{array}{llllllll}24 & 20 & 41 & 11.54 & 17.398 \mathrm{~S} & 167.826 \mathrm{E} & 34 \mathrm{~km}\end{array}$ $5.3 \mathrm{mb}(16$ obs.) $5.5 \mathrm{Msz}(19$ obs.) VANUATU ISLANDS

CENTROID, MOMENT TENSOR (HRV)

Doto Used: GOSN

L.P. B. $17 \mathrm{~S}, 37 \mathrm{C}$

Centraid Location:

Origin Time 20:41:15.7 0.4

Lot $17.44 \mathrm{~S} 0.03$ Lon $167.60 \mathrm{E} \quad 0.03$

Dep 27.71 .8 Holf-durotion 3.1

Principol Axes:

Scole $10 \cdot 17 \mathrm{Nm}$

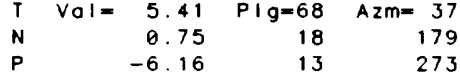

Best Dauble Cauple:Ma=5.8*10**17

NP1: Strike $=26$ Dip=36 SIip= 122

NP2: $\quad 168 \quad 60 \quad 69$

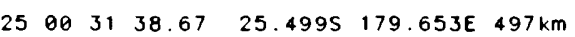

$5.0 \mathrm{mb}$ ( 27 obs.)

SOUTH OF FIJI ISLANDS

CENTROID, MOMENT TENSOR

( HRV)

Doto Used: GDSN

L.P.B. 15S, 27C

Centroid Locotion:

$00: 31: 49.0 \quad 1.0$

Lat 25.38S 0.08 Lon 179.27E 0.09

Dep $520.33 .5 \mathrm{Half}$-duratian 1.8

Principal Axes:

Scole $10 * 16 \mathrm{Nm}$

T $\quad$ al $=11.07 \quad \mathrm{Plg}=42 \quad$ Azm $=62$

$\begin{array}{rrrr}N & 1.04 & 35 & 192 \\ P & -12.11 & 28 & 303\end{array}$

Best Dauble Couple:Ma=1.2*10*17

NP1:Strike $=84$ Dip=36 SI ip $=166$

NP2: $\quad 186 \quad 82 \quad 55$

$\begin{array}{lllllll}25 & 02 & 13 & 20.83 & 30.048 \mathrm{~N} & 99.419 \mathrm{E} \quad 8 \mathrm{~km}\end{array}$

$6.2 \mathrm{mb}$ ( 84 obs.) $6.0 \mathrm{Msz}$ ( 16 obs.)

SICHUAN PROVINCE, CHINA

FAULT PLANE SOLUTION: P-WOVES

NP1: Strike $=75$ Dip=45 Si ip $=-90$

NP2:

255

Principal Axes:

$\begin{array}{lrr}T & P I g=0 & A z m=165 \\ P & 90 & 0\end{array}$

Camment: The facal mechanism is

poorly controlled and

correspands ta narmal

foulting. The preferred fault

plane is not determined.

RADIATED ENERGY

No. of sta: 7 Facal mech. F

Energy $\quad 5.1 \pm 0.7 * 10 * 13 \mathrm{Nm}$

MOMENT TENSOR SOLUTION

Dep 7 No. af sta: 15

Principal Axes:

Scale $10 * 18 \mathrm{Nm}$

$T \quad V O I=1.92 \quad P I g=12 \quad$ Azm $=338$

$\begin{array}{rrrr}N & -0.24 & 21 & 73 \\ P & -1.68 & 65 & 221\end{array}$

Best Double Couple: $M O=1.8 * 10 * * 18$

NP 1: Strike $=43$ Dip $=38$ SI ip $=-126$

NP2: $\quad 266 \quad 60 \quad 65$

CENTROID, MOMENT TENSOR (HRV)

DOto UsEd: GDSN

L.P.B.: 13S, 34C M.W.: 15S, $24 \mathrm{C}$

Centroid Locotion

Origin Time

$02: 13: 31.20 .1$

Lot $29.90 \mathrm{~N} 0.02$ Lon $99.41 \mathrm{E} 0.03$

Dep 15.0 BDY Holf-duration 4.6

Principol Axes:

Scole $10 * 18 \mathrm{Nm}$

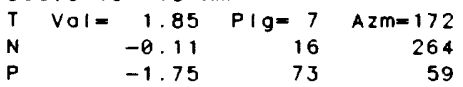

Best Double Couple:Mo=1.8*10**18

NP1: Strike=245 Dip=40 S। i $p=-114$

NP2: $\quad 96 \quad 54 \quad-71$

$\begin{array}{lllllll}25 & 03 & 11 & 17.46 & 17.420 \mathrm{~S} & 167.776 \mathrm{E} & 15 \mathrm{~km}\end{array}$

5.0mb ( 7 obs.) $4.8 \mathrm{Msz}$ ( 1 obs.)

VANUATU ISLANDS

CENTROID, MOMENT TENSOR (HRV)

Do to Used: GDSN

L.P.B.: 11S, $22 \mathrm{C}$

Centroid Locotion:

Origin Time $03: 11: 21.8 \quad 1.0$

Lot 17.2350 .08 Lon $167.69 E \quad 0.10$

Dep 20.44 .4 Holf-durotion 2.4

Principol Axes:

Scole $10 * 17 \mathrm{Nm}$

T $\quad$ O I $=3.00 \quad \mathrm{Plg}=62 \quad$ Azm $=9$

$\begin{array}{rrrr}N & -0.62 & 27 & 174 \\ P & -2.37 & 6 & 267\end{array}$

Best Double Couple:Mo=2.7.10*17

NP 1: Strike $=24$ Dip=46 SI ip= 129

NP2: $\quad 155 \quad 56 \quad 57$

$\begin{array}{llllll}2505 \quad 13 & 34.07 & 5.108 \mathrm{~S} & 143.501 \mathrm{E} \quad 110 \mathrm{~km}\end{array}$ $5.5 \mathrm{mb}$ ( 43 ObS.)

PAPUA NEW GUINEA

CENTROID, MOMENT TENSOR

Doto Used: GDSN
(HRV)
P. B.: 185, $38 \mathrm{C}$

Centraid Location:

Origin Time $05: 13: 39.60 .3$

Lat 5.1550 .03 Lon 143.26E 0.03

Dep 105.92 2.3 Half-duration 3.0 Principol Axes:

Scale $10 * 17 \mathrm{Nm}$

T $\quad \mathrm{Val}=5.60 \quad \mathrm{Plg}=11 \quad$ Azm $=249$

$\begin{array}{rrrr}N & 0.49 & 59 & 140 \\ P & -6.09 & 28 & 345\end{array}$

Best Double Cauple:Mo=5.9*10*17

NP1: Strike $=23$ Dip=62 SI i $p=-13$

$\begin{array}{llll}\text { NP2: } & 120 & 78 & -151\end{array}$

$\begin{array}{llllllll}25 & 06 & 11 & 32.28 & 11.073 \mathrm{~N} & 86.761 \mathrm{~W} & 46 \mathrm{~km}\end{array}$ 4.8mb ( 8 ODS.) 4.5MsZ ( 4 ODs.) NEAR COAST OF NICARAGUA

CENTROID. MOMENT TENSOR

(HRV)

Doto Used: GDSN

.P.B.: 10S, 15C

Centroid Lacation:

Origin Time

$06: 11: 25.7 \quad 1.3$

Lat $11.32 \mathrm{~N} 0.18$ Lan 86.00W 0.29

Dep $15.0 \mathrm{FIX}$ Half-duration 1.5

Principol Axes:

Scale $10 * 16 \mathrm{Nm}$

$T \quad$ Vol $=11.65$ PIg=32 Azm= 73

$\begin{array}{llll}\mathrm{N} & -0.20 & 12 & 335\end{array}$

Best Double Couple: $M a=1.2 * 10 * * 17$

NP 1 : Strike=199 Dip=17 SI i $p=-45$

$\begin{array}{llll}\text { NP2 : } & 332 & 78 & -103\end{array}$

$\begin{array}{lllllll}25 & 14 & 29 & 00.51 & 16.773 \mathrm{~N} & 99.328 \mathrm{~W} & 19 \mathrm{~km}\end{array}$ $6.2 \mathrm{mb}$ ( 56 obs.) $6.8 \mathrm{Msz}$ ( 29 Obs.) NEAR COAST OF GUERRERO, MEXICO

FAULT PLANE SOLUTION: P-WaveS

NP1: Strike=121 Dip=77 SIip= 90 NP2 301

90

Principol Axes:

$\begin{array}{lrr}T & P I g=58 & A z m= \\ P & 32 & 211\end{array}$

Comment: The facal mechanism is

poorly controlled and

correspands to reverse

faulting. The preferred fault plane is NP2

RADIATED ENERGY

No. of sta: 9 Facal mech. C

Energy 2.5 $2.8 * 10 * 14 \mathrm{Nm}$

CENTROID, MOMENT TENSOR (HRV)

Data Used: GDSN

L.P.B.: 20S, 54C M.W.: 16S, 32C

Centraid Location

Origin Time

$14: 29: 5.90 .1$

Lot $16.83 \mathrm{~N} 0.01$ Lon $99.12 w 0.01$

Dep 15.0 BDY Holf-durotion 9.0

Principol Axes:

Scole $10 *+19 \mathrm{Nm}$

$T \quad V O I=2.40 \quad P I g=54 \quad A z m=36$

$\begin{array}{rrrr}N & -0.03 & 4 & 300 \\ P & -2.38 & 36 & 207\end{array}$

Best Double Couple: $M O=2.4 * 10 * * 19$

NP1: Strike $=276$ Dip=10 S।ip= 66

$\begin{array}{rrrr}\text { NP2: } & 121 & 81 & 94 \\ \text { GEOSCOPE MOMENT } & \text { TENSOR } & \text { (PAR) }\end{array}$

DeP 15.0 Holf-durotion 20.0

Best Double Couple:Mo=2.6*10**19

NP1: Strike=164 Dip=83 Si ip= 101

NP2: $\quad-73 \quad 13 \quad 33$

$\begin{array}{llllllll}27 & 02 & 20 & 04.74 & 30.601 \mathrm{~N} & 140.589 \mathrm{E} \quad 85 \mathrm{~km}\end{array}$

$6.1 \mathrm{mb}$ ( 86 obs.)

SOUTH OF HONSHU, JAPAN

FAULT PLANE SOLUTION: P-WOVES

NP1: Strike=178 Dip=87 S1 i $=106$

NP2

Principol Axes:

T PIg=46 Azm=104

Comment: The focol mechonism is

poorly controlled and

corresponds to reverse

foulting with o moderote left-

loterol strike-slip component

The preferred foult plone is NP2 2

RADIATED ENERGY

No. of sto: 7 Focol mech. C

Energy $5.1 \pm 1.7 * 10 * 13 \mathrm{Nm}$

MOMENT TENSOR SOLUTION

Dep 91 No. of sta: 14

Principol Axes 
Scole $10 *+18 \mathrm{Nm}$

I $\quad V_{0} l=1.57 \quad$ PIg $=51 \quad$ Azm $=89$

$\begin{array}{rrrr}N & 0.16 & 15 & 346 \\ P & -1.73 & 35 & 239\end{array}$

Best Double Couple: $M O=1.7 * 10 * 18$

NP1: Strike $=279$ Dip=17 Siip= 28

$\begin{array}{llll}\text { NP2: } & 162 & 82 & 105\end{array}$

CENTROID, MOMENT TENSOR (HRV)

Dato Used: GDSN

L.P.B.: 16S, 45C M.W.: 11S, $16 \mathrm{C}$

Centraid Lacation:

Origin Time $02: 20: 7.40 .1$

Lot $36.48 \mathrm{~N} 0.01$ Lon $140.41 \mathrm{E} 0.01$

Dep 78.01 .3 Half-duration 5.3

Principol Axes:

Scole $10 *+18 \mathrm{Nm}$

I $\quad \mathrm{VOl}=3.25 \quad \mathrm{Plg}=56 \quad$ Azm $=169$

$\begin{array}{rrrr}N & -0.27 & 3 & 203 \\ P & -2.98 & 34 & 295\end{array}$

Best Double Couple: $M O=3.1 * 16 * 18$

NP1:Strike $=37$ Dip=12 SIip= 104

$\begin{array}{llll}\text { NP2: } & 203 & 79 & 87\end{array}$

$27 \quad 09 \quad 3458.64 \quad 23.950 S \quad 179.764 w 524 \mathrm{~km}$ $5.1 \mathrm{mb}$ ( 28 obs.)

SOUTH OF FIJI ISLANDS

CENTROID, MOMENT TENSOR

Doto Used: GDSN

L.P.B.: 10S, $19 \mathrm{C}$

Centraid Lacation:

Origin Time 69:35: $9.6 \quad 1.4$

Lat $23.79 \$ \quad 0.15$ Lan 179.75E 0.14

Dep 543.8 6.4 Half-duration 1.6

Principal Axes:

Scale $10 * * 16 \mathrm{Nm}$

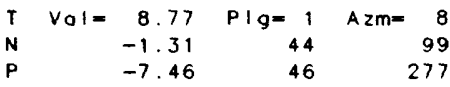

Best Dauble Cauple:Ma=8.1*10*16

NP1: Strike $=63$ Dip=59 Si i $p=-145$

NP2: $\quad 313 \quad 60 \quad-37$

$\begin{array}{llllllll}27 & 16 & 39 & 36.98 & 56.419 \mathrm{~N} & 164.122 \mathrm{E} \quad 33 \mathrm{~km}\end{array}$ $4.9 \mathrm{mb}$ ( 12 obs.) 4.8Msz ( 3 abs.) KOMANDORSKY ISLANDS REGION

CENTROID, MOMENT TENSOR (HRV)

Doto Used: GDSN

L.P.B.: 16S, 32C

Centraid Lacatian:

origin Time

$10: 39: 40.6 \quad 0.4$

Lot $56.53 \mathrm{~N} 0.05$ Lan $164.14 \mathrm{E} 0.09$

Dep 34.85 .4 Holf-duration 1.7

Principol Axes:

Scale $10 * * 16 \mathrm{Nm}$

I $\quad$ Ool $=12.77 \quad$ Plg $=28 \quad$ Azm $=242$

$\begin{array}{rrrr}N & -1.90 & 59 & 37 \\ P & -10.87 & 11 & 146\end{array}$

Best Dauble Couple: $M O=1.2 * 10 * 17$

NP1: Strike $=281$ D i $p=62$ Si i $p=167$

NP2

79

$27 \quad 23 \quad 66 \quad 52.24 \quad 37.627 \mathrm{~N} \quad 28.180 \mathrm{E} \quad 14 \mathrm{~km}$ $5.3 \mathrm{mb}$ ( 44 obs.) 5.6Msz ( 7 abs.) TURKEY

CENTROID, MOMENT TENSOR (HRV)

Doto Used: GDSN

L.P.B.: 13S, $21 \mathrm{C}$

Centroid Lacation:

Origin Time 23:06:55.3 0.6 Lot $36.71 N 0.05$ Lan $27.75 E 0.08$ Dep $15.0 \mathrm{FIX}$ Holf-durotion 2.2

Principol Axes

Scole $10 * * 17 \mathrm{Nm}$

$\begin{aligned} & \text { T } \\ & N\end{aligned} \quad \mathrm{Ol}=\begin{array}{r}2.26 \\ -6.53\end{array} \quad \mathrm{PIg}=\begin{aligned} & 9 \\ & 2\end{aligned} \quad$ Azm $=\begin{array}{r}4 \\ 95\end{array}$

$\begin{array}{lllr} & -0.53 & 2 & 95 \\ P & -1.73 & 81 & 200\end{array}$

Best Double Cauple: $M O=2.6 * 10 * * 17$

NP1: Strike $=92$ Dip=36 Si $P=-94$

NP2 :

$276 \quad 54$

$-87$

$\begin{array}{llllllll}28 & 62 & 34 & 25.31 & 17.830 \mathrm{~N} & 105.174 \mathrm{~W} & 10 \mathrm{~km}\end{array}$ $5.3 \mathrm{mb}$ ( 20 ODS.) $5.2 \mathrm{Msz}$ ( 6 ODs.) OFF COAST OF JALISCO, MEXICO

CENTROIO, MOMENT TENSOR (HRV)

Doto Used: GOSN

L.P.B.: 18S, $37 \mathrm{C}$

Centraid Lacation:

Origin Time $\quad 02: 34: 27.10 .3$ Lat $17.96 \mathrm{~N} 0.04$ Lan $105.22 \mathrm{~W} 0.04$ Dep 15.0 FIX Half-duration 2.5 Principol Axes:

Scale $10 * 17 \mathrm{Nm}$

$T \quad$ Val $=3.28 \quad \mathrm{Plg}=7 \quad$ Azm $=246$
$\begin{array}{llll}N & -0.08 & 72 & 357 \\ P & -3.19 & 17 & 153\end{array}$

Best Dauble Couple: $M a=3.2 * 10 * * 17$

NP1: Strike=291 Dip=73 Si i $p=-173$

$\begin{array}{llll}\text { NP2 } & 198 & 83 & -17\end{array}$

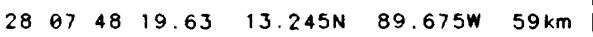

$5.2 \mathrm{mb}$ ( 44 abs.)

EL SALVADOR

CENTROID, MOMENT TENSOR (HRV)

Dato USEd: GDSN

L.P.B.: 17S, $28 \mathrm{C}$

Centroid Location:

Origin Time $\quad 07: 48: 17.80 .6$

Lot $12.81 \mathrm{~N} 0.09$ Lon $90.10 \mathrm{~W} 0.10$

Dep 29.54 .6 Holt-duration 1.7

Principol Axes:

Scale $10 * * 16 \mathrm{Nm}$

$\begin{array}{rrrr}T & V a l=8.03 & 81 g=9 & A z m=216 \\ N & 0.58 & 17 & 123\end{array}$

$\begin{array}{llll}N & 0.58 & 17 & 123 \\ P & -8.61 & 71 & 334\end{array}$

Best Double Couple:Mo=8.3*10**16

NP 1 : Strike=326 o i $p=39$ Si i $p=-62$

NP2: $\quad 112 \quad 56 \quad-110$

$\begin{array}{llllllll}28 & 69 & 13 & 46.23 & 26.163 \$ & 67.478 E & 10 \mathrm{~km}\end{array}$

5. $2 \mathrm{mb}$ ( 11 abs.) 4.9Msz ( 1 abs.)

$M I D-I N D I A N$ RISE

CENTROID, MOMENT TENSOR (HRV)

Doto Used: GDSN

L.P.B.: 15S, $25 \mathrm{C}$

Centroid Location:

Origin Time $\quad 09: 13: 54.80 .7$

Lat 20.305 0.10 Lan 67.12E 0.10

Dep $15.0 \mathrm{FIX}$ Half-duration 1.5

Principol Axes:

Scale $10 * * 16 \mathrm{Nm}$

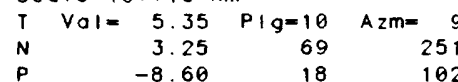

Best Double Couple: $M O=7.0 * 10 * * 16$

$\begin{array}{rrrr}\text { NP 1 : Strike=145 Dip=70 Si i }=r & -6 \\ \text { NP2: } & 237 & 85 & -160\end{array}$

$\begin{array}{llllllll}28 & 09 & 23 & 07.34 & 16.606 \mathrm{~S} & 173.645 \mathrm{~W} \quad 93 \mathrm{~km}\end{array}$

5. $4 \mathrm{mb}$ ( 24 abs.)

TONGA ISLANDS

CENTROID, MOMENT TENSOR (HRV)

Dato Used: GDSN

L.P. B.: 15S, $26 \mathrm{C}$

Centroid Location

Origin Time $\quad 09: 23: 14.20 .7$

Lat $16.525 \quad 0.12$ Lan $173.69 \mathrm{~W} 0.08$

Dep 91.13 .4 Half-duration 1.6

Principol Axes:

Scale $10 * 16 \mathrm{Nm}$

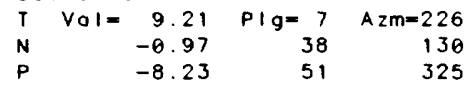

Best Double Couple:Ma=8.7*10**16

NP 1: Strike $=351$ Di $p=50$ SI i $p=-37$

$\begin{array}{llll}\text { NP2: } & 107 & 63 & -134\end{array}$

$\begin{array}{lllllll}28 & 13 & 30 & 19.24 & 37.004 \mathrm{~N} & 28.139 \mathrm{E} & 17 \mathrm{~km}\end{array}$ $5.1 \mathrm{mb}$ ( 39 abs.) 5.1Msz ( 4 abs.) TURKEY

CENTROID, MOMENT TENSOR (HRV)

Doto Used: GDSN

L.P. B : 15S, $28 \mathrm{C}$

Centraid Lacation:

Origin Time 13:30:22.8 0.5

Lat 36.95N 0.64 Lan 27.79E 0.06

Dep 15.6 FIX Half-duration 2.2

Principol Axes:

Scole $10 * * 17 \mathrm{Nm}$

$T \quad V a I=2.55 \quad \dot{P} / g=4 \quad$ Azm $=8$

$\begin{array}{llll}N & -0.39 & 7 & \text { A } 2 m= \\ P & -2.16 & 89\end{array}$

Best Dauble Couple:Ma=2.4*10**17

NP1: Strike $=90$ Dip=41 SIip=-101

NP2: $\quad 285 \quad 50 \quad-80$

$\begin{array}{llllllll}28 & 17 & 38 & 26.42 & 4.354 \mathrm{~S} & 161.413 \mathrm{E} & 27 \mathrm{~km} \text { । }\end{array}$ $5.4 \mathrm{mb}$ ( 25 abs.) 5.8Msz ( 30 abs.) SOUTHERN SUMATERA

CENTROID. MOMENT TENSOR (HRV)

Dato Used: GDSN

L.P.B.: 18S, $42 \mathrm{C}$

Centraid Lacation:

Origin Time

$17: 38: 29.0 \quad 0.4$

Lat 4.75S 0.04 Lan 101.12E 0.05

Dep 15.0 Bor Half-duration 3.9

Principol Axes:
Scole $10 * * 18 \mathrm{Nm}$

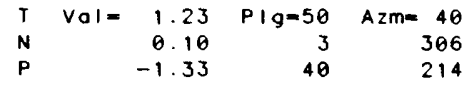

Best Double Couple: $M O=1.3 * 10 * * 18$ NP1: Strike $=277$ Dip= 6 SIip= 61 $\begin{array}{llll}\text { NP2: } & 127 & 85 & 93\end{array}$

$\begin{array}{lllllll}28 & 20 & 08 & 51.59 & 4.294 \mathrm{~S} & 101.363 \mathrm{E} & 30 \mathrm{~km}\end{array}$ $5.2 \mathrm{mb}$ ( 21 obs.) $5.6 \mathrm{Msz}$ ( 17 abs.) SOUTHERN SUMATERA

CENTROID. MOMENT TENSOR

(HRV)

Dato Used: GDSN

L.P. B. 20S $38 \mathrm{C}$

Centroid Lacation

$26: 68: 55.6 \quad 0.7$

Lat 4.86S 0.06 Lan 101.18E 0.07

Dep 15.6 FIX Half-duration 2.7

Principol Axes:

Scole $10 * 17 \mathrm{Nm}$

T $\mathrm{Vol}=3.58 \quad \mathrm{Plg}=62 \quad \mathrm{Azm}=18$

$\begin{array}{rrrr}N & 0.80 & 5 & 118 \\ P & -4.38 & 27 & 211\end{array}$

Best Double Couple: $M 0=4.0 * 10 * * 17$

NPI: Strike=315 Dip=19 Stip=107

NP2: $\quad 116 \quad 32 \quad 84$

$\begin{array}{lllllll}28 & 20 & 26 & 17.94 & 59.515 \mathrm{~S} & 29.397 w \quad 22 \mathrm{~km}\end{array}$

$5.5 \mathrm{mb}$ ( 10 obs.) 5.7Msz ( 2 obs.)

SOUTH SANDWICH ISLANDS REGION

CENTROID, MOMENT TENSOR (HRV)

Doto Used: GDSN

L.P.B.: $175,38 \mathrm{C}$

Centraid Location:

Origin Time $20: 26: 25.7 \quad 0.2$

Lat $59.47 \mathrm{~S} 0.03$ Lon $29.54 \mathrm{~W} 0.06$

Dep $15.6 \mathrm{FIX} \mathrm{Half-duration} 3.8$

Principol Axes:

Scale $10 * 17 \mathrm{Nm}$

I $\quad \mathrm{O} O \mathrm{l}=11.52 \quad \mathrm{P} / \mathrm{g}=6 \quad \mathrm{Azm}=290$

$\begin{array}{rrrr}N & 0.25 & 83 & 136 \\ P & -11.77 & 3 & 21\end{array}$

Best Double Couple: $M O=1.2 * 10 * 18$

NP1:Strike=66 Dip=84 SIip= 2

NP2: $\quad 335 \quad 88$

$\begin{array}{llllll}28 & 2169 & 57.66 & 4.210 \mathrm{~S} & 101.370 \mathrm{E} \quad 32 \mathrm{~km}\end{array}$ $5.4 \mathrm{mb}$ ( 27 obs.) 5.7Msz ( 21 obs.) SOUTHERN SUMATERA

CENTROID, MOMENT TENSOR (HRV)

Doto Used: GDSN

L.P.B.: 16S, $31 \mathrm{C}$

Centraid Lacatian:

Origin Time 21:10:4.4 1.6

Lat 4.21S FIX; Lon 101.39E FIX

Dep $15.0 \mathrm{FIX}$ Half-duration 2.7

Principal Axes:

Scale $10 * * 18 \mathrm{Nm}$

I $\quad$ ol $=1.46 \quad P \mid g=50 \quad$ Azm $=34$

$\begin{array}{lrrr}N & 0.12 & 2 & 126\end{array}$

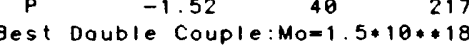

NP1:Strike=324 Dip= 5 Si ip= 108

NP2: $\quad 126 \quad 85$

$\begin{array}{llllll}29 & 0103 & 06.85 & 5.494 \mathrm{~S} & 102.976 \mathrm{E} \quad 38 \mathrm{~km}\end{array}$

5. $4 \mathrm{mb}$ ( 26 obs.) 5.1Msz ( 13 obs.)

SOUTHERN SUMATERA

CENTROID, MOMENT TENSOR

(HRV)

Doto Used: GDSN

L.P.B.: 15S, $24 \mathrm{C}$

Centraid Lacation

Origin Time $01: 03: 10.30 .7$

Lat $5.47 \mathrm{~S}$ FIX:Lan $102.99 \mathrm{E}$ FIX

Dep 33.26 .5 Half-duration 1.6

Principal Axes:

Scale $10 * * 16 \mathrm{Nm}$

$T \quad$ Vol $=7.67 \quad \mathrm{Plg}=61 \quad$ Azm=133

$\begin{array}{rrrr}N & 1.04 & 29 & 326\end{array}$

Best Dauble Cauple:Mo=8.2*10**16

NP 1: Strike=295 Dip=47 S1 ip= 49

NP2:

$\begin{array}{lll}167 & 57 & 125\end{array}$

$\begin{array}{lllllllll}29 & 06 & 25 & 39.12 & 57.190 \mathrm{~N} \quad 122.069 \mathrm{E} \quad 30 \mathrm{~km}\end{array}$

5.3mb ( 47 obs.) 5.1Msz (10 abs.)

EASTERN USSR

CENTROID, MOMENT TENSOR

(HRV)

Data USEA: GDSN

L.P.B.: $15 \mathrm{~S}, 28 \mathrm{C}$

Centroid Lacotion:

Origin Time

$86: 25: 43,9: 4$

Lot $57.13 N \quad 0.04$ Lan $121.80 E 0.10$ 


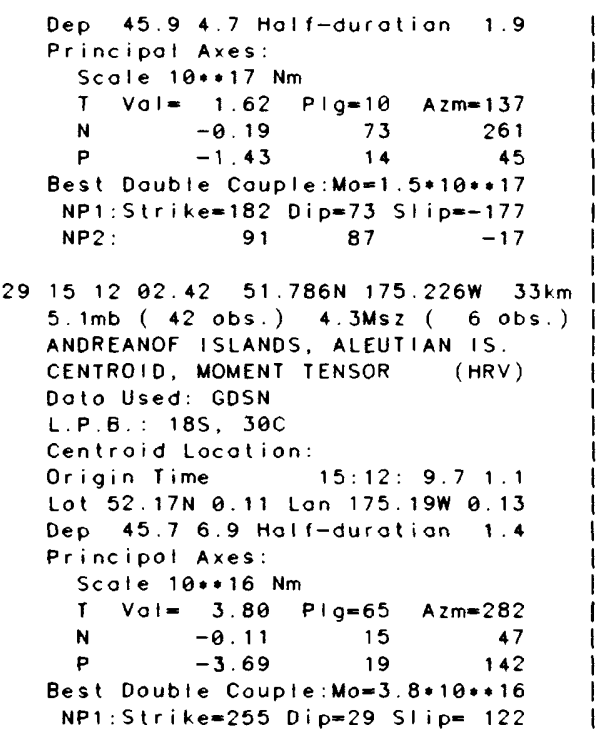

Dep 45.9 4.7 Holf-durotion 1.9 Principol Axes: Scole $10 \ldots 17 \mathrm{Nm}$

$T \quad$ VOl $=1.62 \quad P 1 g=10 \quad$ A2m $=137$

$\begin{array}{llll}N & -0.19 & 73 & 261 \\ P & -1.43 & 14 & 45\end{array}$

Best Double Couple:Mo=1.5*10*:17

NP1: Strike $=182$ Dip $=73$ S1 i $p=-177$

NP2 :

91

87

$-17$

$29 \quad 15 \quad 1202.42 \quad 51.786 \mathrm{~N} \quad 175.226 \mathrm{~W} \quad 33 \mathrm{~km}$ 5. $1 \mathrm{mb}$ ( 42 obs.) 4.3MSZ ( 6 obs.) ANDREANOF ISLANDS, ALEUTIAN IS

CENTROID, MOMENT TENSOR (HRV) Doto UsEo: GDSN

L.P.B.: $18 \mathrm{~S}, 30 \mathrm{C}$

Centroid Locotion:

Origin Time $15: 12: 9.71 .1$

Lot $52.17 \mathrm{~N} 0.11$ Lon 175.19W 0.13

Dep 45.76 .9 Holf-durotion 1.4 Principot Axes

Scole $10 * 16 \mathrm{Nm}$

I $V_{0} l=3.80 \quad P \mid g=65 \quad$ Azm $=282$

$\begin{array}{rrrr}N & -0.11 & 15 & 47 \\ P & -3.69 & 19 & 142\end{array}$

Best Double Couple:Mo=3.8*10**16

NP 1: Strike $=255$ Dip=29 S1 ip= 122

NP2 :

74

$\begin{array}{llllll}3008 & 22 & 54.01 & 10.960 \mathrm{~N} & 68.325 \mathrm{~W} & 20 \mathrm{~km}\end{array}$ 5. $9 \mathrm{mb}$ ( 62 obs.) 6.OMsz ( 31 obs.) । NEAR COAST OF VENEZUELA

RADIATED ENERGY

No. of sta: 5 focal mech. M Energy $4.3 \pm 1.5 * 10 *+13 \mathrm{Nm}$ MOMENT TENSOR SOLUTION

Dep 15 No. of sto: 10

Principol Axes:

Scole $10 *+17 \mathrm{Nm}$

$T \quad V_{0} l=8.30 \quad$ PIg $=53 \quad$ A2m $=231$

$\begin{array}{rrrr}N & -0.38 & 30 & 10 \\ P & -7.91 & 20 & 112\end{array}$

Best Double Couple: Mo $=8 \cdot 1 * 10 * 17$

NP1: Strike $=242$ Dip=36 Slip= 147

NP2: $\quad 359 \quad 71 \quad 58$

CENTROID, MOMENT TENSOR (HRV)

Doto Used: GOSN

L.P.B.: 19S, 46C

Centraid Lacotion:

Origin Time $68: 22: 59.90 .3$

Lot $10.88 \mathrm{~N} 0.04$ Lon $68.08 \mathrm{~W} 0.03$

Dep 15.0 FIX Half-durotion 3.7

Principol Axes:

Scale $10 * 17 \mathrm{Nm}$

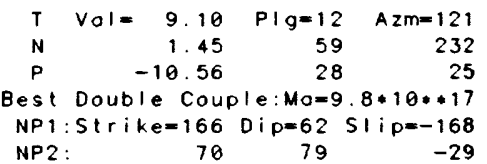

$\begin{array}{llllll}30 & 15 & 33 & 52.73 & 17.912 S \quad 178.665 W \quad 570 k m\end{array}$ 5. $4 \mathrm{mb}$ ( 46 Obs.)

FIJI ISLANDS REGION

CENTROID, MOMENT TENSOR

(HRV)

Do to Used: GDSN

L.P.B.: 175,410

Centroio Locotion

origin Time Lat 17.6750 .03 Lon 179.15 W 0.03 Dep 591.2 1.3 Holf-duration 4.0 Principol Axes:

Scole $10 *+17 \mathrm{Nm}$

$T$ Vol $=13.01 \quad$ Plg $=22 \quad$ Azm $=340$

$\begin{array}{rrrr}N & 0.06 & 37 & 88 \\ P & -13.08 & 45 & 227\end{array}$

Best Double Couple: $M 0=1,3 * 10 * 18$

NP 1: Strike $=25$ D i $p=40$ SI i $p=-158$

$\begin{array}{llll}\text { NP2 : } & 278 & 76 & -52\end{array}$

Compiled by Willis $S$. Jocobs, Leonord E. Kerry, Jahn H. Minsch, Russell E. Needham, Woverly J. Person, Bruce W. Presgrave and Williom $H$. Schmieder.

EXPLANATION OF THE ENTRIES "GEOSCOPE MOMENT TENSOR (PAR)"

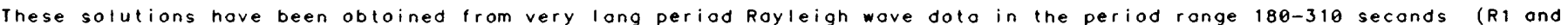

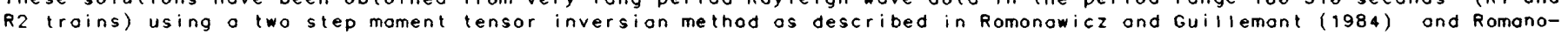

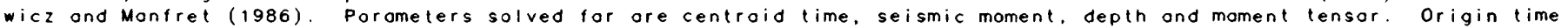

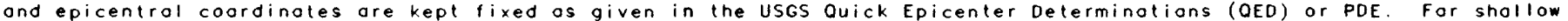
earthquakes the precision on depth is in generol no greoter thon $+/-10 \mathrm{~km}$.

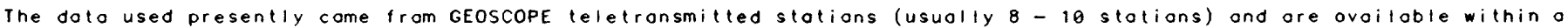

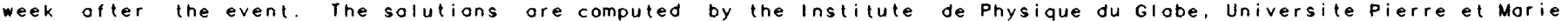
Curie, Poris, France.

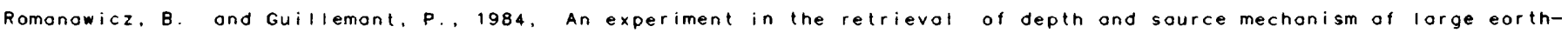
quakes using very lang-period Rayleigh wove dota: Bulletin of the Seismological Society of America, $v$. 74 , na. 2, p. 417-437.

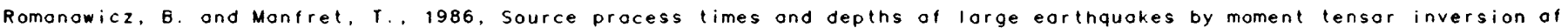
mantle wave doto and the effect of loterot heterogeneity: Annoles de Geophysique, v. B4, no. 3, p. $271-282$. 


\section{April 1989 08:05:57.10 \\ Vanuatu Islands}

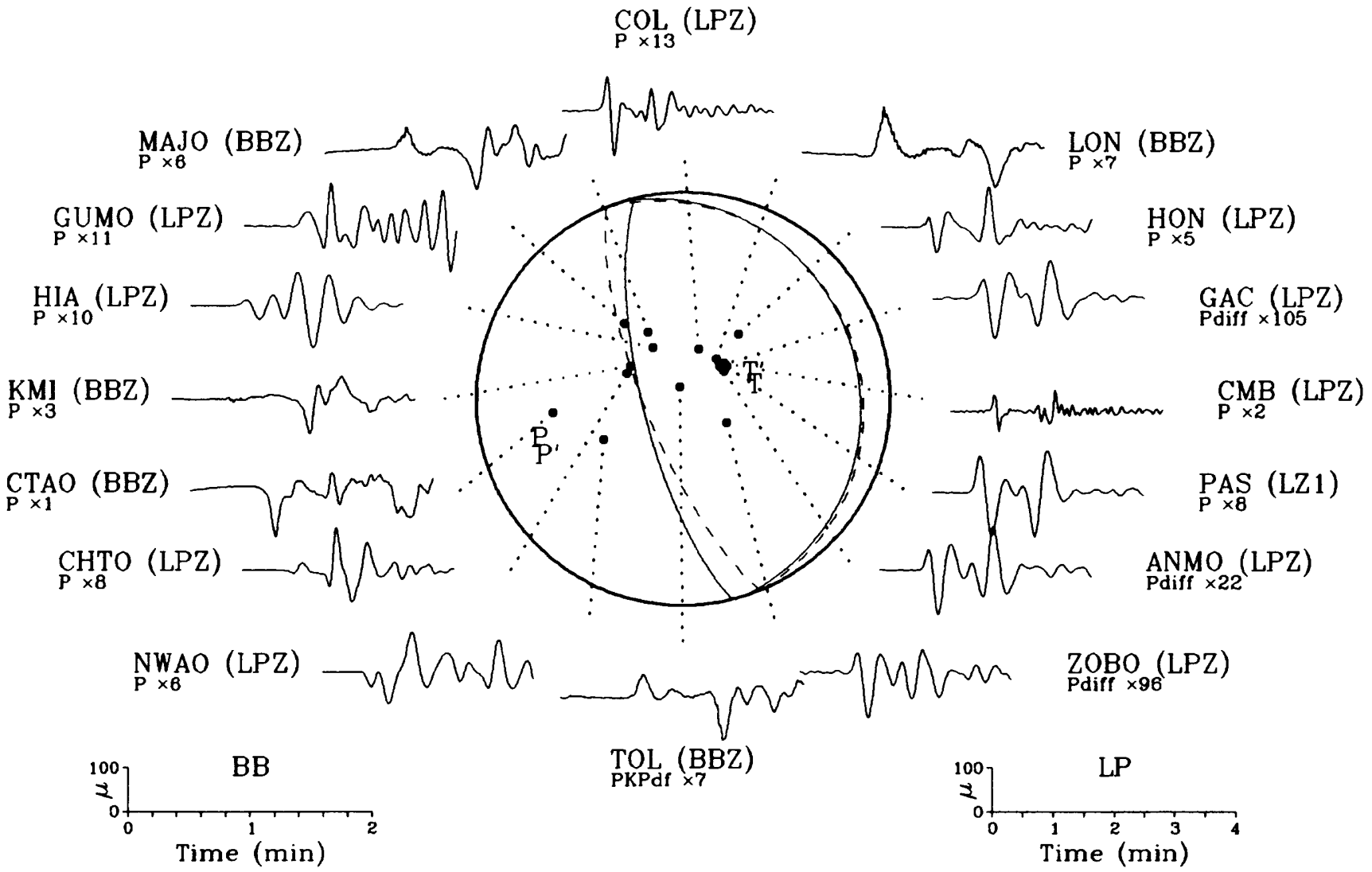

\section{April 1989 03:56:36.91 \\ Kuril Islands Region}

$\underset{\mathrm{P} \times 5}{\mathrm{GDH}}(\mathrm{LPZ})$

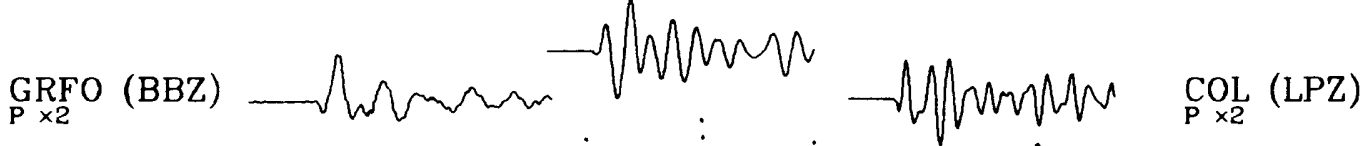

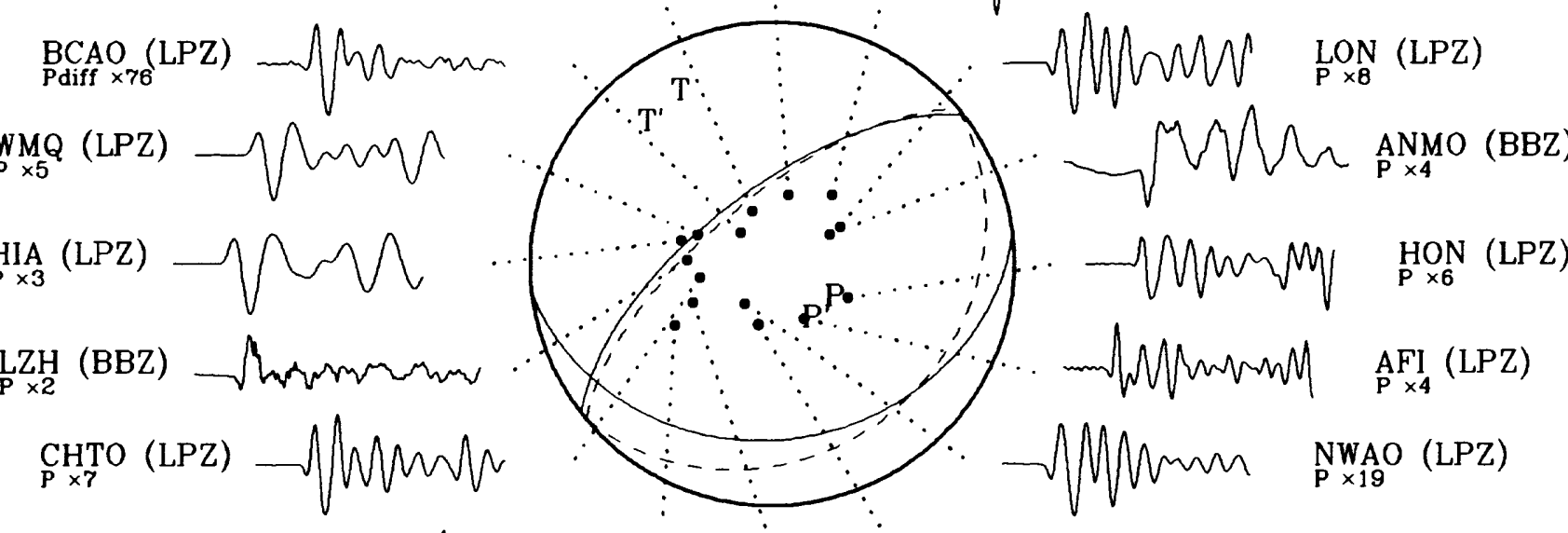

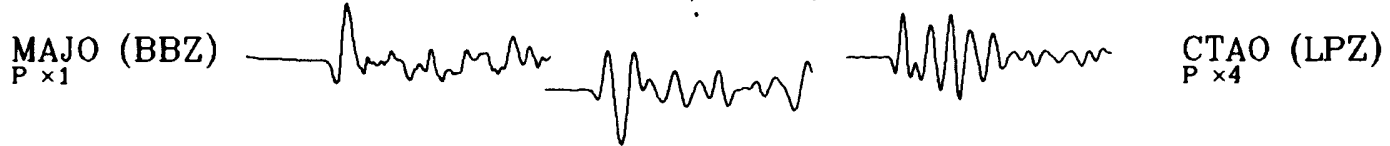

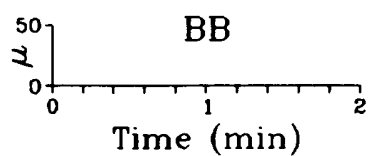

$\underset{P \times 11}{\text { TATO }}(\mathrm{LPZ})$

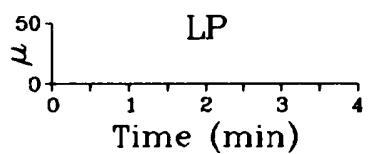




\section{April 1989 20:34:08.93 \\ Sichuan Province, China}

$\underset{\mathrm{P} \times \bar{B}}{\mathrm{C}} \mathrm{L}(\mathrm{BBZ})$

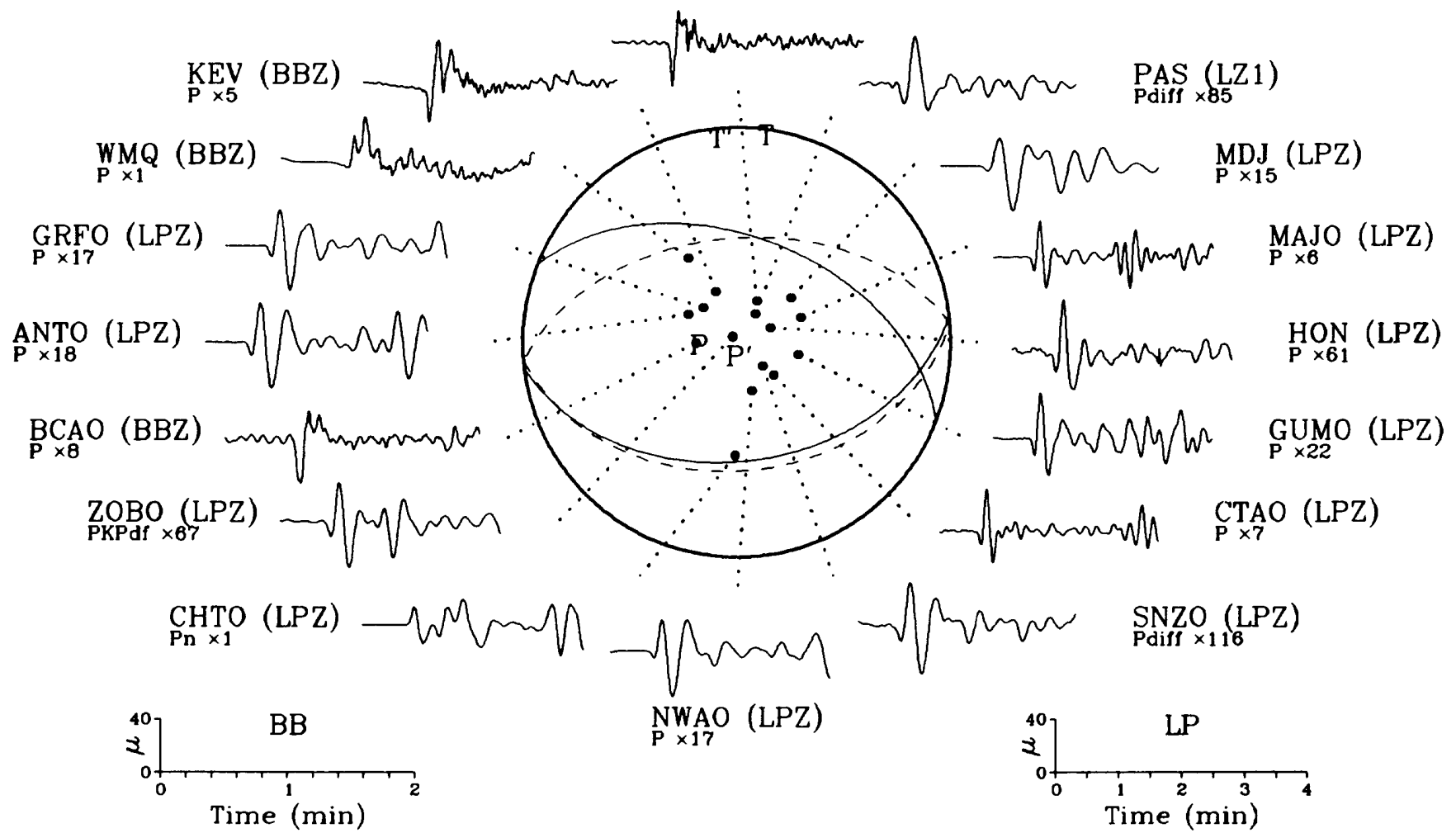

19 April 1989 00:08:19.65

Kermadec Islands Region

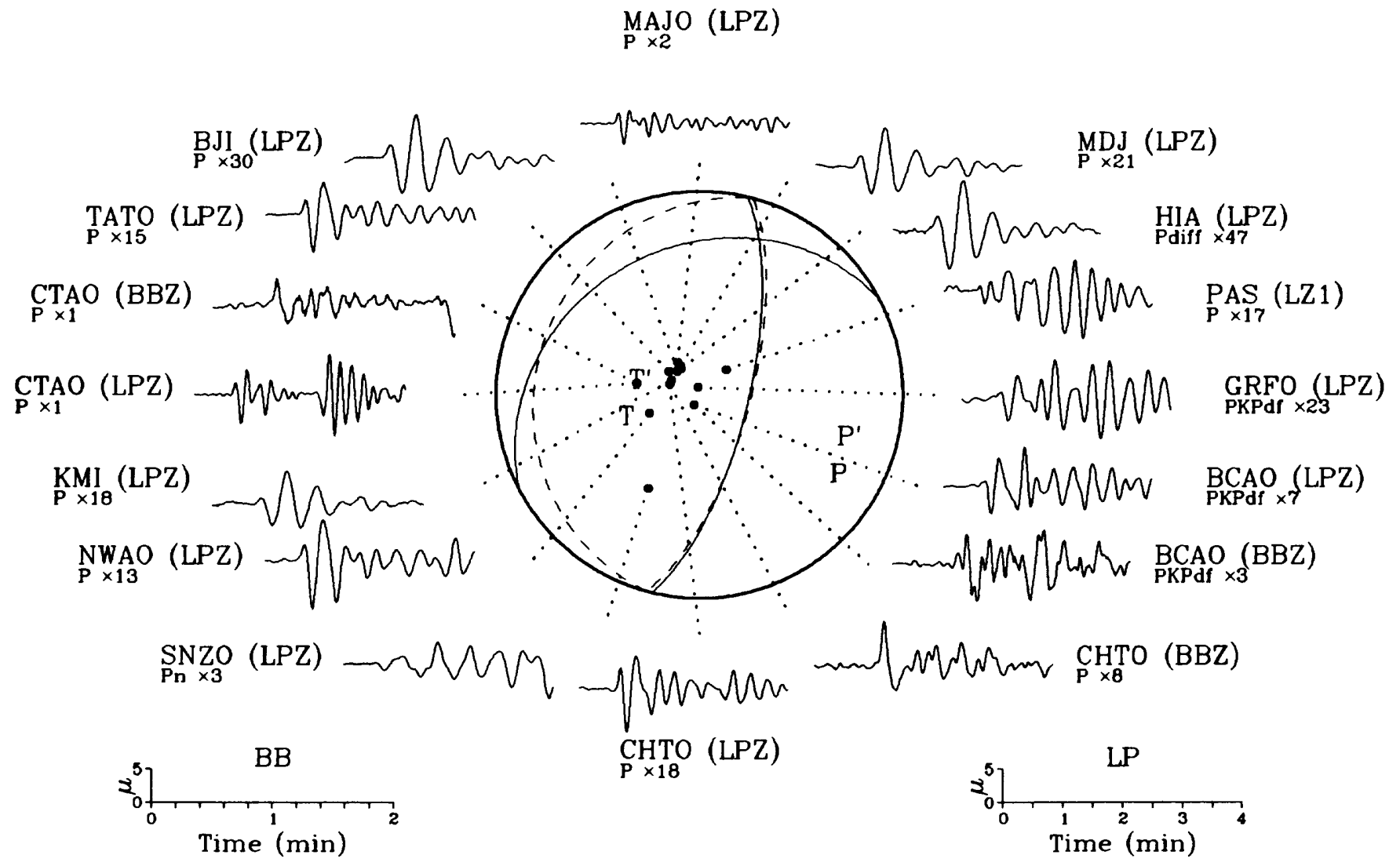




\section{April 1989 22:59:54.07 \\ Eastern USSR}

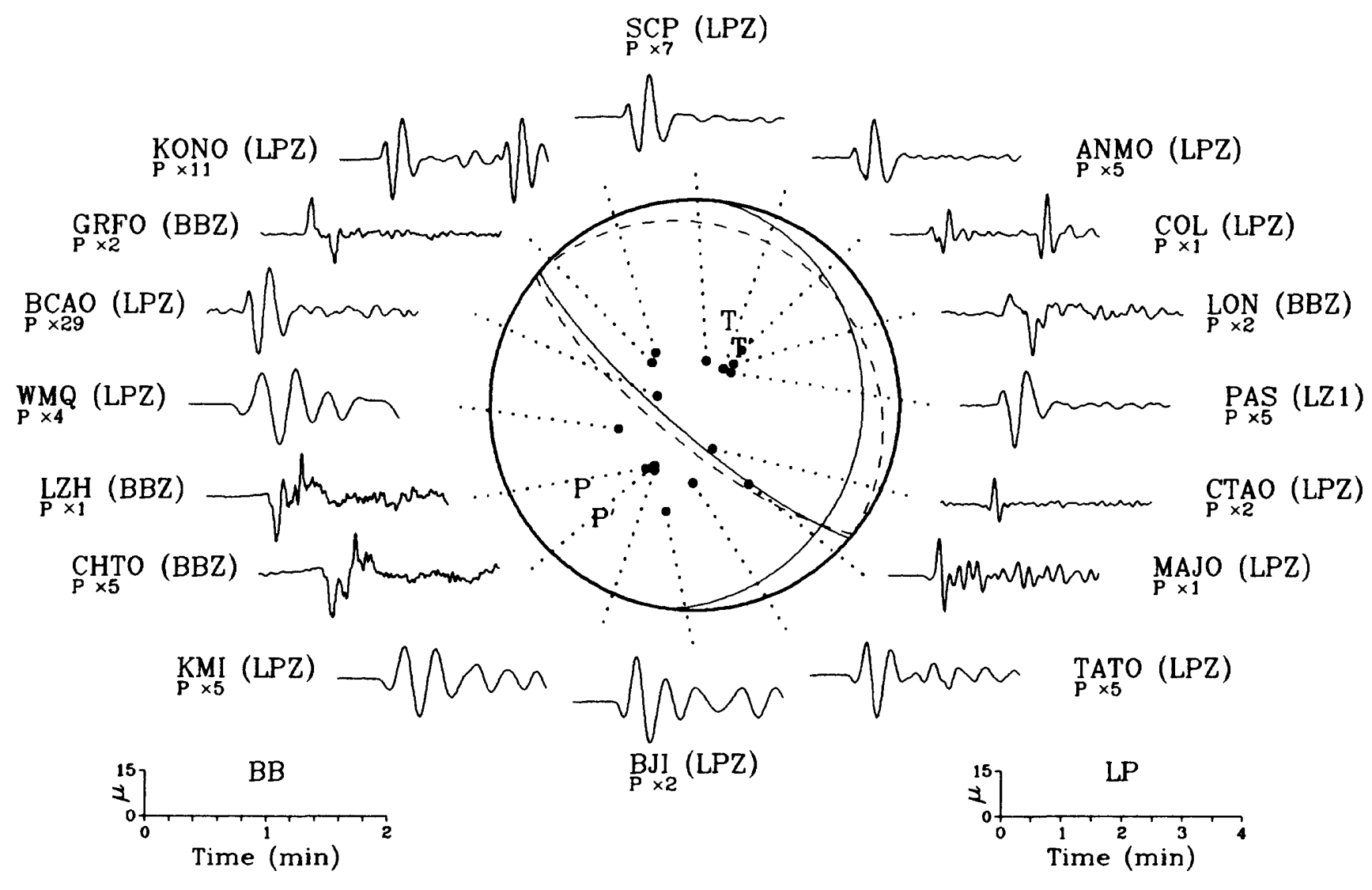

25 April 1989 02:13:20.83

Sichuan Province, China

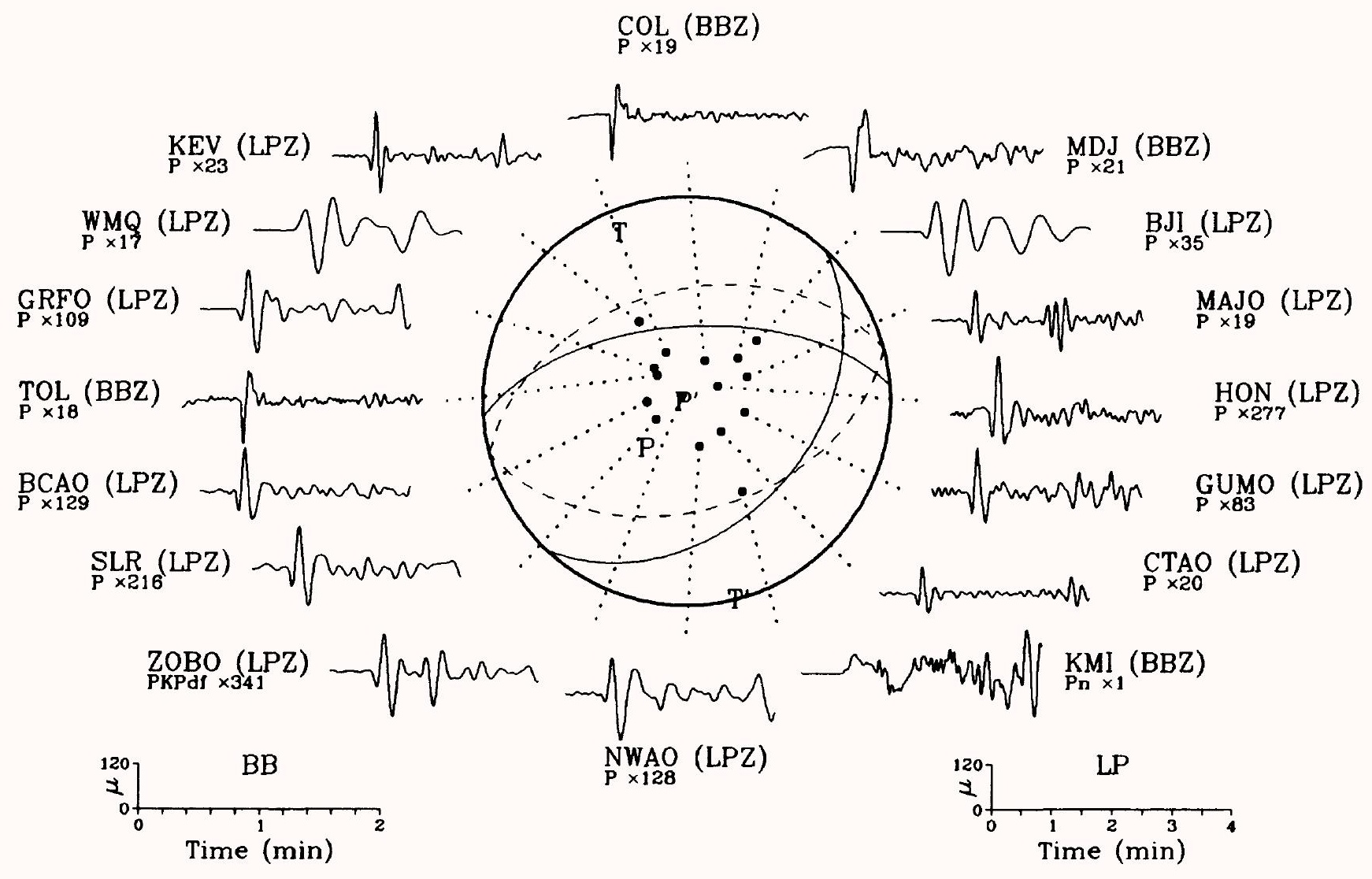




\section{April 1989 14:29:00.51}

\section{Near Coast of Guerrero, Mexico}

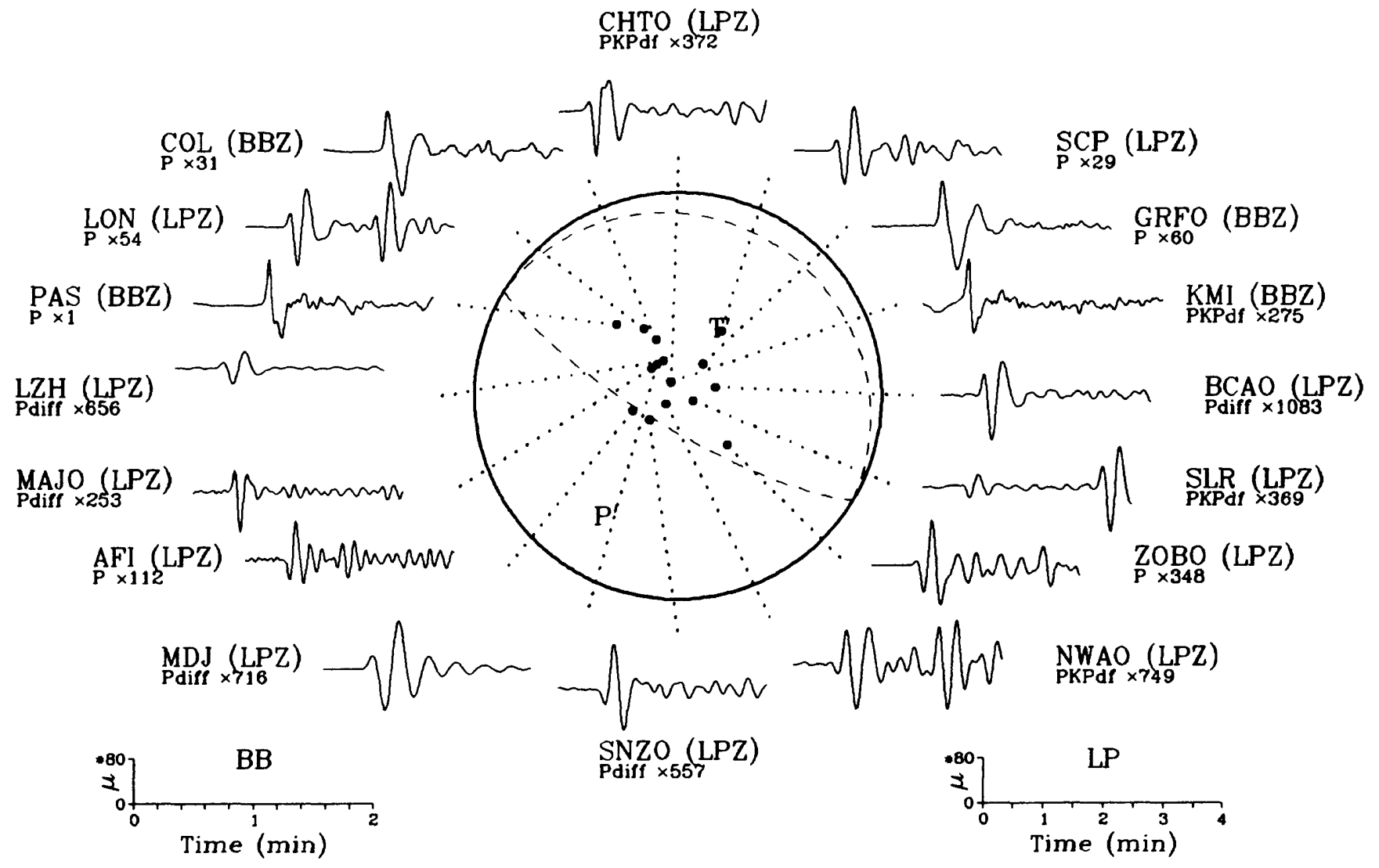

27 April 1989 02:20:04.74

South of Honshu, Japan

$\underset{P}{\mathrm{KEV}} \times 18(\mathrm{LPZ})$

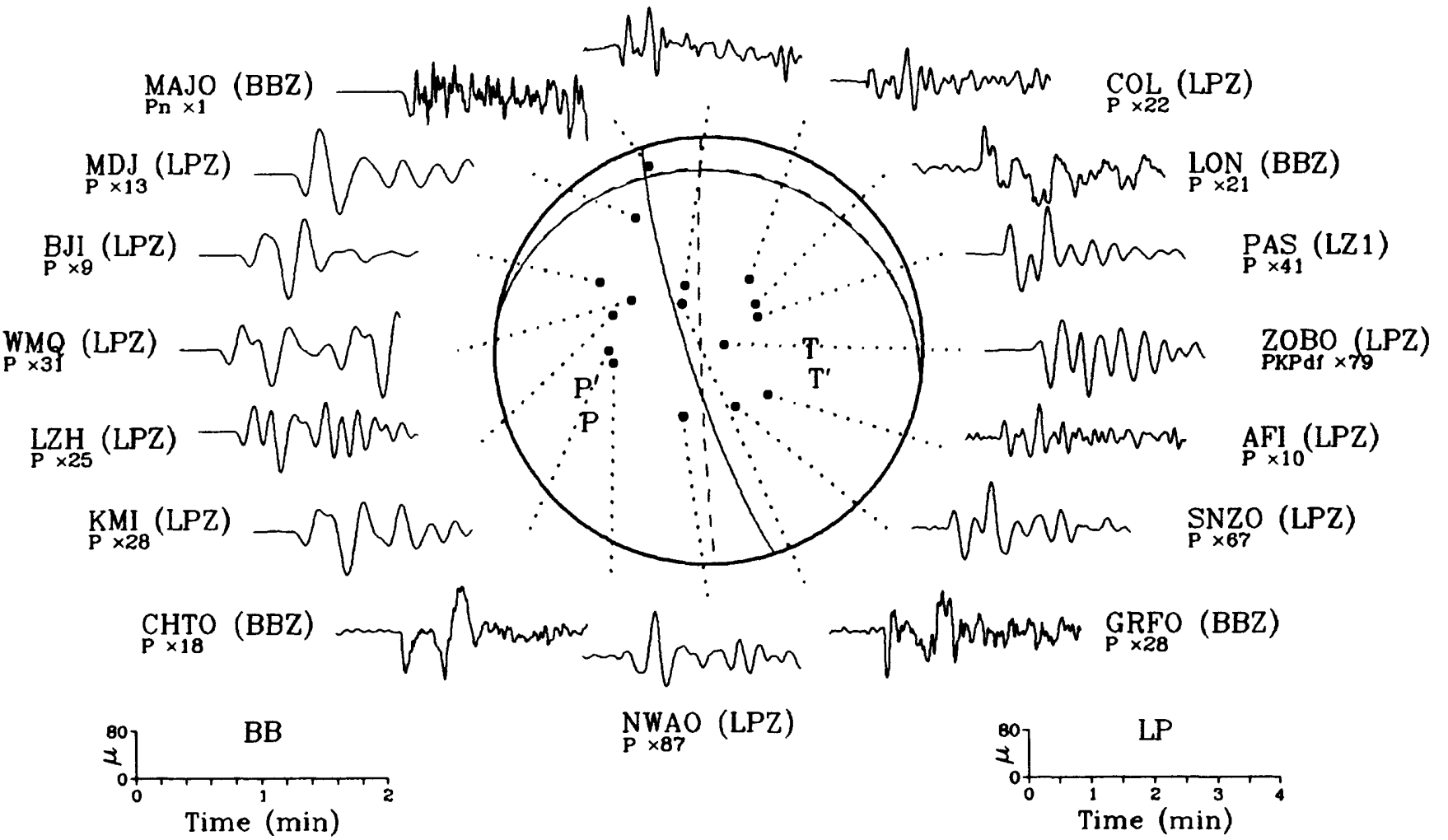




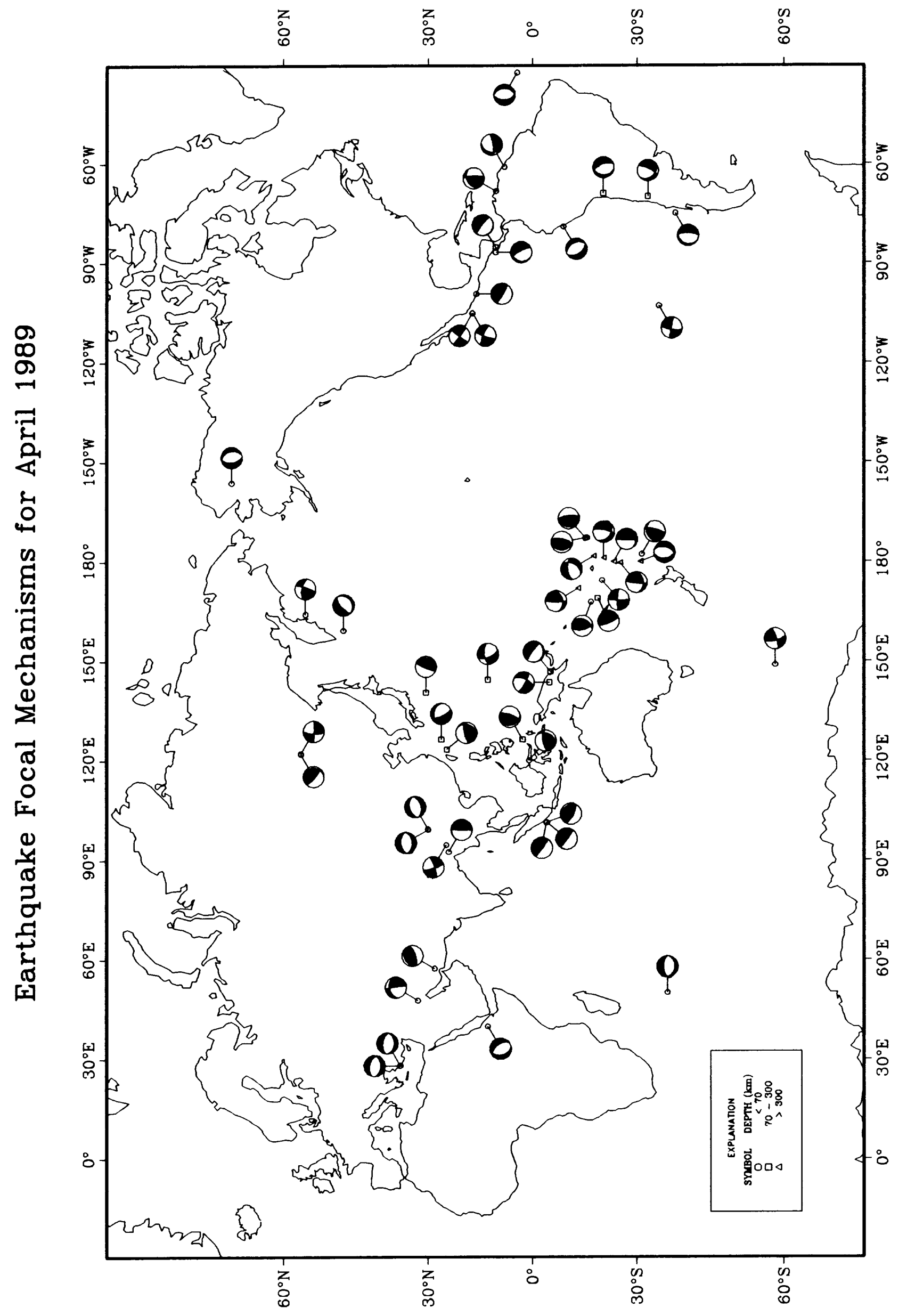




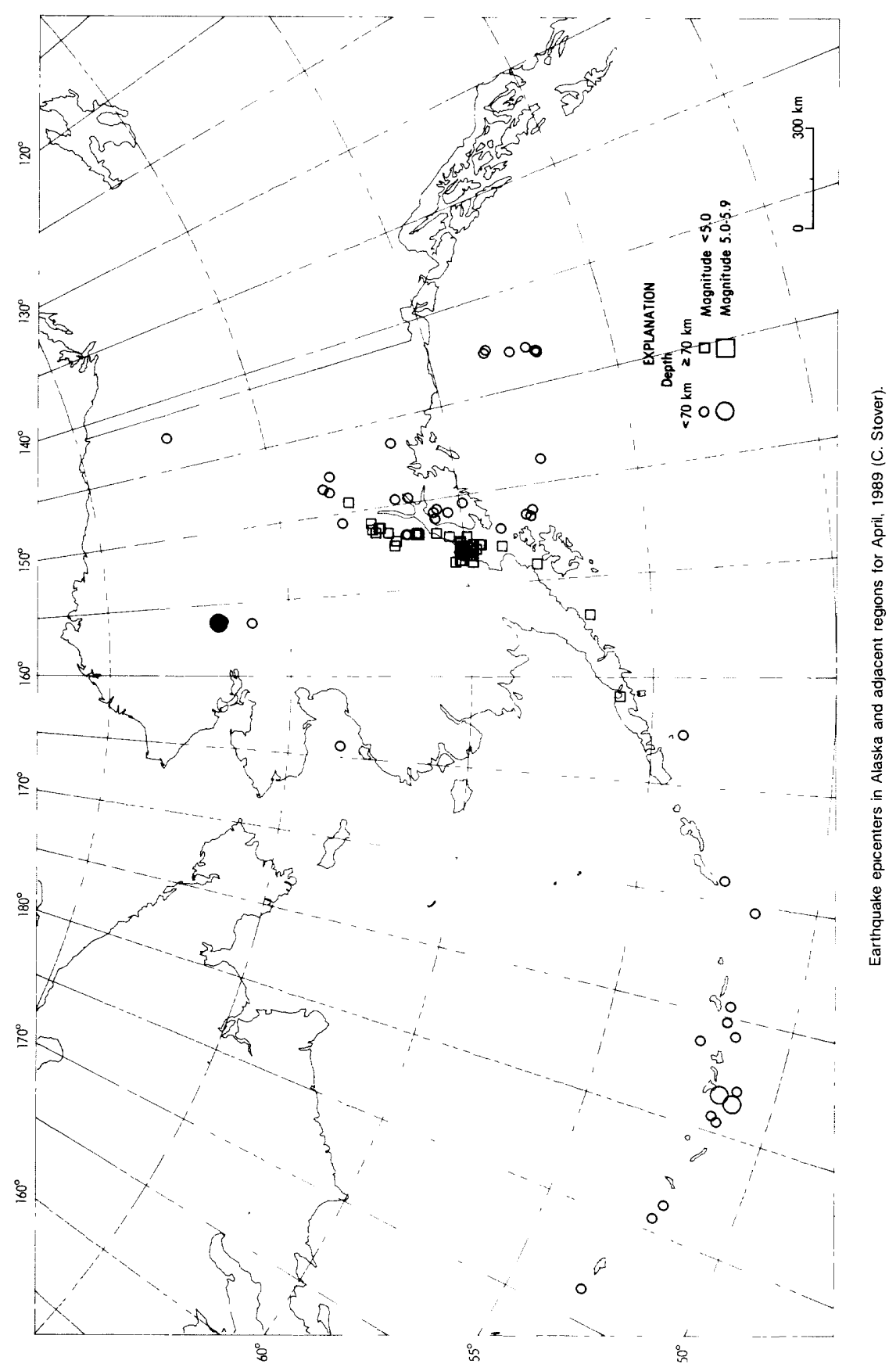




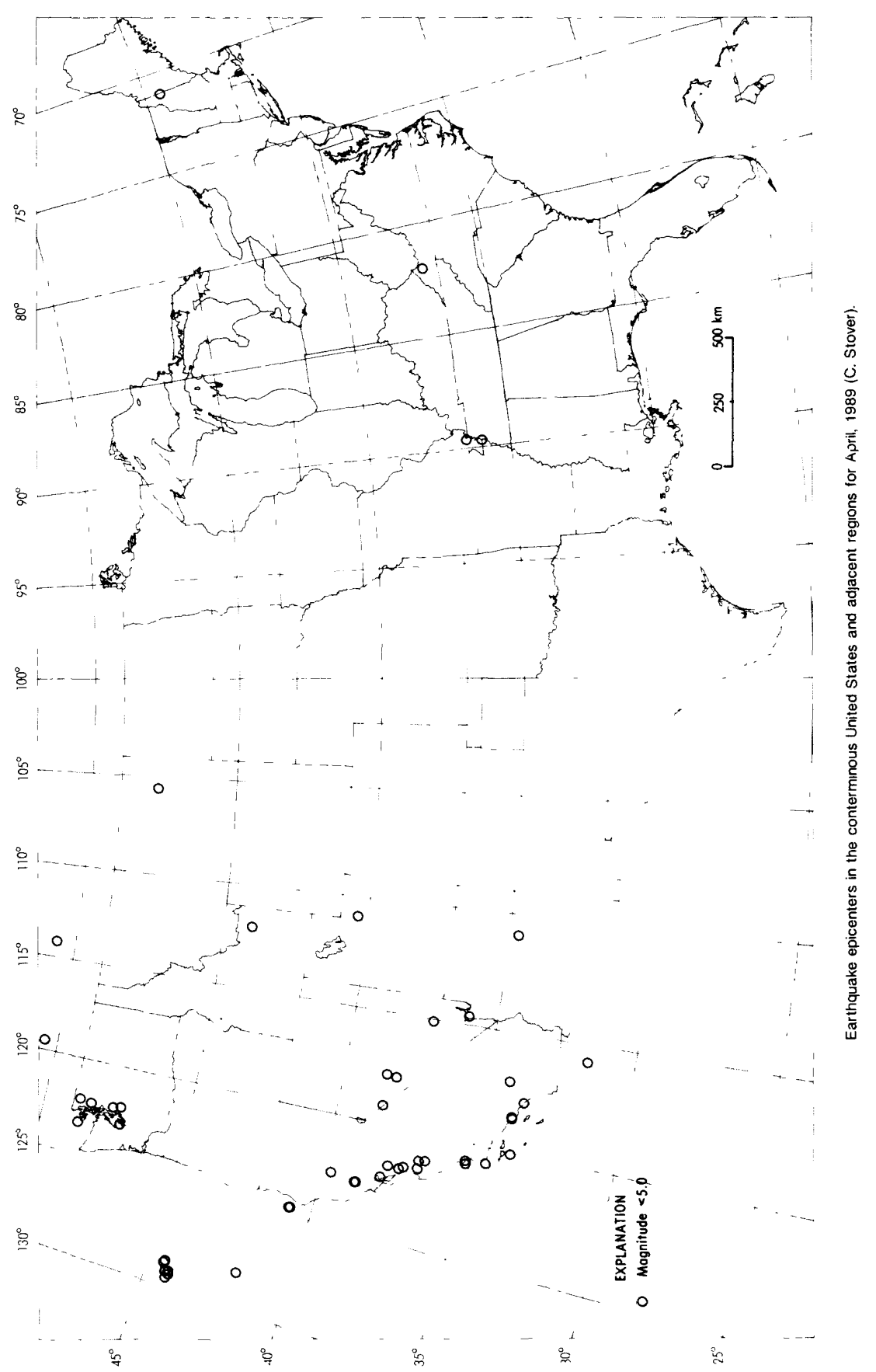




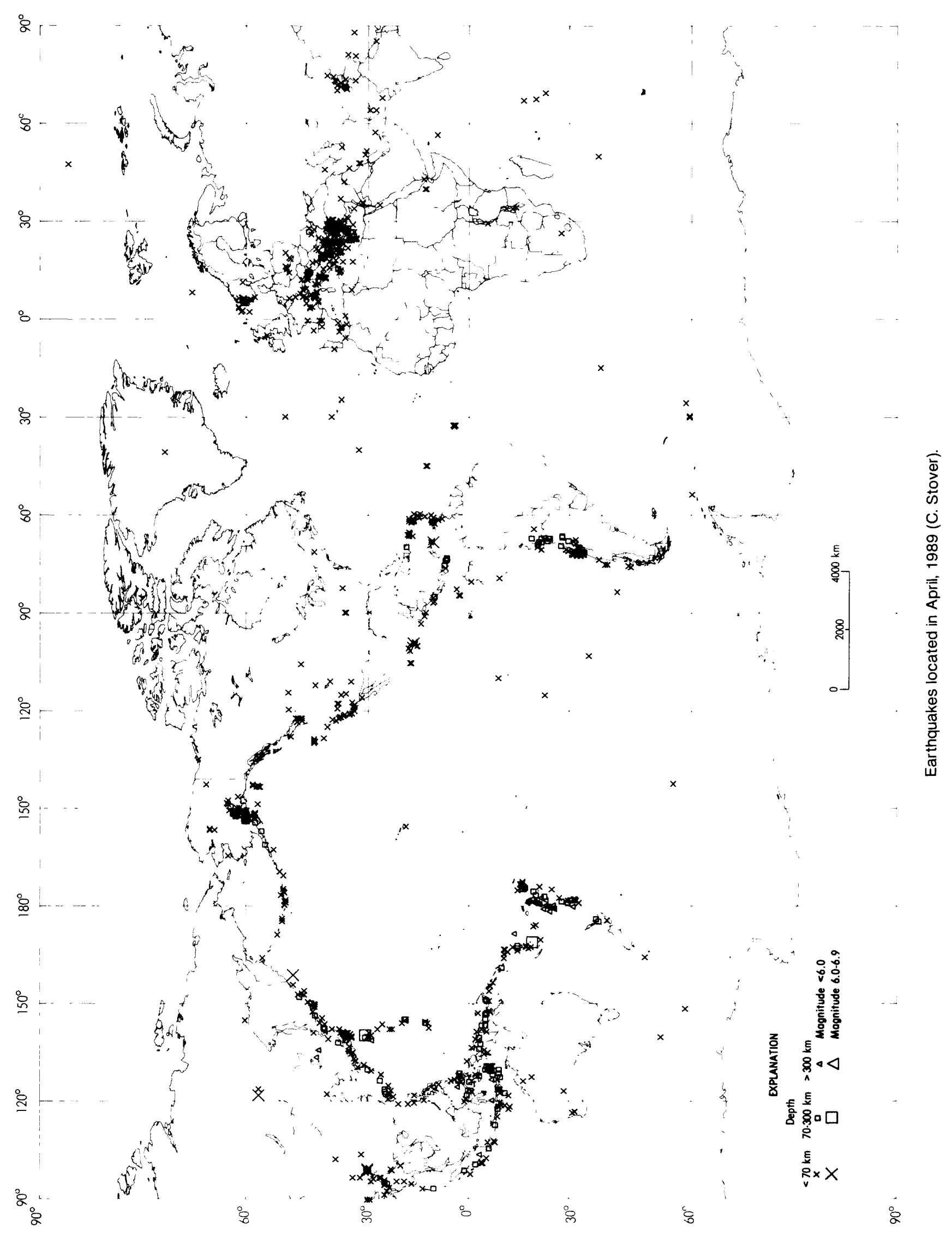




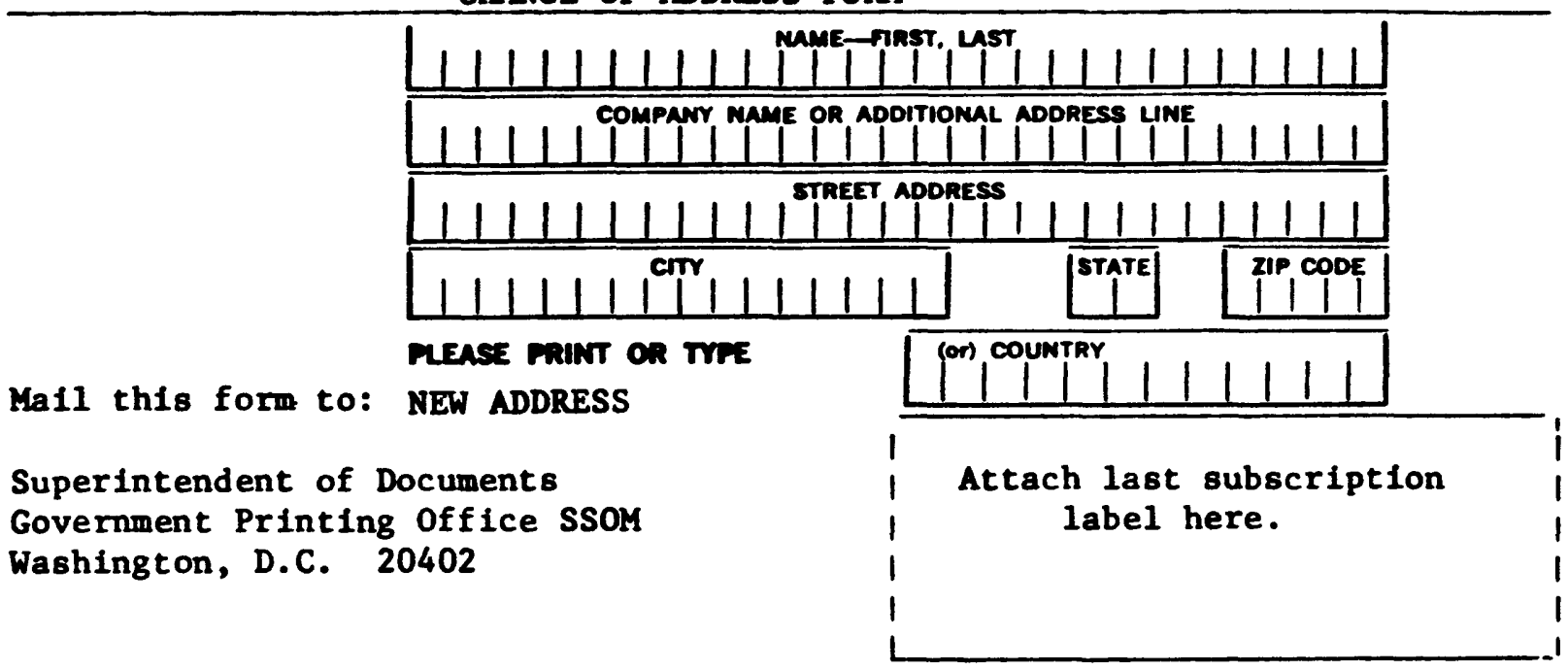

SUUSCRIMTON ORDER FORA

ENTER MY SUBSCRIPTION TO:

(8) Domestic; \& $\$$ Foreign.

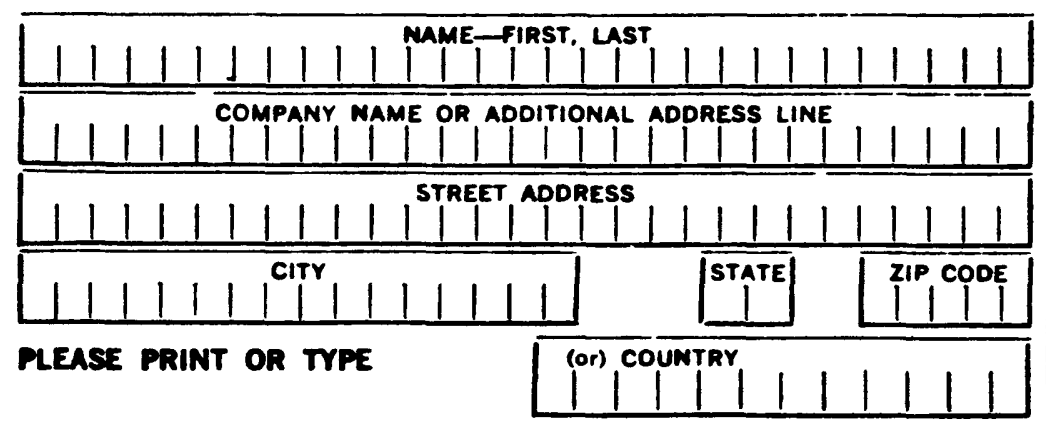

Remittence Enclosed (Make chocks payable to Superin. tendent of Documents)

$\square$ Cheres to my Doposit Account No. my Dopost

MAIL OROER FORM TO: Superintendent of Documents Government Printing Office Washington, D.C. 20402 

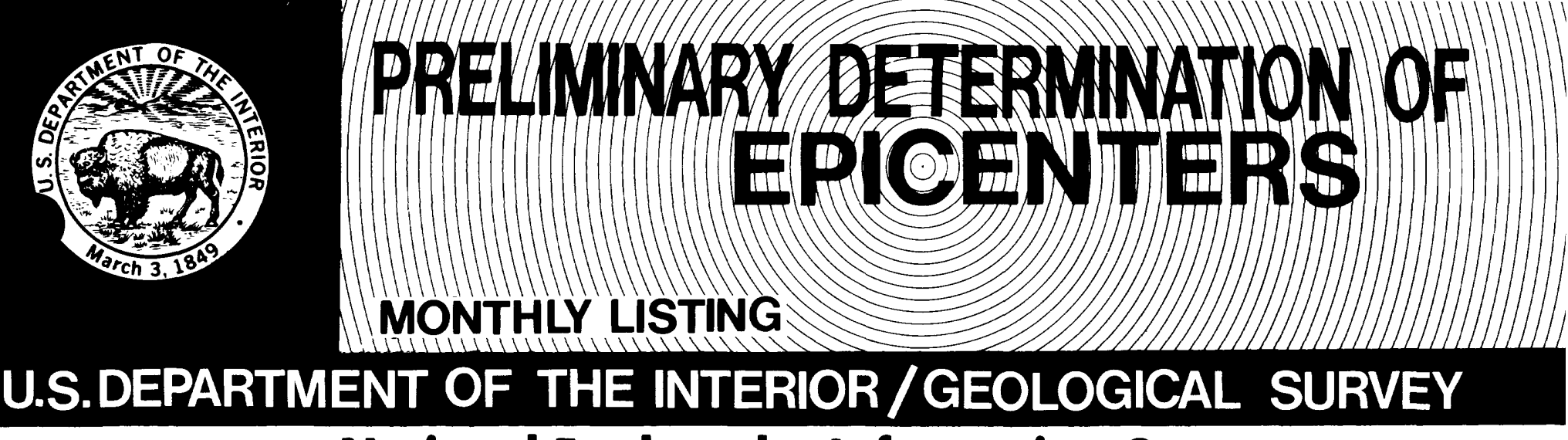
National Earthquake Information Cenfer

\section{MAY 1989}

\begin{tabular}{|c|c|c|c|c|c|c|c|c|c|c|c|c|c|c|}
\hline $\begin{array}{l}\text { K DAY } \\
\text { E }\end{array}$ & OR & $\begin{array}{ll}1611 \\
\text { UT }\end{array}$ & N TIME & $\begin{aligned} G E C \\
\text { COOF }\end{aligned}$ & $\begin{array}{l}\text { OGR } \\
\text { RDI }\end{array}$ & $\begin{array}{l}\text { APHIC } \\
\text { NATES }\end{array}$ & & DEPTH & & $\begin{array}{l}\text { MAGN I } \\
\text { GS }\end{array}$ & ITUDES & SD & $\begin{array}{l}\text { NO. } \\
\text { STA }\end{array}$ & CONTRIBUTED \\
\hline $\mathbf{Y}$ & HR & $M N$ & SEC & LAT & & LONG & & & & MB & Msz & & USED & \\
\hline 01 & 01 & 24 & 42.9 & 25.694 & $\mathbf{N}$ & 142.785 & $\mathbf{E}$ & 33 & $\mathbf{N}$ & 5.8 & 4.0 & 1.1 & 36 & VOLCANO ISLANDS REGION \\
\hline 01 & 82 & 45 & $08.9 ?$ & 36.34 & $\mathbf{N}$ & 70.63 & $E$ & 234 & $?$ & 4.2 & & 1.0 & 14 & HINDU KUSH REGION \\
\hline 01 & $\theta 2$ & 46 & 18.8 & 63.001 & $\mathbf{N}$ & 150.225 & $w$ & 33 & $\mathbf{N}$ & & & 0.4 & 6 & CENTRAL ALASKA. ML 3.0 (PMR). \\
\hline 01 & 83 & 03 & 10. $9 ?$ & 38.97 & $\mathbf{N}$ & 23.56 & $\mathbf{E}$ & 10 & G & & & 0.6 & 7 & GREECE \\
\hline 01 & 03 & 22 & 44.9 & 66.980 & $\mathbf{N}$ & 156.383 & $\mathbf{w}$ & 5 & G & & & 0.3 & 17 & ALASKA. ML 3.5 (PMR). \\
\hline 01 & 03 & 22 & $52.8 *$ & 50.500 & $\mathbf{N}$ & 18.894 & $E$ & 10 & G & & & 0.5 & 5 & POLAND. ML 3.7 (KRA), 3.3 (VKA). \\
\hline 01 & 83 & 42 & 57.38 & 59.788 & $N$ & 149.454 & $\mathbf{w}$ & 27 & & & & & 49 & KENAI PENINSULA, ALASKA. LAGS-P>. ML 3.2 (PMR). \\
\hline 01 & 84 & 19 & 10.1 & 39.164 & $\mathbf{N}$ & 21.871 & $\mathbf{E}$ & 24 & & & & 1.0 & 16 & GREECE. MD 3.4 (ATH). \\
\hline 01 & 85 & 11 & $40 \cdot 4$ & 66.906 & $\mathbf{N}$ & 156.228 & $w$ & 5 & G & & & 0.4 & 18 & ALASKA. ML 3.5 (PMR) \\
\hline 01 & 85 & 32 & $28.2 \%$ & 39.950 & $N$ & 23.331 & $\mathbf{E}$ & 10 & G & & & 0.1 & 5 & AEGEAN SEA \\
\hline 01 & 86 & 38 & $28 \cdot 3 *$ & 67.031 & $\mathbf{N}$ & 156.455 & $w$ & 5 & G & & & 0.4 & 11 & ALASKA \\
\hline 01 & 07 & 24 & 34.4 & 39.649 & $\mathbf{N}$ & 21.289 & $\mathbf{E}$ & 5 & G & 3.4 & & 1.4 & 19 & GREECE. MD 3.6 (ATH) \\
\hline 81 & 07 & 27 & $53.2 \%$ & 38.917 & $N$ & 27.591 & $E$ & 10 & G & & & 0.4 & 5 & TURKEY \\
\hline 01 & 07 & 38 & $10.3 ?$ & 19.45 & $\mathbf{N}$ & 66.00 & $\mathbf{w}$ & 10 & G & & & 0.3 & 6 & PUERTO RICO REGION \\
\hline 01 & 07 & 57 & $23.0 ?$ & 44.62 & $\mathbf{N}$ & 7.03 & $E$ & 10 & $\mathbf{G}$ & & & 0. 1 & 4 & NORTHERN ITALY. ML 1.9 (GEN). \\
\hline 01 & 08 & 39 & $25.9 ?$ & 37.19 & $\mathrm{~N}$ & 28.03 & $\bar{E}$ & 5 & G & & & 1.3 & 5 & TURKEY \\
\hline 001 & 88 & 45 & 21.6 & 4.200 & $\mathbf{S}$ & 101.366 & $\bar{E}$ & 31 & D & 5.6 & 5.6 & 1.0 & 235 & SOUTHERN SUMATERA \\
\hline 01 & 08 & 54 & 30.0 & 9.469 & $\mathbf{N}$ & 84.898 & $\mathbf{w}$ & 33 & N & & & 0.5 & 16 & $\begin{array}{l}\text { COSTA RICA. MD } 4.4 \text { (SJR), } 4.3 \text { (HDC). Felt (111) at Joco } \\
\text { ond Esterillos; (11) ot orotino. }\end{array}$ \\
\hline 01 & 89 & 04 & $46.8 ?$ & 44.76 & $\mathbf{N}$ & 6.46 & $E$ & 10 & G & & & 0. 2 & 5 & FRANCE. ML 2.0 (GEN). \\
\hline 01 & 09 & 36 & $49.3 *$ & 20.285 & $\mathbf{s}$ & 67.045 & $E$ & 10 & G & 4.6 & & 0.7 & 20 & MID-INDIAN RISE \\
\hline 01 & 89 & 41 & 05.2 & 39.240 & $\mathbf{N}$ & 23.523 & $\bar{E}$ & 10 & G & & & 0.5 & 11 & AEGEAN SEA. MD 3.0 (ATH). \\
\hline 81 & 09 & 43 & $01.2 ?$ & 28.77 & $\mathbf{N}$ & 140.76 & $E$ & 33 & $\mathbf{N}$ & 4.4 & & 1.2 & 6 & BONIN ISLANDS REGION \\
\hline 01 & 10 & 36 & $52.0 \%$ & 37.127 & $\mathbf{N}$ & 28.095 & $\bar{E}$ & 10 & G & & & 0.4 & 5 & TURKEY \\
\hline 81 & 11 & 34 & 56.6 & 37.118 & $\mathbf{N}$ & 27.890 & $E$ & 10 & G & & & 0.8 & 14 & TURKEY \\
\hline 81 & 12 & 27 & $51.4 ?$ & 32.08 & $s$ & 178.83 & $E$ & 458 & $?$ & 3.9 & & 0. 9 & 21 & SOUTH OF KERMADEC ISLANDS \\
\hline 01 & 13 & 23 & $05.4 \pi$ & 44.639 & $\mathrm{~N}$ & 7.080 & $E$ & 10 & G & & & 0.5 & 5 & NORTHERN ITALY. ML 2.1 (GEN) \\
\hline 01 & 13 & 45 & $28.5 ?$ & 61.62 & $\mathbf{N}$ & 4.47 & $\vec{E}$ & 10 & G & & & 0.7 & 4 & SOUTHERN NORWAY. MD 1.9 (BER). \\
\hline 01 & 14 & 00 & $10.0 ?$ & 37.17 & $\mathbf{N}$ & 27.98 & $E$ & 5 & $\mathbf{G}$ & & & 1.4 & 5 & TURKEY \\
\hline 01 & 14 & 37 & $08.4 ?$ & 18.71 & s & 172.83 & $w$ & 33 & $\mathbf{N}$ & 5.1 & & 0.9 & 9 & TONGA ISLANDS REGION \\
\hline 01 & 15 & 33 & 03.0 & 66.992 & $\mathbf{N}$ & 156.227 & $w$ & 5 & G & 4.1 & & 0.8 & 28 & ALASKA. ML 3.8 (PMR). FeIt (IV) at Kabuk. \\
\hline 01 & 16 & 08 & $18.0 \%$ & 42.403 & $\mathbf{N}$ & 19.821 & $E$ & 10 & G & & & 0.6 & 6 & YUGOSLAVIA. MD 2.2 (TTG). \\
\hline 01 & 16 & 28 & 26.9 & 39.094 & $\mathbf{N}$ & 23.202 & $\mathbf{E}$ & 13 & & & & 0.5 & 15 & AEGEAN SEA. ML 3.1 (ATH). \\
\hline 01 & 16 & 54 & $56 \cdot 6 \pi$ & 44.672 & N & 7.161 & $E$ & 10 & G & & & 0.2 & 5 & NORTHERN ITALY. ML 2.0 (GEN). \\
\hline 81 & 17 & 34 & 09.7 & 29.960 & $\mathbf{N}$ & 99.370 & $\bar{E}$ & 10 & G & 4.9 & & 1.2 & 15 & SICHUAN PROVINCE, CHINA \\
\hline 01 & 17 & 46 & 00.6 & 31.592 & $\mathbf{N}$ & 96.679 & $\bar{E}$ & 33 & $\mathbf{N}$ & 4.1 & & 0.5 & 7 & (a) \\
\hline 01 & 18 & 10 & 19.0. & 20.627 & s & 68.178 & $w$ & 33 & $\mathbf{N}$ & & & 0.9 & 5 & CHILE-BOLIVIA BORDER REGION \\
\hline 01 & 18 & 35 & 05.68 & 37.142 & $\mathbf{N}$ & 113.815 & $w$ & 1 & & & & & 28 & $\begin{array}{l}\text { UTAH. } \text { SLC-P>. CL } 3.7 \text { (SLC). ML } 3.5 \text { (NE|S). Felt (V) at } \\
\text { St. George and ( }|1| \text { ) at Santa Claro. }\end{array}$ \\
\hline 01 & 19 & 04 & 43. 0. & 20.350 & $\mathrm{~s}$ & 168.102 & E & 29 & D & $4 \cdot 8$ & & 1.1 & 46 & LOYALTY ISLANDS \\
\hline 01 & 19 & 06 & $26.3 *$ & 17.141 & $\mathbf{N}$ & 62.337 & $w$ & 60 & $?$ & & & 0.7 & 9 & LEEWARD ISLANDS \\
\hline 01 & 20 & 12 & 07.9 & 66.909 & $\mathbf{N}$ & 156.314 & $w$ & 5 & G & & & 0.7 & 18 & ALASKA. ML 3.5 (PMR). \\
\hline 01 & 20 & 16 & $35.5 *$ & 45.077 & $\mathbf{N}$ & 15.106 & $E$ & 10 & G & & & 0.7 & 8 & YUGOSLAVIA. MD 3.2 (LJU), 2.7 (TRI). ML 2.2 (KBA). \\
\hline 01 & 21 & 03 & 38.6 & 37.212 & $\mathbf{N}$ & 21.145 & $\bar{E}$ & 10 & G & 4.8 & & 1.4 & 209 & $\begin{array}{l}\text { SOUTHERN GREECE. ML } 4.6 \text { (ATH), } 4.6 \text { (TTG). Felt in } \\
\text { southwestern Greece. }\end{array}$ \\
\hline 01 & 21 & 26 & 38.6 & 51.934 & $\mathbf{N}$ & 178.917 & $E$ & 129 & * & 4.5 & & 0.9 & 35 & $\begin{array}{l}\text { southwestern Greece. } \\
\text { RAT ISLANDS. ALEUTIAN ISLANDS }\end{array}$ \\
\hline 01 & 21 & 38 & $57.7 ?$ & 40.76 & $\mathbf{N}$ & 30.49 & $E$ & 10 & G & & & 1.3 & 7 & TURKEY \\
\hline 01 & 22 & 29 & 34.0 & 19.218 & $\mathbf{N}$ & 64.389 & $\mathbf{w}$ & 38 & $\cdot$ & 4.0 & & 1.1 & 34 & VIRGIN ISLANDS. ML 4.8 (FDF). \\
\hline 01 & 22 & 39 & $44.2 ?$ & 4.24 & $\mathrm{~s}$ & 101.51 & $\underline{E}$ & 33 & $\mathbf{N}$ & 3.9 & & 0.5 & 10 & SOUTHERN SUMATERA \\
\hline 01 & 23 & 14 & 13. 1? & 6.89 & $\mathrm{~s}$ & 130.59 & $E$ & 110 & $?$ & 4.3 & & 1.2 & 13 & BANDA SEA \\
\hline 01 & 23 & 30 & $51.7 \%$ & 18.245 & $\mathrm{~N}$ & 66.310 & $w$ & 10 & G & & & 0.2 & 6 & PUERTO RICO REGION \\
\hline 02 & $0 \theta$ & 20 & $55.5 ?$ & 50.74 & $\mathbf{N}$ & 179.50 & $E$ & 33 & N & 5.1 & & 1.9 & 13 & RAT ISLANDS, ALEUTIAN ISLANDS. ML 4.5 (PMR). \\
\hline 02 & 01 & 06 & 12.3. & 7.238 & $\mathbf{N}$ & 76.371 & $w$ & 89 & $\bullet$ & 3.6 & & 0.7 & 8 & NORTHERN COLOMBIA \\
\hline 82 & 01 & 42 & 12.3 & 38.406 & $N$ & 22.890 & $E$ & 10 & G & & & 0.8 & 21 & GREECE. ML 3.2 (ATH) \\
\hline 82 & 01 & 49 & 50.0 & 6.436 & $\mathrm{~S}$ & 147.622 & $\bar{E}$ & 80 & * & 4.4 & & 1.1 & 17 & EAST PAPUA NEW GUINEA REGION \\
\hline 02 & 02 & 25 & $10.9 \%$ & 46.398 & $\mathbf{N}$ & 3.466 & $\bar{E}$ & 10 & G & & & 0.7 & 5 & FRANCE. ML 1.8 (LDG) \\
\hline 82 & 02 & 30 & 19.0 & 3.858 & N & 126.391 & $E$ & 33 & $\mathrm{~N}$ & 5.1 & & 0.8 & 69 & TALAUD I SLANDS \\
\hline 02 & 02 & 33 & 30.6 & 41.737 & $\mathrm{~s}$ & 73.589 & $w$ & 29 & 0 & 5.1 & 4.5 & 1.0 & 47 & NEAR COAST OF SOUTHERN CHILE \\
\hline
\end{tabular}

Annual Subscriptions: Superintendent of Documents. U.S. Government Printing 0ffice, Woshington, D.C. 20402 Bock issues: Boaks and Open-file Reports Section, U.S. Geolagical Survey, Bax 25425 , Denver, Co 80225. 


\begin{tabular}{|c|c|c|c|c|c|c|c|c|c|c|c|c|c|}
\hline 02 & 04 & 02 & 42.8 & 39.141 & $N$ & 23.287 & $E$ & 17 & & & & 0.7 & 20 \\
\hline 02 & 05 & 11 & 01.2 & 66.945 & $\mathbf{N}$ & 156.160 & $w$ & 5 & G & & & 1.0 & 22 \\
\hline 02 & 05 & 12 & $19.6 *$ & 45.187 & $N$ & 28.261 & $w$ & 10 & G & 4.3 & & 1.0 & 15 \\
\hline 02 & 05 & 22 & 47.7 & 45.130 & $N$ & 28.203 & $w$ & 10 & G & $4: 6$ & & 1.0 & 69 \\
\hline 02 & 05 & 40 & 24.5 & 66.914 & $N$ & 156.173 & $w$ & 5 & G & & & 0.3 & 15 \\
\hline 02 & 05 & 49 & $36.7 *$ & 0.410 & $N$ & 80.422 & $w$ & 33 & $N$ & 4.3 & & 1.0 & 11 \\
\hline 02 & 05 & 51 & $35.2 ?$ & 37.13 & $N$ & 28.10 & $\mathbf{E}$ & 10 & G & & & 1.2 & 5 \\
\hline 02 & 06 & 11 & $00.5 *$ & 45.018 & $N$ & 28.104 & $w$ & 10 & G & 4.5 & & 0.9 & 26 \\
\hline 02 & 06 & 25 & 33.3 & 45.134 & $N$ & 28.031 & $w$ & 10 & G & 5.0 & & 1.1 & 179 \\
\hline 02 & 06 & 27 & 28.2 & 45.060 & $N$ & 28.141 & $w$ & 10 & G & 5.2 & 5.6 & 1.1 & 194 \\
\hline 02 & 06 & 58 & $41.3 *$ & 26.521 & $\mathrm{~S}$ & 67.466 & $w$ & 139 & $\bullet$ & 5.1 & & 1.4 & 21 \\
\hline 02 & 07 & 68 & 16.8 & 35.276 & $\mathbf{N}$ & 136.576 & $E$ & 54 & & 4.7 & & 1.0 & 52 \\
\hline 02 & 07 & 42 & 49.3 & 66.933 & $\mathbf{N}$ & 156.227 & $w$ & 5 & G & & & 0.4 & 12 \\
\hline 02 & 08 & 24 & $12.6 \%$ & 46.074 & $\mathbf{N}$ & 2.858 & $E$ & 10 & G & & & 0.3 & 9 \\
\hline 02 & 08 & 25 & $27.0 ?$ & 46.05 & $\mathbf{N}$ & 2.92 & $E$ & 10 & G & & & 0.2 & 4 \\
\hline 02 & 08 & 45 & $16.4 *$ & 46.268 & $\mathbf{N}$ & 12.787 & $E$ & 10 & G & & & 0.9 & 5 \\
\hline 02 & 09 & 27 & $35.8 *$ & 44.857 & $\mathbf{N}$ & 28.153 & $w$ & 10 & G & 4.6 & & 1.1 & 37 \\
\hline 62 & 09 & 30 & 15.5 & 16.747 & $\mathbf{N}$ & 99.343 & $w$ & 10 & G & 5.4 & 4.9 & 1.0 & 189 \\
\hline 02 & 09 & 30 & 58.9 & 44.968 & $\mathbf{N}$ & 28.025 & $w$ & 10 & G & 5.0 & 5.5 & 1.0 & 197 \\
\hline 02 & 89 & 39 & $31.7 ?$ & 47.43 & $\mathbf{N}$ & 7.72 & $w$ & 10 & G & & & 0.7 & 15 \\
\hline 02 & 10 & 01 & 33.5 & 44.948 & $N$ & 28.128 & $w$ & 10 & G & 4.7 & & 1.0 & 84 \\
\hline 02 & 10 & 05 & $36.4 *$ & 44.832 & $N$ & 28.098 & $w$ & 10 & G & 4.6 & & 1.1 & 31 \\
\hline 02 & 10 & 12 & $58.1 ?$ & 31.58 & $\mathrm{~S}$ & 68.20 & $w$ & 10 & G & 4.1 & & 1.3 & 12 \\
\hline 62 & 10 & 39 & $14.4 *$ & 50.963 & $N$ & 6.542 & $\mathbf{E}$ & 10 & G & & & 1.5 & 5 \\
\hline 02 & 11 & 52 & $30.5 *$ & 53.219 & $N$ & 173.029 & $\mathbf{E}$ & 33 & $\mathbf{N}$ & 5.1 & & 1.2 & 21 \\
\hline 02 & 12 & 19 & $23.1 ?$ & 17.99 & $N$ & 65.80 & $w$ & 10 & G & & & 0.7 & 6 \\
\hline 02 & 12 & 21 & $34.8 \%$ & 42.760 & $N$ & 19.181 & $\mathbf{E}$ & 10 & G & & & 0.1 & 5 \\
\hline 02 & 13 & 37 & $06.4 ?$ & 62.25 & $N$ & 4.59 & $\mathbf{E}$ & 10 & G & & & 0.6 & 6 \\
\hline 02 & 13 & 37 & 14.48 & 32.730 & $N$ & 118.060 & $w$ & 6 & G & & & & 10 \\
\hline 02 & 13 & 41 & $38.5 ?$ & 62.26 & $\mathbf{N}$ & 4.43 & $\mathbf{E}$ & 10 & G & & & 0.6 & 7 \\
\hline 02 & 14 & 11 & 49.1 & 40.791 & $N$ & 28.039 & $\mathbf{E}$ & 10 & G & & & 1.5 & 10 \\
\hline 02 & 15 & 06 & $30.4 ?$ & 42.88 & $\mathbf{N}$ & 13.00 & $\mathbf{E}$ & 10 & G & & & 0.1 & 4 \\
\hline 02 & 16 & 51 & 31.68 & 59.983 & $N$ & 153.463 & $w$ & 135 & & & & & 14 \\
\hline 02 & 16 & 56 & $18.0 ?$ & 31.41 & $\mathrm{~S}$ & 70.39 & $w$ & 33 & $N$ & & & 1.2 & 9 \\
\hline 02 & 17 & 39 & 11.8 & 37.246 & $\mathbf{N}$ & 28.099 & $\mathbf{E}$ & 10 & G & & & 1.6 & 5 \\
\hline 02 & 17 & 42 & $59.2 *$ & 35.531 & $N$ & 44.353 & $\mathbf{E}$ & 33 & $N$ & 3.9 & & 1.2 & 5 \\
\hline 02 & 18 & 02 & $38.7 *$ & 36.779 & $\mathbf{N}$ & 28.025 & $E$ & 10 & G & & & 1.2 & 7 \\
\hline 02 & 18 & 26 & $58 \cdot 6$ & 39.328 & $N$ & 23.547 & $\mathbf{E}$ & 12 & & & & 1.1 & 33 \\
\hline 02 & 19 & 23 & $05.8 \&$ & 61.808 & $\mathbf{N}$ & 149.784 & $w$ & 35 & & & & & 45 \\
\hline 02 & 19 & 28 & $21.4 \%$ & 46.437 & $\mathbf{N}$ & 0.970 & $w$ & 10 & G & & & 1.5 & 7 \\
\hline 02 & 19 & 38 & $33.4 *$ & 2.452 & $\mathbf{N}$ & 128.461 & $E$ & 33 & $\mathbf{N}$ & 4.7 & & 0.6 & 14 \\
\hline 02 & 19 & 52 & $39.4 ?$ & 50.31 & $\mathbf{N}$ & 159.05 & $\mathbf{E}$ & 39 & $D$ & 4.6 & & 1.3 & 14 \\
\hline 02 & 20 & 53 & 04.7 & 40.386 & $\mathbf{N}$ & 25.981 & $\mathbf{E}$ & 10 & G & & & 0.9 & 13 \\
\hline 02 & 21 & 36 & $48.4 \&$ & 62.975 & $\mathbf{N}$ & 149.974 & $w$ & 91 & & & & & 8 \\
\hline 02 & 22 & 11 & $22.0 ?$ & 4.32 & $\mathrm{~S}$ & 101.19 & E & 52 & $?$ & 4.3 & & 1.2 & 9 \\
\hline 02 & 22 & 13 & 03.48 & 60.201 & $\mathbf{N}$ & 153.187 & $w$ & 138 & & & & & 26 \\
\hline 02 & 22 & 14 & $04.9 *$ & 17.298 & $\mathbf{N}$ & 62.326 & $w$ & 33 & $\mathbf{N}$ & & & 1.2 & 8 \\
\hline 02 & 22 & 52 & 44.4 & 66.970 & $\mathbf{N}$ & 156.218 & $w$ & 5 & G & & & 0.9 & 19 \\
\hline 02 & 23 & 01 & 10.2 & 53.806 & $\mathbf{N}$ & 160.561 & $E$ & 60 & 0 & 5.3 & & 0.9 & 214 \\
\hline 02 & 23 & 09 & 14.2 & 39.275 & $\mathbf{N}$ & 23.551 & $E$ & 18 & & & & 0.9 & 43 \\
\hline 02 & 23 & 16 & 22.1 & 39.246 & $N$ & 23.576 & $E$ & 13 & & 3.8 & & 0.8 & 52 \\
\hline 03 & 00 & 26 & 01.7 & 43.192 & $N$ & 14.454 & $\mathbf{E}$ & 33 & $\mathbf{N}$ & & & 1.4 & 98 \\
\hline 03 & 02 & 54 & $32.6 *$ & 44.999 & $N$ & 28.110 & $w$ & 10 & G & 4.2 & & 1.0 & 28 \\
\hline 03 & 02 & 56 & $25.5 \&$ & 60.129 & $N$ & 146.980 & $w$ & 8 & & 4.9 & & & 152 \\
\hline 03 & 02 & 57 & 48.7 & 42.633 & $\mathbf{N}$ & 13.026 & $E$ & 10 & G & & & 0.4 & 6 \\
\hline 03 & 03 & 03 & 13.1 & 44.595 & $\mathbf{N}$ & 27.087 & $w$ & 10 & G & 4.2 & & 0.6 & 15 \\
\hline 03 & 93 & 86 & $54.3 ?$ & 37.17 & $\mathbf{N}$ & 28.00 & $E$ & 10 & G & & & 1.6 & 5 \\
\hline 03 & 03 & 09 & $21.7 \&$ & 60.115 & $N$ & 147.056 & $w$ & 9 & & & & & 35 \\
\hline 03 & 03 & 33 & 08.78 & 64.476 & $N$ & 146.914 & $w$ & 8 & & & & & 14 \\
\hline 03 & 03 & 40 & 48.0 & 1.057 & $\mathrm{~s}$ & 78.251 & $w$ & 10 & G & 4.4 & & 1.2 & 13 \\
\hline 03 & 04 & 01 & 24.2 & 39.257 & $\mathbf{N}$ & 23.513 & $\mathbf{E}$ & 13 & & & & 1.2 & 34 \\
\hline 03 & 04 & 40 & $38.8 ?$ & 3.68 & $\mathrm{~s}$ & 134.40 & $E$ & 33 & $\mathbf{N}$ & $4 \cdot 3$ & & 1.1 & 5 \\
\hline 63 & 05 & 04 & 26.1 & 42.840 & $\mathbf{N}$ & 111.611 & $w$ & 5 & G & & & 1.1 & 8 \\
\hline 03 & 05 & 15 & $11.1 ?$ & 20.73 & $\mathrm{~S}$ & 170.54 & $E$ & 104 & $?$ & 4.6 & & 0.1 & 5 \\
\hline 03 & 05 & 25 & 15.3 & 50.659 & $\mathbf{N}$ & 129.564 & $w$ & 10 & G & 4.6 & 3.8 & 1.0 & 78 \\
\hline 03 & 05 & 42 & 49.4 . & 61.486 & $\mathbf{N}$ & 1.414 & $E$ & 10 & G & & & 0.9 & 12 \\
\hline 03 & 05 & 53 & 01.1 & 30.091 & $\mathbf{N}$ & 99.475 & $E$ & 14 & G & 6.1 & 6.1 & 1.0 & 422 \\
\hline 03 & 06 & 10 & 43.4 & 32.994 & $\mathrm{~s}$ & 178.821 & $w$ & 10 & G & 4.8 & & 1.1 & 32 \\
\hline 03 & 06 & 17 & 21.7 & 67.099 & $N$ & 156.562 & $w$ & 5 & G & & & 0.4 & 12 \\
\hline 03 & 06 & 41 & 33.0 & 30.001 & $\mathbf{N}$ & 99.403 & E & 10 & G & 5.1 & & 1.2 & 89 \\
\hline 03 & 07 & 44 & 10.5 & 46.382 & $\mathbf{N}$ & 2.421 & $E$ & 16 & & & & 0.7 & 40 \\
\hline 03 & 08 & 12 & 04.9 & 30.085 & $\mathbf{N}$ & 99.285 & $E$ & 10 & G & 4.4 & & 1.3 & 31 \\
\hline 03 & 08 & 19 & 07.0 & 30.046 & $\mathbf{N}$ & 99.363 & $E$ & 10 & G & 4.3 & & 1.4 & 20 \\
\hline 03 & 88 & 19 & $27.1 ?$ & 19.27 & $\mathrm{~S}$ & 178.04 & $w$ & 428 & $?$ & 4.5 & & 1.0 & 15 \\
\hline 03 & 08 & 37 & $50.1 *$ & 30.145 & $\mathbf{N}$ & 99.307 & $E$ & 10 & G & 4.8 & & 1.5 & 15 \\
\hline 03 & 09 & 12 & 41.8 & 29.857 & $\mathbf{N}$ & 51.797 & $E$ & 10 & G & 4.9 & & 1.2 & 182 \\
\hline 03 & 09 & 13 & 24.2 & 29.964 & $\mathbf{N}$ & 51.655 & $E$ & 33 & $\mathbf{N}$ & 5.1 & & 1.1 & 61 \\
\hline 03 & 09 & 28 & 45.7 & 30.086 & $\mathbf{N}$ & 99.316 & $E$ & 10 & G & 4.8 & & 1.2 & 26 \\
\hline 03 & 09 & 52 & 04.4 & 30.163 & $\mathbf{N}$ & 99.148 & $\mathbf{E}$ & 10 & G & 4.9 & & 1.5 & 36 \\
\hline 03 & 11 & 22 & $48.5 \&$ & 60.129 & $\mathbf{N}$ & 147.014 & $w$ & 1 & & & & & 32 \\
\hline 03 & 11 & 52 & 36.3 & 42.567 & $\mathbf{N}$ & 12.545 & E & 10 & G & & & 0.8 & 13 \\
\hline 03 & 12 & 16 & 40.7 & 29.893 & $N$ & 51.819 & $E$ & 10 & G & 4.8 & & 1.0 & 27 \\
\hline 03 & 12 & 59 & 22.4 & 35.336 & $\mathbf{N}$ & 27.723 & $E$ & 10 & G & & & 1.5 & 16 \\
\hline 03 & 13 & 00 & 25.9 & 3.519 & $\mathbf{N}$ & 125.850 & $E$ & 140 & * & 5.0 & & 0.7 & 35 \\
\hline 03 & 13 & 1 & 32.2 & 1.936 & $\mathbf{N}$ & 128.748 & $\mathbf{E}$ & 33 & $N$ & 5.0 & & 1.0 & 25 \\
\hline
\end{tabular}




\begin{tabular}{|c|c|c|c|c|c|c|c|c|c|c|c|c|c|c|}
\hline 83 & 13 & 22 & $46.6 \%$ & 60.722 & N & 5.552 & $E$ & 10 & G & & & 0.5 & 7 & SOUTHERN NORWAY. MO I.9 (BER). \\
\hline 03 & 14 & 20 & 33.3 & 45.552 & $\mathbf{N}$ & 151.030 & $\boldsymbol{E}$ & 33 & $\mathbf{N}$ & 5.0 & 4.1 & 0.8 & 88 & KURIL ISLANDS \\
\hline 03 & 14 & 20 & 42.7 & 26.025 & $\mathbf{N}$ & 124.732 & $\mathbf{E}$ & 191 & D & 4.7 & & 0.9 & 49 & NORTHEAST OF TAIWAN \\
\hline 83 & 15 & 41 & 30.8 & 30.053 & $\mathbf{N}$ & 99.499 & E & 8 & G & 5.8 & 5.9 & 1.0 & 385 & $\begin{array}{l}\text { SICHUAN PROVINCE, CHINA. Depth fram broodbond } \\
\text { displacement seismograms. }\end{array}$ \\
\hline 03 & 15 & 50 & $50.7 ?$ & 18.01 & $\mathbf{N}$ & 65.85 & $w$ & 10 & G & & & 0.5 & 6 & PUERTO RICO REGION \\
\hline 03 & 17 & 20 & $19.2 ?$ & 67.19 & $\mathbf{N}$ & 156.38 & $w$ & 5 & G & & & 0.4 & 11 & ALASKA \\
\hline 83 & 17 & 22 & $10.5 *$ & 30.343 & $\mathbf{N}$ & 99.492 & $E$ & 10 & G & 4.2 & & 1.2 & 9 & SICHUAN PROVINCE, CHINA \\
\hline 03 & 17 & 28 & 21.0 & 30.073 & $\mathbf{N}$ & 99.432 & $\boldsymbol{E}$ & 10 & G & 5.3 & 4.6 & 1.0 & 159 & SICHUAN PROVINCE, CHINA \\
\hline 03 & 17 & 28 & 39.7 & 6.944 & $\mathbf{N}$ & 94.778 & $E$ & 33 & $\mathbf{N}$ & $4 \cdot 4$ & & 0.9 & 13 & NICOBAR ISLANDS REGION \\
\hline 03 & 17 & 33 & 27.5 & 51.567 & $\mathbf{N}$ & 173.643 & $w$ & 48 & D & 4.8 & & 0. 9 & 116 & ANDREANOF ISLANDS, ALEUTIAN IS. \\
\hline 03 & 17 & 37 & $21.2 *$ & 51.536 & $N$ & 173.651 & $w$ & 50 & D & 4.7 & & 1.1 & 36 & ANDREANOF ISLANDS, ALEUTIAN IS \\
\hline 03 & 17 & 53 & $46.5 *$ & 51.929 & $\mathbf{N}$ & 173.824 & w & 33 & $\mathbf{N}$ & 4.5 & & 1.2 & 42 & ANDREANOF ISLANDS, ALEUTIAN IS. \\
\hline 83 & 17 & 54 & $46.1 *$ & 30.092 & $\mathbf{N}$ & 99.321 & $E$ & 10 & G & & & 1.4 & 9 & SICHUAN PROVINCE, CHINA \\
\hline 03 & 18 & 19 & 31.9 & 29.937 & $\mathbf{N}$ & 99.260 & $\boldsymbol{E}$ & 10 & G & $4 \cdot 3$ & & 1.0 & 11 & SICHUAN PROVINCE, CHINA \\
\hline 63 & 18 & 44 & 54.2 & 6.955 & $\mathbf{N}$ & 94.592 & $E$ & 33 & $\mathbf{N}$ & 4. 9 & 4.8 & 1.1 & 103 & NICOBAR ISLANDS REGION \\
\hline 03 & 19 & 27 & $53.0 \%$ & 11.244 & $\mathbf{N}$ & 60.900 & $\mathbf{w}$ & 33 & $*$ & & & 1.1 & 10 & WINOWARD ISLANDS. MO 3.5 (TRN). \\
\hline 03 & 19 & 29 & 27.4 & 37.936 & $\mathbf{N}$ & 29.113 & $E$ & 10 & G & & & 1.0 & 16 & TURKEY. MD 3.4 (ATH). \\
\hline 03 & 19 & 59 & 18.4 & 38.776 & $\mathbf{N}$ & 70.711 & $\boldsymbol{E}$ & 33 & $\mathbf{N}$ & 4.7 & & 0.9 & 65 & $\begin{array}{l}\text { AFGHANISTAN-USSR BORDER REGION. FeIl (IV) ot Garm, } \\
\text { Deonasu, Nurek, Komarou and Gissar: (III) ot Obigarm, } \\
\text { Dzhirgatal, Dushonbe and Tashkent. USSR. }\end{array}$ \\
\hline 83 & 20 & 20 & $25.6 ?$ & 5.36 & $\mathrm{~S}$ & 131.33 & $\boldsymbol{E}$ & 126 & $?$ & & & 1.8 & 5 & BANDA SEA \\
\hline 03 & 20 & 37 & $48.0 *$ & 39.000 & $\mathbf{N}$ & 28.213 & w & 10 & G & 4. 1 & & 0.7 & 15 & $\begin{array}{l}\text { AZORES ISLANOS. FeIt (IV) on Graciaso and (III) on Sao } \\
\text { Jorge. }\end{array}$ \\
\hline 03 & 21 & 07 & $22.1 *$ & 30.180 & $\mathbf{N}$ & 99.277 & $E$ & 10 & G & 4.5 & & 1.5 & 13 & SICHUAN PROVINCE, CHINA \\
\hline 03 & 21 & 11 & 01.3 & 49.150 & $\mathbf{N}$ & 6.932 & $\boldsymbol{E}$ & 10 & G & & & 1.0 & 13 & GERMANY. MO 2.6 (UCC). \\
\hline 03 & 22 & 20 & 47.52 & 62.910 & $\mathbf{N}$ & 151.296 & w & 114 & & & & & 24 & CENTRAL ALASKA. $\angle A G S-P>$. \\
\hline 03 & 22 & 38 & $59.0 ?$ & 31.06 & S & 68.45 & $w$ & 115 & $?$ & & & 1.5 & 12 & SAN JUAN PROVINCE, ARGENTINA \\
\hline 03 & 23 & 06 & $31.5 *$ & 3.294 & $\mathbf{N}$ & 126.743 & $\boldsymbol{E}$ & 33 & $\mathbf{N}$ & 4. 1 & & 0.4 & 8 & TALAUD ISLANDS \\
\hline 03 & 23 & 26 & $40.5 *$ & 26.990 & S & 26.651 & $E$ & 5 & G & & & 1.4 & 14 & REPUBLIC OF SOUTH AFRICA \\
\hline 03 & 23 & 47 & 52.2 & 37.107 & N & 27.870 & $E$ & 5 & $\mathbf{G}$ & & & 1.0 & 9 & TURKEY \\
\hline 04 & 00 & 22 & 06.7 & 11.038 & N & 68.270 & w & 16 & & 5.4 & 5.2 & 1.0 & 227 & $\begin{array}{l}\text { NEAR COAST OF VENEZUELA. More than } 2,000 \text { people mode } \\
\text { homeless in the Tucacas area. Felt strongly in the } \\
\text { states of Falcon and Caraboba. Also felt at Caracos and } \\
\text { in parts of Aragua and Mirando. }\end{array}$ \\
\hline $\begin{array}{l}04 \\
04\end{array}$ & $\theta 0$ & 43 & 45.5 & 39.291 & $\mathbf{N}$ & 23.488 & $E$ & 10 & G & & & 0.4 & 9 & AEGEAN SEA. ML 3.0 (ATH). \\
\hline 04 & $\theta 0$ & 49 & $53.4 \%$ & 37.188 & $\mathbf{N}$ & 28.000 & $E$ & 10 & G & & & 1.0 & 8 & TURKEY \\
\hline 04 & 01 & 06 & 51.4 & 39.735 & $\mathbf{N}$ & 30.792 & $E$ & 10 & G & & & 1.0 & 14 & TURKEY \\
\hline 04 & 01 & 31 & 19.9 & 29.999 & $\mathbf{N}$ & 99.490 & E & 10 & G & 5.0 & 4.3 & 1.1 & 94 & SICHUAN PROVINCE, CHINA \\
\hline 84 & 02 & 24 & $13.6 ?$ & 40.84 & $\mathbf{N}$ & 27.85 & $E$ & 10 & G & & & 1.6 & 5 & TURKEY \\
\hline 04 & 02 & 35 & 47.68 & 60.839 & $\mathbf{N}$ & 151.419 & $w$ & 71 & & & & & 30 & KENAI PENINSULA, ALASKA. <AGS-P>. \\
\hline 04 & 02 & 40 & $01.5 ?$ & 6.22 & $\mathrm{~s}$ & 134.26 & $E$ & 33 & $\mathbf{N}$ & 4. 2 & & 1.0 & 6 & AROE ISLANDS REGION \\
\hline 04 & 83 & 37 & 39.28 & 40.537 & $\mathbf{N}$ & 127.437 & w & 5 & G & 5.0 & 4.1 & & 173 & OFF COAST OF NORTHERN CALIFORNIA. $\angle B R K\rangle, M L 4.7$ (BRK). \\
\hline 84 & 84 & 52 & $51.8 ?$ & 56.04 & $\mathbf{N}$ & 157.00 & E & 41 & 0 & 4.6 & & 1.3 & 25 & KURIL ISLANDS \\
\hline 04 & 05 & 30 & 46.1 & 30.053 & $\mathbf{N}$ & 99.340 & $E$ & 10 & G & 5.1 & & 1.1 & 20 & SICHUAN PROVINCE, CHINA \\
\hline 04 & 05 & 33 & $19.0 *$ & 40.585 & $\mathbf{N}$ & 21.398 & $E$ & 10 & G & & & 0.6 & 7 & GREECE. ML 2.9 (SKO). \\
\hline 04 & 05 & 41 & $14.3 \%$ & 11.790 & $\mathbf{N}$ & 61.102 & $w$ & 30 & $\dot{G}$ & & & 1.1 & 10 & $\begin{array}{l}\text { WINOWARD ISLANDS. MO } 3.5 \text { (TRN). } \\
\text { GERMANY }\end{array}$ \\
\hline 04 & $\begin{array}{l}05 \\
05\end{array}$ & $\begin{array}{l}48 \\
54\end{array}$ & $44.4 ?$ & $\begin{array}{l}49.16 \\
37.076\end{array}$ & $\begin{array}{l}\mathbf{N} \\
\mathbf{N}\end{array}$ & $\begin{array}{l}6.93 \\
27.984\end{array}$ & $\begin{array}{l}E \\
E\end{array}$ & $\begin{array}{l}10 \\
10\end{array}$ & $\begin{array}{l}G \\
G\end{array}$ & & & 1.5 & 5 & $\begin{array}{l}\text { GERMANY } \\
\text { TURKEY }\end{array}$ \\
\hline 04 & 05 & 56 & $\begin{array}{l}20.6 \\
48.5\end{array}$ & 40.577 & $\mathrm{~N}$ & 21.410 & $E$ & 10 & G & & & 0.8 & 5 & GREECE. ML 2.5 (SKO). \\
\hline 04 & 86 & 41 & 25.08 & 63.498 & $\mathbf{N}$ & 149.178 & $w$ & 93 & & & & & 15 & CENTRAL ALASKA. <AGS-P>. \\
\hline 04 & 07 & 13 & $27.9 \&$ & 64.049 & $\mathbf{N}$ & 146.822 & $w$ & 14 & & & & & 18 & CENTRAL ALASKA. ¿AGS-P>. \\
\hline 84 & 88 & 06 & $00.5 \%$ & 37.807 & $\mathbf{N}$ & 29.271 & $E$ & 10 & G & & & 1.1 & 5 & TURKEY \\
\hline 84 & 88 & 25 & 50.8 & 29.947 & $\mathbf{N}$ & 99.153 & $E$ & 10 & G & 4.4 & & 1.0 & 13 & SICHUAN PROVINCE, CHINA \\
\hline 84 & 89 & 03 & $33.6 *$ & 37.112 & $\mathbf{N}$ & 21.094 & $E$ & 28 & & 3.8 & & 1.1 & 16 & SOUTHERN GREECE \\
\hline 04 & 10 & 30 & 06.9 & 6.609 & $\mathrm{~S}$ & 75.760 & $w$ & 33 & $\mathbf{N}$ & 5.5 & 5.1 & 0.8 & 238 & NORTHERN PERU \\
\hline 04 & 10 & 56 & 58.6 & 29.968 & $\mathbf{N}$ & 99.396 & E & 10 & G & 4.4 & & 1.3 & 12 & SICHUAN PROVINCE, CHINA \\
\hline 04 & 11 & 00 & 33.6 & 43.116 & $\mathbf{N}$ & 13.461 & $\mathbf{E}$ & 10 & G & & & 1.4 & 7 & CENTRAL ITALY. MD 3.1 (TRI), 2.6 (SSO) \\
\hline 04 & 11 & 26 & 41.1 & 43.083 & $\mathbf{N}$ & 13.475 & $\mathbf{E}$ & 10 & G & & & 1.2 & 13 & CENTRAL ITALY, MD 3.4 (TRI), 3.3 (SSO) \\
\hline 04 & 11 & 27 & 42.0 & 37.630 & $\mathbf{N}$ & 118.822 & w & 5 & G & & & 0.6 & 33 & $\begin{array}{l}\text { CALIFORNIA-NEVADA BORDER REGION. ML } 3.1 \text { (NEIS), } 3.1 \\
\text { (PAS). }\end{array}$ \\
\hline 04 & 11 & 53 & $01.8 ?$ & 4. 96 & $\mathrm{~S}$ & 144.32 & $E$ & 126 & $*$ & 4.1 & & 0.7 & 11 & NEAR N COAST OF PAPUA NEW GUINEA \\
\hline 04 & 12 & 17 & 06.5 & 23.249 & $\mathbf{S}$ & 66.921 & W & 163 & & 4.2 & & 0.5 & 20 & JUJUY PROVINCE, ARGENTINA \\
\hline 04 & 12 & 22 & $15.7 ?$ & 31.35 & S & 68.09 & $w$ & 33 & $\mathbf{N}$ & & & 1.2 & 5 & SAN JUAN PROVINCE, ARGENTINA \\
\hline 04 & 12 & 46 & $38.1 *$ & 5.364 & $\mathbf{S}$ & 147.856 & $E$ & 33 & $\mathbf{N}$ & 4.3 & & 0.9 & 7 & EAST PAPUA NEW GUINEA REGION \\
\hline 04 & 13 & 15 & 08.0 & 21.963 & $S$ & 179.305 & $w$ & 591 & 0 & 5.4 & & 0.9 & 242 & FIJI ISLANOS REGION \\
\hline $\begin{array}{l}04 \\
04\end{array}$ & $\begin{array}{l}14 \\
14\end{array}$ & 15 & 10.0 & $\begin{array}{l}39.121 \\
29.987\end{array}$ & $\begin{array}{l}\mathbf{N} \\
\mathbf{N}\end{array}$ & $\begin{array}{l}24.598 \\
99.349\end{array}$ & $\begin{array}{l}E \\
E\end{array}$ & $\begin{array}{r}8 \\
10\end{array}$ & & & & $\begin{array}{l}1.1 \\
1.2\end{array}$ & $\begin{array}{l}18 \\
44\end{array}$ & $\begin{array}{l}\text { AEGEAN SEA. ML } 3.1 \text { (ATH). } \\
\text { SICHUAN PROVINCE. CHINA }\end{array}$ \\
\hline 04 & 14 & 34 & $\begin{array}{l}59.1 \\
48.1 *\end{array}$ & $\begin{array}{l}29.987 \\
27.841\end{array}$ & $\mathbf{N}$ & $\begin{array}{l}99.349 \\
56.359\end{array}$ & $E$ & 33 & $\mathbf{N}$ & $\begin{array}{l}4.7 \\
4.0\end{array}$ & 3.9 & $\begin{array}{l}1.2 \\
0.4\end{array}$ & $\begin{array}{r}4+4 \\
7\end{array}$ & $\begin{array}{l}\text { SICHUAN PROVINCE, CHINA } \\
\text { SOUTHERN IRAN }\end{array}$ \\
\hline 04 & 14 & 37 & $01.8 ?$ & 37.12 & $\mathbf{N}$ & 27.97 & $E$ & 10 & G & & & 1.1 & 5 & TURKEY \\
\hline 04 & 15 & 29 & 55.7 & 9.341 & $N$ & 56.977 & $\boldsymbol{E}$ & 10 & G & 4.9 & & 0.8 & 36 & CARLSBERG RIDGE \\
\hline 04 & 16 & 26 & 13.2 & 27.503 & $\mathbf{N}$ & 106.311 & $\boldsymbol{E}$ & 10 & $\mathbf{G}$ & 4.2 & & 1.3 & 11 & EASTERN CHINA. ML 4.6 (BJI). \\
\hline 04 & 16 & 46 & $46.8 *$ & 8.804 & N & 147.718 & $\boldsymbol{E}$ & 33 & $\mathbf{N}$ & 4.0 & & 0.8 & 13 & CAROLINE ISLANDS REGION \\
\hline 04 & 16 & 54 & 38.98 & 60.537 & $\mathbf{N}$ & 147.380 & $w$ & 12 & & & & & 26 & SOUTHERN ALASKA. 〈AGS-P>. \\
\hline 04 & 16 & 55 & $56.9 \%$ & 37.938 & $\mathbf{N}$ & 29.091 & $\boldsymbol{E}$ & 10 & G & & & 1.2 & 6 & TURKEY \\
\hline 04 & 17 & 38 & $48 \cdot 4 \&$ & 59.400 & N & 157.002 & $\mathbf{w}$ & $\theta$ & & & & & 8 & SOUTHERN ALASKA. 〈AGS-P>. \\
\hline 04 & 18 & 10 & 28.7 & 30.046 & $\mathbf{N}$ & 99.483 & $\boldsymbol{E}$ & 10 & G & 4.7 & & 1.3 & 47 & SICHUAN PROVINCE, CHINA \\
\hline 04 & 18 & 23 & 00.0 & 39.521 & $\mathbf{N}$ & 23.519 & $E$ & 10 & G & & & 0.6 & 8 & AEGEAN SEA \\
\hline 04 & 18 & 32 & 55.6 & 13.039 & $\mathrm{~S}$ & 76.195 & w & 72 & & 5.3 & & 1.0 & 118 & NEAR COAST OF PERU. Felt $(1 \mid 1)$ at 1 Co and Lima. \\
\hline 04 & 19 & 31 & 15.1 & 37.104 & $\mathbf{N}$ & 27.912 & $\boldsymbol{E}$ & 10 & G & & & 1.0 & 11 & TURKEY. MO 3.4 (ATH). \\
\hline 04 & 20 & 00 & $03.7 ?$ & 37.08 & $\mathbf{N}$ & 27.89 & $\boldsymbol{E}$ & 5 & G & & & 1.5 & 5 & TURKEY \\
\hline 04 & 20 & 10 & $02.7 ?$ & 37.65 & N & 20.56 & $E$ & 10 & G & & & 1.4 & 8 & IONIAN SEA. ML 3.5 (ATH). \\
\hline 04 & 20 & 22 & 03.1 & 2.156 & $\mathbf{S}$ & 80.588 & $\mathbf{w}$ & 33 & N & & & 1.4 & 7 & NEAR COAST OF ECUADOR \\
\hline 04 & 20 & 22 & $16.9 *$ & 34.534 & $\mathbf{N}$ & 29.893 & $\boldsymbol{E}$ & 10 & G & & & 0. 7 & 6 & EASTERN MEDITERRANEAN SEA \\
\hline 04 & 20 & 41 & $53.1 *$ & 13.078 & $\mathbf{N}$ & 90.216 & $w$ & 34 & * & 4.6 & & 1.3 & 36 & NEAR COAST OF GUATEMALA \\
\hline 04 & 20 & 53 & 31.9 & 40.834 & $\mathbf{N}$ & 22.932 & $\boldsymbol{E}$ & 10 & G & & & 0.4 & 9 & GREECE \\
\hline 04 & 21 & 27 & 06.1 & 42.363 & $\mathbf{N}$ & 19.904 & $\boldsymbol{E}$ & 10 & G & & & 0.6 & 9 & YUGOSLAVIA. MD 2.5 (TTG). \\
\hline 84 & 22 & 15 & $30.4 ?$ & 31.48 & $\mathrm{~S}$ & 68.14 & $w$ & 33 & N & & & 1.4 & 6 & SAN JUAN PROVINCE, ARGENTINA \\
\hline 04 & 22 & 25 & 26.0 & 38.194 & $\mathbf{N}$ & 72.432 & $\mathbf{E}$ & 57 & * & 4.8 & & 1.2 & 43 & $\begin{array}{l}\text { TAJIK SSR. Felt (111) at kharog, Rushon, Vanch, Vir, } \\
\text { Savnob and Ishkashim. }\end{array}$ \\
\hline 84 & 23 & 42 & 20.6 & 37.082 & N & 27.943 & $E$ & 14 & & & & 1.3 & 16 & TURKEY. MD 3.6 (ATH). \\
\hline 05 & 00 & 54 & 11.6 & 37.005 & $\mathbf{N}$ & 27.965 & E & 20 & & & & 1.5 & 17 & $\begin{array}{l}\text { TURKEY. MD } 3.8 \text { (ATH) } \\
\text { NEAR COAST OF CENTRAL }\end{array}$ \\
\hline
\end{tabular}




\begin{tabular}{|c|c|c|c|c|c|c|c|c|c|c|c|c|c|c|}
\hline 05 & 02 & 47 & 31 & $4 ?$ & 10.43 & $N$ & 60.62 & $w$ & 56 & $?$ & & 0.2 & 9 & TRINIDAD. MD 3.6 (TRN) \\
\hline 05 & 86 & 16 & 59. & $.1 *$ & 17.988 & 5 & 131.408 & $E$ & 10 & G & & 1.1 & 7 & NORTHERN TERRITORY, AUSTRALIA. ML 4.1 (OIS). \\
\hline 05 & 86 & 19 & 08. & 4 & 31.286 & $N$ & 104.440 & $E$ & 10 & G & & 0. 7 & 7 & SICHUAN PROVINCE, CHINA \\
\hline 05 & 06 & 54 & 49 & $.3 \%$ & 44.630 & $N$ & 7.075 & $E$ & 10 & $G$ & & 0.1 & 6 & NORTHERN ITALY. ML 1.8 (GEN). \\
\hline 05 & 08 & 11 & 38 & 9 & 3. 189 & N & 76.331 & $w$ & 10 & & 3.2 & 1.0 & 11 & COLOMBIA \\
\hline 05 & 08 & 33 & 18 & $2 \%$ & 18.247 & $N$ & 66.311 & $w$ & 10 & G & & 0.4 & 6 & PUERTO RICO REGION \\
\hline 05 & 09 & 01 & 07. & $1 ?$ & 38.35 & $N$ & 20.02 & E & 10 & G & & 1.4 & 7 & GREECE. MD 3.7 (ATH) \\
\hline 05 & 09 & 17 & 17 & $8 ?$ & 28.79 & N & 86.74 & $\mathbf{E}$ & 10 & G & & 1.4 & 5 & TIBET \\
\hline 05 & 09 & 44 & 20 & 7 & 44.519 & $N$ & 7.344 & $\mathbf{E}$ & 10 & & & 0.9 & 33 & NORTHERN ITALY. ML 3.1 (GEN). \\
\hline 05 & 10 & 20 & 01. & 28 & 37.955 & $\mathbf{N}$ & 122.350 & $\mathbf{w}$ & 6 & & & & 14 & $\begin{array}{l}\text { CENTRAL CALIFORNIA. } \angle B R K\rangle \text {. ML } 2.5 \text { (BRK). MO=2.6*10**13 } \\
\text { Nm (BRK). Felt at EI Sobrante, Pinole, Richmond and Son } \\
\text { Pobla. }\end{array}$ \\
\hline 05 & 10 & 25 & 54. & $1 \%$ & 18.246 & N & 66.312 & $w$ & 10 & G & & 0.5 & 5 & PUERTO RICO REGION \\
\hline 05 & 10 & 35 & 32 & $.5 *$ & 17.444 & N & 100.680 & $\mathbf{w}$ & 33 & N & & 1.3 & 8 & GUERRERO, MEXICO \\
\hline 05 & 10 & 44 & 34 & $2 ?$ & 42.27 & N & 22.71 & $\mathbf{E}$ & 10 & G & & 0.9 & 5 & BULGAR IA \\
\hline 05 & 11 & 23 & 39 & .58 & 39.817 & $\mathbf{N}$ & 123.225 & $w$ & 12 & & & & 10 & $\begin{array}{l}\text { NEAR COAST OF NORTHERN CALIF. <BRK>. ML } 2.9 \text { (BRK). FeIt } \\
(111) \text { at Cavelo. }\end{array}$ \\
\hline 05 & 11 & 53 & 35 & $0 \%$ & 33.924 & $s$ & 71.125 & $\mathbf{w}$ & 33 & $N$ & & 0.4 & 7 & NEAR COAST OF CENTRAL CHILE \\
\hline 05 & 12 & 21 & 51. & $\theta \cdot$ & 13.999 & s & 166.273 & $E$ & 33 & $\mathrm{~N}$ & 4.7 & 1.4 & 17 & VANUATU ISLANDS \\
\hline 05 & 13 & 30 & 40. & & 31.984 & S & 178.658 & $w$ & 10 & G & 5.34 .9 & 1.0 & 120 & KERMADEC ISLANDS REGION \\
\hline 05 & 14 & 11 & 55 & 18 & 32.950 & $N$ & 117.740 & $w$ & 6 & G & & & 24 & $\begin{array}{l}\text { CALIFORNIA-MEXICO BORDER REGION. SPAS-P>. ML } 3.4 \text { (PAS). } \\
\text { Felt ( } 11 \text { ) ot Son Diego, Colifornio. }\end{array}$ \\
\hline 05 & 16 & 32 & 22 & $7 \&$ & 61.737 & $N$ & 149.755 & $w$ & 41 & & & & 34 & SOUTHERN ALASKA, 〈AGS-P>. \\
\hline 05 & 16 & 58 & 47. & $6 *$ & 30.035 & $\mathbf{N}$ & 99.270 & $\mathbf{E}$ & 10 & $G$ & 4.7 & 1.3 & 13 & SICHUAN PROVINCE, CHINA \\
\hline 05 & 17 & 11 & 07. & 1 & 29.993 & $N$ & 99.255 & $\mathbf{E}$ & 10 & G & & 1.3 & 14 & SICHUAN PROVINCE, CHINA. ML 3.8 (BJI) \\
\hline 05 & 17 & 11 & 58. & 6 & 41.840 & $N$ & 19.284 & $\mathbf{E}$ & 17 & & & 1.0 & 23 & ALBANIA. ML 3.0 (TTG). MD 3.4 (ATH). \\
\hline 05 & 18 & 28 & 39. & 4 & 8.281 & s & 71.381 & $w$ & 593 & G & 6.4 & 1.0 & 589 & 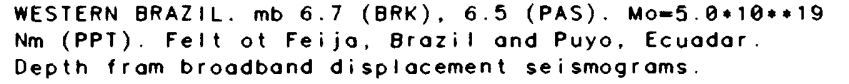 \\
\hline 05 & 19 & 10 & 45. & $\theta ?$ & 7.25 & $\$$ & 71.08 & w & 600 & G & 4.9 & 0.8 & 16 & WESTERN BRAZIL \\
\hline 05 & 20 & 22 & 28. & $4 ?$ & 26.13 & S & 178.82 & $\mathbf{E}$ & 501 & $?$ & 4.6 & 1.5 & 8 & SOUTH OF FIJI ISLANDS \\
\hline 05 & 20 & 39 & 56. & & 43.225 & $N$ & 13.318 & $\mathbf{E}$ & 10 & G & & 1.1 & 9 & CENTRAL ITALY. MD 2.6 (SSO) \\
\hline 06 & 00 & 31 & 07. & $4 \%$ & 40.505 & $N$ & 22.624 & $\bar{E}$ & 10 & G & & 0.1 & 5 & GREECE \\
\hline 06 & 01 & 41 & 29.9 & & 66.971 & $N$ & 156.229 & $w$ & 5 & G & & 0.7 & 9 & ALASKA. ML 4.0 (PMR). \\
\hline 06 & 02 & 40 & 56.2 & & 22.471 & N & 99.776 & E & 10 & G & 4.74 .5 & 1.3 & 54 & BURMA-CHINA BORDER REGION \\
\hline 06 & 02 & 43 & 30.9 & $9 *$ & 8.472 & s & 67.869 & $\mathbf{E}$ & 10 & G & & 0.6 & 9 & $M I D-I N D I A N$ RISE \\
\hline 06 & 02 & 45 & 26.3 & $3 \%$ & 39.259 & $N$ & 28.905 & $\mathbf{E}$ & 10 & G & & 0.4 & 6 & TURKEY \\
\hline 06 & 03 & 54 & 05.6 & & 6.332 & $s$ & 130.766 & $E$ & 86 & * & 4.5 & 0.9 & 24 & $\begin{array}{l}\text { BANDA SEA } \\
\text { DODECANESE ISLANDS }\end{array}$ \\
\hline 06 & 04 & 12 & 15.7 & & 36.475 & N & 28.190 & E & 77 & $?$ & & 0.5 & 7 & $\begin{array}{l}\text { DODECANESE ISLANDS } \\
\text { SICHUAN PROVINCE, CHINA }\end{array}$ \\
\hline $\begin{array}{l}06 \\
06\end{array}$ & $\begin{array}{l}04 \\
04\end{array}$ & $\begin{array}{l}12 \\
53\end{array}$ & $\begin{array}{l}42.5 \\
47.5\end{array}$ & $\begin{array}{l}5 \\
5 *\end{array}$ & $\begin{array}{l}29.726 \\
40.975\end{array}$ & $\begin{array}{l}N \\
N\end{array}$ & $\begin{array}{l}99.329 \\
22.160\end{array}$ & $\begin{array}{l}E \\
E\end{array}$ & $\begin{array}{l}10 \\
10\end{array}$ & $\begin{array}{l}G \\
G\end{array}$ & 4.8 & $\begin{array}{l}1.0 \\
0.5\end{array}$ & $\begin{array}{r}13 \\
6\end{array}$ & $\begin{array}{l}\text { SICHUAN PROVINCE, CHINA } \\
\text { GREECE. ML } 1.2(\text { SKO). }\end{array}$ \\
\hline 06 & 06 & 27 & 41.8 & 8 & 12.438 & N & 120.972 & E & 33 & N & 4.74 .3 & 1.0 & 39 & MINDORO, PHILIPPINE ISLANDS \\
\hline$\theta 6$ & 07 & 26 & 46.4 & $4 *$ & 24.458 & $N$ & 94.214 & $\mathbf{E}$ & 10 & G & & 0.4 & 8 & BURMA-INDIA BORDER REGION \\
\hline 06 & 09 & 57 & 58.7 & & 2.005 & s & 151.458 & E & 33 & $N$ & 4.3 & 1.4 & 5 & NEW IRELAND REGION \\
\hline 06 & 11 & 19 & 01.7 & $7 ?$ & 36.08 & $N$ & 24.90 & $E$ & 33 & $N$ & & 0.7 & 5 & SOUTHERN GREECE. MD 3.5 (ATH). \\
\hline 06 & 11 & 31 & 14. & $\theta *$ & 8.497 & $s$ & 125.014 & $E$ & 292 & $*$ & 5.3 & 1.1 & 14 & T I MOR \\
\hline 06 & 12 & 02 & 21.6 & & 51.263 & $N$ & 178.252 & E & 39 & D & 4. 9 & 1.1 & 73 & RAT ISLANDS, ALEUTIAN ISLANDS. ML 4.7 (PMR). \\
\hline 06 & 12 & 30 & 43.8 & 8. & 53.239 & N & 160.293 & E & 100 & G & 4.4 & 0. 8 & 16 & NEAR EAST COAST OF KAMCHATKA \\
\hline 06 & 13 & 07 & 18.8 & $\theta \%$ & 38.852 & N & 112.989 & E & 10 & G & & 1.0 & 5 & NORTHEASTERN CHINA. ML 3.1 (BJI). \\
\hline 06 & 14 & 32 & 29.6 & & 41.836 & N & 12.727 & E & 10 & G & & 0.5 & 9 & SOUTHERN ITALY \\
\hline 06 & 14 & 32 & 52.8 & & 15.853 & N & 95.098 & $w$ & 27 & & 4.8 & 1.1 & 70 & NEAR COAST OF OAXACA. MEXICO \\
\hline 06 & 16 & 25 & 07.9 & & 23.846 & N & 122.001 & E & 33 & $N$ & 3.5 & 1.1 & 9 & TAIWAN REGION \\
\hline 06 & 16 & 35 & 55.7 & & 66.905 & N & 156.077 & $w$ & 5 & G & & 0.8 & 18 & $\begin{array}{l}\text { ALASKA } \\
\text { FASTFPN MFOITERPANFAN SFA }\end{array}$ \\
\hline 06 & 17 & 02 & 03.1 & & 34.458 & N & 27.457 & $\mathbf{E}$ & 33 & N & & 0.6 & 7 & EASTERN MEDITERRANEAN SEA. MD 3.7 (ATH). \\
\hline 06 & 17 & 11 & 15.8 & & 39.781 & $N$ & 25.585 & E & 5 & & & 0.6 & $\begin{array}{r}19 \\
4\end{array}$ & $\begin{array}{l}\text { AEGEAN SEA } \\
\text { GREECE. ML } 2.9 \text { (ATH). }\end{array}$ \\
\hline $\begin{array}{l}06 \\
06\end{array}$ & $\begin{array}{l}17 \\
18\end{array}$ & $\begin{array}{l}51 \\
38\end{array}$ & $\begin{array}{l}26.3 \\
09.6\end{array}$ & $\begin{array}{l}3 ? \\
6\end{array}$ & $\begin{array}{l}38.04 \\
37.118\end{array}$ & $\begin{array}{l}N \\
N\end{array}$ & $\begin{array}{l}22.00 \\
27.979\end{array}$ & $\begin{array}{l}\mathbf{E} \\
\mathbf{E}\end{array}$ & $\begin{array}{l}10 \\
10\end{array}$ & G & & $\begin{array}{l}0.1 \\
1.5\end{array}$ & $\begin{array}{r}4 \\
11\end{array}$ & $\begin{array}{l}\text { GREECE. ML } 2.9 \text { (ATH). } \\
\text { TURKEY. MD } 3.3 \text { (ATH). }\end{array}$ \\
\hline 86 & 18 & 52 & 12.2 & & 67.146 & $N$ & 156.578 & $w$ & 5 & G & & 0.6 & 6 & ALASKA \\
\hline 86 & 19 & 04 & 25.2 & & 36.441 & $\mathbf{N}$ & 71.694 & E & 85 & * & 4.6 & 0.6 & 15 & AFGHANISTAN-USSR BORDER REGION \\
\hline$\theta$ & 19 & 50 & 28.8 & $\theta$. & 37.114 & $\mathrm{~N}$ & 27.853 & $\mathbf{E}$ & 10 & G & & 1.4 & 7 & TURKEY \\
\hline 06 & 20 & 03 & 45.3 & 3 & 51.296 & N & 178.214 & E & 42 & $D$ & 4.9 & 1.0 & 49 & RAT ISLANDS, ALEUTIAN ISLANDS \\
\hline 86 & 21 & 01 & 59.1 & $1 *$ & 21.839 & $N$ & 143.298 & $\mathbf{E}$ & 32 & $?$ & 4.3 & 1.0 & 18 & MARIANA ISLANDS REGION \\
\hline 86 & 21 & 14 & 57.4 & 4 & 36.477 & $N$ & 70.115 & $\mathbf{E}$ & 219 & $D$ & 4.8 & 1.0 & 190 & $\begin{array}{l}\text { HINDU KUSH REGION. Felt ( } 111 \text { ) at Obigarm, Dushanbe and } \\
\text { Lyangar; ( } 11 \text { ) at Kulyab, USSR. }\end{array}$ \\
\hline 06 & 21 & 40 & 41.5 & & .947 & $N$ & 156.262 & $w$ & 5 & G & & .5 & 13 & ALASKA \\
\hline 06 & 23 & 00 & 13.7 & & 40.505 & $N$ & 22.617 & $\mathbf{E}$ & 10 & G & & .3 & 5 & GREECE \\
\hline 06 & 23 & 29 & 33.2 & & 40.918 & N & 22.092 & $\mathbf{E}$ & 10 & G & & 1.0 & 22 & GREECE. MD 3.3 \\
\hline 06 & 23 & 37 & 43.5 & & 16.225 & N & 95.250 & $w$ & 11 & & 4.9 & 1.1 & 61 & OAXACA, MEXICO \\
\hline 06 & 23 & 46 & 13.0 & & 64.719 & $N$ & 17.322 & w & 10 & G & 4.24 .1 & 1.2 & 7 & ICELAND \\
\hline 07 & 00 & 38 & 18.5 & & 23.553 & N & 99.526 & $\mathbf{E}$ & 33 & $N$ & 5.35 .6 & 1.1 & 266 & $\begin{array}{l}\text { BURMA-CHINA BORDER REGION. At least one person killed. } \\
91 \text { injured and } 5,300 \text { hauses destroyed in the Gengma } \\
\text { oreo. China. Felt strangly in Lancang and Menglion } \\
\text { Caunties. Direct econamic lasses of mare thon } 54 \\
\text { million dollars were sustained. }\end{array}$ \\
\hline 07 & 1 & 32 & 19.4 & $4 *$ & 37.563 & N & 71.870 & E & 33 & N & 4.3 & .5 & 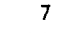 & AFGHANISTAN-USSR BORDER REGION \\
\hline 07 & 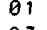 & 49 & 05.2 & 2 & 37.740 & N & 15.104 & E & 10 & G & & 0.9 & 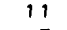 & SICILY \\
\hline 07 & 03 & $\theta 0$ & 24.9 & & 29.909 & N & 99.318 & E & 10 & G & 4.9 & 1.3 & 17 & SICHUAN PROVINCE, CHINA \\
\hline 07 & 03 & 02 & 45.2 & & 41.478 & N & 142.051 & E & 72 & & 4.6 & 1.1 & 52 & $\begin{array}{l}\text { HOKKAIDO, JAPAN REGION. Felt (I JMA) ot Hachinohe. } \\
\text { Honshu. }\end{array}$ \\
\hline 07 & 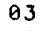 & 55 & 02.9 & $9 *$ & 30.064 & N & 99.254 & E & 10 & G & & .4 & 7 & SICHUAN PROVINCE, CHINA \\
\hline 07 & bs & 57 & 38.4 & & 5. 22 & S & 145.91 & $E$ & 33 & N & 4.3 & .3 & $r$ & EAST PAPUA NEW GUINEA REGION \\
\hline 07 & 04 & 43 & 47.3 & & 38.309 & N & 22.782 & E & 10 & G & & 0.9 & 22 & GREECE. ML 3.4 (ATH). \\
\hline 07 & 04 & 58 & 55.8 & & 37.082 & N & 27.877 & $E$ & 10 & G & & 1.3 & 7 & TURKEY \\
\hline 07 & 05 & 47 & $45 \cdot 2$ & $2 \&$ & 61.981 & N & 150.413 & $w$ & 43 & & & & 29 & SOUTHERN ALASKA. 〈AGS-P>. \\
\hline
\end{tabular}


$\begin{array}{llllll}07 & 06 & 23 & 35.986 & 62.277 \mathrm{~N}\end{array}$ $07 \quad 06 \quad 37595 ? 33.76 \quad \mathrm{~S}$ $065203.1 \& 60.677 \mathrm{~N}$ $065628.0 \quad 34.965 \mathrm{~N}$

$070150.2 ? \quad 54.53 \mathrm{~N}$ $08 \quad 37 \quad 37.8$ ? $38.29 \mathrm{~N}$ $\begin{array}{lllll}08 & 55 & 58.1 & 27.424 \mathrm{~N}\end{array}$ $\begin{array}{lllll}10 & 36 & 21.4 & 38.304 \mathrm{~N}\end{array}$ $104650.138 .320 \mathrm{~N}$ $\begin{array}{lllllll}11 & 36 & 49.7 ? & 38.29 \quad \mathrm{~N}\end{array}$

$120259.6 * 38.281 \mathrm{~N}$ $\begin{array}{lllll}12 & 27 & 32.9 & 8.690 \mathrm{~S}\end{array}$ $\begin{array}{lllll}12 & 28 & 21.3 & 7.149 \mathrm{~S}\end{array}$ $\begin{array}{lll}13 & 05 & 22.7 * 36.895 \mathrm{~N}\end{array}$ $134044.7 \quad 39.693 \mathrm{~N}$

$\begin{array}{llllll}08 & 13 & 48 & 34 & 4 * 6.384 & 5\end{array}$ $08 \quad 140156.4 * 11.7325$ $\begin{array}{lllllll}0 & 08 & 14 & 28 & 30.9 & 23.427 & \mathrm{~S}\end{array}$ $\begin{array}{llllll}08 & 14 & 58 & 32.6 \% & 37.774 & N\end{array}$ $\begin{array}{llllll}08 & 15 & 25 & 40.98 & 32.350 & \mathrm{~N}\end{array}$ $08 \quad 16 \quad 1908.8 \& 63.171 \mathrm{~N}$ $08 \quad 1620 \quad 32.8 * 23.550 \mathrm{~S}$ $08 \quad 170414.0 * 17.720 \mathrm{~N}$ $88 \quad 1733 \quad 48.4 * 60.630 \mathrm{~N}$
$149.742 \mathrm{~W}$

$\begin{array}{llr}178.50 & E & 161 \\ 150.718 & W & 48\end{array} \quad 4.6$

$135.573 \mathrm{E} \quad 18$

$161.74 w$

22.59

$127.748 \mathrm{E}$

$22.789 \mathrm{E}$

$22.740 \mathrm{E}$

$22.61 \quad E$

$22.583 \mathrm{E}$

$75.443 \mathrm{~W}$

129.215

$71.580 \mathrm{E}$

$20.283 \mathrm{E}$

$121.550 \mathrm{~W}$

$27.838 \mathrm{E}$

$150.721 \mathrm{~W}$

$156.269 \mathrm{w}$

$174.974 W$

$17.612 \mathrm{E}$

$10.47 \mathrm{E}$

$122.158 \mathrm{E}$

$175.474 \mathrm{~W}$

$5.09 \quad W$

$27.427 \mathrm{E}$

$68.765 \mathrm{E}$

$172.847 \mathrm{~W}$

$141.993 \mathrm{E}$

$25.843 \mathrm{E}$

$139.039 \mathrm{E}$

$71.172 \mathrm{E}$

$79.785 \mathrm{E}$

$28.01 \mathrm{E}$ $140.70 \mathrm{E}$ $101.94 \mathrm{~W}$

$156.316 \mathrm{~W}$

$27.896 \mathrm{E}$

$14.981 \mathrm{E}$ $130.930 \mathrm{E}$

$22.45 \mathrm{E}$

$99.344 E$

$178.845 \mathrm{~W} 531 \mathrm{D} 5.4$

$153.484 W \quad 158 \quad 4.6$

$27.97 \mathrm{E} \quad 10 \mathrm{G}$

60.06 W 24

28.381 E $10 \mathrm{G}$

124.13 E $226 ? 4.5$

$106.491 \mathrm{E} \quad 10 \mathrm{G} \quad 3.9$

$22.45 \mathrm{E} \quad 10 \mathrm{G}$

$126.696 \mathrm{E} \quad 76 \cdot 5.1$

$127.24 \mathrm{E} \quad 33 \mathrm{~N} \quad 4.3$

$167.185 \mathrm{E} 150 * 4.8$

$28.500 \mathrm{E}$

$32.381 \mathrm{E}$

$152.867 \mathrm{~W}$

$152.532 w$

$114.31 W$

8. $79 \mathrm{E}$

$153.599 \mathrm{E}$

5. $750 \mathrm{~W}$

$21.365 \mathrm{E}$

$172.91 \mathrm{~W}$

$96.937 \mathrm{E}$

$27.93 \mathrm{E}$

$149.77 \mathrm{E}$

$147.092 \mathrm{~W}$

$145.457 \mathrm{E}$

$117.990 \mathrm{~W}$

$62.365 \mathrm{~W}$

$23.031 \mathrm{E}$

$28.092 \mathrm{E}$

$156.243 \mathrm{~W}$

153.505

$117.172 \mathrm{E}$

$179.953 \mathrm{~W}$

$30.564 \mathrm{E}$

$115.250 \mathrm{~W}$

$150.864 \mathrm{~W}$

$179.777 \mathrm{E}$

$95.051 \mathrm{~W}$

$6.231 \mathrm{E}$

$140.060 \mathrm{E}$
$150 *$
$33 \mathrm{~N}$

$33 \mathrm{~N}$

464.6

\section{$5 \mathrm{G}$}

$10 \mathrm{G}$

$58 * 4.8$

$10 \mathrm{G}$

$28 * 3.5$

$33 \mathrm{~N} 5.0$

$33 \mathrm{~N}$

$10 \mathrm{G}$

$33 \mathrm{~N} 4.1$

17

$200 \quad 4.6$

56

$10 \mathrm{G}$

$10 \mathrm{G}$

$10 \mathrm{G}$

$5 \mathrm{G}$

$79 \cdot 4.4$

$33 \mathrm{~N}$

548 D 5.6

$5 \mathrm{G}$

$6 \mathrm{G}$

136

600 G 4.5

$33 \mathrm{~N}$

$10 \mathrm{G}$

$82 \quad 4.9$
38 CENTRAL ALASKA. LAGS-P>

14 SOUTH OF KERMADEC ISLANDS

57 KENAI PENINSULA, ALASKA. LAGS-P>. ML 3.6 (PMR)

0.58 NEAR S. COAST OF SOUTHERN HONSHU. MG 3.6 (JMA). FEIt ( I I JMA) Ot KYOtO.

1.014 ALASKA PENINSULA

0.44 GREECE. ML 2.8 (ATH)

1.333 RYUKYU ISLANDS. FEIt

26 GREECE. ML 3.5 (ATH)

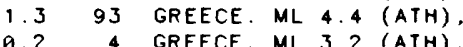

0.24 GREECE. ML 3.2 (ATH)

$\begin{array}{lrl}0.7 & 5 & \text { GREE } \\ 0.7 & 45 & \text { PERU }\end{array}$

$1.3 \quad 34$ BANDA SEA

1.5 23 AFGHANISTAN-USSR BORDER REGION

(KBA), 45 (TTG). MD 4.1 (ATH)

16 CENTRAL CALIFORNIA. ¿BRK>. ML 3.2 (BRK). FeIt (III) ot Gilroy and Son Martin. Also felt ot Margon Hill.

1.510 TURKEY. MD 3.3 (ATH)

37 KENAI PENINSULA. ALASKA. 〈AGS-P>.

11 ALASKA

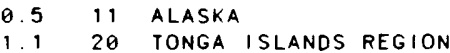

1.259 ADRIATIC SEA. ML 4.1 (KBA), 4.1 (ZAG), 4.0 (TTG), 3.4 (LJU). MD 4.2 (TRI). 4.0 (BEO).

0.84 SWEDEN. ML 2.2 (BER).

1.1105 EASTERN USSR

1.1 62 TONGA ISLANDS

1.34 MOROCCO. MD 3.7 (TIO)

1.410 TURKEY

1.09 HINDU KUSH REGION

0.514 ANDREANOF ISLANDS, ALEUTIAN IS

0.617 VOLCANO ISLANDS REGION

1.213 AEGEAN SEA

0.8135 HONSHU, JAPAN. FeIt (I I JMA) ot Utsunomiyo; (I JMA) ot Mi to, Kofu. Tokyo ond on Oshimo.

0.6 17 AFGHANISTAN-USSR BORDER REGION

1.033 EASTERN KAZAKH SSR. Felt (IV) ot Amanbakter and (II) ot AIMOATO.

1.25 TURKEY

1. 17 SOUTH OF HONSHU, JAPAN

1.36 GUERRERO, MEXICO

0.315 ALASKA. ML 3.6 (PMR)

1.97 TURKEY

0.86 SICILY

0.15 KYUSHU, JAPAN. Felt $(111$ JMA) in the Mt. Aso oreo.

0.45 GREECE

0. 913 BURMA-CHINA BORDER REGION

1. 1198 FIJI ISLANDS REGION

64 SOUTHERN ALASKA. 〈AGS-P>

1.65 TURKEY

0.210 WINDWARD ISLANDS. ML. 3.1 (FDF)

$0.3 \quad 8$ TURKEY

$1.1 \quad 10$ TIMOR

0.56 NORTHERN CHINA. ML 4.0 (BJI)

1.56 GREECE. ML 2.3 (SKO)

1.3 54 MOLUCCA PASSAGE

1.07 TALAUD ISLANDS

1.039 VANUATU ISLANDS

0. 48 EASTERN MEDITERRANEAN SEA

1.3

65 SOUTHERN ALASKA. 〈AGS-P>

75 KODIAK ISLAND REGION. LAGS-P>. ML 4.2 (PMR). FEIt (IV) at KOdiOK.

0.1 6 WESTERN IDAHO. ML 3.2 (BUT)

1.45 NORTHERN ITALY

1.324 NEW BRITAIN REGION

1.56 STRAIT OF GIBRALTAR

1.111 SOUTHERN GREECE. ML 3.3 (ATH)

1.612 ANDREANOF ISLANDS, ALEUTIAN IS. ML 3.7 (PMR)

0. 6 7 TIBET

1.54 TURKEY

1.37 NEW BRITAIN REGION

23 SOUTHERN ALASKA <AGS-P>.

0.518 MARIANA ISLANDS

0.46 SOUTHERN CALIFORNIA. ML 3.0 (NEIS). Felt at Anoheim, Breo. Bueno Park ond Fullerton.

0.46 LEEWARD ISLANDS. ML 2.8 (FDF).

0.65 GREECE

1.27 TURKEY

0.728 ALASKA. ML 4.3 (PMR). Felt (IV) at Kabuk ond (111) at Shungnok.

1. 11 NEW BRITAIN REGION

1.36 SOUTH OF SUMBAWA ISLAND

1.1352 SOUTH OF FIJI ISLANDS. Mb 5.7 (BRK).

1.46 TURKEY

5 CALIFORNIA-MEXICO BORDER REGION. LPAS-P>. ML 3.3 (PAS).

29 CENTRAL ALASKA. LAGS-P>

0.512 SOUTH OF FIJI ISLANDS

1.17 OAXACA, MEXICO

0.68 SOUTHERN NORWAY. MD 1.7 (BER)

1.1104 NEAR EAST COAST OF HONSHU, JAPAN. FEIt $(111$ JMA) at Ut sunomiyo: ( 1 I JMA) ot Mito and Kumogoya: (1 JMA) at 


\begin{tabular}{|c|c|c|c|c|c|c|c|c|c|c|c|c|}
\hline 08 & 17 & 54 & 43.5 & & 36.225 & $\mathrm{~N}$ & 28. & .086 & $\mathrm{E}$ & 87 & & 9 \\
\hline 08 & 18 & 28 & 59.0 & & 7.004 & $\mathrm{~s}$ & 147. & 415 & E & 84 & 4. & 9 \\
\hline 08 & 19 & 21 & 40.1 & & 62.161 & $\mathrm{~N}$ & 149. & .395 & w & 36 & & \\
\hline 88 & 20 & 07 & 45.4 & & 43.047 & $\mathrm{~N}$ & 13. & .319 & E & 10 & G & \\
\hline 88 & 20 & 37 & 50.8 & . 3 & 37.459 & $\mathrm{~N}$ & 11. & 685 & E & 10 & G & \\
\hline 08 & 20 & 48 & 06.8 & $\% 4$ & 40.540 & $\mathrm{~N}$ & 15. & .833 & E & 10 & G & \\
\hline 88 & 21 & 21 & 11.9 & & 50.698 & $\mathrm{~N}$ & 129. & .746 & $w$ & 10 & $G$ & 2 \\
\hline 08 & 21 & 30 & 36.7 & - 1 & 15.781 & $\mathrm{~s}$ & 177. & .738 & E & 22 & D & 9 \\
\hline 08 & 22 & 31 & 31.3 & ** 1 & 15.059 & $\mathrm{~N}$ & 60. & .136 & w & 33 & $\mathrm{~N}$ & \\
\hline 88 & 22 & 35 & 06.8 & * 1 & 17.977 & s & 178 & .825 & w & 680 & 4. & .7 \\
\hline 08 & 23 & 18 & 43.6 & & 32.282 & $\mathrm{~N}$ & 137. & .626 & $E$ & 390 & & 1 \\
\hline 08 & 23 & 28 & 28.8 & * 3 & 34.982 & $\mathrm{~N}$ & 45. & .000 & $E$ & 47 & 4. & .7 \\
\hline 09 & $\theta \theta$ & 35 & 07.7 & & 45.554 & $\mathrm{~N}$ & 3. & .554 & E & 5 & G & \\
\hline 09 & 01 & 28 & 42.6 & & 41.901 & $\mathrm{~N}$ & 20. & 560 & $E$ & 5 & G & \\
\hline 89 & 02 & $\theta 0$ & 54.0 & & 41.890 & $\mathrm{~N}$ & 20. & .668 & $E$ & 10 & G & \\
\hline 89 & 02 & 30 & 37.8 & & 28.071 & $\mathrm{~N}$ & 16. & 156 & $w$ & 23 & D & .24 .6 \\
\hline 89 & 03 & 23 & 09.4 & & 35.465 & $\mathrm{~N}$ & 27. & .900 & $E$ & 10 & G & \\
\hline 09 & 04 & 19 & 41.2 & & 42.697 & $\mathrm{~N}$ & 12. & .719 & $\mathbf{E}$ & 10 & G & \\
\hline 09 & 04 & 39 & 25.3 & $\% 6$ & 60.074 & $\mathrm{~N}$ & 5. & .218 & $\mathbf{E}$ & 10 & G & \\
\hline 09 & 05 & 02 & 03.8 & ? 1 & 18.10 & $\mathrm{~s}$ & 70. & 18 & $w$ & 122 & $?$ & \\
\hline 09 & 05 & 16 & 05.1 & * 3 & 36.608 & $\mathrm{~N}$ & 70. & .976 & E & 165 & 4. & 4 \\
\hline 09 & 05 & 17 & 47.5 & & 38.478 & $\mathrm{~N}$ & 28. & 105 & $\mathbf{E}$ & 10 & G & \\
\hline 09 & 05 & 51 & 13.3 & $? 1$ & 17.86 & $\mathrm{~N}$ & 60. & .88 & $w$ & 33 & $\mathrm{~N}$ & \\
\hline 09 & 06 & 01 & 24.9 & * 3 & 35.547 & $\mathrm{~N}$ & 27. & .702 & $E$ & 10 & G & \\
\hline 09 & 06 & 15 & 53.0 & & 38.317 & $\mathrm{~N}$ & 22. & .737 & $\mathbf{E}$ & 10 & G & \\
\hline 09 & 06 & 23 & 38.6 & & 38.348 & $\mathrm{~N}$ & 22. & .760 & $\mathbf{E}$ & 33 & $\mathrm{~N}$ & \\
\hline 89 & 06 & 25 & 13.1 & & 41.876 & $\mathrm{~N}$ & 20. & 582 & E & 10 & G & \\
\hline 09 & 07 & 42 & 06.5 & & 59.678 & $\mathrm{~N}$ & 153. & .362 & $w$ & 127 & & \\
\hline 89 & 08 & 24 & 19.1 & & 10.015 & $\mathrm{~s}$ & 161. & .022 & $E$ & 33 & 4. & .34 .4 \\
\hline 89 & 10 & 22 & 43.0 & & 38.358 & $\mathrm{~N}$ & 22. & 590 & E & 10 & G & \\
\hline 09 & 10 & 27 & 34.5 & & 20.064 & $S$ & 133. & 997 & $\mathbf{E}$ & 10 & G & \\
\hline 09 & 10 & 38 & 16.9 & & 19.365 & $\mathrm{~N}$ & 104. & .801 & $w$ & 65 & 4. & .5 \\
\hline 89 & 11 & 41 & 41.0 & & 11.627 & $N$ & 125. & 488 & $w$ & 5 & G & \\
\hline 09 & 13 & 11 & 50.1 & & 88.19 & $\mathrm{~N}$ & & .37 & $\mathbf{E}$ & 10 & G & \\
\hline 89 & 13 & 24 & 54.9 & & 17.69 & $\mathrm{~S}$ & 179. & 09 & $w$ & 692 & 4. & \\
\hline 89 & 15 & 34 & 09.6 & & 52.991 & $s$ & 159. & 386 & $E$ & 10 & G & 3 \\
\hline 89 & 16 & 12 & 24.3 & & 54.642 & $N$ & 156. & 258 & $m$ & $\theta$ & & 0 \\
\hline 89 & 16 & 17 & & & 22.879 & $\mathbf{N}$ & 92. & 504 & $t$ & 105 & 4. & 0 \\
\hline 09 & 16 & 30 & 55 & & 34.10 & S & 71. & 10 & 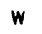 & 33 & N & \\
\hline 09 & 16 & 18 & 18.8 & & 2.857 & $\mu$ & 148. & 221 & & 67 & & \\
\hline 9 & 10 & & & & $10.2 J 6$ & & 119. & נס & & 16 & & \\
\hline
\end{tabular}

09

09
09

09

09

09

a 10

10

10

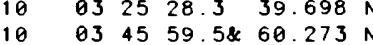

$10 \quad 0350 \quad 47.6 * 31.561 \mathrm{~S}$

10

10

10

$\begin{array}{llll}97 & 59 & 35.7 \% & 45.56 \theta N\end{array}$ $\begin{array}{llll}08 & 23 & 24.5 & 2.688 \mathrm{~N}\end{array}$ $0902 \quad 27.8460 .423 \mathrm{~N}$ $\begin{array}{llll}10 & 06 & 19.8 & 40.230 \mathrm{~N}\end{array}$ $10 \quad 08 \quad 03.5 * 11.543 \mathrm{~N}$ $\begin{array}{llllll}10 & 36 & 31.6 \% & 37.281 & N\end{array}$ $\begin{array}{llll}10 & 47 & 03.6 \% & 39.900\end{array}$ $110039.5 * 8.266 \mathrm{~S}$ $\begin{array}{llll}12 & 19 & 18.1 & 41.651 \mathrm{~N}\end{array}$ $13 \quad 36 \quad 20.5 \% 39.730 \mathrm{~N}$ $135917.9 * 30.032 \mathrm{~N}$ $\begin{array}{lllll}14 & 59 & 05.7 & 43.161 \mathrm{~N}\end{array}$ $153628.7 \& 62.343 \mathrm{~N}$ $\begin{array}{llll}15 & 45 & 06.3 & 32.865\end{array}$ $\begin{array}{llll}16 & 13 & 49.0 \% & 60.567\end{array}$ $\begin{array}{llll}16 & 15 & 16.0 \% & 39.754 \mathrm{~N}\end{array}$ $\begin{array}{llllll}16 & 24 & 44.38 & 58.784 & N\end{array}$ $\begin{array}{llllll}17 & 13 & 05.1 ? & 39.58 \quad \mathrm{~N}\end{array}$ $185155.7 \& 62.52 \theta \mathrm{N}$ $193239.5 * 5.498 \mathrm{~S}$

30

$27.910 \mathrm{E}$

$148.802 \mathrm{~W}$

$19 \quad 4.23 .5$

$121.630 \mathrm{~W}$

$121.630 \mathrm{~W}$
$69.420 \mathrm{E}$

$150.428 \mathrm{~W}$

$4.930 \mathrm{~W}$
$7.865 \mathrm{E}$

$10 \mathrm{G} \quad 4.3$

$128.424 \mathrm{E} \quad 54$ ? 5.2

$147.680 \mathrm{~W}$

$143.739 \mathrm{E}$

$87.060 \mathrm{~W}$

$27.822 \mathrm{E}$

$27.914 \mathrm{E}$

$129.522 \mathrm{E}$

$20.443 \mathrm{E}$

$27.853 \mathrm{E}$

$99.393 \mathrm{E}$

$25.400 \mathrm{E}$

$150.083 \mathrm{~W}$

$141.779 \mathrm{E}$

$5.034 \mathrm{E}$

$27.865 \mathrm{E}$

$139.016 \mathrm{~W}$

$19.48 \mathrm{E}$

20

$\begin{array}{llll}33 \mathrm{~N} & 5.1 & 4.7\end{array}$

$52 ? 4.43 .6$

$10 \mathrm{G}$

$10 \mathrm{G}$

$176 ? 4.4$

$\begin{array}{ll}10 & \mathrm{G} \\ 10 & \mathrm{G}\end{array}$

9

$34 * 5.04 .0$

$10 \mathrm{G}$

$10 \mathrm{G}$

0

$10 \mathrm{G}$

$149.362 \mathrm{~W}$
$145.757 \mathrm{E}$
57
127

$127 ? 3.3$
Yokohamo. Ajiro, kowaguchı-ko ond on Oshimo.

$\begin{array}{rr}1.1 & 31 \\ 0.6 & 18 \\ & 36 \\ 1.0 & 10 \\ 0.3 & 8 \\ 0.7 & 5 \\ 0.8 & 34 \\ 1.0 & 26 \\ 0.4 & 17 \\ 0.6 & 35 \\ 1.0 & 250\end{array}$

DODECANESE ISLANDS

EAST PAPUA NEW GUINEA REgION

CENTRAL ALASKA. LAGS-P>

CENTRAL ITALY. MD 2.7 (SSO).

SICILY

SOUTHERN I TALY

VANCOUVER ISLAND REgION

FIJI ISLANDS

LEEWARD ISLANDS. ML 2.8 (FDF). MD 2.9 (TRN).

1.0250 SOUTH OF HONSHU. JAPAN. Felt ( 11 JMA) at Utsunomiyo and ( I JMA) at Tokyo

0.7 7 IRAO

1.118 FRANCE. ML 2.9 (LDG)

1.311 ALBANIA. ML 2.7 (SKO). MD 2.5 (TTG)

0.56 ALBANIA. ML 2.0 (SKO).

1.0202 CANARY ISLANDS REGION. FeIt (V) an Tenerife and Gron Canaria.

1.511 DODECANESE ISLANDS. MD 3.7 (ATH)

0.7 7 CENTRAL ITALY. MD 2.4 (SSO).

1.09 SOUTHERN NORWAY. ML 1.8 (BER).

0.95 NEAR COAST OF NORTHERN CHILE

0.7 10 HINDU KUSH REGION

1.36 TURKEY

0.67 LEEWARD ISLANDS. MD 3.2 (FDF)

1.07 DODECANESE ISLANDS. MD 3.5 (ATH).

1.36 GREECE. ML 3.3 (ATH).

0.3 6 GREECE, ML 2.7 (ATH)

0.76 ALBANIA. ML 2.5 (OHR)

49 SOUTHERN ALASKA. LAGS-P>

$\begin{array}{rrl}1.5 & 12 & \text { SOLOMON ISLANDS } \\ 1.4 & 8 & \text { GREECE. ML } 3.2 \text { (ATH) }\end{array}$

0.95 NORTHERN TERRITORY. AUSTRALIA

1.440 NEAR COAST OF JALISCO. MEXICO

9 OFF COAST OF NORTHERN CALIFORNIA. CBRK>. ML 3.4 (BRK).

0.5 5 SOUTHERN NORWAY. MD 2.3 (BER).

0.8 14 FIJI ISLANDS REGION

1.524 MACOUARIE ISLANDS REGION

32 CENTRAL ALASKA. LAGS-P>. ML 4.8 (PMR). Felt (IV) at Galena and Ruby.

0.66 INDIA-BANGLADESH BORDER REGION

0.810 NEAR COAST OF CENTRAL CHILE

52 CENTRAL ALASKA. LAGS-P>.

45 WASHINGION. $\angle S E A>$. ML 4.4 (SEA). SIight damage (VI) at Okanagan. Felt (V) at Chelan. Caulee Dam, Malatt. Manson, Methaw and Winthrop; (IV) ot Ardenvoir.

Brewster, Bridgeport, Carltan, Canconully. Dryden,

Electric City, Elmer City. Entiat, Grand Caulee.

Hartline, Omak, Orondo. Pateras. Stehekin. Twisp. Waterville and Wenatchee.

20 CENTRAL CALIFORNIA. <BRK>. ML 3.0 (BRK). FeIt (IV) at Las Altos and $(111)$ at Mauntain View.

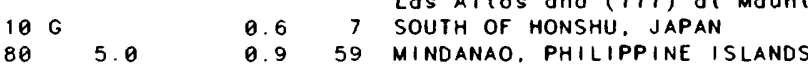

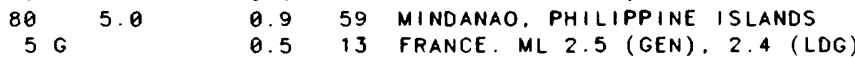

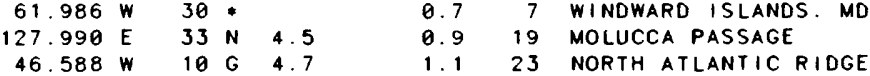

$\begin{array}{rllllll}127.990 & \mathrm{E} & 33 \mathrm{~N} & 4.5 & 0.9 & 19 & \text { MOLUCCA PASSAGE } \\ 46.588 \mathrm{~W} & 10 \mathrm{G} & 4.7 & 1.1 & 23 & \text { NORTH ATLANTIC RIDGE }\end{array}$

141.99 E $33 \mathrm{~N} \quad 0.1$ 6 WEST CAROLINE ISLANDS

$69.303 \mathrm{E} \quad 10 \mathrm{G} \quad 5.25 .1 \quad 0.8 \quad 94$ MID-INDIAN RISE

0.7

WINDWARD ISLANDS. MD 3.3 (TRN)

$\begin{array}{rllllll}127.990 & \mathrm{E} & 33 \mathrm{~N} & 4.5 & 0.9 & 19 & \text { MOLUCCA PASSAGE } \\ 46.588 \mathrm{~W} & 10 \mathrm{G} & 4.7 & 1.1 & 23 & \text { NORTH ATLANTIC RIDGE }\end{array}$

$\begin{array}{llllll}26.671 \mathrm{E} & 36 & 4.1 & 3.4 & 1.3 & 81 \\ \text { CRETE. ML } 4.4 \text { (ATH) }\end{array}$

$\begin{array}{rlllllr}69.191 & \mathrm{~W} & 33 \mathrm{~N} & & & 1.3 & 8\end{array}$

CHILE-ARGENTINA BORDER REGION

114 TURKEY. ML 4.6 (ATH). Felt at Balikesir.

33 TURKEY. ML 4.1 (ATH). Felt at Balikesir.

30 KENAI PENINSULA. ALASKA. 〈AGS-P>.

$\begin{array}{rrrr}8 & & & 30 \\ 23 & 3.1 & 1.1 & 16\end{array}$

$\begin{array}{rrrrr}7 & & & & \\ 10 & 6 & 5.0 & 4.7 & 0.6\end{array}$

13
19

ArgentinO.

19

DINDIAN RISE

CENTRAL ALASKA. LAGS-P>. ML 3.2 (PMR). Felt (1) ot

Palmer.

1.0

28

NORTH OF SVALBARD

$\begin{array}{rrrr}10 & G & 4.4 & 1.4 \\ 5 & G & & 1.4\end{array}$
1.0

27

1.1

0. 5

1. 5

1.4

0. 9

0.3
1.2

1.0

1.1
HALMAHERA

SOUTHERN ALASKA. LAGS-P>.

OFF EAST COAST OF HONSHU, JAPAN

NEAR COAST OF NICARAGUA

TURKEY

TURKEY

TIMOR SEA

ALBANIA. ML 2.4 (SKO)

TURKEY

SICHUAN PROVINCE, CHINA

BULGARIA

35 CENTRAL ALASKA. LAGS-P>

SOUTH OF HONSHU, JAPAN

SOUTHERN NORWAY. MD 1.3 (BER)

TURKEY

12 OFF COAST OF SOUTHEASTERN ALASKA. 〈AGS-P>

8 GREECE-ALBANIA BORDER REGION

6 CENTRAL ALASKA. ¿AGS-P>

6 EAST PAPUA NEW GUINEA REgION 


10
10
10
10
10
10
10
10
11
11
11
11
11
11
11
11
11
11
11
11
11
11
11
11
11
11
11
11
11
$\begin{array}{lllll}20 & 19 & 21.9 & 33.221 \mathrm{~N}\end{array}$

$21 \quad 1900.5 ? 22.34$ $\begin{array}{lllll}22 & 18 & 46.1 & 23.282 \mathrm{~S}\end{array}$

$\begin{array}{llll}22 & 23 & 36.5 & 37.052 \mathrm{~N}\end{array}$ $\begin{array}{lllll}22 & 35 & 22.7 & 37.046 & N\end{array}$ $\begin{array}{llll}22 & 44 & 55.9 \% & 39.261 \mathrm{~N}\end{array}$ $\begin{array}{llllll}23 & 27 & 32.9 & 37.16 & \mathrm{~N}\end{array}$ $\begin{array}{llllll}00 & 36 & 15.5 & 44.305 \mathrm{~N}\end{array}$ $00 \quad 4700.98 \quad 36.923 \mathrm{~N}$

$\begin{array}{llll}01 & 24 & 26.9 & 42.856 \mathrm{~N}\end{array}$ $014245.9 \& 60.038 \mathrm{~N}$ $\begin{array}{lllllll}02 & 21 & 02 & 2 ? & 15 & 30 & N\end{array}$ $\begin{array}{lllll}02 & 47 & 44.3 & 51.828 \mathrm{~N}\end{array}$ 025214.5 ? $16.25 \mathrm{~N}$ $034910.5 ? 66.46 \mathrm{~N}$ $0432 \quad 11.0 \% \quad 37.036 \mathrm{~N}$ $05 \quad 00 \quad 11.3 \& \quad 59.748 \mathrm{~N}$ $\begin{array}{lllll}05 & 42 & 25.4 & 38.766 \mathrm{~N}\end{array}$ $054459.4 * 14.806 \mathrm{~N}$ $073553.5 * 30.389 \mathrm{~S}$ $\begin{array}{lllll}09 & 00 & 19.5 \% & 42.767 \mathrm{~N}\end{array}$ $094728.0 ? \quad 13.81 \mathrm{~N}$ $\begin{array}{llllll}11 & 40 & 32.8 \% & 44.643 \mathrm{~N}\end{array}$ $\begin{array}{lllll}12 & 18 & 02.8 & 42.453 \mathrm{~N}\end{array}$ $122449.9 * 29.265 \mathrm{~S}$ $\begin{array}{llll}12 & 34 & 11.5 ? & 37.48 \mathrm{~N}\end{array}$ $\begin{array}{llllll}13 & 09 & 29.2 \% & 43.955 \mathrm{~N}\end{array}$ $\begin{array}{llll}16 & 02 & 07.1 & 43.508 \mathrm{~N}\end{array}$

$\begin{array}{lllll}16 \quad 02 & 18.7 ? & 67.20 \mathrm{~N}\end{array}$ $\begin{array}{llll}16 & 4458.2 * 21.853 \mathrm{~S}\end{array}$ $\begin{array}{llllll}18 & 30 & 27 & 1 ? 55.24 \mathrm{~N}\end{array}$ $205351.0 \quad 8.946 \mathrm{~N}$ $223728.0 * 2.609 \mathrm{~N}$ $\begin{array}{lllll}23 & 32 & 27.1 & 37.143 \mathrm{~N}\end{array}$ $00 \quad 0205.3 * 21.316 \mathrm{~S}$ $00 \quad 40 \quad 48.5 \quad 32.260 \mathrm{~N}$ $02 \quad 3728.6 \quad 39.662 \mathrm{~N}$ 03 $58 \begin{array}{lllll}24 & 2 ? & 37.10 & \mathrm{~N}\end{array}$ $053341.6 * 20.035 \mathrm{~S}$ $05 \quad 46 \quad 32.2 \& 59.826 \mathrm{~N}$ $\begin{array}{llll}06 & 07 & 27.3 & 17.013 \mathrm{~N}\end{array}$ $06 \quad 3801.8 \quad 39.294 \mathrm{~N}$ $072811.0 * 11.877 \mathrm{~S}$ $0730 \quad 05.6 * 16.456 \mathrm{~N}$ $\begin{array}{llll}08 & 07 & 12.8 \% & 36.141 \mathrm{~N}\end{array}$ $\begin{array}{lllllllll}09 & 27 & 38 & 4 & 44.631 & N\end{array}$ $110131.2 * 39.755 \mathrm{~N}$ $114538.9 * 18.772 \mathrm{~S}$ $\begin{array}{llll}15 & 04 & 14.2 & 43.477 \mathrm{~N}\end{array}$ $1505013.43 .415 \mathrm{~N}$ $\begin{array}{llll}16 & 43 & 19.6 & 13.310 \mathrm{~N}\end{array}$ $\begin{array}{llll}16 & 59 & 51.4 \% & 44.093 \mathrm{~N}\end{array}$ $1700 \quad 11.9 * 40.270 \mathrm{~N}$ $\begin{array}{llll}18 & 12 & 13.3 * 37.501 \mathrm{~N}\end{array}$ $\begin{array}{llllll}19 & 26 & 25.3 ? & 59.85 \mathrm{~N}\end{array}$ $\begin{array}{llll}20 & 07 & 04.8 & 67.122 \mathrm{~N}\end{array}$ $210136.1 * 66.916 \mathrm{~N}$ $221554.2 * 36.067 \mathrm{~N}$ $\begin{array}{llll}22 & 25 & 25.8 & 12.952 \mathrm{~N}\end{array}$ $\begin{array}{llllll}23 & 03 & 37.8 \% & 33.440 \mathrm{~S}\end{array}$ $\begin{array}{llllll}23 & 07 & 41.5 ? & 35.05 & \mathrm{~N}\end{array}$ $\begin{array}{lllll}23 & 14 & 58.8 & 28.810 N\end{array}$ $\begin{array}{llllll}00 & 14 & 48.7 ? & 12.26 \mathrm{~N}\end{array}$ $00 \quad 43 \quad 39.9 \& 60.494 \mathrm{~N}$ $010556.6 \& 59.129 \mathrm{~N}$ $\begin{array}{lllll}01 & 10 & 03.4 & 42.548 \mathrm{~N}\end{array}$ $02 \quad 02 \quad 33.2 \& 35.040 \mathrm{~N}$ $021957.9 * 36.635 \mathrm{~N}$ 03 $1254.4 \& 66.899 \mathrm{~N}$ $\begin{array}{llll}03 & 35 & 02.8 & 50.103 \mathrm{~N}\end{array}$ $\begin{array}{llll}04 & 18 & 32.3 & 46.038 \mathrm{~N}\end{array}$ $0436 \quad 32.8 \& 59.464 \mathrm{~N}$ $044120.6 ? 52.44 \mathrm{~N}$ $\begin{array}{llll}06 & 25 & 22.0 & 39.199 \mathrm{~N}\end{array}$ $06 \quad 4230.3 \% 56.321 \mathrm{~N}$ $113914.5 * 21.400 \mathrm{~S}$ $114430.3 * 25.603 \mathrm{~N}$ $\begin{array}{llll}12 & 15 & 04.1 & 18.983\end{array}$ $123143.7 * 14.194 \mathrm{~N}$ $\begin{array}{lllll}12 & 39 & 46.1 & 41.194 \mathrm{~N}\end{array}$ $133938.1 * 7.543 \mathrm{~S}$ $\begin{array}{llll}13 & 42 & 55.4 & 15.231 \mathrm{~N}\end{array}$ $\begin{array}{llll}16 & 08 & 13.9 \% & 15.895 \mathrm{~N}\end{array}$ $\begin{array}{llllll}18 & 48 & 51.5 \% & 44.572 \mathrm{~N}\end{array}$ $\begin{array}{llll}19 & 37 & 40.7 & 66.933 \mathrm{~N}\end{array}$ $210148.8 \& 38.473 \mathrm{~N}$ $\begin{array}{lllrl}21 & 35 & 39.3 & 4.154 & N\end{array}$
$75.447 \mathrm{E}$ $75.519 \mathrm{E}$

$69.14 \mathrm{E}$ $69.190 \mathrm{~W}$

$27.897 \mathrm{E}$ $27.939 \mathrm{E}$ $29.532 \mathrm{E}$ $27.93 \mathrm{E}$ $6.470 \mathrm{E}$ $121.673 \mathrm{~W}$

$17.701 \mathrm{E}$ $152.978 \mathrm{~W}$ $99.51 \mathrm{~W}$ $170.093 \mathrm{w}$ 99.75 W $13.10 \mathrm{E}$ $27.854 \mathrm{E}$ $150.626 \mathrm{~W}$ $22.173 \mathrm{E}$ $55.673 \mathrm{E}$ $72.621 \mathrm{~W}$ $19.125 \mathrm{E}$ $60.08 \mathrm{~W}$ $6.873 \mathrm{E}$ $24.265 E$ $71.465 \mathrm{~W}$ $71.39 \mathrm{E}$ $7.467 \mathrm{E}$ $147.607 \mathrm{E}$

156.62 w $139.006 \mathrm{~W}$ $162.31 \mathrm{~W}$ $39.982 \mathrm{E}$ $127.235 \mathrm{E}$ $27.900 \mathrm{E}$ $179.530 \mathrm{~W}$ $71.095 \mathrm{E}$ $27.917 \mathrm{E}$ $27.91 \mathrm{E}$ $69.348 W$ $151.033 \mathrm{~W}$ $62.308 \mathrm{~W}$ 23.653 $124.838 \mathrm{E}$ $94.238 \mathrm{~W}$ $139.879 \mathrm{E}$

$7.304 E$ $23.187 \mathrm{E}$ $168.663 \mathrm{E}$ $110.737 \mathrm{~W}$ $110.707 \mathrm{w}$ $59.536 \mathrm{w}$ $6.941 \mathrm{E}$ 25.880 E $69.614 \mathrm{E}$ $2.37 \mathrm{E}$ 156.588 $156.298 \mathrm{~W}$ $29.379 \mathrm{E}$ $87.947 \mathrm{~W}$ 70.590 $23.10 \mathrm{E}$ $142.373 \mathrm{E}$ $143.83 \mathrm{E}$ $144.790 \mathrm{~W}$ $138.110 \mathrm{~W}$ $13.271 \mathrm{E}$ $118.920 \mathrm{~W}$ $23.619 \mathrm{E}$ $156.090 \mathrm{~W}$ $105.360 \mathrm{E}$ $14.746 \mathrm{E}$ $152.671 \mathrm{~W}$ $168.51 \mathrm{~W}$ $23.702 \mathrm{E}$ $148.193 \mathrm{~W}$ $178.822 W$ $142.470 \mathrm{E}$ $167.519 \mathrm{~W}$ $146.702 \mathrm{E}$ $22.462 \mathrm{E}$ 129.519 $61.103 \mathrm{~W}$ $60.251 \mathrm{w}$ $7.130 \mathrm{E}$ $156.195 \mathrm{~W}$ $108.924 \mathrm{~W}$ $122.547 \mathrm{E}$
$33 \mathrm{~N} 3.9$ $2 * 4.840$

$10 \mathrm{G} 4.4$

10 G 3.7

$10 \mathrm{G}$

$10 \mathrm{G}$

$10 \mathrm{G}$

$10 \mathrm{G}$

$10 \mathrm{G}$

112

$33 \mathrm{~N}$

$\begin{array}{lllll}33 & \mathrm{~N} & 5.0 & 4.5 \\ 33 & \mathrm{~N} & & & \end{array}$

$10 \mathrm{G}$

$10 \mathrm{G}$

27

$10 \mathrm{G}$

$10 \mathrm{G}$

$33 \mathrm{~N}$

$10 \mathrm{G}$

$23 *$

$10 \mathrm{G}$

$10 \mathrm{G}$

$33 \mathrm{~N}$

$33 \mathrm{~N}$

$10 \mathrm{G}$

68

$5 \mathrm{G}$

$0 \mathrm{G}$

$33 \mathrm{~N} 4.3$

10 G 4.94 .7

$33 \mathrm{~N} 4.9$

$10 \mathrm{G}$

641.5 .0

$33 \mathrm{~N} 4.7$

$10 \mathrm{G}$

$10 \mathrm{G}$

113 .

39

24

$10 \mathrm{G}$

$33 \mathrm{~N} 4$

$33 N$

$33 \mathrm{~N}$

$10 \mathrm{G}$

$10 \mathrm{G}$

$112 *$

$5 \mathrm{G}$

5

$10 \mathrm{G}$

$10 \mathrm{G}$

$10 \mathrm{G}$

33
10
5

50

$10 \mathrm{G}$

$\begin{array}{ll}57 & \mathrm{D} \\ 33 & \mathrm{~N}\end{array}$

$10 \mathrm{G}$

$33 \mathrm{~N} 5$.

$33 \mathrm{~N}$

0

$10 \mathrm{G}$

12
$33 \mathrm{~N}$
10

10

$36 \mathrm{D} \quad 5.6 \quad 5.6$

$10 \mathrm{G}$

82

$33 \mathrm{~N} 4.8$

$10 \mathrm{G}$

12

$33 \mathrm{~N} \quad 4.6$

$\begin{array}{llll}33 & N & 4.9 & 4.9\end{array}$

80 .

$10 \mathrm{G}$

$33 \mathrm{~N}$

106.

$10 \mathrm{G}$

$10 \mathrm{G}$

$5 \mathrm{G}$

8

617 *

5

$0.6 \quad 10$

. 25

1. 19

1. 3

0. 8

1. 8

1.1

1. 1

0

1. 3

1.4

0.7
0.6

a. 5

1. 1

1.0

1.0

0. 4

1. 3

2. 3

1. 1

0.7 
$\begin{array}{lllllll}0 & 13 & 23 & 19 & 41.8 & 35 & 220 \mathrm{~N}\end{array}$ $\begin{array}{llllll}13 & 23 & 42 & 34.3 & 41.069 & N\end{array}$ $13 \quad 2352 \quad 21.0861 .540 \mathrm{~N}$ $140014 \quad 41.0860 .111 \mathrm{~N}$ $\begin{array}{llll}00 & 16 & 09.58 & 36.740 \mathrm{~N}\end{array}$ $20.152 \mathrm{E}$ $149.985 \mathrm{~W}$ $152.493 \mathrm{~W}$

$\begin{array}{rrrrrr}33 & N & 4 & 9 & 4 & .9 \\ 10 & G & & & & \\ 37 & & & & & \\ 78 & & & & & \\ 2 & & & & & \end{array}$
1. 3
76

ALBANIA. ML 3 . (ITG)

24 SOUTHERN ALASKA. ¿AGS-P>.

26 SOUTHERN ALASKA. 〈AGS-P>.

43 NEW MADRID. MISSOURI REGION. CSLM>. MOLG 3.7 (NEIS). 3.7 (TUL). Felt (V) at Hayti; (IV) at Canalou and Mathews; ( 111 ) at Cooter. Daniphan, Fredericktown. Grayridge, Kewanee. New Madrid, Risco, Senath and Sikeston. Alsa felt $(1 / 1)$ ot Pollard. Arkonsas and Bardwell, Hickman and Kevil, Kentucky

$14004109.0 \quad 41.051 \mathrm{~N}$

20.177 E 10 G 3.4

$1 \quad 14$ $00 \quad 5950.4 \quad 30.523$ S 178.414 w

44 G 5.96 .6

Librazhd and $(111)$ I

KERMADEC ISLANDS. MS 6.4 (BRK), 6.3 (PAS)

$M O=2.5 * 10 * 19 \mathrm{Nm}$ (PPT). Felt (iv) on Raoul. Two events about 5 seconds apart. Depth from braodband

displacement seismagrams based on second event.

$\begin{array}{llllll}01 & 34 & 54.6 ? & 30.72 & \mathrm{~S} \\ 02 & 13 & 30.07 & 44.439 & \mathrm{~N} \\ 02 & 31 & 41.9 ? & 13.32 & \mathrm{~N} \\ 04 & 28 & 37.58 & 61.214 & \mathrm{~N} \\ 06 & 32 & 09.7 & 38.209 & \mathrm{~N} \\ 06 & 34 & 20.8 * & 32.720 & \mathrm{~S} \\ 06 & 52 & 55.67 & 61.235 & \mathrm{~N} \\ 07 & 32 & 48.2 ? & 22.55 & \mathrm{~S} \\ 07 & 49 & 22.2 & 45.62 \theta & \mathrm{N} \\ 08 & 03 & 31.0 & 25.231 & \mathrm{~S} \\ 08 & 41 & 45.0 * & 16.431 & \mathrm{~N} \\ 08 & 55 & 39.4 * & 15.534 & \mathrm{~N} \\ 09 & 10 & 25.3 & 2.922 & \mathrm{~S} \\ 09 & 40 & 46.5 & 13.733 & \mathrm{~S} \\ 11 & 32 & 56.88 & 60.409 & \mathrm{~N} \\ 11 & 46 & 55.9 * & 50.844 & \mathrm{~N} \\ 11 & 58 & 30.28 & 60.204 & \mathrm{~N} \\ 12 & 32 & 09.1 ? & 41.21 & \mathrm{~N} \\ 12 & 47 & 10.98 & 11.253 & \mathrm{~N} \\ 12 & 59 & 00.1 & 39.164 & \mathrm{~N} \\ 15 & 17 & 08.68 & 8.344 & \mathrm{~N} \\ 17 & 05 & 05.1 & 7.439 & \mathrm{~S} \\ 18 & 18 & 44.2 ? & 44.25 & \mathrm{~N} \\ 19 & 12 & 39.7 ? & 16.56 & \mathrm{~N} \\ 20 & 35 & 16.0 ? & 18.69 & \mathrm{~N} \\ 21 & 35 & 19.18 & 61.701 & \mathrm{~N} \\ 21 & 58 & 16.9 & 46.268 & \mathrm{~N} \\ 22 & 53 & 36.2 & 52.671 & \mathrm{~N} \\ 23 & 13 & 13.3 . & 35.252 & \mathrm{~N} \\ 00 & 23 & 19.1 ? & 9.26 & \mathrm{~S} \\ 00 & 57 & 28.2 * & 17.648 & \mathrm{~S} \\ 01 & 10 & 29.98 & 62.653 & \mathrm{~N} \\ 01 & 34 & 37.0 * & 10.550 & \mathrm{~S} \\ 02 & 15 & 12.88 & 60.680 & \mathrm{~N} \\ 04 & 43 & 39.6 & 52.220 & \mathrm{~N} \\ 05 & 10 & 22.5 * & 5.143 & \mathrm{~S} \\ 06 & 13 & 31.3 & 18.365 & \mathrm{~S} \\ 06 & 49 & 54.3 ? & 28.19 & \mathrm{~N} \\ 08 & 33 & 27.1 & 45.097 & \mathrm{~N} \\ 09 & 22 & 42.6 & 38.311 & \mathrm{~N}\end{array}$

$177.96 w$

$7.344 \mathrm{E}$

$33 \mathrm{~N}$

59.98 W 10 G

$146.913 W \quad 3$

$15.038 \mathrm{E} \quad 10 \mathrm{G}$

$71.407 \mathrm{~W} \quad 10 \mathrm{G}$

$3.858 \mathrm{E} \quad 18 \mathrm{G}$

66.35 W $317 ?$

27.681 E 10 G

$179.971 \mathrm{E} \quad 512 \quad 5.1$

$62.481 \mathrm{~W} 10 \mathrm{G}$

$93.333 \mathrm{~W}$

$127.669 \mathrm{E}$

$66.248 \mathrm{E}$

$147.749 \mathrm{~W}$

$51.243 \mathrm{E}$

$153.170 \mathrm{~W}$

24.50 E

$85.595 \mathrm{~W}$

$23.567 \mathrm{E}$

$82.896 \mathrm{~W}$

$128.048 \mathrm{E}$

$148.84 \quad E$

$146.33 \mathrm{E}$

$146.684 \mathrm{~W}$

$13.220 \mathrm{E}$

$35.037 \mathrm{~W}$

$26.869 \mathrm{E}$

$66.70 \mathrm{E}$

$178.707 \mathrm{~W}$

$151.300 \mathrm{~W}$

$165.040 \mathrm{E}$

$152.163 \mathrm{~W}$

$157.369 \mathrm{E}$

$129.711 \mathrm{E}$
$67.937 \mathrm{~W}$

$67.937 \mathrm{~W}$
$128.43 \mathrm{E}$

$6.781 \mathrm{E}$

$21.816 \mathrm{E}$

$6937 \quad 12.5 ? 35.97 \mathrm{~N}$

$\begin{array}{lllll}09 & 48 & 38.8 & 37.749 \mathrm{~N}\end{array}$ $0950 \quad 42.5 * 37.035 \mathrm{~N}$ $095439.7 \quad 43.118 \mathrm{~N}$

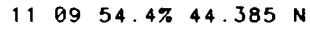
$\begin{array}{lll}11 & 25 & 32.9 * 52.968 \mathrm{~N}\end{array}$ $113931.8 * 52.848 \mathrm{~N}$ $115600.7 \% 58.282 \mathrm{~N}$ $\begin{array}{lllll}11 & 58 & 34.0 & 71.140 \mathrm{~N}\end{array}$ $121018.0 \cdot 38.255 \mathrm{~N}$ $131000.0837 .108 \mathrm{~N}$

\section{$0.81 \mathrm{~W}$}

$14.312 \mathrm{E}$

$27.842 \mathrm{E}$

$6.345 \mathrm{E}$

$167.186 \mathrm{~W}$

$167.132 \mathrm{~W}$

155. $106 \mathrm{~W}$

$6.729 \mathrm{~W}$

$21.741 \mathrm{E}$

$116.121 \mathrm{~W}$
$147.967 \mathrm{E}$

$33 \mathrm{~N}$

$33 \mathrm{~N} 5.44 .7$

$10 \mathrm{G} 5.25 .6$

15

$33 \mathrm{~N} 4.5$

145

$10 \mathrm{G}$

162

$10 \mathrm{G}$

38

$112 \quad 5.3$

$33 \mathrm{~N} 4.9$

$10 \mathrm{G}$

91.

14

$10 \mathrm{G}$

$10 \mathrm{G} \quad 5.05 .4$

$10 \mathrm{G}$

$10 \mathrm{G}$

$654 ? 4.8$

94.5 .1

85

$89 ? 4.9$

$219 ? 4.4$

203 D 4.8

$33 \mathrm{~N} 3.9$

100

$34 \quad 4.84 .5$

\section{$10 \mathrm{G}$}

$10 \mathrm{G}$

$10 \mathrm{G}$

$33 * 5.04 .0$

$10 \mathrm{G}$

$33 \mathrm{~N} \quad 4.7$

$33 \mathrm{~N} \quad 4.6$

0

10

$\begin{array}{ll}10 & G \\ 0 & 4.4\end{array}$

KERMADEC ISLANDS

$\begin{array}{rrrr}1.5 & 12 & \text { KERMADEC ISLANDS } \\ 0.4 & 7 & \text { NORTHERN ITALY. ML } 2.0 \text { (GEN) }\end{array}$

0.29 WINDWARD ISLANDS. ML 3.1 (FDF)

22 SOUTHERN ALASKA. $\angle A G S-P\rangle$.

1.17 SICILY

1.49 NEAR COAST OF CENTRAL CHILE

1.16 NORWEGIAN SEA. MD 2.0 (BER)

0.67 JUJUY PROVINCE, ARGENTINA

0.9 9 ROMANIA

1.289 SOUTH OF FIJI ISLANDS

1.46 LEEWARD ISLANDS. ML 2.9 (FDF)

1.47 NEAR COAST OF CHIAPAS, MEXICO

1.4115 CERAM SEA

1.3172 MID-INDIAN RISE

33 SOUTHERN ALASKA. 〈AGS-P>.

1 WESTERN KAZAKH SSR

29 SOUTHERN ALASKA. 〈AGS-P>.

6 GREECE-BULGARIA BORDER REGION

7 NICARAGUA. SHDC>. MD 4.4 (HDC)

0.412 AEGEAN SEA. ML 2.9 (ATH).

20 PANAMA-COSTA RICA BORDER REgION. LHDC>. MD 4.3 (HDC).

1.297 BANDA SEA

1.512 KURIL ISLANDS

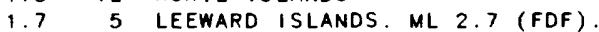

0.38 MARIANA ISLANDS

23 SOUTHERN ALASKA. CAGS-P>

$1.0 \quad 9$

AUSTRIA. MD 2.7 (LJU), 2.7 (TRI). ML 2.3 (KBA)

1.5168 NORTH ATLANTIC OCEAN

$\begin{array}{lll}1.6 & 8 & \text { CRETE. MD } 3.9 \text { (ATH) } \\ 1.0 & 6 & \text { MID-INDIAN RISE }\end{array}$

0.724 FIJI ISLANDS REGION

18 CENTRAL ALASKA. LAGS-P>.

$1.0 \quad 32$ SANTA CRUZ ISLANDS

23 SOUTHERN ALASKA. 〈AGS-P>

$1.0 \quad 50$ KAMCHATKA

0.813 BANDA SEA

1.199 BOLIVIA

1.66 RYUKYU ISLANDS

1.418 FRANCE. ML 2.5 (LDG) 2.4 (GEN)

$1.4 \quad 174$

GREECE. ML 4.5 (TTG), 4.3 (ATH). Minor damoge at Patras.

0.6 6 ALGERIA. mbLg 3.5 (MDD).

0.45 TURKEY

$\begin{array}{lrl}0.4 & 5 & \text { TURKEY } \\ 0.9 & 71 & \text { KURIL ISLANDS }\end{array}$

0. 4

1.0

0.8

(LDG)

12 FOX ISLANDS. ALEUTIAN ISLANDS

14 FOX ISLANDS. ALEUTIAN ISLANDS

21 ALASKA PENINSULA. CAGS-P>. ML 3.3 (PMR).

JAN MAYEN ISLAND REGION

7 GREECE. MD 3.3 (ATH).

48 SOUTHERN NEVADA. CDOE>. ML 4.4 (BRK). 37'06' $27.46^{\prime \prime}$ N., $116^{\circ} \theta 7^{\circ} 15.19^{*}$ W. . Surface Elev. $1365 \mathrm{~m}$. , Depth of Burial $400 \mathrm{~m}$., Shat Time 131000.087, "PALISADE," Nevada Test Site (Dept. of Energy).

$134547.0 .51 .507 \mathrm{~N}$ $153938.0836 .690 \mathrm{~N}$ $1540 \quad 03.8 .42 .590 \mathrm{~N}$ $\begin{array}{lll}16 & 03 & 32.4 \cdot 17.359 \mathrm{~N}\end{array}$ $171427.3 ? 44.64 \mathrm{~N}$ $\begin{array}{llllll}18 & 02 & 27.1 & 32.58 \mathrm{~S}\end{array}$ $\begin{array}{lllll}18 & 05 & 38.2 & 24.087 \mathrm{~N}\end{array}$ $\begin{array}{lllll}18 & 12 & 55.2 & 16.643 \mathrm{~S}\end{array}$ $\begin{array}{llll}18 & 16 & 16.8 & 1.550 \mathrm{~N}\end{array}$ $\begin{array}{llll}19 & 24 & 26.3 & 43.212 \mathrm{~N}\end{array}$ $\begin{array}{llllll}19 & 43 & 43.1 & 4.612 \mathrm{~S}\end{array}$ $\begin{array}{llllll}19 & 53 & 44.1 & 7.844 \mathrm{~N}\end{array}$ $20 \quad 15 \quad 58.6 ? 32.54 \mathrm{~S}$ $\begin{array}{lllll}20 & 36 & 41.6 & 39.279 & \mathrm{~N}\end{array}$ $\begin{array}{llll}20 & 42 & 19.38 & 61.014 \mathrm{~N}\end{array}$ $\begin{array}{lllll}20 & 48 & 29.4 & 19.299 \mathrm{~N}\end{array}$ $210258.2 * 42.971 \mathrm{~N}$ $\begin{array}{lllll}21 & 23 & 51.4 & 45.472 \mathrm{~N}\end{array}$ $\begin{array}{llll}22 & 03 & 04.5 \& & 46.282 \mathrm{~N}\end{array}$

$169.302 w$ $121.353 \mathrm{~W}$ $26.000 \mathrm{E}$ $100.774 \mathrm{~W}$ $6.70 \mathrm{E}$ $31.22 \mathrm{E}$ $122.278 \mathrm{E}$ $173.872 \mathrm{~W}$ $127.268 \mathrm{E}$ $147.879 \mathrm{E}$ $154.946 \mathrm{E}$ $126.903 \mathrm{E}$ $71.93 \mathrm{~W}$ $23.417 \mathrm{E}$ $150.429 \mathrm{~W}$ $145.290 \mathrm{E}$ 148.158 E $151.693 \mathrm{E}$ $122.102 \mathrm{~W}$ $232011.1 .49 .782 \mathrm{~N} \quad 97.520$ $\begin{array}{lllllllll}0 & 15 & 23 & 34 & 33.6 & 9.803 \mathrm{~S} & 159.531 \mathrm{E}\end{array}$

\begin{tabular}{|c|c|c|c|c|c|}
\hline 33 & $\mathbf{N}$ & 4.7 & & 1.1 & 11 \\
\hline 2 & & & & & 26 \\
\hline 10 & G & & & 1.4 & 6 \\
\hline 10 & G & & & 1.3 & 11 \\
\hline 0 & $G$ & & & 0.3 & 4 \\
\hline 10 & G & & & 1.5 & 8 \\
\hline 47 & & 5.2 & & 0.9 & 131 \\
\hline 86 & * & 5.5 & & 0.9 & 152 \\
\hline 106 & * & 5.5 & & 1.1 & 91 \\
\hline 33 & $\mathbf{N}$ & 5.2 & 4.9 & 1.0 & 169 \\
\hline 495 & & 5.1 & & 0.9 & 89 \\
\hline 33 & $\mathbf{N}$ & 4.7 & & 0.8 & 22 \\
\hline 21 & & & & 0.4 & 9 \\
\hline 22 & & & & 1.1 & 25 \\
\hline 4 & & & & & 23 \\
\hline 199 & & 4.8 & & 0.8 & 34 \\
\hline 33 & $\mathbf{N}$ & 4.6 & & 1.5 & 10 \\
\hline 33 & $\mathbf{N}$ & 5.3 & 4.9 & 1.0 & 176 \\
\hline 12 & & & & & 9 \\
\hline 33 & $\mathbf{N}$ & & & 1.2 & 9 \\
\hline 24 & G & 5.9 & 5.9 & 1.0 & 263 \\
\hline
\end{tabular}
FOX ISLANDS, ALEUTIAN ISLANDS. ML 4.4 (PMR).

26 CENTRAL CALIFORNIA. CBRK>. ML 3.4 (BRK). BULGARIA

GUERRERO, MEXICO

FRANCE. ML 2.3 (GEN)

OFF COAST OF SOUTH AFRICA

TAIWAN REGION

TONGA ISLANDS

HALMAHERA

KURIL ISLANDS. Felt (I JMA) ot Hiroo, Hokkaido.

SOLOMON ISLANDS

MINDANAO, PHILIPPINE ISLANDS

NEAR COAST OF CENTRAL CHILE

AEGEAN SEA. ML 3.1 (ATH).

SOUTHERN ALASKA. 〈AGS-P>.

MARIANA ISLANDS

OFF COAST OF HOKKAIDO, JAPAN

KURIL ISLANDS

NORTHERN CALIFORNIA. ¿BRK>. ML 3.1 (BRK).

USSR-MONGOLIA BORDER REGION

SOLOMON ISLANDS. MS 5.7 (BRK). MO=1.3*10*18 Nm (PPT). Depth from braadband displacement seismograms. 


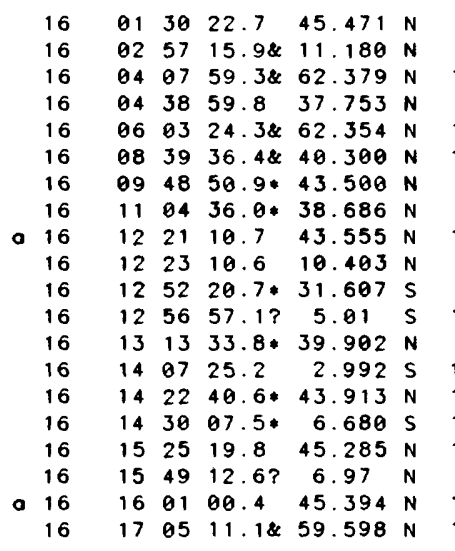

$\begin{array}{llllll}\text { a } & 16 \quad 17 \quad 22 & 52.9 & 56.329 \mathrm{~s}\end{array}$ $\begin{array}{llllll}16 & 20 & 03 & 22.7 ? & 10.27 \mathrm{~N}\end{array}$ $16203800.0 * 16.027 \mathrm{~N}$ $\begin{array}{lllllll}16 & 21 & 07 & 54.6 ? & 49.29 \quad \mathrm{~N}\end{array}$ $\begin{array}{lllll}16 & 21 & 11 & 03.7 * 76.746 \mathrm{~N}\end{array}$ $\begin{array}{llllll}16 & 21 & 14 & 50.3 \% & 37.949 \mathrm{~N}\end{array}$ $\begin{array}{lllllll}16 & 21 & 19 & 33.7 ? & 51.53 \mathrm{~N} \\ 16 & 21 & 44 & 53.58 & 61.486 \mathrm{~N}\end{array}$ $\begin{array}{lllll}16 & 22 & 15 & 30.6 * 35.943 \mathrm{~N}\end{array}$ $\begin{array}{llllll}16 & 22 & 46 & 18.8 & 42.109 \mathrm{~N}\end{array}$

$26.225 \mathrm{E}$
$85.632 \mathrm{~W}$
$142.750 \mathrm{~W}$
$21.105 \mathrm{E}$
$142.760 \mathrm{~W}$
$124.400 \mathrm{~W}$
$83.625 \mathrm{E}$
$14.357 \mathrm{E}$
$127.632 \mathrm{~W}$
$93.439 \mathrm{E}$
$71.871 \mathrm{~W}$
$147.28 \mathrm{E}$
$25.600 \mathrm{E}$
$138.317 \mathrm{E}$
$149.551 \mathrm{E}$
$130.361 \mathrm{E}$
$151.808 \mathrm{E}$
73.15
$151.772 \mathrm{~W}$
$151.699 \mathrm{E}$

$139.125 W$

$39.125 W$ $98.448 \mathrm{~W}$ $6.90 \mathrm{E}$

5. $728 E$

$1.513 \mathrm{~W}$

$16.21 \mathrm{E}$

$151.033 \mathrm{~W}$

$92.045 \mathrm{E}$

$21.339 \mathrm{E}$

$140.754 \mathrm{E}$

$168.308 \mathrm{~W}$ $152.258 \mathrm{~W}$

$6.914 \mathrm{E}$

$7.334 \mathrm{E}$

$161.29 \mathrm{E}$

$69.565 \mathrm{~W}$

90.79 W

$129.432 \mathrm{E}$

$6.876 \mathrm{E}$

$7.799 \mathrm{E}$

$6.705 \mathrm{E}$

$71.587 \mathrm{~W}$

$66.386 \mathrm{~W}$

$94.982 \mathrm{E}$

$66.419 \mathrm{~W}$

$156.238 \mathrm{E}$

$24.576 \mathrm{E}$

$7.148 \mathrm{E}$

$2.51 \mathrm{E}$

$18.782 \mathrm{E}$

109.294 W

$122.18 \mathrm{E}$

154.634

$140.005 \mathrm{E}$

$2.97 \mathrm{E}$

$159.638 \mathrm{E}$

$19.297 \mathrm{E}$

$127.025 \mathrm{E}$

$154.192 \mathrm{E}$

$156.796 \mathrm{E}$

$99.329 \mathrm{E}$

$85.531 \mathrm{~W}$

$30.091 \mathrm{E}$

$30.217 \mathrm{E}$

$70.978 \mathrm{~W}$

$62.315 \mathrm{~W}$

$20.287 \mathrm{E}$

$27.759 E$

$93.163 \mathrm{E}$

$35.391 \mathrm{E}$

$151.695 \mathrm{~W}$

$74.493 \mathrm{~W}$

$151.644 \mathrm{~W}$

$20.389 \mathrm{E}$

$127.822 \mathrm{E}$

$7.055 \mathrm{E}$

$7.047 \mathrm{E}$

$7.206 \mathrm{E}$

$120.336 \mathrm{~W}$

66.38
167.58

$167.87 \mathrm{~W}$

$26.506 \mathrm{E}$

$153.095 \mathrm{~W}$

$132.145 \mathrm{E}$

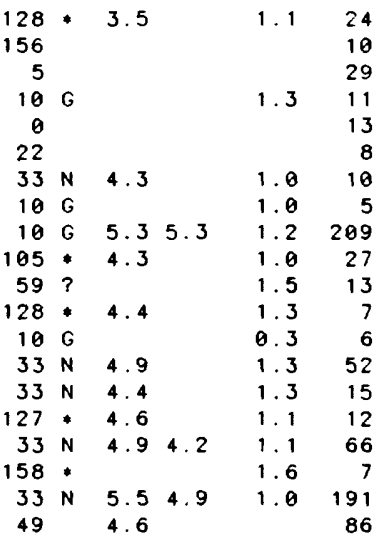

$10 \quad \mathrm{G} \quad 5.8 \quad 5.8$

27 *

$33 \mathrm{~N}$

$10 \mathrm{G}$

$10 \mathrm{G}$

10

$10 \mathrm{G}$

61

$33 \mathrm{~N} 4.2$

$10 \mathrm{G}$

$61 * 4.4$

$33 \mathrm{~N} \quad 4.9$

73

$10 \mathrm{G}$

80 .

154

$33 \mathrm{~N}$

$310 \quad 5.6 \quad 5.9$

$111 \cdot 5.0$

$10 \mathrm{G}$

$10 \mathrm{G}$

257

$59 ?$
279 ?

142

142
20.

$10 \mathrm{G}$

$10 \mathrm{G}$

106

$10 \mathrm{G} \quad 4.95 .0$

$33 \mathrm{~N} 4.5$

$10 \mathrm{G} \quad 5.05 .3$

$104 ? 4.4$

$10 \mathrm{C}$

$10 \mathrm{G}$

$33 \mathrm{~N} 4.54 .2$

$10 \mathrm{G}$

$48 ? 4.94 .0$

$33 \mathrm{~N} 5.04 .3$

488

$33 \mathrm{~N}$

163

$10 \mathrm{G}$

$10 \mathrm{C}$

$33 \mathrm{~N}$

$10 \mathrm{G}$
$10 \mathrm{G}$

$10 \mathrm{G}$

$33 \mathrm{~N} 1$

$10 \mathrm{G}$

59

$94 * 4.4$
$160 * 4.8$

72

$10 \mathrm{G}$

140 ?

$10 \mathrm{G}$

106

$10 \mathrm{G}$

$5 \mathrm{G}$
$10 \mathrm{G}$

$\begin{array}{lll}10 & G & \\ 33 & N & 4.8 \\ 33 & N & 4.7\end{array}$

$33 \mathrm{~N}$

124

$53 \cdot 4.2$

ROMANIA

NICARAGUA. LHDC>. MD 4.3 (HDC)

CENTRAL ALASKA. 〈AGS-P>. ML 3.9 (PMR)

SOUTHERN GREECE. ML 3.5 (ATH).

3 CENTRAL ALASKA. LAGS-P>.

NEAR COAST OF NORTHERN CALIF. ¿BRK>. ML 2.6 (BRK).

NORTHERN XINJIANG, CHINA

SICILY

OFF COAST OF OREGON

ANDAMAN ISLANDS REGION

NEAR COAST OF CENTRAL CHILE

EAST PAPUA NEW gUINEA REgION

AEGEAN SEA. MD 3.0 (ATH).

CERAM

KURIL ISLANDS REgION

BANDA SEA

6 KURIL ISLANDS

NORTHERN COLOMBIA

KURIL ISLANDS

6 KENAI PENINSULA, ALASKa. LAGS-P>. ML 4.3 (PMR). Felt (IV) at Homer

1.1118 SOUTH PACIFIC CORDILLERA. Mo $=2.0 * 10 * 18 \mathrm{Nm}$ (PPT)

1.010 TRINIDAD. MD 3.5 (TRN).

1.111 NEAR COAST OF GUERRERO, MEXICO

1.25 GERMANY

1.214 SVALBARD REGION

0.45 SPAIN. MbLg 2.8 (MDD)

0.68 POLAND. ML 3.7 (VKA).

22 SOUTHERN ALASKA. 〈AGS-P>.

1.515 OINGHAI PROVINCE, CHINA

1.57 YUgOSLAVIA. ML 2.3 (SKO)

1.430 NEAR EAST COAST OF HONSHU, JAPAN. Felt (I JMA) at Ut sunomiyo.

1.0103 FOX ISLANDS, ALEUTIAN ISLANDS

23 SOUTHERN ALASKA. 〈AGS-P>.

0.415 FRANCE. ML 2.3 (GEN). 2.0 (LOG).

0.25 NORTHERN ITALY. ML 1.8 (GEN).

6 SOLOMON ISLANDS

0.9 12 PERU-BOLIVIA BORDER REGION

1.311 NEAR COAST OF GUATEMALA

1.1373 EASTERN USSR. MS 5.8 (BRK).

1.042 BANDA SEA

0.1 8 FRANCE. ML 2.5 (GEN).

1.05 NORTHERN ITALY. ML 1.8 (GEN).

0.210 FRANCE. ML 2.2 (GEN).

1. 11 NEAR COAST OF CENTRAL CHILE

1.22 JUJUY PROVINCE, ARGENTINA

1.421 NICOBAR ISLANOS REGION

1.513 SOUTHERN BOLIVIA

1.036 SOLOMON ISLANDS

0.67 BULGARIA 


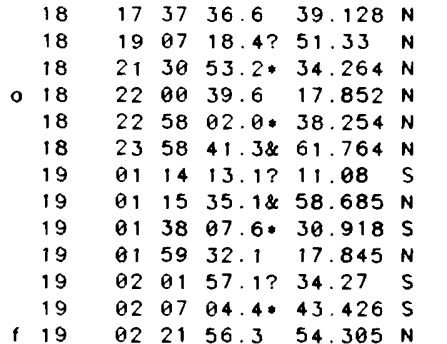

$21.921 \mathrm{E}$

$\begin{array}{rl}176.60 & W \\ 26.773 & E\end{array}$

$147.009 \mathrm{E}$

$23.142 \mathrm{E}$

$151.673 \mathrm{~W}$

$166.99 \mathrm{E}$

$155.965 \mathrm{~W}$

$68.977 W$

$146.940 \mathrm{E}$

$71.56 \mathrm{~W}$

$92.120 \mathrm{E}$

022156.3
$10 \mathrm{G}$

$33 \mathrm{~N} 47$

$93 ? 3.4$

$56 \cdot 5.55 .1$

$10 \mathrm{G}$

99

$75 ? 4.4$

156

$10 G$

$66 *$

$10 \mathrm{G} 5.0$

104 G 6.1
1.320 GREECE. MD 3.5 (ATH)

158 ANDREANOF ISLANDS, ALEUTIAN IS. Felt on Adok.

1. 1 13 CRETE

$\begin{array}{lrl}1.1 & 13 & \text { CRETE } \\ 1.1 & 162 & \text { MARIANA ISLANDS REGION }\end{array}$

15162 MARIANA ISLANDS REGION

$\begin{array}{lll} & 39 & \text { SOUTHERN ALASKA. } \\ 1.3 & 22 & \text { SANTA CRUZ ISLANOS }\end{array}$

ALASKA PENINSULA. 〈AGS-P〉.

1.310 SAN JUAN PROVINCE, ARGENTINA

1.0 38 MARIANA ISLANDS

0.7 8 NEAR COAST OF CENTRAL CHILE

0.923 SOUTHEAST INDIAN RISE

1.0 611 FOX ISLANDS, ALEUTIAN ISLANDS, mb 5.4 (BRK)

$M O=3.0 * 10 * 18 \mathrm{Nm}$ (PPT). Felt (V) on Unolosko ond Akuton and (IV) ot Cold Boy and Folse Pass. Depth from broodbond displacement seismogroms.

$\begin{array}{llllll}02 & 43 & 43.4 \% & 44.734 \mathrm{~N}\end{array}$ $02 \quad 45 \quad 37.0 * 17.776 \mathrm{~S}$ $\begin{array}{lllll}02 & 46 & 47.9 & 1.710 \mathrm{~s}\end{array}$ $033408.130 .094 \mathrm{~N}$ $\begin{array}{lllll}05 & 49 & 36.4 & 15.672 \mathrm{~N}\end{array}$ $\begin{array}{llll}06 & 23 & 20.7 & 40.162 \mathrm{~N}\end{array}$

$7.239 E-10$

168.128 E 10

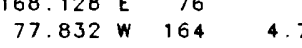

99.568 E $\quad 33 \mathrm{~N} \quad 4.8$

$\begin{array}{rlll}60.432 & W & 10 \mathrm{G} & \\ 141.924 \mathrm{E} & 76 & 4.5\end{array}$

$\begin{array}{llll}07 & 14 & 07.2 & 42.068 \mathrm{~N}\end{array}$ $101041.3 ? 45.62 \mathrm{~N}$ $1016 \quad 05.0$ ? $18.97 \mathrm{~N}$ $1022 \quad 12.0 .39 .112 \mathrm{~N}$ $104723.1836 .808 \mathrm{~N}$ $115006.9 * 2.306 \mathrm{~N}$

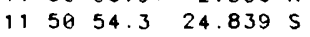
122058.2 ? $23.20 \mathrm{~N}$ $123650.5 * 34.122 \mathrm{~S}$ $125141.1 * 4.102 \mathrm{~S}$ $\begin{array}{llll}13 & 42 & 17.9 & 10.088 \mathrm{~N}\end{array}$

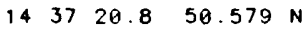
$161054.5 * 0.170 \mathrm{~N}$ $\begin{array}{llll}16 & 28 & 49.1 ? 66.99 \mathrm{~N}\end{array}$ $\begin{array}{llll}18 & 00 & 37.9 & 36.882 \mathrm{~N}\end{array}$ $\begin{array}{lllll}18 & 47 & 41.7 & 5.464 \mathrm{~N}\end{array}$ $\begin{array}{lllll}19 & 09 & 12.1 & 39.611 \mathrm{~N}\end{array}$ $\begin{array}{lllll}19 & 37 & 45.5 & 8.489 & 5\end{array}$ $\begin{array}{llllll}21 & 43 & 25.4 ? & 29.25 & \mathrm{~S}\end{array}$ $\begin{array}{lllll}22 & 20 & 39.78 & 59.580 \mathrm{~N}\end{array}$ $\begin{array}{lllll}22 & 21 & 40.2 & 42.749 \mathrm{~N}\end{array}$ $2310 \quad 88.6 \cdot 37.191 \mathrm{~N}$ $\begin{array}{lllll}00 & 19 & 10.8 & 40.681 \mathrm{~N}\end{array}$ $012804.2 * 36.848 \mathrm{~N}$ 05 $59 \begin{array}{llll}057 & 30 & 20.0095 & 5\end{array}$ $\begin{array}{lllll}07 & 10 & 17.9 & 40.221 \mathrm{~N}\end{array}$ $081432.1 ? .32 .12 \mathrm{~S}$ $08 \quad 1553.7 * 43.438 \mathrm{~N}$ $085726.8 \% 36.578 \mathrm{~N}$ $104629.3 * 7.158 \mathrm{~s}$ $\begin{array}{llllll}10 & 50 & 29.6 \% & 43.971 \mathrm{~N}\end{array}$ $\begin{array}{llllll}12 & 07 & 39.8 & 38.741 & \mathrm{~N}\end{array}$ $\begin{array}{llllll}12 & 28 & 43 & 1 & 23.333 & \mathrm{~N}\end{array}$ $\begin{array}{llll}13 & 02 & 02.3 & 36.485\end{array}$

$130839.7 * 17.076 \mathrm{~N}$ $\begin{array}{lllll}14 & 18 & 20.9 & 34.996 \mathrm{~N}\end{array}$ $152850.4 * 44.551 \mathrm{~N}$ $\begin{array}{llll}15 & 35 & 41.5 & 6.727 \mathrm{~s}\end{array}$

$\begin{array}{llllll}16 & 01 & 43.6 & 30.508 \mathrm{~S}\end{array}$

19.167 E 10 G

6.31 E $10 \mathrm{C}$

$66.79 \mathrm{~W} 10 \mathrm{G}$

23.389 E $10 \mathrm{C}$

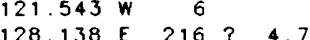

$70.019 \mathrm{~W} \quad 52 \mathrm{D} \quad 5.5$

141.59 E $33 \mathrm{~N} \quad 4.6$

$70.666 \mathrm{~W} 71$

141.847 E $109 \cdot 4.7$

$93.047 \mathrm{E} \quad 33 \mathrm{~N} \quad 4.6$

$5.538 \mathrm{E} \quad 14$

122.006 E $217 ? 4.6$

$\begin{array}{llrll}156.24 & W & 5 & \\ 23.076 & E & 89 & & 4.1\end{array}$

$127.422 \mathrm{E} \quad 33 \mathrm{~N} \quad 4.9$

27.766 E $10 \mathrm{G}$

$114.634 \mathrm{E} 141 * 4.9$

$72.76 \mathrm{~W} 33 \mathrm{~N}$

$150.682 W$

$13.006 \mathrm{E}$

$28.033 \mathrm{E}$

$23.355 \mathrm{E}$

$26.279 \mathrm{E}$

$169.268 \mathrm{E}$

$63.765 \mathrm{E}$

$70.16 \mathrm{~W}$

$12.980 \mathrm{E}$

121.212 W

$151.223 \mathrm{E}$

$8.023 \mathrm{E}$

$23.489 \mathrm{E}$

$\begin{array}{rrrrr}125.822 & E & 33 & N & 5.1 \\ 70.837 & E & 213 & & 4.8\end{array}$

120.778 E $33 N \quad 4.8$

29.327 E $10 \mathrm{G}$

141.360 E $227 ? 4.2$

$75.752 \mathrm{~W} \quad 33 \mathrm{~N} 5.0$

$178.270 W \quad 29 \quad 6 \quad 5.75 .9$

$\begin{array}{llll}16 & 35 & 05.1 & 43.645 \mathrm{~N}\end{array}$

$127.441 \mathrm{~W}$

$10 \mathrm{G} \quad 4.3$

$1636 \quad 48.2 * 36.263 \mathrm{~N}$

$\begin{array}{llllll}16 & 44 & 13.6 \% & 15.711 \mathrm{~N}\end{array}$

$\begin{array}{llll}16 & 58 & 05.8 & 17.274 \mathrm{~N}\end{array}$

$175904.6860 .321 \mathrm{~N}$

$\begin{array}{lllll}18 & 09 & 27.5 & 11.954 \mathrm{~N}\end{array}$

$\begin{array}{llll}20 & 44 & 02.2 \quad 39.553 \mathrm{~N}\end{array}$

$213723.0 .58 .169 \mathrm{~N}$ $2140 \quad 00.8 ? \quad 9.68$ $\begin{array}{lllll}22 & 03 & 19.2 & 53.636 \mathrm{~N}\end{array}$ $\begin{array}{lllll}22 & 49 & 04.3 & 42.574 \mathrm{~N}\end{array}$ $011408.3 ? 41.69 \mathrm{~N}$ $015557.3 ? 22.08 \mathrm{~S}$ 02 i5 14.3\& $36.200 \mathrm{~N}$

$\begin{array}{lllll}02 & 15 & 41.0 & 45.497 \mathrm{~N}\end{array}$ $\begin{array}{llll}02 & 57 & 08.2 & 35.270 \mathrm{~N}\end{array}$

$03 \quad 35 \quad 13.7 ? \quad 20.34 \mathrm{~s}$ $\begin{array}{lllll}03 & 40 & 53.9 & 44.478 & N\end{array}$ $\begin{array}{lllllll}03 & 51 & 10.6 ? & 40.01 \mathrm{~N}\end{array}$ $0353 \quad 38.8 \quad 38.282 \mathrm{~N}$ $\begin{array}{lllll}04 & 03 & 42.2 & 31.111 \mathrm{~S}\end{array}$ $042203.5 \& 62.918 \mathrm{~N}$ $043941.3 ? \quad 16.15 \mathrm{~N}$ $\begin{array}{lllll}04 & 46 & 22.0 \% & 16.206 \mathrm{~N}\end{array}$ $06 \quad 2118.7 * 31.277 \mathrm{~s}$
41. 391

$62.280 w$

$151.025 \mathrm{~W}$

$57.817 \mathrm{E}$

40.172E

$32.147 w$

$124.44 \mathrm{E}$

$169.338 \mathrm{E}$

$13.146 \mathrm{E}$

$12.86 \quad E$

$68.83 \mathrm{~W}$

$120.300 w$

$26.359 \mathrm{E}$ $136.886 \mathrm{E}$

$174.99 w$

$10.890 \mathrm{E}$

$27.46 \mathrm{E}$

$21.679 \mathrm{E}$

$177.801 \mathrm{~W}$

$151.295 \mathrm{~W}$

$60.44 W$

$60.559 w$

$177.635 \mathrm{~W}$ $\begin{array}{ll}10 & 6 \\ 28 & 4.7\end{array}$

$10 \quad G$

$10 \mathrm{G}$

44

$19 G 4.7-1.2$

$\begin{array}{llllll}38 & 5.0 & 5.1 & 1.2 & 26\end{array}$

$10 \quad 6 \quad 4.3$

$128 ? 4.5$

$\begin{array}{llll}33 \mathrm{~N} & 4.7 & 4.6\end{array}$

$10 \mathrm{G}$

$10 \mathrm{G}$

$33 \mathrm{~N}$

$148 \cdot 4 \cdot 3$

47 .

$750 \quad 4.6$

13

$10 \mathrm{G} 3.4$

50 D 5.3

14

$10 \mathrm{G}$

$10 \mathrm{G}$

4005.04 .6
0.4 10 NORTHERN ITALY ML 2.3 (GEN).

0.915 VANUATU ISLANDS

0.767 ECUADOR

103 SICHUAN PROVINCE, CHINA

8 LEEWARD ISLANDS. ML 2.B (FDF)

1.242 NEAR EAST COAST OF HONSHU, JAPAN. FEIt (III JMA) Ot Miyako: (II JMA) ot Mita. Morioko and ofunoto.

1.17 YUGOSLAVIA. MO 2.0 (TTG)

1.16 FRANCE. ML 2.3 (GEN).

0.9 6 PUERTO RICO REGION

1.65 AEGEAN SEA. ML 2.8 (ATH)

16 CENIRAL CALIFORNIA. 〈BRK>. ML 2.9 (BRK)

1.218 HALMAHERA

1.0155 NEAR COAST OF NORTHERN CHILE. Fell (IV) at Antofagasto.

0.6 6 VOLCANO ISLANDS REGION

612 CHILE-ARGENTINA BORDER REGION

1.210 PAPUA NEW GUINEA

0.920 ANDAMAN ISLANDS REGION

0.4 7 BELGIUM. MO 2.1 (DOU)

21 MINAHASSA PENINSULA

0. 3 ALASKA

1.3106 SOUTHERN GREECE

$\begin{array}{ll}1 & 28 \\ 1 & 8\end{array}$

1.28

PHILIPPINE ISLANDS REGION

TURKEY

BALI ISLAND REgION

OFF COAST OF CENTRAL CHILE

EENINSULA, ALASKA. LAGS-P>.

CENTRAL ITALY. MD 2.2 (SSO).

5 TURKEY

7 DODECANESE ISLANDS. MD 3.4 (ATH).

VANUATU ISLANDS

4 UZBEK SSR. FeIt (111) at GaZIi

5 CENTRAL I TALY

5 CENTRAL CALIFORNIA. <BRK>. ML 2.8 (BRK).

5 NEW BRITAIN REGION

7 CORSICA. ML 2.2 (GEN).

68 SOUTHWESTERN RYUKYU ISLANDS

0.9164 HINDU KUSH REGION. Felt (111) at Ishkashim, Khorag and Dushanbe; (11) ot Toshkent, USSR.

1.527 LUZON, PHILIPPINE ISLANDS

0.8 9 EASTERN MEDITERRANEAN SEA

0.922 HOKKAIDO, JAPAN REGION

1.342 NORTHERN PERU

1.3335 KERMADEC ISLANDS MS 6 (BRK), 5.4 (PAS). Depth from broadband displacement seismograms.

0.431 OFF COAST OF OREGON

1.421 NEAR EAST COAST OF HONSHU, JAPAN. Felt ( 1 I JMA) at Mito and (I JMA) at Onahamo.

0.87 LEEWARD ISLANDS. ML 2.6 (FDF)

0.718 LEEWARD ISLANDS. ML 4.0 (FDF)

36 KENAI PENINSULA. ALASKA. 〈AGS-P>

ARABIAN SEA

TURKEY. Twenty hauses were damaged and several cattle killed in the Erzincan orea.

1.19 NORTH ATLANTIC OCEAN

1.410 TIMOR

1.0 57 KOMANDORSKY ISLANDS REGION

0.311 CENTRAL ITALY. MD 2.6 (SSO)

0.1 4 SOUTHERN ITALY

D 8 NORTHERN CHILE

14 CENTRAL CALIFORNIA. LBRK>. ML 2.9 (BRK). Felt (11) at COOI IngO

Q.9 65 ROMANIA

0.610 SOUTHERN HONSHU, JAPAN. MG 3.4 (JMA). Felt ( I JMA) at Nogaya

1.26 TONGA ISLANDS

1.233 NORTHERN ITALY. MD 3.3 (ROM). ML 3.1 (KBA)

Q 74 TURKEY

0.98 GREECE. MD 3.4 (ATH).

1.147 KERMADEC ISLANDS REGION

20 CENTRAL ALASKA. CAGS-P>

0.36 LEEWARD ISLANDS. ML 2.6 (FDF)

0.68 LEEWARD ISLANDS. ML 2.8 (FDF)

1.320 KERMADEC ISLANDS REGION
9. 35

15 GPEECE. MD 3.3 (ATH), ML 3.2 (SKO)

9 CHILE-ARGENTINA BORDER REGION

12 GREECE MD 3.2 (AIH) 


\begin{tabular}{|c|c|c|c|c|c|c|c|c|c|}
\hline & 21 & 06 & 47 & 41.3 & 40.395 & $\mathrm{~N}$ & 15.555 & $E$ & 11 \\
\hline & 21 & 07 & 11 & $10.4 *$ & 4.571 & $S$ & 128.641 & $E$ & 179 \\
\hline & 21 & 08 & 36 & 01.38 & 40.813 & $N$ & 124.420 & $w$ & 24 \\
\hline & 21 & 08 & 57 & $50.2 \%$ & 39.138 & $N$ & 27.580 & $E$ & 10 \\
\hline & 21 & 09 & 30 & $20.9 \pi$ & 39.199 & $\mathbf{N}$ & 27.502 & $E$ & 10 \\
\hline & 21 & 09 & 40 & 48.28 & 58.204 & $\mathbf{N}$ & 151.170 & $w$ & 65 \\
\hline & 21 & 10 & 38 & $40.3 *$ & 17.485 & $s$ & 66.535 & $E$ & 10 \\
\hline & 21 & 11 & 09 & $48.9 ?$ & 24.33 & $N$ & 121.09 & $\mathbf{E}$ & 66 \\
\hline & 21 & 11 & 26 & 17.7 & 37.085 & $N$ & 27.953 & $E$ & 10 \\
\hline & 21 & 11 & 46 & $37.5 ?$ & 39.15 & $\mathbf{N}$ & 27.61 & $E$ & 10 \\
\hline & 21 & 12 & 14 & $38.7 ?$ & 49.56 & $\mathbf{N}$ & 0. 10 & $E$ & 10 \\
\hline & 21 & 12 & 51 & $08.9 *$ & 16.782 & $N$ & 99.404 & $w$ & 33 \\
\hline a & 21 & 13 & 07 & $34.3 *$ & 15.988 & $S$ & 178.262 & $E$ & 33 \\
\hline & 21 & 13 & 55 & 51.8 & 15.106 & $N$ & 60.307 & $w$ & 29 \\
\hline & 21 & 14 & 52 & 27.3 & 40.574 & $\mathbf{N}$ & 20.830 & $E$ & 10 \\
\hline & 21 & 17 & 48 & 12.3 & 1.538 & $N$ & 99.742 & $\mathbf{E}$ & 189 \\
\hline & 21 & 17 & 54 & $27.6 *$ & 43.453 & $\mathbf{N}$ & 4.184 & $E$ & 10 \\
\hline & 21 & 18 & 41 & $31.9 *$ & 5.003 & $S$ & 144.850 & $E$ & 63 \\
\hline & 21 & 18 & 49 & $30.2 *$ & 34.935 & $N$ & 132.261 & $E$ & 10 \\
\hline $\mathbf{a}$ & 21 & 19 & 23 & 41.1 & 2.586 & $\mathbf{N}$ & 126.702 & $E$ & 58 \\
\hline a & 21 & 19 & 30 & 07.1 & 2.545 & $N$ & 126.537 & $E$ & 77 \\
\hline & 21 & 21 & 00 & 05.58 & 61.910 & $N$ & 147.432 & $w$ & 9 \\
\hline & 21 & 21 & 23 & 02.6 & 2.620 & $\mathbf{N}$ & 126.629 & $E$ & 58 \\
\hline & 21 & 21 & 44 & $06.9 *$ & 24.587 & $S$ & 70.278 & $w$ & 53 \\
\hline & 21 & 21 & 45 & $38.7 *$ & 39.042 & $N$ & 21.723 & $E$ & 10 \\
\hline$a$ & 21 & 21 & 56 & 48.6 & 17.952 & $S$ & 178.593 & $w$ & 584 \\
\hline & 21 & 22 & 02 & 52.68 & 60.089 & $N$ & 153.546 & $w$ & 170 \\
\hline & 21 & 23 & 03 & $48.3 *$ & 59.740 & $\mathbf{N}$ & 30.070 & $w$ & 10 \\
\hline & 21 & 23 & 05 & $30.4 \%$ & 39.334 & $\mathbf{N}$ & 29.063 & E & 10 \\
\hline & 21 & 23 & 29 & $19.6 ?$ & 13.80 & $\mathbf{N}$ & 60.19 & $w$ & 33 \\
\hline & 21 & 23 & 41 & 57.38 & 59.504 & $\mathbf{N}$ & 152.639 & $w$ & 75 \\
\hline & 21 & 23 & 53 & 51.4 & 36.845 & $\mathbf{N}$ & 115.931 & $w$ & 7 \\
\hline & 22 & 00 & 14 & 14.0 & 59.834 & $N$ & 29.846 & $w$ & 10 \\
\hline & 22 & 00 & 17 & $00.1 ?$ & 60.06 & $N$ & 29.78 & $w$ & 10 \\
\hline & 22 & 01 & 82 & $25.5 \%$ & 31.689 & $\mathbf{N}$ & 35.797 & $E$ & 10 \\
\hline & 22 & 01 & 14 & $27.3 *$ & 59.978 & $N$ & 29.922 & $w$ & 10 \\
\hline & 22 & 01 & 52 & 20.7 & 60.184 & $N$ & 29.384 & $w$ & 10 \\
\hline a & 22 & 02 & 02 & 04.0 & 59.802 & $\mathbf{N}$ & 29.809 & $w$ & 10 \\
\hline & 22 & 02 & 09 & $20.7 ?$ & 59.62 & $\mathbf{N}$ & 30.17 & $w$ & 10 \\
\hline & 22 & 02 & 18 & 36.1 & 59.954 & $\mathbf{N}$ & 29.736 & $w$ & 10 \\
\hline & 22 & 02 & 29 & 14.3 & 59.941 & $\mathbf{N}$ & 29.710 & $w$ & 10 \\
\hline & 22 & 02 & 36 & 45.5 & 59.776 & $\mathbf{N}$ & 29.608 & $w$ & 10 \\
\hline & 22 & 02 & 38 & 37.6 & 59.858 & $\mathbf{N}$ & 29.740 & $w$ & 10 \\
\hline & 22 & 02 & 46 & 36.7 & 59.869 & $N$ & 29.638 & $w$ & 10 \\
\hline & 22 & 02 & 49 & 31.38 & 34.940 & $\mathbf{N}$ & 116.780 & $w$ & 3 \\
\hline & 22 & 02 & 58 & 26.6 & 59.288 & $\mathbf{N}$ & 30.161 & $w$ & 10 \\
\hline & 22 & 03 & 06 & 34.8 & 36.710 & $\mathrm{~N}$ & 12.865 & $\mathbf{E}$ & 33 \\
\hline & 22 & 03 & 09 & 03.5 & 59.674 & $\mathbf{N}$ & 29.808 & $w$ & 10 \\
\hline & 22 & 03 & 12 & 54.7 & 59.949 & $\mathbf{N}$ & 29.656 & $w$ & 10 \\
\hline & 22 & 03 & 16 & 13.98 & 63.339 & $N$ & 150.253 & $w$ & 14 \\
\hline & 22 & 03 & 20 & $54.1 *$ & 59.641 & $\mathbf{N}$ & 29.868 & $w$ & 10 \\
\hline & 22 & 03 & 23 & 16.0 & 36.775 & $\mathbf{N}$ & 12.892 & $E$ & 33 \\
\hline & 22 & 03 & 31 & $08.6 ?$ & 60.06 & $\mathbf{N}$ & 29.67 & $w$ & 10 \\
\hline & 22 & 03 & 37 & $01.8 *$ & 59.420 & $\mathbf{N}$ & 30.076 & $w$ & 10 \\
\hline & 22 & 03 & 57 & $35.8 *$ & 67.007 & $\mathbf{N}$ & 156.419 & $w$ & 5 \\
\hline & 22 & 04 & 66 & 21.9 & 59.950 & $\mathbf{N}$ & 29.657 & $w$ & 10 \\
\hline & 22 & 04 & 18 & 30.3 & 66.923 & $N$ & 156.233 & $w$ & 5 \\
\hline & 22 & 04 & 20 & 53.1 & 59.655 & $\mathrm{~N}$ & 29.916 & $w$ & 10 \\
\hline & 22 & 04 & 36 & $25.5 *$ & 5.294 & $S$ & 145.771 & $E$ & 110 \\
\hline & 22 & 04 & 37 & 01.1 & 59.945 & $\mathbf{N}$ & 29.634 & $w$ & 10 \\
\hline & 22 & 04 & 42 & $41.1 *$ & 59.995 & $\mathbf{N}$ & 29.800 & $w$ & 10 \\
\hline & 22 & 04 & 45 & 34.4 & 59.855 & $N$ & 29.699 & $w$ & 10 \\
\hline & 22 & 04 & 52 & $08.2 \%$ & 40.638 & $\mathbf{N}$ & 27.626 & $E$ & 10 \\
\hline & 22 & 05 & 00 & $27.2 *$ & 59.601 & $N$ & 29.842 & $w$ & 10 \\
\hline & 22 & 05 & 15 & $48.3 *$ & 59.915 & $\mathbf{N}$ & 29.568 & $w$ & 10 \\
\hline & 22 & 05 & 25 & $18.9 *$ & 59.810 & $\mathbf{N}$ & 29.726 & $w$ & 10 \\
\hline & 22 & 05 & 41 & $41.4 *$ & 36.210 & $N$ & 139.724 & E & 10 \\
\hline & 22 & 05 & 59 & $22.7 *$ & 38.348 & $\mathbf{N}$ & 126.166 & $E$ & 33 \\
\hline & 22 & 06 & 01 & $30.4 *$ & 43.367 & $N$ & 19.186 & $E$ & 10 \\
\hline & 22 & 06 & 03 & 38.1 & 59.627 & $N$ & 29.900 & $w$ & 10 \\
\hline & 22 & 06 & 40 & 31.0 & 41.942 & $\mathbf{N}$ & 20.379 & $E$ & 10 \\
\hline & 22 & 07 & 04 & $26.5 \%$ & 18.108 & $\mathbf{N}$ & 66.750 & $w$ & 33 \\
\hline & 22 & 07 & 21 & 36.7 & 52.798 & $\mathbf{N}$ & 173.539 & $w$ & 156 \\
\hline & 22 & 08 & 07 & 34.4 & 60.009 & $N$ & 29.683 & $w$ & 10 \\
\hline & 22 & 08 & 09 & $14.1 *$ & 32.672 & $\mathbf{S}$ & 70.188 & $w$ & 121 \\
\hline & 22 & 09 & 27 & 59.9 & 59.633 & $\mathbf{N}$ & 29.937 & $w$ & 10 \\
\hline & 22 & 09 & 33 & $18.2 \&$ & 65.710 & $\mathbf{N}$ & 147.926 & $w$ & $\theta$ \\
\hline & 22 & 09 & 34 & 35.4 & 53.779 & $\mathbf{N}$ & 161.439 & $E$ & 33 \\
\hline & 22 & 09 & 41 & 21.58 & 63.512 & $\mathbf{N}$ & 147.995 & $w$ & 10 \\
\hline & 22 & 09 & 48 & $13.3 *$ & 53.863 & $\mathbf{N}$ & 161.381 & $E$ & 33 \\
\hline & 22 & 10 & 23 & 31.4 & 44.355 & $N$ & 7.300 & E & 10 \\
\hline & 22 & 10 & 33 & $01.9 \%$ & 60.633 & $\mathbf{N}$ & 6.229 & $E$ & 10 \\
\hline & 22 & 10 & 50 & 29.9 & 59.781 & $N$ & 29.876 & $w$ & 10 \\
\hline a & 22 & 11 & 08 & 06.0 & 53.597 & $\mathbf{N}$ & 161.657 & $E$ & 41 \\
\hline & 22 & 11 & 35 & $04.4 *$ & 59.740 & $N$ & 29.729 & $w$ & 10 \\
\hline & 22 & 13 & 26 & 32.3 & 54.119 & $N$ & 160.543 & $E$ & 33 \\
\hline & 22 & 14 & 19 & $39.7 ?$ & 54.02 & $N$ & 161.03 & E & 33 \\
\hline & 22 & 14 & 24 & $00.6 ?$ & 54.50 & $N$ & 159.23 & $E$ & 33 \\
\hline & 22 & 14 & 40 & $21.2 ?$ & 1.67 & $\mathbf{S}$ & 149.17 & $E$ & 33 \\
\hline
\end{tabular}




$\begin{array}{llllll}22 & 15 & 29 & 32.3 ? & 17.23 & \mathrm{~N} \\ 22 & 15 & 43 & 16.3 & 13.105 & \mathrm{~S} \\ 22 & 17 & 16 & 01.8 * 46.197 & \mathrm{~N} \\ 22 & 18 & 05 & 20.8 & 44.621 & \mathrm{~N} \\ 22 & 18 & 11 & 17.7 * 18.512 & \mathrm{~N} \\ 22 & 19 & 24 & 34.9 & 27.243 & \mathrm{~N} \\ 22 & 20 & 00 & 28.8 * & 59.877 & \mathrm{~N} \\ 22 & 20 & 16 & 18.3 & 44.023 & \mathrm{~N} \\ 22 & 20 & 24 & 12.8 * & 2.275 & \mathrm{~S} \\ 22 & 20 & 31 & 50.1 & 59.731 & \mathrm{~N} \\ 22 & 21 & 11 & 16.3 & 44.378 & \mathrm{~N} \\ 22 & 21 & 45 & 36.5 * & 59.628 & \mathrm{~N} \\ 22 & 22 & 04 & 42.7 & 33.373 & \mathrm{~N} \\ 22 & 22 & 16 & 23.28 & 59.533 & \mathrm{~N} \\ 22 & 23 & 05 & 43.2 * & 22.344 & \mathrm{~N} \\ 22 & 23 & 13 & 25.3 & 36.648 & \mathrm{~N} \\ 22 & 23 & 48 & 11.9 ? & 10.53 & \mathrm{~N} \\ 23 & 03 & 44 & 45.1 \% & 66.728 & \mathrm{~N} \\ 23 & 03 & 48 & 03.3 * & 48.565 & \mathrm{~S} \\ 23 & 04 & 31 & 58.3 & 30.532 & \mathrm{~N} \\ 23 & 04 & 53 & 02.7 \% & 41.786 & \mathrm{~N} \\ 23 & 05 & 47 & 00.2 & 45.261 & \mathrm{~N} \\ 23 & 07 & 14 & 03.5 * & 21.304 & \mathrm{~S} \\ 23 & 07 & 50 & 55.4 * & 0.666 & \mathrm{~S} \\ 23 & 09 & 28 & 48.4 ? & 18.25 & \mathrm{~N} \\ 23 & 09 & 47 & 23.2 & 59.815 & \mathrm{~N} \\ 23 & 10 & 54 & 46.3 & 52.341 \mathrm{~S}\end{array}$

61.01
167.054
16.066
7.059
147.055
87.886
29.647
7.669
$\mathrm{~W}$
$140.533 \mathrm{E}$
$29.675 \mathrm{~W}$
$7.243 \mathrm{E}$
$29.974 \mathrm{~W}$
$132.105 \mathrm{E}$
$153.176 \mathrm{~W}$
$104.465 \mathrm{E}$
$25.839 \mathrm{E}$
93.14
$13.230 \mathrm{E}$
$121.096 \mathrm{E}$
$35.177 \mathrm{E}$
$12.738 \mathrm{E}$
$110.378 \mathrm{~W}$
$67.375 \mathrm{~W}$
$123.596 \mathrm{E}$
$67.43 \mathrm{~W}$
$29.770 \mathrm{~W}$
$160.568 \mathrm{E}$

223 .

$10 \mathrm{G}$

$10 \mathrm{G}$

$69 ? 4.5$

$34 \cdot 5.0$

10 G 4.2

9

$33 \mathrm{~N} \quad 5.1 \quad 4.5$

$10 \mathrm{G} \quad 4.3$

$10 \mathrm{G}$

$10 \mathrm{G} \quad 4.3$

$33 \mathrm{~N}$

101

$33 \mathrm{~N} 4.1$

$41 \cdot 4.0$

$33 \mathrm{~N} \quad 4.3$

$10 \mathrm{G}$

$10 \mathrm{G} \quad 4.1 \quad 4.1$

$10 \mathrm{G}$

$10 \mathrm{G}$

$5 \mathrm{G}$

$33 * 4.0$

$33 \mathrm{~N}$

$10 \mathrm{G} \quad 4.5$

$10 \mathrm{G} \quad 6.4 \quad 8.2$

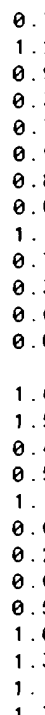

0.7
1.2
0.9
0.3
0.7
0.9
0.8
0.6
1.1
0.7
0.3
0.6
0.6
1.0
1.5
0.4
0.5
1.1
0.6
0.2
0.6
0.5
1.0
1.3
1.1
1.3

5 LEEWARD ISLANDS. ML 2.7 (FDF).

4 VANUATU I SLANDS

YUgOSLAVIA. ML 2.6 (KBA).

NORTHERN ITALY. ML 2.3 (GEN), 2.3 (LDG).

4 MARIANA ISLANDS REgION

NEPAL. Felt in eostern Nepol.

NORTH ATLANTIC OCEAN

NORTHERN ITALY. ML 2.8 (GEN), 2.8 (LDG).

NEAR N. COAST OF WEST IRIAN

NORTH ATLANTIC OCEAN

NORTHERN ITALY. ML 2.1 (GEN).

NORTH ATLANTIC OCEAN

SHIKOKU, JAPAN. Fell (11 JMA) ot UWajima.

SOUTHERN ALASKA. 〈AGS-P>.

YUNNAN PROVINCE, CHINA

DODECANESE ISLANDS. ML 4.1 (ATH).

ANDAMAN I SLANDS REGION

NORTHERN NORWAY. MD 2.8 (BER).

SOUTH OF AUSTRALIA

DEAD SEA REGION

SOUTHERN ITALY

MONTANA. ML 3.0 (BUT)

CHILE-BOLIVIA BORDER REGION

MINAHASSA PENINSULA

MONA PASSAGE

NORTH ATLANTIC OCEAN

MACOUARIE ISLANDS REGION. MS 8.0 (BRK), 7.8 (PAS) $M O=2.5 * 10 * * 21 \mathrm{Nm}$ (PPT). Fell (V) on Campbell is land. Also felt an Macquarie island. Small tsunami reported along the sautheastern caast of Tasmania and in Jervis Bay and Sydney Harbour. Australia. Camplex event, observed an braadbond displacement seismograms.

$\begin{array}{lllllll}23 & 11 & 24 & 17.5 ? & 51.37 & 5\end{array}$ $1148 \quad 28.5 \% 60.272 \mathrm{~N}$ $121117.059 .863 \mathrm{~N}$ $\begin{array}{lllll}13 & 33 & 36.2 & 44.481 \mathrm{~N}\end{array}$ $\begin{array}{llll}135359.7 & 9.305 \mathrm{~S}\end{array}$ $140935.3 * 52.087 \mathrm{~S}$ $\begin{array}{lllll}14 & 23 & 10.9 & 38.672 \mathrm{~N}\end{array}$ $\begin{array}{llll}15 & 33 & 12.2 \% & 14.796 \mathrm{~N}\end{array}$ $\begin{array}{llllll}16 & 07 & 55.1 & 52.117 & 5\end{array}$ $165833.3841 .180 \mathrm{~N}$

$\begin{array}{rrrrr}161.45 & E & 10 & G & 5.6 \\ 5.417 & E & 10 & G & \\ 29.653 & W & 10 & G & 4.6 \\ 112.187 & W & 5 & G & \\ 119.415 & E & 51 & ? & 4.9 \\ 161.100 & \text { E } & 10 & G & 5.5 \\ 30.635 & E & 10 & G & \\ 61.055 & \text { W } & 130 & G & \\ 160.741 & E & 10 & G & 5.4 \\ 121.960 & \text { W } & 7 & & \end{array}$

$1.0 \quad 16$

0.87

$\begin{array}{lr}0.6 & 31 \\ 0.5 & 8\end{array}$

1.330

$1.3 \quad 47$

0.7

0.3

1.1

$121.960 \mathrm{~W}$

$7.414 \mathrm{E} \quad 10 \mathrm{G}$

$121.950 \mathrm{~W} \quad 12$

23

- 23

23

23

23

23

23

23
23

24

24

24

$24 \quad 0253 \quad 44.1 ? 46.01 \mathrm{~N}$

$\begin{array}{llllll}24 & 02 & 16 & 57.5 & 52.503 & \mathrm{~S}\end{array}$

$\begin{array}{lllll}24 & 03 & 08 & 32.7 & 49.803 \mathrm{~N}\end{array}$

$24 \quad 05 \quad 18 \quad 57.8 * 59.936 \mathrm{~N}$

$240538 \quad 09.0861 .539 \mathrm{~N}$

$\begin{array}{lllll}24 & 05 & 58 & 53.34 & 40.358 \mathrm{~N}\end{array}$

$24 \quad 06 \quad 58 \quad 47.4 * 39.348 \mathrm{~N}$

$24 \quad 0700 \quad 09.1 * 53.478 \mathrm{~S}$

$24070441.5 * 36.681 \mathrm{~N}$

$\begin{array}{llllll}24 & 07 & 44 & 18.1 & 52.217 & \mathrm{~S}\end{array}$

$\begin{array}{lllllll}0 & 24 & 08 & 05 & 47.6 & 3.748 \mathrm{~S}\end{array}$

$24 \quad 0851 \quad 45.0 ? \quad 18.36 \quad \mathrm{~N}$

$24 \quad 123230.5 * 3.604 \mathrm{~S}$

$24 \quad 123400.1 \% 14.930 \mathrm{~N}$

f $24133114.456 .177 \mathrm{~N}$

$24 \quad 13 \quad 32 \quad 27.5 * 38.030 \mathrm{~N}$

$24 \quad 14 \quad 17 \quad 12.5 \% 44.366 \mathrm{~N}$

$24 \quad 144258.2 * 35.334 \mathrm{~N}$

$24 \quad 1451 \quad 14.7 * 55.746 \mathrm{~N}$

$\begin{array}{llllll}24 & 15 & 21 & 04.5 & 51.783 \mathrm{~S}\end{array}$

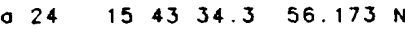

$24 \quad 16 \quad 27 \quad 51.5 ? \quad 36.90 \quad \mathrm{~N}$

$24 \quad 16 \quad 43 \quad 33.0 \quad 85.116 \mathrm{~N}$

$24 \quad 173019.8 * 52.571 \mathrm{~S}$

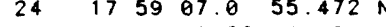

$\begin{array}{llllll}24 & 18 & 49 & 42.6 ? & 1.48 \quad \mathrm{~N}\end{array}$

$\begin{array}{lllll}24 & 19 & 13 & 33.6 & 55.493 \mathrm{~N}\end{array}$

$\begin{array}{lllll}24 & 19 & 33 & 40.6 \% & 58.740 \mathrm{~N}\end{array}$

$\begin{array}{llllll}24 & 19 & 59 & 26.3 & 55.543 \mathrm{~N}\end{array}$

24

$20 \quad 2255.7 \% 60.729 \mathrm{~N}$
$160.563 E$

$21.029 \mathrm{E}$

$27.297 \mathrm{E}$

$77.431 \mathrm{~W}$

$158.68 \mathrm{E}$

$68.915 \mathrm{~W}$

$145.70 \mathrm{E}$

$128.64 \mathrm{~W}$

$178.44 W$

$82.564 \mathrm{~W}$

$65.033 \mathrm{w}$

$14.73 \mathrm{E}$

$159.888 E$

$160.584 \mathrm{E}$

$147.641 \mathrm{E}$

$29.663 \mathrm{~W}$

$149.852 W$

$124.603 \mathrm{~W}$

$23.490 \mathrm{E}$

$160.434 E$

28. $177 \mathrm{E}$

$159.564 \mathrm{E}$

$123.256 \mathrm{E}$

$103.08 \mathrm{~W}$

$149.796 \mathrm{E}$

$60.952 \mathrm{~W}$

164.264 E

$31.917 \mathrm{E}$

$7.363 \mathrm{E}$

$136.899 \mathrm{E}$

166. $178 \mathrm{E}$

$161.425 \mathrm{E}$

$164.185 \mathrm{E}$

$20.55 \mathrm{E}$

$95.611 \mathrm{E}$

$160.083 \mathrm{E}$

$35.140 \mathrm{~W}$

$127.14 \mathrm{E}$

$35.165 \mathrm{~W}$

5. $961 \mathrm{E}$

$35.212 \mathrm{~W}$

$5.576 \mathrm{E}$
$10 \mathrm{G} \quad 5.96 .0$

20
75

$15 * 4.5$

10 G 5.55 .2

$138 ?$

$88 * 4.5$

$10 \mathrm{G}$

$88 ? 4.8$

$10 \mathrm{G}$

10

$12 ?$

$10 \mathrm{G} 5$

$10 \mathrm{G} \quad 5.75 .2$

$596 ? 4.1$

$10 \mathrm{G} \quad 4.4$

44

$14 \mathrm{G} \quad 3.7$

$10 \mathrm{G} \quad 4.6$

$10 \mathrm{G}$

$10 \mathrm{G} 5.25 .1$

$22 \quad 5.65 .0$

$33 \mathrm{~N} 4.2$

$10 \mathrm{G} \quad 4.3$

30 .

$19 \mathrm{G} 5.96 .1$

$10 \mathrm{G}$

$14 *$

8

13

22

5

29

150

29

31

27

$\begin{array}{llllll}36 & 5.5 & 5.4 & 1.0 & 277\end{array}$

$16 * 3.7$

$10 \mathrm{G} \quad 4.8$

10 G 4.95 .0

$28 ? 4.5$

$10 \mathrm{G} \quad 4.5$

$10 \mathrm{G}$

$10 \mathrm{G}$

$10 \mathrm{G}$
1. 423

0. 956

1. $2 \quad 11$

0. 610

$\begin{array}{ll}0.6 & 10 \\ 0.9 & \end{array}$

1. 2

1. 4
0. 67
MORTH OF MACOUARIE ISLAND

SOUTHERN NORWAY. MD 1.6 (BER)

NORTH ATLANTIC OCEAN

EASTERN IDAHO. ML 3.2 (BUT)

SUMBA I SLAND REGION

MACOUARIE ISLANDS REGION

TURKEY

WINDWARD ISLANDS

49 MACOUARIE ISLANDS REGION

10 NORTHERN CALIFORNIA. <BRK>. ML 3.5 (BRK). Felt (IV) at Big Bend ond Pondoso. Felt $(111)$ ot Dunsmuir and $M t$. Shosto. Also fell ot Castello ond McCloud.

9 SOUTHERN NORWAY. MD 2.0 (BER)

9 NORTHERN CALIFORNIA. <BRK>. ML 3.7 (BRK). FeIt ot Dunsmuir. MCCloud and Weed.

NORTH OF MACOUARIE ISLAND. MS 6.0 (BRK).

SOUTHERN GREECE. MD 3.8 (ATH)

DODECANESE ISLANDS. MD 3.6 (ATH)

PERU-ECUADOR BORDER REGION

NORTH OF MACOUARIE ISLAND

CHILE-BOLIVIA BORDER REGION

MARIANA ISLANDS

KERMADEC ISLANDS

PANAMA-COSTA RICA BORDER REGION. MD 4.1 (UPA) Borlovento.

NEAR COAST OF NORTHERN CALIF. ¿BRK>. ML 3.0 (BRK).

AEGEAN SEA. MD 3.2 (ATH).

7 MACOUARIE ISLANDS REGION

5 DODECANESE ISLANDS

41 MACOUARIE ISLANDS REGION

171 SULAWESI

10 NEAR COAST OF MICHOACAN, MEXICO

13 BISMARCK SEA

9 WINDWARD ISLANDS. ML 2.4 (FDF).

426 KOMANDORSKY ISLANDS REGION. MS 5.8 (BRK), 5.6 (PAS). $M O=6.0 * 10 * * 18 \mathrm{Nm}$ (PPT). Depth from broadband

displacement seismograms.

6 TURKEY

7 NORTHERN ITALY. ML 2.3 (GEN).

5 SOUTHERN HONSHU, JAPAN. MG 3.1 (JMA). Felt (I JMA) at Gifu.

$33 \mathrm{~N} \quad 4.6 \quad 1.0 \quad 21$ KOMANDORSKY ISLANDS REGION

$10 \mathrm{G} \quad 5.6 \quad 4.8 \quad 1.2 \quad 35$ NORTH OF MACOUARIE ISLAND

10 G 4.6
KOMANDORSKY ISLANDS REGION.

MEDITERRANEAN SEA. ML 3.6 (ATH).

NORTH OF SEVERNAYA ZEMLYA

MACOUARIE ISLANOS REgION

NORTH ATLANTIC OCEAN

HALMAHERA

NORTH ATLANTIC OCEAN

5 NORTH ATLANTIC OCEAN

SOUTHERN NORWAY. MD 1.7 (BER)
SOUTHERN NORWAY. MD 1.6 (BER) 


24
025
25
25
25
25
25
25
25
25
25
25
25
25
25
25
25
25
25
25
25
25
25
25
25
25
25
25
25
25
25
25
25

$234123.8 * 52.645 \mathrm{~S} \quad 160.247 \mathrm{E}$

01 $24 \quad 16.0 \% \quad 37.723 \mathrm{~N}$ $012728.9847 .373 \mathrm{~N}$ $\begin{array}{lllll}01 & 48 & 47.8 & 40.640 \mathrm{~N}\end{array}$ $03 \quad 3304.5 \quad 8.882 \mathrm{~N}$ $\begin{array}{llll}03 \quad 4756.4 & 37.796 \mathrm{~N}\end{array}$ $\begin{array}{lllll}04 & 03 & 22.7 & 38.254 \mathrm{~N}\end{array}$ $043146.4 * 31.455 \mathrm{~S}$ $\begin{array}{llll}04 & 43 & 10.7 & 52.334 \mathrm{~S}\end{array}$ $045441.5 \% \quad 0.056 \mathrm{~N}$ $045742.0 ? \quad 31.52 \mathrm{~S}$ $055651.8 * 33.449 \mathrm{~N}$ $064626.9 * 31.029 \mathrm{~S}$ $072415.5 ? 54.98 \mathrm{~S}$ $\begin{array}{llll}07 & 43 & 18.5 & 30.846 \mathrm{~N}\end{array}$ $075225.9 * 11.455 \mathrm{~S}$ 080307.9 ? $1.76 \mathrm{~N}$ $\begin{array}{llllll}08 & 06 & 32.6 & 5.850 \mathrm{~s}\end{array}$ $084537.5 * 43.535 \mathrm{~N}$ 093442.5 ? $29.77 \mathrm{~N}$ $\begin{array}{lllll}09 & 39 & 25.4 & 52.071 & \mathrm{~S}\end{array}$ $102135.1 \% 60.602 \mathrm{~N}$ $\begin{array}{lllll}10 & 35 & 47.6 ? & 2.30 \mathrm{~N}\end{array}$ $113733.7 * 52.143 \mathrm{~S}$ 114348.6 ? 39.10 N $114349.5 \quad 59.681 \mathrm{~N}$ $1151 \quad 13.7 \quad 44.515 \mathrm{~N}$ $115621.6 \quad 7.332 \mathrm{~S}$ $123449.4 * 85.603 \mathrm{~N}$ $1240 \quad 09.3 \& 35.862 \mathrm{~N}$
$4.038 w$ $122.689 w$ $21.623 \mathrm{E}$ $62.014 W$ $29.256 \mathrm{E}$ $21.792 \mathrm{E}$ $68.057 \mathrm{~W}$ $160.748 \mathrm{E}$ $78.610 \mathrm{~W}$ $179.14 \mathrm{~W}$ $104.625 \mathrm{E}$ $177.764 \mathrm{~W}$ $129.20 \mathrm{~W}$ $109.332 \mathrm{~W}$ $118.090 \mathrm{E}$ $122.86 \mathrm{E}$ $148.300 \mathrm{E}$ $7.850 \mathrm{E}$ $83.78 \mathrm{E}$ $159.931 \mathrm{E}$ $6.221 \mathrm{E}$ $126.66 \mathrm{E}$ $160.868 \mathrm{E}$ $27.58 \quad E$ $29.771 \mathrm{~W}$ $7.150 \mathrm{E}$ 128.539 $90.492 \mathrm{E}$ $120.398 \mathrm{~W}$
$10 \mathrm{G} \quad 4.8$

$10 \mathrm{G}$

20

$10 \mathrm{G}$

$33 \mathrm{~N}$

$10 \mathrm{G}$

$10 \mathrm{G}$
$115 \%$

$115 *$

$10 \mathrm{G}$

190 ?

$33 \mathrm{~N}$

$\begin{array}{lllll}33 & N & 4.8 & 4.8\end{array}$

10 G 5.25 .1

$\begin{array}{lll}5 & G & 4.6\end{array}$

$33 \mathrm{~N} 4.1$

$33 \mathrm{~N} 4.7$

1125.9

$10 \mathrm{G}$

$33 \mathrm{~N}$

$10 \mathrm{G}$

$10 \mathrm{G}$

$91 ? 4$.

10 G 5.45 .0

$10 \mathrm{G}$

$33 \mathrm{~N} \quad 4.6$

$10 \mathrm{G}$

171

$10 \mathrm{G} \quad 4.7$

10

\begin{tabular}{|c|c|c|c|c|c|c|c|c|c|c|c|c|c|}
\hline \multirow[t]{2}{*}{02} & 25 & 13 & 01 & $34.2 *$ & 36.961 & $s$ & 177.434 & E & \multicolumn{2}{|l|}{287} & \multicolumn{2}{|l|}{4.9} & 1.1 \\
\hline & 25 & 13 & 15 & $33.4 ?$ & 58.03 & $N$ & 6.39 & $E$ & $\theta$ & G & & & 0.6 \\
\hline & 25 & 13 & 28 & $16.1 ?$ & 32.85 & $S$ & 179.69 & $E$ & 169 & $?$ & 4. 9 & & 1.0 \\
\hline & 25 & 13 & 53 & $30.8 ?$ & 44.13 & $\mathbf{N}$ & 127.54 & $w$ & 10 & G & & & 0.4 \\
\hline & 25 & 15 & 19 & $43.0 ?$ & 45.12 & $\mathbf{N}$ & 7.06 & $E$ & 10 & G & & & 0.6 \\
\hline & 25 & 15 & 30 & $21.7 *$ & 1.392 & $s$ & 79.285 & $w$ & 38 & $*$ & 4.9 & & 1.2 \\
\hline & 25 & 16 & 12 & $24.8 *$ & 21.814 & $S$ & 68.482 & $w$ & 122 & $*$ & & & 1.4 \\
\hline & 25 & 16 & 58 & 10.5 & 44.519 & $N$ & 7.153 & $E$ & 10 & G & & & 0.4 \\
\hline & 25 & 17 & 46 & $10.9 *$ & 37.208 & $\mathbf{N}$ & 70.789 & $E$ & 33 & $N$ & 3.6 & & 0.8 \\
\hline & 25 & 18 & 04 & 23.9 & 38.553 & $N$ & 20.552 & $E$ & 13 & & & & 1.3 \\
\hline & 25 & 18 & 14 & $36.5 *$ & 9.404 & $\mathbf{N}$ & 126.102 & $E$ & 33 & $N$ & 5.0 & & 0.9 \\
\hline & 25 & 18 & 32 & $16.5 \%$ & 60.701 & $N$ & 5.505 & $E$ & 10 & G & & & 0.7 \\
\hline & 25 & 18 & 52 & 26.5 & 13.998 & $N$ & 91.900 & $w$ & 68 & & 4.8 & & 1.1 \\
\hline & 25 & 18 & 55 & 04.2 & 17.105 & $\mathbf{N}$ & 62.301 & $w$ & 30 & $\bullet$ & & & 0.9 \\
\hline & 25 & 19 & 01 & 37.6 & 60.126 & $N$ & 29.525 & $w$ & 10 & $G$ & 4.3 & & 1.0 \\
\hline 0 & 25 & 19 & 49 & 15.9 & 15.259 & $s$ & 172.811 & $w$ & 73 & 0 & 5.0 & & 1.1 \\
\hline & 25 & 19 & 59 & 56.8 & 44.406 & $N$ & 6.433 & $E$ & 12 & & & & 0.4 \\
\hline$a$ & 25 & 20 & 13 & 33.4 & 51.677 & $N$ & 172.128 & $E$ & 33 & $N$ & 5.3 & 4.6 & 1.0 \\
\hline & 25 & 21 & 33 & $38.2 ?$ & 15.11 & $N$ & 60.34 & $w$ & 10 & G & & & 0.3 \\
\hline a & 25 & 21 & 36 & 21.2 & 13.968 & $N$ & 91.804 & $w$ & 69 & & 5.0 & & 0.9 \\
\hline & 25 & 22 & 57 & $06.2 ?$ & 45.52 & $N$ & 3.78 & $E$ & 10 & G & & & 0.3 \\
\hline & 25 & 23 & 28 & $30.9 ?$ & 44.81 & $N$ & 6.54 & $E$ & 10 & G & & & 0.6 \\
\hline & 25 & 23 & 43 & 56.2 & 15.442 & $N$ & 61.332 & $w$ & 10 & G & & & 0.1 \\
\hline & 26 & 01 & 08 & 45.8 & 39.345 & $N$ & 75.436 & $E$ & 35 & * & 4.8 & 4.4 & 1.0 \\
\hline & 26 & 01 & 24 & 26.3 & 17.034 & $N$ & 62.282 & $w$ & 11 & & & & 1.2 \\
\hline & 26 & 01 & 54 & $16.2 *$ & 53.230 & $s$ & 159.282 & $E$ & 10 & G & 5.2 & 4.5 & 1.2 \\
\hline & 26 & 02 & 16 & 16.6 & 15.432 & $N$ & 61.299 & $w$ & 10 & G & & & 0.3 \\
\hline & 26 & 02 & 34 & $27.9 ?$ & 34.16 & $\mathrm{~N}$ & 135.18 & $\mathrm{E}$ & 10 & G & & & 0.4 \\
\hline & 26 & 03 & 54 & $02.6 \%$ & 60.153 & $N$ & 140.646 & $w$ & 4 & & & & \\
\hline & 26 & 04 & 18 & $33.8 ?$ & 53.30 & $N$ & 164.90 & $w$ & 33 & $N$ & 4.4 & & 2.1 \\
\hline & 26 & 06 & 57 & $37.8 *$ & 52.645 & $s$ & 160.150 & $E$ & 10 & G & 4. 9 & & 1.4 \\
\hline & 26 & 06 & 58 & $19.8 \%$ & 39.569 & $N$ & 34.014 & $E$ & 10 & G & & & 1.2 \\
\hline נ & 26 & 07 & 00 & 01.7 & 52.583 & $s$ & 160.106 & $E$ & 10 & G & 5.6 & 5.3 & 1.1 \\
\hline & 26 & 07 & 13 & 29.4 & 17.997 & $N$ & 61.517 & $w$ & 41 & * & & & 0.6 \\
\hline & 26 & 07 & 20 & 22.7 & 38.391 & $N$ & 25.135 & $E$ & 17 & & & & 1.0 \\
\hline & 26 & 08 & 01 & 05.0 & 38.360 & $N$ & 25.190 & $E$ & 10 & G & & & 0.7 \\
\hline & 26 & 08 & 44 & $46.1 *$ & 33.318 & $s$ & 70.566 & $w$ & 82 & $?$ & & & 1.1 \\
\hline & 26 & 09 & 08 & $10.7 *$ & 30.326 & $N$ & 109.144 & $w$ & 5 & G & & & 0.6 \\
\hline & 26 & 09 & 23 & $47.7 *$ & 15.915 & $S$ & 172.917 & $w$ & 33 & $\mathrm{~N}$ & 4.9 & 4.8 & 1.3 \\
\hline & 26 & 11 & 20 & 55.3 & 1.421 & $S$ & 119.853 & $E$ & 33 & N & 4.7 & 4.0 & 1.2 \\
\hline & 26 & 11 & 32 & $02.6 *$ & 32.336 & $\mathrm{~s}$ & 69.335 & $w$ & 33 & N & & & 1.0 \\
\hline & 26 & 11 & 45 & 29.4 & 18.537 & $N$ & 145.696 & $E$ & 197 & * & 4.8 & & 0.9 \\
\hline & 26 & 12 & 08 & 18.3 & 40.807 & $N$ & 20.705 & $\bar{E}$ & 20 & & 3.4 & & 1.2 \\
\hline & 26 & 12 & 56 & $18 \cdot 3 *$ & 51.926 & $s$ & 161.386 & $E$ & 10 & G & 5.3 & 4.9 & 1.2 \\
\hline 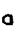 & 26 & 14 & 28 & $37.8 *$ & 4.406 & $s$ & 105.270 & $w$ & 10 & G & 4.7 & 5.2 & 1.4 \\
\hline & 26 & 16 & 09 & 20.4 & 36.088 & $N$ & 26.679 & $E$ & 144 & * & & & 0.8 \\
\hline & 26 & 18 & 02 & $07.9 \%$ & 44.504 & $N$ & 7.293 & $\bar{E}$ & 10 & G & & & 0.3 \\
\hline & 26 & 18 & 07 & 00.08 & 37.086 & $N$ & 116.055 & $w$ & 0 & & & & \\
\hline
\end{tabular}
$194830.7 * 36.579 \mathrm{~N}$ $200837.9 * 30.205 \mathrm{~N}$
17.983 E 10 71.035 E $182 ? \quad 3.6$ 50.576 E 33 N

0

1.4

1. 3

0.5

1. 5

1.4

1. 1

1. 1

0. 2

0. 9

1.0

1.0

1.0

1.0

1. 4

1. 2

1.0

0. 2

0. 2

1. 1

1. 5

0.6

1. 3

0. 6

0.6

0.3

0. 8

1. 2
20 MACOUARIE ISLANDS REGION MACOUARIE ISLANDS REGION. MS 5.4 (BRK). MO=1.25*10**18 $\mathrm{Nm}$ (PPT)

6 SPAIN mbLg 3 O (MOD)

48 WASHINGTON. $\angle S E A\rangle . C L 2.8$ (SEA)

GREECE. MD 3.3 (ATH).

VENEZUELA

TURKEY

13 GREECE. ML 3.1 (ATH).

16 SAN JUAN PROVINCE, ARGENTINA

55 MACOUARIE ISLANDS REGION

COLOMOIA-ECUADOR BORDER REGION

KERMADEC ISLANDS REGION

6 GANSU PROVINCE, CHINA. ML 3.7 (BJI).

12 KERMADEC ISLANDS REGION

10 SOUTH PACIFIC CORDILLERA

43 NORTHWESTERN MEXICO. ML 4.3 (NEIS)

8 SOUTH OF SUMBAWA ISLAND

6 minahassa peninsula

7 NEW BRITAIN REGION

9 NEAR SOUTH COAST OF FRANCE. ML 2.1 (GEN)

6 NEPAL

85 MACOUARIE ISLANDS REGION

8 SOUTHERN NORWAY. MD 1.7 (BER)

11 MOLUCCA PASSAGE

31 MACOUARIE ISLANDS REGION

4 TURKEY

44 NORTH ATLANTIC OCEAN

15 NORTHERN ITALY. ML 2.4 (GEN).

235 BANDA SEA

8 NORTH OF SEVERNAYA ZEMLYA

CENTRAL CALIFORNIA LGS) ML 3.6 (BRK), 3.8 (PAS). Felt (IV) at Shandan and Templeton: (III) at Lockwaod and Paso Robles. Also felt at Parkfield. Coalingo and Creston.

57 OFF E. COAST OF N. ISLAND, N.Z. Felt throughout North Island

7 SOUTHERN NORWAY. MD 2.3 (BER). Prabable explosion.

9 SOUTH OF KERMADEC ISLANDS

30 OFF COAST OF OREGON. CL 3.4 (SEA).

4 NORTHERN ITALY. ML 2.1 (GEN).

39 ECUADOR. Felt in central Ecuadar.

9 CHILE-BOLIVIA BORDER REGION

23 NORTHERN ITALY. ML 2.8 (LDG), 2.7 (GEN).

7 AFGHANISTAN-USSR BORDER REGION

26 GREECE. ML 3.6 (ATH).

20 MINDANAO, PHILIPPINE ISLANDS

9 SOUTHERN NORWAY. MD 1.7 (BER)

45 NEAR COAST OF GUATEMALA

9 LEEWARD ISLANDS. ML 2.8 (FDF)

25 NORTH ATLANTIC OCEAN

72 SAMOA ISLANDS REGION

21 FRANCE. ML 2.7 (LDG)

92 NEAR ISLANDS. ALEUTIAN ISLANDS

7 LEEWARD ISLANDS. ML 2.6 (FDF)

38 NEAR COAST OF GUATEMALA

4 FRANCE. ML 1.9 (LDG)

4 FRANCE. ML 2.2 (GEN)

6 LEEWARD ISLANDS. ML 2.1 (FDF).

91 SOUTHERN XINJIANG, CHINA

18 LEEWARD ISLANDS. ML 3.8 (FDF)

21 MACOUARIE ISLANDS REGION

10 LEEWARD ISLANDS. ML 2.3 (FDF)

NEAR S. COAST OF SOUTHERN HONSHU. MG 2.7 (JMA). FeIt (I JMA) at WOKOYOMO

14 SOUTHEASTERN ALASKA. LAGS-P>.

8 UNIMAK ISLAND REGION

20 MACOUARIE I SLANDS REGION

TURKEY

2 MACOUARIE ISLANDS REgION

29 LEEWARD ISLANDS. ML 4.4 (FDF).

16 AEGEAN SEA. ML 3.7 (ATH)

7 AEGEAN. SEA. ML 3.1 (ATH).

11 CHILE-ARGENTINA BORDER REGION

3 NORTHWESTERN MEXICO. ML 3.5 (NEIS)

43 SAMOA ISLANDS REGION

22 SULAWESI

8 MENDOZA PROVINCE, ARGENTINA

69 MARIANA ISLANDS

50 GREECE-ALBANIA BORDER REGION. ML 4.4 (ATH), 3.5 (TTG) Felt (IV) ot Pogradec and Malia: (III) at Korca. Albania.

27 NORTH OF MACOUARIE ISLAND

24 NORTHERN EASTER I. CORDILLERA

13 DODECANESE ISLANDS

5 NORTHERN ITALY. ML 1.6 (GEN).

28 SOUTHERN NEVADA. LDOE>. ML 3.7 (NEIS). 37 $05^{\circ} 09.12^{\circ}$ N. . 116 $113^{\circ} 18.47^{\prime \prime}$ W. Surface Elev. $1257 \mathrm{~m}$. . Depth of Burial $400 \mathrm{~m}$. Shot Time 180700.021, "TULIA." Nevado Test Site (Dept. of Energy).

1.257 YUGOSLAVIA.MD 3.8 (ATH) ML 3.7 (TTG).

0.910 AFGHANISTAN-USSR BORDER REGION

$\begin{array}{rrr}1.9 & 10 & \text { AFGHAN } \\ 1.3 & 9 & \text { IRAN }\end{array}$ 


$\begin{array}{rrrllll}26 & 20 & 33 & 37.6 & 41.857 & \mathrm{~N} \\ 26 & 22 & 19 & 16.6 \% & 38.219 & \mathrm{~N} \\ 26 & 22 & 31 & 20.7 \% & 40.664 & \mathrm{~N} \\ 27 & 00 & 16 & 20.4 & 44.798 & \mathrm{~N} \\ 27 & 00 & 43 & 47.4 & 42.511 & \mathrm{~N} \\ 27 & 02 & 03 & 10.2 ? & 45.14 & \mathrm{~N} \\ 27 & 02 & 44 & 09.8 & 13.612 & \mathrm{~N} \\ 0 & 27 & 03 & 01 & 24.9 & 55.279 & \mathrm{~S} \\ & & & & & \\ 27 & 04 & 14 & 43.0 & 29.925 & \mathrm{~N} \\ 27 & 06 & 49 & 11.6 & 40.125 & \mathrm{~N} \\ & & & & & \\ 27 & 07 & 34 & 09.68 & 57.627 & \mathrm{~N} \\ 27 & 08 & 07 & 27.48 & 57.419 & \mathrm{~N} \\ 0 & 27 & 08 & 31 & 18.7 & 3.451 & \mathrm{~S} \\ 27 & 11 & 54 & 41.4 * & 7.940 & \mathrm{~N} \\ 27 & 12 & 59 & 33.0 * & 17.129 & \mathrm{~N} \\ 27 & 13 & 39 & 47.4 \% & 45.857 & \mathrm{~N} \\ 0 & 27 & 14 & 27 & 46.4 & 50.236 & \mathrm{~S} \\ 27 & 15 & 56 & 42.9 * & 30.925 & \mathrm{~S} \\ 27 & 16 & 38 & 17.4 \% & 40.609 & \mathrm{~N} \\ 27 & 16 & 48 & 24.2 * & 16.870 & \mathrm{~S} \\ 27 & 16 & 51 & 21.5 ? & 7.57 & \mathrm{~S} \\ 27 & 17 & 18 & 46.5 \% & 42.288 & \mathrm{~N} \\ 27 & 17 & 34 & 39.2 & 46.358 & \mathrm{~N} \\ 27 & 17 & 52 & 29.4 * & 30.090 & \mathrm{~N} \\ 0 & 27 & 20 & 08 & 37.3 & 30.167 & \mathrm{~N}\end{array}$

$\begin{array}{llllll}27 & 20 & 36 & 05.2 & 30.209 & \mathrm{~N} \\ 27 & 20 & 39 & 58.5 * 30.482 & \mathrm{~N} \\ 27 & 21 & 31 & 57.2 & 29.942 & \mathrm{~N} \\ 27 & 21 & 43 & 04.48 & 63.717 & \mathrm{~N} \\ 27 & 22 & 40 & 39.5 * & 30.220 & \mathrm{~N} \\ 27 & 23 & 25 & 51.3 & 42.026 & \mathrm{~N} \\ 27 & 23 & 36 & 15.0 ? & 18.78 & \mathrm{~N} \\ 28 & 00 & 27 & 44.5 & 34.680 & \mathrm{~N}\end{array}$

$012209.1 * 15.560 \mathrm{~s}$ $015435.7 * 53.225 \mathrm{~S}$ $02 \quad 2828.1 \quad 42.920 \mathrm{~N}$ $\begin{array}{llll}02 & 55 & 19.6 & 25.053 \mathrm{~S}\end{array}$

$032345.9 * 51.200 \mathrm{~S}$ $\begin{array}{lllll}03 & 30 & 52.4 & 42.914 \mathrm{~N}\end{array}$ $034741.6 ? \quad 38.48 \quad \mathrm{~N}$ $\begin{array}{lllll}04 & 03 & 23.5 & 9.228 \mathrm{~S}\end{array}$ $\begin{array}{llllll}04 & 53 & 44.5 ? & 34.05 & \mathrm{~S}\end{array}$ $05 \quad 35 \quad 27.7 * 34.575 \mathrm{~N}$ $\begin{array}{llll}05 & 52 & 22.9 \% & 35.310 \mathrm{~N}\end{array}$ $0608 \quad 45.0 .30 .190 \mathrm{~N}$ $09 \quad 2920.0 \quad 39.349 \mathrm{~N}$ $\begin{array}{lllll}09 & 46 & 28.2 & 16.714 \mathrm{~S}\end{array}$

$\begin{array}{lllll}10 & 20 & 00.3 & 16.723 \mathrm{~S}\end{array}$ $\begin{array}{llllllll}10 & 32 & 17 & 9 ? & 46.64 & \end{array}$ $\begin{array}{llllll}11 & 25 & 21.8 & 53.50 \mathrm{~S} & \\ 11 & 51 & 15.3 & 30.198 & \end{array}$ $\begin{array}{llll}11 & 51 & 15.3 & 30.198 \\ 11 & 56 & 29.0 & 44.636\end{array}$ $\begin{array}{llll}12 & 16 & 03.2 & 6.508 \mathrm{~S}\end{array}$ $123640.6 ? 44.65 \mathrm{~N}$ 140730.9 ? $41.81 \quad \mathrm{~N}$ $\begin{array}{llll}14 & 28 & 41.3 .4 .996 & 4\end{array}$ $\begin{array}{llll}15 & 01 & 11.4 & 5.960 \mathrm{~s}\end{array}$ $\begin{array}{lllll}15 & 19 & 07.1 & 6.515 \mathrm{~S}\end{array}$ $\begin{array}{llllll}15 & 26 & 30.7 ? & 6.68 & S\end{array}$ $\begin{array}{llllll}15 & 52 & 54.2 & 51.053 & \mathrm{~s}\end{array}$ $\begin{array}{llll}16 & 28 & 43.0 & 30.151\end{array}$ $\begin{array}{llll}16 & 35 & 57.3 & 38.343 \mathrm{~N}\end{array}$ $164405.2 * 36.467 \mathrm{~N}$ $170916.8 ? 6.58 \mathrm{~S}$ $\begin{array}{llllll}17 & 25 & 46.6 ? & 44.20 \mathrm{~N}\end{array}$ 182208.7 ? $6.43 \mathrm{~S}$ $\begin{array}{lllll}18 & 29 & 27.7 \% & 45.192 & N\end{array}$ $\begin{array}{llll}1843 & 17.2 * 7.032\end{array}$ $\begin{array}{llll}20 & 01 & 57.7 ? & 39.80 \mathrm{~N}\end{array}$ $200505.0 * 40.130 \mathrm{~N}$ $\begin{array}{lllll}20 & 19 & 45.9 & 44.644 \mathrm{~N}\end{array}$ $2051141.539 .043 \mathrm{~N}$ $210125.6 * 0.384 \mathrm{~N}$ $\begin{array}{llll}21 & 03 & 06.7 & 18.635\end{array}$ $213448.5858 .098 \mathrm{~N}$ $\begin{array}{lllll}22 & 29 & 11.6 \% & 31.485 & N\end{array}$ $00 \quad 2728.7 ? \quad 1.56 \quad \mathrm{~N}$ $\begin{array}{lllll}01 & 08 & 13.2 & 44.717 \mathrm{~N}\end{array}$ $0113040 ? 17.37 \mathrm{~N}$ $01 \quad 1505.7 \quad 36.058 \mathrm{~N}$ $\begin{array}{llll}01 & 29 & 38.0 & 6.667 \mathrm{~S}\end{array}$ $\begin{array}{lllll}02 & 12 & 27.8 & 48.914 & 5\end{array}$ $\begin{array}{lllll}03 & 42 & 34.3 & 6.499 & 5\end{array}$ $040252.4 \quad 28.066 \mathrm{~N}$
$20.611 \mathrm{E}$ $15.079 \mathrm{E}$ $29.091 \mathrm{E}$ $6.761 \mathrm{E}$ $18.496 \mathrm{E}$ $14.58 \mathrm{E}$ $120.908 \mathrm{E}$ $133.192 \mathrm{~W}$

$99.347 \mathrm{E}$ $19.773 \mathrm{E}$

$\begin{array}{rlrlll}154.094 & W & 40 & 4.4 & \\ 154.539 & W & 23 & & & \\ 138.855 & E & 49 & & 5.4 & 4.4 \\ 82.859 & W & 34 & ? & 4.1 & \\ 62.319 & W & 32 & . & & \\ 0.361 & W & 10 & G & & \\ 158.073 & E & 10 & G & 5.5 & 4.9 \\ 71.605 & W & 128 & ? & 4.2 & \\ 14.987 & E & 10 & G & & \\ 177.211 & W & 33 & N & 4.9 & 5.0 \\ 129.56 & E & 245 & ? & 4.4 & \\ 18.722 & E & 10 & G & & \\ 12.898 & E & 10 & G & & \\ 51.005 & E & 33 & N & 4.8 & \\ 50.921 & E & 31 & & 5.6 & 5.8\end{array}$
$50.968 \mathrm{E}$

$50.803 \mathrm{E}$ $148.832 \mathrm{~W}$ $50.685 \mathrm{E}$ $19.780 \mathrm{E}$ $67.59 \mathrm{~W}$ $135.586 \mathrm{E}$ $\begin{array}{llll}10 & 33 & 44.5 .39 .516 \mathrm{~N}\end{array}$
$73.918 W 110 ? 4.6$

$160.012 \mathrm{E} \quad 10 \mathrm{G} \quad 4.4$

$17.942 \mathrm{E}$ $130.781 \mathrm{E}$

$159.341 \mathrm{E}$ $17.997 \mathrm{E}$ $23.89 \mathrm{E}$ $158.879 \mathrm{E}$ 68.25 $141.583 \mathrm{E}$ $118.888 \mathrm{E}$ $50.837 \mathrm{E}$

$23.272 \mathrm{E}$ $173.278 \mathrm{~W}$

173.247

$0.49 w$ $117.833 w$ $158.35 \mathrm{E}$

$50.868 \mathrm{E}$

$7.297 \mathrm{E}$ $147.747 \mathrm{E}$ $6.97 \mathrm{E}$

$12.74 \mathrm{E}$ $151.298 \mathrm{E}$ $147.799 \mathrm{E}$ $147.75 \mathrm{E}$ $159.682 \mathrm{E}$ $50.647 \mathrm{E}$ $22.721 \mathrm{E}$ $38.351 \mathrm{E}$ $147.66 \mathrm{E}$ $148.62 \mathrm{E}$ $147.64 \mathrm{E}$

$7.469 \mathrm{E}$ $147.721 \mathrm{E}$

$20.98 \mathrm{E}$ $20.665 \mathrm{E}$

$7.300 \mathrm{E}$ $21.748 \mathrm{E}$ $28.914 \mathrm{~W}$ $177.913 \mathrm{~W}$ $143.172 \mathrm{~W}$ $36.070 \mathrm{E}$ $92.05 \mathrm{~W}$ $7.277 \mathrm{E}$ $61.85 \mathrm{~W}$ $27.455 \mathrm{E}$ $147.821 \mathrm{E}$ $121.346 \mathrm{E}$ $147.730 \mathrm{E}$ $130.590 \mathrm{E}$

$\begin{array}{rr}10 & G \quad 4.4\end{array}$

$10 \mathrm{G}$

$10 \mathrm{G}$

$10 \mathrm{G} \quad 5.1$

$10 \mathrm{G}$

$41 \cdot 4.7$

$33 \mathrm{~N}$

$33 \mathrm{~N} 3.9$

$10 \mathrm{G}$

50 D 5.

$10 \mathrm{G}$

$5 \mathrm{G}$

$10 \mathrm{G} 4.2$

$28 \cdot 4.3$

14

$10 \mathrm{G}$

$10 \mathrm{G}$

$102 * 4.7$

$92 \cdot 5.0$

$80 ? 4.5$

$10 G 5.3$

$65 \cdot 4.7$

$10 \mathrm{G}$

$10 \mathrm{G} \quad 3.9$

$33 \mathrm{~N} 5.0$

$10 \mathrm{G}$

$10 \mathrm{G}$

$10 \mathrm{G}$

11

$10 \mathrm{G}$

570

$10 \mathrm{G}$

$10 \mathrm{G}$

$10 \mathrm{G}$

$33 \mathrm{~N}$

33 . $148.435 \mathrm{E}$

$10 \mathrm{G}$

$10 \mathrm{G}$

$10 \mathrm{G}$

$10 \mathrm{C}$

$10 \mathrm{G}$

$175 \quad 4.6$

$10 \mathrm{G} \quad 5.55 .7$

$10 \mathrm{G} 5.85 .1$

$\begin{array}{llll}10 & G & 4.7 & 4.3\end{array}$

$\begin{array}{llll}35 & G & 5.7 & 5.5\end{array}$

$46 \cdot 4.64 .4$

$33 \quad 5.55 .5$

$\begin{array}{llll}56 & ? & 4.5 & 3.9\end{array}$

$\begin{array}{llll}33 & N & 4.8 & 3.7\end{array}$

$33 \mathrm{~N} \quad 4.7 \quad 4.4$

$10 G \quad 4.6 \quad 4.8$

$\begin{array}{lllll}12 & D & 4.7 & 4.3\end{array}$

$10 \mathrm{G} 5.25 .2$

$40 \quad 5.4 \quad 4.8$
0.76 ALBANIA. ML 2.5 (SKO)

0.98 SICILY

086 TURKEY

0.37 FRANCE. ML 1.7 (GEN)

0.911 YUGOSLAVIA. MD 2.5 (TTG)

1.26 YUGOSLAVIA. MD 2.6 (LJU)

0.952 MINDORO. PHILIPPINE ISLANDS

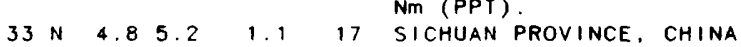

$183.5 \quad 1.5 \quad 40$ ALBANIA. MD 3.9 (ATH). Felt (IV) at Borsh, Dhermi, Carraj, KUC and Himare.

1 KODIAK ISLAND REGION. LAGS-P>. ML 3.3 (PMR).

8 KODIAK ISLAND REGION. 〈AGS-P>.

1.1145 WEST IRIAN

0.822 SOUTH OF PANAMA

6 LEEWARD ISLANDS. MD 2.9 (TRN)

1.311 FRANCE. ML 2.8 (LDG)

1.1 112 NORTH OF MACOUARIE ISLAND

0.712 NEAR COAST OF CENTRAL CHILE

0.5 SOUTHERN ITALY

1.022 FIJI ISLANDS REGION

9 BANDA SEA

6 YUGOSLAVIA. MD 2.0 (TTG)

0. 5

0. $8 \quad 9$

1.0405

$\begin{array}{rrrrrr}28 & & 4.6 & & 1.0 & 87 \\ 33 & N & 4.5 & & 1.5 & 19 \\ 33 & N & 4.5 & & 1.0 & 34 \\ 107 & & & & & 16 \\ 33 N & 4.1 & 3.7 & 1.8 & 8 \\ 10 & G & & & 0.7 & 13 \\ 25 & * & & & 0.3 & 6 \\ 7 & & & 1.0 & 8\end{array}$

$48 * 4.64 .4$

$26 \quad 5.0 \quad 4.8$

NORTHERN ITALY. ML 3.3 (FUR), 3.2 (LDG), 2.8 (KBA).

IRAN

IRAN. MS 5.4 (BRK). Seventeen people injured, several hauses damaged and about 100 cattle killed in the Do Gabadan area. Felt in the Bushehr-Shiraz orea and at Mamasani. Also felt in Kuwait.

IRAN

IRAN

SOUTHERN IRAN

CENTRAL ALASKA. 〈AGS-P>

IRAN

YUGOSLAVIA. ML 2.4 (TTG)

MONA PASSAGE

NEAR S. COAST OF SOUTHERN HONSHU. MG 2.9 (JMA). Fele (II JMA) at OSOKa.

$\begin{array}{lrl}1.4 & 16 & \text { SOUTHERN PERU } \\ 0.1 & 5 & \text { MACOUARIE ISLANDS REGION }\end{array}$

1.233 ADRIATIC SEA. MD 3.0 (TTG). ML 3.4 (KBA)

$\begin{array}{lrl}1.2 & 33 & \text { ADRIATIC SEA. MD 3.0 (TTG). ML } 3.4 \text { (KBA). } \\ 0.9 & 132 & \text { NORTHERN TERRITORY, AUSTRALIA. Felt strongly in the }\end{array}$ UIUTU ar EO.

1.4 11 NORTH OF MACOUARIE ISLAND

0.919 ADRIATIC SEA. MD 2.8 (TTG).

0.14 GREECE. ML 2.7 (ATH)

55 SOLOMON ISLANDS. Felt ot HONIara.

13 MENDOZA PROVINCE, ARGENTINA

2 OFF EAST COAST OF HONSHU, JAPAN

5 EASTERN CHINA. ML 3.8 (BJI).

IRAN

AEGEAN SEA. ML 3.2 (ATH)

4 TONGA ISLANDS. MS 5.8 (BRK). MO=4.6*10**17 Nm (PPT).

Depth fram braadband displacement seismograms.

1.0166

TONGA ISLANDS

FRANCE. ML 2.2 (LDG)

5 NEVADA. ML 2.5 (BRK)

8 MACOUARIE ISLANDS REGION

20 IRAN

61 NORTHERN ITALY. ML 3.7 (LDG), 3.5 (GEN).

27 EAST PAPUA NEW GUINEA REGION

4 FRANCE. ML 1.7 (GEN).

4 SOUTHERN ITALY

24 NEW BRITAIN REGION 
$\begin{array}{llllllll}29 & 04 & 05 & 24.98 & 58.481 \mathrm{~N}\end{array}$ $\begin{array}{llllll}29 & 05 & 10 & 02.9 & 30.011 \mathrm{~N}\end{array}$ $\begin{array}{llllllll}29 & 05 & 45 & 35.6 ? & 35.43 & \mathrm{~N}\end{array}$ $\begin{array}{llllll}29 & 05 & 46 & 41.9 & 30.088 \mathrm{~N}\end{array}$ $\begin{array}{lllllll}29 & 05 & 55 & 42.4 & 59.597 & \mathrm{~N}\end{array}$ $29 \quad 06 \quad 12 \quad 04.8 * 33.468 \mathrm{~S}$

$\begin{array}{lllllll}29 & 07 & 48 & 23.5 ? & 39.11 \quad \mathrm{~N}\end{array}$ $\begin{array}{llllll}29 & 08 & 27 & 05.1 \% & 41.387 \mathrm{~N}\end{array}$

$\begin{array}{lll}25 & 46.2 & 6.589 \mathrm{~S}\end{array}$ $\begin{array}{rrrlrrr}29 & 09 & 52 & 45.4 & 6.517 & \mathrm{~S}\end{array}$ $\begin{array}{lllllll}29 & 10 & 29 & 37.0 & 6.632 & 5\end{array}$ $\begin{array}{lllllll}29 & 11 & 19 & 12.5 & 40.586 & \mathrm{~N}\end{array}$ $\begin{array}{llllllll}29 & 11 & 22 & 04.5 \% & 38.444 & \mathrm{~N}\end{array}$ $\begin{array}{llll}11 & 22 & 10.38 & 59.776 \mathrm{~N}\end{array}$ $\begin{array}{llllllll}29 & 12 & 16 & 52.47 & 38.306 & \mathrm{~N}\end{array}$ $29 \quad 122438.0 \quad 36.653 \mathrm{~N}$ $29 \quad 124453.8 * 38.520 \mathrm{~S}$ $29 \quad 16 \quad 00 \quad 03.5 * 56.623 \mathrm{~N}$

$17 \quad 1708.0 \% 61.816 \mathrm{~N}$ $185156.2 ? 31.67 \mathrm{~S}$ $\begin{array}{llllll}19 & 06 & 14.1 & 49.164 \mathrm{~N}\end{array}$ $1938 \quad 01.1 \% 60.313 \mathrm{~N}$ $\begin{array}{llllll}20 & 37 & 05.2 ? & 33.93 \mathrm{~N}\end{array}$ $2044 \quad 16.7 \% \quad 40.149 \mathrm{~N}$ $\begin{array}{lllllll}21 & 49 & 19.4 & 15.788 \mathrm{~N}\end{array}$ $\begin{array}{lllll}22 & 07 & 11.8 & 10.204 \mathrm{~S}\end{array}$ $\begin{array}{lllll}22 & 22 & 30.7 & 23.897 & \mathrm{~S}\end{array}$

$\begin{array}{llllll}00 & 10 & 49.2 & 38.585 & \mathrm{~N}\end{array}$ 010319.0 ? $10.55 \mathrm{~N}$ $055932.5 * 44.568 \mathrm{~S}$ $\begin{array}{llll}06 & 28 & 07.9 & 43.225 \mathrm{~N}\end{array}$

$06 \quad 56 \quad 53.1 \quad 42.030 \mathrm{~N}$ $06 \quad 58 \quad 36.7 \quad 42.033 \mathrm{~N}$ $07 \quad 23 \quad 44.6 \quad 42.049 \mathrm{~N}$ $0725 \quad 40.2 ? \quad 24.20$ $08 \quad 45 \quad 03.9812 .120 \mathrm{~N}$ $\begin{array}{llll}10 & 04 & 56.1 & 42.031 \mathrm{~N}\end{array}$ $\begin{array}{llll}10 & 41 & 42.1 * 28.889 \mathrm{~N}\end{array}$ $\begin{array}{llll}11 & 28 & 20.8 & 19.924\end{array}$ $122328.2863 .748 \mathrm{~N}$ $\begin{array}{llll}12 & 29 & 47.4 & 19.250 \mathrm{~N}\end{array}$

$\begin{array}{lllllll}13 & 39 & 17 & 1 & 17 & 851 \mathrm{~s}\end{array}$ $\begin{array}{llll}13 & 50 & 56.2 & 17.401 \mathrm{~N}\end{array}$

$\begin{array}{llll}13 & 5250.1 & 21.272\end{array}$ $\begin{array}{llll}15 & 07 & 58.5 \% & 39.245\end{array}$ $\begin{array}{llll}16 & 50 & 45.9 & 6.469 \mathrm{~S}\end{array}$ $165134.1 * 37.089 \mathrm{~N}$

$\begin{array}{lllllll}17 & 08 & 28.9 ? & 21.02 \quad \mathrm{~S}\end{array}$ 171744.5 ? $47.71 \mathrm{~N}$ $\begin{array}{lllllll}17 & 43 & 53.9 ? & 41.74 \mathrm{~N}\end{array}$ $\begin{array}{llll}18 & 22 & 44.9 & 34.078 \mathrm{~N}\end{array}$

$\begin{array}{llllll}20 & 11 & 03.5 \% & 46.537 \mathrm{~N}\end{array}$ $201306.9 * 0.530 \mathrm{~N}$ $\begin{array}{llllll}20 & 13 & 43.4 & 29.781 \mathrm{~N}\end{array}$ $\begin{array}{lllll}20 & 16 & 28.6 & 42.038 & \mathrm{~N}\end{array}$ $\begin{array}{llllll}20 & 58 & 21.4 & 37.20 \quad \mathrm{~N}\end{array}$ $\begin{array}{llllll}21 & 04 & 35.48 & 62.493 \mathrm{~N}\end{array}$ $\begin{array}{llllll}21 & 20 & 45.6 ? & 32.63\end{array}$ $223723.3 * 39.661 \mathrm{~N}$ $\begin{array}{llll}23 & 15 & 50.3 & 40.426 \mathrm{~N}\end{array}$ $\begin{array}{llllll}23 & 16 & 57.87 & 59.935 \mathrm{~N}\end{array}$ $00 \quad 46 \quad 40.58 \quad 58.961 \mathrm{~N}$ Q $1413.8 * 15.355 \mathrm{~S}$ $02 \quad 56 \quad 17.3 \quad 38.115 \mathrm{~N}$ $0324 \quad 26 \quad 98 \quad 59.799 \mathrm{~N}$ 040406.2 ? $50.33 \mathrm{~N}$ $\begin{array}{llll}04 & 15 & 14.8 & 6.804 N\end{array}$ $\begin{array}{llll}04 & 41 & 42.6 * 36.386 \mathrm{~N}\end{array}$ $05 \quad 49 \quad 56.6859 .947 \mathrm{~N}$ $055420.5 \quad 45.383 \mathrm{~S}$
$06 \quad 05 \quad 27.7 \& \quad 61.329 \mathrm{~N}$ $\begin{array}{llll}06 & 41 & 07.5 ? \quad 40.08\end{array}$ $\begin{array}{lllll}08 & 10 & 33.2 ? & 37.83 & \end{array}$ $\begin{array}{llllll}09 & 36 & 07 & 5 \% & 39 & 124 \mathrm{~N}\end{array}$ $095246.1 * 42.778$ $1000 \quad 02.5 * 31.190 \mathrm{~S}$
$153.956 W$ $99.504 \mathrm{E}$ $22.86 \quad E$ $50.908 \mathrm{E}$ $29.904 W$ $70.893 w$

27.56 $29.236 \mathrm{E}$ 142.215

$147.765 \mathrm{E}$

$7.520 \mathrm{E}$

$147.769 \mathrm{E}$

$147.792 \mathrm{E}$

15. $758 \mathrm{E}$

$26.574 \mathrm{E}$

$153.440 \mathrm{~W}$

$26.543 \mathrm{E}$

$71.109 \mathrm{E}$

176.024

$156.885 W$

77

$33 \mathrm{~N} 5.0$

$10 \mathrm{G}$

$40 \quad 5.04 .3$

$10 \mathrm{G} \quad 4.5$

51

7.433

69.67

$6.951 \mathrm{E}$

$7.252 \mathrm{E}$

$140.42 \mathrm{E}$

$28.860 \mathrm{E}$

$89.948 W$

$161.395 \mathrm{E}$

$70.337 \mathrm{~W}$

$26.883 \mathrm{E}$

$60.57 \mathrm{~W}$

174.314

$0.410 \mathrm{~W}$

$19.778 \mathrm{E}$

19.755

$19.737 \mathrm{E}$

$66.78 \mathrm{~W}$

86.910

$19.757 \mathrm{E}$

$52.169 \mathrm{E}$

$175.537 \mathrm{~W}$

$148.972 W$

$64.547 w$

$173.043 \mathrm{~W}$

$94.645 \mathrm{~W}$

174.099

$27.763 \mathrm{E}$

$147.765 \mathrm{E}$

$59.788 \mathrm{E}$

68.66

8. $11 \mathrm{E}$

12. 58 E

135.226

2. $124 \mathrm{E}$

$126.694 \mathrm{E}$

$99.187 \mathrm{E}$

$19.758 \mathrm{E}$

$28.63 \mathrm{E}$

$151.232 \mathrm{~W}$

$71.32 \mathrm{~W}$

$20.974 \mathrm{E}$

$63.390 \mathrm{E}$

$6.299 \mathrm{E}$

154.362

$177.295 \mathrm{~W}$

$20.629 \mathrm{E}$

$152.357 \mathrm{~W}$

$18.98 \mathrm{E}$

$73.023 \mathrm{~W}$

$35.531 \mathrm{E}$

52.509 W

$167.086 \mathrm{E}$
$10 \mathrm{G}$

$10 \mathrm{G}$

735.0

$55 \quad 4.7$

$10 \mathrm{G}$

$51 * 4.3$

$43 \cdot 5.0$

$25 \quad 4.1$

$10 \mathrm{G}$

127

$10 \mathrm{G}$

$268 * 4.2$

$33 \mathrm{~N}$

$33 \mathrm{~N}$

$33 \mathrm{~N}$

$10 \mathrm{G}$

$166 ?$

$10 \mathrm{G}$

$10 \mathrm{G}$

$33 \mathrm{~N}$

$24 \quad 5.0$

$86 \mathrm{D} 5.6$

$\begin{array}{llll}32 & D & 5.5 & 4.9\end{array}$

$10 \mathrm{G}$

$33 \mathrm{~N}$

$33 \mathrm{~N} 4.8$

$10 \mathrm{G}$

11

$10 \mathrm{G}$

$177 ?$

$54 \mathrm{G}$

$10 \mathrm{G}$

$33 \mathrm{~N} 4.3$

21705.0

0

39

4. $6 \quad 4.2$

$\begin{array}{llll}38 & 0 & 5.0 & 4.7\end{array}$

$\begin{array}{llll}33 & \mathrm{~N} & 5.1 & 5.1 \\ 10 & \mathrm{G} & & \\ 49 & & 5.2 & 4.6 \\ 10 & \mathrm{G} & & \end{array}$

$33 \mathrm{~N}$

$10 \mathrm{G}$

$10 \mathrm{C}$

10

$75 ?$

$33 \mathrm{~N} \quad 4.4$

$10 \mathrm{G}$

$10 \mathrm{G}$

84

$55 ?$

$10 \mathrm{G}$

$33 \mathrm{~N} 4.7$

$10 \mathrm{G}$

$135 \quad 3.6$

$380 \cdot 5.0$

16

$10 \mathrm{G}$

172 D 5.1

$33 \mathrm{~N}$

23 G 5.86 .3 13805.2

23 KODIAK ISLAND REGION, 〈AGS-P>

1.1113 SICHUAN PROVINCE, CHINA

0.55 MEDITERRANEAN SEA. MD 3.9 (ATH)

1.2119 IRAN. Felt at Gach Saran and Mamasani.

0.735 NORTH ATLANTIC OCEAN

$\begin{array}{rrl}0.7 & 35 & \text { NORTH ATLANTIC OCEAN } \\ 0.5 & 8 & \text { CHILE-ARGENTINA BORDER REGION. FeIt (IV) in the }\end{array}$ Santiago area and ( 111 ) at Valparaisa.

0. 4 TURKEY

5 TURKEY

1.0 151 HOKKAIDO, JAPAN REGION. FeIt (II JMA) at Hirao, Tamakamai and Urakowa; (I JMA) at Kushiro and Obihiro. Alsa felt (I I JMA) at Hachinahe. Hanshu.

1.131 EAST PAPUA NEW GUINEA REGION

0.25 NORTHERN ITALY. ML 2.0 (GEN).

0.817 EAST PAPUA NEW GUINEA REGION

0.914 EAST PAPUA NEW GUINEA REGION

1.090 SOUTHERN ITALY. ML 4.5 (TTG). MD 4.3 (ATH).

0.9 7 AEGEAN SEA

27 SOUTHERN ALASKA. LAGS-P>.

1.56 AEGEAN SEA

0.9 19 AFGHANISTAN-USSR BORDER REGION

1.0 10 NORTH ISLAND, NEW ZEALAND

1.05 ALASKA PENINSULA. ML 3.5 (PMR)

0.66 COSTA RICA. MD 3.5 (SJR). Felt in the Golto de Nicoya area.

1.0 8 SOUTHERN NORWAY. MD 2.1 (BER)

0.411 SAN JUAN PROVINCE, ARGENTINA

1.111 GERMANY. MD 2.2 (UCC) 


\begin{tabular}{|c|c|c|c|c|c|c|c|c|c|c|c|c|c|c|c|}
\hline & 31 & 10 & 01 & $07.3 \%$ & 37.743 & $N$ & 29.172 & $E$ & 10 & G & & & 0.5 & 5 & TURKEY \\
\hline & 31 & 10 & 03 & $41.0 ?$ & 42.71 & $N$ & 19.10 & $E$ & 10 & G & & & 0.4 & 4 & YUGOSLAVIA. MD 2.1 (TTG) \\
\hline & 31 & 10 & 31 & 01.2? & 41.61 & $\mathbf{N}$ & 12.53 & $E$ & 10 & G & & & 0.3 & 6 & SOUTHERN ITALY \\
\hline & 31 & 10 & 38 & 04.7 & 18.177 & S & 168.044 & $E$ & 55 & * & 4.9 & 4.3 & 1.1 & 40 & VANUATU ISLANDS \\
\hline & 31 & 11 & 54 & $53.0 *$ & 32.877 & S & 71.229 & $w$ & 57 & $?$ & & & 0.3 & 10 & NEAR COAST OF CENTRAL CHILE \\
\hline & 31 & 12 & 14 & 59.0 & 34.719 & $\mathrm{~N}$ & 32.237 & E & 16 & & & & 0.9 & 12 & CYPRUS. ML 3.5 (CSS). Felt (IV) in the Pophos oreo. \\
\hline & 31 & 12 & 24 & $28.7 \&$ & 60.501 & $\mathbf{N}$ & 152.301 & $w$ & 93 & & & & & 27 & SOUTHERN ALASKA. 〈AGS-P>. \\
\hline & 31 & 12 & 46 & $57.4 \&$ & 47.952 & N & 121.894 & $w$ & 18 & & & & & 30 & WASHINGTON. $\angle S E A\rangle$. CL 2.6 (SEA) \\
\hline o & 31 & 14 & 20 & 24.1 & 3.006 & $\mathbf{s}$ & 142.078 & $\mathbf{E}$ & 33 & $\mathbf{N}$ & 5.2 & 4.5 & 1.1 & 67 & NEAR N COAST OF PAPUA NEW GUINEA \\
\hline & 31 & 16 & 18 & 08.2 & 44.490 & N & 7.313 & $\mathbf{E}$ & 10 & $G$ & & & 0.4 & 9 & NORTHERN ITALY. ML 2.0 (GEN). \\
\hline & 31 & 16 & 25 & $03.1 ?$ & 44.45 & $N$ & 7.26 & $\mathbf{E}$ & 10 & G & & & 0.1 & 4 & NORTHERN ITALY. ML 1.7 (GEN). \\
\hline & 31 & 16 & 51 & 05.0 & 27.998 & $\mathrm{~s}$ & 66.601 & $w$ & 168 & & 4.5 & & 1.4 & 42 & CATAMARCA PROVINCE, ARGENTINA \\
\hline & 31 & 17 & 12 & $07.3 ?$ & 34.14 & $N$ & 135.26 & $\mathbf{E}$ & 10 & $G$ & & & 0.3 & 4 & $\begin{array}{l}\text { NEAR S. COAST OF SOUTHERN HONSHU. MG } 1.9 \text { (JMA) } \\
\text { JMA) at WOKOYamo. }\end{array}$ \\
\hline & 31 & 17 & 24 & $48 \cdot 3 *$ & 22.666 & $\mathrm{~N}$ & 94.372 & $E$ & 106 & * & 4.2 & & 1.4 & 13 & BURMA \\
\hline & 31 & 17 & 30 & $47.2 ?$ & 41.76 & $\mathbf{N}$ & 12.73 & $\mathbf{E}$ & 10 & $G$ & & & 0.2 & 4 & SOUTHERN ITALY \\
\hline o & 31 & 19 & 10 & 37.0 & 6.512 & $\mathrm{~s}$ & 147.817 & $\bar{E}$ & 43 & & 5.2 & 4.8 & 1.0 & 62 & EAST PAPUA NEW GUINEA REGION \\
\hline & 31 & 20 & 53 & $18.8 ?$ & $44 \cdot 18$ & $N$ & 12.31 & $E$ & 10 & $G$ & & & 0.3 & 4 & NORTHERN ITALY \\
\hline & 31 & 22 & 33 & $42.9 *$ & 6.689 & $\mathrm{~s}$ & 147.747 & $\mathbf{E}$ & 60 & $?$ & 4.5 & 4.0 & 1.4 & 10 & EAST PAPUA NEW GUINEA REGION \\
\hline & 31 & 23 & 43 & 24.2 & 39.620 & $N$ & 27.795 & $E$ & 9 & & & & 1.0 & 40 & TURKEY \\
\hline
\end{tabular}

ADDITIONAL SOURCE PARAMETERS

$\begin{array}{lllllll}010845 & 21.60 & 4.200 \mathrm{~S} & 101.366 \mathrm{E} & 31 \mathrm{~km}\end{array}$ $5.6 \mathrm{mb}(42$ obs.) $5.6 \mathrm{Msz}$ ( 28 obs.) SOUTHERN SUMATERA

CENTROID, MOMENT TENSOR (HRV)

Doto Used: GDSN

L.P.B.: $14 \mathrm{~S}, 35 \mathrm{C}$

Centroid Location:

Origin Time $\quad 8: 45: 22.00 .6$

Lat 4.98S 0.05 Lon 101.01E 0.05

Dep 23.12 .8 Holf-duration 3.0 Principal Axes:

Scale 10**17 $\mathrm{Nm}$

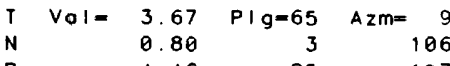

$\begin{array}{llrr}P & -4.46 & 25 & 197\end{array}$

NP1: Strike=294 Dip=21 Siip= 99

$\begin{array}{llll}\text { NP2 : } & 105 & 70 & 87\end{array}$

$0206 \quad 2728.26 \quad 45.060 \mathrm{~N} \quad 28.141 \mathrm{~W} \quad 10 \mathrm{~km}$ $5.2 \mathrm{mb}$ ( 50 obs.) 5.6Msz ( 10 obs.) NORTH ATLANTIC RIDGE

CENTROID, MOMENT TENSOR (HRV)

Doto Used: GDSN

L.P.B.: $13 \mathrm{~S}, 26 \mathrm{C}$

Centroid Lacation:

Origin Time $06: 27: 31.2 \quad 0.5$

Lat $44.54 \mathrm{~N} 0.10$ Lon $28.12 \mathrm{~W} 0.04$

Dep 15. $\mathrm{FIX}$ Holf-duration 2.4

Principol Axes:

Scole $10 * * 17 \mathrm{Nm}$

T $\quad \mathrm{O}$ I $=2.02 \quad \mathrm{PIg}=\theta \quad A z \mathrm{~m}=9 \theta$

$\begin{array}{rrrr}N & & 2.02 & P l g= \\ P & -0.05 & 0 & 180 \\ P & -1.97 & 90 & 180\end{array}$

Best Double Couple: $M_{0}=2 . \theta * 1 \theta * * 17$

NP 1: Strike=180 D i $p=45$ SI i $p=-90$

NP2: $\quad \theta \quad 45 \quad-90$

$\begin{array}{lllllll}02 & 09 & 30 & 15.52 & 16.747 \mathrm{~N} & 99.343 \mathrm{w} & 10 \mathrm{~km}\end{array}$ $5.4 \mathrm{mb}$ ( 57 Obs.) $4.9 \mathrm{Msz}$ ( 4 Obs.) NEAR COAST OF GUERRERO, MEXICO

CENTROID, MOMENT TENSOR (HRV)

Doto Used: GDSN

L.P.B.: 135, 30C

Centroid Locotion:

Origin Time $\quad 09: 30: 17.60 .5$

Lot $16.82 \mathrm{~N}$ FIX; Lon $99.35 \mathrm{~W}$ FIX

Dep 47.95.7 Half-duration 2.0

Principol Axes:

Scole $10 * 17 \mathrm{Nm}$

$T \quad V 0 I=1.57 \quad P I g=52 \quad A z m=94$

$\begin{array}{rrrr}N & 0.68 & 32 & 311 \\ P & -2.25 & 18 & 209\end{array}$

Best Double Couple:Mo=1.9*10**17

NP 1 : Strike $=260$ Dip=39 Si ip= 33

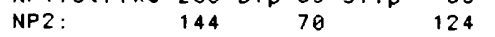

$020930 \quad 58.97 \quad 44.968 \mathrm{~N} \quad 28.025 \mathrm{~W} \quad 10 \mathrm{~km}$ । $5.0 \mathrm{mb}$ ( 57 obs.) 5.5Msz ( 11 obs.) NORTH ATLANTIC RIDGE

CENTROID, MOMENT TENSOR

( HRV)

Doto Used: GDSN

L.P.B.: 12S, $22 \mathrm{C}$

Centroid Locotion:

Origin Time $\quad 09: 31: 3.10 .5$

Lot $44.75 \mathrm{~N} 0.09$ Lon $27.82 \mathrm{~W} 0.09$

Dep 15.0 Fix Holf-durotion 1.8

Principol Axes:

Scale $10 * 17 \mathrm{Nm}$

\begin{tabular}{|c|c|c|c|c|}
\hline$T$ & Val $=$ & 1.91 & $P \mid g=17$ & $A z m=271$ \\
\hline N & & -0.40 & 16 & 6 \\
\hline $\mathbf{P}$ & & -1.51 & 67 & 137 \\
\hline
\end{tabular}

Best Double Couple: MO=1.7*10**17

NP 1 : Strike=339 Dip=31 Si ip=-121

NP2: $\quad 194 \quad 64 \quad 63$

$0305 \quad 53 \quad 01.17 \quad 30.091 \mathrm{~N} \quad 99.475 \mathrm{E} \quad 14 \mathrm{~km}$

$6.1 \mathrm{mb}$ ( 77 obs.) 6.1Msz ( 15 obs.)

SICHUAN PROVINCE, CHINA

FAULT PLANE SOLUTION: P-Woves

NP1: Strike=111 Dip=75 S1 ip= -38

NP2: $\quad 212 \quad 54 \quad-161$

Principol Axes:

$$
\begin{array}{lrr}
T & P \mid g=14 & A z m=166 \\
P & 37 & 65
\end{array}
$$

Camment: The focal mechanism is

poorly contralled and

corresponds to strike-slip

foulting with o large normal

component. The preferred foult

plane is not determined.

RADIATED ENERGY

No. of sto: 10 Focal mech. C

Energy $0.5 \pm 0.1 * 10 * * 14 \mathrm{Nm}$

CENTROID. MOMENT TENSOR (HRV)

Data Used: GDSN

L.P.B.: 14S, 33C M.W.: 14S, $24 \mathrm{C}$

Centroid Locotion:

Origin Time

$05: 53: 12.20 .1$

Lat $30.00 N 0.02$ Lon $99.69 E 0.03$

Dep 15.0 FIX Half-duration $5 . \theta$

Principol Axes

Scole $10 *+18 \mathrm{Nm}$

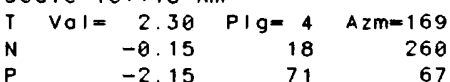

Best Double Couple:Mo=2.2*10**18

NP1: Strike $=240$ Dip=44 Si ip $=-117$

NP2 :

95

$\begin{array}{llllll}03 & 09 & 13 & 24.23 & 29.964 \mathrm{~N} & 51.655 \mathrm{E} \quad 33 \mathrm{~km}\end{array}$

5. $1 \mathrm{mb}$ ( 12 ODS.)

SOUTHERN IRAN

CENTROID, MOMENT TENSOR (HRV)

Doto Used: GDSN

L.P.B.: $14 \mathrm{~S}, 25 \mathrm{C}$

Centroid Locotion:

Origin Time $\quad 09: 13: 20.41 .4$

Lot $29.64 \mathrm{~N} 0.16$ Lon $51.42 E$ 0.15

Dep 15.0 Fix Half-duration 1.7

Principol Axes:

Scole $10 * * 16 \mathrm{Nm}$

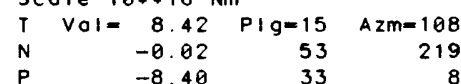

Best Double Couple:Ma=8.4*18**16

NP 1 : Strike=153 Dip=55 SI ip=-166

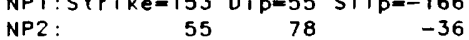

$\begin{array}{llllll}03 & 1541 & 30.88 & 30.053 \mathrm{~N} & 99.499 E & 8 \mathrm{~km}\end{array}$

$5.8 \mathrm{mb}$ ( 84 obs.) $5.9 \mathrm{Msz}$ ( 14 obs.)

SICHUAN PROVINCE, CHINA

FAULT PLANE SOLUTION: P-WaVES

NP 1: Strike=111 Dip=68 SI ip= -31

NP2: 214
Principol Axes:

$\begin{array}{lrr}T & & \\ P & P I g= & A z m=164 \\ 37 & 70\end{array}$

Comment: The focol mechonism is paorly controlled and

corresponds to strike-slip foulting with o lorge normal companent. The preferred foult plane is not determined.

RADIATED ENERGY

No. of sto: 8 Focal mech. F Energy $0.4 \pm 0.1 * 10 * * 14 \mathrm{Nm}$ MOMENT TENSOR SOLUTION

DeP 6 No. of sta: 6 Principal Axes:

Scale $10 * * 17 \mathrm{Nm}$

$T \quad V a I=6.61 \quad \mathrm{PIg}=8 \quad$ Azm=350

$\begin{array}{rrrr}N & -2.38 & 48 & 251 \\ P & -4.23 & 41 & 87\end{array}$

Best Dauble Cauple:Ma=5.4*10*17 NP 1: Strike $=120$ Dip $=56$ Si ip $=-27$ NP2: $\quad 225 \quad 68 \quad-143$ CENTROID, MOMENT TENSOR (HRV)

Doto Used: GOSN

L.P.B.: $14 \mathrm{~S}, 32 \mathrm{C}$

Centraid Locotion:

Origin Time 15:41:40.5 0.2

Lat $29.83 \mathrm{~N} 0.02$ Lon $99.43 \mathrm{E} 0.05$

Dep 15.0 FIX Holf-durotion $3 . \theta$

Principol Axes:

Scole $10 * 17 \mathrm{Nm}$

$T \quad V O I=7.30 \quad P / g=0 \quad A Z m=181$

$\begin{array}{rrrr}N & -3.39 & 0 & 91 \\ P & -3.90 & 90 & 180\end{array}$

Best Double Couple:Mo=5.6*10**17 NP1: Strike $=271$ Dip $=45$ Si ip $=-90$ NP2 : $91 \quad 45$

$-90$

$\begin{array}{lllllll}03 & 18 & 44 & 54.26 & 6.955 \mathrm{~N} & 94.592 \mathrm{E} & 33 \mathrm{~km}\end{array}$ 4. $9 \mathrm{mb}$ ( 31 obs.) 4.8Msz ( 2 obs.) NICOBAR ISLANDS REGION

CENTROID. MOMENT TENSOR

Doto Used: GDSN

L.P.B.: 14S, 25C

Centroid Lacation:

Origin Time 18:44:58.1 1.0

Lot $6.90 \mathrm{~N} 0.11$ Lon $95.13 \mathrm{E} \quad 0.12$

Dep $33.0 \mathrm{FIX} \mathrm{Holf-duration} 1.6$ Principal Axes:

Scale $10 *+16 \mathrm{Nm}$

$T \quad V a l=6.43 \quad \mathrm{Plg}=26 \quad$ Azm $=274$

$\begin{array}{llll}N & -0.52 & 49 & 149\end{array}$

$\begin{array}{cccr}P & -5.91 & 29 & 20 \\ \text { Best Double Couple:Mo=6.2*10*16 }\end{array}$

NP 1: Strike $=56$ Dip $=49$ SI i $=-2$

NP2: $\quad 148 \quad 89 \quad-139$

$04002206.74 \quad 11.038 \mathrm{~N} \quad 68.270 \mathrm{~W} \quad 16 \mathrm{~km}$ $5.4 \mathrm{mb}$ ( 62 obs.) 5.2MsZ (9 Obs.) NEAR COAST OF VENEZUELA

CENTROID. MOMENT TENSOR

(HRV)

Doto Used: GDSN

L.P.B.: 18S, $33 \mathrm{C}$

Centroid Locotion:

Origin lime $\theta 0: 22: 11.90 .8$

Lot $10.96 \mathrm{~N} 0.07$ Lon $68.42 \mathrm{~W} 0.06$

Dep 15.0 FIX Holf-duration 2.1

Principol Axes:

Scale $10 * * 17 \mathrm{Nm}$

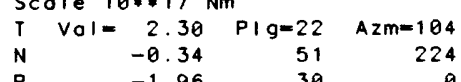


Best Double Couple:Mo=2,1*10*17 NP 1: Strike=145 Dip=51 SI ip=-174 NP2 :

51

85

$-39$

$\begin{array}{llllllll}04 & 03 \quad 37 & 39.20 & 40.537 \mathrm{~N} & 127.437 \mathrm{~W} & 5 \mathrm{~km}\end{array}$ $5.0 \mathrm{mb}$ ( 33 abs.) 4.1Msz (1 Obs.) OFF COAST OF NORTHERN CALIFORNIA CENTROID, MOMENT TENSOR (HRV) Dota Used: GDSN

L.P.B.: 16S, $23 \mathrm{C}$

Centroid Location

Origin Time $03: 37: 45.41 .0$ Lot $40.46 \mathrm{~N} 0.12$ Lon $127.72 \mathrm{~W} 0.11$ DeP 15.0 Fix Holf-durotion 1.5 Principol Axes:

Scale $10 * 16 \mathrm{Nm}$

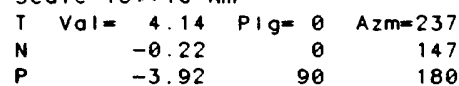

Best Double Couple:Mo=4.0*10**16 NP 1: Strike $=327$ Dip=45 Si $\quad$ p $=-90$ NP2: $\quad 147 \quad 45 \quad-90$

$0410 \quad 3006.96 \quad 6.609 \mathrm{~S} \quad 75.760 \mathrm{~W} \quad 33 \mathrm{~km}$ $5.5 \mathrm{mb}$ ( 64 obs.) $5.1 \mathrm{Msz}$ ( 4 obs.) NORTHERN PERU

CENTROID, MOMENT TENSOR (HRV)

Doto Used: GDSN

L.P.B.: 15S, $24 \mathrm{C}$

Centroid Location

Origin Time $\quad 10: 30: 13.40 .5$

Lat 6.3150 .08 Lon $76.18 w 0.10$

DeP 36.35 .5 Half-duration 1.4

Principal Axes:

Scale $10 * 16 \mathrm{Nm}$

T $\quad \mathrm{Val}=5.92 \quad \mathrm{Plg}=72 \quad$ Azm $=287$

$\begin{array}{rrrr}N & 0.16 & 13 & 150 \\ P & -6.08 & 12 & 57\end{array}$

Best Dauble Couple:Ma=6 $0 * 10 * 16$ NP1:Strike $=130$ Dip=35 S1ip= 66 $\begin{array}{llll}\text { NP2: } & 338 & 58 & 106\end{array}$

$04 \quad 13 \quad 1508.06 \quad 21.963 \mathrm{~S} \quad 179.305 w 591 \mathrm{~km}$ $5.4 \mathrm{mb}$ ( 49 abs.)

FIJI ISLANDS REGION

CENTROID, MOMENT TENSOR

(HRV)

L.P. 19S $41 \mathrm{C}$

Centraid Lacation:

Origin Time 13:15:14.9 0.4

Lat $21.96 \mathrm{~S} 0.03$ Lan 179.34W 0.03

Dep 616.4 1.4 Half-duration 2.8

Principal Axes:

Scale $10 * 17 \mathrm{Nm}$

$T$ Val $=4.21 \quad P / g=68 \quad A z m=169$

$\begin{array}{rrrr}N & 0.59 & 15 & 38 \\ P & -4.81 & 16 & 303\end{array}$

Best Dauble Cauple:Mo=4.5*10**17

NP 1: Strike $=12$ Dip $=32$ Slip= 60

NP2: $\quad 226 \quad 63 \quad 107$

$04 \quad 18 \quad 32 \quad 55.69 \quad 13.039 \mathrm{~S} \quad 76.195 \mathrm{~W} \quad 72 \mathrm{~km}$

5. $3 \mathrm{mb}(19$ abs.)

NEAR COAST OF PERU

CENTROID. MOMENT TENSOR (HRV)

Data Used: GDSN

L.P.B.: 17S, 26C

Centroid Location:

Origin Time 18:33: 2.30 .5

Lat $12.84 \mathrm{~S} 0.06$ Lon $76.20 \mathrm{~W} 0.09$

Dep 84.06 .2 Holf-duration 1.4

Principal Axes:

Scale $10 * 16 \mathrm{Nm}$

I $\quad$ Oal $=5.88 \quad$ PIg $=65 \quad$ Azm $=239$

$\begin{array}{rrrr}N & -0.92 & 5 & 339 \\ P & -4.96 & 24 & 71\end{array}$

Best Double Cauple:Mo=5.4+10*16

NP1: Strike=172 Dip=21 Si i $=103$

NP2: $\quad 337 \quad 70 \quad 85$

$\begin{array}{llllll}05 & 18 & 28 & 39.45 & 8.281 \mathrm{~s} & 71.381 w \quad 593 \mathrm{~km}\end{array}$ $6.4 \mathrm{mb}$ ( 71 abs.)

WESTERN BRAZIL

FAULT PLANE SOLUTION: P-WaVES

NP1: Strike=145 Di $p=60$ SI i $p=-103$

$\begin{array}{lll}350 & 32 & -69\end{array}$

Principal Axes:

$\begin{array}{crr}T & P I g=14 & \text { Azm=244 } \\ P & 72 & 24 \\ \text { Comment: The focal mechanism is }\end{array}$

poorly controlled and

corresponds to narmal faulting

with a small right-lateral strike-slip component. The

preferred foult plone is NP RADIATED ENERGY

No. of sto: 7 Focal mech. M Energy $0.9 \pm 0.3 * 10 * 15 \mathrm{Nm}$ MOMENT TENSOR SOLUTION

DeP 597

Scole $10 * 19 \mathrm{Nm}$

$\begin{array}{rrrr}T & \text { VOl }= \\ T & 3.93 & P I g=8 & A z m=235 \\ N & 0.00 & 5 & 326 \\ P & -3.94 & 81 & 88\end{array}$

Best Double Couple: $M O=3.9 * 10 * * 19$

NP 1 : Strike=319 Dip=37 Si i $=-98$ NP2 : 149

CENTROID, MOMENT TENSOR (HRV)

Doto Used: GDSN

L.P.B.: 24S, 63C M.W.: 15S, $35 \mathrm{C}$ Centroid Location:

Origin Time

$18: 28: 49.4 \quad 0.1$

Lat $8.05 \mathrm{~S} 0.01$ Lon $71.49 \mathrm{~W} 0.01$

Dep 606.00 .7 Holf-durotion 12.0 Principal Axes:

Scale $10 * * 19 \mathrm{Nm}$

$T \quad V a l=4.58 \quad P / g=15 \quad A z m=249$

$\begin{array}{rrrr}N & 0.42 & 3 & 158 \\ P & -5.00 & 75 & 57\end{array}$

Best Double Couple:Mo $=4.8 * 10 * * 19$

NP1: Strike=344 Dip=31 SI ip= -84

NP2: $\quad 157 \quad 60 \quad-93$

$07 \quad 00 \quad 38 \quad 18.53 \quad 23.553 \mathrm{~N} \quad 99.526 \mathrm{E} \quad 33 \mathrm{~km}$ $5.3 \mathrm{mb}$ ( 67 abs.) 5.6Msz ( 10 abs.) BURMA-CHINA BORDER REGION

CENTROID, MOMENT TENSOR

(HRV)

Data Used: GOSN

L.P.B.: 15S, $29 \mathrm{C}$

Centroid Lacation:

Origin Time $\quad 00: 38: 20.30 .4$

Lat $23.46 \mathrm{~N} 0.04$ Lon $99.65 \mathrm{E} 0.06$

Dep 15.0 FIX Half-durotian 2.5 Principal Axes:

Scale $10 * 17 \mathrm{Nm}$

T $\quad \mathrm{Val}=3.26 \quad \mathrm{Plg}=18 \quad$ A $2 \mathrm{~m}=292$

$\begin{array}{rrrr}N & 0.13 & 72 & 103 \\ P & -3.39 & 3 & 201\end{array}$

Best Dauble Cauple:Ma=3 3*10*17 NP 1: Strike $=335$ Di $p=76$ SI i $p=169$

NP2: $\quad 68 \quad 79$

$0803 \quad 36 \quad 38.45 \quad 18.891 \mathrm{~s} \quad 178.845 \mathrm{~W} \quad 531 \mathrm{~km}$

$5.4 \mathrm{mb}(44$ obs.)

FIJI ISLANDS REGION

CENTROID, MOMENT TENSOR

(HRV)

Data Used: GDSN

L.P.B.: 15S, $27 \mathrm{C}$

Centroid Locotion

Origin Time $\quad 03: 36: 45.60 .6$

Lot $18.72 \mathrm{~S} 0.07$ Lon $179.37 \mathrm{~W} 0.05$

Dep $516.83 .3 \mathrm{Half}$-duration 2.3

Principol Axes:

Scale $10 * 17 \mathrm{Nm}$

$T \quad V a l=2.66 \quad P / g=5 \quad A z m=203$

$\begin{array}{rrrr}N & -0.19 & 73 & 95 \\ P & -2.47 & 16 & 294\end{array}$

Best Dauble Couple:Mo=2.6*10**17

NP 1: Strike=337 Dip=75 Slip= -8

NP2: $\quad 69 \quad 82 \quad-165$

$\begin{array}{lllllll}08 \quad 06 \quad 20 & 02.09 & 0.019 \mathrm{~N} & 126.696 \mathrm{E} \quad 76 \mathrm{~km}\end{array}$

$5.1 \mathrm{mb}$ ( 13 abs.)

MOLUCCA PASSAGE

CENTROID', MOMENT TENSOR

(HRV)

Data Used: GDSN

L.P. 8.: 16S, 24C

Centraid Lacatian:

Origin 1 ime

$06: 20: 3.20 .6$

Lat $0.04 \mathrm{~S} 0.07$ Lon $126.09 \mathrm{E} \quad 0.06$

Dep $33.0 \mathrm{FIX} \mathrm{Half-duratian} 1.9$

Principal Axes

Scale $10 *+17 \mathrm{Nm}$

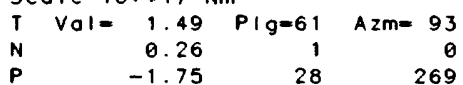

$\begin{array}{cccr}P & -1.75 & 28 & 269 \\ \text { Best Dauble Couple:Mo=1.6*10*117}\end{array}$

NP1: Strike=355 Dip=17 SIip= 85

NP2

$181 \quad 73$

92

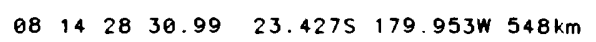
$5.6 \mathrm{mb}$ ( 41 abs.)

SOUTH OF FIJI ISLANDS

CENTROID, MOMENT TENSOR

(HRV)
L B : 19S $49 \mathrm{C}$

Centroid Locotion:

Origin Time 14:28:39.3 0.2

Lat 23.625 0.02 Lon 180.01E 0.02

Dep 558.1 1.1 Malf-duration 5.1

Principal Axes:

Scale $10 * * 18 \mathrm{Nm}$

$T \quad V a l=2.93 \quad P l g=63 \quad$ Azm $=137$

$\begin{array}{rrrr}N & -0.03 & 0 & 228\end{array}$

Best Double Couple:Ma=2.9*10*118

NP1: Strike= 49 Dip=18 SIip= 91

$\begin{array}{llll}\text { NP2: } & 228 & 72 & 98\end{array}$

$\begin{array}{lllllllll}08 & 23 & 18 & 43.61 & 32.282 \mathrm{~N} & 137.626 \mathrm{E} & 390 \mathrm{~km}\end{array}$ $5.1 \mathrm{mb}$ ( 65 obs.)

SOUTH OF HONSHU, JAPAN

CENTROID, MOMENT TENSOR

Data Used: GDSN

L.P.B.: 11S, $22 \mathrm{C}$

Centraid Lacatian:

$23: 18: 46.5 \quad 0.5$

Lat 32.12N 0.04 Lan $137.46 \mathrm{E} 0.09$

Dep $381.32 .9 \mathrm{Hal}$-duratian 1.9

Principal Axes:

Scale $10 * * 17 \mathrm{Nm}$

T $\quad \mathrm{Oal}=1.48 \quad \mathrm{Plg}=39 \quad$ Azm $=97$

$\begin{array}{rrrr}N & -0.01 & 4 & 4 \\ P & -1.48 & 51 & 269\end{array}$

Best Double Cauple:Ma=1.5*10**17

NP1: Strike $=218$ Dip= 7 SIip= -55

NP2: $\quad 3 \quad 84 \quad-94$

$0902 \quad 30 \quad 37.86 \quad 28.071 \mathrm{~N} \quad 16.156 \mathrm{~W} \quad 23 \mathrm{~km}$ $5.2 \mathrm{mb}$ ( 51 obs.) 4.6Msz ( 1 obs.) CANARY ISLANDS REGION

CENTROID. MOMENT TENSOR

( HRV)

Doto Used: GDSN

L.P.B.: 13S, 19C

Centraid Locotion:

Origin Time $02: 30: 32.21 .3$

Lat $28.03 \mathrm{~N}$ FIX; Lan $16.17 \mathrm{~W}$ FIX

Dep $15.0 \mathrm{FIX} \mathrm{Half-duration} 1.5$

Principol Axes:

Scale $10 * 16 \mathrm{Nm}$

$T \quad V a I=4.53 \quad P I g=60 \quad$ A $2 \mathrm{~m}=91$

$\begin{array}{llll}N & -0.91 & 24 & 312 \\ P & -3.62 & 18 & 214\end{array}$

Best Double Cauple:Mo=4.1*10*16

NP1:Strike=273 Dip=34 SIip= 45

NP2. $\quad 143 \quad 67 \quad 116$

$09 \quad 15 \quad 34 \quad 09.65 \quad 52.991 \mathrm{~S} \quad 159.386 \mathrm{E} \quad 10 \mathrm{~km}$ $5.3 \mathrm{mb}$ ( 12 obs.) 4.8Msz (1 abs.) MACOUARIE ISLANDS REGION

CENTROID, MOMENT TENSOR

(HRV)

Data Used: GDSN

L.P.B.: 15S, 33C

Centraid Lacatian

Origin Time 15:34:16.7 0.4

Lat $52.94 \mathrm{~S}$ FIX: Lan 158.73E FiX Dep 15.0 FIX Half-duration 2.1 Principal Axes:

Scale $10 * * 17 \mathrm{Nm}$

$T$ Val $=1.94 \quad P 1 g=1 \quad$ Azm=167

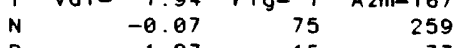

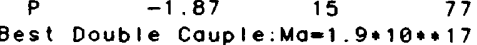

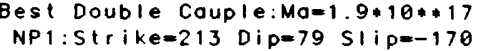

NP2: $\quad 121 \quad 80 \quad-11$

$\begin{array}{lllllll}10 & 0106 & 49.67 & 22.146 \mathrm{~S} & 69.303 \mathrm{E} & 10 \mathrm{~km}\end{array}$ $5.2 \mathrm{mb}$ ( 29 abs.) $5.1 \mathrm{Msz}$ ( 4 abs.) MID-INDIAN RISE

CENTROID, MOMENT TENSOR

(HRV)

Data Used: GDSN

L.P.B.: 13S, $19 \mathrm{C}$

Centroid Lacation:

Origin Time $01: 06.5350 .7$

Lat $22.23 \mathrm{~S} F I X$ :Lan 69.27E FIX

Dep 15.0 Fix Half-duration 1.5

Principal Axes:

Scale 10*16 $\mathrm{Nm}$

$T$ Vol $=6.91 \quad P / g=\theta \quad A=m=238$

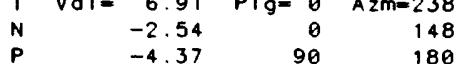

Best Dauble Couple:Ma=5.6*10**16

NP 1 : Strike $=328$ Dip=45 SI $p=-90$

NP2: $\quad 148 \quad 45 \quad-90$

$\begin{array}{llllllll}12 & 22 & 25 & 25.87 & 12.952 \mathrm{~N} & 87.947 \mathrm{~W} & 57 \mathrm{~km}\end{array}$ $5.4 \mathrm{mb}(7$ ODS.)

NEAR COAST OF NICARAGUA 
CENTROID, MOMENT TENSOR

(HRV)

Data Used: GOSN

L.P.B.: 16S, 29C

Centraid Lacatian

Origin Time 22.25:30.9 0.5 Lat $12.89 \mathrm{~N}$ FIX:Lan $87.78 \mathrm{~W}$ FIX Dep $63.34 .8 \mathrm{Half}$-duration 1.7 Principal Axes:

Scale $10 * 16 \mathrm{Nm}$

$\begin{array}{rrrr}T & \text { Val }=11.02 & \text { PIg=55 } & \text { Azm }=62 \\ N & 0.16 & 27 & 283\end{array}$

P $\quad-11.18 \quad 20 \quad 183$

Best Dauble Cauple: $\mathrm{Ma}=1.1 * 10 * * 17$

NPI : Strike $=236$ Dip=35 Slip= 37

NP2 :

70

$1303 \quad 3502.85 \quad 50.103 \mathrm{~N} \quad 105.360 \mathrm{E} \quad 36 \mathrm{~km}$ $5.6 \mathrm{mb}$ ( 73 obs.) $5.6 \mathrm{Msz}$ ( 13 obs.) USSR-MONGOLIA BORDER REGION

CENTROID, MOMENT TENSOR (HRV)

Dota Used: GDSN

L.P.B.: 145, $34 \mathrm{C}$

Centraid Locatian:

Origin Time $\quad 03: 35: 4.30 .4$ Lat $50.32 \mathrm{~N} 0.05$ Lan 105.93E 0.09 Dep 15.0 FIX Half-duration 2.7 Principol Axes:

Scale $10 * 17 \mathrm{Nm}$

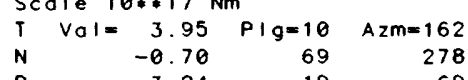

P $\quad-3.24 \quad 19 \quad 69$

Best Dauble Cauple:Ma=3.6*10**17 NP 1: Strike=206 D i $p=70$ SI i $p=-174$ $\begin{array}{llll}\text { NP2 : } & 114 & 84 & -20\end{array}$

$\begin{array}{lllllll}13 & 12 & 15 & 04.17 & 18.983 \mathrm{~N} & 107.519 \mathrm{~W} \quad 33 \mathrm{~km}\end{array}$ $4.9 \mathrm{mb}$ ( 16 abs.) 4.9Msz (1 abs.) OFF COAST OF JALISCO, MEXICO CENTROID, MOMENT TENSOR

(HRV)

Data Used: GDSN

L.P.B.: 15S, $28 \mathrm{C}$

Centraid Lacation:

Origin Time 12:15:6.0 0.8 Lat $19.25 \mathrm{~N} 0.10$ Lan 108.24W 0.09 Dep 15.0 FIX Hali-duratian 1.5 Principal Axes:

Scale $10 * * 16 \mathrm{Nm}$

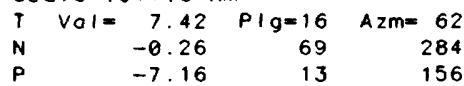

Best Dauble Cauple:Mo=7.3*10**16 NP 1: Strike=199 Dip=69 SIip= 2 $\begin{array}{llll}\text { NP2 : } & 109 & 88 & 159\end{array}$

$\begin{array}{lllllll}13 & 23 & 19 & 41.82 & 35.220 \mathrm{~N} & 91.575 \mathrm{E} & 33 \mathrm{~km}\end{array}$ 4.9mb ( 20 obs.) 4.9Msz ( 3 abs.) QINGHAI PROVINCE, CHINA

CENTROID, MOMENT TENSOR

(HRV)

Doto Used: GDSN

L.P.B.: 13S, $24 \mathrm{C}$

Centraid Lacatian:

Origin Time 23:19:47.8 0.6

Lat $35.69 \mathrm{~N} 0.06$ Lon $91.65 \mathrm{E} 0.07$

Dep $15.0 \mathrm{FIX} \mathrm{Half-duratian} 1.8$ Principal Axes:

Scale $10 * 16 \mathrm{Nm}$

T $\quad V a l=9.63 \quad$ Plg $=18 \quad$ Azm $=110$

$\begin{array}{rrrr}N & 0.89 & 66 & 333 \\ P & -10.52 & 15 & 205\end{array}$

Best Dauble Cauple:Ma=1.0*10**17

NP 1: Strike $=248$ Dip=66 SIip= 2

$\begin{array}{llll}\text { NP2: } & 157 & 88 & 156\end{array}$

$\begin{array}{llllll}1400 & 59 & 50.45 & 30.523 \mathrm{~S} & 178.414 \mathrm{~W} \quad 44 \mathrm{~km}\end{array}$ $5.9 \mathrm{mb}$ ( 44 abs.) 6.6Msz ( 21 abs.) KERMADEC ISLANDS

FAULT PLANE SOLUTION: P-Waves

NP1:Strike $=5$ Dip=60 Slip= 90

NP2: $\quad 185 \quad 30 \quad 90$

Principol Axes:

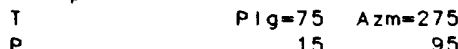

Camment: The facal mechanism is

paorly cantrolled and

correspands to reverse

faulting. The preferred foult

Plane is NP2.

RADIATED ENERGY

No. of sto: 5 focal mech. F

Energy $0.4 \pm 0.1 * 10 * 14 \mathrm{Nm}$

MOMENT TENSOR SOLUTION

Dep 32 Na. of sta: 15

Principol Axes:
Scale $10 * 19 \mathrm{Nm}$

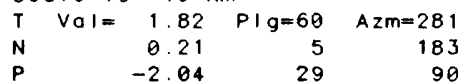

Best. Dauble Couple: $M a=1.9 * 10 * 19$ NP 1: Strike=167 Dip=16 Si $i p=73$ NP2 :

CENTROID, MOMENT TENSOR (HRV) Dota Used: GDSN

L.P.B.: 15S, 41C M.W.: 10S, $23 \mathrm{C}$ Centraid Lacatian

Origin Time

$01: 00: 2.002$

Lat 30.8450 .01 Lan $177.87 \mathrm{~W} 0.01$

Dep 44.20 .7 Half-duration 10.5 Principal Axes:

Scole 10**19 Nm

$T \quad V a I=2.49 \quad P \mid g=73 \quad A Z m=266$

$\begin{array}{rrrr}N & 0.00 & 5 & 12 \\ P & -2.50 & 16 & 104\end{array}$

Best Double Cauple:Ma=2.5*10**19 NP 1: Strike=201 D i $p=29$ SI ip $=100$ NP2 :

$$
961
$$

84
(PAR)

DeP 50 (

Best Dauble Cauple:Ma=3.8*10**19 NP 1: Strike $=221$ Dip=65 Si ip $=106$ NP2 :$$
29
$$

$\begin{array}{llllllll}14 & 09 & 10 & 25.37 & 2.922 \mathrm{~S} & 127.669 \mathrm{E} \quad 33 \mathrm{~km}\end{array}$ 5.4mb ( 24 abs.) 4.7Msz ( 7 abs.) CERAM SEA

CENTROID MOMENT TENSOR

(HRV)

Data Used: GDSN

L.P.B.: 15S, 25C

Centraid Location:

Origin Time $09: 10: 29.00 .6$

Lat 2.8350 .08 Lon $127.65 \mathrm{E} \quad 0.07$

Dep $21.37 .8 \mathrm{Half}$-duratian 2.2

Principal Axes:

Scale $10 * * 17 \mathrm{Nm}$

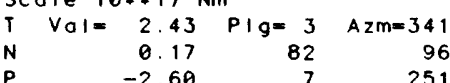

Gest Dauble Cauple:Ma=2.5*10**17

NP 1: Strike $=26$ Dip $=83$ S1ip $=-177$

296

87

$\begin{array}{lllllll}14 & 09 & 40 & 46.57 & 13.7335 & 66.248 \mathrm{E} & 10 \mathrm{~km}\end{array}$ $5.2 \mathrm{mb}$ ( 41 abs.) $5.6 \mathrm{Msz}$ ( $17 \mathrm{abs}$.) MID-INDIAN RISE

CENTROID, MOMENT TENSOR

(HRV)

Dota Used: GDSN

L.P.B.: 20S, $44 \mathrm{C}$

Centroid Lacatian:

Origin Time $\quad 09: 40: 54.70 .3$

Lat 13.4550 .04 Lon $66.06 \mathrm{E} 0.03$

Dep $15.0 \mathrm{FIX} \mathrm{Half-duratian} 3.4$ Principol Axes:

Scale $10 * * 17 \mathrm{Nm}$

$\begin{array}{rrrr}T & V a I=10.77 & P \mid g=13 & A z m=98 \\ N & -2.35 & 72 & 237\end{array}$

$\begin{array}{lllr}\mathrm{P} & -8.42 & 11 & 6\end{array}$

Best Dauble Cauple:Ma=9.6*10**17

NP1: Strike=142 Dip=72 S1 i $p=179$

NP2: $\quad 232 \quad 89$

179
18

$\begin{array}{llllll}1705 & 05.14 & 7.4395 & 128.048 \mathrm{E} & 112 \mathrm{~km}\end{array}$

$5.3 \mathrm{mb}$ ( 15 obs.)

BANDA SEA

CENTROID, MOMENT TENSOR (HRV)

Data Used: GDSN

L.P.B.: 16S, $28 \mathrm{C}$

Centroid Lacation

Origin Time 17:05: 8.7 1.3

Lat 7.8050 .12 Lan $127.97 E 0.09$

Dep 144.0 2.7 Half-duration 1.7 Principal Axes:

Scale $10 * * 16 \mathrm{Nm}$

T $\quad$ a l $=10.36 \quad$ PIg=55 Azm=219

$\begin{array}{rrrr}N & -1.38 & 27 & 82 \\ P & -8.98 & 20 & 342\end{array}$

Best Double Couple:Mo=9.7*10*16

NP1:Strike $=34$ Dip=34 S1ip= 37

NP2: $\quad 273 \quad 70 \quad 119$

$\begin{array}{llllll}22 & 53 & 36.25 & 52.671 \mathrm{~N} \quad 35.037 \mathrm{~W} \quad 10 \mathrm{~km} & \end{array}$ 5. Omb ( 50 abs.) 5.4Msz ( 7 obs.) NORTH ATLANTIC OCEAN

CENTROID, MOMENT TENSOR

(HRV)

Data Used: GDSN

L.P.B.: $15 \mathrm{~S}, 38 \mathrm{C}$

Centroid Locatian:

Origin Time

$22: 53: 41.30 .6$
Lat $52.46 \mathrm{~N} 0.05$ Lan $34.60 \mathrm{~W}$. 06

Dep $15.0 \mathrm{~F}$ ix Half-duratian 2.6 Principol Axes

Scale $10 * 17 \mathrm{Nm}$

T $\quad \mathrm{Val}=3.99 \quad \mathrm{Plg}=0 \quad$ A $\mathrm{Zm}=229$

$\begin{array}{rrrr}N & -0.87 & 90 & 180 \\ P & -3.03 & 0 & 139\end{array}$

Best Dauble Cauple:Mo=3 $5 * 10 * * 17$ NP1: Strike $=274$ Dip $=90$ SIip $=180$ NP2: $\quad 4 \quad 90$

$\begin{array}{llllllll}5 & 18 & 05 & 38.29 & 24.087 \mathrm{~N} & 122.278 \mathrm{E} & 47 \mathrm{~km}\end{array}$

$5.2 \mathrm{mb}$ ( 41 obs.)

TAIWAN REGION

CENTROID, MOMENT TENSOR

(HRV)

Data Used: GDSN

L.P.B : 9S, $15 \mathrm{C}$

Centraid Lacotian:

$18: 05: 38.90 .8$

Lat $23.93 \mathrm{~N} 0.07$ Lan $122.25 \mathrm{E} 0.18$

Dep 69.76 .4 Holf-durotian 1.5

Principol Axes:

Scale $10 * 16 \mathrm{Nm}$

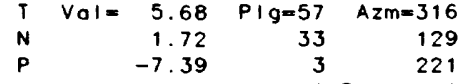

Best Dauble Couple:Mo=6.5*10**16 NP1: Strike=341 Dip=51 SI i $p=134$ $\begin{array}{llll}\text { NP2: } & 103 & 56 & 49\end{array}$

$\begin{array}{lllllll}15 & 18 & 16 & 16.87 & 1.550 \mathrm{~N} & 127.268 \mathrm{E} & 106 \mathrm{~km}\end{array}$ $5.5 \mathrm{mb}(28$ abs.

HALMAHERA

CENTROID, MOMENT TENSOR

(HRV)

L.P. B. 16S, 28C

Centroid Lacation

Origin Time

$18: 16: 16.90 .8$

Lat $1.75 \mathrm{~N} 0.08$ Lan $127.24 \mathrm{E} 0.08$

Dep $80.94 .9 \mathrm{Half}$-duratian 1.8

Principal Axes:

Scale $10 * * 16 \mathrm{Nm}$

T $\quad V a l=10.92 \quad P \mid g=60 \quad$ Azm $=215$

$\begin{array}{rrrr}N & -0.54 & 30 & 39 \\ P & -10.38 & 2 & 308\end{array}$

Best Double Cauple:Ma=1.1*10**17

NP 1: Strike $=11$ Dip=51 SI i $p=50$

NP2: $\quad 244 \quad 54 \quad 128$

$\begin{array}{llllllll}15 & 19 & 24 & 26.35 & 43.212 \mathrm{~N} & 147.879 \mathrm{E} & 33 \mathrm{~km}\end{array}$ 5. $2 \mathrm{mb}$ ( 62 abs.) 4.9Msz ( 4 abs.) KURIL ISLANDS

CENTROID, MOMENT TENSOR

(HRV)

Dato Used: GDSN

L.P. B : 14S, 25C

Centraid Location:

$19: 24: 31.5 \quad 0.7$

$54 \mathrm{~N} 0.10$ Lon $147.46 \mathrm{E} 0.18$

Dep $15.0 \mathrm{FIX} \mathrm{Holf-durotion} 1.4$

Principol Axes:

Scale $10 * 16 \mathrm{Nm}$

T $\quad V a l=8.24 \quad P$ Ig $=57 \quad$ Azm $=292$

$\begin{array}{rrrr}N & -1.00 & B & 34\end{array}$

Best Dauble Cauple:Ma=7.7*10**16

NP1: Strike $=243$ Di $p=15$ SI i $p=120$

NP2: $32 \quad 77$

$\begin{array}{llllll}15 & 21 & 23 & 51.46 & 45.472 \mathrm{~N} & 151.693 \mathrm{E} \quad 33 \mathrm{~km}\end{array}$ 5.3mb ( 47 abs.) 4.9Msz ( 12 abs.) KURIL ISLANDS

CENTROID. MOMENT TENSOR

(HRV)

Dota Used: GDSN

.P.B.: 16S, $28 \mathrm{C}$

Centraid Lacation:

Origin Time $21: 23: 53.4 \quad 0.6$ Lot $45.63 \mathrm{~N} 0.08$ Lon $152.26 \mathrm{E} \quad 0.13$ Dep $15.0 \mathrm{FIX} \mathrm{Half-duration} 1.5$ Principol Axes:

Scale $10 * 16 \mathrm{Nm}$

$T$ Val $=9.06 \quad$ PIg $=70 \quad$ Azm $=270$

$\begin{array}{llll}N & 0.38 & 12 & 36\end{array}$

$\begin{array}{cccr}P & -9.44 & 16 & 130 \\ \text { Best Double Cauple:Mo=9.3*10**16}\end{array}$ NP 1 : Strike $=237$ Di $p=31$ SI i $p=114$

NP2: $\quad 30 \quad 62 \quad 76$

$\begin{array}{lllllll}15 & 23 & 34 & 33.65 & 9.803 \mathrm{~S} & 159.531 \mathrm{E} & 24 \mathrm{~km}\end{array}$ $5.9 \mathrm{mb}$ ( 48 obs.) $5.9 \mathrm{Msz}$ ( 21 abs.) SOLOMON ISLANDS

FAULT PLANE SOLUTION: P-WOVES NP1: Strike $=95$ Dip $=85$ SIip $=16$ NP2: $\quad 4 \quad 74 \quad 175$ 
Principol Axes:

$$
\begin{array}{rrr}
T & P I g=15 & A z m=320 \\
P & 8 & 228
\end{array}
$$

Comment: The focol mechonism is

moderotely well cantrolled and corresponds ta strike-slip

foulting with o moderote

reverse component. The

preferred foult plone is not determined.

RADIATED ENERGY

No. of sto: 8 Focol mech. M Energy MOMENT TENSOR SOLUTION

Dep 9 No. of sta: 11 Principol Axes:

Scole $10 * 18 \mathrm{Nm}$

$$
\text { I } \quad \mathrm{O} O \mathrm{l}=1.61 \quad \mathrm{Plg}=27 \quad \text { Azm }=307
$$$$
\begin{array}{llll}
N & 0.03 & 33 & 56
\end{array}
$$

Best Double Couple:Mo=1.6*10**18 NP 1: Strike=349 Di $p=35$ Si i $p=-161$ NP2: $243 \quad 79 \quad-57$

CENTROLD, MOMENT TENSOR (HRV)

Dota Used: GDSN

L.P.B.: 16S, 38C

Centraid Lacotion:

Origin Time 23:34:40.3 0.3 Lot $9.94 \mathrm{~S} 0.03$ Lan $159.56 \mathrm{E} \quad 0.04$ Dep 28.12 .5 Holf-durotion 3.3 Principol Axes:

Scale $10 * 17 \mathrm{Nm}$

$T \quad$ Vol $=7.45 \quad \mathrm{Plg}=27 \quad$ Azm=332

$\begin{array}{rrrr}N & 1.93 & 29 & 79 \\ P & -9.38 & 48 & 207\end{array}$

Best Double Cauple: Mo=8.4*10**17 NP 1: Strike $=15$ Dip=32 SIip=-158 NP2: $\quad 266 \quad 79 \quad-60$

$\begin{array}{lllllll}16 & 12 & 21 & 10.74 & 43.555 \mathrm{~N} & 127.632 \mathrm{~W} & 10 \mathrm{~km}\end{array}$ $5.3 \mathrm{mb}$ ( 54 obs.) 5.3Msz ( 11 abs.) OFF COAST OF OREGON

CENTROID, MOMENT TENSOR (HRV)

Doto Used: GDSN

L.P.B.: 15S, 30C

Centroid Locotion.

Origin Time $12: 21: 13.40 .6$

Lot $43.38 \mathrm{~N} 0.07$ Lon $128.12 \mathrm{~W} 0.05$ Dep $15.0 \mathrm{FIX} \mathrm{Holf-durotion} 2.4$ Principol Axes:

Scole $10 * * 17 \mathrm{Nm}$

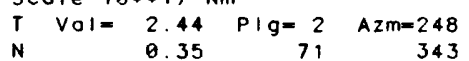

$\begin{array}{llll}P & -2.80 & 18 & 158\end{array}$

Best Double Couple:Mo=2.6*10**17

NP 1 : Strike $=295$ Dip $=76$ SI i $p=-168$

NP2

$202 \quad 78$

$-14$

$\begin{array}{llllllll}16 \quad 16 & 01 & 00.45 & 45.394 \mathrm{~N} & 151.772 \mathrm{E} & 33 \mathrm{~km}\end{array}$ $5.5 \mathrm{mb}$ ( 54 obs.) $4.9 \mathrm{Msz}$ ( 7 obs.) KURIL ISLANDS

CENTROID, MOMENT TENSOR (HRV)

Doto Used: GOSN

L.P.B.: 14S, $24 \mathrm{C}$

Centraid Lacation:

Origin rime $16: 01.4 .41 .1$

Lot $45.73 \mathrm{~N} 0.07$ Lon $152.24 \mathrm{E} 0.11$

Dep 33.34 .8 Holf-duration 1.6 Principol Axes:

Scole $10 * 16 \mathrm{Nm}$

I $\quad \mathrm{VOI}=6.89 \quad \mathrm{PIg}=78 \quad \mathrm{Azm}=293$

$\begin{array}{rrrr}N & 2.46 & 3 & 36 \\ P & -9.35 & 11 & 126\end{array}$

Best Double Couple:Mo=8.1*10**16

NP 1 : Strike=220 Dip=34 SIip= 95

$\begin{array}{llll}\text { NP2 : } & 34 & 56 & 87\end{array}$

$\begin{array}{lllllllll}16 & 17 & 22 & 52.97 & 56.329 S & 139.125 W & 10 \mathrm{~km}\end{array}$ $5.8 \mathrm{mb}$ ( 7 ODS.) $5.8 \mathrm{MSz}$ ( 5 ObS.) SOUTH PACIFIC CORDILLERA

FAULT PLANE SOLUTION: P-WOVES

NP1: Strike $=10$ Dip=65 Slip=-179

NP2:

280

89

$-25$

Principol Axes:

T PIg=17 Azm=328

Comment: The focal mechonism is poorly contralled and

correspands to strike-slip

foulting with o moderote

normol component. The

preferred foult plone is not

determined.
CENTROID, MOMENT TENSOR

(HRV)

DOta Used: GDSN

Centroid Locotion:

Origin Time

$17: 23: 0.90 .2$

Lot $56.48 \mathrm{~S} 0.03$ Lon $138.91 \% 0.05$

Dep 15.0 FIX Holf-durotion 3.4

Principol Axes:

Scole $10 * 17 \mathrm{Nm}$

$T \quad$ Vol $=6.96 \quad$ PIg $=2 \quad$ Azm $=335$

$\begin{array}{llll}N & 2.67 & 70 & 71\end{array}$

Best Double Couple:Mo=8.3*10**17

NP1:Strike $=21$ Dip=74 Slip=-167

$\begin{array}{llll}\text { NP2: } & 288 & 78 & -16\end{array}$

$170504 \quad 35.94 \quad 57.886 \mathrm{~N} \quad 122.018 \mathrm{E} \quad 31 \mathrm{~km}$ $5.6 \mathrm{mb}$ ( $85 \mathrm{abs}$ ) $5.9 \mathrm{Msz}$ ( $19 \mathrm{abs}$ ) EASTERN USSR

CENTROID, MOMENT TENSOR (HRV)

Doto Used: GDSN

L.P.B.: 14S, 34C

Centroid Lacotion:

Origin Time

$05: 04: 39.8 \quad 0.2$

Lot $57.07 \mathrm{~N} 0.03$ Lon $122.03 \mathrm{E} 0.06$

Dep 29.0 FlX Holf-durotion 3.5

Principol Axes:

Scole $10 * * 17 \mathrm{Nm}$

$T \quad V 0 I=9.05 \quad P / g=19 \quad$ Azm $=334$

$\begin{array}{rrrr}N & 1.86 & 45 & 83\end{array}$

Best Double Couple: $M O=1.0 * 10 *+18$

NP1: Strike $=18$ Dip=48 Si ip $=-163$

$\begin{array}{llll}\text { NP2: } & 277 & 77 & -43\end{array}$

$17153142.50 \quad 8.660 \mathrm{~S} \quad 109.294 \mathrm{~W} \quad 10 \mathrm{~km}$ 4.9mb ( 6 obs.) $5.0 M S z$ ( 1 abs.) NORTHERN EASTER I. CORDILLERA

CENTROID, MOMENT TENSOR (HRV)

Doto Used: GOSN

L.P.B.: 13S, $22 \mathrm{C}$

Centroid Locotion:

Origin Iime $\quad 15: 31: 43.71 .2$

Lot 9.1650 .88 Lon $109.38 w 0.09$

Dep 15.2 Flx Holf-durotion 1.6

Principol Axes:

Scole $10 * * 16 \mathrm{Nm}$

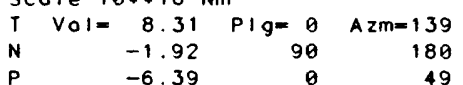

Best Double Couple: $M 0=7.4 * 10 * 16$

NP1: Strike=184 Dip=90 Si i $=180$

NP2

$274 \quad 99$

$17 \quad 16 \quad 1254.5162 .1045 \quad 154.634 \mathrm{E} \quad 10 \mathrm{~km}$ $5.0 \mathrm{mb}$ ( 5 obs.) 5.3MSZ ( 4 obs.) BALLENY ISLANDS REGION

CENTROID, MOMENT TENSOR

(HRV)

Doto Used: GDSN

L.P.B.: 13S. $29 \mathrm{C}$

Centraid Lacotion:

Origin Time $16: 13: 180.3$ Lot 62.345 FIX; Lon 153.74E FIX Dep 15.0 FIX Holf-durotion 2.8 Principal Axes:

Scole $10 *+17 \mathrm{Nm}$

I $\mathrm{VOl}=4.48 \mathrm{Plg}=2 \quad \mathrm{~A}=\mathrm{m}=199$

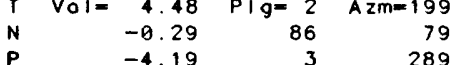

Best Double Couple:Mo=4.3*10**17

NP1: Strike=334 Dip=86 Si $\mathrm{S}=-1$

NP2: $\quad 64 \quad 89 \quad-176$

$\begin{array}{llllllll}17 & 18 & 16 & 14.62 & 7.825 \mathrm{~N} & 127.025 \mathrm{E} \quad 48 \mathrm{~km}\end{array}$

4.9mb ( 6 obs.) 4.0MSZ ( 1 obs.)

PHILIPPINE ISLANDS REGION

CENTROID. MOMENT TENSOR

(HRV)

Doto Used: GDSN

L.P.B.: 11s, $17 \mathrm{C}$

Centraid Lacation

Origin Time $18: 16: 10.60 .8$

Lot $8.21 \mathrm{~N} 0.12$ Lan $125.88 E 0.16$

Dep 15.0 FIX Holf-durotion 1.5 Principol Axes:

Scole $10 * 17 \mathrm{Nm}$

$T \quad V O I=1.36 \quad P / g=38 \quad$ Azm=382

$\begin{array}{rrrr}N & -0.12 & 33 & 181 \\ P & -1.25 & 35 & 65\end{array}$

Best Double Couple:Mo=1.3*10**17

NP1: Strike= 96 Dip=33 Slip=

NP2: $\quad 3 \quad 88 \quad 123$

$\begin{array}{llllll}18 & 22 & 00 & 39.63 & 17.852 \mathrm{~N} & 147.009 \mathrm{E} \quad 56 \mathrm{~km}\end{array}$
$5.5 \mathrm{mb}(33$ obs.) $5.1 \mathrm{Msz}$ ( 17 Obs.)

MARIANA ISLANDS REGION

CENTROID, MOMENT TENSOR

(HRV)

Doto Used: GDSN

L.P.B.: 16S, 30C

Centraid Locotion:

Origin Time

$22: 00: 41.10 .3$

Lot $17.89 \mathrm{~N} 0.04$ Lan $147.34 \mathrm{E} 0.03$

Dep 15.0 BDY Half-durotion 2.2 Principol Axes:

Scole $10 * * 17 \mathrm{Nm}$

T $\quad V_{0}=2.31 \quad$ Plg $=76 \quad$ A $m=250$

$\begin{array}{rrrr}N & 0.60 & 5 & 1 \\ P & -2.91 & 13 & 93\end{array}$

Best Double Couple:Ma=2.6*10**17 NP1: Strike $=190$ Dip=32 Si ip $=100$ NP2: $\quad 358 \quad 58 \quad 84$

$19022156.38 \quad 54.305 \mathrm{~N} \quad 165.574 \mathrm{~W} 104 \mathrm{~km}$ 6. $1 \mathrm{mb}$ ( 100 ODs.)

FOX ISLANDS, ALEUTIAN ISLANDS

FAULT PLANE SOLUTIDN: P-WOVES

NP 1:Strike $=65$ Dip=83 S1 ip= -83

NP2 :

200

$10-135$

Principol Axes

$T \quad P 1 g=38 \quad A z m=149$

Comment: The focol mechonism is

poarly cantrolled and

correspands to normol foulting

with o smoll right-loterol

strike-slip component. The

preferred foult plone is NP1.

RADIATED ENERGY

No. of sto: 8 focal mech.

Energy

8 Focal mech. C
$0.2 \pm 0.1 * 10 * 14 \mathrm{Nm}$

MOMENT TENSOR SOLUTION

DEP 106 No of $s t 0: 18$

Principol Axes:

Scole $10 *+18 \mathrm{Nm}$

T $\quad V_{0 l}=3.05 \quad \mathrm{Plg}=41 \quad$ AZm=149

$\begin{array}{rrrr}N & -0.59 & 2 & 58 \\ P & -2.46 & 49 & 326\end{array}$

Best Double Couple: $M_{0}=2.8 * 10 * 18$ NP1: Strike=263 Dip= 4 S1ip= -65

NP2: 58 B 86

CENTROID, MOMENT TENSOR (HRV)

Doto Used: GOSN

L.P.B.: 17S, 44C M.W.: 11S, $16 \mathrm{C}$

Centroid Locotion:

Origin Time

$02: 22: 1.00 .1$

Lot $54.25 \mathrm{~N} 0.01$ Lon $165.65 \mathrm{~W} 0.02$

Dep 103.91 .0 Holf-durotion 4.7

Principol Axes:

Scole $10 * 18 \mathrm{Nm}$

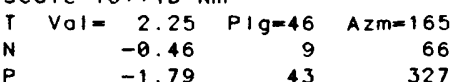

Pest Double Couple:Mo=2 $2.0 * 10 *+18$

NP1 : Strike $=344$ Dip=9 Slip= 8

NP2: $\quad 246 \quad 89 \quad 99$

$\begin{array}{llllll}19 & 1150 & 54.38 & 24.8395 \quad 70.019 W \quad 52 \mathrm{~km}\end{array}$ $5.5 \mathrm{mb}$ ( 49 ODS.)

NEAR COAST OF NORTHERN CHILE

CENTROID, MOMENT TENSOR (HRV)

Doto Used: GDSN

L.P.B.: 14S, 32C

Centraid Locotion

Origin rime $11: 51: 130.3$

Lot 24.865 FIX; Lon 70.07W Fix

Dep 50.45 .0 Holf-durotion 2.1

Principol Axes:

Scole $10 *+17 \mathrm{Nm}$

$\begin{array}{rrrr}T & \text { Vol }= & 1.61 & \text { Plg=51 } \\ N & -0.15 & 18 & A z m= \\ P & -1.45 & 33 & 173 \\ P & -1.45\end{array}$

Best Double Couple:Mo=1.5*10*17

NP1:Strike $=52$ Dip=21 S1ip=151

$\begin{array}{llll}\text { NP2: } & 169 & 80 & 71\end{array}$

$20160143.6130 .508 \mathrm{~s} \quad 178.270 \mathrm{~W} 29 \mathrm{~km}$ $5.7 \mathrm{mb}$ ( 34 obs.) $5.9 \mathrm{Msz}$ ( 23 obs.) KERMADEC ISLANDS

FAULT PLANE SOLUTION: P-WOVES

NP1:Strike $=10$ Dip=68 Siip= 90

NP2: $\quad 190 \quad 22 \quad 90$

Principol Axes:

$\begin{array}{rrr}T & P 1 g=67 & A z m=280 \\ P & 23 & 100\end{array}$

Comment: The focol mechonism is

poorly contralled ond

corresponds to reverse 
foulting. The preferred foult plane is NP2

RADIATED ENERGY

No. of sto: 8 Focal mech. C Energy $\quad 0.5 \pm 0.1 * 10 * 13 \mathrm{Nm}$ MOMENT TENSOR SOLUTION

Dep 35

Scole $10 * * 18 \mathrm{Nm}$

$T$ VOI $=2.64 \quad$ PIg=69 Azm=282

$\begin{array}{lrrr}N & 0.05 & 1 & 15\end{array}$

Best Double Couple: $M O=2.7 * 10 * 18$ NP1: Strike=197 Dip=24 SIip= 93 NP2:

CENTROID, MOMENT TENSOR (HRV) Doto Used: GDSN

L.P.B.: 175, $48 \mathrm{C}$ M.W.: $115,21 \mathrm{C}$ Centraid Locotion:

Origin Time 16:01:53.8 0.2 Lot $30.64 \mathrm{~S} 0.01$ Lon 178.15W 0.01 Dep 49.20 .8 Holf-duration 5.1 Principol Axes

Scole $10 * * 18 \mathrm{Nm}$

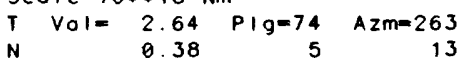

P $\quad-3.02 \quad 15 \quad 104$

Best Double Couple:Mo=2.8*10**18 NP1: Strike=282 Dip=31 Slip=101 NP2 :

$$
60
$$

84

$\begin{array}{lllllllll}20 & 20 & 44 & 02.26 & 39.553 \mathrm{~N} & 40.172 \mathrm{E} & 38 \mathrm{~km}\end{array}$ $5.0 \mathrm{mb}$ ( 56 obs.) $5.1 \mathrm{Msz}$ ( 15 obs.) TURKEY

CENTROID, MOMENT TENSOR (HRV) Dato Used: GDSN

L.P.B.: 16S, $31 \mathrm{C}$

Centraid Lacation:

Origin Time 20:44:1.60.5 Lot $38.87 \mathrm{~N} 0.06$ Lon $40.01 E 0.05$ Dep 15.0 Fix Half-duration 2.2 Principol Axes:

Scale $10.17 \mathrm{Nm}$

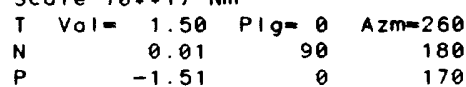

Best Double Couple: $M a=1.5 * 10 * * 17$ NP1: Strike $=305$ Dip $=90$ Slip $=180$ NP2 :

$35 \quad 90$

$210403 \quad 42.23 \quad 31.1115 \quad 177.801 \mathrm{w} 50 \mathrm{~km}$ $5.3 \mathrm{mb}$ ( 5 abs.)

KERMADEC ISLANDS REGION

CENTROID, MOMENT TENSOR

Doto Used: GDSN

L.P.B.: $14 \mathrm{~S}, 22 \mathrm{C}$

Centraid Lacation

Origin Time $\quad 04: 03: 43.90 .6$ Lot 31.2650 .06 Lan 177.53w 0.07 Dep 25.74 .0 Holf-duration 1.8 Principol Axes:

Scale $10 * * 16 \mathrm{Nm}$

$T \quad V O I=10.38 \quad$ PIg=68 Azm=281

$\begin{array}{rrrr}N & 1.65 & 0 & 191 \\ P & -12.04 & 22 & 101\end{array}$

Best Double Cauple:Ma=1,1*10*17

NP1: Strike=190 Dip=23 Siip= 89

NP2: $\quad 11 \quad 67 \quad 90$

$21 \quad 1307 \quad 34.37 \quad 15.988 \mathrm{~S} \quad 178.262 \mathrm{E} \quad 33 \mathrm{~km}$ $4.8 \mathrm{mb}$ ( 9 obs.)

FIJI ISLANDS

CENTROID, MOMENT TENSOR (HRV)

Doto Used: GDSN

L.P.B.: 11S, 22C

Centraid lacation

Origin Time $\quad 13: 07: 35.01 .8$

Lot $16.27 \mathrm{~S} 0.10$ Lan 178.26E 0.13

Dep 15.0 FIX Half-duration 1.5

Principol Axes:

Scole $10 * * 16 \mathrm{Nm}$

$T \quad V O I=6.81 \quad P I g=0 \quad$ Azm $=113$

$\begin{array}{rrrr}N & -2.56 & 0 & 23 \\ P & -4.25 & 90 & 180\end{array}$

Best Double Couple: $M_{0}=5.5 * 10 * 16$ NP1 : Strike=203 Dip=45 SIip= -90 NP2: $\quad 23 \quad 45 \quad-90$

$\begin{array}{lllllllll}21 & 19 & 23 & 41.18 & 2.586 \mathrm{~N} & 126.702 \mathrm{E} & 58 \mathrm{~km}\end{array}$ 5. $3 \mathrm{mb}$ ( 29 obs.)

MOLUCCA PASSAGE

CENTROID, MOMENT TENSOR

(HRV)
L.P. $125,24 \mathrm{C}$

Centroid Lacatian

Origin Time

$19: 23: 47.9 \quad 0.8$

Lot $2.81 \mathrm{~N} 0.07$ Lan 126.49E 0.12

Dep $15.0 \mathrm{FIX} \mathrm{Half-duration} 1.9$

Principal Axes:

Scale $10 * 17 \mathrm{Nm}$

$T \quad$ VOI $=1.58 \quad \mathrm{Plg}=69 \quad$ Azm $=97$

$\begin{array}{rrrr}N & 0.04 & 9 & 342 \\ P & -1.62 & 19 & 249\end{array}$

Best Double Couple:Mo=1.6*10**17

NP1:Strike=323 Dip=27 S1ip=69

NP2 : $\quad 166 \quad 65 \quad 100$

$\begin{array}{llllllll}21 & 19 & 30 & 07.19 & 2.545 \mathrm{~N} & 126.537 \mathrm{E} & 77 \mathrm{~km}\end{array}$ $5.4 \mathrm{mb}$ ( 13 abs.)

MOLUCCA PASSAGE

CENTROID. MOMENT TENSOR

(HRV)

Dota Used: GOSN

L.P.B : $11 \mathrm{~S}, 14 \mathrm{C}$

Centroid Lacation

Origin Time

$19: 30: 14.7 \quad 1.4$

Lot 2.53N FIX: Lon 126.56E FIX

Dep 15.0 FIX Holf-durotion 1.6

Principol Axes:

Scole 10**17 $\mathrm{Nm}$

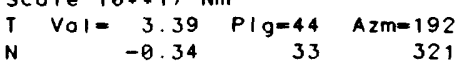

$\begin{array}{rrrr}N & -0.34 & 33 & 321 \\ P & -3.05 & 28 & 71\end{array}$

Best Double Couple:Ma=3.2*10**17

NP1: Strike=211 Dip=34 SIip= 163

NP2: $\quad 315 \quad 80 \quad 57$

$\begin{array}{lllllll}21 & 21 & 56 & 48.63 & 17.952 S \quad 178.593 w \quad 584 k m\end{array}$

$5.7 \mathrm{mb}$ ( 49 obs.)

FIJI ISLANDS REGION

FAULT PLANE SOLUTION: P-Waves

NPI: Strike $=302$ Dip=68 Slip= -32

NP2: $\quad 45 \quad 61 \quad-155$

Principal Axes:

$\begin{array}{lrr}T & P I g=5 & A 2 m=355 \\ P & 38 & 261\end{array}$

Comment: The focal mechonism is

paarly cantralled and

corresponds to strike-slip

foulting with a large normol

component. The preferred foult

Plane is not determined.

RADIATED ENERGY

No. of sta: 8 Facal mech.

Energy $0.2 \pm 0.1 * 10 * 14 \mathrm{Nm}$

MOMENT TENSOR SOLUTION

Dep 599 No. of sto: 9

Principal Axes

Scale $10 * * 17 \mathrm{Nm}$

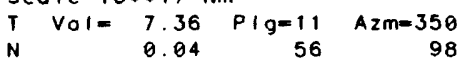

$\begin{array}{lrrr}N & 0.04 & 56 & 98 \\ P & -7.48 & 32 & 253\end{array}$

Best Double Couple:Ma=7.4*10**17

NP1: Strike $=36$ Dip $=59$ SI i $p=-164$ NP 2 :

$$
\begin{array}{rrr}
36 & \text { D I } p=59 & \text { SI i } p=-164 \\
298 & 77 & -32
\end{array}
$$

CENTROID, MOMENT TENSOR

(HRV)

Doto Used: GDSN

L.P.B.: 15S, $34 \mathrm{C}$

Centroid Location

Origin Time 21:56:57.2 0.4 Lat 17.6450 .03 Lan 178.91w 0.03

Dep 599.21 .7 Holf-duration 3.2 Principol Axes:

Scole $10 * * 17 \mathrm{Nm}$

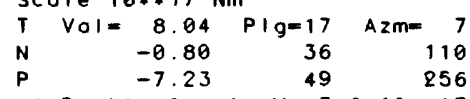

Best Double Couple:Mo=7.6*10*17

NP1: Strike $=56$ D i $p=42$ Si i $p=-151$

NP2: $\quad 304 \quad 71 \quad-52$

$22020204.00 \quad 59.802 \mathrm{~N} \quad 29.809 \mathrm{~W} \quad 10 \mathrm{~km}$ $5.0 \mathrm{mb}$ ( 46 Obs.) 5.OMsz ( 8 abs.) NORTH ATLANTIC OCEAN

CENTROID, MOMENT TENSOR

(HRV)

Doto Used: GOSN

L.P.B.: 7S, $12 \mathrm{C}$

Centraid Lacotion

Origin Time $\quad 02: 01: 51.20 .5$

Lat $59.78 \mathrm{~N} F I X$ iLon $29.82 \mathrm{~W}$ FIX

Dep $15.0 \mathrm{FIX}$ Holf-durotion 1.7

Principal Axes:

Scole $10 * * 16 \mathrm{Nm}$

$\begin{array}{rrrr}T & V a l=7.22 & P l g=0 & A z m=187 \\ N & 2.54 & 90 & 180 \\ P & -9.76 & 0 & 97\end{array}$

1

$22110806.07 \quad 53.597 \mathrm{~N} 161.657 \mathrm{E} \quad 41 \mathrm{~km}$ $5.0 \mathrm{mb}$ ( 53 obs.) $5.2 \mathrm{Msz}$ ( 14 obs.) OFF EAST COAST OF KAMCHATKA

CENTROID, MOMENT TENSOR

Dota Used: GDSN

L.P.B.: 13S. $21 \mathrm{C}$

Centraid Lacation

Origin Time

$11: 08: 11.90 .6$

Lat $53.75 \mathrm{~N} \theta 10$ Lan $162.02 \mathrm{E} 0.14$

Dep 15.0 FIX Half-duration 1.5 Principal Axes:

Scale $10 * * 16 \mathrm{Nm}$

$T \quad V_{0 I}=8.45 \quad \mathrm{Plg}=60 \quad$ Azm $=274$

$\begin{array}{rrrr}N & 0.25 & 6 & 14 \\ P & -8.70 & 30 & 107\end{array}$

Best Double Couple:Mo=8.6*10*16 NP 1: Strike $=214$ Di $p=16$ SI ip $=111$ $\begin{array}{llll}\text { NP2: } & 13 & 75 & 84\end{array}$

$\begin{array}{llllllll}23 & 10 & 54 & 46.32 & 52.341 \mathrm{~s} \quad 160.568 \mathrm{E} \quad 10 \mathrm{~km}\end{array}$ $6.4 \mathrm{mb}$ ( 28 obs.) $8.2 \mathrm{Msz}(12$ abs.) MACOUARIE ISLANDS REGION

FAULT PLANE SOLUTION: P-Waves

NP 1: Strike $=120$ Dip=85 SI

NP2:

210

Principol Axes:

T PIg=4 Azm=345

Comment: The focal mechanism is

moderately well cantralled and

corresponds to strike-slip

faulting. The preferred foult

plone is not delermined.

RADIATED ENERGY

No. of sto: 4 Focal mech.

Energy $\quad 1.0 \pm 0.1 * 10 * * 17 \mathrm{Nm}$

MOMENT TENSOR SOLUTION

Dep 15 No. of sta: 5

Principal Axes:

Scale 10**21 $\mathrm{Nm}$

$T \quad V_{01}=1.51 \quad$ Plg $=0 \quad$ Azm $=354$

$\begin{array}{rrrr}N & 1.51 & P l g=0 & A 2 m=354 \\ P & 0.04 & 89 & 105\end{array}$

Best Double Cauple:Mo=1.5*10**21 NP1: Strike $=39$ Dip=89 Si ip $=-179$ NP2 : 309

CENTROID, MOMENT TENSOR (HRV)

Doto Used: GDSN

L.P.B.: 1S, 3C M.W.: 215,610 Centraid Lacatian:

Origin Time

$10: 55: 12.20 .2$

Lat $52.15 \mathrm{~S} 0.01$ Lan 160.41E 0.02

Dep $15.0 \mathrm{FIX}$ Holf-duration 18.0 Principal Axes:

Scale $10 *+21 \mathrm{Nm}$

$T$ Vol $=1.39 \quad$ PIg=21 Azm $=352$

$\begin{array}{rrrr}N & -0.06 & 67 & 151 \\ P & -1.32 & 8 & 259\end{array}$

Best Double Cauple:Mo=1.4*10**21 NP 1 : Strike $=34$ Dip=69 Si ip $=170$ NP2

MOMENT TENSOR

Half-durotian 29.0 Best Dauble Cauple:Ma=2,1*10**21 NP1:Strike $=36$ Dip=85 Siip $=197$ NP2: $\quad 305 \quad 73 \quad-6$

$\begin{array}{lllllll}23 & 17 & 11 & 42.85 & 51.8865 & 160.563 \mathrm{E} & 10 \mathrm{~km}\end{array}$ $5.9 \mathrm{mb}$ ( 30 Obs.) 6.OMsz ( 12 obs.) NORTH OF MACOUARIE ISLAND

FAULT PLANE SOLUTION: P-Woves

NP1 : Strike $=128$ Dip=89 SIip=

NP2: 218

Principol Axes:

$T \quad P I g=1$ Azm=353

Comment: The focal mechanism is moderately well controlled and corresponds to strike-slip

foulting. The preferred foult

plone is not determined.

$\begin{array}{lllllll}2408 & 05 & 47.61 & 3.7485 & 123.256 \mathrm{E} & 22 \mathrm{~km}\end{array}$ $5.6 \mathrm{mb}$ ( 29 obs.) $5.0 \mathrm{Msz}$ ( 13 obs.) SULAWES I

CENTROID, MOMENT TENSOR

(HRV)

Dota Used: GDSN

L.P.B.: 12S, $20 \mathrm{C}$

Centroid Locotion

Origin Time

$08: 05: 50.90 .6$ 
Lat $4.045 \quad 0.08$ Lan $123.32 \mathrm{E} \quad 0.09$ Dep 15.0 Bor Half-duratian 2.8 Principal Axes:

Scole $10 * 17 \mathrm{Nm}$

$$
\begin{array}{rrrr}
i & V a l=6.56 & P \mid g=57 & A 2 m=213 \\
N & 0.01 & 20 & 337 \\
P & -6.57 & 25 & 77
\end{array}
$$

Best Double Couple:Mo*6.6*10**17 NP1: Strike=204 Dip=27 S1ip=140 NP2 :

330

73

$\begin{array}{llllllll}24 & 13 & 31 & 14.48 & 56.177 \mathrm{~N} & 164.264 \mathrm{E} & 19 \mathrm{~km}\end{array}$ $5.9 \mathrm{mb}$ ( 87 obs.) 6.1Msz ( 35 abs.) KOMANDORSKY ISLANOS REGION

FAULT PLANE SOLUTION: P-WaVES NP 1: Strike $=300$ Dip=90 SIip=177 NP2 30 87 360 Principol Axes:

$$
\begin{array}{lrr}
T & P I g=2 & A z m=255 \\
P & & 345
\end{array}
$$

Camment: The focal mechanism is moderately well contralled and correspands to strike-slip faulting. The preferred foult plane is not determined.

RADIATED ENERGY

Na. of sta: 10 Facal mech. Energy $\quad 0.4 \pm 0.1 * 10 * 15 \mathrm{Nm}$ MOMENT TENSOR SOLUTION

DeP 15 Na of sta: 15 Principol Axes:

Scale $10 * 18 \mathrm{Nm}$

$T \quad V a l=3.14 \quad P I g=6 \quad$ A2m $=244$

$\begin{array}{rrrr}N & 0.52 & 78 & 124 \\ P & -3.66 & 10 & 335\end{array}$

Best Double Couple: $M a=3.4 * 10 * 18$ NP1:Strike $=19$ Dip $=79$ Slip $=-3$ NP2: $\quad 110 \quad 87 \quad-169$

CENTROID, MOMENT TENSOR (HRV)

Data Used: GDSN

L.P.B.: 17S, $49 \mathrm{C}$

Centroid Locotion:

Origin Time $\quad 13: 31: 20.40 .2$

Lat $56.42 \mathrm{~N} 0.02$ Lan 164.05E 0.04 Dep 43.62 .5 Half-duration 5.6 Principal Axes:

Scale $10 * * 18 \mathrm{Nm}$

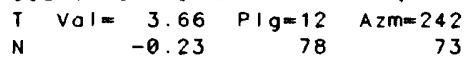

$\begin{array}{lrrr}\mathrm{N} & -0.23 & 78 & 73 \\ \mathrm{P} & -3.43 & 2 & 332\end{array}$

Best Double Couple:Mo*3.5*10**18

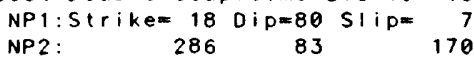

$\begin{array}{llllllll}15 & 43 & 34.39 & 56.173 \mathrm{~N} & 164.185 \mathrm{E} \quad 36 \mathrm{~km}\end{array}$ $5.5 \mathrm{mb}(86$ Obs.) $5.4 \mathrm{Msz}(18 \mathrm{abs}$.) KOMANDORSKY ISLANDS REGION

CENTROID, MOMENT TENSOR (HRV)

Data Used: GOSN

L.P.B.: 15S, 35C

Centraid Lacation:

Origin Time $\quad 15: 43: 38.20 .3$

Lat $56.42 \mathrm{~N} 0.03$ Lan 164.09E 0.06

Dep 43.03 .7 Half-ouratian 3.2 Principal Axes:

Scale $10 *+17 \mathrm{Nm}$

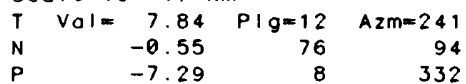

Best Dauble Couple:Ma*7.6*10**17

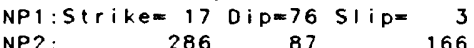

$$
\begin{array}{llll}
\text { NP2: } & 286 & 87 & 166
\end{array}
$$

$24175907.05 \quad 55.472 \mathrm{~N} \quad 35.140 \mathrm{~W} \quad 10 \mathrm{~km}$ $4.9 \mathrm{mb}$ ( 43 abs.) $5.0 \mathrm{MSZ}$ ( 4 abs.) NORTH ATLANTIC OCEAN

CENTROID, MOMENT TENSOR (HRV)

DOtO Used: GOSN

L.P.B.: 10S, $19 \mathrm{C}$

Centraid Lacation:

Origin Time $\quad 17: 59: 9.60 .5$

Lat $54.72 \mathrm{~N}$ 0.11 Lan $35.25 \mathrm{~W} 0.07$

Dep $15.0 \mathrm{FIX}$ Holf-duration 1.7

Principal Axes:

Scale $10.017 \mathrm{Nm}$

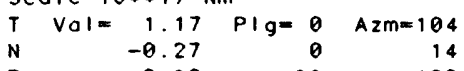

$\begin{array}{llrr}\mathrm{N} & -0.27 & 0 & 14 \\ \mathrm{P} & -0.90 & 90 & 180\end{array}$

Best Double Couple: $M O=1.0 * 10 * 17$

NP 1: Strike=194 Dip=45 SI ip $=-90$

NP2: $\quad 14 \quad 45 \quad-90$

$25005452.13 \quad 52.272 \mathrm{~S} \quad 159.833 \mathrm{E} \quad 10 \mathrm{~km}$
$5.7 \mathrm{mb}$ ( 32 abs.) $5.6 \mathrm{Msz}$ ( 12 obs.) MACOUARIE ISLANDS REGION

CENTROID, MOMENT TENSOR

(HRV)

Data UsEd: GOSN

L.P.B.: 19S, 42C

Centroid Lacation:

Origin Time

$00: 55: 2.6 \quad 0.2$

Lat 51.9050 .03 Lan 159.10E 0.04

Dep 15.0 FIX Half-duratian 3.7

Principal Axes:

Scale 10**17 $\mathrm{Nm}$

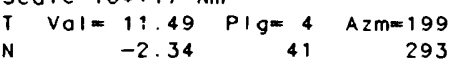

$\begin{array}{llll}N & -9.15 & 49 & 105\end{array}$

Best Double Couple:Ma=1.0*10**18

NP 1: Strike $=255$ D i $p=54$ SI i $p=-144$

$\begin{array}{llll}\text { NP2 : } & 142 & 61 & -41\end{array}$

$\begin{array}{lllllll}25 & 04 & 43 & 10.70 & 52.3345 & 160.748 \mathrm{E} & 10 \mathrm{~km}\end{array}$ $5.5 \mathrm{mb}$ ( 23 ODS.) 4.8MSZ ( 3 ObS.) MACOUARIE ISLANDS REGION

CENTROID, MOMENT TENSOR

(HRV)

Data Used: GDSN

L.P.B.: 13S, $20 \mathrm{C}$

Centraid Location

Origin Time $04: 43: 7.30 .7$

Lat $52.51 \mathrm{~S} 0.14$ Lan $160.84 E 0.22$

Dep 15.0 FIX Half-duration 1.6

Principol Axes:

Scale 10**16 Nm

$\begin{array}{rrrr}T & V a l=6.17 & P \mid g=56 & \text { Azm }=299 \\ N & 3.69 & 8 & 40\end{array}$

$\begin{array}{llll}P & -9.86 & 32 & 135\end{array}$

Best Dauble Cauple:Ma $=8.0 * 10 * 116$ NP1 : Strike $=252$ Dip=14 SI ip= 122 $\begin{array}{lrrr}\text { NP2: } & 39 & 78 & 82\end{array}$

$\begin{array}{lllllllll}25 & 07 & 24 & 15.56 & 54.98 & \mathrm{~S} & 129.20 \mathrm{~W} & 10 \mathrm{~km}\end{array}$ $5.2 \mathrm{mb}$ ( 4 Obs.) $5.1 \mathrm{MSZ}$ ( 2 obs.) SOUTH PACIFIC COROILLERA

CENTROID, MOMENT TENSOR

Data Used: GDSN

L.P. 8.: 16S, $27 \mathrm{C}$

Centraid Lacatian:

Origin Time

07: $24: 25.6 \quad 0.5$

Lat 55.5650 .10 Lan $128.74 \% 0.09$

Dep 15.0 FIX Half-duration 1.7

Principol Axes:

Scale $10 * * 16 \mathrm{Nm}$

$\begin{array}{rrrr}T & V a l= \\ N & 9.25 & P l g=0 & \text { Azm }=156 \\ -0.47 & 90 & 180\end{array}$

$\begin{array}{rrrr}P & -8.78 & 0 & 66 \\ \text { Best Dauble Cauple: } & \text { Ma=9.0*10*.16 }\end{array}$

NP1: Strike $=201$ Dip $=90$ SI i $=180$

NP2: $\quad 291 \quad 90$

$\begin{array}{llllllll}25 & 08 & 06 & 32.64 & 5.850 \mathrm{~S} & 148.300 \mathrm{E} & 112 \mathrm{~km}\end{array}$ $5.9 \mathrm{mb}$ ( 32 abs.)

NEW BRITAIN REGION

FAULT PLANE SOLUTION: P-Waves

NP1: Strike $=215$ Dip=52 SIip $=180$

NP2: 125

$$
\begin{array}{lrr}
T & P I g=26 & \text { Azm }=177 \\
P & 26 & 73
\end{array}
$$

Comment: The facal mechanism is

paarly cantrolled and

carrespands ta strike-slip

faulting with a large norma

campanent. The preferred fault

plane is nat determined.

MOMENT TENSOR SOLUTION

DeP 105 No. af sta: 6

Principal Axes:

Scale 10**17 $\mathrm{Nm}$

$T \quad$ Vol $=4.24 \quad$ PIg=22 A2m=168

$\begin{array}{rrrr}N & 0.01 & 54 & 292 \\ P & -4.24 & 27 & 66\end{array}$

Best Dauble Cauple:Mo=4.2*10**17

NP1: Strike=209 Di $p=54$ Si i $p=-176$

NP2: $\quad 116 \quad 87 \quad-36$

CENTROID, MOMENT TENSOR (HRV)

Data Used: GOSN

L.P.B.: 17S, 30C

Centraid Lacatian:

Origin Time $\quad 08: 06: 38.30 .3$

Lat $5.97 \mathrm{~S} 0.03$ Lon $148.51 \mathrm{E} 0.04$

Dep 96.32 .6 Half-duration 3.4

Principol Axes:

Scole $10 * 17 \mathrm{Nm}$

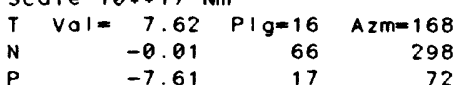

Best Double Couple:Ma=7.6*10**17 NP 1: Strike $=210$ Di $p=66$ SI i $p=-179$

NP2: $\quad 120 \quad 89 \quad-24$

$\begin{array}{lllllll}25 & 09 & 39 & 25.49 & 52.071 \mathrm{~S} & 159.931 \mathrm{E} & 10 \mathrm{~km}\end{array}$ 5.5mb ( 18 abs.) 5.5Msz ( 8 abs.) MACOUARIE ISLANDS REGION

CENTROID, MOMENT TENSOR

(HRV)

Data Used: GOSN

P.B.: 18S, 4

Centraio Locatian:

Origin Time

$09: 39: 34.5 \quad 0.3$

Lat $51.96 \mathrm{~S} \quad 0.04$ Lan 159.22E 0.05

Dep 15.0 FIX Half-duratian 3.0

Principal Axes:

Scale $10 * 17 \mathrm{Nm}$

$T \quad V a l=7.64 \quad P I g=6 \quad$ Azm $=27$

$\begin{array}{llll}N & -2.14 & 57 & 288\end{array}$

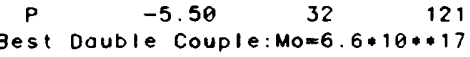

NP1 : Strike $=159$ Dip $=63$ SI ip $=-20$

NP2: $\quad 258 \quad 72 \quad-152$

$\begin{array}{llllll}25 & 1156 & 21.67 & 7.332 \mathrm{~S} & 128.539 \mathrm{E} \quad 171 \mathrm{~km}\end{array}$ $5.5 \mathrm{mb}$ ( 40 abs.)

BANDA SEA

FAULT PLANE SOLUTION: P-Waves

NP1: Strike $=70$ Dip=60 Slip $=90$

Principal Axes:

$P \mid g=75 \quad A=m=340$ $P \quad 15 \quad 160$

paarly controlled mechan

corresponds to reverse

faulting. The preferred foult plane is NP2

CENTROID, MOMENT TENSOR

(HRV)

Data Used: GDSN

L.P.B.: 14S, $23 C$

Centroid Lacotion:

Origin Time $11: 56: 24.00 .8$

Lat 7.635 0.07 Lon 128.26E 0.07

Dep 170.71 .8 Half-duration 2.1 Principal Axes:

Scale $10 * 17 \mathrm{Nm}$

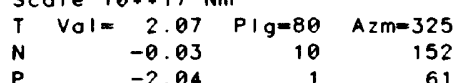

Best Dauble Cauple:Ma $=2.1 * 10 * 17$ NP1:Strike=142 Dip=45 SIip= 76 NP2: $\quad 341 \quad 47 \quad 103$

$\begin{array}{llllll}25 & 1301 & 34.23 & 36.961 \mathrm{~S} & 177.434 \mathrm{E} & 287 \mathrm{~km}\end{array}$

$4.9 \mathrm{mb}(12$ abs.)

OFF E. COAST OF N. ISLAND, N.Z

CENTROID, MOMENT TENSOR (HRV)

DOto UsEd: GDSN

L.P.B.: 16S, $29 \mathrm{C}$

Centroid Lacatian

Origin Time

$13: 01: 39.7: 7$

Lat $36,3850.08$ Lon $176.91 E$ o.10

Dep 265.1 3.6 Half-duratian 1.7 Principal Axes:

Scale $10 * 16 \mathrm{Nm}$

$T \quad V O I=10.88 \quad \mathrm{Plg}=33 \quad$ Azm $=234$

$\begin{array}{rrrr}N & 0.73 & 51 & 19 \\ \mathrm{P} & -11.62 & 17 & 132\end{array}$

Best Double Couple:Ma=1,1*10.*17

NP 1: Strike $=268$ Dip=53 Slip=167

$\begin{array}{lll}\text { NP2 : } & 6 & 80\end{array}$

$\begin{array}{lllllll}25 & 19 & 49 & 15.90 & 15.259 \mathrm{~S} & 172.811 \mathrm{w} & 73 \mathrm{~km}\end{array}$

5. $0 \mathrm{mb}$ ( 9 abs.)

SAMOA ISLANDS REGION

CENTROID, MOMENT TENSOR

Dato Used: GDSN

L.P. B.: 15S, 26C

Centroid Locotion

Origin Time

$19: 49: 18.50 .6$

Lat 15.395 0.09 Lon 173.07W 0.07

Dep $53.45 .9 \mathrm{Hal}$-duration 1.7

Principal Axes:

Scale $10 * 16 \mathrm{Nm}$

$T \quad V a l=5.99 \quad P I g=62 \quad$ Azm $=207$

$\begin{array}{rrrr}N & 2.81 & 10 & 317 \\ P & -8.81 & 26 & 51\end{array}$

Best Double Cauple:Ma*7.4*10**16

NP1:Strike=164 Dip=21 SIip=119

NP2: $313 \quad 72 \quad 80$

$2520 \quad 13 \quad 33.42 \quad 51.677 \mathrm{~N} \quad 172.128 \mathrm{E} \quad 33 \mathrm{~km}$ $5.3 \mathrm{mb}$ ( 70 abs.) $4.6 \mathrm{Msz}$ ( 4 abs.) 
NEAR ISLANDS. ALEUTIAN ISLANDS CENTROID, MOMENT TENSOR (HRV) Doto Used: GDSN

L.P.B.: $14 \mathrm{~S}, 28 \mathrm{C}$

Centraid Lacotion:

Origin Time $20: 13: 36.40 .4$

Lat $51.47 \mathrm{~N} 0.09$ Lon 171.44E 0.09

Dep $15.0 \mathrm{~F}$ IX Half-duration 1.5

Principol Axes:

Scole $10 * 16 \mathrm{Nm}$

$T \quad V O l=7.53 \quad P / g=20 \quad A z m=58$

$\begin{array}{llll}N & -0.93 & 11 & 324 \\ P & -6.60 & 67 & 207\end{array}$

Best Dauble Cauple:Ma=7.1*10**16

NP1: Strike=167 Dip=27 SIip= -65

NP2: $\quad 319 \quad 66 \quad-102$

$\begin{array}{lllllll}25 & 21 & 36 & 21.27 & 13.968 \mathrm{~N} & 91.804 \mathrm{~W} & 69 \mathrm{~km}\end{array}$ 5. Omb ( 25 ODS.)

NEAR COAST OF GUATEMALA

CENTROID, MOMENT TENSOR

Dato Used: GOSN

L.P.B.: $175,32 \mathrm{C}$

Centraid Lacotion:

Origin Time $21: 36: 17.80 .5$

Lot $13.68 \mathrm{~N} 0.06$ Lon $92.26 \mathrm{~W} 0.06$

Dep $32.23 .5 \mathrm{Half}$-duration 1.8

Principol Axes:

Scale $10 * 16 \mathrm{Nm}$

T $\quad \mathrm{O} O \mathrm{I}=11.59 \quad \mathrm{PIg}=70 \quad$ AZm $=54$

$\begin{array}{rrrr}N & 1.05 & 8 & 300 \\ P & -12.64 & 18 & 207\end{array}$

Best Dauble Cauple:Mo=1.2*10**17

NP1:Strike $=284$ Dip=28 Slip= 72

NP2: $\quad 124 \quad 64 \quad 99$

$26070001.71 \quad 52.583 \mathrm{~S} \quad 160.106 \mathrm{E} \quad 10 \mathrm{~km}$ $5.6 \mathrm{mb}$ ( 14 obs.) 5.3Msz ( 10 abs.) MACOUARIE ISLANDS REGION

CENTROID, MOMENT TENSOR

(HRV)

Doto Used: GDSN

L.P.B.: 15S, $26 \mathrm{C}$

Centraid Locotion:

Origin Time $07: 00: 10.50 .6$

Lot 52.005 0.09 Lon 160.32E 0.13

Dep $15.0 \mathrm{Fl}$ Holf-duration 2.1

Principol Axes:

Scale $10 * 17 \mathrm{Nm}$

T $\quad V O 1=3.44 \quad P \mid g=51 \quad$ Azm $=102$

$\begin{array}{lrrr}N & 0.00 & 7 & 201 \\ P & -3.43 & 38 & 296\end{array}$

Best Dauble Cauple: Ma=3.4*10**17

NP1:Strike $=68$ Dip $=9$ SIip $=138$

NP2: $\quad 200 \quad 84 \quad 83$

$\begin{array}{lllllll}26 & 12 & 56 & 18.32 & 51.9265 & 161.386 E & 10 \mathrm{~km}\end{array}$ 5.3mb ( 8 ODS.) 4.9MSZ ( 2 abs.) NORTH OF MACOUARIE ISLAND

CENTROID, MOMENT TENSOR (HRV)

Doto Used: GDSN

L.P.B.: $15 \mathrm{~S}, 30 \mathrm{C}$

Centraid Location:

Origin Time 12:56:29.5 0.7

Lat 51.8750 .11 Lan 160.43E 0.08 Dep $15.0 \mathrm{FIX} \mathrm{Half-duration} 1.5$ Principol Axes:

Scole $10 * * 16 \mathrm{Nm}$

I VOI $=4.75 \quad \mathrm{Plg}=0 \quad$ A $\mathrm{Zm}=178$

$\begin{array}{rrrr}N & 0.67 & 90 & 180\end{array}$

Best Dauble Cauple:Mo=5.1*10*16 NP 1: Strike $=223$ Dip=90 SI ip $=180$ NP2 : $\quad 313 \quad 90$

$\begin{array}{lllllll}26 & 14 & 28 & 37.82 & 4.4065 & 105.270 \mathrm{~W} & 10 \mathrm{~km}\end{array}$ 4. $7 \mathrm{mb}(10$ obs.) $5.2 \mathrm{MsZ}$ ( 4 Obs.) NORTHERN EASTER 1. CORDILLERA

CENTROID, MOMENT TENSOR (HRV)

Dota Used: GDSN

L.P.B.: $17 \mathrm{~S}$. $35 \mathrm{C}$

Centraid Lacation:

Origin Time $\quad 14: 28: 42.10 .3$

Lot 4.725 0.82 Lan 105.09w 0.03

Dep 15.0 Fix Half-duration 2.6 Principol Axes:

$$
\begin{aligned}
& \text { Scale } 10.117 \mathrm{Nm} \\
& \text { T Val }=2.86 \quad P / g=0 \quad A z m=142 \\
& \begin{array}{rrrr}
N & -0.44 & 90 & 180 \\
P & -2.43 & 0 & 52
\end{array}
\end{aligned}
$$

Best Double Couple:Mo=2.6*10*17 NP 1: Sirike $=187$ Dip=90 SI ip $=180$ $\begin{array}{rrr}\text { NP1: Strike }=187 & \text { Di } P=90 \\ & 277 & 90\end{array}$
$270301 \quad 24.92 \quad 55.279 \mathrm{~S} \quad 133.192 \mathrm{~W} \quad 10 \mathrm{~km}$ $5.5 \mathrm{mb}$ ( 19 Obs.) 5.7MSZ ( 11 Obs.) SOUTH PACIFIC CORDILLERA

CENTROID, MOMENT TENSOR (HRV)

Doto Used: GOSN

L.P.B.: 18S, $44 \mathrm{C}$

Centraid Locatian:

Origin Time $03: 01: 33.50 .2$
Lot 55.0650 .03 Lon $132.89 \mathrm{~W} 0.05$

Dep $15.0 \mathrm{FIX} \mathrm{Half-duration} 4.2$

Principol Axes:

Scole $10 * * 18 \mathrm{Nm}$

T
$N$$\quad \begin{array}{rrr}1.53 & \text { PIg }=12 & \text { Azm }=346 \\ 0.05 & 3 & 77\end{array}$

$\begin{aligned} & \mathrm{N} \\ & \mathrm{P}\end{aligned}-1.05 \quad 38 \quad 77$

Best Dauble Couple: Mo=1.6*10**18 NP 1: Strike $=72$ Dip $=33$ SI i $p=-95$ $\begin{array}{llll}\text { NP2 : } & 258 & 57 & -87\end{array}$

$\begin{array}{lllllll}2708 & 31 & 18.76 & 3.4515 & 138.855 E \quad 49 \mathrm{~km}\end{array}$ $5.4 \mathrm{mb}(20$ abs.) 4.4Msz ( 3 abs.) WEST IRIAN

CENTROID, MOMENT TENSOR (HRV)

Dota Used: GOSN

L.P.B.: 14S, $23 \mathrm{C}$

Centraid Lacotian:

Origin Time $\quad 08.31: 25.20 .7$

Lot 3.49S 0.05 Lon 138.73E 0.06

Dep 71.25 .6 Holf-duration 1.5 Principal Axes:

Scole $10 * * 16 \mathrm{Nm}$

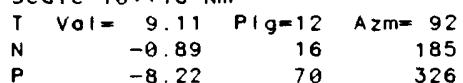

Best Dauble Couple:Ma=8.7*10**16

NP 1: Strike=162 Dip=36 Si i $p=-117$

NP2: $\quad 15 \quad 59 \quad-72$

$27 \quad 142746.44 \quad 50.2365 \quad 158.073 \mathrm{E} \quad 10 \mathrm{~km}$ $5.5 \mathrm{mb}$ ( 23 ODS.) $4.9 \mathrm{MSZ}$ ( 5 Obs.) NORTH OF MACQUARIE ISLAND

CENTROID. MOMENT TENSOR

(HRV)

DOto Used: GDSN

L.P.B.: 16S, $33 \mathrm{C}$

Centroid Locotion:

Origin Time $\quad 14: 27: 55.20 .7$

Lat 50.4750 .06 Lon 156.84E 0.11

Dep 15.0 Fix Half-duration 1.7

Principal Axes:

Scale $10 *+16 \mathrm{Nm}$

T $\quad$ O I $=9.98 \quad P$ Ig $=14 \quad$ Azm $=35$

$\begin{array}{rrrr}N & 3.45 & 65 & 158 \\ P & -13.42 & 20 & 299\end{array}$

Best Dauble Cauple:Ma=1,2*18*17

NP1: Strike $=78$ Dip $=65$ Slip $=-176$

NP2: $\quad 346 \quad 86 \quad-25$

$2720 \quad 08 \quad 37.31 \quad 30.167 \mathrm{~N} \quad 50.921 \mathrm{E} \quad 31 \mathrm{~km}$ $5.6 \mathrm{mb}$ ( 87 abs.) 5.8Msz ( 26 abs.) I RAN

CENTROID, MOMENT TENSOR

(HRV)

Doto Used: GDSN

L.P.B.: 185, $37 \mathrm{C}$

Centroid Lacation

Origin Time

$20: 08: 41.40 .4$

Lot $29.86 \mathrm{~N} 0.05$ Lon $50.69 \mathrm{E} 0.04$ Dep 15.0 BDY Half-duration 3.7 Principol Axes:

Scale $10 * * 18 \mathrm{Nm}$

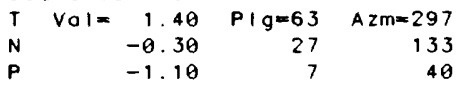

Best Dauble Cauple: $M a=1.3 \cdot 10 * 18$

NP1:Strike $=103$ Dip=45 SIip= 51

$\begin{array}{llll}\text { NP2 : } & 332 & 57 & 122\end{array}$

$\begin{array}{lllllll}28 & 09 & 46 & 28.27 & 16.714 \mathrm{~S} & 173.278 \mathrm{w} & 35 \mathrm{~km}\end{array}$ $5.7 \mathrm{mb}(42$ abs.) $5.5 \mathrm{Msz}$ ( 20 obs.) TONGA ISLANDS

FAULT PLANE SOLUTION: P-Woves NP1: Strike=132 Dip=83 Si $p=147$ NP2: 227

Principol Axes:

$\begin{array}{rrr}T & P I g=28 & A z m= \\ P & 17 & 184\end{array}$

Comment: The focal mechanism is paorly controlled and corresponds to strike-slip foulting with o lorge reverse component. The preferred foult plone is not determined.

MOMENT TENSOR SOLUTION

DeP 39
Principal Axes:

Scale $10 * 17 \mathrm{Nm}$

$T \quad V a l=4.95 \quad P / g=48 \quad A z m=88$

$\begin{array}{rrrr}N & 0.11 & 38 & 297 \\ P & -5.96 & 15 & 195\end{array}$

Best Dauble Cauple:Ma=5.0*10**17

NP1:Strike $=245$ Dip=45 Slip= 29

NP2 :

CENTROID. MOMENT TENSOR (HRV)

Data Used: GDSN

L.P.B.: $175,33 \mathrm{C}$

Centroid Lacation:

at 16.0450 .03 Lan $172.84 \mathrm{~W} 0.04$

Dep $39.92 .5 \mathrm{Half}$-duration 2.9

Principol Axes:

Scale $10 * 17 \mathrm{Nm}$

T $V a l=5.97 \quad P I g=43 \quad$ AZm $=65$

$\begin{array}{rrrr}N & -0.96 & 43 & 274 \\ P & -5.01 & 15 & 169\end{array}$

Best Dauble Couple:Mo=5.5*10**17

NP1: Strike $=217$ Dip=49 Siip= 23

NP2: $\quad 111 \quad 73 \quad 136$

$\begin{array}{lllllll}28 & 10 & 20 & 00.35 & 16.723 \mathrm{~S} & 173.247 \mathrm{~W} \quad 50 \mathrm{~km}\end{array}$

$5.2 \mathrm{mb}$ ( 30 obs.)

TONGA ISLANDS

CENTROID, MOMENT TENSOR

(HRV)

Doto Used: GDSN

L.P.B.: 12S, $23 \mathrm{C}$

Centraid Lacation:

origin Time

$10: 20: 8.80 .9$

Lot 16.2450 .09 Lon 173.40w 0.09

Dep $38.85 .8 \mathrm{Holf}$-durotion 1.8

Principol Axes:

Scole $10 * * 16 \mathrm{Nm}$

$T \quad V_{0 l}=14.75 \quad P / g=58 \quad$ AZm $=63$

$\begin{array}{lrrr}N & -3.32 & 23 & 292\end{array}$

$\begin{array}{cccr}P & -11.43 & 22 & 192 \\ \text { Best Double Couple: } M o=1.3 * 10 * 17\end{array}$

NP1: Strike=248 Dip=31 Siip= 41

NP2: $\quad 120 \quad 70 \quad 114$

$\begin{array}{llllllll}28 & 15 & 19 & 07.19 & 6.5155 & 147.799 E & 33 \mathrm{~km}\end{array}$

$5.5 \mathrm{mb}$ ( 30 abs.) 5.5MsZ ( 20 obs.)

EAST PAPUA NEW GUINEA REGION

CENTROID, MOMENT TENSOR (HRV)

Doto UsEd: GDSN

L.P.B.: $17 \mathrm{~S}, 38 \mathrm{C}$

Centraid Lacation:

Origin Time 15:19:15.4 0.4

Lat 6.7550 .04 Lan $147.90 \mathrm{E} \quad 0.05$

Dep 15.0 FIX Half-durotion 3.0

Principal Axes:

Scale $10 * * 17 \mathrm{Nm}$

I Val $=6.02 \quad \mathrm{Plg}=55 \quad$ Azm $=272$

$\begin{array}{rrrr}N & 1.44 & 10 & 168 \\ P & -7.46 & 33 & 72\end{array}$

Best Dauble Cauple:Ma=6.7*10*17

NP 1: Strike=127 Dip=15 Slip= 48

NP2: $\quad 350 \quad 79 \quad 100$

$\begin{array}{llllllll}28 & 15 & 52 & 54.28 & 51.053 \mathrm{~S} & 159.682 \mathrm{E} & 10 \mathrm{~km}\end{array}$

$5.3 \mathrm{mb}$ ( 7 ObS.)

NORTH OF MACOUARIE ISLAND

CENTROID, MOMENT TENSOR

Doto Used: GDSN

L.P.B.: $11 \mathrm{~s}, 23 \mathrm{C}$

Centroid Lacotion

Origin Time

$15 \cdot 53 \cdot 7.0,1.0$

Lot 50.545 O. 12 Lon 158.29E 0.16

Dep 15.0 FIX Holf-durotion 1.9 Principol Axes:

Scale $10 * 16 \mathrm{Nm}$

$T \quad$ Vol $=15.56 \quad \mathrm{Plg}=23 \quad$ Azm $=19$

$\begin{array}{rrrr}N & -3.87 & 63 & 233 \\ P & -11.68 & 14 & 114\end{array}$

Best Dauble Cauple:Ma=1,4*10*17 NP1: Strike=158 Dip=64 Si ip= ?

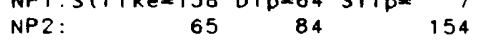

$\begin{array}{lllllllll}29 & 02 & 12 & 27.88 & 48.9145 & 121.346 \mathrm{E} & 10 \mathrm{~km}\end{array}$ $5.2 \mathrm{mb}(7$ obs.) 5.2Msz ( 1 obs.) SOUTH OF AUSTRALIA

CENTROID, MOMENT TENSOR

Dota Used: GDSN

L.P.B.: 145,220

Centraid Location

Origin Time

02:12:33.2 0.5

Let 48.675 0.94 Lan 121.31E 0.09

DeP 15. Fix Half-duration 2.2

Principol Axes:

Scale 10**17 $\mathrm{Nm}$ 


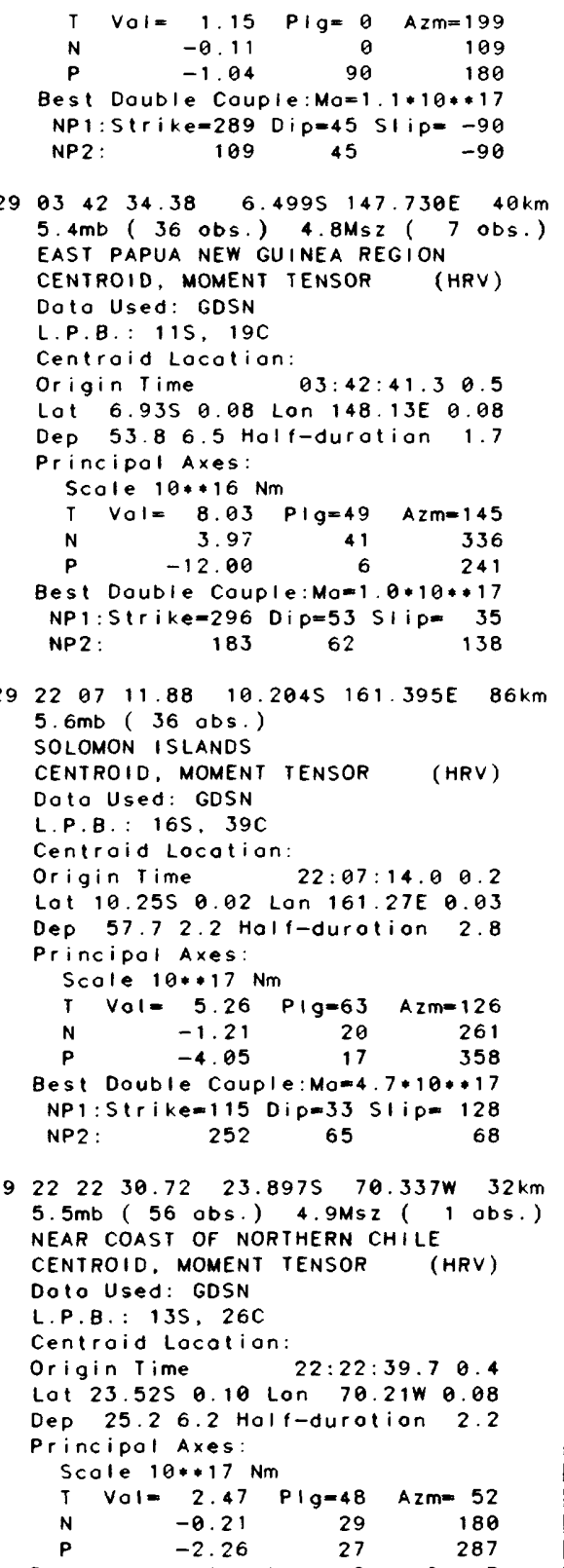

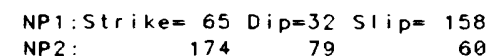

$\begin{array}{llllllll}30 & 13 & 39 & 17.14 & 17.851 \mathrm{~S} & 173.043 \mathrm{~W} & 38 \mathrm{~km}\end{array}$ 5. Omb ( 18 obs.) 4.7Msz (4 Obs.) । TONGA ISLANDS

CENTROID. MOMENT TENSOR (HRV)

Doto Used: GDSN

L.P.B.: 12S, $22 \mathrm{C}$

Centraid Locotion:

Origin Time 13:39:15.11.4

Lot $18.125 \quad 0.13$ Lon $172.56 \mathrm{~W} 0.14$

Dep 15.0 Fix Holf-duration 1.7 Principol Axes:

Scale $10 * 16 \mathrm{Nm}$

$T \quad$ Vol $=12.74 \quad$ Plg $=55 \quad$ Azm $=276$

$\begin{array}{rrrr}N & -3.46 & 8 & 18 \\ P & -9.28 & 33 & 113\end{array}$

Best Double Cauple:Mo=1,1*10**17

NP1: Strike=233 Dip=14 Slip= 126

$\begin{array}{llll}\text { NP2 : } & 17 & 79 & 82\end{array}$

$\begin{array}{llllll}30 & 13 \quad 50 & 56.26 & 17.401 \mathrm{~N} & 94.645 \mathrm{~W} & 138 \mathrm{~km}\end{array}$ $5.2 \mathrm{mb}$ ( 65 obs.)

CHIAPAS, MEXICO

CENTROID, MOMENT TENSOR (HRV)

Doto Used: GDSN

L.P.B.: 15S, 31C

Centraid Lacation:

Origin Time 13:51:0.90.4

Lat $17.63 \mathrm{~N} 0.04$ Lan $94.84 \mathrm{~W} 0.04$

Dep 142.41 .1 Half-duration 3.2

Principol Axes:

Scale $10 * 17 \mathrm{Nm}$

$T$ Val $=7.37 \quad$ PIg $=4 \quad$ Azm= 62

$\begin{array}{lllr}N & -0.49 & 43 & 328 \\ P & -6.87 & 46 & 156\end{array}$

Best Double Couple:Ma $=7,1 * 10 * * 17$

NP 1 : Strike $=188$ Dip $=56$ si ip $=-34$

$\begin{array}{llll}\text { NP2: } & 298 & 63 & -141\end{array}$

$\begin{array}{lllllll}3105 & 054 & 20.56 & 45.3835 & 167.086 \mathrm{E} & 23 \mathrm{~km}\end{array}$ 5. $8 \mathrm{mb}$ ( 29 obs.) 6.3Msz ( 29 obs.) SOUTH ISLAND, NEW ZEALAND

FAULT PLANE SOLUTION: P-Woves

NP 1: Strike=105 Dip=84 SIip= 90

NP2:

285

Principol Axes:

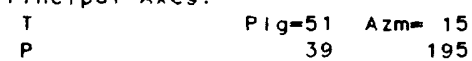

Comment: The focol mechonism is

poorly controlled and

corresponds to reverse

foulting. The preferred fould

Plone is NP2.

RADIATEO ENERGY

No. of sto: 4 focal mech. C

Energy $0.1+0.0 * 10 * * 15 \mathrm{Nm}$

MOMENT TENSOR SOLUTION

DeP 12 No. of $510: 11$

Principol Axes:
Scale $10 * 18 \mathrm{Nm}$

T $\quad$ O I $=4.14 \quad P \mid g=49 \quad$ A $z m=343$

$\begin{array}{llll}N & -1.02 & 27 & 108\end{array}$

$\begin{array}{llll}P & -3.12 & 28 & 214\end{array}$

Best Dauble Cauple: Mo=3.6*10**18 NP1:Strike=351 Dip=29 SIip=155 NP2: $\quad 102 \quad 78 \quad 63$ CENTROID, MOMENT TENSOR (HRV) Doto Used: GOSN

L.P.B.: 18S, $47 \mathrm{C}$ M.W.: 9S, $21 \mathrm{C}$ Centraid Location:

Origin Time 05:54:27.4 0.2 Lat $45.20 \mathrm{~S} 0.02$ Lan 166.59E 0.02 Dep 18.0 BDY Holf-durotion 6.7 Principol Axes:

Scale $10 * 18 \mathrm{Nm}$

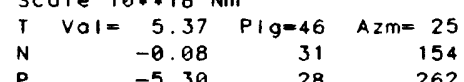

Best Double Couple:Mo=5.3*10**18

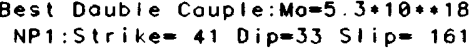

147

80

$\begin{array}{lllllll}31 & 14 & 20 & 24.18 & 3.006 \mathrm{~S} & 142.078 \mathrm{E} \quad 33 \mathrm{~km}\end{array}$ $5.2 \mathrm{mb}$ ( 14 obs.) $4.5 \mathrm{MsZ}$ ( 7 obs.) NEAR N COAST OF PAPUA NEW GUINEA CENTROID. MOMENT TENSOR (HRV) Doto Used: GDSN

L.P.B.: 9S, 19C

Centraid Locotion:

Origin Time 14:20:28.20.5

Lot 3.14S 0.07 Lan 142.37E 0.08

Dep 28.16 .0 Holf-durotion 1.6 Principol Axes:

Scole $10 * 16 \mathrm{Nm}$

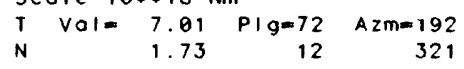

$\begin{array}{lrrr}\mathrm{P} & -8.74 & 14 & 53\end{array}$

Best Double Cauple:Mo=7.9*10*16 NP1:Strike=159 Dip=33 SIip= 112 NP2: $\quad 314 \quad 60 \quad 76$

$\begin{array}{lllllll}31 & 19 & 10 & 37.09 & 6.512 S & 147.817 \mathrm{E} \quad 43 \mathrm{~km}\end{array}$ $5.2 \mathrm{mb}$ ( 21 abs.) 4.8Msz ( 6 abs.) EAST PAPUA NEW GUINEA REGION

CENTROID, MOMENT TENSOR (HRV)

Doto Used: GDSN

L.P.B.: 13S, $24 \mathrm{C}$

Centraid Lacotian:

Origin Time $19: 10: 39.01 .0$

Lot 7.00 S 0.09 Lon 148.17E 0.10

Dep $15.0 \mathrm{Fix}$ Holf-durotion 1.4 Principol Axes:

Scale $10 * 16 \mathrm{Nm}$

$\begin{array}{rrrr}T & V O I=11.39 & P I g=38 & A z m=310 \\ N & 0.40 & 31 & 192\end{array}$

$\begin{array}{llll}P & -11.79 & 36 & 76\end{array}$

Best Double Couple:Mo=1.2*10**17 NP 1:Strike=104 Dip=31 Slip= 2 $\begin{array}{llll}\text { NP2 : } & 13 & 89 & 121\end{array}$ 
03 May 1989 05:53:01.17

Sichuan Province, China

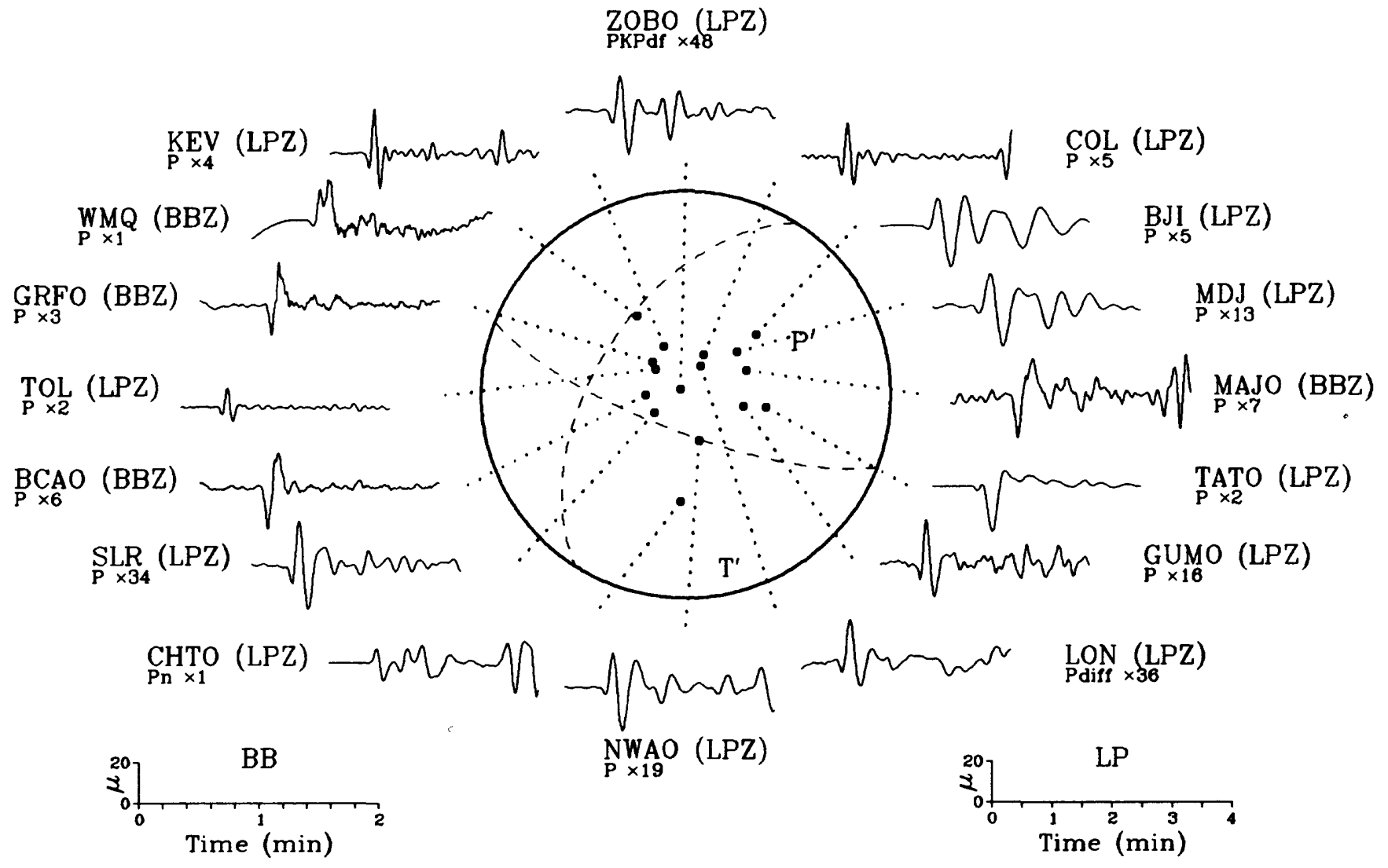

05 May 1989 18:28:39.45

Western Brazil

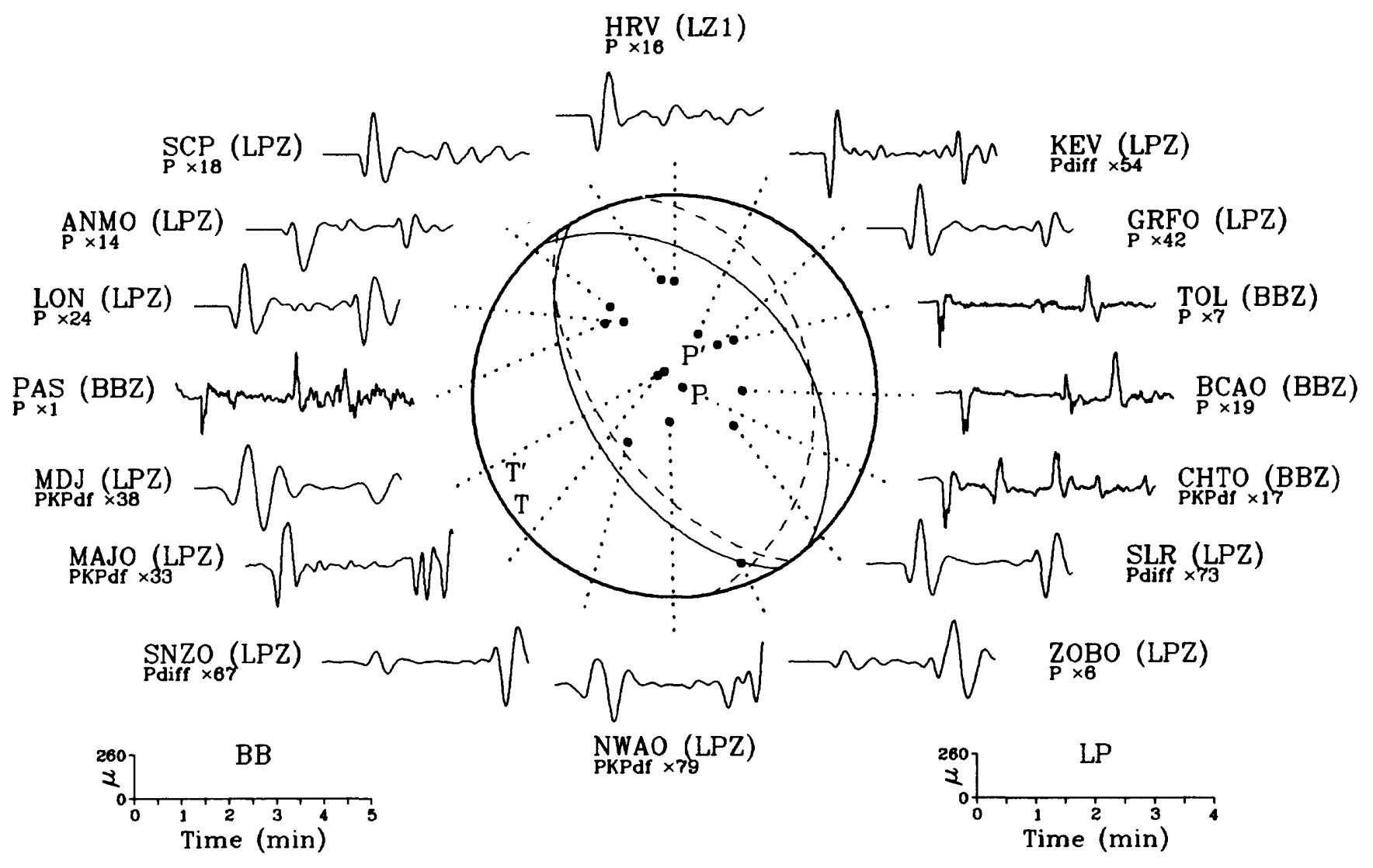




\section{May 1989 00:59:50.45 \\ Kermadec Islands}

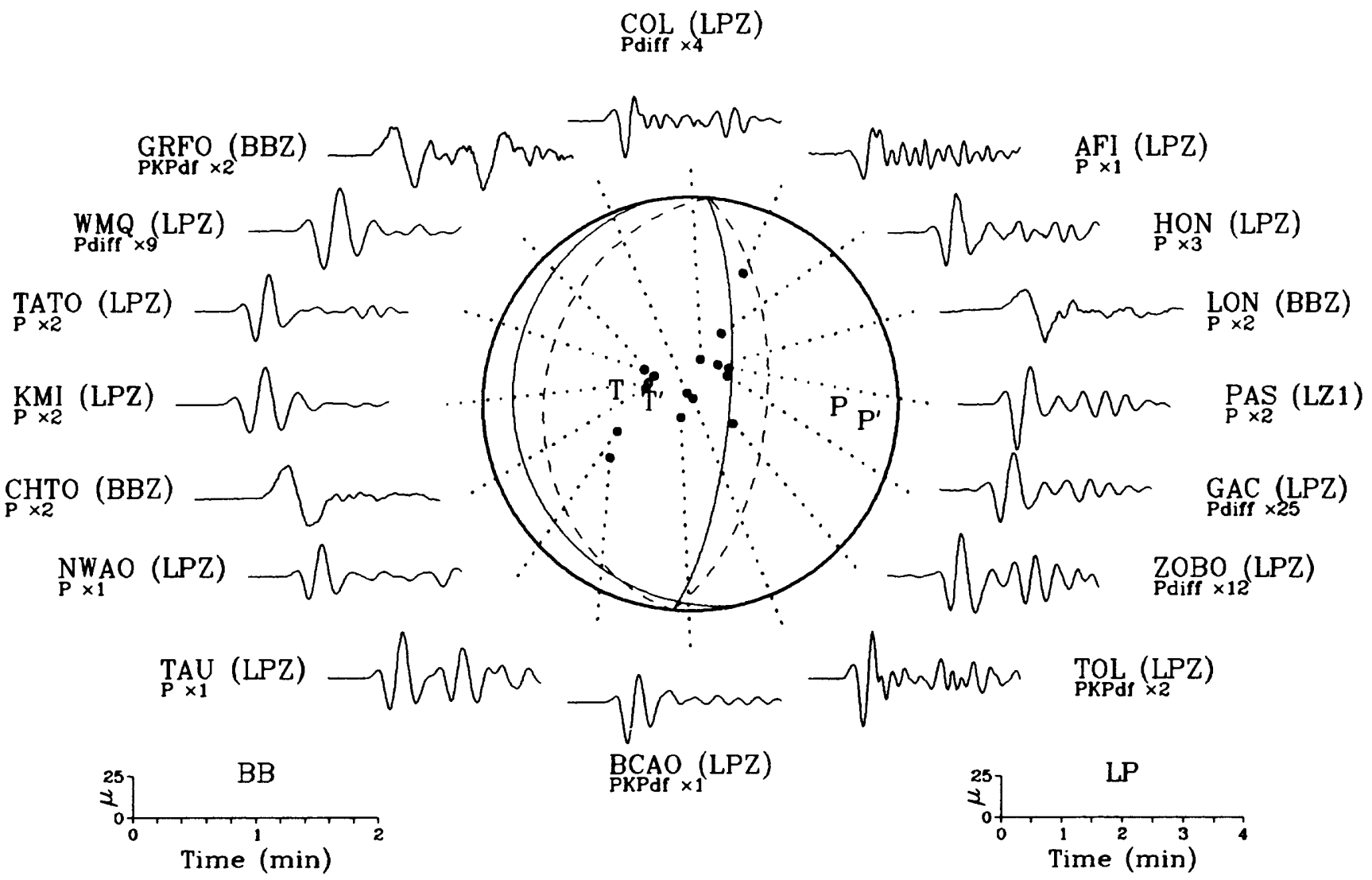

\section{May 1989 02:21:56.38 \\ Fox Islands, Aleutian Islands}

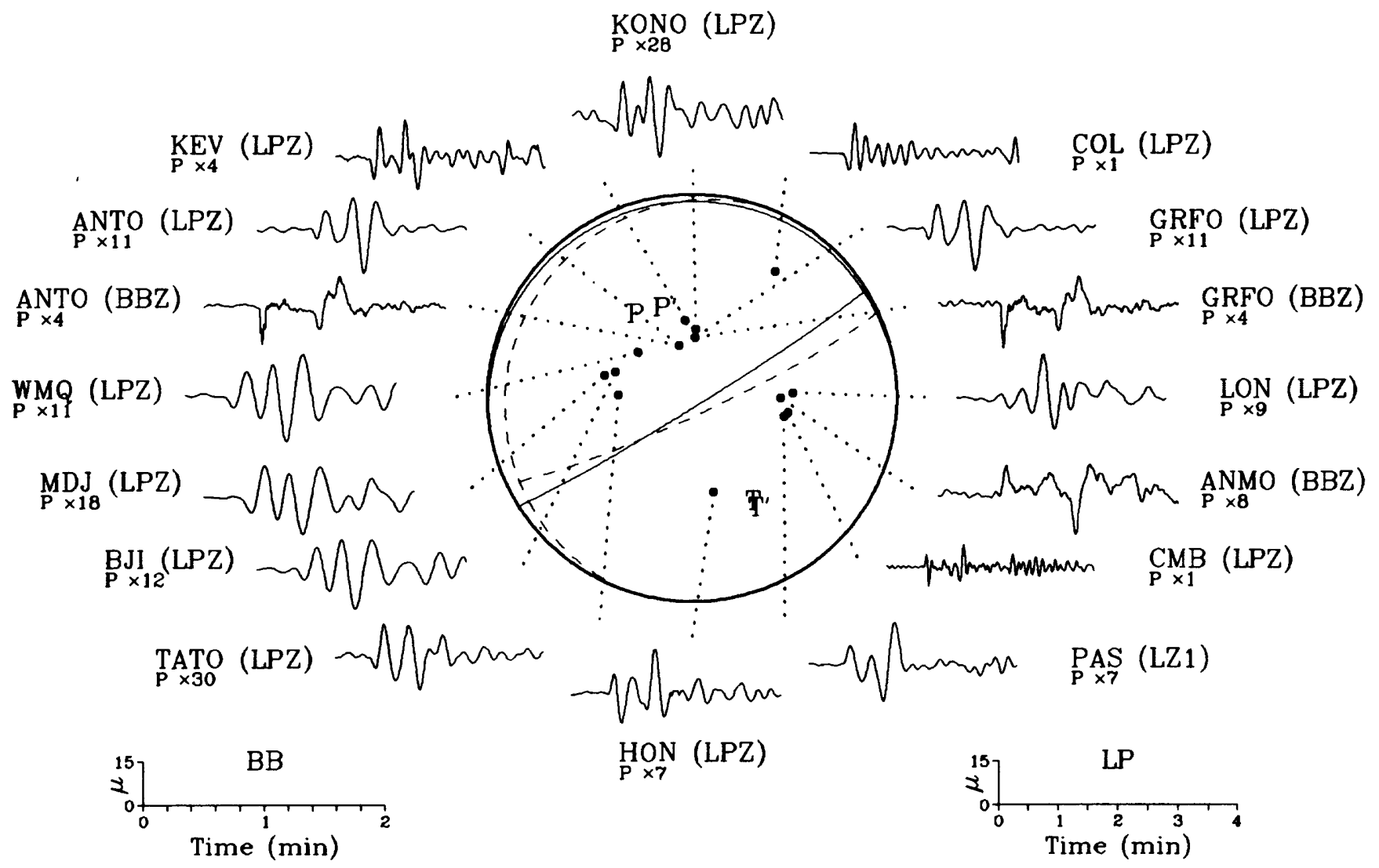




\section{May 1989 16:01:43.61 \\ Kermadec Islands}

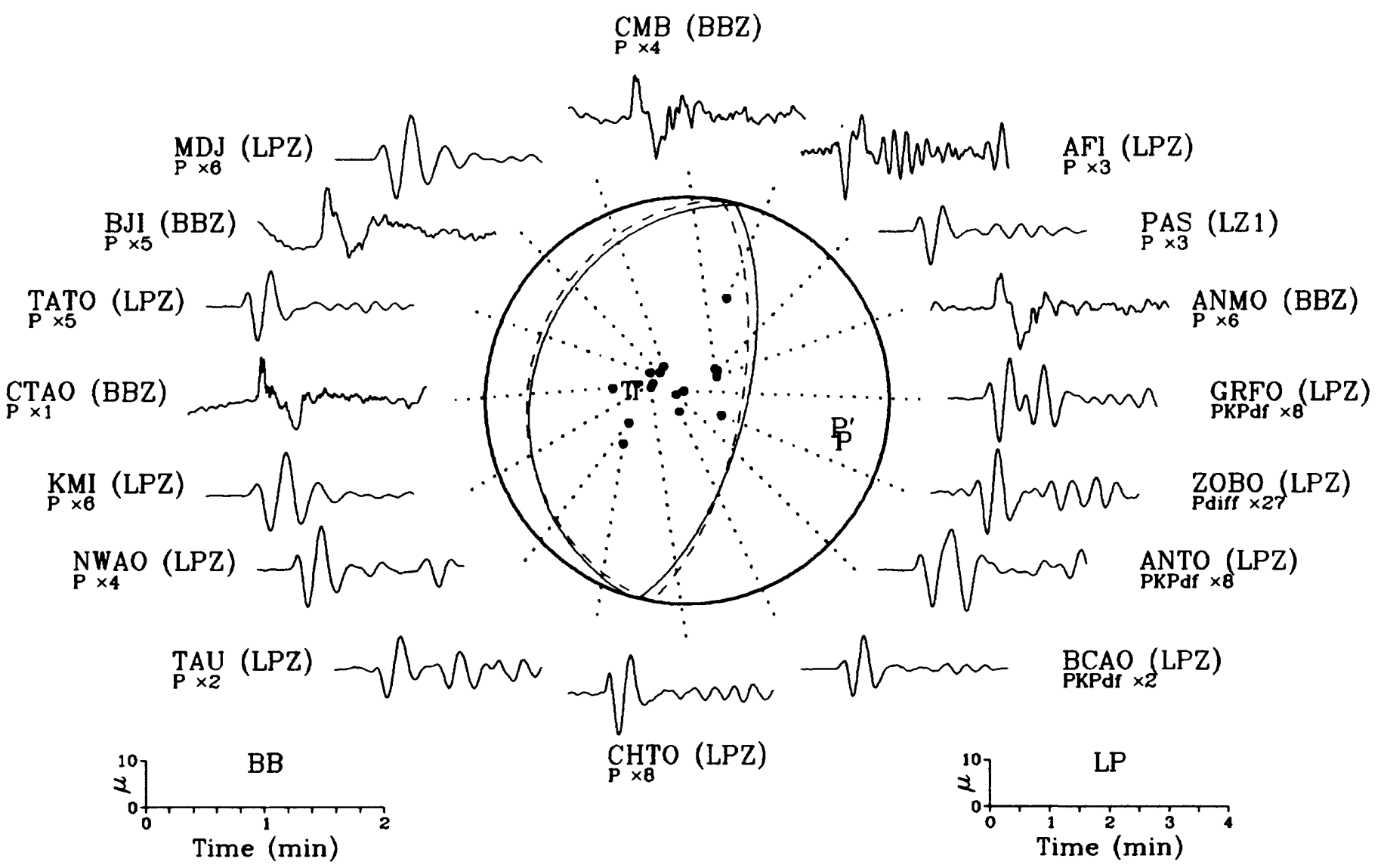

23 May 1989 10:54:46.32
Macquarie Islands Region

$\underset{\text { Pdiff }}{\mathrm{COL}}(\mathrm{LPZ})$

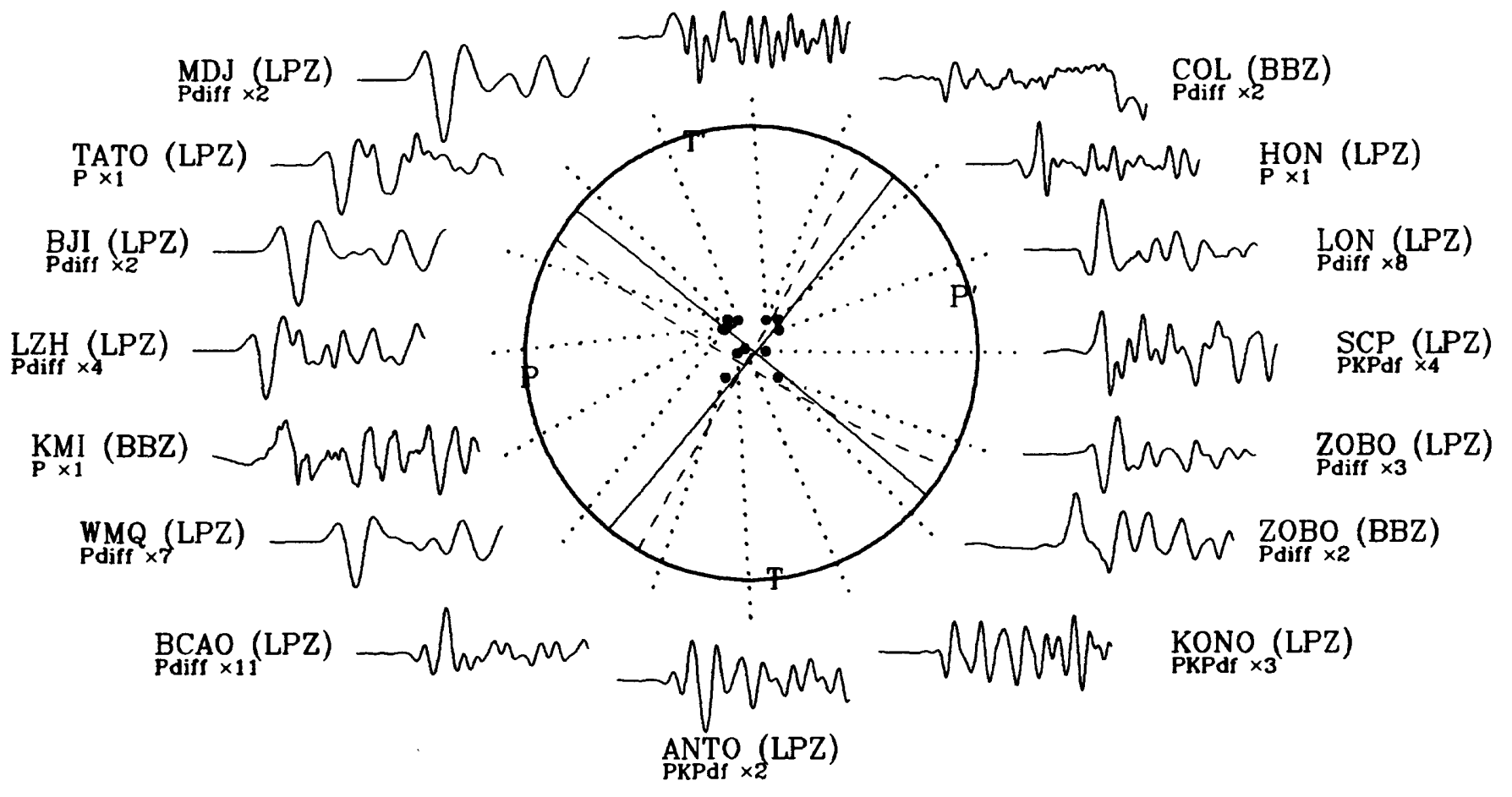




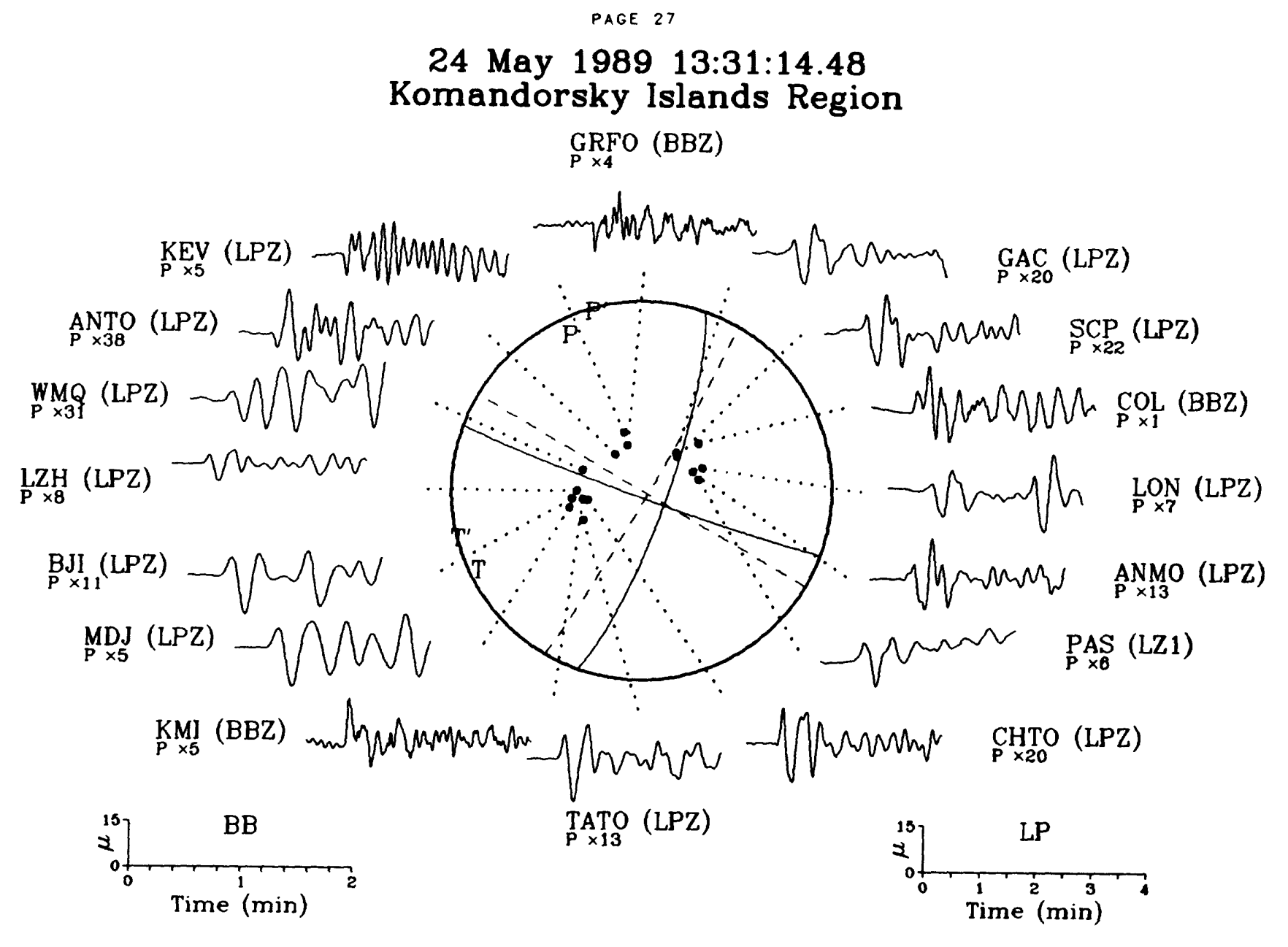

\section{May 1989 05:54:20.56 \\ South Island, New Zealand}

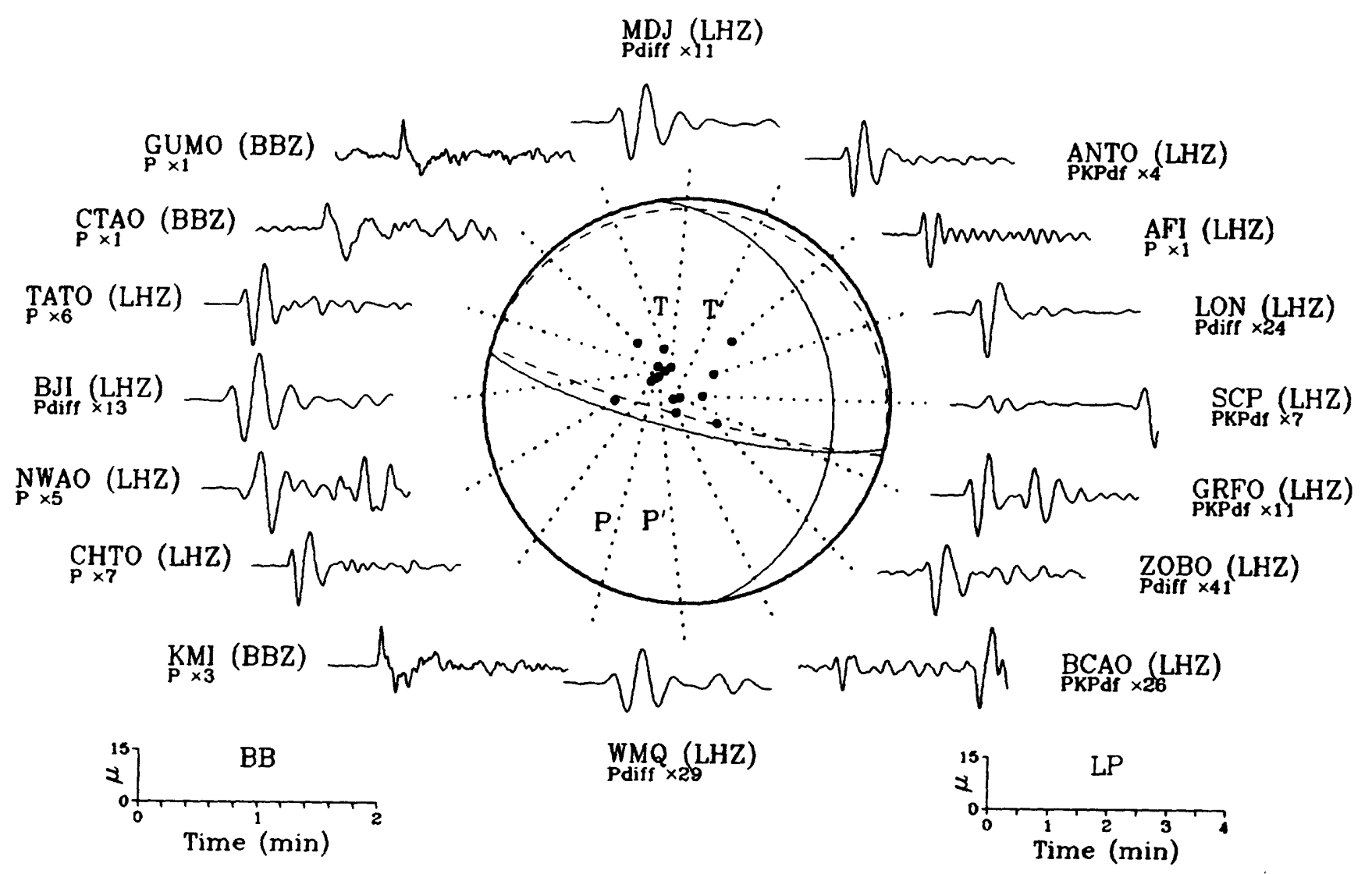




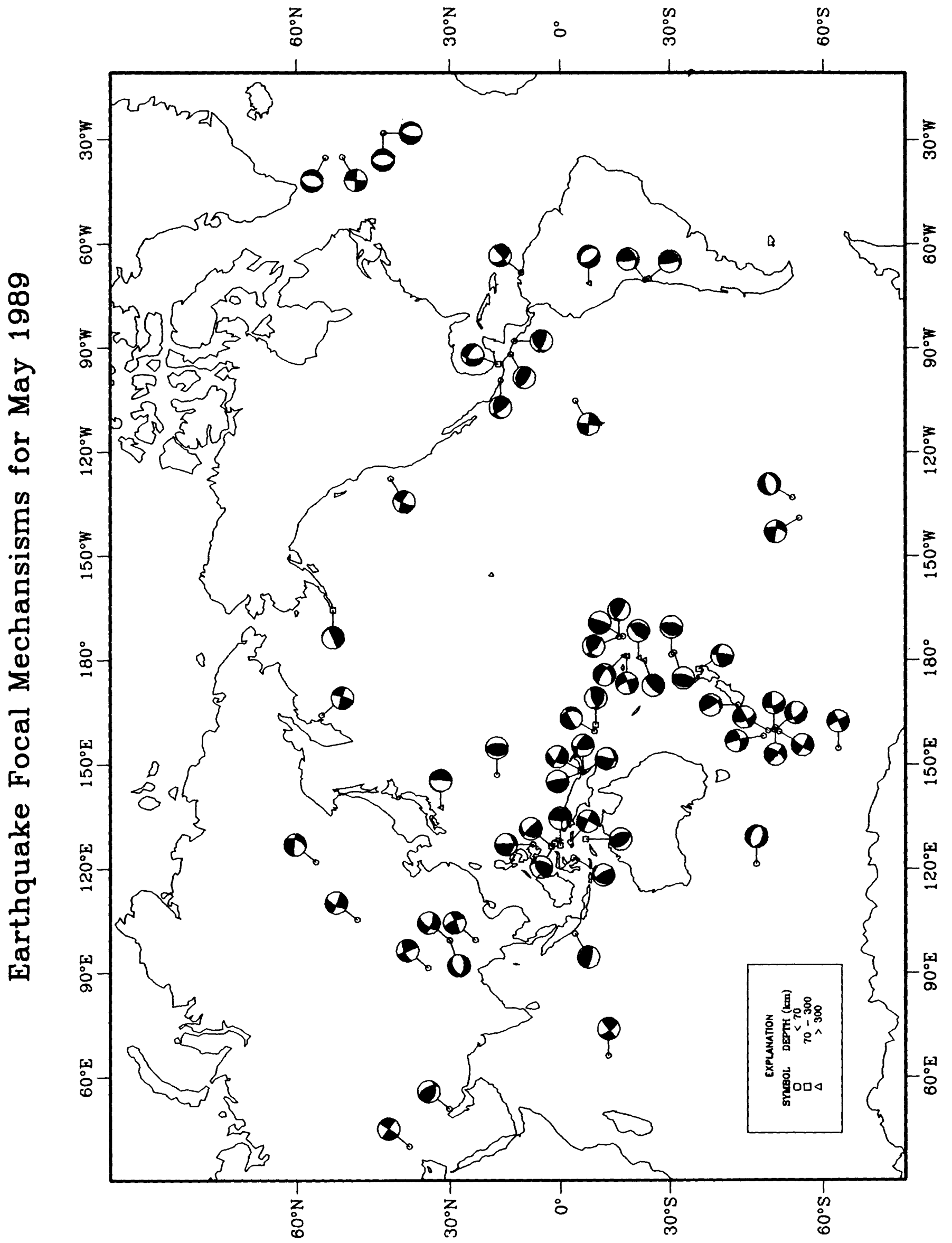




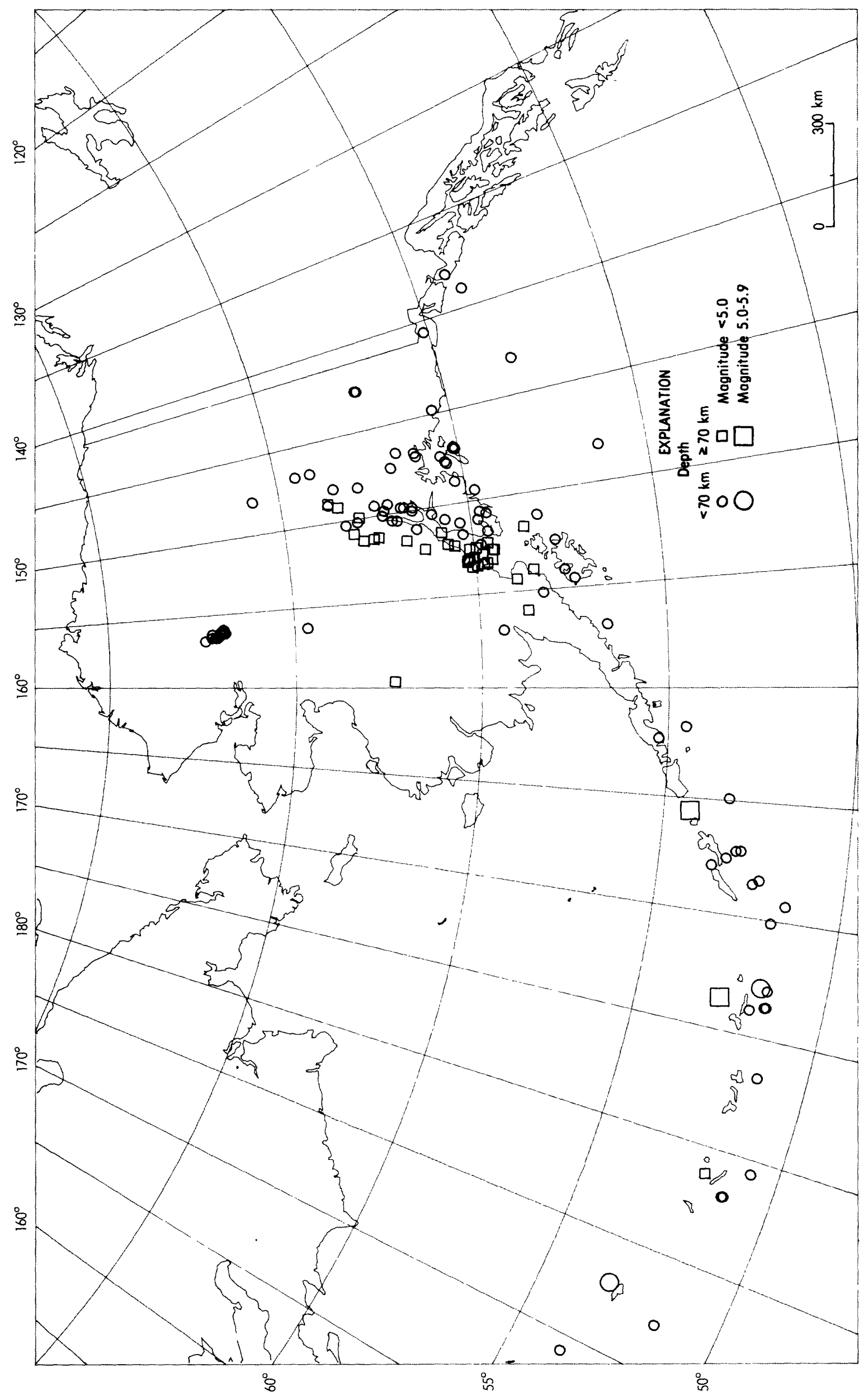

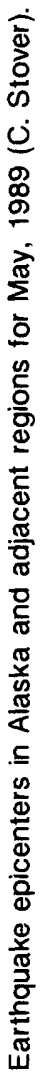




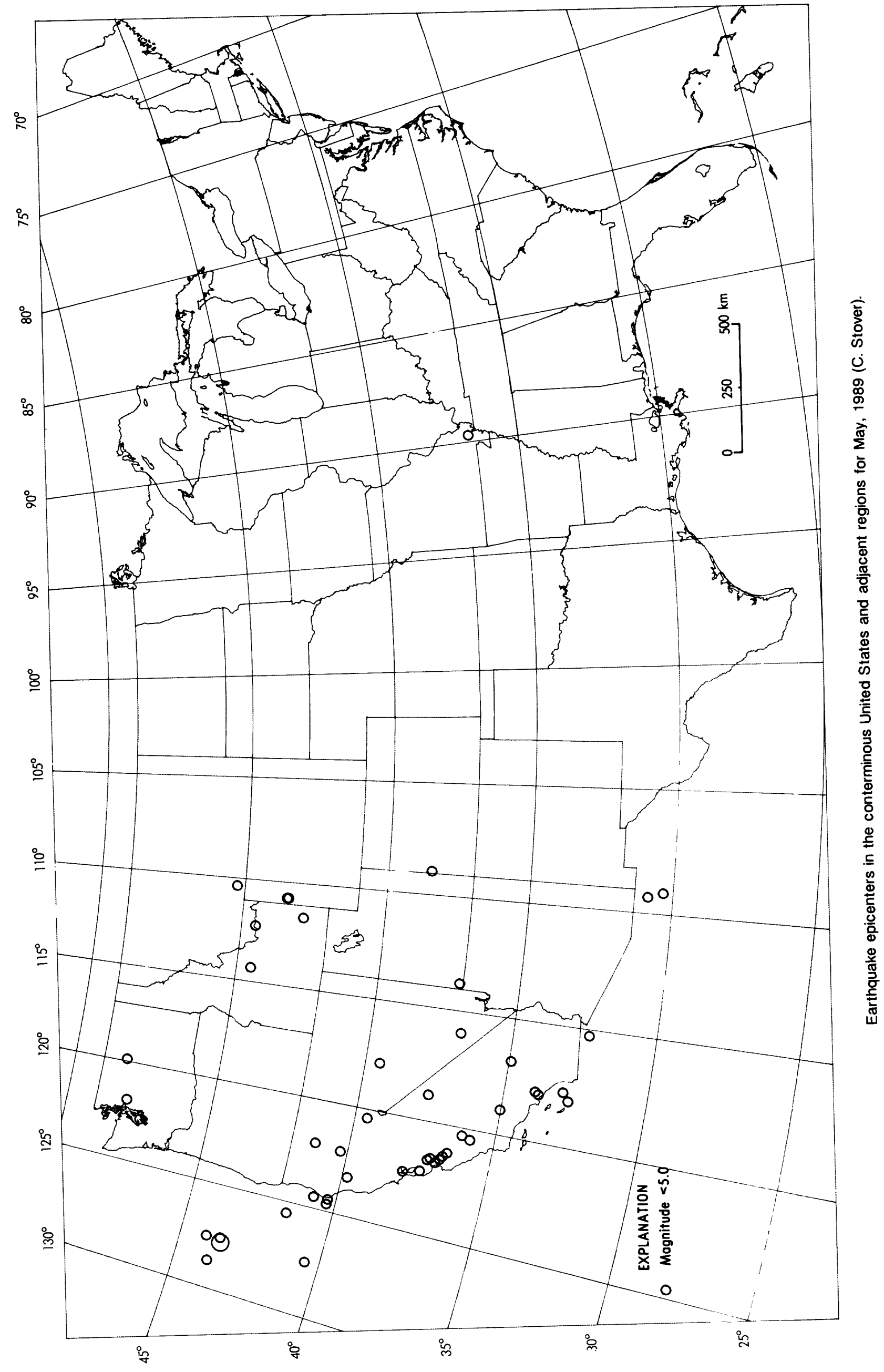




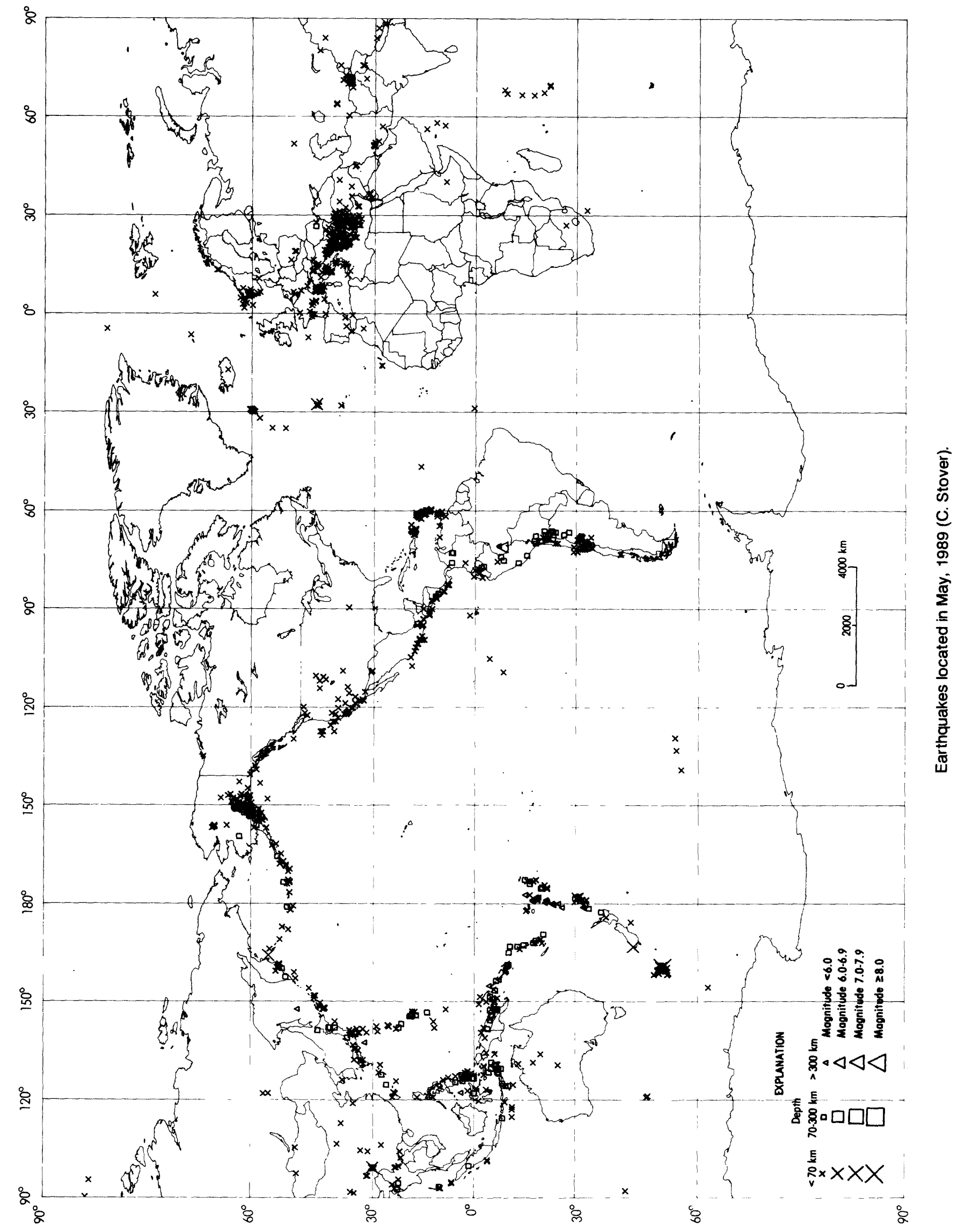




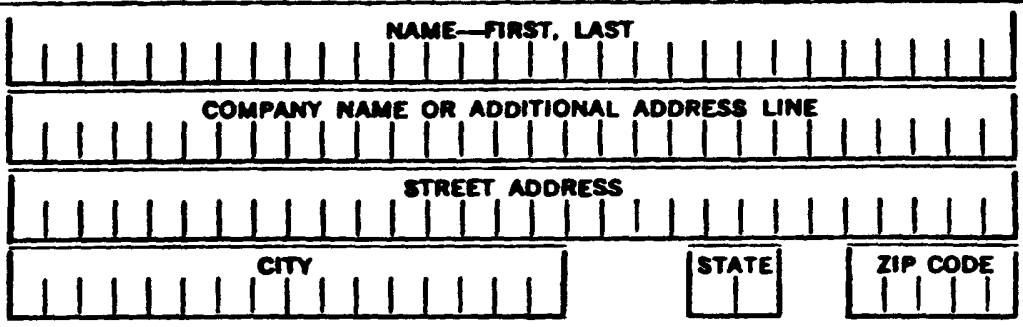

MLEASE PAINT OR TMrE

Ma11 this form to: NEW ADDRESS

Superintendent of Documents Government Printing office SSOM

Washington, D.C. 20402

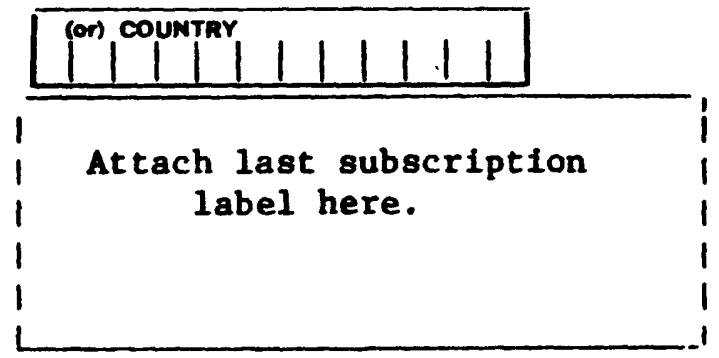

sueschipton Onden rona

ENTER MY SUBSCRIPTION TO:

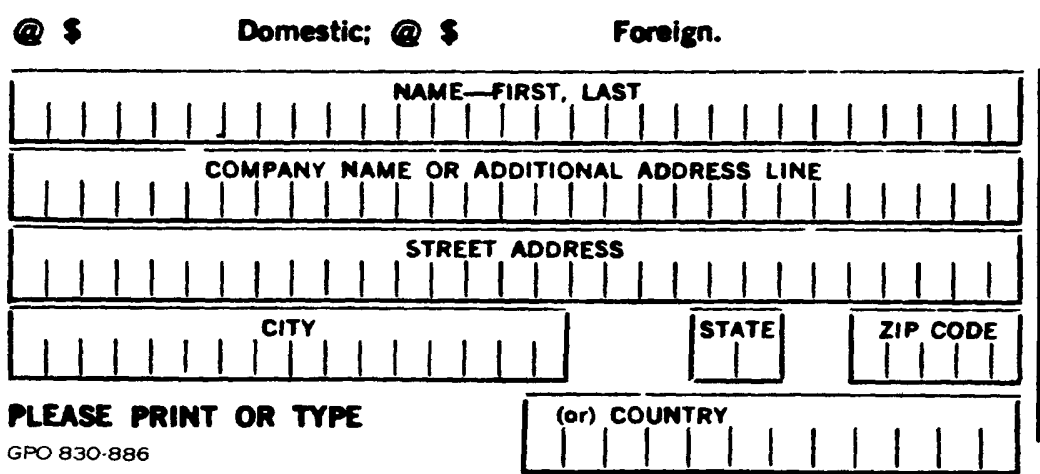

$\square$ Remittence Enclosed (Make checks payable to Suparin. tondent of Documents)

$\square$ Cheree to my Depost Account No. ..........................

MAIL ORDER FORM TO: Superintendent of Documents Governmemt Printing Office Woshington. D.C. 20402 


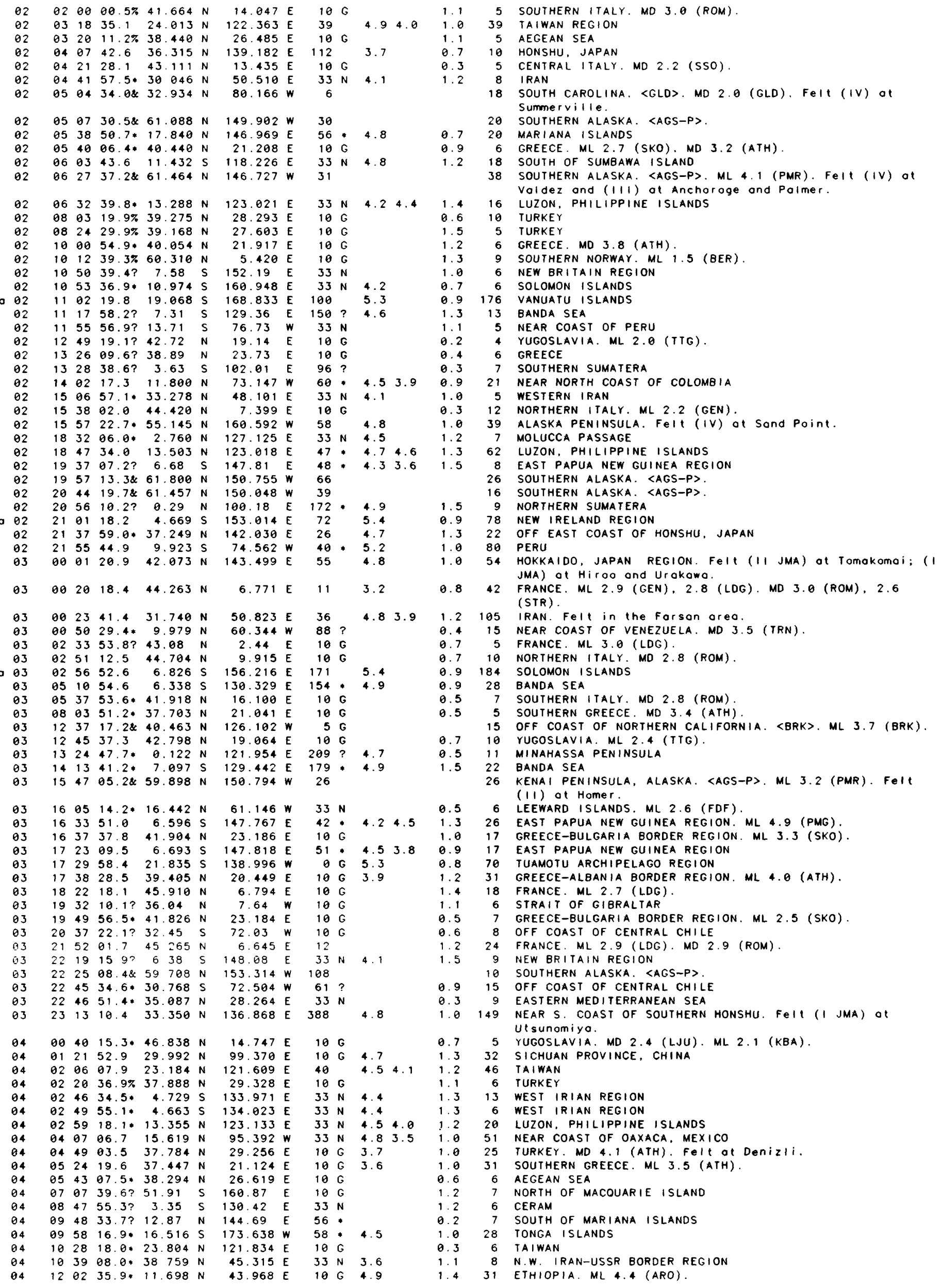




$\begin{array}{llllllllllllll}04 & 13 & 33 & 32.7 ? & 16.55 & \mathrm{~S} & 174.17 & \mathrm{~W} & 76 & ? & 4.3 & 1.3 \\ 04 & 15 & 00 & 26.6 * & 43.765 & \mathrm{~N} & 17.492 & \mathrm{E} & 10 & \mathrm{G} & & & 1.0 \\ 04 & 15 & 07 & 46.3 & 23.443 & \mathrm{~N} & 99.325 & \mathrm{E} & 10 & \mathrm{G} & 5.0 & 1.2 \\ 04 & 16 & 35 & 59.4 * & 7.074 & \mathrm{~S} & 129.813 & \mathrm{E} & 131 & * & 5.0 & 1.2 \\ 04 & 18 & 34 & 36.4 ? & 36.79 & \mathrm{~N} & 98.52 & \mathrm{E} & 10 & \mathrm{G} & & & 1.6 \\ 04 & 19 & 13 & 11.4 & 39.674 & \mathrm{~N} & 20.013 & \mathrm{E} & 21 & & 4.1 & 1.4 \\ 04 & 19 & 25 & 45.8 & 37.089 & \mathrm{~N} & 29.014 & \mathrm{E} & 10 & \mathrm{G} & & & 0.9 \\ 04 & 19 & 39 & 19.1 ? & 18.90 & \mathrm{~S} & 173.43 & \mathrm{~W} & 33 & \mathrm{~N} & 4.7 & 1.1 \\ 04 & 20 & 29 & 45.8 * & 52.738 & \mathrm{~S} & 160.713 & \mathrm{E} & 10 & \mathrm{G} & 5.2 & 4.6 & 1.0 \\ 04 & 21 & 33 & 59.78 & 34.600 & \mathrm{~N} & 116.840 & \mathrm{~W} & 2 & 4.2 & & \end{array}$

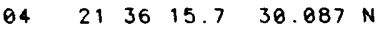
$\begin{array}{llllll}04 & 22 & 43 & 28.5 & 27.586\end{array}$ $05 \quad 002427.6 \quad 30.226 \mathrm{~N}$ $05 \quad 013157.6 \quad 30.112 \mathrm{~N}$ $\begin{array}{llll}02 & 53 & 43.5 & 40.874 \mathrm{~N}\end{array}$ 034355.1 ? $37.86 \mathrm{~N}$ $044927.2 * 43.144 \mathrm{~N}$ $070003.7 * 44.899 \mathrm{~N}$ $071346.0861 .676 \mathrm{~N}$ $073209.0 * 46.284 \mathrm{~N}$ $\begin{array}{llll}08 & 56 & 30.2 \% & 39.254 \mathrm{~N}\end{array}$ $100920.3 * 37.253 \mathrm{~N}$ $10 \quad 28 \quad 40.3 \% 60.635 \mathrm{~N}$ $111243.0 * 18.665 \mathrm{~N}$ $133732.1 ? 17.69 \mathrm{~N}$

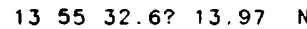
$\begin{array}{lllll}15 & 56 & 19.0 & 43.415 \mathrm{~N}\end{array}$ 155924.5 ? $16.14 \mathrm{~N}$ $\begin{array}{llll}16 & 23 & 40.4 & 12.002 \mathrm{~S}\end{array}$ $175013.0 * 35.228 \mathrm{~N}$ $175436.5 * 84.823 \mathrm{~N}$ $190911.0 * 16.981 \mathrm{~N}$ $2000 \quad 42.3 \quad 5.943 \mathrm{~S}$ $\begin{array}{llllll}20 & 12 & 44.3 & 16.54 \mathrm{~N}\end{array}$ $\begin{array}{lllll}20 & 38 & 48.1 & 39.628 \mathrm{~N}\end{array}$ 203915.0 ? $9.84 \mathrm{~S}$ $\begin{array}{llll}21 & 50 & 11.7 & 37.765 \mathrm{~N}\end{array}$ $\begin{array}{llll}21 & 56 & 26.2 & 46.008\end{array}$ $\begin{array}{llll}22 & 21 & 17.3 & 60.103 \mathrm{~N}\end{array}$ 224055.5 ? $44.98 \mathrm{~N}$ $224254.2860 .523 \mathrm{~N}$ $233329.9862 .575 \mathrm{~N}$ $\begin{array}{llll}23 & 54 & 26.6 & 37.773 \mathrm{~N}\end{array}$ BO 5507.1 ? $16.10 \mathrm{~N}$ $011744.3 * 17.502 \mathrm{~N}$ $\begin{array}{lllll}02 & 41 & 17.7 & 17.517 \mathrm{~s}\end{array}$ $025145.4 * 4.221 \mathrm{~N}$ $034503.9 ? \quad 40.35 \mathrm{~N}$ $072022.1851 .111 \mathrm{~N}$ $\begin{array}{llll}07 & 24 & 56.6 & 18.457 \mathrm{~N}\end{array}$ 073325.4 ? $18.51 \mathrm{~N}$ 075650.7 ? $61.88 \mathrm{~N}$ $0822 \quad 47.6 * 29.933 \mathrm{~N}$ $\begin{array}{llllll}08 & 28 & 31.8 \% & 33.740 \mathrm{~S}\end{array}$ $\begin{array}{lllll}09 & 00 & 29.4 & 37.764 \mathrm{~N}\end{array}$ $\begin{array}{lllll}09 & 11 & 34.1 & 43.711 \mathrm{~N}\end{array}$ $095310.0859 .887 \mathrm{~N}$ $\begin{array}{llll}10 & 41 & 43.8 \% & 68.842 N\end{array}$

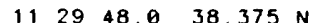
$\begin{array}{llll}12 & 48 & 38.8 & 15.632 \mathrm{~N}\end{array}$ $\begin{array}{lll}13 & 1755.2 * 30.5855\end{array}$ $\begin{array}{llll}14 & 04 & 29.0 ? & 52.87\end{array}$ $\begin{array}{lllll}14 & 09 & 17.9 & 6.628\end{array}$ $1435158 * 50441 \mathrm{~N}$ $\begin{array}{llllll}16 & 36 & 33 & 7.21838 \mathrm{~N}\end{array}$ $\begin{array}{llllll}16 & 59 & 11.9 & 45.994 \mathrm{~N}\end{array}$ $\begin{array}{llllll}17 & 06 & 35.1 ? & 61.78 \mathrm{~N}\end{array}$ $171521.2 * 31.228 \mathrm{~s}$ $\begin{array}{llll}18 & 0421.7 * 37.780 \mathrm{~N}\end{array}$ $\begin{array}{llll}19 & 03 & 29.7 \% & 43.758 \mathrm{~N}\end{array}$ $\begin{array}{llllll}19 & 45 & 26.98 & 61.878 \mathrm{~N}\end{array}$ $\begin{array}{llll}20 & 02 & 34.2 & 44.789 \mathrm{~N}\end{array}$ $213137.0 \quad 52.673 \mathrm{~N}$ $\begin{array}{llllll}23 & 27 & 05 & 18 & 61 & 900 \mathrm{~N}\end{array}$ $\begin{array}{lllll}00 & 07 & 25.9 & 37.178 \mathrm{~N}\end{array}$ $\begin{array}{llll}00 & 12 & 49.2 & 37.252 \mathrm{~N}\end{array}$ $\begin{array}{llll}00 & 18 & 19.2 & 48.779 \mathrm{~N}\end{array}$

$\begin{array}{llll}00 & 28 & 34.5 & 37.150 \mathrm{~N}\end{array}$ $013520.5 .60 .126 \mathrm{~N}$ $015422.4 \quad 37.312 \mathrm{~N}$ $015740.2 \quad 37.279 \mathrm{~N}$ $0233 \quad 38.7 \% 37.337 \mathrm{~N}$ $02 \quad 4747.0 \quad 43.919 \mathrm{~N}$ $024808.9 * 7.085 \mathrm{~s}$ $\begin{array}{lllll}02 & 59 & 13.6 & 37.312 \mathrm{~N}\end{array}$ $033122.2 \% 38.928 \mathrm{~N}$
$50.717 \mathrm{E}$ 140.882 51.012 $99.225 E$ 22.989 29.57 13.941 $14.747 \mathrm{E}$ $150.826 \mathrm{~W}$ $13.335 \mathrm{E}$ $27.720 \mathrm{E}$ $4.535 \mathrm{~W}$ $6.225 \mathrm{E}$ $142.692 \mathrm{E}$ 61.99 W $121.01 \mathrm{E}$

$5.433 \mathrm{E}$ $99.70 \mathrm{~W}$ $14.563 \mathrm{E}$ $22.249 E$

$5.990 \mathrm{E}$ $62.319 \mathrm{~W}$ $105.443 \mathrm{E}$ 99.59 W $27.802 \mathrm{E}$ $161.76 \mathrm{E}$ 29.242 $6.609 \mathrm{E}$ $29.525 \mathrm{~W}$ $6.27 \mathrm{E}$ $150.589 \mathrm{~W}$ $149.075 \mathrm{~W}$ $29.236 \mathrm{E}$ $99.91 \mathrm{~W}$ $145.893 \mathrm{E}$ $178.934 \mathrm{~W}$ $82.046 \mathrm{w}$ $126.79 \mathrm{~W}$ $124.543 \mathrm{~W}$ $146.302 E$ $144.39 E$ 4.30 $138.708 \mathrm{E}$ $71.224 \mathrm{~W}$ 26.712 $18.737 \mathrm{E}$ $153.370 \mathrm{~W}$

$6.417 \mathrm{E}$ $119.319 \mathrm{w}$ 60.581 $178.337 \mathrm{~W}$ $159.39 \mathrm{E}$ $147.730 \mathrm{E}$ $6.155 \mathrm{E}$ $143.110 \mathrm{E}$

6.768 $7.43 \mathrm{E}$ $71.041 \mathrm{~W}$ $26.714 \mathrm{E}$ $12.543 \mathrm{E}$ $147.722 \mathrm{w}$ $111.512 \mathrm{w}$ $34.836 \mathrm{~W}$ $147.714 \mathrm{~W}$ $4.451 \mathrm{~W} 10$

$4.490 \mathrm{~W} 10 \mathrm{G}$

$19.210 \mathrm{E} \quad 16$

$4.527 w$ $29.367 w$

$4.534 W$

4. $557 w$

$4.558 W$

$6.470 \mathrm{~W}$

$129.195 \mathrm{E}$

4.549 W $27.713 \mathrm{E}$

10

$10 \mathrm{G}$

35

106

$10 \mathrm{G}$

580

$10 \mathrm{G}$

$453 ?$

$10 \mathrm{G}$

$10 \mathrm{G}$

126

$10 \mathrm{G}$

$5 \mathrm{G}$

$10 \mathrm{C}$

24

$10 \mathrm{G}$

$10 \mathrm{G}$

$10 \mathrm{G}$

106

$10 \mathrm{G}$

$10 \mathrm{G}$

$10 \mathrm{G}$
$26 * 4.3$

$38 * 4.74 .4$

$37 * 4.0$

10 G 4.5

$10 \mathrm{G}$

$10 \mathrm{G}$

$10 \mathrm{G}$

$10 \mathrm{G}$

$10 \mathrm{G}$

$10 \mathrm{G}$

$10 \mathrm{G}$

$33 \mathrm{~N}$

$33 \mathrm{~N}$

$33 \mathrm{~N}$

$10 \mathrm{G}$

$33 \mathrm{~N}$

$10 \mathrm{G}$

$10 \mathrm{G}$

$33 \mathrm{~N}$

104 ?

$10 \mathrm{G}$

118 ?

$10 \mathrm{G}$

$10 \mathrm{G}$

171 *

$10 \mathrm{G}$

$20 \mathrm{G}$

114 ?

$18 \mathrm{G}$

$5 \mathrm{G}$
$10 \mathrm{G}$

$242 ? 5.0$

10 G 4.6

$45 * 4.9$

$236 ?$

$1 \mathrm{G}$
$33 \mathrm{~N}$

$10 \mathrm{G}$

$5 \mathrm{G}$

7 D 4.7

$G$

$69 ? 5.1$

4. 8

5.1

4.73 .7

5. 4

4.6

4. 1
1.2

1.5

0.6

0.9

1. 1

0.9

0. 4

0. 5

1. 2
1. 3

9. 4

1.2
0.3

0. 3

0.6

0.9

๑. 1

a. 7

12 TONGA ISLANDS

YUgOSLAVIA. ML 3.2 (ZAG), 2.5 (LJU). MD 3.1 (TRI). Felt in the Knin areo

16 BURMA-CHINA BORDER REGION. ML 4.6 (BJI)

25 BANDA SEA

5 OINGHAI PROVINCE, CHINA

7 GREECE-ALBANIA BORDER REGION. ML 4.4 (ATH), 4.0 (TTG)

TURKEY

TONGA ISLANDS

10 MACOUARIE ISLANDS REGION

43 SOUTHERN CALIFORNIA. LPAS-P>. ML 4.5 (PAS), 4.2 (BRK). Felt (V) ot Apple Volley; (IV) ot Burbank; (III) ot Barstow, Cothedral City, Doggett, Fownskin. Hesperia, Lucerne volley. Mentone, Newberry Springs, Polm Desert. Posodeno, Riolto ond wilmington.

22

50 BONIN ISLANDS REGION. Felt (I JMA) on Chichi-shimo.

13 IRAN

SICHUAN PROVINCE, CHINA

5 TURKEY

8 CENTRAL ITALY. MD 2.8 (SSO), 2.7 (ROM)

7 ADRIATIC SEA. MD 2.5 (TRI), 2.5 (LJU)

20 SOUTHERN ALASKA. LAGS-P>.

5 AUSTRIA. MD 2.9 (LJU), 2.6 (ROM), 2.3 (TRI).

TURKEY

SPAIN

SOUTHERN NORWAY. MD 1.8 (BER)

MARIANA ISLANDS REGION

LEEWARD ISLANDS. ML 2.9 (FDF).

MINDORO, PHILIPPINE ISLANDS

8 NEAR SOUTH COAST OF FRANCE. MD 2.5 (STR).

5 NEAR COAST OF GUERRERO, MEXICO

5 ANGOLA

2 MEDITERRANEAN SEA. MD 4.2 (ATH)

11 NORTH OF SVALBARD

5 LEEWARD ISLANDS. MD 2.6 (TRN).

39 SUNDA STRAIT

8 NEAR COAST OF GUERRERO, MEXICO

16 TURKEY. MD 3.2 (ATH). Felt at Bandirma.

6 SOLOMON ISLANDS

8 TURKEY

19 SWITZERLAND. ML 2.8 (LDG)

40 NORTH ATLANTIC OCEAN

6 FRANCE. ML 2.6 (LDG)

16 KENAI PENINSULA, ALASKA. 〈AGS-P>

21

$\begin{array}{rr}1.0 & 168 \\ 1.1 & 8\end{array}$ 


$\begin{array}{lllllll}07 & 05 & 07 & 47.6 * & 37.102 & \mathrm{~N} \\ 07 & 05 & 08 & 47.3 ? & 57.89 & \mathrm{~S} \\ 07 & 05 & 10 & 48.6 & 37.331 & \mathrm{~N} \\ 07 & 05 & 25 & 44.5 ? & 16.28 & \mathrm{~N} \\ 07 & 06 & 00 & 24.5 & 37.314 & \mathrm{~N} \\ 07 & 07 & 04 & 41.0 & 37.315 & \mathrm{~N} \\ 07 & 07 & 38 & 15.4 * 37.321 & \mathrm{~N} \\ 07 & 09 & 58 & 16.2 * & 8.956 & \mathrm{~S} \\ 07 & 10 & 03 & 19.2 * & 16.311 & \mathrm{~S} \\ 07 & 11 & 17 & 41.5 & 60.701 & \mathrm{~N} \\ 07 & 11 & 50 & 00.98 & 57.348 & \mathrm{~N} \\ 07 & 12 & 19 & 48.3 & 14.131 & \mathrm{~N} \\ 07 & 13 & 10 & 29.0 * 33.242 & \mathrm{~N} \\ 07 & 14 & 35 & 30.5 * & 51.258 & \mathrm{~N} \\ 07 & 15 & 08 & 25.1 & 34.596 & \mathrm{~N} \\ 07 & 15 & 17 & 48.3 & 6.591 & \mathrm{~S} \\ 07 & 15 & 42 & 07.88 & 63.605 & \mathrm{~N} \\ 07 & 16 & 08 & 12.3 & 37.257 & \mathrm{~N} \\ 07 & 16 & 31 & 49.3 & 36.150 & \mathrm{~N} \\ 07 & 17 & 40 & 04.9 * & 9.202 & \mathrm{~S} \\ 07 & 18 & 03 & 40.28 & 61.596 & \mathrm{~N} \\ 07 & 18 & 26 & 19.1 & 37.035 & \mathrm{~N} \\ 07 & 18 & 30 & 06.1 * 21.803 & \mathrm{~N} \\ 07 & 18 & 42 & 33.08 & 64.460 & \mathrm{~N} \\ 07 & 18 & 53 & 12.6 * 40.048 \mathrm{~S} \\ 07 & 19 & 15 & 12.3 ? & 51.70 & \mathrm{~N} \\ 07 & 19 & 45 & 53.7 & 38.057 \mathrm{~N}\end{array}$

$\begin{array}{lllllll}07 & 21 & 01 & 48.8 & 39.366 & \mathrm{~N} \\ 07 & 21 & 47 & 35.0 & 5.632 & \mathrm{~N} \\ 07 & 23 & 51 & 23.4 * & 34.262 & \mathrm{~N} \\ 08 & 00 & 02 & 06.0 & 47.855 & \mathrm{~N} \\ 08 & 00 & 17 & 58.3 ? & 35.12 & \mathrm{~N} \\ 08 & 01 & 11 & 12.8 & 37.965 & \mathrm{~N} \\ 08 & 01 & 24 & 56.7 & 14.678 & \mathrm{~S} \\ 08 & 01 & 33 & 48.2 & 44.167 & \mathrm{~N} \\ 08 & 01 & 37 & 25.7 ? & 14.42 & \mathrm{~N} \\ 08 & 01 & 41 & 49.8 * & 29.906 & \mathrm{~N} \\ 08 & 02 & 22 & 33.58 & 58.269 & \mathrm{~N} \\ 08 & 02 & 48 & 28.1 & 53.748 & \mathrm{~N} \\ 08 & 04 & 07 & 44.3 * & 55.550 & \mathrm{~S} \\ 08 & 04 & 48 & 59.4 & 37.269 & \mathrm{~N} \\ 08 & 04 & 55 & 09.6 * & 35.438 & \mathrm{~N} \\ 08 & 06 & 05 & 27.2 & 46.465 & \mathrm{~N} \\ 08 & 06 & 20 & 40.78 & 57.082 & \mathrm{~N} \\ 08 & 06 & 24 & 09.6 & 6.837 & \mathrm{~N} \\ & & & & & \\ 08 & 08 & 38 & 39.2 * & 13.798 & \mathrm{~N} \\ 08 & 09 & 09 & 28.28 & 61.779 & \mathrm{~N} \\ 08 & 09 & 25 & 57.7 & 30.179 & \mathrm{~N} \\ 08 & 09 & 41 & 35.97 & 59.876 & \mathrm{~N} \\ 08 & 09 & 51 & 56.8 & 19.531 & \mathrm{~S} \\ 08 & 10 & 07 & 26.4 ? & 9.87 & \mathrm{~S} \\ 08 & 10 & 54 & 32.9 & 37.751 \mathrm{~N} \\ 08 & 12 & 25 & 57.3 ? & 1.29 & \mathrm{~S} \\ 08 & 13 & 29 & 16.7 * & 18.057 & \mathrm{~N} \\ 08 & 13 & 46 & 28.0 & 23.496 \mathrm{~N} \\ 08 & 14 & 14 & 22.9 ? & 42.04 & \mathrm{~N} \\ 08 & 15 & 31 & 14.07 & 41.084 & \mathrm{~N} \\ 08 & 15 & 41 & 13.07 & 45.565 & \mathrm{~N} \\ 08 & 16 & 35 & 50.1 \% & 61.779 & \mathrm{~N} \\ 08 & 17 & 17 & 41.7 & 41.299 & \mathrm{~N} \\ 08 & 18 & 18 & 43.3 & 39.165 \mathrm{~N}\end{array}$

$4.418 \mathrm{~W}$ $4.494 W$ $62.51 W$ $4.560 w$ 4. $555 \mathrm{~W}$

$4.546 \mathrm{~W}$ $106.549 \mathrm{E}$ $5.509 \mathrm{E}$ $155.206 \mathrm{~W}$ $51.745 \mathrm{E}$ $141.372 \mathrm{E}$ $15.727 \mathrm{E}$ $27.994 \mathrm{E}$ $147.836 \mathrm{E}$ $146.213 \mathrm{~W}$ $4.557 \mathrm{~W}$ $120.081 \mathrm{~W}$ $78.940 \mathrm{~W}$ $151.760 \mathrm{~W}$ $4.552 \mathrm{~W}$ $121.393 \mathrm{E}$ $149.472 \mathrm{~W}$ $74.623 \mathrm{~W}$ $16.44 \mathrm{E}$ $21.620 \mathrm{E}$ $173.487 \%$

$\begin{array}{rlll}10 & G & & \\ 33 & N & 4.4 & \\ 10 & G & & \\ 33 & N & & \\ 10 & G & & \\ 10 & G & & \\ 10 & G & & \\ 33 & N & 5.0 & 3.5 \\ 48 & O & 5.1 & \\ 10 & G & & \\ 54 & & & \\ 10 & G & 4.7 & 4.5 \\ 33 & N & 4.5 & \\ 5 & G & & \\ 33 & N & & \\ 48 & * & 5.0 & 4.5 \\ O & & & \\ 10 & G & & \\ 5 & G & & \\ 67 & O & 5.0 & \\ 76 & & & \\ 10 & G & & \\ 75 & * & 3.9 & \\ 16 & & & \\ 33 & N & 4.9 & \\ 10 & G & & \\ 25 & & 5.0 & 4.8\end{array}$

28.140 125.732 $91.930 \mathrm{E}$ $7.187 \mathrm{E}$ $21.17 \mathrm{E}$ $21.491 E$ 167.215 59.51 W $99.472 \mathrm{E}$ $142.663 \mathrm{~W}$ $162.535 \mathrm{E}$ $28.029 \mathrm{~W}$ $4.544 \mathrm{~W}$ $27.504 \mathrm{E}$ $10.641 \mathrm{E}$ $149.483 \mathrm{~W}$ $37.878 \mathrm{E}$

$91.736 \mathrm{~W}$ $149.690 \mathrm{~W}$ $50.991 \mathrm{E}$ $5.736 E$ $173.736 \mathrm{~W}$ $124.31 \mathrm{E}$ $26.749 \mathrm{E}$ $101.42 \mathrm{E}$

$145.559 \mathrm{E}$ $121.493 \mathrm{E}$ 19.31 $29.207 \mathrm{E}$ 26. $281 \mathrm{E}$ $7.367 E$ 22.795 $99.477 \mathrm{~W}$

\begin{tabular}{|c|c|c|}
\hline $\begin{array}{l}10 \\
70\end{array}$ & G & 5.1 \\
\hline 10 & G & 3.5 \\
\hline 10 & G & \\
\hline $\begin{array}{l}33 \\
10\end{array}$ & N & \\
\hline 32 & 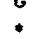 & 5.1 \\
\hline$\theta$ & & \\
\hline 10 & G & \\
\hline 10 & G & 5.3 \\
\hline 10 & G & \\
\hline 33 & N & 5.0 \\
\hline 25 & $D$ & 5.2 \\
\hline 10 & 6 & \\
\hline 10 & G & \\
\hline 10 & G & \\
\hline 10 & G & 59. \\
\hline 19 & & 3.04 .8 \\
\hline 33 & N & 4.33 .4 \\
\hline $\begin{array}{l}43 \\
33 \\
3\end{array}$ & $\mathrm{~N}$ & \\
\hline 10 & G & \\
\hline 23 & 0 & $5.5 \quad 5.4$ \\
\hline 80 & ? & 4.4 \\
\hline $\begin{array}{l}13 \\
167\end{array}$ & & \\
\hline 33 & N & $\begin{array}{l}4.5 \\
4.9\end{array}$ \\
\hline 27 & D & 5.04 .0 \\
\hline 10 & G & \\
\hline 10 & & \\
\hline 10 & 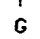 & \\
\hline 10 & & \\
\hline & & \\
\hline
\end{tabular}

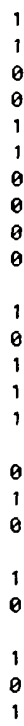

1.4
1.0
0.6
0.6
1.3
1.3
0.4
0.9
0.9
0.7
1.1
0.4
1.2
1.4
1.4
0.7
1.1
0.9
1.3
0.9
1.4
0.4
1.4

10 SPAIN. MbLg 2.9 (MDD).

12 SOUTH SANOWICH ISLANDS REgION

SPAIN. MbLg 2.6 (MDD)

LEEWARD ISLANDS

SPAIN. MbLg 2.7 (MDD)

SPAIN. MbLg 2.9 (MDD)

SPAIN. mbLg 2.7 (MDD)

SOUTH OF JAVA

TONGA ISLANDS

SOUTHERN NORWAY. ML 1.7 (BER)

- ALASKA PENINSULA. 〈AGS-P>.

57 EASTERN GULF OF ADEN

3 OFF EAST COAST OF HONSHU, JAPAN

9 POLAND. ML 3.6 (VKA), 3.0 (KRA).

- EASTERN MEDITERRANEAN SEA

43 EAST PAPUA NEW GUINEA REGION

33 CENTRAL ALASKA. $\angle A G S-P$. ML 3.6 (PMR).

8 SPAIN. mbLg 2.7 (MDD).

CENTRAL CALIFORNIA. ML 2.7 (BRK).

17 NEAR COAST OF NORTHERN PERU. Feli (IV) al Chimbote.

SOUTHERN ALASKA. LAGS-P>.

10 SPAIN. MbLG 2.9 (MDO)

6 TAIHAN REGION

27 CENTRAL ALASKA. 〈AGS-P>

18 OFF COAST OF SOUTHERN CHILE

POLAND. ML 3.3 (VKA).

282 GREECE. ML 5.0 (TTG), 4.7 (ATH). MD 5.1 (VKA), 4.8

(KBA). Crocks in many buildings in the Koto Akhaia oreo. Felt widely an narthern Peloponnisos and an Zakinthas and Kefallinia.

1.2

13

1.0 95 MINDANAO, PHILIPPINE ISLANDS

1.518 OINGHAI PROVINCE, CHINA

0.88 SWITZERLAND. ML 2.5 (LDG). MO 1.6 (STR).

1.35 MEDITERRANEAN SEA. MD 3.5 (ATH).

1.18 SOUTHERN GREECE. ML 3.2 (ATH)

1.164 VANUATU ISLANDS

1.536 NORTHERN ITALY. ML 3.1 (LOG). MO 2.9 (ROM).

0.2 5 WINOWARD ISLANDS. ML 3.0 (FDF)

1.415 SICHUAN PROVINCE, CHINA

15 GULF OF ALASKA. 〈AGS-P>

32 OFF EAST COAST OF KAMCHATKA

0.815 SOUTH SANDWICH ISLANDS REGION

0.916 SPAIN. mbLg 3.3 (MOD).

0.96 DODECANESE ISLANOS. MD 3.7 (ATH)

1.013 NORTHERN ITALY. MD 2.7 (ROM)

41 GULF OF ALASKA. ¿AGS-P>. ML 4.0 (PMR)

41 GULF OF ALASKA.
91 ETHIOPIA. Minor injuries to a few people and damage at Sodo.

28 NEAR COAST OF GUATEMALA

28 SOUTHERN ALASKA. LAGS-P>.

1.011 IRAN

0.6 8 SOUTHERN NORWAY. MD 1.8 (BER)

1.1178 TONGA ISLANOS. Mo=1.0*10*18 Nm (PPT). Ms 5.4 (BRK).

0.57 TIMOR

1.315 DODECANESE ISLANOS. ML 3.6 (ATH)

1.413 SOUTHERN SUMATERA

0.2 9 MARIANA ISLANDS

1.477 TAIWAN

0.34 YUGOSLAVIA. ML 2.1 (TTG)

1.17 TURKEY

1.119 ROMANIA

1.16 SOUTHERN NORWAY. ML 2.1 (BER).

0.67 YUGOSLAVIA. ML 1.9 (SKO).

0.714 KANSAS. mbLg 4.0 (NEIS), 3.8 (TUL). Felt (V) ot Domar, Palco and Zurich: (111) at Bogue Plainville and Wokeeney; (11) of Hays, Ogollah and Strutigort. Also felt in the stockton areo.

$96.61 \mathrm{E} \quad 1 \mathrm{Q}$

$77992 \mathrm{~W} \quad 14 \mathrm{C}$

$150.941 \mathrm{~W} 110$

$118.590 \mathrm{~W} 8$

47.82 E $10 \mathrm{G}$

$106.159 \mathrm{~W} 72 * 3.9$

70.197 E 223 D 4.5

$68.18 \mathrm{~W} 153$ ?

20.95 E $10 \mathrm{G}$

161.154

$21.453 \mathrm{E}$

W $10 \mathrm{G}$

$20.89 \mathrm{E} \quad 10 \mathrm{G}$

151.278 E $157 \quad 5.1$

$3.855 \mathrm{~W} \quad 10 \mathrm{G} \quad 4.93 .3$

$93.346 \mathrm{E}$

$4.378 \mathrm{~W}$

$29.491 E$

$92.774 \mathrm{E}$

$92.45 \mathrm{~W}$

$6.236 \mathrm{E}$

$149.757 \mathrm{~W}$

121.65

$16.35 \mathrm{E} \quad 10 \mathrm{G}$

$27.931 \mathrm{E} \quad 10 \mathrm{G}$
$33 \mathrm{~N} 4.3$

$\begin{array}{llll}10 & G & 4.7\end{array}$

$10 \mathrm{G}$

$33 \mathrm{~N} 4.8$

$10 \mathrm{G}$
$33 \mathrm{~N} 4.3$
B 9

TIBET

1.211 NEAR COAST OF PERU, Felt (11) ot Limo

24 CENTRAL ALASKA. 〈AGS-P>

11 CENTRAL CALIFORNIA. SPAS-P>. ML 3.0 (PAS).

0.4

0.8

1.1

1.0

1.0

1.3

1.2
0.8

0. 8

1.0

1.0
1.2
1.5

1.5

1.2

1.0

0.9
1.4

1.4
0.6

1.3

$\begin{array}{ll}0 & 3 \\ 0 & 7\end{array}$

NORTHWESTERN IRAN

27 OFF COAST OF JALISCO, MEXICO

HINOU KUSH REGION

9 SAN JUAN PROVINCE, ARGENTINA

12 SOLOMON ISLANDS

8 SOUTHERN GREECE. ML 3.3 (ATH).

6 SPAIN. MbLg 2.9 (MDD)

4 ALBANIA. ML 2.6 (SKO)

44 NEW BRITAIN REGION

60 JAN MAYEN ISLAND REGION

30 JAN MAYEN ISLAND REgION

6 TURKEY

54 ANDAMAN ISLANDS REGION

9 OFF COAST OF CHIAPAS. MEXICO

7 SOUTHERN NORWAY, MD 1.8 (BER)

28 CENTRAL ALASKA. 〈AGS-P>. Felt (11) of Willaw.

7 TAIWAN

6 POLAND ML $=9$ (KRA)
4 ALBANIA. ML 2.5 (SKO).

13 ANDAMAN ISLANDS REGION

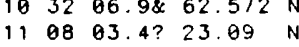

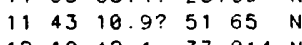




\begin{tabular}{|c|c|c|c|c|c|c|c|c|c|c|}
\hline 0 & 09 & 12 & 19 & 35.7 & 71.432 & $N$ & 4. 371 & $w$ & 10 & $G$ \\
\hline & 09 & 12 & 25 & $56.7 *$ & 71.699 & $N$ & 3717 & $w$ & 10 & G \\
\hline & 09 & 13 & 02 & $00.5 \%$ & 60.699 & $N$ & 5.517 & $E$ & 10 & G \\
\hline & 09 & 13 & 09 & $44.0 *$ & 10.608 & $S$ & 119.517 & $E$ & 33 & $N$ \\
\hline & 09 & 13 & 38 & 06.9 & 41.673 & $N$ & 19.961 & $E$ & 10 & G \\
\hline & 09 & 13 & 39 & $55.5 *$ & 10.566 & $S$ & 119.460 & $E$ & 33 & $N$ \\
\hline & 09 & 13 & 55 & 49.38 & 60.713 & $N$ & 151.857 & $w$ & 73 & \\
\hline & 09 & 14 & 46 & 04.9 & 29.395 & $N$ & 102.302 & $E$ & 10 & G \\
\hline & 09 & 15 & 06 & 32.98 & 60.913 & $N$ & 149.283 & $w$ & 17 & \\
\hline o & 09 & 15 & 34 & 11.6 & 7.865 & $S$ & 117.517 & $E$ & 245 & G \\
\hline & 09 & 16 & 29 & $44.9 *$ & 23.384 & $N$ & 121.413 & $E$ & 10 & $G$ \\
\hline & 09 & 16 & 38 & 13.4 & 8.112 & $S$ & 117.581 & $E$ & 242 & $*$ \\
\hline 0 & 09 & 16 & 52 & 12.1 & 20.260 & $N$ & 145.147 & $E$ & 149 & D \\
\hline & 09 & 18 & 04 & 05.7 & 39.671 & $N$ & 74.490 & $E$ & 33 & $N$ \\
\hline & 09 & 18 & 08 & $19.9 ?$ & 45.95 & $N$ & 2.72 & $E$ & 10 & G \\
\hline & 09 & 18 & 43 & 43.0 & 7.968 & $N$ & 127.211 & $E$ & 33 & $N$ \\
\hline & 09 & 19 & 27 & 46.4 & 38.284 & $N$ & 22.707 & $E$ & 10 & G \\
\hline & 09 & 21 & 11 & 23.4 & 25.265 & $N$ & 101.976 & $E$ & 37 & • \\
\hline & 09 & 21 & 52 & $51.7 \%$ & 39.191 & $N$ & 29.547 & $E$ & 10 & $G$ \\
\hline & 89 & 22 & 14 & $01.3 \%$ & 37.972 & $N$ & 0.181 & $E$ & 10 & G \\
\hline & 10 & 00 & 28 & $02.2 \%$ & 38.365 & $N$ & 27.078 & $E$ & 10 & G \\
\hline & 10 & 02 & 06 & $32.3 \%$ & 37.234 & $\mathbf{N}$ & 4.533 & $w$ & 5 & G \\
\hline & 10 & 02 & 06 & 38.9 & 17.121 & $S$ & 166.731 & $E$ & 33 & $N$ \\
\hline & 10 & 92 & 56 & $41.8 *$ & 4.770 & $\mathrm{~S}$ & 102.496 & $E$ & 33 & $N$ \\
\hline & 10 & 04 & 53 & 21.5 & 71.484 & $N$ & 3.800 & $w$ & 10 & G \\
\hline & 10 & 06 & 52 & $12.8 \&$ & 61.801 & $N$ & 150.675 & $w$ & 58 & \\
\hline & 10 & 07 & 15 & $06.9 \%$ & 42.105 & $N$ & 7.754 & $w$ & 10 & G \\
\hline & 10 & 08 & 44 & 55.3 & 30.044 & $S$ & 71.218 & $w$ & 104 & $*$ \\
\hline & 10 & 09 & 09 & $53.9 ?$ & 1.69 & $S$ & 77.70 & $w$ & 183 & - \\
\hline & 10 & 09 & 10 & $01.4 *$ & 38.899 & $N$ & 20.716 & $E$ & 10 & G \\
\hline & 10 & 10 & 37 & 18.6 & 40.596 & $N$ & 22.170 & $E$ & 10 & G \\
\hline & 10 & 10 & 43 & $12.4 \%$ & 37.662 & $N$ & 30.006 & $E$ & 10 & G \\
\hline & 10 & 11 & 43 & $18.4 ?$ & 61.88 & $N$ & 3.95 & $E$ & 10 & G \\
\hline & 10 & 12 & 02 & 03.0. & 6.373 & $S$ & 154.814 & $E$ & 66 & $?$ \\
\hline & 10 & 13 & 40 & 47.8 & 16.393 & $N$ & 61.277 & $w$ & 75 & $*$ \\
\hline & 10 & 14 & 08 & 32.9 & 38.387 & $N$ & 27.140 & $E$ & 9 & \\
\hline & 10 & 14 & 43 & $52.3 *$ & 4.012 & $S$ & 139.592 & $E$ & 74 & $?$ \\
\hline & 10 & 17 & 07 & $35.8 \&$ & 59.793 & $N$ & 153.277 & $w$ & 121 & \\
\hline & 10 & 17 & 29 & 58.1 & 22.252 & $\$$ & 138.734 & $w$ & 0 & G \\
\hline & 10 & 17 & 38 & 22.5 & 36.689 & $N$ & 22.877 & $E$ & 25 & $*$ \\
\hline & 10 & 17 & 59 & 09.28 & 58.117 & $N$ & 153.732 & $w$ & 62 & \\
\hline & 10 & 18 & 26 & $28.7 *$ & 36.464 & $N$ & 22.693 & $E$ & 10 & $G$ \\
\hline & 10 & 18 & 35 & $00.8 \&$ & 41.947 & $N$ & 125.034 & $w$ & 5 & $\mathbf{G}$ \\
\hline & 10 & 19 & 09 & 16.3 & 36.682 & $\mathrm{~N}$ & 22.924 & $E$ & 15 & \\
\hline & 10 & 19 & 39 & $54.7 ?$ & 51.51 & $N$ & 6.68 & $E$ & 10 & $G$ \\
\hline & 10 & 21 & 22 & 06.0 & 37.174 & $N$ & 4.566 & $w$ & 21 & $*$ \\
\hline & 10 & 21 & 50 & 56.9 & 36.369 & $N$ & 31.965 & $E$ & 112 & \\
\hline & 10 & 22 & 32 & $23.1 \%$ & 42.790 & $N$ & 12.675 & $E$ & 10 & $G$ \\
\hline & 11 & $\theta \theta$ & 51 & $26.7 \&$ & 36.250 & $N$ & 120.300 & $w$ & 6 & G \\
\hline & 11 & 01 & 18 & $03.5 *$ & 43.824 & $N$ & 8.263 & $E$ & 10 & G \\
\hline & 11 & 81 & 46 & $44.6 \&$ & 59.778 & $N$ & 153.418 & $w$ & 130 & \\
\hline & 11 & 84 & 44 & 51.6 & 36.494 & $N$ & 71.262 & $\boldsymbol{E}$ & 224 & \\
\hline & 11 & 85 & 03 & $42.3 *$ & 36.620 & $N$ & 27.974 & $E$ & 10 & G \\
\hline & 11 & 86 & 05 & 07.9 & 47.173 & $N$ & 7.492 & $\mathbf{E}$ & 10 & $G$ \\
\hline & 11 & 86 & 45 & $47.3 *$ & 6.415 & $S$ & 148.081 & $\mathbf{E}$ & 33 & $N$ \\
\hline & 11 & 86 & 57 & $30.5 *$ & 30.261 & $N$ & 51.643 & $E$ & 33 & $N$ \\
\hline & 11 & 86 & 59 & $50.5 *$ & 41.622 & $N$ & 72.902 & $E$ & 33 & $N$ \\
\hline & 11 & 87 & 08 & $30.8 *$ & 59.679 & $N$ & $29 . \mathrm{BOO}$ & $w$ & 10 & G \\
\hline & 11 & 89 & 29 & $18.2 \&$ & 38.845 & $N$ & 122.808 & $w$ & 4 & \\
\hline & 11 & 09 & 53 & 30.9. & 13.348 & $N$ & 144.274 & $\mathbf{E}$ & 126 & \\
\hline & 11 & 10 & 08 & 27.2 & 59.605 & $N$ & 29.815 & $w$ & 10 & G \\
\hline & 11 & 10 & 38 & $10.8 *$ & 60.032 & $N$ & 29.706 & $w$ & 10 & G \\
\hline & 11 & 11 & 26 & $09.2 *$ & 59.103 & $N$ & 30.212 & $w$ & 10 & G \\
\hline & 11 & 11 & 33 & $04.3 *$ & 11.289 & $N$ & 126.123 & $E$ & 33 & $N$ \\
\hline & 11 & 11 & 34 & 49.18 & 57.459 & $N$ & 142.885 & $w$ & 10 & $G$ \\
\hline & 11 & 12 & $\theta 0$ & $32.9 *$ & $44 \quad 477$ & $N$ & $124 \quad 895$ & $w$ & 10 & G \\
\hline a & 11 & 12 & 21 & $497 ?$ & 5175 & $S$ & $158 \quad 85$ & $E$ & 10 & G \\
\hline & 11 & 12 & 42 & 162 & 59606 & $N$ & 29871 & $w$ & 10 & c. \\
\hline & 11 & 12 & 44 & $53.3 *$ & 59400 & $N$ & $30 \quad 159$ & $w$ & 10 & $G$ \\
\hline & 11 & 12 & 53 & $25.6 *$ & 59.961 & $N$ & 29.686 & $w$ & 10 & $G$ \\
\hline & 11 & 13 & 98 & $51.7 \%$ & 42.131 & $N$ & 7769 & $w$ & 10 & G \\
\hline & 11 & 13 & 16 & 55.4 & 66.936 & $\mathrm{~N}$ & $156 \quad 375$ & $w$ & 5 & $G$ \\
\hline a & 11 & 13 & 24 & 32.0 & 35.107 & $N$ & 35.008 & $w$ & 10 & G \\
\hline & 11 & 13 & 42 & 44.1 & 26.419 & $N$ & 90.763 & $\mathbf{E}$ & 33 & $N$ \\
\hline & 11 & 14 & 52 & 33.7 & 19.960 & $\$$ & 133.731 & $E$ & 5 & G \\
\hline & 11 & 14 & 55 & $26.7 ?$ & 11.80 & $S$ & 77.73 & $w$ & 33 & $N$ \\
\hline & 11 & 16 & 49 & $49.2 *$ & 6.598 & $\mathrm{~S}$ & 147.909 & $E$ & 33 & $N$ \\
\hline & 11 & 16 & 55 & 35.2 & 37.330 & $N$ & 20.735 & $E$ & 10 & G \\
\hline & 11 & 17 & 22 & $46.5 \&$ & 32.410 & $N$ & 115.370 & $w$ & 6 & G \\
\hline & 11 & 18 & 11 & $54.0 *$ & 34.180 & $N$ & 24.563 & $E$ & 10 & $G$ \\
\hline & 11 & 18 & 22 & $22.2 \%$ & 37.332 & $\mathrm{~N}$ & 2.144 & $w$ & 10 & G \\
\hline & 11 & 19 & 34 & $24.6 *$ & 59.601 & $N$ & 30.003 & $w$ & 10 & $G$ \\
\hline & 11 & 20 & 03 & 51.3 & 35.183 & $N$ & 140.821 & $E$ & 67 & * \\
\hline & 11 & 21 & 46 & 05.4 & 43.880 & $N$ & 12.0002 & $E$ & 9 & G \\
\hline & 11 & 21 & 54 & 28.38 & 60.288 & $N$ & 140.448 & $w$ & 13 & \\
\hline & 11 & 23 & 14 & 23.08 & 61.940 & $N$ & 150.770 & $w$ & 0 & \\
\hline 0 & 11 & 23 & 24 & 39.0 & 6.463 & $S$ & 147.857 & $E$ & 49 & \\
\hline & $\begin{array}{l}11 \\
12\end{array}$ & $\begin{array}{l}23 \\
00\end{array}$ & $\begin{array}{l}57 \\
04\end{array}$ & & $\begin{array}{ll}12 & 519 \\
21 & 861\end{array}$ & $\begin{array}{l}\mathrm{S} \\
\mathrm{N}\end{array}$ & $\begin{array}{r}129.558 \\
89.763\end{array}$ & $\begin{array}{l}E \\
E\end{array}$ & $\begin{array}{r}10 \\
6\end{array}$ & $\begin{array}{l}G \\
G\end{array}$ \\
\hline
\end{tabular}




12
$0 \quad 12$
12
12
12
12
12
12
12
12
12
12
12
12
12
12

$\begin{array}{lllllll}00 & 07 & 46.6 & 6.492 & S & 147.955 E\end{array}$ $\begin{array}{llllll}05 & 47 & 35.7 & 15.283 \mathrm{~S} & 167.572 \mathrm{E}\end{array}$ $0550 \quad 42.6 \& 63.060 \mathrm{~N}$ $063411.7 \& 63.391 \mathrm{~N}$ $0813 \quad 36.3 \% 11.006 \mathrm{~N}$ $085748.7 ? 23.50 \mathrm{~S}$

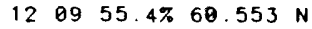
$\begin{array}{lllll}12 & 12 & 08.9 & 19.388 \mathrm{~N}\end{array}$ $122615.2 * 6.629 \mathrm{~S}$ $\begin{array}{lllll}13 & 11 & 51.5 & 30.169 \mathrm{~S}\end{array}$ $\begin{array}{lll}14 & 12 & 48.6 * 50.383 \mathrm{~N}\end{array}$ 145123.4 ? $44.67 \mathrm{~N}$ $\begin{array}{llll}14 & 57 & 43.3 & 41.518 \mathrm{~N}\end{array}$ $16 \quad 36 \quad 59.3 * 31.689 \mathrm{~S}$ $\begin{array}{lllll}16 & 40 & 41.8 \% & 39.647 \mathrm{~N}\end{array}$ $1657 \quad 18.4 \% 34.030 \mathrm{~N}$

$150.993 \mathrm{~W} \quad 122$

$149.818 W 104$

$61.671 \mathrm{~W} 10 \mathrm{G}$

$66.63 w$

$5.206 \mathrm{E}$

$145.447 \mathrm{E}$

$147.924 \mathrm{E}$

$178.918 \mathrm{~W}$

$6.059 \mathrm{E}$

8. $13 \mathrm{E}$

$20.771 \mathrm{E}$

$66.857 \mathrm{~W}$

$27.807 \mathrm{E}$

$118.180 \mathrm{~W}$

$\begin{array}{rlll}33 & N & 4.1 & 4.2 \\ 247 & & 5.4 & \\ 122 & & \\ 104 & & \\ 10 & G & \\ 237 & ? & 4.2 \\ 10 & G & \\ 150 & * & 4.9 \\ 46 & * & 4.2 \\ 82 & * & 5.6 \\ 10 & G & \\ 10 & G & \\ 10 & G & \\ 144 & ? \\ 10 & G & \\ 16 & & 4.3\end{array}$

1. 120

1. 2102

11

27

1.0
1.2
0.4

0. 4

1. 1.4

1.4
0.3

0. 3

0. 1

1.2

0.9

minor damage in the Bonaripara area. Fett throughout eastern Bangladesh fram Chittagang to Rangpur. Also felt in Megholoyo, India. Depth from broodbond

displocement sei smogroms.

EAST PAPUA NEW GUINEA REgION

VANUATU ISLANDS

CENTRAL ALASKA. 〈AGS-P>

WINDWARD ISLANDS

13 JUJUY PROVINCE, ARGENTINA

6 SOUTHERN NORWAY. MD 1.5 (BER)

54 MARIANA ISLANDS

13 EAST PAPUA NEW GUINEA REgION

77 KERMADEC ISLANDS

5 GERMANY. MD 2.1 (UCC)

6 NORTHERN ITALY. ML 2.1 (GEN)

6 ALBANIA. ML 2.4 (SKO)

16 LA RIOJA PROVINCE, ARGENTINA

11 TURKEY

55 SOUTHERN CALIFORNIA. LPAS-P>. ML 4.4 (PAS), 4.5 (BRK). Slight domoge ( $V I$ ) ot Bell Gardens and in the downtown Los Angeles areo. Felt (V) at Acton, Arcodia, Bell,

Bellflawer. Burbank. Commerce, Campton, Cavina, Dawney. Duarte, El Serena, Gardena, Glendara, Harbar City.

Howthorne, Highland Pork, Inglewood, Lokewood, Lang

Beach, Los Nietos, Lynmood. Montebello, Mount Wilson.

Narwalk. Pasadena, Sierro Madre, South Gate and Whittier. Felt in Las Angeles, Oronge, Riverside, San Bernardino ond Ventura Counties.

$\begin{array}{lllllllll}18 & 21 & 03.3 & 22.554 & \mathrm{~S} & 175.826 \mathrm{~W}\end{array}$

$183455.5 \cdot 33.284 \mathrm{~N}$

$184103.3 * 5.053 \mathrm{~N}$ $\begin{array}{llll}20 & 31 & 20.5 & 40.916 \mathrm{~N}\end{array}$

$204522.0 * 5.687 \mathrm{~s}$

$223035.3 * 24.753 \mathrm{~N}$

223211.0 ? $16.24 \mathrm{~N}$

$00 \quad 36 \quad 09.4 * 42.376 \mathrm{~N}$

$00 \quad 4732.1 \% 59.643 \mathrm{~N}$

$013436.6 \% 19.433 \mathrm{~N}$

014251.1 ? $8.28 \mathrm{~N}$

$014916.7 \% 16.056 \mathrm{~N}$

$\begin{array}{llllll}03 & 31 & 43.0 \% & 43.899 \mathrm{~N}\end{array}$

$034910.2 ? 44.13 \mathrm{~N}$

$054528.2 * 28.969 \mathrm{~S}$

$06 \quad 40 \quad 45.5 \& 37.738 \mathrm{~N}$

$\begin{array}{llll}08 & 52 & 42.8 \quad 30.935 \mathrm{~N}\end{array}$ 092928.9 ? $23.89 \mathrm{~N}$ $\begin{array}{llll}10 & 04 & 51.2 & 19.398 \mathrm{~N}\end{array}$ $\begin{array}{lllllll}10 & 17 & 35.4 ? & 13.61 \mathrm{~N}\end{array}$ $\begin{array}{llll}11 & 2553.1 & 0.059 & \mathrm{~S}\end{array}$ $1156 \quad 14.3 \% 60.295 \mathrm{~N}$ $121738.6 * 39.581 \mathrm{~N}$ $1220 \quad 32.1 \& 61.703 \mathrm{~N}$ $\begin{array}{llll}12 & 2905.3 & 42.490 \mathrm{~N}\end{array}$ $122921.2 * 3.851 \mathrm{~S}$ $\begin{array}{llll}13 & 16 & 06.9 & 35.599 \mathrm{~N}\end{array}$

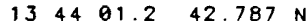
$144938.0 * 47.046 \mathrm{~N}$ $\begin{array}{llll}15 & 14 & 26.1 ? & 12.78 \mathrm{~N}\end{array}$ $160440.0 \% 63.129 \mathrm{~N}$ $\begin{array}{lllll}17 & 49 & 40.5 & 3.670 \mathrm{~S}\end{array}$ $\begin{array}{llll}18 & 19 & 03.0 & 42.596 \mathrm{~N}\end{array}$ $184034.2 * 19.815 \mathrm{~N}$ $\begin{array}{lllll}19 & 19 & 33 & 4 * 26.533 \mathrm{~N}\end{array}$ $\begin{array}{lllllll}19 & 52 & 2 & 3 * & 9 & 4525\end{array}$ $223123161822 \mathrm{~N}$ $225020.0861 .816 \mathrm{~N}$ $225933.4 * 43.342 \mathrm{~S}$ $001651.5 * 42.544 \mathrm{~N}$ $00 \quad 1708.3 \quad 37.082 \mathrm{~N}$

$00 \quad 2900.0 \quad 51.488 \mathrm{~N}$ $003558.2 \quad 51.547 \mathrm{~N}$ $\begin{array}{llll}01 & 21 & 36.2 & 44.486 \mathrm{~N}\end{array}$ $04 \quad 4000.3 \quad 51.583 \mathrm{~N}$ $0450 \quad 34.3 \& 59.543 \mathrm{~N}$ $050554.7 \quad 51.640 \mathrm{~N}$ $\begin{array}{llll}05 & 11 & 05.2 & 17.085 \mathrm{~N}\end{array}$ $062558.2 * 41.974 \mathrm{~N}$ $\begin{array}{lllll}07 & 15 & 41.8 & 40.459 \mathrm{~N}\end{array}$ $\begin{array}{lllll}10 & 17 & 35.2 & 12.875 & N\end{array}$ $\begin{array}{llllll}12 & 31 & 41 & 1 & 39.297 \mathrm{~N}\end{array}$ $123524.8 ? 51.26 \mathrm{~N}$ $\begin{array}{lllllll}12 & 40 & 07.3 \% & 39.357 & \mathrm{~N}\end{array}$ $125441.4 * 10.359 \mathrm{~S}$ $\begin{array}{llllll}13 & 33 & 06 & 6 & 51.801 \mathrm{~N}\end{array}$ $162110.0 * 22.191 \mathrm{~S}$
$82.575 \mathrm{w}$ $19.921 \mathrm{E}$ $130.979 E$ $95.154 \mathrm{E}$ $101.85 \mathrm{~W}$ $153.124 \mathrm{~W}$ $155.589 \mathrm{~W}$ $82.02 \mathrm{~W}$ $61.297 \mathrm{~W}$

$7.505 \mathrm{E}$ $7.24 \mathrm{E}$ $71.604 \mathrm{~W}$ $122.132 \mathrm{~W}$

138.398 $122.50 \mathrm{E}$ $143.48 \mathrm{E}$ $123.370 \mathrm{E}$ $5.099 \mathrm{E}$ $20.525 \mathrm{E}$ $151.588 \mathrm{~W}$ $24.260 \mathrm{E}$ $138.517 \mathrm{E}$ $27.233 \mathrm{E}$ $12.728 \mathrm{E}$ $9.154 \mathrm{E}$ $49.29 \mathrm{E}$ $150.763 \mathrm{~W}$ $140.139 \mathrm{E}$ $13.078 \mathrm{E}$ $71.140 \mathrm{~W}$ $129.379 \mathrm{E}$ $124256 \mathrm{E}$ $7448 \mathrm{E}$ $151155 \mathrm{~W}$ $38.969 \mathrm{E}$ $13.111 \mathrm{E}$ $141.072 \mathrm{E}$

174.300 174.316 w $174.173 \mathrm{~W}$ $152.995 \mathrm{~W}$ $174.236 w$ $62.251 \mathrm{~W}$ $23.159 \mathrm{E}$ $120.790 \mathrm{~W}$ $143.351 \mathrm{E}$ 28.992 $16.03 \mathrm{E}$ $29.056 \mathrm{E}$ $78.618 w$ $174.480 \mathrm{~W}$ $68.718 \mathrm{~W}$ $10.479 \mathrm{E}$

\begin{tabular}{|c|c|c|}
\hline 16 & & 4.2 \\
\hline 71 & D & 5.5 \\
\hline 185 & $*$ & 4.0 \\
\hline 33 & $N$ & 4.54 .2 \\
\hline 10 & G & \\
\hline 65 & - & 4.6 \\
\hline 33 & $N$ & \\
\hline 33 & $N$ & \\
\hline 10 & G & \\
\hline 116 & & \\
\hline 17 & & \\
\hline 33 & $N$ & \\
\hline 10 & $G$ & \\
\hline 10 & G & \\
\hline 10 & G & \\
\hline 33 & $N$ & \\
\hline 2 & & \\
\hline 394 & & 5.1 \\
\hline 10 & $G$ & \\
\hline 33 & $N$ & 4.8 \\
\hline 104 & D & \\
\hline 164 & $\bullet$ & 4.8 \\
\hline 10 & G & \\
\hline 10 & $G$ & \\
\hline 93 & & \\
\hline 10 & G & \\
\hline 106 & $?$ & 4.5 \\
\hline 22 & & \\
\hline 10 & G & \\
\hline 10 & G & \\
\hline 10 & $G$ & 4.6 \\
\hline 114 & & \\
\hline 71 & D & 5.4 \\
\hline 10 & G & \\
\hline 10 & G & \\
\hline 33 & $\mathbf{N}$ & 4.5 \\
\hline 118 & $?$ & 4.6 \\
\hline 10 & $G$ & \\
\hline 68 & & \\
\hline 10 & G & 5.05 .0 \\
\hline 10 & G & \\
\hline & & 4. 9 \\
\hline
\end{tabular}

$33 \mathrm{~N} 5.24 .5$ $33 \mathrm{~N} 5.34 .9$ $10 \mathrm{G}$

$33 \mathrm{~N} \quad 4.94 .3$

924.0

$33 \mathrm{~N} \quad 4.9$

$10 \mathrm{G}$

$10 \mathrm{G}$

5

126

5.5

$10 \mathrm{G}$

$10 \mathrm{G}$

$33 \mathrm{~N} 4.7$

$33 \mathrm{~N} 45$ $\begin{array}{lr}1.3 & 159 \\ 1.3 & 30\end{array}$

SOUTHERN CALIFORNIA. 〈PAS-P>. ML 4.1 (PAS), 3.9 (BRK). Felt in the los Angeles orea.

30 TONGA ISLANDS REGION

JMA) Ot Utsunomiyo.

17 SOUTH OF PANAMA

9 ALBANIA. ML 2.7 (SKO).

1.216 BANDA SEA

$0.7 \quad 7$ BURMA

1.47 NEAR COAST OF GUERRERO, MEXICO

1.06 YUGOSLAVIA

28 SOUTHERN ALASKA. 〈AGS-P>

45 HAWAII. ¿HVO-P〉. MD 3.9 (HVO).

0.35 PANAMA-COSTA RICA BORDER REGION

0.66 LEEWARD ISLANDS. ML 2.2 (FDF)

0. 35 NEAR SOUTH COAST OF FRANCE

0.15 NORTHERN ITALY. MD 1.0 (STR)

1.514 NEAR COAST OF CENTRAL CHILE

9 CENTRAL CALIFORNIA. <BRK>. ML 1.8 (BRK). Felt ot OaKland and San Leandro.

0.9176 SOUTH OF HONSHU, JAPAN

1.066 TAIWAN REGION

1.056 REVILLA GIGEDO ISLANDS REGION

0.610 SOUTH OF MARIANA ISLANDS

0.8 11 MINAHASSA PENINSULA

0.37 SOUTHERN NORWAY. MD 1.4 (BER).

1.1 6 GREECE-ALBANIA BORDER REGION

11 SOUTHERN ALASKA. 〈AGS-P>

1.17 BULGARIA

1.219 WEST IRIAN

1.312 DODECANESE ISLANDS, MD 4.0 (ATH)

6 CENTRAL ITALY. MD 2.5 (SSO), 2.2 (ROM)

0.25 GERMANY

0.4 11 EASTERN GULF OF ADEN

18 CENTRAL ALASKA. LAGS-P>.

Q 163 WEST IRIAN

0.87 CENTRAL ITALY. MD 2.3 (ROM), 2.3 (SSO)

0.6 7 DOMINICAN REPUBLIC REGION

$1.1 \quad 15$ RYUKYU ISLANDS

1311 SOUTHERN NORWA, MD 2.0 (BER)

11 SOUTHERN ALASKA. \AGS-P>

1.331 PRINCE EDWARD ISLANDS REGION

0. 8

NEAR EAST COAST OF HONSHU, JAPAN. FEI $t$ (II JMA) ot Mito, Shirokowo, Onohomo and Fukushimo; (I JMA) ot Utsunomiyo.

a 9195 ANDREANOF ISLANDS, ALEUTIAN IS. Felt on Adok.

1.0171 ANDREANOF ISLANDS, ALEUTIAN is. Felt on Adok.

0.67 NORTHERN ITALY. MD 2.3 (ROM).

0.976 ANDREANOF ISLANDS, ALEUTIAN IS

47 SOUTHERN ALASKA. 〈AGS-P>

0.980 ANDREANOF ISLANDS, ALEUTIAN IS

1.66 LEEWARD ISLANDS

0.85 GREECE-BULGARIA BORDER REGION

1.17

1. 1229

1. 10

NORTHERN CALIFORNIA ML 26 (BRK).

SOUTH OF MARIANA ISLANDS. Felt (IV) on Guam.

TURKEY

POLAND

6 TURKEY

14

1028 ANDREANOF ISLANDS, ALEUTIAN IS.

NEAR COAST OF PERU

0.8 10 NORTHERN CHILE. Felt ( 111 ) ot Calamo. Tocopillo and OUillohuo. 


\begin{tabular}{|c|c|c|c|c|c|c|c|c|c|c|c|c|}
\hline 14 & 17 & 29 & $51.4 *$ & 66. & 958 & $\mathrm{~N}$ & 20.614 & $L^{2}$ & 10 & G & & \\
\hline o 14 & 18 & 86 & 38.2 & 34 & 289 & $\mathrm{~N}$ & 26.055 & $E$ & 14 & & 5.2 & 51 \\
\hline 14 & 18 & 26 & 20.8 & 34 & 194 & $\mathrm{~N}$ & 26.180 & $E$ & 40 & * & 4.3 & \\
\hline 14 & 18 & 29 & 26.0 & 33 & 972 & $\mathrm{~N}$ & 26.201 & E & 10 & G & 4.4 & \\
\hline 14 & 19 & 47 & $47.0 ?$ & 44 & 32 & $\mathrm{~N}$ & 142.74 & $E$ & 225 & * & 4.6 & \\
\hline 14 & 21 & 44 & 35.1 & 40 & 085 & $\mathrm{~N}$ & 144.609 & $E$ & 33 & $N$ & 5.3 & 4.6 \\
\hline 14 & 21 & 49 & 43.48 & & 042 & $\mathrm{~N}$ & 124.218 & $w$ & 23 & & & \\
\hline - 14 & 22 & 37 & 11.3 & 14 & 013 & s & 65.945 & $E$ & 10 & $G$ & 5.1 & 4.9 \\
\hline 14 & 22 & 49 & 41.6 & 10 & 206 & $\mathrm{~N}$ & 60.021 & $w$ & 10 & G & & \\
\hline 15 & 01 & 27 & 57.3 & & 851 & $\mathrm{~N}$ & 28.361 & $E$ & 10 & $G$ & & \\
\hline 15 & 01 & 40 & $19.4 ?$ & 43 & 67 & $\mathrm{~N}$ & 13.75 & $E$ & 10 & $G$ & & \\
\hline 15 & 01 & 59 & 51.9 & 43 & 035 & $\mathrm{~N}$ & 12.984 & $E$ & 10 & $G$ & & \\
\hline 15 & 02 & 10 & $01.6 *$ & 2 & 438 & $\mathrm{~N}$ & 128.352 & $E$ & 33 & $N$ & 4.9 & \\
\hline 15 & 03 & $\theta 0$ & $10.7 ?$ & & .84 & $\mathrm{~s}$ & 174.20 & $w$ & 33 & $N$ & 4.9 & 4.0 \\
\hline 15 & 04 & 11 & $18.9 ?$ & & 25 & $N$ & 177.96 & $E$ & 33 & $N$ & 4.5 & \\
\hline 15 & 07 & 13 & $08.1 *$ & 39 & .964 & N & 71.032 & $E$ & 33 & $N$ & 4.6 & 4.0 \\
\hline 15 & 08 & 42 & 55.12 & 62 & 822 & $\mathrm{~N}$ & 148.195 & $w$ & 54 & & & \\
\hline 15 & 09 & 57 & $59.8 \%$ & 67 & 346 & $\mathrm{~N}$ & 157.398 & $w$ & 37 & & & \\
\hline 15 & 10 & 30 & 53.9 & 42 & 347 & $\mathrm{~N}$ & 20.094 & $E$ & 10 & $G$ & & \\
\hline 15 & 11 & 10 & $17.4 ?$ & 34 & 36 & $\mathbf{N}$ & 26.40 & $E$ & 10 & G & & \\
\hline 15 & 12 & 10 & $17.7 ?$ & 23 & .84 & $S$ & 179.79 & $w$ & 558 & * & 4.7 & \\
\hline 15 & 12 & 11 & 54.7 & 51 & 625 & $N$ & 174.280 & $w$ & 33 & $\mathrm{~N}$ & 4.9 & 4.4 \\
\hline 15 & 12 & 36 & $18.8 *$ & 29 & 923 & s & 70.143 & $w$ & 33 & $\mathrm{~N}$ & & \\
\hline 15 & 12 & 42 & $14.2 \%$ & 60 & 637 & $N$ & 6.207 & $E$ & 10 & $G$ & & \\
\hline 15 & 15 & 45 & $44.3 *$ & 45 & 698 & $N$ & 11.096 & $E$ & 10 & $G$ & & \\
\hline - 15 & 15 & 51 & 58.6 & & .800 & $N$ & 73.019 & $w$ & 162 & $D$ & 5.1 & \\
\hline 15 & 16 & 54 & $56.2 ?$ & 51 & .04 & $\mathrm{~N}$ & 177.90 & $E$ & 33 & $N$ & 4.4 & \\
\hline 15 & 16 & 59 & $41.2 *$ & 33 & .943 & $\mathrm{~N}$ & 26.302 & $E$ & 10 & $G$ & & \\
\hline 15 & 18 & 14 & $26.9 *$ & 33 & .053 & $s$ & 117.627 & $E$ & 10 & G & & \\
\hline 015 & 19 & 32 & 45.6 & & .824 & $\mathrm{~N}$ & 82.309 & $w$ & 10 & G & 4.9 & 4.9 \\
\hline 15 & 19 & 39 & $39.2 \%$ & 15 & .944 & $\mathrm{~N}$ & 60.966 & $w$ & 24 & $*$ & & \\
\hline 15 & 20 & 01 & $05.5 \%$ & 60 & .720 & $N$ & 5.524 & $E$ & 10 & G & & \\
\hline 15 & 20 & 08 & $50.3 *$ & 23 & .504 & $\mathrm{~N}$ & 94.989 & $E$ & 33 & $N$ & & \\
\hline 15 & 21 & 15 & 28.3 & 34 & .727 & $N$ & 101.442 & $E$ & 18 & - & 4.7 & 4.3 \\
\hline 15 & 21 & 38 & $48.7 *$ & 34 & 293 & $\mathrm{~N}$ & 26.730 & $E$ & 10 & $G$ & & \\
\hline 15 & 22 & $0 \theta$ & 56.78 & 58 & .674 & $\mathrm{~N}$ & 142.927 & w & 10 & $G$ & & \\
\hline 15 & 22 & 22 & $14.9 \%$ & 32 & .892 & $\mathrm{~s}$ & 71.223 & $w$ & 33 & $N$ & & \\
\hline 15 & 22 & 41 & $33.9 \%$ & 61 & .217 & $N$ & 3.532 & $E$ & 10 & $G$ & & \\
\hline 15 & 23 & 07 & 39.0 & 15 & 916 & $N$ & 60.959 & $w$ & 10 & $G$ & & \\
\hline 16 & 01 & 33 & 57.68 & 61 & .421 & $\mathrm{~N}$ & 152.041 & $w$ & 106 & & 3.5 & \\
\hline 16 & 02 & 12 & 53.4 & 10 & .379 & $s$ & 119.530 & $E$ & 33 & $N$ & 5.1 & \\
\hline 16 & 04 & 15 & 40.1 & 44 & .826 & $\mathrm{~N}$ & 9.040 & $E$ & 10 & $G$ & & \\
\hline 16 & 04 & 19 & 45.2 & 52 & .407 & $\mathrm{~N}$ & 159.572 & $E$ & 33 & $\mathrm{~N}$ & 4.9 & 4.2 \\
\hline 16 & 07 & 00 & 47.58 & 34 & .830 & $N$ & 121.010 & $w$ & 6 & $G$ & & \\
\hline 16 & 07 & 08 & $28.4 ?$ & 44 & .34 & $N$ & 6.67 & $E$ & 10 & G & & \\
\hline o 16 & 07 & 18 & 35.4 & 13 & .224 & $N$ & 145.154 & $E$ & 68 & & 5.5 & \\
\hline 16 & 88 & 38 & 43.58 & 37 & .037 & $\mathbf{N}$ & 121.477 & w & 7 & & & \\
\hline 16 & 89 & 09 & $49.9 \%$ & 41 & .681 & $\mathrm{~N}$ & 13.694 & $E$ & 10 & $\mathbf{G}^{\prime}$ & & \\
\hline 16 & 09 & 26 & $30.8 *$ & 44 & .310 & $\mathrm{~N}$ & 8.189 & $E$ & 10 & $G$ & & \\
\hline 16 & 09 & 27 & 42.58 & 63 & .544 & $N$ & 151.269 & w & 18 & & & \\
\hline 16 & 89 & 55 & $46.0 *$ & 44 & .233 & $N$ & 7.434 & $E$ & 10 & G & & \\
\hline 16 & 10 & 27 & 56.3 & 36 & .907 & $\mathrm{~N}$ & 5.240 & $w$ & 10 & G & & \\
\hline 16 & 10 & 35 & 16.8 & 36 & 926 & $\mathrm{~N}$ & 291 & 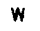 & 10 & G & & \\
\hline 16 & 10 & 36 & $59.6 *$ & 20 & .713 & s & 174.428 & $w$ & 152 & $?$ & 4. & \\
\hline 16 & 10 & 39 & $47.4 \%$ & 36 & .831 & $\mathrm{~N}$ & 5.301 & $w$ & 10 & $G$ & & \\
\hline-1 & & & & & $7: 4$ & & $800 ?$ & & 58 & & & \\
\hline
\end{tabular}

$105121.5857 .755 \mathrm{~N}$

$\begin{array}{rr}0.5 & 5 \\ 1.2 & 322 \\ 1.3 & 84 \\ 1.2 & 21 \\ 1.1 & 9 \\ 0.9 & 227 \\ & 9\end{array}$

0.9150

0.911

1.4

1. 6

0.6
0.8
1.5

1.3

1.3

5 SWEDEN. MD 3,1 (BER)

CRETE. ML 4.8 (ATH)
CRETE

EASTERN MEDITERRANEAN SEA. MD 4.3 (ATH).

HOKKAIDO, JAPAN REGION

OFF EAST COAST OF HONSHU, JAPAN. Felt $(11$ JMA $)$ ot Miyako and (I JMA) ot Morioka.

NEAR COAST OF NORTHERN CALIF, <BRK>. Fell (IV) at Eureko and Samoa. Alsa felt of Arcoto, Bayside and Trinidod.

MID-INDIAN RISE

TRINIDAD

GREECE-ALBANIA BORDER REGION. ML 1.4 (SKO).

5 CENTRAL ITALY. MD 2.7 (ROM).

9 CENTRAL ITALY. MD 2.5 (ROM), 2.4 (SSO).

16 HALMAHERA

24 TONGA ISLANDS

13 RAT ISLANDS, ALEUTIAN ISLANDS

16 TAJIK SSR. Felt (V) at Isfora. Neftebad, Oftubruy, Shurab and Kanibodam; (III) at Fergana. Tashkent and Andizhan: (11) at Nomangon.

38 CENTRAL ALASKA. LAGS-P>.

7 ALASKA. $\angle A G S-P\rangle$

1.314 YUGOSLAVIA. ML 2.5 (TTG).

0.95 CRETE. MD 3.6 (ATH).

1.417 SOUTH OF FIJI ISLANDS

73 ANDREANOF ISLANDS, ALEUTIAN IS.

10 CENTRAL CHILE

8 SOUTHERN NORWAY. MD 1.8 (BER).

6 NORTHERN ITALY. MD 2.5 (ROM).

218 NORTHERN COLOMBIA. Felt ot Bogoto, Bucaramongo,

Medellín. Cucuto and Tunja.

8 RAT I SLANDS, ALEUTIAN ISLANDS

6 EASTERN MEDITERRANEAN SEA. MD 3.9 (ATH).

5 WESTERN AUSTRALIA

103 SOUTH OF PANAMA MS 5.1 (BRK).

6 LEEWARD ISLANDS. ML 2.1 (FDF).

8 SOUTHERN NORWAY. MD 1.6 (BER).

8 BURMA-INDIA BORDER REGION

2 OINGHAI PROVINCE, CHINA

CRETE. MD 3.8 (ATH)

GULF OF ALASKA. LAGS-P>.

$\begin{array}{lrl}0.5 & 8 & \text { NEAR COAST OF CENTRAL CHILE } \\ 1.6 & 10 & \text { NORWEGIAN SEA. MD } 1.9 \text { (BER). } \\ 0.8 & 8 & \text { LEEARD ISLAN. }\end{array}$

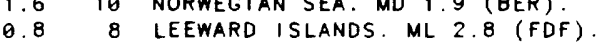

29 SOUTHERN ALASKA. $\angle A G S-P>$.

44 SUMBA ISLAND REGION

0.610 NORTHERN ITALY. ML 2.6 (GEN). MD 2.2 (ROM)

$\begin{array}{lll}0.6 & 10 & \text { NORTHERN ITALY. ML } 2.6 \text { (GEN) } \\ 1.0 & 67 & \text { OFF EAST COAST OF KAMCHATKA }\end{array}$

17 OFF COAST OF CALIFORNIA. SPAS-P>. ML 3.3 (PAS)

0.97

1.1199 MARIANA ISLANDS. Felt (IV) on GUOm.

14 CENTRAL CALIFORNIA. 〈BRK>. ML 2.8 (BRK).

5 SOUTHERN ITALY. MD 2.6 (ROM)

6 NORTHERN ITALY. ML 2.1 (GEN)

24 CENTRAL ALASKA. $\angle A G S-P>$. ML 3.7 (PMR)

0.75 NORTHERN TTALY. MD 2.5 (ROM).

1.525 STRAIT OF GIBRALTAR. mbLg 3.7 (MDD). MD 3.5 (TAF). Felt (III) in the Algodonales-Ronda orea, Spain.

1.412 STRAIT OF GIBRALTAR. MDLg 3.0 (MDD)

0.614 TONGA ISLANDS

1.05 STRAIT OF GIBRALTAR, mbLg 2.6 (MDD).

511 KODIAK ISLAND REGION. ¿AGS-P>. SI ight damage (v) at Kodiak and Larsen Boy. Felt (iv) ot Akniak. Karluk and Part Lians: (111) at Homer, Kenai and Ninilichik. Depth $51.1 \mathrm{kilameters}$ from broodband displacement se i smograms

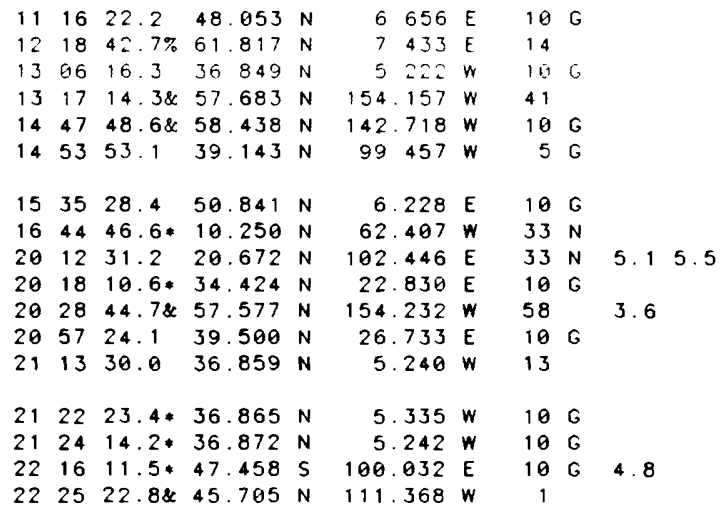

0

68

0.7

0.38

GERMANY. ML 1.9 (JCK)

1.2117 SOUTHEAST ASIA

1.419 MEDITERRANEAN SEA. ML 3.7 (ATH).

26 KODIAK ISLAND REGION. $\angle A G S-P\rangle$.

1.121 TURKEY. MD 3.5 (ATH).

1.224 STRAIT OF GIBRALTAR. mbLg 3.5 (MDD). MD 3.5 (TAF). Fell at Rondo, Spoin.

1.15 STRAIT OF GIBRALTAR

1.36 STRAIT OF GIBRALTAR

1.111 SOUTHEAST INDIAN RISE

12 MONTANA. <BUT>. ML 3.6 (BUT). Felt (111) ot Belgrade. Ennis and Gallatin Gatewoy. Alsa felt in the Amsterdam areo

$29.287 \mathrm{~W}$ $100.080 \mathrm{E}$ $100.156 \mathrm{E}$ $150628 \mathrm{~W}$ $137.982 \mathrm{E}$ $\begin{array}{lll}10 & G & 4.3\end{array}$

10 G 5.3

10 G $5.4 \quad 5 \quad 4$ 21

$360 \div 59$
1.0 11 AZORES ISLANDS REGION

1.241 SOUTHEAST INDIAN RISE

37 SOUTHEAST INDIAN RISE

10 CENTRAL ALASKA $\angle A G S-P>$

0.9484 SOUTH OF HONSHU, JAPAN. mb 6.2 (BRK). Fett (111 JMA) at 


\begin{tabular}{|c|c|c|c|c|c|c|c|c|c|c|c|}
\hline 17 & $\theta 0$ & 13 & $25.6 *$ & 2.902 & $\mathrm{~s}$ & 143.462 & 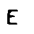 & 33 & $\mathrm{~N}$ & 5.3 & 4.7 \\
\hline 17 & 00 & 48 & $01.7 ?$ & 17.27 & $\mathrm{~N}$ & 62.48 & $\mathbf{w}$ & 32 & * & & \\
\hline 17 & 01 & 84 & 17.3 & 51.469 & $\mathbf{N}$ & 174.115 & w & 33 & $\mathbf{N}$ & 4.8 & \\
\hline 17 & 01 & 49 & 10.6 & 35.978 & $\mathrm{~N}$ & 27.607 & E & 10 & G & & \\
\hline 17 & 04 & 00 & $49.4 *$ & 3.109 & s & 147.333 & E & 33 & $\mathrm{~N}$ & 4.8 & 4.9 \\
\hline 17 & 04 & 38 & 25.6 & 41.807 & $\mathbf{N}$ & 142.695 & E & 63 & & 4.3 & \\
\hline 17 & 05 & 24 & $31.7 \%$ & 34.027 & s & 70.810 & $w$ & 33 & $\mathrm{~N}$ & & \\
\hline 17 & 05 & 52 & $00.3 *$ & 45.159 & $\mathrm{~N}$ & 6.507 & E & 10 & G & & \\
\hline 17 & 07 & 47 & $03.2 *$ & 3.082 & $\mathrm{~s}$ & 147.176 & $E$ & 33 & $\mathrm{~N}$ & 4.7 & 4.5 \\
\hline 17 & 08 & 31 & $10.0 *$ & 9.646 & s & 108.877 & E & 28 & D & 5.0 & 4.5 \\
\hline 17 & 10 & 06 & $31.5 \%$ & 33.626 & $\mathrm{~s}$ & 71.706 & $w$ & 18 & $*$ & & \\
\hline 17 & 10 & 09 & $16.6 \%$ & 33.619 & $\mathrm{~s}$ & 71.785 & $w$ & 26 & & & \\
\hline 17 & 10 & 14 & 39.3 & 60.879 & $\mathrm{~N}$ & 3.677 & E & 10 & G & & \\
\hline 17 & 10 & 45 & 52.0 & 42.059 & $\mathbf{N}$ & 19.671 & E & 10 & G & & \\
\hline 17 & 11 & 26 & $09.8 \%$ & 60.904 & $\mathrm{~N}$ & 3.445 & E & 10 & G & & \\
\hline 17 & 12 & 21 & 46.28 & 61.896 & $\mathrm{~N}$ & 152.089 & $w$ & 117 & & & \\
\hline 17 & 13 & 16 & 35.28 & 63.557 & $\mathrm{~N}$ & 150.782 & $w$ & $\theta$ & & & \\
\hline 17 & 14 & 39 & $16.9 *$ & 12.318 & $\mathrm{~N}$ & 143.735 & E & 12 & & 5.0 & 3.9 \\
\hline 17 & 14 & 57 & 48.4 & 31.419 & $\mathrm{~s}$ & 67.550 & $w$ & 28 & & 5.3 & 4.5 \\
\hline 17 & 15 & 30 & $02.4 *$ & 31.505 & $\mathrm{~s}$ & 179.951 & $w$ & 284 & $*$ & 4.2 & \\
\hline 17 & 15 & 36 & $08.6 *$ & 39.850 & $\mathrm{~N}$ & 21.050 & $E$ & 19 & $*$ & & \\
\hline 17 & 16 & 52 & 54.9 & 29.856 & $\mathrm{~N}$ & 59.706 & E & 33 & N & 4.8 & 4.6 \\
\hline 17 & 16 & 58 & $52.3 \%$ & 33.549 & $\mathrm{~s}$ & $71.37 \theta$ & $w$ & 33 & $\mathbf{N}$ & & \\
\hline 17 & 17 & 49 & $48.2 *$ & 38.849 & $\mathrm{~N}$ & 23.319 & E & 10 & G & & \\
\hline 17 & 18 & 17 & 22.8 & 43.430 & $\mathrm{~N}$ & 12.776 & E & 10 & G & & \\
\hline 17 & 18 & 28 & 08.6 & 40.494 & $\mathrm{~s}$ & 74.674 & $w$ & 33 & $\mathrm{~N}$ & 5.6 & 5.0 \\
\hline 17 & 19 & 24 & $36.0 \%$ & 36.278 & $\mathrm{~N}$ & 27.499 & E & 10 & G & & \\
\hline 17 & 19 & 47 & $01.0 *$ & 52.304 & $\mathrm{~s}$ & 160.542 & $E$ & 10 & G & 4.9 & 4.5 \\
\hline 17 & 20 & 08 & 43.7 & 60.736 & $\mathrm{~N}$ & 4.267 & E & 22 & * & & \\
\hline 17 & 20 & 47 & 14.6 & 1.437 & $\mathrm{~s}$ & 24.169 & $w$ & 10 & G & 5.1 & 4.9 \\
\hline 17 & 20 & 56 & 10.0 & 37.997 & $\mathrm{~N}$ & 21.976 & E & 40 & $?$ & 4.1 & \\
\hline 17 & 21 & 28 & $21.2 \%$ & 59.859 & $\mathrm{~N}$ & 5.930 & E & 10 & $\mathrm{G}$ & & \\
\hline 17 & 22 & 13 & 37.1 & 50.331 & $N$ & 7.366 & E & 10 & G & & \\
\hline 17 & 23 & 02 & $51.9 ?$ & 46.62 & $\mathbf{N}$ & 10.01 & E & 10 & G & & \\
\hline 18 & 00 & 30 & 46.5 & 35.373 & $N$ & 27.424 & E & 13 & & & \\
\hline 18 & 01 & 07 & 31.3 & 44.554 & $\mathbf{N}$ & 6.883 & E & 10 & G & & \\
\hline 18 & 01 & 58 & 30.3 & 2.502 & $\mathrm{~N}$ & 126.581 & E & 33 & $\mathrm{~N}$ & 5.0 & \\
\hline 18 & 02 & 20 & $41.2 *$ & 23.207 & $\mathrm{~s}$ & 70.003 & $w$ & 103 & $?$ & 4.3 & \\
\hline 18 & 03 & 15 & 51.9 & 38.297 & $N$ & 20.437 & E & 31 & & 4.0 & \\
\hline 18 & 04 & 00 & 31.1 & 36.864 & $\mathrm{~N}$ & 5.253 & $w$ & 10 & G & & \\
\hline 18 & 04 & 02 & $33.1 \%$ & 58.479 & $N$ & 152.699 & $w$ & 56 & & & \\
\hline 18 & 04 & 16 & $49.7 *$ & 10.622 & $s$ & 165.960 & $E$ & 146 & $?$ & 4.4 & \\
\hline 18 & 04 & 38 & 13.98 & 63.239 & $N$ & 150.514 & $w$ & 121 & & & \\
\hline 18 & 04 & 54 & $42.8 *$ & 18.211 & $\mathrm{~s}$ & 177.911 & $w$ & 424 & $*$ & 4.6 & \\
\hline 18 & 05 & 09 & 30.2 & 78.935 & N & 3.520 & E & 10 & G & 4.8 & 4.6 \\
\hline 18 & 05 & 54 & 58.8 & 44.408 & $\mathrm{~N}$ & 14.879 & E & 10 & 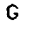 & & \\
\hline 18 & 06 & 09 & 39.0 & 41.241 & $\mathrm{~N}$ & 19.355 & $F$ & 10 & G & & \\
\hline 18 & 07 & 28 & $44.5 ?$ & 59.97 & N & 2.79 & E & 10 & G & & \\
\hline 18 & 09 & 10 & $58.8 \%$ & 60.006 & $\mathbf{N}$ & 6.623 & & 19 & & & \\
\hline 18 & 09 & 32 & $32.3 ?$ & 28.52 & $s$ & 68.79 & $w$ & 70 & 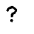 & & \\
\hline 18 & 09 & 45 & $47.88 c$ & 58.360 & $\mathrm{~N}$ & 142.838 & $w$ & 9 & & & \\
\hline 18 & 10 & 47 & 37.08 & 63.114 & $\mathbf{N}$ & 148.417 & $w$ & 81 & & & \\
\hline 18 & 11 & 34 & $04.0 ?$ & 40.26 & $\mathrm{~N}$ & 125.01 & $w$ & 10 & G & & \\
\hline 18 & 12 & 43 & 08.78 & 63.840 & 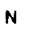 & 148.417 & & 102 & & & \\
\hline 18 & 12 & 44 & $41.3 ?$ & 20.98 & $\mathrm{~s}$ & 178.66 & $w$ & 575 & * & 5.0 & \\
\hline 18 & 13 & 09 & $37.9 \&$ & 36.577 & $\mathrm{~N}$ & 121.223 & $w$ & 7 & & & \\
\hline 18 & 14 & 06 & 28.8 & 17.761 & $\mathbf{N}$ & 68.811 & $w$ & 62 & G & 5.9 & \\
\hline
\end{tabular}

$\begin{array}{rrlllrrr}18 & 14 & 51 & 56 & 6 * & 31.776 & \mathrm{~S} \\ 18 & 15 & 97 & 22 & 1 \% & 36 & 847 & \mathrm{~N} \\ 18 & 15 & 40 & 45 & 4 \% & 63 & 034 & \mathrm{~N} \\ 0 & 18 & 15 & 49 & 14 & 1 & 12.449 & \mathrm{~S} \\ 18 & 16 & 48 & 04.0 & 2.414 & \mathrm{~S} \\ 18 & 17 & 00 & 04 & 5 * & 5.162 & \mathrm{~N} \\ 18 & 17 & 08 & 33.08 & 37.580 & \mathrm{~N} \\ 18 & 17 & 38 & 20.5 * & 6.745 & \mathrm{~S} \\ 18 & 18 & 20 & 58.9 \% & 36.866 & \mathrm{~N} \\ 18 & 18 & 32 & 29.4 & 36.869 & \mathrm{~N} \\ 18 & 18 & 57 & 29.5 * & 35.646 & \mathrm{~N} \\ 18 & 19 & 04 & 03.5 ? & 34.03 & \mathrm{~N} \\ 18 & 19 & 28 & 29.1 & 2.671 & \mathrm{~S} \\ 18 & 19 & 37 & 49.88 & 58.115 & \mathrm{~N} \\ 18 & 20 & 06 & 17.1 * & 43.970 & \mathrm{~N} \\ 18 & 20 & 38 & 37.38 & 47.410 & \mathrm{~N}\end{array}$

\begin{tabular}{|c|c|c|c|c|c|c|}
\hline 71.447 & w & 33 & N & & 0.8 & 3 \\
\hline 5.275 & w & 10 & G & & 12 & \\
\hline 5.1764 & w & 118 & & & & 8 \\
\hline 1520 & E & 33 & $\mathrm{~N}$ & 5.14 .2 & 1.1 & 55 \\
\hline 88.772 & $E$ & 33 & N & 4.83 .9 & 0.9 & 3 \\
\hline 5.771 & E & 33 & $\mathbf{N}$ & 4.8 & 1.4 & 30 \\
\hline 18.688 & w & 3 & & & & 19 \\
\hline 7.707 & E & 55 & * & 4.9 & 1.4 & \\
\hline 5.237 & $w$ & 10 & G & & 1.5 & 6 \\
\hline 5.333 & $w$ & 10 & G & & 0.9 & \\
\hline 24.534 & $E$ & 10 & $\mathbf{G}$ & & 0.8 & 5 \\
\hline 23.39 & $E$ & 10 & G & & 1.4 & 7 \\
\hline 39.781 & E & 33 & $\mathbf{N}$ & 4.53 .8 & 1.4 & 26 \\
\hline 4.535 & w & 86 & & & & 17 \\
\hline .515 & $E$ & 10 & G & & 0.4 & \\
\hline 22.776 & w & 45 & & 4.1 & & \\
\hline
\end{tabular}

$\begin{array}{ccccccc}20 & 38 & 57.5 ? & 45.52 & N \\ 21 & 11 & 58.8 * & 2.023 & N\end{array}$ $\begin{array}{rrrrr}21 & 11 & 58.8 * & 2.023 \mathrm{~N} \\ 21 & 16 & 51.0 & 15.900 \mathrm{~N}\end{array}$
$9.12 \mathrm{E}$ $79.017 \mathrm{~W}$ $95.431 \mathrm{~W}$
100

$\begin{array}{lllll}33 & \mathrm{~N} & 4.7 & 4.2\end{array}$

$38 * 5.0$

Fukushima, Mito, Toteyoma, Tokyo, Utsunomiyo and Yokohomo: ( 1 I JMA) of Kumagoyo and on Hochija-jimo; ( JMA) ot Kofu. Kowoguchi-ko, Mishimo and on Oshimo. Depth from broodband displacement seismograms.

39 10 6 15 8 APUA NEW GUINEA REGION

LEEWARD ISLANDS. ML 3.4 (FDF).

ANDREANOF ISLANDS, ALEUTIAN IS

DODECANESE ISLANDS. MO 3.5 (ATH).

BISMARCK SEA

HOKKAIDO, JAPAN REGION

CHILE-ARGENTINA BORDER REGION

FRANCE. ML 2.4 (GEN)

BISMARCK SEA

SOUTH OF JAVA

NEAR COAST OF CENTRAL CHILE

NEAR COAST OF CENTRAL CHILE

NORTH SEA. MD 2.4 (BER)

YUGOSLAVIA. MD 2.2 (TTG)

NORTH SEA. MD 1.6 (BER)

SOUTHERN ALASKA. 〈AGS-P>.

CENTRAL ALASKA. 〈AGS-P>

SOUTH OF MARIANA ISLANOS

SAN JUAN PROVINCE, ARGENTINA. Felt (V) at caucete ond (IV) at Son Juon.

KERMADEC I SLANDS REGION

GREECE, MD 3.1 (ATH)

SOUTHERN IRAN

NEAR COAST OF CENTRAL CHILE

GREECE. ML 3.3 (ATH)

CENTRAL ITALY. MD 2.3 (ROM)

OFF COAST OF SOUTHERN CHILE

DODECANESE ISLANDS. MD 3.4 (ATH)

MACOUARIE ISLANDS REgION

SOUTHERN NORWAY. MO 1.5 (BER).

CENTRAL MID-ATLANTIC RIDGE

SOUTHERN GREECE. ML 3.9 (ATH)

SOUTHERN NORWAY. MD 1.5 (BER)

GERMANY. ML 2.5 (JCK).

NORTHERN ITALY

DODECANESE ISLANDS. ML 4.1 (ATH)

FRANCE. ML 2.6 (GEN), 2.5 (LDG).

MOLUCCA PASSAGE

NEAR COAST OF NORTHERN CHILE

55 GREECE. ML 4.0 (ATH). 3.7 (ROM)

15 STRAIT OF GIBRALTAR. MbLg 2.8 (MDD)

KODIAK ISLAND REGION. LAGS-P>

SANTA CRUZ I SLANDS

9 CENTRAL ALASKA. <AGS-P>

FIJI ISLANDS REGION

GREENLAND SEA

ADRIATIC SEA. ML 3.8 (ROM), 3.7 (TTG), 3.6 (KBA). MD 3.8 (VKA). Felt in the Zadar areo. Yugoslavia.

ALBANIA. ML 3.7 (ROM). MD 3.6 (ATH), 3.0 (TTG).

NORTH SEA. MD 2.1 (BER).

SOUTHERN NORWAY. MD 1.3 (BER)

LA RIOJA PROVINCE. ARGENTINA

GULF OF ALASKA. 〈AGS-P>

CENTRAL ALASKA. 〈AGS-P>

OFF COAST OF NORTHERN CALIFORNIA. ML 3.1 (BRK)

CENTRAL ALASKA. ¿AGS-P>

FIJI ISLANDS REGION

CENTRAL CALIFORNIA. <BRK>. ML 2.5 (BRK) Republic ond throughout Puerto Rico. Also felt ot Caracas and in tawns alang the central caost of Venezuela. Depth from braodbond displacement se i smograms.

NEAR COAST OF CENTRAL CHILE

SIRAIT OF GIBRALTAR

CENTRAL ALASKA 〈AGS-P>

SOUTH OF TIMOR

WEST IRIAN

MINDANAO, PHILIPPINE ISLANDS

CALIFORNIA-NEVADA BORDER REGION. CBRK>. ML 3.1 (BRK), 3.1 (PAS)

EAST PAPUA NEW GUINEA REgION

STRAIT OF GIBRALTAR

STRAIT OF GIBRALTAR

CRETE. MD 3.7 (ATH)

CRETE. MD 3.8 (ATH)

NEAR $N$. COAST OF WEST IRIAN

ALASKA PENINSULA. LAGS-P>.

CENTRAL I TALY. MD 2.2 (ROM)

WASHINGTON. ¿SEA>. ML 4.4 (SEA). Felt (V) ot Kingston ond Poulsbo. Felt (IV) ot Bremerton, Burley, Clinton. Dockton, Gig Harbar, Grohom, Hansville, Indianola, Lokeboy, Lilliwoup, Littlerock. Longbronch. Monchester. Motlock. Port Ludlow. Seattle, Seobeck, Sultan, Sumner. Suquomish. Trocyton, Voshon ond Wilkeson. Felt os for os Sidney ond Victorio, British Columbio, Conodo. NORTHERN ITALY. ML 2.1 (GEN). MD 1.8 (ROM) NORTHERN ITALY.

NEAR COAST OF OAXACA, MEXICO 


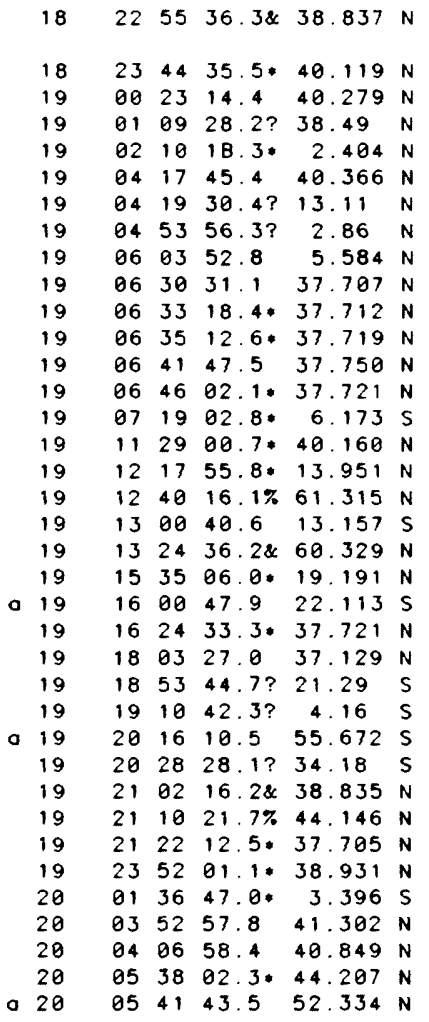

$20 \quad 06 \quad 19463 \quad 3564 \mathrm{~N}$ $\begin{array}{llllll}20 & 06 & 32 & 12.1 & 40.868 \mathrm{~N}\end{array}$ $20115725.0833 .500 \mathrm{~N}$

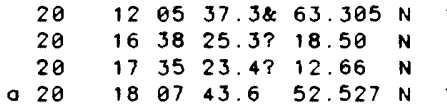

$20 \quad 1941 \quad 18.4 \% 38.844 \mathrm{~N}$ $20 \quad 21 \quad 1151.2 * 12.685 \mathrm{~N}$ $20 \quad 214949.9 * 36.302 \mathrm{~N}$ $20 \quad 2204 \quad 16.9 * 41.027 \mathrm{~N}$ $\begin{array}{lllllll}20 & 23 & 24 & 44.7 \% & 42.026 & N \\ 20 & 23 & 49 & 38.8 & 3.867 & \mathrm{~S}\end{array}$ $\begin{array}{lllll}23 & 40 & 38.8 & 3.867 \mathrm{~S}\end{array}$ $20 \quad 235550.4$ ? $18.66 \quad \mathrm{~N}$

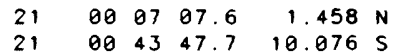
$210059 \quad 18.0837 .612 \mathrm{~N}$ $21012853.4837 .383 \mathrm{~N}$

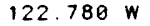

$123.548 \mathrm{~W}$ 25. $132 \mathrm{E}$ $25.10 \mathrm{E}$ $126.592 \mathrm{E}$ $24.019 \mathrm{E}$ $90.27 \mathrm{~W}$

$72.88 \mathrm{~W}$

$74.386 \mathrm{~W}$ $14.979 \mathrm{E}$ $14.975 \mathrm{E}$ $14.932 \mathrm{E}$ $14.952 E$ $14.936 \mathrm{E}$ $130.214 \mathrm{E}$ $113.026 \mathrm{E}$ $61.317 \mathrm{~W}$ 10.280 E $167.128 \mathrm{E}$ $153.577 \mathrm{~W}$ $107.903 \mathrm{~W}$ 67.559 w $14.909 \mathrm{E}$ $27.868 \mathrm{E}$ $143.47 \mathrm{E}$ $28.247 \mathrm{~W}$

$71.16 \mathrm{~W}$ $122.785 \mathrm{~W}$ $9.937 \mathrm{E}$

$16.692 \mathrm{E}$

$23.217 \mathrm{E}$

$129.689 \mathrm{E}$ $20.068 \mathrm{E}$

$20.373 \mathrm{E}$

$11.441 \mathrm{E}$ $174.184 \mathrm{E}$

$124.424 \mathrm{E}$ $20.352 \mathrm{E}$ 116.450 N

$149.591 \mathrm{~W}$ 61.99 W $41.93 \mathrm{E}$ $172.508 \mathrm{E}$

$29.141 \mathrm{E}$ $41.832 \mathrm{E}$ $143.128 \mathrm{~W}$

$70.674 \mathrm{E}$

$22.845 \mathrm{E}$

$12.839 \mathrm{E}$

86. $993 \mathrm{E}$

$67.85 \mathrm{~W}$ $126.408 \mathrm{E}$ $116.140 \mathrm{E}$ $119.055 \mathrm{~W}$ $121.737 \mathrm{~W}$

$14.86 E$ $62.336 \mathrm{~W}$ $146.189 \mathrm{E}$ 45. $868 \mathrm{E}$ $144.283 \mathrm{E}$ $69.663 \mathrm{E}$

$67.06 \mathrm{~W}$ $179789 \mathrm{~W}$ $150719 \mathrm{~W}$ $160350 \mathrm{E}$

$6.248 \mathrm{E}$ $147.035 \mathrm{~W}$ $112.40 \mathrm{E}$

$12.949 \mathrm{E}$

$62.06 \mathrm{~W}$

$6.37 \mathrm{E}$

$5.562 \mathrm{E}$

$150.677 \mathrm{~W}$

5. $217 \mathrm{E}$ $126.836 \mathrm{E}$ $15.843 \mathrm{E}$ $157.171 \mathrm{E}$ $75.32 \mathrm{~W}$ $170.006 \mathrm{E}$ $139.853 \mathrm{E}$ $69.03 \mathrm{~W}$

50

$10 \mathrm{G}$

$10 \mathrm{G}$

$99 ? 4.7$

$10 \mathrm{C}$

$33 \mathrm{~N} 4.8$

$33 \mathrm{~N}$

117

$10 \mathrm{G}$

10

$10 \mathrm{C}$

106

108

10

32 .

$10 \mathrm{C}$

$239 \cdot 5.8$

$33 \mathrm{~N} \quad 4.4$

$189 \mathrm{D} 5.5$

10

10

177 ?

$110 ? 4.6$

$33 \mathrm{~N} \quad 5.65 .1$

$33 N$

18

100

$10 \mathrm{G}$

$33 \mathrm{~N} 4$

$10 \mathrm{G}$

$10 \mathrm{G}$

$10 \mathrm{G}$

$\begin{array}{llll}48 & D & 5.2 & 4.3\end{array}$

$310 * 5.0$

$10 \mathrm{G}$

92

$10 \mathrm{G}$

$10 \mathrm{G}$

$33 \mathrm{~N}$

$\begin{array}{ll}10 & G \\ 10 & G\end{array}$

$10 \mathrm{G}$

145

$10 \mathrm{G}$

$10 \mathrm{G} \quad 5.2 \quad 4.6$

$33 \mathrm{~N}$

$46 * 5.05 .0$

55

7

$10 \mathrm{O}$

$33 \mathrm{~N}$

$219 \cdot 4.3$

$33 \mathrm{~N}$

$33 \mathrm{~N} \quad 4.3$

$33 \mathrm{~N} 4.2$

$33 \mathrm{~N}$

620
5

$10 G \quad 5.247 \quad 1.1$

$10 G$

6
43

$\begin{array}{ll}43 & ? \\ 10 & 6\end{array}$

9

O G

$10 \mathrm{G}$

08

$10 \mathrm{G}$

$33 \mathrm{~N} 4.6$

$10 \mathrm{G}$

$10 \mathrm{G} \quad 4.8$

$150 ?$

$\begin{array}{lllll}33 \mathrm{~N} & 5.0 & 4.7\end{array}$

$33 \mathrm{~N}$

$112373 \mathrm{~W} \quad 8$

2

0.6

15 NORTHERN CALIFORNIA. CBRK>. ML 3.6 (BRK). MO=1.2*10*14 $\mathrm{Nm}$ (BRK)

0.8 7 NORTHERN CALIFORNIA. ML 2.6 (BRK)

1.0 i5 AEGEAN SEA. MD 3.1 (ATH).

4 AEGEAN SEA. ML 2.8 (ATH)

28 MOLUCCA PASSAGE

17 AEGEAN SEA. ML 3.5 (ATH)

29 NEAR COAST OF GUATEMALA

5 COLOMBIA

25 'COLOMBIA

6 SICILY. MD 2.5 (ROM)

5 SICILY. MD 2.7 (ROM)

5 SICILY. MD 2.5 (ROM)

7 SICILY. MD 2.8 (ROM).

5 SICILY. MD 2.7 (ROM).

18 BANDA SEA

5 NORTHEASTERN CHINA. ML 3.3 (BJI).

10 WINDWARD ISLANDS. ML 3.0 (FDF)

4 SOUTHERN NORWAY. MD 2.3 (BER).

73 VANUATU ISLANDS

19 SOUTHERN ALASKA. 〈AGS-P>

$\begin{array}{lrl}1.4 & 21 & \text { OFF COAST OF JALISCO, MEXICO } \\ 1.1 & 246 & \text { CHILE-BOLIVIA BORDER REGION }\end{array}$

1.35 SICILY. MD 2.6 (ROM)

1.58 TURKEY

8

NORTHERN CHILE

19 PAPUA NEW GUINEA

8

SOUTH SANDWICH ISLANDS REGION

NEAR COAST OF CENTRAL CHILE

6 IONIAN SEA. MD 2.6 (ROM).

1 GREECE. ML 3.0 (ATH).

5 CERAM

7 ALBANIA. ML 1.8 (SKO)

8 GREECE-ALBANIA BORDER REGION, IL 2.2 (SKO)

0.9231

NEAR ISLANDS, ALEUTIAN ISLANDS. ML 5.2 (PMR). FeIt (IV) On ShemYo.

1.166 CELEBES SEA

1.26 GREECE-ALBANIA BORDER REGION. ML 2.3 (SKO)

4 SOUTHERN CALIFORNIA. 〈PAS-P>. ML 3.0 (PAS). FeIt at Polm SPrings.

12 CENTRAL ALASKA. LAGS-P>

8 LEEWARD ISLANDS. ML 3.1 (FDF)

8 ETHIOPIA. ML 3.3 (ARO)

0.38

NEAR ISLANDS, ALEUTIAN ISLANDS. ML 4.9 (PMR). FeIt

( 111$)$ on $A t$ tu

1.06 TURKEY

9 ETHIOPIA. ML 4.5 (ARO)

4 GULF OF ALASKA. 〈AGS-P〉.

18

6 YUgOSLAVIA. ML 2.7 (SKO)

5 CENTRAL ITALY. MD 2.3 (ROM)

78

5

69 MOLUCCA PASSAGE

45 SOUTH OF SUMBAWA ISLAND

14 CENTRAL CALIFORNIA. <BRK> ML 3.1 (BRK), 3.0 (PAS)

15 CENTRAL CALIFORNIA. <BRK>. ML 3.5 (BRK). MO=2.4*10*14 $\mathrm{Nm}$ (BRK). Felt ( 1 II) at San Jase

4 SOUTHERN ITALY. MD 2.7 (ROM).

9 LEEWARD ISLANDS. ML 2.9 (FDF)

32 KURIL ISLANDS

9 IRAN-IRAQ BORDER REGION

12 MARIANA ISLANDS REGION

10 HINDU KUSH REGION

MONA PASSAGE

36 FIJI ISLANDS REGION

20 CENTRAL ALASKA $\angle A G S-P\rangle$ ML 3.0 (PMR)

28 MACQUARIE ISLANDS REGION

SOUTHERN NORWAY. MD 1.7 (BER).

28 SOUTHERN ALASKA. LAGS-P>.

5 SOUTH OF JAVA

NORTHERN I TALY, MD 2.6 (ROM) 2.5 (TRI)

12 LEEWARD ISLANDS. ML 3.7 (FDF).

7 SOUTHERN NORWAY. MD 2.2 (BER). Prabable explosion

7 SOUTHERN NORWAY. MD 1.6 (BER)

22 CENTRAL ALASKA. ২AGS-P>

5 SOUTHERN NORWAY. MD 1.8 (BER)

19 MINDANAO. PHILIPPINE ISLANDS

6 SICILY. MD 2.6 (ROM)

12 MACQUARIE ISLANDS REGION

5 PERU

39 LOYALTY ISLANDS REgION 


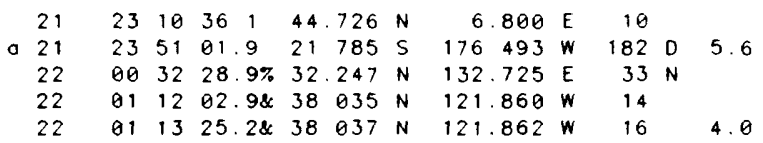

\begin{tabular}{|c|c|c|c|c|c|c|c|c|c|c|}
\hline 22 & 01 & 29 & $23.1 ?$ & 30.72 & $N$ & 49.85 & $E$ & 33 & $N$ & 4.1 \\
\hline 22 & $\theta 2$ & 37 & 21.7 & 45.702 & $N$ & 26.622 & $E$ & 108 & $*$ & \\
\hline 22 & 03 & 08 & 14.5 & 37.601 & $N$ & 141.341 & $E$ & 59 & & 4.6 \\
\hline 22 & 06 & 51 & $54.7 ?$ & 39.60 & $N$ & 21.69 & $E$ & 10 & $G$ & \\
\hline 22 & 06 & 53 & 04.4 & 40.241 & $N$ & 21.514 & $E$ & 10 & $G$ & \\
\hline 22 & 98 & 23 & 17.98 & 60.445 & $N$ & 142.869 & $w$ & 2 & & \\
\hline 22 & 08 & 27 & $04.7 *$ & 5.393 & $s$ & 152.494 & $E$ & 58 & $*$ & 4.83 .9 \\
\hline 22 & 08 & 39 & $27.3 \&$ & 58.442 & $N$ & 142.778 & $w$ & 10 & $G$ & \\
\hline 22 & 10 & 07 & $10.3 \%$ & 10.654 & $N$ & 60.840 & $w$ & 33 & $N$ & \\
\hline 22 & 10 & 44 & $08.7 \&$ & 62.112 & $N$ & 151.755 & $w$ & 0 & & \\
\hline 22 & 10 & 46 & $35.9 ?$ & 61.32 & $N$ & 6.78 & $E$ & 10 & G & \\
\hline 22 & 11 & 06 & 06.8 & 28.252 & $N$ & 130.644 & $E$ & 33 & $D$ & 5.3 \\
\hline 22 & 11 & 12 & $54.9 *$ & 34.518 & $N$ & 140.254 & $E$ & 28 & $*$ & 4.4 \\
\hline 22 & 12 & 04 & $55.7 \%$ & 15.674 & $N$ & 60.957 & $w$ & 24 & $*$ & \\
\hline 22 & 12 & 05 & $58.2 *$ & 47.899 & $N$ & 18.299 & $E$ & 12 & & \\
\hline 22 & 12 & 11 & $17.4 ?$ & 31.39 & $S$ & 70.05 & $w$ & 170 & $?$ & \\
\hline 22 & 12 & 25 & 25.6 & 20.303 & $\mathrm{~s}$ & 71.028 & $w$ & 33 & $N$ & 4.5 \\
\hline 22 & 12 & 44 & $52.9 *$ & 16.803 & $N$ & 62.240 & $w$ & 33 & $N$ & \\
\hline 22 & 13 & 14 & $05.7 \&$ & 61.801 & $N$ & 149.464 & $w$ & 12 & & \\
\hline 22 & 13 & 27 & 53.8 & 42.791 & $N$ & 12.917 & $E$ & 10 & $G$ & \\
\hline 22 & 13 & 45 & $54.4 ?$ & 36.78 & $N$ & 29.09 & $E$ & 10 & $G$ & \\
\hline 22 & 14 & 04 & 54.9 & 47.989 & $N$ & 6.659 & $E$ & 12 & & \\
\hline 22 & 14 & 34 & $58.0 *$ & 61.596 & $N$ & 5.300 & $E$ & 10 & $G$ & \\
\hline 22 & 15 & 08 & 46.1 & 6.704 & $s$ & 81.593 & $w$ & 32 & $D$ & 4.9 \\
\hline 22 & 16 & 30 & $15.2 \&$ & 60.470 & $N$ & 142.933 & $w$ & $\theta$ & & \\
\hline 22 & 16 & 31 & $30.2 \%$ & 38.264 & $N$ & 15.043 & $E$ & 10 & $G$ & \\
\hline 22 & 17 & 43 & 06.58 & 60.233 & $N$ & 152.430 & $w$ & 82 & & \\
\hline 22 & 19 & 51 & 48.5 & 27.171 & $s$ & 71.369 & $w$ & 36 & 0 & 5.1 \\
\hline 22 & 21 & 06 & $01.8 *$ & 36.395 & $N$ & 114.262 & $w$ & 10 & G & 4.6 \\
\hline 22 & 21 & 15 & 00.88 & 37.283 & $N$ & 116.412 & $w$ & 0 & & 5.34 .8 \\
\hline
\end{tabular}

\begin{tabular}{|c|c|c|c|c|}
\hline 114.204 & $w$ & 10 & $G$ & 4.0 \\
\hline 16.072 & $E$ & 10 & G & \\
\hline 152.413 & $w$ & 126 & & \\
\hline 114.252 & $w$ & 10 & G & 4.5 \\
\hline 15.079 & $E$ & 10 & G & \\
\hline 14.447 & $\mathbf{E}$ & 10 & G & \\
\hline 22.823 & $\mathbf{E}$ & 10 & G & \\
\hline 172.656 & $w$ & 33 & $N$ & 4.95 .0 \\
\hline 114.292 & $w$ & 10 & G & 4.5 \\
\hline 24.327 & $E$ & 33 & $\mathbf{N}$ & 4.0 \\
\hline 162.78 & $E$ & 135 & $?$ & \\
\hline 20.789 & $E$ & 10 & $G$ & \\
\hline 30.413 & $E$ & 10 & G & \\
\hline 62.290 & $w$ & 33 & $N$ & \\
\hline 20.591 & $E$ & 25 & & 3.7 \\
\hline 6.573 & $E$ & 10 & G & \\
\hline 143.031 & $w$ & 10 & G & \\
\hline 65.604 & $w$ & 15 & & 4.5 \\
\hline 14.355 & $\mathbf{E}$ & 10 & $G$ & \\
\hline 65.864 & $w$ & 10 & $G$ & \\
\hline 65.608 & $w$ & 10 & $G$ & \\
\hline 91177 & $w$ & 95 & $\star$ & 4.3 \\
\hline 17873 & $w$ & 651 & $?$ & 4.8 \\
\hline 8316 & $E$ & 19 & 6 & \\
\hline 744 & $E$ & 10 & $G$ & \\
\hline 66.167 & $w$ & 10 & $G$ & \\
\hline 6.246 & $E$ & 10 & $G$ & \\
\hline 125.55 & $E$ & 33 & $\mathbf{N}$ & \\
\hline 142.996 & $E$ & 170 & 0 & 5.3 \\
\hline 29.228 & $E$ & 10 & $G$ & \\
\hline 5.279 & $E$ & 10 & $G$ & \\
\hline 14.35 & $E$ & 10 & $G$ & \\
\hline 175.74 & $w$ & 211 & $?$ & 4.3 \\
\hline 175.45 & $\mathbf{E}$ & 33 & $N$ & \\
\hline 6.014 & $E$ & 10 & $G$ & \\
\hline 129.37 & $E$ & 173 & $?$ & \\
\hline 54.442 & $E$ & 33 & $N$ & \\
\hline 65.81 & $w$ & 10 & $G$ & \\
\hline 7.28 & $E$ & 10 & $G$ & \\
\hline 25.382 & $w$ & 33 & $\mathbf{N}$ & 5.3 \\
\hline 29.205 & $E$ & 10 & $G$ & \\
\hline 141.750 & $E$ & 10 & G & 3.7 \\
\hline 140.845 & $E$ & 20 & & 4.5 \\
\hline 20.265 & $E$ & 24 & & 3.8 \\
\hline
\end{tabular}

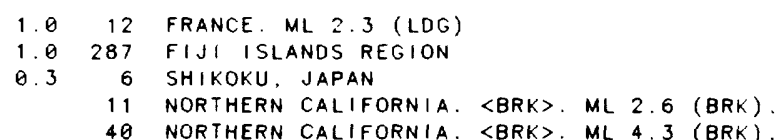

$\mathrm{Nm}$ (BRK). Fell (V) ot Albany. Pittsburg. Rio Vista and Travis Air Farce Base. Felt (IV) at Antioch, Benicio, Birds Landing, Byron, Daly City. Isletan. Oakley. Part Costa, San Anselmo. Son Pablo and South San Francisco. Felt from San Francisco to Vacoville to Stockton.

0.8 10 WESTERN IRAN

0.9 16 ROMANIA

1. 148 NEAR EAST COAST OF HONSHU, JAPAN. FeIt ( I JMA) at Fukustima, Mita, Onahamo, Ofunato, Shirokawa and Ut sunomiya.

1.35 GREECE

1.315 GREECE. MD 3.6 (ATH)

21 SOUTHERN ALASKA. LAGS-P>

20 NEW BRITAIN REGION

7 GULF OF ALASKA. 〈AGS-P>.

6 TRINIDAD

29 CENTRAL ALASKA. LAGS-P>. ML 3.3 (PMR)

4 SOUTHERN NORWAY. MO 2.1 (BER)

1.0143 RYUKYU ISLANDS. FeIt (I JMA) ot NOZE.

0.7 12 NEAR EAST COAST OF HONSHU, JAPAN

0.3 8 LEEWARD ISLANDS. ML 2.3 (FDF)

1.37 HUNGARY. ML 2.6 (BRA), 2.9 (VKA). Felt ot Komorno, Marcelovo and Hurbanovo, Czechoslavakia.

0.5 10 CHILE-ARGENTINA BORDER REGION

1.311 OFF COAST OF NORTHERN CHILE

1.39 LEEWARD ISLANDS. ML 3.0 (FDF)

8 SOUTHERN ALASKA. LAGS-P>

1.08 CENTRAL ITALY. MO 2.6 (SSO), 2.3 (ROM)

Q.9 4 TURKEY

0.911 FRANCE. ML 2.9 (LDG)

0.56 SOUTHERN NORWAY. MD 1.7 (BER)

1

NEAR COAST OF NORTHERN PERU

15 SOUTHERN ALASKA, LAGS-P>

0.25 SICILY. MD 2.4 (ROM)

21 SOUTHERN ALASKA. LAGS-P>

1.228 NEAR COAST OF NORTHERN CHILE

1.321 GULF OF CALIFORNIA

221 SOUTHERN NEVADA. CDOE>. ML 5.2 (BRK). $37^{\circ} 16^{\circ} 58.35^{\circ}$ N., 116' $24^{\circ} 44^{\circ} .30^{\prime \prime}$ W. Surface Elev. $2007 \mathrm{~m}$. . Depth Burial $500 \mathrm{~m}$., Shat Time 211500.83 , "CONTACT," Nevodo Test Site (Dept. of Energy).

1.215 BAJA CALIFORNIA

0.610 POLANO. ML 2.9 (VKA)

12 SOUTHERN ALASKA. SAGS-P>

0.9 13 GULF OF CALIFORNIA

1.25 SICILY. MO 2.7 (ROM)

1.15 SICILY. MD 2.4 (ROM)

0.77 GREECE. MD 3.2 (ATH). ML 3.0 (SKO).

1.176 TONGA ISLANDS REGION

1.3 28 GULF OF CALIFORNIA

D.9 12 CRETE. MD 4.2 (ATH)

0.55 SOLOMON ISLANDS

1.46 GREECE-ALBANIA BOROER REGION. ML 2.8 (SKO).

1.48 TURKEY

0.47 LEEWARD ISLANDS. ML 2.8 (FDF)

1.26 IONIAN SEA. ML 3.6 (ATH), 3.4 (ROM).

0.317 FRANCE. ML 2.8 (LDG).

11 GULF OF ALASKA. $\angle A G S-P\rangle$

1.0 34 PUERTO RICO REGION

0.49 YUGOSLAVIA. MO 2.9 (LJU), 2.6 (ROM).

0.66 PUERTO RICO REGION

1.38 PUERTO RICO REGION

1426 GUATEMALA

a 7

b. 1

$\begin{array}{ll}0 & 5 \\ 1 & 4\end{array}$

1. $1 \quad 171$

1. 1

$\begin{array}{ll}0 . & 0 \\ 6 & 9\end{array}$

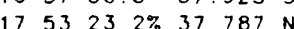

$\begin{array}{llll}18 & 49 & 24.0 * 2.754 \mathrm{~S}\end{array}$

$\begin{array}{llll}20 & 04 & 50.4 \quad 39.675 \mathrm{~N}\end{array}$
6 717 FIJI ISLANOS REGION

1.0

1.4

1. 0

1.0

1. 1

1. 5

a. 5

A NORTHERN ITAL, ML $=7$ (GEN). MD 2.5 (ROM).

NORTHERN ITALY

PUERTO RICO REGION

SOUTHERN NORWAY. MD 2.0 (BER).

1 VOLCANO ISLANDS REGION

8 TURKEY

8 SOUTHERN NORWAY. MO 1.8 (BER)

8 ADRIATIC SEA. MD 2.7 (SSO).

8 TONGA ISLANOS

- NORTH ISLAND, NEW ZEALAND

5 GERMANY

7 TIMOR SEA

Q IRAN. ML 4.0 (BMU)

5 PUERTO RICO REGION

4 NORTHERN ITALY. ML 1.8 (GEN).

45 SOUTH SANDWICH ISLANDS REGION

TURKEY

8 NEAR $N$ COAST OF PAPUA NEW GUINEA

22 HONSHU, JAPAN. Fell (111 JMA) at Sendai, (11 JMA) ot Yamogato and (I JMA) ot Ishinomaki and Shirakawa.

1353 GREECE-ALBANIA BORDER REGION. ML 4.0 (ATH) 3.7 (TTG). Felt (IV) ot Koraq. Grave. Grazhdan and Zminic Albonio
5 MOLUCCA PASSAGE 


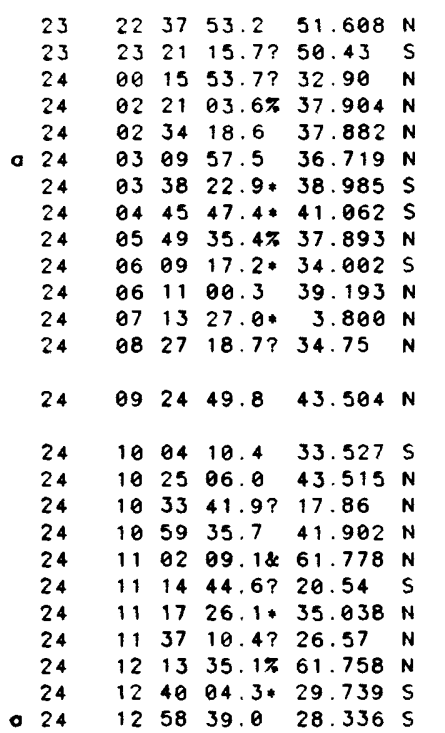

24
24
24
24
24
24
24
24
24
24
24
24
24
24
24
24
24
24
25
25
25
25
25
25
25
25
25
25

25

$680949,42803 \mathrm{~N}$ $\begin{array}{lllllllll}5 & 98 & 47 & 45 & 7 ? & 39 & 53 & \mathrm{~N}\end{array}$ $25 \quad 0906 \quad 38.2 \quad 32 \quad 968 \mathrm{~N}$ $\begin{array}{lllllll}25 & 09 & 10 & 46 & 1 & 46.357 \mathrm{~N}\end{array}$ $25 \quad 991321.1 * 37.786 \mathrm{~N}$ $\begin{array}{lllllll}25 & 09 & 31 & 24.58 & 61.937 & \mathrm{~N}\end{array}$

$095001.9 * 21.454 \mathrm{~N}$ $1044 \quad 52.0859 .883 \mathrm{~N}$ $111559.3 \quad 32.912 \mathrm{~N}$ $123005.9 \quad 6.113 \mathrm{~s}$ $123741.3 * 38.798 \mathrm{~N}$ 1248 05.0? $10.58 \mathrm{~S}$ 125427.3 ? $17.07 \mathrm{~N}$ $141303.5 * 35.517 \mathrm{~N}$ $\begin{array}{llllll}14 & 13 & 40 & 6 & 44.500 \mathrm{~N}\end{array}$ $143236.1 ? 38.32 \mathrm{~N}$ $\begin{array}{llll}15 & 03 & 17.9 \% & 32.046 \mathrm{~N}\end{array}$ $\begin{array}{lllll}16 & 11 & 54.1 & 40.679 \mathrm{~N}\end{array}$ $\begin{array}{llllll}16 & 13 & 03 & 0 & 42.819 \mathrm{~N}\end{array}$ $\begin{array}{lllllll}17 & 52 & 34 & 7 & 40 & 422 & N\end{array}$ $\begin{array}{lllll}18 & 16 & 33.8 \& & 59.962 & N\end{array}$
$16.122 \mathrm{E}$ $164.74 \mathrm{E}$ $88.52 \mathrm{E}$ $14.665 \mathrm{E}$ $14.785 \mathrm{E}$ $35.943 \mathrm{E}$ $14.713 \mathrm{~W}$ $88.302 w$ $14.705 \mathrm{E}$ $70.133 \mathrm{~W}$ $26.505 \mathrm{E}$ $126.416 \mathrm{E}$ $139.33 \mathrm{E}$ $110.758 \mathrm{w}$ $71.012 \mathrm{~W}$ $110.772 \mathrm{~W}$ $65.72 \mathrm{~W}$ $20.237 \mathrm{E}$ $149.884 w$ $179.10 \mathrm{~W}$ 24.289 E $142.30 \mathrm{E}$ $7.487 \mathrm{E}$ $179.147 \mathrm{~W}$ $66.312 w$

$124.982 \mathrm{E}$ $150.012 \mathrm{~W}$ $28.084 \mathrm{E}$ $79.066 \mathrm{~W}$ $142.365 \mathrm{E}$ $126.863 \mathrm{~W}$ $110.752 \mathrm{~W}$ $150.967 \mathrm{~W}$ $5.273 \mathrm{~W}$ 5. $202 \mathrm{~W}$ $5.287 \mathrm{~W}$ 5. $189 \mathrm{~W}$ $155.34 \mathrm{E}$ $149.565 \mathrm{~W}$ $5.18 \mathrm{~W}$ $110.753 \mathrm{~W}$ 173.87 $20.365 \mathrm{E}$ $149.720 \mathrm{~W}$ $170.356 \mathrm{~W}$ $124.700 \mathrm{~W}$ $22.672 \mathrm{E}$ $142.054 \mathrm{E}$ $19.803 \mathrm{E}$ $149.632 \mathrm{~W}$ $26.141 \mathrm{E}$ $145.022 \mathrm{E}$

$169.345 \mathrm{E}$ $85.851 \mathrm{~W}$ $13.047 \mathrm{E}$ $143.333 \mathrm{E}$ $12.977 \mathrm{E}$ $178.167 \mathrm{~W}$ $68.492 \mathrm{~W}$ $12.989 \mathrm{E}$ $118.404 W$

$150499 \mathrm{~W}$ 13 กดด $E$ $2605 \mathrm{E}$ $39668 \mathrm{~W}$ $153.391 \mathrm{E}$ $141.128 \mathrm{E}$ $150.406 \mathrm{~W}$

$122.308 \mathrm{w}$

$93.492 E$ $153.324 \mathrm{~W}$ $39.609 \mathrm{~W}$ $149.062 \mathrm{E}$ $31.712 \mathrm{E}$ $120.88 \mathrm{E}$ $101.44 \mathrm{~W}$ $27.486 \mathrm{E}$ $7.115 \mathrm{E}$ $24.02 \mathrm{E}$ $35.857 \mathrm{E}$ $22.687 \mathrm{E}$ $12.992 \mathrm{E}$ $27.640 \mathrm{E}$ 153. $496 \mathrm{~W}$
5

$\begin{array}{rrrrrr}10 & G & 4.4 & 4.7 & 1.1 & 1 \\ 10 & G & & & 1.2 & \\ 17 & * & & & 1.5 & \\ 10 & G & & & 1.1 & 1 \\ 42 & & 4.9 & 4.2 & 1.0 & 22 \\ 10 & G & 5.0 & & 1.0 & 4 \\ 10 & G & 5.0 & 4.4 & 1.1 & 2 \\ 14 & & & & 0.9 & 1 \\ 10 & G & & & 0.3 & \\ 10 & G & & & 1.2 & 1 \\ 16 & ? & 4.1 & & 1.0 & 1 \\ 27 & * & & & 0.6 & \\ & & & & & \\ 5 & G & & & 0.7 & 3 \\ & & & & & \\ 67 & ? & & & 0.1 & 1 \\ 5 & G & & & 0.8 & 3 \\ 10 & G & & & 0.2 & \\ 10 & G & & & 1.4 & \\ 38 & & & & & 27 \\ 86 & ? & 4.2 & & 0.6 & 1 \\ 10 & G & 4.0 & & 1.2 & 1 \\ 33 & \mathrm{~N} & 4.5 & & 0.8 & \\ 10 & G & & & 1.2 & 10 \\ 84 & * & 3.9 & & 0.9 & 29 \\ 22 & \mathrm{D} & 5.4 & 5.2 & 1.0 & 17\end{array}$

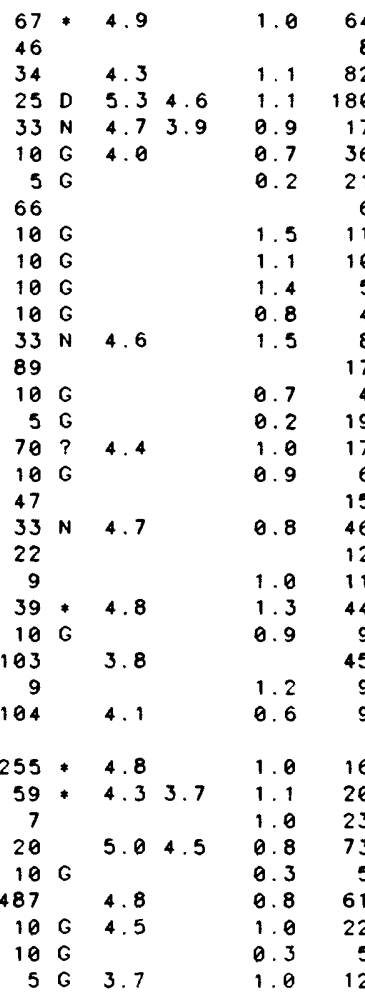

98

106

106

$10 \mathrm{G} \quad 4.94 .3$

$33 \mathrm{~N} 4$

$33 \mathrm{~N}$

\section{8}

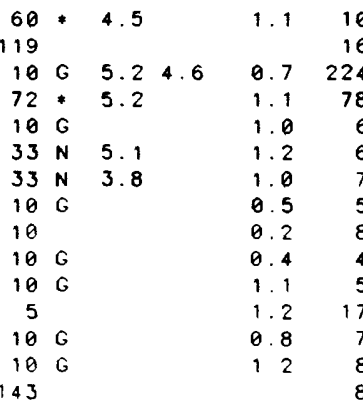

POLAND. ML 3.9 (GRF), 3.8 (VKA), 3.5 (KBA). AUCKLAND ISLANDS REGION TIBET

SICILY. MD 2.7 (ROM).

2 SICILY. MD 2.9 (ROM).

220 TURKEY

5 TRISTAN DA CUNHA REGION

WEST CHILE RISE

4 SICILY. MD 3.1 (ROM)

CHILE-ARGENTINA BORDER REGION

3 TURKEY. MD 3.4 (ATH).

10 TALAUD ISLANDS

6 NEAR S. COAST OF HONSHU, JAPAN. MG 3.1 (JMA). Felt (1 JMA ) on Oshima.

33 WYOMING. ML 3.8 (NEIS). Felt strongly at Jockson. Felt (111) at Kelly

10 NEAR COAST OF CENTRAL CHILE

33 WYOMING. ML 3.7 (NEIS). Felt strongly at Jockson.

PUERTO RICO REGION

ALBANIA

7 SOUTHERN ALASKA, 〈AGS-P>.

4 FIJI ISLANDS REGION

2 CRETE. MD 3.9 (ATH)

BONIN ISLANDS REGION

SOUTHERN NORWAY. MD 2.1 (BER)

20 KERMADEC ISLANDS REGION

CATAMARCA PROVINCE, ARGENTINA. Felt in Catamarca, Cordobo, La Rioja, Santiago del Estero and Tucuman Provinces.

4 MINDANAO, PHILIPPINE I SLANDS

SOUTHERN ALASKA. LAGS-P>.

2 TURKEY. ML 4.2 (ATH).

NEAR COAST OF ECUADOR. Felt in southwestern Colombia.

BONIN ISLANDS REGION

OFF COAST OF OREGON

WYOMING. ML 3.0 (NEIS).

SOUTHERN ALASKA, LAGS-P>

1 STRAIT OF GIBRALTAR

- STRAIT OF GIBRALTAR. mbLg 2.6 (MDD).

STRAIT OF GIBRALTAR

STRAIT OF GIBRALTAR

KURIL ISLANDS REGION

7 CENTRAL ALASKA. LAGS-P>.

STRAIT OF GIGRALTAR

WYOMING. ML 3.0 (NEIS).

TONGA ISLANDS

6 ALBANIA. ML 2.3 (SKO)

5 SOUTHERN ALASKA. $\angle A G S-P\rangle$

46 FOX ISLANDS, ALEUTIAN ISLANDS

12 NEAR COAST OF NORTHERN CALIF. <BRK>. ML 3.1 (BRK).

11 GREECE. MO 3.2 (ATH)

OFF EAST COAST OF HONSHU, JAPAN

9 ALBANIA. MD 3.1 (ATH), 2.8 (ROM). ML 2.7 (SKO).

45 CENTRAL ALASKA. LAGS-P>. Felt (ii) at Cantwell.

9 TURKEY. MD 2.9 (ATH).

HOKKAIDO, JAPAN REGION. Felt (I JMA) at Nemuro and Kushiro.

16 VANUATU ISLANDS

20 COSTA RICA

23 CENTRAL ITALY. MD 3.0 (SSO), 2.9 (ROM)

73 SOUTH OF MARIANA ISLANDS

5 CENTRAL ITALY. MO 2.2 (SSO), 2.1 (ROM)

61 FIJI ISLANDS REGION

22 MENDOZA PROVINCE, ARGENTINA. FeIt (IV) at Mendoza.

CENTRAL ITALY. MD 2.1 (SSO).

2 CALIFORNIA-NEVADA GORDER REGION. ML 3.7 (NEIS). 3.0 (PAS).

34 CENTRAL ALASKA CAGS-P>.

5 CENTRAL ITALY. MD 2.3 (ROM), 2.3 (SSO)

5 TURKEY

25 NORTH ATLANTIC RIDGE

62 KURIL ISLANOS

7 NEAR EAST COAST OF HONSHU, JAPAN

24 SOUTHERN ALASKA. CAGS-P>. ML 3.6 (PMR). Felt (IV) at WIIIOW.

9 NORTHERN CALIFORNIA, LBRK>. ML 2.9 (BRK), MO=1.6*10*14 $\mathrm{Nm}$ (BRK). Felt (IV) at Hercules and Son Poblo. Also felt at Pinole and Richmond.

10 8URMA

6 SOUTHERN ALASKA $\angle A G S-P>$

NORTH ATLANTIC RIDGE

NEW BRITAIN REGION

TURKEY

SUMBA ISLAND REGION

NEAR COAST OF GUERRERO, MEXICO

DOOECANESE ISLANDS. MO 3.7 (ATH)

NORTHERN I TALY

AEGEAN SEA. ML 2.7 (ATH)

DEAO SEA REGION

GREECE. MO 3.4 (ATH)

CENTRAL ITALY. MD 2.5 (SSO), 2.4 (ROM)

TURKEY

SOUTHERN ALASKA. <AGS-P> 
$25 \quad 193430.1 * 28.034 \mathrm{~S}$

$\begin{array}{rrrr}25 & 195005.0 ? 37.73 \mathrm{~S} \\ 25 & 203732.4\end{array}$

1.218 LA RIOJA PROVINCE, ARGENTINA

0.320 NORTH ISLAND. NEW ZEALAND

10364 NEAR COAST OF ECUADOR. MS 6.0 (BRK), 6.0 (PAS). IhirtY people injured and 12 homes domoged (VII) in the Esmeraldos orea. Fell (111) of Guayoquil and (11) at Quita. Felt throughout Ecuador and sauthwestern Colambio. Depth fram broadband displacement se i smogroms.

$\begin{array}{lllll}20 & 43 & 34.5 & 42.821 \mathrm{~N}\end{array}$ $210023.7 * 37.155 \mathrm{~N}$ $\begin{array}{llll}21 & 57 & 38.2 & 42.824 \mathrm{~N}\end{array}$ $\begin{array}{lllll}22 & 55 & 06.7 & 41.065 \mathrm{~N}\end{array}$ $2356 \quad 39.5858 .284 \mathrm{~N}$ $00 \quad 25 \quad 44.0 \quad 44.480 \mathrm{~N}$ $00 \quad 52 \quad 11.6 ? \quad 32.73 \quad S$ $02 \quad 27 \quad 17.4 * 38.049 \mathrm{~N}$ $022850.1 * 17.739 \mathrm{~N}$

12.963 12.985 $20.068 \mathrm{E}$ $142.768 \mathrm{~W}$ $7.437 \mathrm{E}$ 70.90 W $16.738 \mathrm{E}$ $65.646 \mathrm{~W}$ $155.083 \mathrm{w}$
$10 \mathrm{G}$

243
$10 \mathrm{G}$

$10 \mathrm{G}$

$10 \mathrm{G} 4.1$

$33 \mathrm{~N}$

$71 ?$

$33 \mathrm{~N}$ $10 \mathrm{G}$
$034103.2 * 41.883 \mathrm{~N}$ $034400.1 * 37.587 \mathrm{~N}$ $043752.8 * 17.839 \mathrm{~N}$ $054209.3 ? \quad 43.50 \mathrm{~N}$ $055850.4 * 38.756 \mathrm{~N}$ $06 \quad 06 \quad 10.6 \& \quad 19.368 \mathrm{~N}$ $\begin{array}{llllll}06 & 52 & 28.1 ? & 32.76 & 5\end{array}$ $072907.0 \quad 44.666 \mathrm{~N}$ $075203.4 * 38.825 N$ $083830.4 * 37.653 \mathrm{~N}$ $085125.7 ? 52.24 \mathrm{~N}$ $\begin{array}{llll}09 & 10 & 37.2 & 32.947 \mathrm{~N}\end{array}$ $0939 \quad 43.9 * 38.762 \mathrm{~N}$ $0958 \quad 29.5 ? 44.22 \mathrm{~N}$ $1038 \quad 39.4 \quad 39.112 \mathrm{~N}$
$22.090 \mathrm{E}$ $31.427 \mathrm{E}$ $65.626 \mathrm{~W}$ $19.27 \mathrm{E}$ $31.859 \mathrm{E}$ $154.974 W$ $68.85 W$ $7.224 \mathrm{E}$

$31.663 \mathrm{E}$ $20.674 \mathrm{E}$ $159.52 \mathrm{E}$

$39.559 \mathrm{~W}$ $31.880 \mathrm{E}$ $7.46 \mathrm{E}$ $28.242 \mathrm{~W}$
$10 \quad \mathrm{G}$

$10 \mathrm{G}$

$12 *$

$10 \mathrm{G}$

$10 \mathrm{G}$

$33 \mathrm{~N}$

$10 \mathrm{G}$

$10 \mathrm{G}$

$10 \mathrm{G}$

$33 \mathrm{~N} \quad 4.7$

$10 \mathrm{G} \quad 4.94 .2$

$10 \mathrm{G}$

$10 \quad G$

$11 G \quad 5.75 .7$
1.2

0.86 CENTRAL ITALY. MD 2.5 (ROM), 2.5 (SSO)

10 ALBANIA ML 2.4 (ROM)

35 GULF OF ALASKA. 〈AGS-P>. ML 4.1 (PMR)

0.725 NORTHERN ITALY. ML 3.0 (LDG), 3.0 (ROM).

0.410 CHILE-ARGENTINA BORDER REGION

0.47 SOUTHERN ITALY

0.7

PUERTO RICO REGION
8

\section{$\begin{array}{llllll}11 & 18 & 13.5 & 31.466 & \mathrm{~S}\end{array}$ $130706.3 * 11.836 \mathrm{~s}$ $135942.9 * 30.691 \mathrm{~N}$ $140114.5 * 2.322 \mathrm{~S}$ $142045.8 * 10.263 \mathrm{~N}$ \\ 73.009 W $163 * 4.7$ \\ $117.321 \mathrm{E} \quad 33 \mathrm{~N} 3.9$ \\ $142.499 \mathrm{E} \quad 10 \mathrm{G} 4.7$ \\ $79.329 \mathrm{~W} 114 * 4.8$ \\ $122.326 \mathrm{E} \quad 33 \mathrm{~N} 4.7$} $145941.8 \quad 35.681 \mathrm{~N}$

$\begin{array}{lll}16 & 2725.9 \& 61.969 N\end{array}$ $\begin{array}{llllll}16 & 52 & 46.1 \% & 43.214 \mathrm{~N}\end{array}$ $\begin{array}{llll}16 & 57 & 20.9 & 46.274 \mathrm{~N}\end{array}$ $\begin{array}{llll}18 & 13 & 47.8 & 20.031 \mathrm{~S}\end{array}$ $\begin{array}{lll}18 & 39 & 42.9 * 36.556 \mathrm{~N}\end{array}$ $\begin{array}{llll}18 & 39 & 59.8 \text { ? } 36.06 \mathrm{~N}\end{array}$ $\begin{array}{llll}18 & 52 & 27.8 \& & 19.339 \mathrm{~N}\end{array}$ 190345.1 ? $38.01 \mathrm{~N}$ $\begin{array}{lllll}21 & 40 & 30.6 & 39.023 \mathrm{~N}\end{array}$ $225341.7 * 28.452 \mathrm{~N}$ $\begin{array}{llll}23 & 09 & 06.7 \& & 37.128 \mathrm{~N}\end{array}$ $\begin{array}{lllllll}23 & 23 & 57.3 & 14 & 637 & \mathrm{~N}\end{array}$ $\begin{array}{lllll}23 & 55 & 57.0 & 34.305 & \mathrm{~N}\end{array}$ $022559.9 \& 62742 \mathrm{~N}$ $02 \quad 4549.4 \& 61.463 \mathrm{~N}$ $\begin{array}{llll}06 & 37 & 55.5 & 18.529 \mathrm{~S}\end{array}$ $07 \quad 10 \quad 22.6 \quad 51.568 \mathrm{~N}$

$07 \quad 16 \quad 46.1 \quad 51.522 \mathrm{~N}$ $071724.5 * 51.834 \mathrm{~N}$ $\begin{array}{lllllll}08 & 10 & 17.0 & 15.948 \mathrm{~S}\end{array}$ $\begin{array}{llllll}08 & 23 & 54.7 \% & 17.586\end{array}$ $084724.1 \% 60.692 \mathrm{~N}$ $090548.1262 .153 \mathrm{~N}$ $\begin{array}{llllll}09 & 07 & 29.1 ? & 17.85 \mathrm{~N}\end{array}$ $\begin{array}{llllll}09 & 18 & 04 & 5 & 42 & 902 \mathrm{~N}\end{array}$ $092422.3 * 37.413 \mathrm{~N}$ $10 \quad 08 \quad 56.9 \% \quad 61.611 \mathrm{~N}$ $\begin{array}{lllll}10 & 59 & 34.1 & 51.522 \mathrm{~N}\end{array}$ $112646.2 * 22.594 \mathrm{~S}$ $\begin{array}{llll}11 & 57 & 48.9 & 17.90 \quad \mathrm{~N}\end{array}$ $\begin{array}{lllll}12 & 29 & 0 & 1.8 \% & 42.727 \mathrm{~N}\end{array}$ $141226.1 * 32.056 \mathrm{~N}$ $\begin{array}{llllll}14 & 47 & 03.28 & 60.128 \mathrm{~N}\end{array}$ $153000.0837 .275 \mathrm{~N}$
138.368 E $10 \quad \mathrm{G}$

$70.543 \mathrm{E} 118 * 4.9$

$151.003 \mathrm{~W}$

$0.169 \mathrm{E}$

$7.333 \mathrm{E}$

$133.743 \mathrm{E}$

$70.113 \mathrm{E}$

$21.07 \mathrm{E}$

$154.956 \mathrm{~W}$

$21.24 \mathrm{E}$

$75.274 \mathrm{E}$

$51.426 \mathrm{E}$

$121.517 \mathrm{~W}$

$60360 \mathrm{~W}$

$70775 \mathrm{E}$

$150.721 \mathrm{~W}$

$151.523 \mathrm{~W}$

$174832 W$

$174339 \mathrm{~W}$

$174.391 \mathrm{~W}$ $174.326 \mathrm{~W}$

$75.086 w$

$94.510 \mathrm{~W}$

5.510 E

$149.614 \mathrm{~W}$

$65.66 \mathrm{~W}$

$13.035 \mathrm{E}$

$142.078 \mathrm{E}$

$5.217 \mathrm{E}$

$174342 \mathrm{~W}$

$175448 \mathrm{~W}$

$6582 \quad W$

19. $127 \mathrm{E}$

$96.633 \mathrm{E}$

$52.819 \mathrm{~W}$

$116.354 W$
75

$10 \mathrm{G}$

14

$5 \mathrm{G}$

$188 ? 4.5$

$10 \mathrm{G}$

2
10

$22 * 4.2$

$33 \mathrm{~N} 4.3$

7

43 ?

$33 \mathrm{~N} 4.5$

89

82

$88 ? 4.7$

$33 \mathrm{~N} 5.25 .0$

$33 \mathrm{~N} \quad 4.8 \quad 4.6$

$33 \mathrm{~N} 4.9$

$\begin{array}{lll}34 & 4.8 & 4.2\end{array}$

33

$10 \mathrm{G}$

44

$10 \mathrm{G}$

$10 \mathrm{G}$

$33 \mathrm{~N}$

$10 \mathrm{G}$

$33 \mathrm{~N} 4943$

$117 ? 4.9$

$10 \mathrm{G}$

$10 \mathrm{G}$

$33 \mathrm{~N} 4.2$

113

049

HAWAII. LHVO-P). MO 6.2 (HVO). MS 6.2 (BRK), 6.2 (PAS) Five peaple injured slightly, 5 hames destroyed ond about 100 hames damaged in the Puna District.

Landslides occurred in several places and blocked o raad ot Honomu. Slight damage ( $V I$ ) of Howai Notionol Park. Hilo, Hanomu and Keoou. Fell (V) ot Honokoo, Kapoou, Kurtistown. Ninole, Oakalo, Poouhau and Valcana; (IV) at Hokalou, Hawi, Halualoa, Honounau, Loupohoehoe and Pohalo. Felt throughout the island of Hawai i. Also felt on Maui and Oahu. A small tsunomi wos generoted with maximum wove heights (peak-to-trough) of $57 \mathrm{~cm}$. at Honuapo. $21 \mathrm{~cm}$. of Kapaha and $14 \mathrm{~cm}$. at $\mathrm{Hilo}$ Two events obout 5 secands oport, abserved on broodbond displacement seismograms.

0. 7

1.0

0.3

1. 5

1. 2

0. 2

0.4

1. 0

0. 5

0.6

0. 6

0. 4

1. 1390 YUGOSLAVIA. ML 1.9 (SKO)

6 TURKEY

6 PUERTO RICO REGION

5 YUGOSLAVIA. ML 2.2 (TTG).

8 TURKEY

34 HAWA II. SHVO P>. MD 4.4 (HVO)

10 MENDOZA PROVINCE, ARGENTINA

3 NORTHERN I TALY

5 TURKEY

7 IONIAN SEA. ML 3.5 (ATH)

1 OFF EAST COAST OF KAMCHATKA

NORTH ATLANTIC RIDGE

TURKEY

4 NORTHERN ITALY. MD 2.4 (ROM).

AZORES ISLANDS. Ms 5.4 (BRK). Severol people injured slightly ond minor domage (VI) on Grocioso. Fell (V) on Terceira and (IV) an Faial. Pico and Soo Jarge. Depth from broodbond displacement seismograms.

1.314 SOUTH AUSTRALIA

1.115 NORTHERN COLOMBIA

0.87 SOUTH OF SUMBAWA ISLAND

1.011 SOUTH OF HONSHU, JAPAN

0.9 23 NEAR COAST OF ECUADOR

0.411 PANAY, PHILIPPINE ISLANDS

1.0116 HINDU KUSH REGION. Fell (11) ot Khorog ond Dushonbe. USSR. Felt Ol Peshowar. Pokistón.

17 SOUTHERN ALASKA. LAGS-P>. 


\begin{tabular}{|c|c|c|c|c|c|c|c|c|c|c|c|c|}
\hline 27 & 15 & 51 & 49.6 & 68 & 41. & .795 & $\mathrm{~N}$ & 112.734 & $w$ & 6 & & \\
\hline 27 & 16 & 28 & 29.2 & 28 & 41. & .795 & $\mathrm{~N}$ & 112.729 & $w$ & 6 & & \\
\hline 27 & 16 & 37 & 47.5 & $5 \&$ & 59 & .265 & $\mathrm{~N}$ & 153.037 & w & 79 & & \\
\hline 27 & 17 & 01 & 36.7 & 7 & 42 & .063 & $\mathrm{~N}$ & 142.633 & E & 63 & 4.0 & \\
\hline 27 & 17 & 09 & 21.2 & $2 ?$ & 10 & .56 & N & 61.84 & w & 10 & G & \\
\hline 27 & 17 & 26 & 33.6 & $6 *$ & 36. & .916 & N & 71.448 & E & 33 & $\mathrm{~N} \quad 4.5$ & \\
\hline 27 & 17 & 43 & $09 . e$ & $\theta ?$ & 63 & .72 & s & 156.10 & $w$ & 10 & 5.4 & 5.5 \\
\hline 27 & 18 & 86 & 44. & $1 \%$ & 15. & .216 & $\mathrm{~N}$ & 60.497 & w & 10 & G & \\
\hline 27 & 18 & 20 & 00.5 & $5 \&$ & 59 & .571 & N & 153.025 & $w$ & 96 & & \\
\hline 27 & 18 & 40 & 26.3 & $3 ?$ & 32 & .14 & s & 71.64 & w & 33 & $\mathrm{~N}$ & \\
\hline 27 & 18 & 40 & $46 . e$ & $\theta$. & 42 & .554 & $\mathrm{~N}$ & 145.746 & E & 33 & $\mathrm{~N} \quad 4.5$ & \\
\hline 27 & 19 & 52 & 59. & $1 \%$ & 10 & .684 & $\mathrm{~N}$ & 61.712 & w & 33 & $\mathrm{~N}$ & \\
\hline 27 & 20 & 26 & 45 . & $7 *$ & & 368 & s & 132.146 & E & 33 & 3.5 & \\
\hline 27 & 21 & 48 & 49. & $\theta \&$ & 61 & 265 & $\mathrm{~N}$ & 152.039 & w & 109 & & \\
\hline 27 & 22 & 15 & 20. & 7? & 41. & .67 & $\mathrm{~N}$ & 12.70 & E & 10 & G & \\
\hline 27 & 23 & 12 & 23 & 38 & 58 & .217 & N & 142.715 & $w$ & 10 & G & \\
\hline 27 & 23 & 24 & 01.5 & $5 \&$ & 57 & .986 & $\mathrm{~N}$ & 143.795 & $w$ & 10 & G & \\
\hline 28 & 00 & 01 & 55. & 1? & 11. & .51 & N & 60.07 & $w$ & 10 & G & \\
\hline 28 & 00 & 14 & 58. & $6 ?$ & 45. & .99 & $\mathrm{~N}$ & 150.63 & E & 33 & $N \quad 4.8$ & 4.0 \\
\hline 28 & 01 & 41 & 13.4 & 4 & 25 & .662 & N & 140.677 & E & 195 & D 4.7 & \\
\hline 28 & 02 & 04 & 26. & $9 *$ & 12 & 214 & $\mathrm{~N}$ & 125.632 & E & 33 & $\mathrm{~N} 5.0$ & \\
\hline 28 & 03 & $\theta 0$ & 28. & 9 & 45. & .096 & $\mathrm{~N}$ & 151.292 & E & 44 & D 5.6 & 5.1 \\
\hline 28 & 03 & 04 & 58.2 & $2 ?$ & 37. & .91 & $\mathrm{~N}$ & 26.49 & w & 10 & G & \\
\hline 28 & 03 & 16 & 33.6 & $6 *$ & 44. & .345 & $\mathrm{~N}$ & 112.365 & $w$ & 5 & G & \\
\hline 28 & 03 & 47 & 06.5 & $9 *$ & 17. & .376 & $\mathrm{~N}$ & 95.020 & $w$ & 116 & $? \quad 3.9$ & \\
\hline 28 & 04 & 56 & 51.7 & 7 & 63. & .243 & $\mathrm{~N}$ & 150.490 & w & 122 & 4.6 & \\
\hline 28 & 88 & 15 & 16.5 & $9 *$ & 38. & .258 & N & 20.489 & E & 10 & G & \\
\hline 28 & $\theta 9$ & 22 & $40 . e$ & $\theta *$ & 36. & .571 & N & 140.441 & E & 33 & N & \\
\hline 28 & 10 & 19 & 48.5 & 98 & 56. & .173 & N & 150.850 & $w$ & $\theta$ & 4.2 & \\
\hline 28 & 10 & 46 & 57.7 & $7 \%$ & 61. & .608 & N & 5.302 & E & 10 & G & \\
\hline 28 & 12 & 05 & 29.7 & $7 ?$ & 51. & .44 & $\mathrm{~N}$ & 174.18 & w & 33 & $\mathrm{~N} \quad 4.5$ & \\
\hline 28 & 12 & 88 & 32. & 4 & 23. & .851 & N & 94.387 & $E$ & 76 & D 5.0 & \\
\hline 28 & 12 & 36 & 28 . & & 23. & .730 & $\mathrm{~N}$ & 91.234 & $E$ & 33 & $\mathrm{~N}$ & \\
\hline 28 & 12 & 42 & 26. & $5 ?$ & 42. & .76 & N & 19.17 & E & 10 & G & \\
\hline 28 & 13 & 35 & 10 & $3 ?$ & & .79 & $\mathrm{~s}$ & 145.64 & $E$ & 85 & 4.5 & \\
\hline 28 & 13 & 39 & 10. & & 44. & .522 & $\mathrm{~N}$ & 26.028 & E & 21 & & \\
\hline 28 & 14 & 53 & 16.8 & $8 *$ & 9 . & .841 & $s$ & 121.757 & E & 71 & $? 4$. & \\
\hline 28 & 15 & 11 & 27. & $1 \%$ & 61. & .613 & $\mathrm{~N}$ & 5.295 & E & 10 & G & \\
\hline 28 & 15 & 16 & 18. & & & .535 & N & 76.066 & $w$ & 129 & 4.7 & \\
\hline 28 & 15 & 40 & 47 & & & 165 & & & & 19 & * & \\
\hline 28 & 16 & $\theta 0$ & 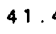 & $4 \&$ & 40 & .655 & $\mathrm{~N}$ & 122.120 & $w$ & 12 & & \\
\hline
\end{tabular}

Burial $600 \mathrm{~m}$., Shot Time 153000 020, "AMARILLO," Nevoda Test Site (Dept. of Energy)

6 UTAH. $\langle S L C-P\rangle$. ML 3.0 (SLC)

6 UTAH. $\angle S L C-P>$. ML 2.9 (SLC).

13 SOUTHERN ALASKA. 〈AGS-P>

HOKKAIDO, JAPAN REGION. Felt (II JMA) at Urakawa and Hiroo.

0.85 TRINIDAD

1.311 AFGHANISTAN-USSR BORDER REGION

1.323 SOUTH PACIFIC CORDILLERA

0.47 LEEWARD ISLANDS. ML 2.6 (FDF).

20 SOUTHERN ALASKA. 〈AGS-P>

0.710 NEAR COAST OF CENTRAL CHILE

1.312 HOKKAIDO, JAPAN REGION

1.35 TRINIDAD

1.25 WEST IRIAN REGION

30 SOUTHERN ALASKA. 〈AGS-P>.

0.8 4 SOUTHERN ITALY. MD 2.2 (ROM)

20 GULF OF ALASKA. LAGS-P>.

11 GULF OF ALASKA. 〈AGS-P>

WINOWARD ISLANDS

KURIL ISLANDS

38 VOLCANO ISLANDS REGION

19 SAMAR, PHILIPPINE ISLANDS

216 KURIL I SLANDS

AZORES ISLANDS

7 EASTERN IDAHO. ML 3.1 (BUT)

8 OAXACA, MEXICO

69 CENTRAL ALASKa. Felt (IV) at Nenano; (III) at Skwentna and Talkeetra.

D.8 7 GREECE. MD 3.5 (ATH)

1.18 NEAR EAST COAST OF HONSHU, JAPAN. MG 3.3 (JMA). Felt ( JMA) at Mito.

21 GULF OF ALASKA. 〈AGS-P>. ML 3.6 (PMR)

0.57 SOUTHERN NORWAY. MO 1.7 (BER)

1.712 ANDREANOF ISLANDS, ALEUTIAN IS

1.0138 BURMA-INDIA BORDER REGION

1.08 INDIA-BANGLADESH BORDER REGION

0.24 YUGOSLAVIA. ML 2.0 (TTG)

1. 7 EAST PAPUA NEW GUINEA REgION

0.7 13 ROMANIA

1.212 SAVU SEA

0.68 SOUTHERN NORWAY. MD 2.1 (BER)

0.940 COLOMBIA. Fell in chaca and valle del cauca Departments.

0.59 LEEWARD ISLANDS. ML 3.3 (FDF).

11 NORTHERN CALIFORNIA. $\angle B R K>$. ML 3.0 (BRK). Fell (IV) at Bella vista, Central valley, Millville, Oak Run, Palo Cedra Project Cily and Whitmare: (III) at Anderson. Cattanwaod, Man:an. Montgomery Creek, Round Mountain and Summit City. Also telt at Redding and Shingletawn.

\begin{tabular}{|c|c|c|c|c|}
\hline 7.795 & $E$ & 10 & G & \\
\hline 126.23 & $E$ & 105 & $?$ & 4.5 \\
\hline 122.30 & E & 10 & G & \\
\hline 146.566 & $w$ & 24 & & \\
\hline 13.89 & $w$ & 10 & G & 4.8 \\
\hline 24.45 & $E$ & 10 & G & \\
\hline 28.383 & $w$ & 10 & 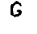 & 4.64 .2 \\
\hline 150.914 & w & 127 & & \\
\hline 147.449 & w & 10 & G & $5.5 \quad 5.7$ \\
\hline 15.185 & E & 10 & G & \\
\hline 7.381 & E & 10 & G & \\
\hline 15.18 & E & 10 & $G$ & \\
\hline 148.814 & $E$ & 50 & D & 5.4 \\
\hline $\begin{array}{r}8.429 \\
127.342\end{array}$ & E & 173 & D & 5.3 \\
\hline 175.319 & $w$ & 35 & D & $5.2 \quad 5.1$ \\
\hline 61.04 & w & 10 & G & \\
\hline 19.654 & E & 47 & * & 3.9 \\
\hline 3.74 & w & 10 & G & \\
\hline 154.817 & E & 82 & $*$ & 5.3 \\
\hline 93.166 & w & 54 & $*$ & 4.8 \\
\hline 7.646 & E & 10 & G & \\
\hline 7.587 & $E$ & 10 & G & \\
\hline 18.628 & $E$ & 10 & G & \\
\hline 71.624 & $w$ & 98 & * & \\
\hline 102.018 & $E$ & 42 & D & 5.35 .0 \\
\hline 89.966 & w & 7 & & 4.94 .0 \\
\hline 25.158 & E & 10 & G & \\
\hline 4.840 & E & 10 & G & \\
\hline 7.242 & E & 11 & & \\
\hline 124.388 & E & 82 & ? & 4.7 \\
\hline 5.788 & w & 10 & G & \\
\hline 28.256 & E & 10 & G & \\
\hline 156.196 & $w$ & 15 & & \\
\hline 91.564 & w & 62 & D & 51 \\
\hline 26 & 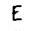 & 33 & 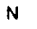 & \\
\hline 9.227 & E & 10 & G & \\
\hline 26.924 & & 33 & & \\
\hline
\end{tabular}

0.1 0.5 1.4

1.6

1.6 $20 \quad 58 \quad 14.2863 .084 \mathrm{~N}$ $21,24 \quad 12.4 * 57,788$ $213630.5 \% 37.681 \mathrm{~N}$ $2152 \quad 16.8 \% 61.816 \mathrm{~N}$ $\begin{array}{llllll}22 & 03 & 39.7 ? & 37.65 & \mathrm{~N}\end{array}$ $\begin{array}{lllll}22 & 11 & 58.9 & 6.223 \mathrm{~S}\end{array}$ $\begin{array}{lllll}22 & 57 & 54.2 & 49.341 \mathrm{~N}\end{array}$ $\begin{array}{llllll}23 & 44 & 513 & 7.604 & 5\end{array}$ $\begin{array}{llllllll}00 & 18 & 39 & 8 & 22 & 960 & \mathrm{~S}\end{array}$ $\begin{array}{lllllll}91 & 21 & 09 & 3 ? & 17 & 40 & \mathrm{~N}\end{array}$ $\begin{array}{lllllll}02 & 4 & 3 & 5 & 39 & 491 \mathrm{~N}\end{array}$

$025854.7 ? 35.10 \mathrm{~N}$ $\begin{array}{llllll}03 & 57 & 33.1 & 6.289 & \mathrm{~S}\end{array}$ $035955.4 * 14.071 \mathrm{~N}$ $04 \quad 1844.3 \quad 43.616 \mathrm{~N}$ $\begin{array}{llllll}94 & 18 & 46.7 & 43.631 & \mathrm{~N}\end{array}$ $04 \quad 56 \quad 34.2 * 49.923 \mathrm{~N}$ $045841.9 * 31.364 \mathrm{~S}$ $05 \quad 25 \quad 51.5 \quad 4.497 \mathrm{~S}$ $965138.7 \quad 15.772 \mathrm{~N}$ 97 $5236.438 .625 \mathrm{~N}$ $10 \quad 32 \quad 54.6 \% 60.524 \mathrm{~N}$ $104002.7 \quad 44.411 \mathrm{~N}$ $\begin{array}{lllll}10 & 55 & 39.8 & 0.405 & \mathrm{~S}\end{array}$ $\begin{array}{llll}11 & 43 & 57.5 \% & 36.588 \mathrm{~N}\end{array}$ $\begin{array}{lllll}12 & 29 & 04.5 & 39.953 \mathrm{~N}\end{array}$ $1232 \quad 31.9859 .404 \mathrm{~N}$ $\begin{array}{lllll}12 & 38 & 37.1 & 14.040 \mathrm{~N}\end{array}$ 


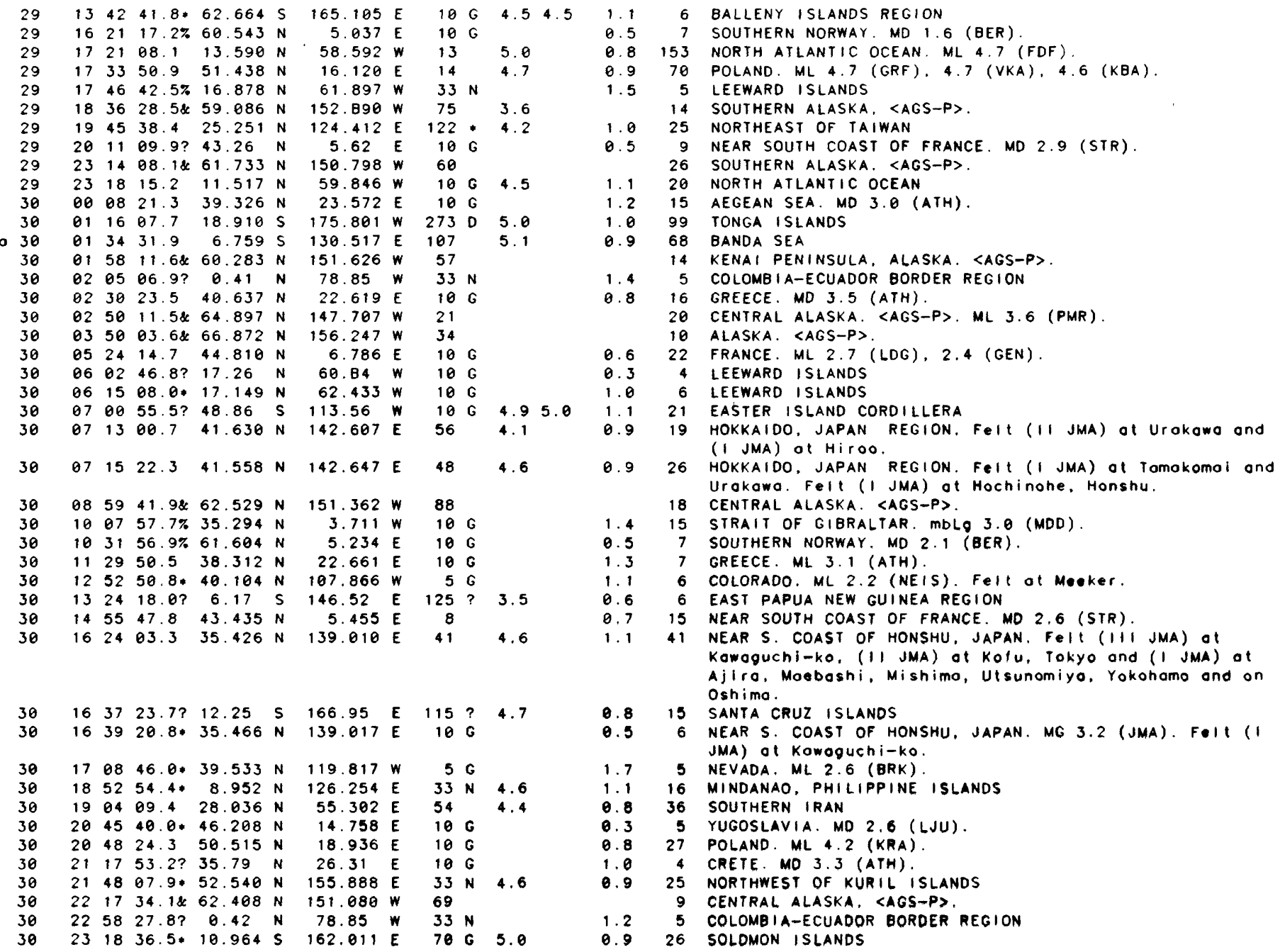

$0104 \quad 08 \quad 18.91 \quad 10.9665 \quad 165.449 \mathrm{E} \quad 33 \mathrm{~km}$ 5. $0 \mathrm{mb}$ ( 7 obs.) $4.8 \mathrm{Msz}$ ( 2 Obs.) SANTA CRUZ ISLANDS

CENTROID, MOMENT TENSOR

(HRV)

Doto Used: GDSN

L.P.B.: $115,20 \mathrm{C}$

Centroid Location:

Origin Time $\quad 04: 08: 21.70 .6$

Lot 11.425 0.11 Lon 165.52E 0.10

Dep 15.0 FIX Half-duration 1.6 Principal Axes:

Scole $10 * 16 \mathrm{Nm}$

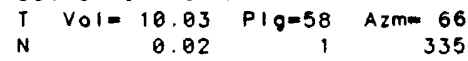

$P \quad-10.05 \quad 32 \quad 244$

NP 1 : Strike=331 Dip=13 Si

$$
\text { NP2: } \quad 155 \quad 77 \quad 91
$$

$01072506.47 \quad 52.004 \mathrm{~S} \quad 159.679 \mathrm{E} \quad 10 \mathrm{~km}$ $5.2 \mathrm{mb}(12$ Obs.) 4.8Msz ( 3 Obs.) MACOUARIE ISLANOS REGION

CENTROID, MOMENT TENSOR

DOtO USEO: GOSN

L.P.8.: 145, 25C

Centroid locotion:

Origin Time $07: 25: 14.20 .5$

Lat 52.125 FIX:Lon 158.56E FIX

Dep 15.0 Fix Holf-durotion 1.5

Principol Axes:

Scole $10 *+16 \mathrm{Nm}$

$T \quad V_{0 I}=5.34 \quad \mathrm{PIg}=0 \quad A \mathrm{Zm}=210$

$\begin{array}{rrrrr}N & 2 & 54 & 90 & 180 \\ P & -7 & 88 & 0 & 120\end{array}$

Best Double Couple: Mo=6.6*10**16

NP1: Strike $=255$ Dip $=90 \quad$ SIip $=180$

NP2: $\quad 345 \quad 90 \quad 0$

$02 \quad 1102 \quad 19.83 \quad 19.068 \$ \quad 168.833 E \quad 100 \mathrm{~km}$
$5.3 \mathrm{mb}(18$ abs.)

VANUATU ISLANDS

CENTROID, MOMENT TENSOR (HRV)

Doto USEd: GDSN

L.P.B.: 17S, $28 \mathrm{C}$

Centroid Locotion:

orloin time 11:02:25.30.7

Lot 18.9550 .06 Lon 168.04E 0.06

Deo 105.1 $3.2 \mathrm{Half}$-duration, 1.7

Principal Axes

Seole $10+16 \mathrm{Nm}$

I $\quad V_{91}=12.05 \quad$ PIg $=18 \quad$ A $2 m=127$

$\begin{array}{rrrr}N & 0.37 & 25 & 226 \\ P & -12.41 & 59 & 5\end{array}$

Best Double Couple Mo=1 $2 \cdot 10 * * 17$

NP 1: $\$ t r, k e=184$ Di $p=35 \quad$ SI: $p=-137$

NP2: $\quad 57 \quad 67 \quad-63$

$02210118.21 \quad 4,6695 \quad 153.014 \quad 72 \mathrm{~km}$

5. $4 m b$ ( 19 Obs.)

NEW IRELAND REGION

CENTROID. MOMENT TENSQR (HRV)

Doto Used: GDSN

L.P.B.: 10S, $18 \mathrm{C}$

Centroid Location

origin Time 21:01:21.50.9

Lat 4.595 0.10 Lon 152.89E 0.1

Dep $21.89 . \mathrm{Half}$-duration 1.5

Principol Axe:

Scale $10 *+16 \mathrm{Nm}$

$T \quad V a l=5.53$ Plg=23 Azm= 55

$\begin{array}{rrrr}N & 0.04 & 10 & 150 \\ P & -5.56 & 65 & 263\end{array}$

Best Double Couple: Mo=5 $5 * 10 * 16$

NP 1 : Strike $=126$ D i $p=24$ Si $p=-116$

$\begin{array}{llll}\text { NP2: } & 334 & 68 & -79\end{array}$

$03025652.60 \quad 6.826 \mathrm{~S} \quad 156 \quad 216 \mathrm{E} \quad 171 \mathrm{~km}$ $5.4 \mathrm{mb}$ ( 38 obs.)
SOLOMON ISLANDS

CENTROID, MOMENT TENSOR (HRV)

Dato Used: CDSN

L.P.B.: 185. 31C

Centroid Locotion:

origin Time

$82: 56: 56.40 .6$

Lot 6.8450 .05 Lan $156.00 E 0.05$

Dep 163.2 1.6 Holfoduration 2.2

Principal axes:

scal: $10 * 17 \mathrm{Nm}$

$r \quad V_{0}=2.02 \quad$ Plq-18 Azm=363

$\begin{array}{rrrr}N & 0.16 & 14 & 208 \\ P & -2.18 & 67 & 82\end{array}$

Best Double Couple:Mo=2.1*10**17

NP1: Strike = 55 Dip=30 Si i $p=-6 B$

$\begin{array}{llll}\text { NP2: } & 201 & 65 & -106\end{array}$

$06072456.64 \quad 18.457 \mathrm{~N} 146.302 \mathrm{E} \quad 58 \mathrm{~km}$ $5.4 m b$ ( 36 obs.)

MARI ANA ISLANDS

CENTROID, MOMENT TENSOR

(HRV)

Doto Used: GOSN

L.P.8.: $145,24 \mathrm{C}$

Centrolo Loegtion

Origin rime 07:25: 130

Lat 18.46N 0.06 Lon 146.25E 0.06

Dep $28.74 .9 \mathrm{Half}$-durotion 1.5

Principol axes:

Scole $10 * 16 \mathrm{Nm}$

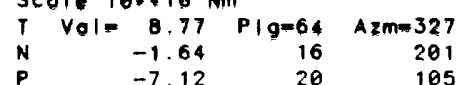

Best Double Couple:Mo=7.9*10**16 NP 1: Strike=169 Dip=29 SI ip= 55 $\begin{array}{lrrr}\text { NP2 : } & 28 & 67 & 108\end{array}$

$\begin{array}{llllllll}07 & 19 & 45 & 53.70 & 38.057 \mathrm{~N} & 21.620 \mathrm{E} & 25 \mathrm{~km}\end{array}$ $5.0 \mathrm{mb}$ ( 58 obs.) $4.8 \mathrm{Msz}$ ( 4 obs.) GREECE 

Doto USEd GDSN

L.P B. 13S, $24 C$

Centrold Location

Origin time $\quad 19: 45.58909$ Lat $38.05 \mathrm{~N} F / X ;$ Lon $21.63 \mathrm{E}$ FIX Dep $25.0 \mathrm{FIX} \mathrm{Half-duration} 1.6$ Principal Axes:

Scale $10 * 16 \mathrm{Nm}$

$T \quad V a l=5.62 \quad P l g=2 \quad A z m=25$

$\begin{array}{rrrr}\mathrm{N} & 2.66 & 54 & 292 \\ \mathrm{P} & -8.29 & 36 & 116\end{array}$

Best Double Couple.Mo=6 $9 * 10 * * 16$

NP 1: Strike=154 Dip=64 S। $p=-26$

$\begin{array}{llll}\text { NP2: } & 256 & 67 & -152\end{array}$

07 $21 \quad 47 \quad 35.05 \quad 5.632 \mathrm{~N} \quad 125.732 \mathrm{E} \quad 170 \mathrm{~km}$ $5.1 \mathrm{mb}(18$ abs.)

MINDANAO, PHILIPPINE ISLANDS

CENTROID. MOMENT TENSOR (HRV)

Data Used: GDSN

L.P.B.: 12S, $23 \mathrm{C}$

Centraid Lacatian

Origin Time $21: 47: 34.2 \quad 1.0$

Lot $5.55 \mathrm{~N} 0.07$ Lan $125.76 \mathrm{E} \quad 0.11$

Dep 158.62 .2 Holf-durotian 1.6

Principal Axes:

Scale $10 * * 16 \mathrm{Nm}$

$T \quad V a l=7.92 \quad P \mid g=67 \quad$ Azm $=257$

N $\quad-2.03 \quad 20 \quad 109$

$\begin{array}{cccr}P & -5.90 & 11 & 15 \\ \text { Best Dauble Couple:Ma=6.9*10*16 }\end{array}$

NPI:Strike $=82$ Dip $=38$ Slip= 57

NP2: $\quad 301 \quad 59 \quad 113$

$\begin{array}{llllllll}08 \quad 06 \quad 24 & 09.61 & 6.837 \mathrm{~N} & 37.878 \mathrm{E} & 19 \mathrm{~km}\end{array}$ $5.0 \mathrm{mb}$ ( 26 obs.) 4.8Msz ( 1 abs.) ETHIOPIA

CENTROID, MOMENT TENSOR (HRV)

Doto Used: GDSN

L.P.B.: $9 \mathrm{~S}, 20 \mathrm{C}$

Centraid Locotion:

Origin Time

$06: 24: 17.5 \quad 1.5$

Lot $7.35 \mathrm{~N} 0.16$ Lon $38.16 \mathrm{E} 0.13$

DeP $15.0 \mathrm{FIX}$ Halt-duration 1.5

Principol Axes:

Scole $10 * 16 \mathrm{Nm}$

T $\quad$ OOI $=3.35 \quad P 1 g=0 \quad$ Azm $=110$

$\begin{array}{rrrr}N & -0.93 & 0 & 20 \\ P & -2.42 & 90 & 180\end{array}$

Best Dauble Couple: $M 0=2.9 * 10 * * 16$ NP 1: Strike $=200$ Di $p=45$ Slip $=-90$ NP2: $\quad 20 \quad 45 \quad-90$

$\begin{array}{lllllll}08 \quad 09 & 51 & 56.84 & 19.531 \mathrm{~s} & 173.736 \mathrm{~W} & 23 \mathrm{~km}\end{array}$ $5.5 \mathrm{mb}$ ( 37 obs.) 5.4Msz ( 28 obs.) TONGA ISLANDS

CENTROID. MOMENT TENSOR (HRV)

Doto Used: GDSN

L.P.B.: 14S, 36C

Centroid Locotion:

Origin Time $09: 52: 4.30 .3$

Lot 19.7350 .03 Lon 173.57w 0.03

Dep 17.7 1.4 Half-durotion 3.0

Principal Axes:

Scale $10 * 17 \mathrm{Nm}$

$T \quad V_{0 l}=5.74 \quad \mathrm{P} / \mathrm{g}=73 \quad$ Azm $=266$

$\begin{array}{rrrr}\mathrm{N} & 0.58 & 4 & 9 \\ \mathrm{P} & -6.32 & 17 & 100\end{array}$

Bes! Double Couple Mo=6 $\quad 0 * 10 * * 17$

NP 1: Strike=196 D i $p=29$ SI ip $=98$

NP2 :

$6 \quad 62$

$\begin{array}{llllllll}09 & 67 & 14 & 28.78 & 5.038 \mathrm{~S} & 151.278 E & 157 \mathrm{~km}\end{array}$ 5. $1 \mathrm{mb}$ ( 22 Obs.)

NEW BRITAIN REGION

CENTROID, MOMENT TENSOR (HRV)

Doto UsEd: GDSN

L.P. B.: 12S, $18 \mathrm{C}$

Centroid Locotion

Origin Time $07: 14: 30.012$

Lat 5.30S 0.12 Lon 151 27E 0.12

Dep 142.34 .3 Half-durotion 1.5 Principol Axes:

Scole $10 * * 16 \mathrm{Nm}$

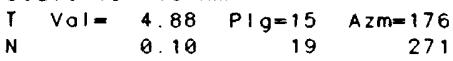

$\begin{array}{lrrr}P & -4.97 & 65 & 51\end{array}$

Best Double Cauple $M o=4.9 * 10 * 16$ NP 1: Strike=241 Di $p=34 \quad S 1$ i $p=-126$

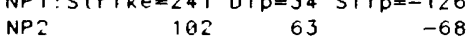

$09 \quad 12 \quad 19 \quad 35.70 \quad 71.432 \mathrm{~N} \quad 4.371 \mathrm{~W} \quad 10 \mathrm{~km}$
$56 \mathrm{mb}$ ( 54 Obs) $54 M \mathrm{Mz}$ ( 19 obs) JAN MAYEN ISLAND REGION

CENTROID, MOMENT TENSOR

(HRV)

Data Used GDSN

L.P.B. 16S, $37 \mathrm{C}$

Centroid Lacation:

Origin Time

$12: 19: 41.50 .3$

Lot $79.99 \mathrm{~N} 0.95$ Lan $3.69 \mathrm{~W} 0.14$

Dep $15.0 \mathrm{~F} / \mathrm{X}$ Half-duration 2.3

Principal Axes:

Scale $10 * * 17 \mathrm{Nm}$

$T \quad V_{0 l}=3.19 \quad P / g=12 \quad A=3=313$

$\mathrm{N} \quad-0.45$

$\begin{array}{rr}12 & A 2 m=313 \\ 68 & 47\end{array}$

Best Double Couple: $M_{0}=3.0 * 10 * * 17$

NP1: Strike= 29 Dip=37 Si $p=-122$

NP2: $\quad 238 \quad 59 \quad-68$

$09 \quad 15 \quad 3411.64 \quad 7.865 \mathrm{~S} \quad 117.517 \mathrm{E} 245 \mathrm{~km}$ $5.8 \mathrm{mb}$ ( 37 abs.)

BALI SEA

FAULT PLANE SOLUTION: P-WaVes

NP1: Strike $=50$ Dip=80 SIip $=90$

NP2 :

230

10

90

Principal Axes:

T PIg=55 AZm=320

$\begin{array}{ccc}P & 35 & 148 \\ \text { Camment: The facal mechonism is }\end{array}$

paorly cantralled and

carresponds ta reverse

foulting. The preferred foult

plone is NP2

RADIATED ENERGY

Na. of sto: 5 facol mech. $M$

Energy

MOMENT TENSOR SOLUTION

DOP 244 of sto: 10

Principol Axes:

Scole $10 *+17 \mathrm{Nm}$

T $\quad \mathrm{O} O \mathrm{I}=3.81 \quad \mathrm{PIg}=50 \quad$ Azm=33!

N

$$
\begin{array}{rrr}
-0.01 & 8 & 231 \\
-3.80 & 39 & 135
\end{array}
$$

Best Double Cauple: $M a=3.8 * 10 * * 17$

NP1: Strike=179 Dip=10 SIip= 37

NP2 :

CENTROID. MOMENT TENSOR (HRV)

DOta UsEd: GDSN

L.P.B.: 17S, $34 \mathrm{C}$

Centroid Lacotian:

Origin Time

$15: 34: 13.90 .4$

Lot 8.0550 .04 Lan $117.29 E 0.04$

Dep 247.6 1.8 Holf-durotion 2.6

Principol Axes

Scole $10 * * 17 \mathrm{Nm}$

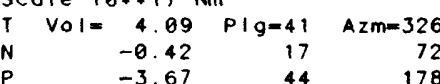

Best Double Cauple:Mo=3,9*10*17

NP1: Strike=346 Dip=17 Si ip $=-175$

NP2: $\quad 252 \quad 89 \quad-73$

$09 \quad 16 \quad 52 \quad 12.10 \quad 20.260 \mathrm{~N} \quad 145.147 \mathrm{E} \quad 149 \mathrm{~km}$

$5.4 \mathrm{mb}$ ( 39 obs.)

MARIANA ISLANDS

CENTRO10. MOMENT TENSOR (HRV)

Doto Used: GDSN

L.P.B.: 135, $23 \mathrm{C}$

Centroid Locotian

Origin Time $\quad 16: 52: 1460.9$

Lot $20.16 \mathrm{~N} 0.06$ Lan $144.73 \mathrm{E} 0.09$

Dep 149.32 .7 Holf-durotion 1.6

Principol Axes:

Scole $10 * 16 \mathrm{Nm}$

$T \quad \mathrm{Val}=9.78 \quad$ PIg $=10 \quad$ Azm $=158$

$\begin{array}{rrrr}N & 2.16 & 43 & 258 \\ P & -11.95 & 45 & 58\end{array}$

lest Double Couple:Mo=1.1*10*17

NP1: Strike $=209$ Dip=51 SI ip=-152

NP2: $\quad 101 \quad 68 \quad-42$

$1122149.71 \quad 51.75 \mathrm{~S} 158.85 \mathrm{E} 10 \mathrm{~km}$ $5.2 \mathrm{mb}$ ( 4 Obs.) 5.1MSZ ( 2 Obs.) NORTH OF MACQUARIE ISLAND

CENTROID, MOMENT TENSOR

(HRV)

Doto Used: GOSN

L. 135, 26C

Centraid Location

Origin Time $12: 21: 55.30 .8$

Lat $51.84 \mathrm{~S} 0.10$ Lon 158.13E 0.12

Dep 15.0 FlX Holf-durotion 1.7

Principol Axes:

Scale $10 * * 16 \mathrm{Nm}$

$T \quad V a I=1036 \quad P I g=12 \quad$ Azm=191
$\begin{array}{rrrr}N & -2.38 & 75 & 332 \\ P & -7.98 & 9 & 99\end{array}$

Best Double Couple Mo=9.2*10*16 NP 1: Strike=235 Dip=75 SIip=178 $\begin{array}{llll}\text { NP2: } & 325 & 88 & 15\end{array}$

$132432.07 \quad 35.107 \mathrm{~N} \quad 35.008 \mathrm{~W} 19 \mathrm{~km}$ $5.8 \mathrm{mb}$ ( 83 abs.) $5.5 \mathrm{Msz}$ ( 17 obs.) NORTH ATLANTIC RIDGE

FAULT PLANE SOLUTION: P-WOVES NPI: Strike=325 Dip=76 SIip= 166 NP2 58 76 14 Principol Axes.

I PIg=20 Azm=282

Camment: The focol mechonism is maderotely well contralled and corresponds to strike-slip

toulting with o smoll reverse

component. The preferred foult plone is not determined.

RADIATEO ENERGY

No. of sta: 6 Focal mech. M Energy 6. Focal mech. M MOMENT TENSOR SOLUTION

DeP 23

Principol Axes

Scole $10 * 18 \mathrm{Nm}$

$T \quad V a l=1.97 \quad P / g=7 \quad A z m=285$

$\begin{array}{rrrr}N & -0.12 & 82 & 76 \\ P & -1.85 & 4 & 194\end{array}$

Best Double Couple:Mo=1.9*10*18 NP 1: Strike $=330$ Dip=82 SIip= 178

NP2 :

CENTROID, MOMENT TENSOR

(HRV)

Doto Used: GDSN

L.P.B.: 135, 37C

Centroid Lacation:

Origin Time

$13: 24: 39.70 .3$

Lot $35.10 \mathrm{~N} 0.04$ Lon $34.88 \mathrm{~W} 0.04$

Dep $15.0 \mathrm{FlX} \mathrm{Halt-duration} 3.0$

Principol Axes:

Scole $10.117 \mathrm{Nm}$

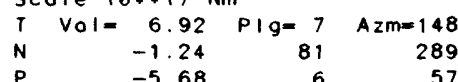

Best Double Couple:Mo=6.3*10*17 NP1: Strike $=192$ Dip=81 S1 ip= 179 NP2:

$\begin{array}{llllll}112324 & 39.00 & 6.463 \mathrm{~S} \quad 147.857 \mathrm{E} \quad 49 \mathrm{~km}\end{array}$ $5.1 \mathrm{mb}$ ( 11 obs.) 4.8Msz ( 6 obs.) EAST PAPUA NEW GUINEA REGION CENTROID. MOMENT TENSOR (HRV) Doto Used: GDSN

L.P.B.: 13S, 22C

Centroid Locotion

Origin Time $23.24: 4150.6$

Lat $6.64 \mathrm{~S} 0.07$ Lon $147.50 \mathrm{E} 0.08$

Dep 47.54 .5 Half-durotion 1.6 Principal Axes:

Scale $10 *+16 \mathrm{Nm}$

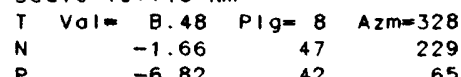

Best Double Couple:Mo=7.6*10**16 NP1: Strike $=97$ Dip=55 S1ip $=-27$ NP2: $\quad 204 \quad 68 \quad-142$

$1200 \quad 04 \quad 09 \quad 76 \quad 21.861 \mathrm{~N} \quad 89.763 \mathrm{E} \quad 61 \mathrm{~m}$ 6. $1 \mathrm{mb}$ ( 61 obs.) 5.1Msz ( 19 obs.) BANGLADESH

FAULT PLANE SOLUTION: P-WOVES

NP1: Strike $=350$ Dip=78 SIip=175

NP2 $81 \quad 85 \quad 12$

Principal Axes:

T PIg=12 Azm=306 $P$. 5 A 215

Comment: The focal mechanism is maderately well controlled and corresponds to strike-slip

faulting with o small reverse

component. The preferred foult plane is not determined.

RADIATED ENERGY

No. of sto: 7 Focol mech. C

Energy MOMENT TENSOR SOLUTION

DeP 18 No of sto:

Principol Axes:

Scole $10 * 17 \mathrm{Nm}$

T $\mathrm{Val}=429 \quad \mathrm{Plg}=40 \quad$ AZm $=322$

$N \quad 0.10 \quad 43 \quad$ A 194 
$\begin{array}{llll}P & -4.40 & 20 & 213\end{array}$ Best Double Couple $M_{0}=4.3 * 10 * 17$ NP1 Strike $=349 \quad$ Dip $=46 \quad$ SI $p=163$ $\begin{array}{lccr}\text { NP2 } & 92 & 78 & 46 \\ \text { CENTRDID, MOMENT } & \text { TENSOR } & \text { (HRV) }\end{array}$ DOto USEd: GDSN

L.P.B.: 10S, $21 \mathrm{C}$

Centroid Location:

Origin Time

$00: 04: 16.3 \quad 0.4$

Lot $22.13 \mathrm{~N} 0.05$ Lan $89.88 \mathrm{E} 0.05$

Dep 15.0 FIX Half-duration $3 . \theta$

Principal Axes

Scale $10 * 17 \mathrm{Nm}$

$T \quad$ VOl $=5.38 \quad \mathrm{Plg}=28 \quad$ AZm $=314$

$\begin{array}{rrrr}N & 0.85 & 62 & 120 \\ P & -6.23 & 6 & 221\end{array}$

Best Double Couple: $M O=5 . B * 10 * * 17$

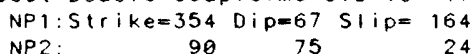

90

$1205 \quad 47 \quad 35.76 \quad 15.2835 \quad 167.572 E \quad 247 \mathrm{~km}$ $5.4 \mathrm{mb}$ ( 19 abs.)

VANUATU ISLANDS

CENTROID, MOMENT IENSOR (HRV)

Data Used: GDSN

L.P.B.: 13S, $23 \mathrm{C}$

Centraid Lacatian:

Origin Time $\quad 05.47: 40.80 .8$

Lat $15.31 \mathrm{~S} 0.06$ Lan $167.56 \mathrm{E} 0.07$

Dep 252.42 .9 Half-duratian 2.2

Principal Axes:

Scale $10 * * 17 \mathrm{Nm}$

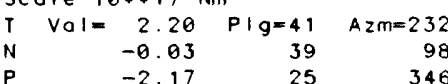

Best Dauble Cauple: $M a=2.2 * 10 * * 17$

NP1:Strike $=27$ Dip=41 S1 ip= 15

NP2: $\quad 286 \quad 80 \quad 130$

$\begin{array}{lllllll}12 & 18 & 21 & 03.30 & 22.554 \mathrm{~S} & 175.826 \mathrm{~W} \quad 71 \mathrm{~km}\end{array}$

5. $5 \mathrm{mb}$ ( 32 abs.)

TONGA ISLANDS REGION

CENTROID, MOMENT TENSOR

(MRV)

Data Used: GDSN

L.P.B.: $155,28 \mathrm{C}$

Centraid Lacatian:

Origin Time $\quad 18: 21: 7.70 .5$

Lat 22.4850 .06 Lon 175.88w 0.06

Dep 67.75 .7 Half-duration 1.8

Principol Axes:

Scale $10 * 17 \mathrm{Nm}$

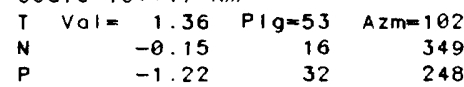

Best Dauble Couple: $M a=1,3 * 10 * * 17$

NP 1 : Strike $=295$ Dip=20 Si ip $=35$

$\begin{array}{lrrr}\text { NP2 : } & 172 & 79 & 107\end{array}$

$\begin{array}{llllll}13 \quad 1749 & 40.57 \quad 3.670 S \quad 140.139 E \quad 71 \mathrm{~km}\end{array}$ $5.4 \mathrm{mb}$ ( 17 abs.)

WEST IRIAN

CENTROID, MOMENT TENSOR (HRV)

Doto Used: GDSN

L.P.B. : $16 \mathrm{~S},-27 \mathrm{C}$

Centraid Lacotian

Origin Time $17: 49: 45.50 .3$

Lat 3.64S 0.02 Lan 140.10E 004

Dep 65993.8 Malf-duration 2.3

Principal Axes

Scale $10 * * 17 \mathrm{Nm}$

$T \quad V a l=2.80 \quad P \mid g=15 \quad$ Azm $=230$

$\begin{array}{rrrr}N & -0.27 & 7 & 322 \\ P & -2.53 & 73 & 76\end{array}$

Best Dauble Cauple: $M O=2.7 * 10 * 17$

NP1 : Strike $=310$ Dip $=31$ SI ip $=-104$

NP2: $\quad 146 \quad 60 \quad-82$

$\begin{array}{llllllll}13 & 22 & 59 & 33.41 & 43.342 \mathrm{~S} & 38.969 \mathrm{E} & 10 \mathrm{~km}\end{array}$ 5. Bmb ( 9 abs.) 5.OMsz ( 1 abs.) PRINCE EDWARD ISLANDS REGION

CENTROID, MOMENT TENSOR (HRV)

Dota Used: GDSN

L.P.B.: $175,38 \mathrm{C}$

Centraid Lacotian

Origin Time

$22: 59: 39.00 .4$

Lat $43.21 \mathrm{~S} 0.04$ Lan $39.49 \mathrm{E} 0.05$

Dep $150 \mathrm{~F} / \mathrm{x}$ Half-duration 2.3

Principal Axes:

Scale $10 * * 17 \mathrm{Nm}$

I $\quad$ Val $=\begin{array}{r}2.46 \\ \text { N }\end{array}$

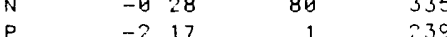

Best Double Couple Mo=2.3*16**17
NP1.Strike=284
NP2
194

$4002900.04 \quad 51488 \mathrm{~N} \quad 174390 \mathrm{~W} \quad 33 \mathrm{~km}$ $5.2 \mathrm{mb}$ ( 50 obs) 4.5MSZ ( 2 obs) ANDREANOF ISLANDS, ALEUTIAN IS

CENTROID, MOMENT TENSOR

(HRV)

Data Used: GOSN

L.P.B.: 12S, $23 \mathrm{C}$

Centroid Location

Origin Time $\quad 00: 29: 3.22 .0$

Lot $51.68 \mathrm{~N} \mathrm{B.18}$ Lon $174.12 \mathrm{~W} 0.25$

Dep $33.0 \mathrm{FlX} \mathrm{Half-durotion} 1.5$

Principol Axes

Scole $10 * 16 \mathrm{Nm}$

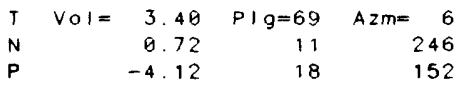

Best Double Couple:Mo $=3.8 * 10 * 16$ NP 1: Strike $=225$ Dip $=29$ SIip $=66$ $\begin{array}{llll}\text { NP2 : } & 71 & 64 & 102\end{array}$

$\begin{array}{llllllllll}14 & 10 & 17 & 35.24 & 12 & 875 \mathrm{~N} & 143.351 \mathrm{E} & 126 \mathrm{~km}\end{array}$ $5.5 \mathrm{mb}$ ( 46 Obs.)

SOUTH OF MARIANA ISLANDS

CENTROID. MOMENT TENSOR

(HRV)

Dato Used: GDSN

L.P.B.: 15S, $29 \mathrm{C}$

Centraid Location:

$10: 17: 39.90 .2$

Lat $12.41 \mathrm{~N} 0.02$ Lan 143.1IE 0.03

Dep $115.31 .6 \mathrm{Half}$-duration 2.6

Principal Axes:

Scole $10 * * 17 \mathrm{Nm}$

T $\quad V a I=3.46 \quad \mathrm{PIg}=5 \quad$ AZm $=232$

$\begin{array}{rrrr}N & 0.12 & 26 & 139 \\ P & -3.58 & 64 & 332\end{array}$

Best Double Couple:Mo=3. $5 * 10 * 17$

NP 1: Strike $=347$ Dip=46 SI ip= -53

NP2: $\quad 120 \quad 55 \quad-122$

$\begin{array}{llllllll}14 & 18 & 06 & 38.26 & 34.289 \mathrm{~N} & 26.055 \mathrm{E} & 14 \mathrm{~km}\end{array}$ $5.2 \mathrm{mb}$ ( 56 abs.) 5.1Msz ( 8 abs.) CRETE

CENTROID, MOMENT TENSOR (HRV)

Doto Used: GDSN

L.P.B.: 12S, $24 \mathrm{C}$

Centraid Lacatian

Origin Time

$18: 06: 40.4 \quad 0.6$

Lot 34.30N FIX; LON 26.10E FIX

Dep $15.0 \mathrm{~F} / \mathrm{X} \mathrm{Half-duration} 1.8$

Principal Axes:

Scole $10 * 17 \mathrm{Nm}$

I $\quad$ ol $=2.42 \quad P 1 g=37 \quad$ Azm=353

$\begin{array}{rrrr}N & -0.26 & 3 & 261 \\ P & -2.16 & 52 & 167\end{array}$

Best Dauble Couple: $M a=2.3 * 10 * 17$

NP1: Strike $=102$ Dip= 8 SI i $p=-68$

$40.085 \mathrm{~N} 144.609 \mathrm{E} \quad 33 \mathrm{~km}$

214435.18 40.085N $144.609 \mathrm{E} \quad 33 \mathrm{~km}$
$5.3 \mathrm{mb}(63$ abs.) $4.6 \mathrm{Msz}$ ( 9 abs.)

OFF EAST COAST OF HONSHU, JAPAN

CENTROID, MOMENT TENSOR (HRV)

Data Used: GDSN

L.P.B.: 10S, $17 \mathrm{C}$

Centraid Location

Origin Time 21:44:33.50

Lot $3982 \mathrm{~N}$ O 14 Lon $14460 \mathrm{E} \quad 0.12$

Dep 33 G F x Holf-durolion 15

Principol Axes:

Scale 10**16 Nm

$\mathrm{T} V \mathrm{VI}=4.63 \quad \mathrm{Plg}=2 \quad A=\mathrm{m}=87$

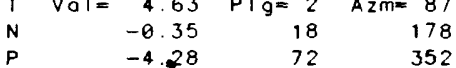

Best Dauble Cauple:Ma=4.5*10**16

NP 1 : Strike $=160$ Dip=46 St ip $=-115$

NP2: $\quad 14 \quad 49 \quad-66$

$1422 \quad 37 \quad 11.36 \quad 14.0135 \quad 65.945 \mathrm{E} \quad 10 \mathrm{~km}$ $5.1 \mathrm{mb}$ ( 32 abs.) 4.9Msz ( 5 abs.) MID-INDIAN RISE

CENTROID, MOMENT TENSOR (MRV)

Doto Used: GDSN

L.P.B.: $9 \mathrm{~S}, 17 \mathrm{C}$

Centraid Lacotion

Origin Time 22:37 19507

Lat 13.74S 9.98 Lon $6578 \mathrm{E} \quad 08$

Dep 150 FIX Half-duration 15

Principol Axes.

Scole $10 *+16 \mathrm{Nm}$

$T \quad \mathrm{Val}=579 \quad P \mid g=72 \quad A 2 m=317$
$\begin{array}{lllll}P & -681 & 12 & 89\end{array}$ Best Double Couple Mo=6 $3 * 10 * 16$ NP1 Strike $=162 \quad 0: p=35 \mathrm{~S} / \mathrm{p}=66$ NP2 $\quad 10 \quad 59 \quad 195$

$15155158.60 \quad 6800 \mathrm{~N} \quad 73.019 \mathrm{~W} 162 \mathrm{~km}$ $5.1 \mathrm{mb}(46$ Obs.)

NORTHERN COLOMBIA

CENTROID. MOMENT TENSOR (HRV)

Data Used: GDSN

L.P.B.: 15S, $25 \mathrm{C}$

Centroid Locotion.

Origin Time

$15: 52: 2.10 .8$

Lot $6.87 \mathrm{~N} 0.08$ Lon $73.09 \mathrm{~W} 0.08$

Dep $156.81 .9 \mathrm{Half}$-duration 1.6

Principol Axes:

Scole $10 *+16 \mathrm{Nm}$

T $\quad$ a l $=9.06 \quad P$ lg $=43 \quad$ Azm $=94$

$\begin{array}{lrrr}N & 0.58 & 8 & 191\end{array}$

Best Double Couple:Mo=9.4*10*16

NP 1: Strike $=109$ Dip= 8 Si ip $=-173$

$\begin{array}{llll}\text { NP2: } & 12 & 89 & -82\end{array}$

$\begin{array}{lllllll}15 & 19 & 32 & 45.63 & 6.824 \mathrm{~N} & 82.309 \mathrm{~W} & 10 \mathrm{~km}\end{array}$

4. $9 \mathrm{mb}$ ( 24 obs.) $4.9 \mathrm{Msz}$ ( 2 abs.)

SOUTH OF PANAMA

CENTROID, MOMENT TENSOR

(HRV)

Data Used: GDSN

L.P.B.: 15S, $29 \mathrm{C}$

Centraid Lacatian :

Origin Time $19: 32: 53.70 .4$

Lat $6.78 \mathrm{~N} 0.04$ Lan $82.29 \mathrm{~W} 0.05$

Dep 15.0 FIX Half-duration 2.3

Principal Axes:

Scale $10 * * 17 \mathrm{Nm}$

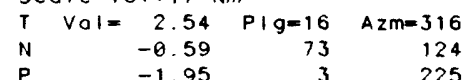

Best Dauble Cauple: $M_{0}=2.3 * 10 * * 17$

NP1.Strike $=0$ Dip=76 Slip=171

NP2 : $\quad 92 \quad 81 \quad 14$

$\begin{array}{lllllll}607 & 18 & 35.45 & 13.224 \mathrm{~N} & 145.154 \mathrm{E} & 68 \mathrm{~km}\end{array}$

$5.5 \mathrm{mb}$ ( 37 abs.)

MARIANA ISLANDS

CENTROID, MOMENT TENSOR

(HRV)

Dota Used: GDSN

L.P.B.: $14 \mathrm{~S}, 20 \mathrm{C}$

Centraid Lacation.

Origin Time $\quad 07: 18: 36.30 .7$

Lat $12.89 \mathrm{~N} 0.08$ Lan 145.34E 0.07

Dep 55.99 .6 Holf-duration 1.7

Principol Axes:

Scale $10 * 16 \mathrm{Nm}$

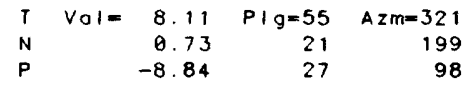

Best Dauble Cauple: $M a=8.5 \cdot 10 * 16$

NP 1: Strike=147 Dip=26 Si $p=36$

$$
\text { NP2: } \quad 24 \quad 75 \quad 112
$$

$\begin{array}{lllllll}6 & 10 & 51 & 21.56 & 57.755 \mathrm{~N} & 153.992 \mathrm{~W} & 58 \mathrm{~km}\end{array}$

$5.8 \mathrm{mb}$ ( 81 abs.)

KODIAK ISLAND REGION

FAULT PLANE SOLUTION: P-WoVES

NP 1: Strike $=240$ Di $p=85$ SI i $p=-65$

NP2 :

341

25

$-168$

Principol Axes

I $\quad P: g=35 \quad$ Azm $=309$

Camment: The facal mechonism is

moderately well cantralled and carrespands ta narmal faulting with a small left-loteral

strike-slip campanent. The

preferred fault plane is NP1.

RADIATED ENERGY

Na. af sta: 4 Facal mech. F

Energy $\quad 0.3 \pm 0.2 * 10 * 14 \mathrm{Nm}$

MOMENT TENSOR SOLUTION

Dep 61

Scole 10.017 Nm

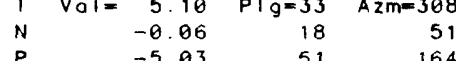

$\begin{array}{lll}-5.03 & 51 & 164\end{array}$

NP 1: Strike $=350$ D i $p=20$ SI i $p=-152$

NP2 : $\quad 234 \quad 81 \quad-72$

CENTROID, MOMENT TENSOR

Dato Used GDSN

L P. B 18S, 35C 
Centroid Location

Origin Time

$105124 \quad 3 \quad 0 \quad 3$ Lat $5770 \mathrm{~N} 6.04$ Lon $15464 \mathrm{~W} 005$ Dep 72.84 .1 Holf-duration 26 Principol Axes:

Scole $10 * 17 \mathrm{Nm}$

$T$ Val $=3.72 \quad$ Plg $=37 \quad$ A $z m=290$

$\begin{array}{rrrr}N & 0.05 & 28 & 44 \\ P & -3.78 & 40 & 161\end{array}$

Best Double Couple:Mo=3 $8 * 10 * * 17$ NP 1: Strike $=319$ Dip=28 SIip=-176 $\begin{array}{lrrr}\text { NP2: } & 225 & 88 & -62\end{array}$

$\begin{array}{llllllll}16 & 20 & 12 & 31.26 & 20.672 \mathrm{~N} & 102 & 446 \mathrm{E} & 33 \mathrm{~km}\end{array}$ 5. $1 \mathrm{mb}$ ( 30 abs.) 5.5Msz ( 6 obs.) SOUTHEAST ASIA

CENTROID, MOMENT TENSOR (HRV)

Data Used: GDSN

L.P.B.: 13S, $25 \mathrm{C}$

Centroid Location

Origin Time $\quad 20: 12: 32.80 .3$

Lat $20.61 \mathrm{~N} 0.04$ Lan $102.61 \mathrm{E} 0.06$

Dep 15.0 FIX Holf-duration 2.5 Principol Axes:

Scale $10 * * 17 \mathrm{Nm}$

$T \quad V a l=2.99 \quad P \mid g=12 \quad$ Azm $=249$

$\begin{array}{rrrr}N & -0.28 & 63 & 135 \\ P & -2.71 & 24 & 345\end{array}$

Best Double Couple:MO=2.9*19*17 NP 1: Strike $=25$ Dip $=65$ SIip $=-9$ $\begin{array}{lrrr}\text { NP2: } & 119 & 82 & -154\end{array}$

$\begin{array}{llllllll}16 & 23 & 42 & 35.12 & 31.807 \mathrm{~N} & 137.982 \mathrm{E} \quad 360 \mathrm{~km}\end{array}$ $5.9 \mathrm{mb}(90$ obs.)

SOUTH OF HONSHU, JAPAN

FAULT PLANE SOLUTION: P-Waves

NP1:Strike $=15$ Dip=85 SIip= -90

NP2: $195 \quad 5 \quad-90$

Principal Axes

$\begin{array}{lrr}T & P I g=40 & A z m=105 \\ P & 50 & 285\end{array}$

Comment: The focal mechanism is moderately well controlled and corresponds to normal

foulting. The preferred fault plane is NPI.

RADIATED ENERGY

No. of sta: 9 Focal mech. M Energy $\quad 0.2 \pm 0.0 * 10 * 14 \mathrm{Nm}$ MOMENT TENSOR SOLUTION

DeP 354 No. af sta: 12

Principal Axes:

Scale $10 * * 18 \mathrm{Nm}$

$T \quad V a l=4.45 \quad P / g=39 \quad A z m=80$

$\begin{array}{rrrr}N & -0.07 & 21 & 188 \\ P & -4.38 & 44 & 299\end{array}$

Best Dauble Couple: $M 0=4.4 * 10 * * 18$ NP 1: Strike=106 Dip=21 Si ip $=-173$ $\begin{array}{llll}\text { NP2 : } & 9 & 87 & -69\end{array}$

CENTROID, MOMENT TENSOR (HRV)

Data Used. GDSN

L.P.B.: 16S, $44 \mathrm{C}$ M.W.: $13 \mathrm{~S}, 25 \mathrm{C}$

Centroid Lacatian

Origin Time 23:42:40.2 0.1

Lat $31.76 \mathrm{~N} 0.01$ Lan $138.11 \mathrm{E} \quad 0.02$

Dep 366.1 0.9 Half-duration 6.0 Principol Axes

Scale $10 * 18 \mathrm{Nm}$

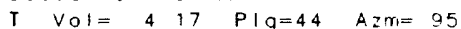

$N \quad 0$ o? $\quad 12 \quad 197$

$\begin{array}{llll}P & -4.19 & 43 & 299\end{array}$

Best Double Couple:Mo=4 $2 * 10 * 18$ NP1. Strike=105 Dip=12 S।ip=178 $\begin{array}{llll}\text { NP2 } & 197 & 90 & 78\end{array}$

$\begin{array}{llllllll}17 & 18 & 28 & 08.62 & 40.494 \mathrm{~S} & 74.674 \mathrm{~W} & 33 \mathrm{~km}\end{array}$ $56 \mathrm{mb}(20$ obs) $5.0 \mathrm{Msz}$ ( 7 obs.) OFF COAST OF SOUTHERN CHILE

CENTROID, MOMENT TENSOR

(HRV)

DIO USEd: GDSN

L.P. B.: 13S, $25 \mathrm{C}$

Centroid Location

Origin Time

$18: 28: 14.4 \quad 0.5$

Lat 41.10S 0.07 Lon $75.21 \mathrm{~W} 0.13$

Dep $15.0 \mathrm{Flx}$ Half-duration 2.0

Principol Axes:

Scale $10 * * 17 \mathrm{Nm}$

T $\quad$ Val $=2.38 \quad P I g=24 \quad$ Azm=115

$\begin{array}{rrrr}N & 0.76 & 29 & 10 \\ P & -314 & 5 i & 237\end{array}$

Best Double Couple. Mo=2.8*10**17

NP $1 \quad s t r 1 t e=247 \quad D_{1} p=34 \quad s \mid 1 P=-28$

NP:

$-121$
$11720471462 \quad 14375 \quad 24169 \mathrm{~W} \quad 101 \mathrm{~m}$ 5 imb । 52 ObS, $4.9 M S z$ ( 6 obs । CENTRAL MID-ATLANTIC RIDGE

CENTROID. MOMENT TENSOR (HRV) Data Used. GDSN

P.B. 17S, 38C

Centroud Lacation:

$20: 47: 24.3 \quad 0.6$

Lat $0.87 \mathrm{~S} 0.05$ Lon $23.93 \mathrm{~W} 0.05$

Dep $15.0 \mathrm{FIX} \mathrm{Half-duration} 2.2$

Principal Axes:

Scale $10 * 17 \mathrm{Nm}$

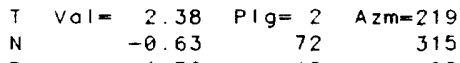

Best Double Couple:Mo=2.1*10*128

NP1:Strike=265 Dip=76 SIi $=-169$

NP2: $\quad 172 \quad 79 \quad-14$

$\begin{array}{lllllll}18 & 14 & 06 & 28.83 & 17.761 \mathrm{~N} & 68.811 \mathrm{~W} & 62 \mathrm{~km}\end{array}$ $5.9 \mathrm{mb}$ ( 69 obs.)

MONA PASSAGE

FAULT PLANE SOLUTION: P-WOVES

NP 1 : Strike $=35$ Dip $=65$ SI i $=-20$

NP2: 134

Principal Axes:

$$
\begin{array}{lrr}
T & P I g=5 & A z m=263 \\
P & 31 & 356
\end{array}
$$

Comment: The focal mechanism is

poorly controlled and

corresponds to strike-slip

faulting with a moderate

normol component. The

preferred foult plone is not

determined.

RADIATED ENERGY

Na. af sta. 6 Facal mech. $C$

Energy $0.7 \pm 0.2 * 10 * * 13 \mathrm{Nm}$

MOMENT TENSOR SOLUTION

Dep 70 No. af sta: 6

Principal Axes:

Scale $10 * * 17 \mathrm{Nm}$

$T \quad V a l=3.39 \quad \mathrm{Plg}=6 \quad$ Azm $=82$

N $\quad 0.06$

$\begin{array}{llll}P & -3.45 & 31 & 348\end{array}$

Best Double Couple: $M O=3.4 * 10 * * 17$

NP 1: Strike $=129$ Di $p=64$ SI i $p=-161$

NP2: $\quad 31 \quad 73 \quad-27$

CENTROID, MOMENT TENSOR (HRV)

Data Used: GDSN

L.P.B.: 16S, $34 \mathrm{C}$

Centraid Location:

Origin Time $\quad 14.96: 30.80 .5$

Lat $17.28 \mathrm{~N} 0.06$ Lon $68.53 \mathrm{~W} 0.05$

Dep 73.24 .0 Half-duration 2.1

Principal Axes:

Scole $10 * * 17 \mathrm{Nm}$

T Val $=1.56 \quad \mathrm{PIg}=4 \quad$ Azm $=263$

$\begin{array}{rrrr}N & 0.39 & 57 & 167\end{array}$

Best Double Couple: $M O=1.8 * 10 * * 17$

NP1: Strike $=34$ Dip=64 SI i $p=-22$

NP2: $\quad 134 \quad 70 \quad-153$

$\begin{array}{lllllllll}18 & 15 & 49 & 14.11 & 12.449 \mathrm{~S} & 121.520 \mathrm{E} \quad 33 \mathrm{~km}\end{array}$ $51 \mathrm{mb}$ ( 18 obs.) 4.2Msz ( 2 obs.) SOUTH OF TIMOR

CENTROID, MOMENT TENSOR (HRV)

DOto USEd GDSN

$L P B$ iss. $20 \mathrm{C}$

Centrold Locotion

Origin Time $15: 49: 17.114$

Lot $12.49 \mathrm{~S} F I X$, Lon $121.48 \mathrm{E}$ FIX

Dep 15.0 FIX Half-duration 1.5 Principal Axes:

Scale 10**16 Nm

$T \quad$ V l $=7.80 \quad$ Plg $=29 \quad$ Azm= 24

$\begin{array}{rrrr}\mathrm{N} & 0.76 & 6 & 117 \\ \mathrm{P} & -8.56 & 60 & 218\end{array}$

Best Double Couple:Mo=8.2*10*16

NP1: Strike $=97$ Di $p=17$ Si i $p=-111$

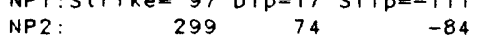

$19 \quad 16 \quad 00 \quad 4794 \quad 22 \quad 113 \mathrm{~S} \quad 67.559 \mathrm{~W} \quad 189 \mathrm{~km}$

$55 \mathrm{mb}(65$ obs.)

CHILE-BOLIVIA BOROER REGION

CENTROID, MOMENT TENSOR

(HRV)

COto Used. GDSN

L $\mathrm{B}, 17 \mathrm{~S}, 34 \mathrm{C}$

Centroid Location

Origin Time

16. $00: 5250.2$

Lot 22 Q5S $\theta 63$ Lon 67 6aW 0 04

Dep $186 \quad 0 \quad 1$, Holf-duration 26
Principol Axes

Scole $10 * 17 \mathrm{Nm}$

T $\quad V a I=3.71 \quad P: g=40 \quad A z m=91$

$\begin{array}{rrrr}\mathrm{N} & 0.05 & 6 & 357 \\ \mathrm{P} & -3.76 & 49 & 260\end{array}$

Best Double Couple:Mo=3,7*10*17 NP1: Strike $=228$ Dip $=7 \mathrm{~S}$ । i $p=-38$

$\begin{array}{llll}\text { NP2 : } & 356 & 86 & -96\end{array}$

$\begin{array}{lllllllll}19 & 20 & 16 & 10.53 & 55.672 \mathrm{~s} & 28 & 247 \mathrm{~W} & 33 \mathrm{~km}\end{array}$ $5.6 \mathrm{mb}$ ( 6 obs.) 5.1MSZ ( 2 obs.) SOUTH SANDWICH ISLANDS REGION CENTROID, MOMENT TENSOR

(HRV)

Data Used GDSN

L.P.B.: 15S, $29 \mathrm{C}$

Centraid Lacation

Origin Time

$20: 16: 16.90 .4$

Lat 55.6450 .06 Lon 28.61W 0.11 Dep 49.96 .5 Half-duration 2.1 Principal Axes

Scale $10 * 17 \mathrm{Nm}$

T $\quad V_{0} l=1.62 \quad \mathrm{Plg}=40 \quad$ Azm $=112$

$\begin{array}{rrrr}N & -0.22 & 18 & 218 \\ P & -1.40 & 44 & 327\end{array}$

Best Dauble Couple:Mo=1.5*19*17

NP1 : Strike=136 Dip=19 S1 i $p=-173$

$\begin{array}{lrrr}\text { NP2: } & 39 & 88 & -72\end{array}$

$\begin{array}{lllllllllll}20 & 05 & 41 & 43.52 & 52.334 \mathrm{~N} & 174.184 \mathrm{E} \quad 48 \mathrm{~km}\end{array}$ $52 \mathrm{mb}$ ( 63 obs.) 4.3Msz ( 5 obs.)

NEAR ISLANDS, ALEUTIAN ISLANDS

CENTROID, MOMENT TENSOR (HRV)

Data Used: GDSN

L.P. B.: 12S, 210

Centroid Location:

Origin Time

$05: 41: 45.7 \quad 0.6$

Li $52.47 \mathrm{~N} 0.12$ Lon $173.72 \mathrm{E} 0.10$

Dep 49.08 .4 Half-duration 1.8

Principal Axes:

Scale $10 * * 16 \mathrm{Nm}$

$T \quad V a I=6.72 \quad P I g=55 \quad$ Azm=162

$\begin{array}{rrrr}N & 0.20 & 6 & 64 \\ P & -6.91 & 35 & 330\end{array}$

Best Double Couple:Mo=6. $8 * 10 * * 16$

NP1:Strike $=35$ Dip=11 SIip=60

$\begin{array}{llll}\text { NP2 : } & 245 & 80 & 96\end{array}$

$\begin{array}{llllllll}20 & 18 & 07 & 43.60 & 52.527 \mathrm{~N} & 172.508 \mathrm{E} & 33 \mathrm{~km}\end{array}$ 5. $2 \mathrm{mb}$ ( 53 abs.) 4.6Msz ( 12 obs.)

NEAR ISLANDS, ALEUTIAN ISLANDS CENTROID, MOMENT TENSOR (HRV) Data Used: GDSN

L B : 15S, $27 \mathrm{C}$

Centroid Locatian

Origin Time

$18: 07: 46.50 .7$

Lat $52.95 \mathrm{~N} 0.06$ Lon $172.38 \mathrm{E} \quad 0.15$

Dep 48.5 5.6 Half-duration 1.7 Principal Axes:

Scale $10 * * 16 \mathrm{Nm}$

$T \quad V a I=8.29 \quad P I g=53 \quad$ AZm $=297$

$\begin{array}{rrrr}N & 0.42 & 28 & 72 \\ P & -8.71 & 22 & 175\end{array}$

Best Double Cauple: $M a=8.5 * 10 * * 16$

NP1: Strike=305 Dip=34 SIip=147

NP2: $63 \quad 73$

$\begin{array}{lllllll}20 & 23 \quad 40 & 38.88 & 3.867 \mathrm{~S} & 86.993 \mathrm{E} \quad 10 \mathrm{~km}\end{array}$ $5.2 \mathrm{mb}$ ( 49 obs.) 4.6Msz ( 3 obs.) SOUTH INDIAN OCEAN

CENTROIO, MOMENT TENSOR (HRV) Data Used: GOSN

P.B.: 13S, 22C

Centroid Locotion:

Origin Time $23: 40: 44.7 \quad 0.8$ Lot 3.3950 .08 Lan $86.89 \mathrm{E} 0.08$ Dep $15.0 \mathrm{FlX} \mathrm{Half-duration} 1.9$ Principal Axes:

Scale $10 * 16 \mathrm{Nm}$

$T \quad V a l=12.37 \quad P \mid g=58 \quad$ A $m=288$

N $\quad-2.13 \quad 23 \quad 59$

$\begin{array}{cccr}P & -10.24 & 22 & 159 \\ \text { Best Double Couple: } M a=1.1 * 10 * 17\end{array}$

NP 1 : Strike=284 Dip=31 Si ip $=139$

NP2: $\quad 51 \quad 70 \quad 66$

$21235101.98 \quad 21.785 S \quad 176.493 w \quad 182 \mathrm{~km}$ $5.6 \mathrm{mb}$ ( 42 obs.)

FIJ) ISLANDS REGION

CENTROIO, MOMENT TENSOR

Data Used. GDSN

P.B : 16S, 38C

Centroid Lacotion

Origin Time

$23: 51,9.20 .3$ 
Lot 21735 मे 04 Lon $176,74 \mathrm{~W} 9.03$ Dep 195.513 Half-duration 26 Principol Axes

Scole $10 * 17 \mathrm{Nm}$

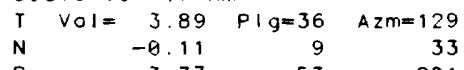

$P \quad-3.77 \quad 53 \quad 291$

Best Double Couple: $M O=3.8 * 10 * 17$ $\begin{array}{rrrr}\text { NP 1 : Strike=258 } & \text { Dip=12 Slip= } & -44 \\ \text { NP2 : } & 32 & 82 & -99\end{array}$

$\begin{array}{llllllll}22 & 1106 & 06.80 & 28.252 \mathrm{~N} & 130.644 \mathrm{E} & 33 \mathrm{~km}\end{array}$ 5. $3 \mathrm{mb}$ ( 31 obs.)

RYUKYU ISLANDS

CENTROID, MOMENT TENSOR (HRV)

Data Used: GDSN

L.P. 8: $12 \mathrm{~S}, 17 \mathrm{C}$

Centraid Locatian:

Drigin Time $\quad 11: 06: 9.60 .6$ Lat $28.02 \mathrm{~N} 0.11$ Lan 130.35E 0.13 Dep $15.0 \mathrm{FIX}$ Halt-duration 1.5 Principal Axes:

Scale $10 * * 16 \mathrm{Nm}$

T $\quad$ Oal $=5.07 \quad P 1 g=7 \quad$ A $2 m=121$

$\begin{array}{rrrr}N & 0.59 & 2 & 212 \\ P & -5.66 & 83 & 316\end{array}$

Best Dauble Cauple:Ma=5. $4 * 10 * * 16$ NP 1: Strike=209 Dip=38 Slip= -93 $\begin{array}{llll}\text { NP2: } & 33 & 52 & -88\end{array}$

$2300 \quad 35 \quad 42.62 \quad 17.098 \mathrm{~S} \quad 172.656 \mathrm{~W} \quad 33 \mathrm{~km}$ 4. $9 \mathrm{mb}$ ( 12 obs.) $5.0 \mathrm{Msz}$ ( 1 obs.) TONGA ISLANOS REGION

CENTROID. MOMENT TENSOR (HRV)

Dala Used: GDSN

L.P. B : 13S, $18 \mathrm{C}$

Centraid Location:

Origin Time $\quad 00: 35: 41.51 .4$

Lot $17.94 \mathrm{~S} 0.17$ Lon $172.84 \mathrm{~W} 0.16$

Dep $15.0 \mathrm{FIX}$ Half-duration 1.5

Principol Axes:

Scale $10 * * 17 \mathrm{Nm}$

I $\quad \mathrm{Val}=1.30 \quad \mathrm{Plg}=49 \quad$ Azm $=277$

$\begin{array}{rrrr}N & 0.68 & 5 & 13 \\ P & -1.38 & 40 & 107\end{array}$

Best Double Cauple: $M O=1,3 * 10 * * 17$

NP1:Strike=237 Dip=7 SIip=134

NP2: $\quad 12 \quad 85 \quad 85$

$\begin{array}{lllllll}23 & 1200 & 53.27 & 22.311 \mathrm{~N} \quad 142.996 \mathrm{E} \quad 170 \mathrm{~km}\end{array}$

$5.3 \mathrm{mb}$ ( 55 Obs.)

VOLCANO ISLANDS REGION

CENTROID, MOMENT TENSOR

(HRV)

Doto Used: GDSN

L B : 13S $18 \mathrm{C}$

Centroid Location:

Origin Time 12:00:55.3 1.0

Lat $22.33 \mathrm{~N} 0.11$ Lon 143.09E 0.11

Dep 189.75.7 Half-duratian 1.7

Principal Axes:

Scale $10 * * 16 \mathrm{Nm}$

T $\quad$ O I $=7.12 \quad \mathrm{PIg}=53 \quad$ Azm $=172$

$\begin{array}{rrrr}N & 0.54 & 20 & 52 \\ P & -7.66 & 29 & 310\end{array}$

Best Double Cauple: $M a=7.4 * 10 * 16$

NP 1 : Strike $=356$ Dip=24 SIip= 32

NP2: $\quad 237 \quad 78 \quad 111$

$\begin{array}{llllllll}24 & 03 & 09 & 57.56 & 36.719 \mathrm{~N} & 35.943 \mathrm{E} & 42 \mathrm{~km}\end{array}$ $49 \mathrm{mb}$ ( 57 obs.) $42 \mathrm{MSZ}$ ( 2 obs) TURKEY

CENTROID, MOMENT TENSOR (HRV)

Doto Used: GDSN

L.P. $12 \mathrm{~S}, 23 \mathrm{C}$

Centraid Location:

Origin time 03:09:58.6 1.1

Lat $36.28 \mathrm{~N} 0.14$ Lon $36.13 E \quad 0.17$

Dep $15.0 \mathrm{FIX}$ Half-duration 1.5

Principal Axes:

Scale $10 * * 16 \mathrm{Nm}$

T. $V a l=4.61 \quad P 1 g=17 \quad$ Azm $=115$

$\begin{array}{rrrr}N & 0.86 & 2 & 206\end{array}$

Best Dauble Cauple:Ma=5.0*10**16 NP i : Strike=203 Dip=28 Si i $p=-93$ NP2: $\quad 27 \quad 62 \quad-88$

$24 \quad 1258 \quad 39.08 \quad 28.336 \mathrm{~S} \quad 66.312 \mathrm{~W} \quad 22 \mathrm{~km}$ $5.4 \mathrm{mb}$ ( 35 Obs.) 5.2MsZ ( 3 obs.) CATAMARCA PROVINCE, ARGENTINA CENTROID. MOMENT TENSOR (HRV)

Dato UsEd GDSN

LP.B: $17 \mathrm{~S}, 35 \mathrm{C}$
Centroid Location

Origin Time $1258 \quad 47 \quad 1 \quad 4$ Lot 28535065 Lon 66 20W 65 Dep $21.0 \mathrm{~F} / \mathrm{X}$ Holf-duration 2.2 Principal Axes

Scale $10 * * 17 \mathrm{Nm}$

T $\quad$ Val $=\begin{array}{r}2.04 \\ -0.01\end{array} \quad$ Plg $=\begin{array}{r}3 \\ 75\end{array} \quad$ Azm= 43

$\begin{array}{llll}N & -0.01 & 75 & 145 \\ P & -2.04 & 15 & 312\end{array}$

Best Dauble Couple: $M a=2.0 * 10 * * 17$

NP 1: Strike= 88 Dip=77 SI i $p=-172$

NP2 :

$357 \quad 82$

$-13$

$251230 \quad 05.91 \quad 6.1135 \quad 149.062 \mathrm{E} \quad 72 \mathrm{~km}$

$5.2 m b$ ( 24 obs.)

NEW 8RITAIN REGION

CENTROID, MOMENT TENSOR (HRV)

Dota Used: GOSN

L.P. 8.: 15S, 31C

Centroid Lacation:

Origin Time 12:30:10.0 0.5

Lat $6.40 \mathrm{~S} 0.04$ Lan 148.92E 0.06

Dep 52.05 .2 Halt-duratian 1.5

Principal Axes:

Scale $10 * * 16 \mathrm{Nm}$

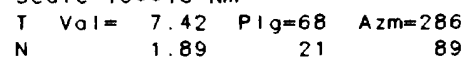

$\begin{array}{rrrr}N & 1.89 & 21 & 89 \\ P & -9.31 & 6 & 181\end{array}$

Best Dauble Cauple: $M O=8.4 * 10 * * 16$ NP 1: Strike=293 Dip=43 Si $i p=121$ NP2: $\quad 73 \quad 54 \quad 64$

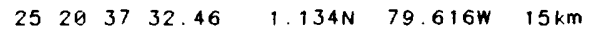
$5.9 \mathrm{mb}$ ( 77 obs.) $6.1 \mathrm{Msz}$ ( 33 obs.) NEAR COAST OF ECUADOR

FAULT PLANE SOLUTION: P-WoVES

NP1: Sirike=193 Dip=73 SIip=90

NP2: $\quad 13 \quad 17 \quad 90$

Principal Axes:

$\begin{array}{rrr}\text { I } & P I g=62 & A z m=103 \\ P & 28 & 283\end{array}$

Comment: The focal mechanism is

paorly controlled and

correspands ta reverse

faulting. The preferred foult plane is NP2.

RADIATED ENERGY

No. of sta: 5 focal mech. F Energy $0.3 \pm 0.1 * 10 * 14 \mathrm{Nm}$ MOMENT TENSOR SOLUTION

Dep 13 Na, of sta: 15 Principal Axes:

Scole $10 * * 18 \mathrm{Nm}$

T $\quad$ Vol $=3.63 \quad P \mid g=61 \quad$ Azm $=107$

$\begin{array}{llll}N & 0.60 & 7 & 209 \\ P & -3.62 & 28 & 303\end{array}$

Best Dauble Couple: Mo $=3.6 * 10 * 18$

NP 1: Strike= 50 Dip=18 SIip= 112

NP2: $\quad 207 \quad 73 \quad 83$

CENTROID, MOMENT TENSOR (HRV)

Doto Used: GDSN

L.P.B.: 17S, 450

Centraid Location:

$\begin{array}{ll}\text { Origin Time } & 20: 37: 43.20 .2 \\ \text { Lat } 0.81 \mathrm{~N} 0.03 \text { Lon } 79.92 \mathrm{~W} 0.03\end{array}$

Dep $16.01 .6 \mathrm{Half}$-duratian 5.2

Principal Axes:

Scale $10 * * 18 \mathrm{Nm}$

$T \quad \vee a l=3.38 \quad P \mid g=64 \quad$ Azm $=63$

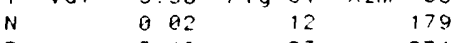

$\begin{array}{lll}-3.49 & 23 & 274\end{array}$

Best Double Couple: $M O=3.4 * 10 * * 18$
NP1:Strike $=27$ Di $p=25$ Si i $p=120$

NP2: $\quad 174 \quad 69 \quad 77$

$\begin{array}{llllllll}26 & 03 & 27 & 03.96 & 19.362 \mathrm{~N} & 155.083 \mathrm{~W} & 9 \mathrm{~km}\end{array}$ $5.8 \mathrm{mb}$ ( 35 obs.) 6.1Msz ( 18 abs.) HAWAII

FALIT PLANE SOLUTION: P-Wove

NP $1:$ Strike $=42$ Dip=86 SIip= 115 NP2 141$$
25
$$

Principol Axes:

$\begin{array}{lrr}T & P I g=44 & A z m=336 \\ P & 36 & 110\end{array}$

Comment: The focal mechanism is

maderately well controlled and

corresponds to reverse

faulting with o moderote

strike-slip component. The

preferred foult plone is not

delermined.

RADIATED ENERG

No of sta 7 Focal mech.
Eriergy

(6) $4 \pm 0 \quad 1 * 16 * * 14 \mathrm{Nm}$

MOMENT TENSOR SOLUTION

Dep 11

Scale $10 * * 18 \mathrm{Nm}$

T $\quad \forall a l=5.45 \quad$ Plg $=43 \quad$ Azm $=331$

$\begin{array}{lrrr}N & -0.01 & 6 & 236\end{array}$

Best Double Couple: Mo $=5.4 * 10 * * 18$

NP 1: Strike $=130$ Dip=6 Si i $=-15$ NP2

$$
\begin{array}{rrr}
130 \\
236
\end{array} \quad \text { Di }=\begin{array}{rr}
6 & \text { SI i } \\
88 & -15
\end{array}
$$

CENTROID, MOMENT TENSOR (HRV)

Dota Used: GDSN

L.P.B: $16 \mathrm{~S}, 38 \mathrm{C}$ M.W.: 14S, $25 \mathrm{C}$ Centraid Locatian:

Origin Time

93:27:16 $6 \quad 0.2$

Lat $19.47 \mathrm{~N} 0.02$ Lon 155.06W 0.02

DeP $15.0 \mathrm{FIX} \mathrm{Half-duration} 5.7$ Principal Axes:

Scale 10**18 Nm

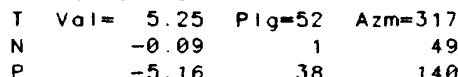

$\begin{array}{lll}-5.16 & 38 & 140\end{array}$

NP1:Strike=238 Dip=7 Siip= 99

$\begin{array}{lrrr}\text { NP2: } & 49 & 83 & 89\end{array}$

$\begin{array}{lllllll}26 & 10 \quad 38 & 39.46 & 39.112 \mathrm{~N} & 28.242 \mathrm{~W} & 11 \mathrm{~km}\end{array}$ $5.7 \mathrm{mb}$ ( 59 abs.) $5.7 \mathrm{Msz}$ ( 13 obs.) AZORES ISLANDS

FAULT PLANE SOLUTION: P-WOVES

NP1 : Strike $=187$ Dip=83 SI ip $=175$

NP2 278
Axes:

Principol Axes:

T $\quad P I g=8 \quad A z m=142$

Comment: The focal mechanism is maderately well cantrolled and carrespands ta strike-slip

foulting with o small normal

component. The preferred fault

plane is not determined.

RADIATED ENERGY

No. of sta: 7 Facal mech. M

Energy MOMENT TENSOR SOLUTION

DEP 33 Na of $510: 13$

Principol Axes:

Scale $10 * * 17 \mathrm{Nm}$

$T \quad V a l=6.65 \quad$ Plg=16 Azm= 51

$\begin{array}{llll}N & 0.18 & 73 & 248\end{array}$

$\begin{array}{llll}P & -6.83 & 5 & 143\end{array}$

Best Dauble Cauple:Mo=6.7*10**17

NP 1 : Strike=188 Dip=75 SIip= 8

NP2: $\quad 96 \quad 82 \quad 165$

CENTROID, MOMENT TENSOR (HRV)

Dota Used: GOSN

L.P.B.: 17S. $44 C$

Centroid Location:

Origin Time $10: 38: 45.70 .3$

Lat $39.11 \mathrm{~N} 0.03$ Lon $28.32 \mathrm{~W} 0.06$ Dep 15.0 Fix Half-duration 3.0 Principal Axes:

Scale $10 * * 17 \mathrm{Nm}$

$T$ Val $=5.57 \quad \mathrm{Plg}=14 \quad$ Azm $=29$

$\begin{array}{rrrr}\mathrm{N} & -0.29 & 11 & \text { A } \mathrm{m}= \\ \mathrm{P} & -5.29 & 122\end{array}$

Best Double Couple:Mo=5.4*10**17

NP1 Sirike=105 Dip=32 Slip=-110

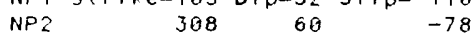

$\begin{array}{llllll}27 & 07 & 10 & 22.62 & 51.568 \mathrm{~N} & 174.339 \mathrm{~W} \quad 33 \mathrm{~km}\end{array}$ $5.2 \mathrm{mb}$ ( 53 abs.) 5.0Msz ( 18 abs.) ANDREANOF ISLANDS. ALEUTIAN IS

CENTROIO, MOMENT TENSOR (HRV)

Dato Used: GDSN

L.P.B.: 14S, $25 \mathrm{C}$

Centroid Location:

Origin Time 07.19 .2750 .7

Lat $51.93 \mathrm{~N} 0.07$ Lon $174.61 \mathrm{w} 0.09$

Dep 23.93 .5 Half-duration 1.9 Principol Axes:

Scale $10 * * 16 \mathrm{Nm}$

$T \quad$ Vol $=11.43 \quad$ Plg $=66 \quad$ Azm $=356$

$\begin{array}{rrrr}N & -0.04 & 8 & 248 \\ P & -1140 & 22 & 155\end{array}$

8est Double Coupte:Mo=1,1*10*17

NP1. Strike=231 Dip=24 Si, $=71$

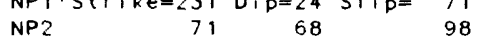

$27174309.0163 .72 \mathrm{~S} 156.10 \mathrm{~W} 10 \mathrm{~km}$ $54 \mathrm{mb}$ ( 4 ODS ) 5 5MSZ ( 3 ODS) SOUTH PACIFIC COROILLERA 
CENTROID, MOMENT TENSOR (HRV) DOto Used GDSN

L.P B 14S, 32C

Centraid Locotion

Origin Time $17: 43: 1430.4$

Lot 63.785 FIX; Lon 156.15W FIX

Dep $15.0 \mathrm{FIX} \mathrm{Holf-durotion} 2.9$

Principal Axes

Scole $10 * * 17 \mathrm{Nm}$

$T \quad \mathrm{Val}=6.72 \quad \mathrm{Plg}=51 \quad$ Azm=327

$\begin{array}{llll}N & -2.98 & 4 & 232\end{array}$

Best Double Couple:Mo=5.2*10**17

NP 1: Strike=196 Dip= 8 Slip= 54

$\begin{array}{llll}\text { NP2: } & 52 & 84 & 94\end{array}$

$\begin{array}{llllllll}28 & 03 & 00 & 28.92 & 45.096 \mathrm{~N} & 151.292 \mathrm{E} & 44 \mathrm{~km}\end{array}$ $5.6 \mathrm{mb}$ ( 61 obs) $5.1 \mathrm{Msz}$ ( 10 obs.) KURIL ISLANDS

CENTROID, MOMENT TENSOR (HRV)

Data Used: GDSN

L.P.B.: 14 S, $27 \mathrm{C}$

Centraid Lacation:

Origin Time $03: 00: 30.60 .8$

Lot $45.28 \mathrm{~N} 0.05$ Lon $151.61 \mathrm{E} 0.09$

Dep $15.0 \mathrm{FIX}$ Half-duration 1.9

Principal Axes:

Scale $10 * 17 \mathrm{Nm}$

$T \quad V a l=1.49 \quad P / g=64 \quad$ A2m $=248$

$\begin{array}{llll}N & -0.01 & 19 & 23\end{array}$

8est Double Couple: $M_{0}=1.5 * 10 * 17$

NP1: Strike $=236$ Dip=32 Si $i p=128$

NP2:

13

$\begin{array}{lllllll}28 & 21 & 24 & 12.45 & 57.788 S & 147.449 \mathrm{~W} & 10 \mathrm{~km}\end{array}$ $5.5 \mathrm{mb}$ ( 8 obs.) 5.7Msz ( 3 obs.) SOUTH PACIFIC CORDILLERA

CENTROID, MOMENT TENSOR (HRV)

Data Used: GDSN

L.P.B.: $14 \mathrm{~S}, 33 \mathrm{C}$

Centroid Location:

Origin Time $21: 24: 19.00 .3$

Lat $58.14 \mathrm{~S} 0.04$ Lan $147.18 \mathrm{~W} 0.07$

DeP $15.0 \mathrm{FIX} \mathrm{Half-duration} 3.0$

Principal Axes:

Scole $10 . .17 \mathrm{Nm}$

$T \quad V a I=5.79 \quad P / g=5 \quad$ Azm $=344$

$\begin{array}{rrrr}N & -0.11 & 82 & 109\end{array}$

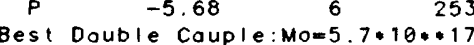

NP1: Strike $=28$ Dip $=82$ Si i $p=-179$

NP2 :

298

89

$28221158.90 \quad 6.2235 \quad 148.814 E \quad 50 \mathrm{~km}$ $5.4 \mathrm{mb}(22$ obs.)
NEW BRITAIN REGION

CENTROID, MOMENT TENSOR (HRV)

Doto Used: GDSN

L.P.B.: 8S, $18 \mathrm{C}$

Centroid Locotion:

Origin time 22:12:4.2 0.5

Lat 6.8150 .07 Lon $148.85 E 0.07$

Dep $15.0 \mathrm{FIX} \mathrm{Half-duration} 2.4$

Principal Axes:

Scole 10.017 Nm

T Val= $2.21 \quad$ Plg $=74 \quad$ Azm= 57

$\begin{array}{rrrr}N & 0.06 & 14 & 268\end{array}$

$\begin{array}{cccc}P & -2.27 & 8 & 176\end{array}$

MP 1: Strike=250 Dip=39 S1ip= 67

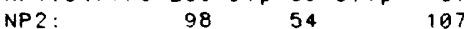

$\begin{array}{llllllll}28 & 23 & 44 & 51.39 & 7.664 \mathrm{~S} & 127.342 \mathrm{E} & 173 \mathrm{~km}\end{array}$ $5.3 \mathrm{mb}$ ( 29 Obs.)

BANDA SEA

CENTROID, MOMENT TENSOR (HRV)

Dota Used: GDSN

L.P.B.: 15S, $26 \mathrm{C}$

Centroid Location:

Origin Time $23: 44: 54.7 \quad 1.2$

Lot $7.53 \mathrm{~S} \theta 09$ Lon $127.15 \mathrm{E}$ 0. 09

Dep 177.92 .5 Half-duration 2.2

Principol Axes:

Scole $10 * 17 \mathrm{Nm}$

T $\quad$ OOI $=2.76 \quad P 1 g=57 \quad$ Azm $=318$

$\begin{array}{rrrr}N & -0.61 & 32 & 158 \\ P & -2.14 & 9 & 62\end{array}$

Best Double Couple: Mo $=2.4 * 10 * * 17$

NP1: Strike $=121$ Di $=45$ SIiP= 43

NP2: $\quad 358 \quad 61 \quad 127$

$\begin{array}{llllllll}29 & 00 & 18 & 39.81 & 22.960 \mathrm{~S} & 175.319 \mathrm{~W} & 35 \mathrm{~km}\end{array}$ 5. $2 \mathrm{mb}$ ( 11 obs.) 5.1Msz ( 7 abs.) TONGA ISLANDS REGION

CENTROID. MOMENT TENSOR (HRV) Dato Used: GDSN

L.P.B.: 11S, $23 \mathrm{C}$

Centroid Location:

Origin Time $\theta 0: 18: 42.31 .1$

Lat 23.0250 .06 Lon 174.79w 0.09

Dep $24.34 .2 \mathrm{Half}$-duration $2 . \theta$ Principal Axes: Scale $10 * 17 \mathrm{Nm}$

$$
T \quad V a l=0.98 \quad P / g=74 \quad \text { Azm=337 }
$$$$
\begin{array}{lrrr}
N & 0.42 & 13 & 194
\end{array}
$$

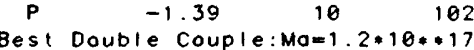

NP 1: Strike $=177$ Dip=37 Slip= 68

NP2: $\quad 23 \quad 56 \quad 106$

$2903 \quad 57 \quad 33.13 \quad 6.2895 \quad 154.817 \mathrm{E} \quad 82 \mathrm{~km}$
5. 3 mit ( 20 Obs

SOLOMON ISLANDS

CENTROID, MOMENT TENSOR (HRV)

Doto Used: GOSN

L.P.B.: 95, $17 \mathrm{C}$

Centraid Location.

Origin Time $03: 57: 40.20 .7$

Lat 6.1350 .12 Lon 154.24E 0.11

Dep 68.57 .7 Holf-duration 1.5 Principol Axes:

Scale $10 * * 16 \mathrm{Nm}$

T Vol $=4.46 \quad \mathrm{PIg}=77 \quad$ Azm $=147$

$\begin{array}{rrrr}N & 0.44 & 11 & 295 \\ P & -4.90 & 7 & 26\end{array}$

Best Double Couple:Mo=4.7*10**16

NP 1 : Strike $=128 \quad$ Dip $=40 \quad$ S I i $p=107$

$\begin{array}{lll}N P 2: & 287 & 52\end{array}$

$\begin{array}{llllllll}29 & 05 & 25 & 51.52 & 4.497 \mathrm{~S} & 102.018 \mathrm{E} & 42 \mathrm{~km}\end{array}$ $5.3 \mathrm{mb}$ ( 22 ObS.) $5.0 \mathrm{Msz}$ ( 10 Obs.) SOUTHERN SUMATERA

CENTROID, MOMENT TENSOR

(HRV)

Data UsEd: GDSN

L.P.B.: 14S, $29 \mathrm{C}$

Centroid Locotion:

Origin Time $\quad 05: 25: 53.80 .8$

Lot 4.93S 0.05 Lon 101.77E 0.07

Dep 44.53 .6 Half-duration 2.0 Principol Axes:

Scale $10 *+17 \mathrm{Nm}$

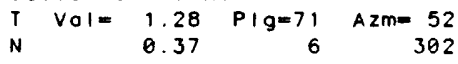

$\begin{array}{lrrr}N & -1.65 & 17 & 210\end{array}$

Best Double Couple:Ma=1.5*10*17 NP1: Strike $=290$ Dip=2B Slip= 77

$\begin{array}{rrr}\text { NP2: } & 126 & 63\end{array}$

$30013431.90 \quad 6.759 \mathrm{~S} \quad 130.517 \mathrm{E} \quad 107 \mathrm{~km}$ $5.1 \mathrm{mb}$ ( 22 abs.)

BANDA SEA

CENTROID, MOMENT TENSOR (HRV)

Data Used: GDSN

L.P.B.: 10S, 210

Centraid Lacation

Origin Time $\quad 01: 34: 36.90 .8$

Lat 6.7850 .08 Lan $130.31 \mathrm{E} 0.14$

Dep 103.74 .8 Half-duration 1.5

Principal Axes

Scale $10 * * 16 \mathrm{Nm}$

$T \quad V a l=5.31 \quad P \mid g=35 \quad$ AZm=311

$\begin{array}{rrrr}N & 2.22 & 55 & 128 \\ P & -7.54 & 1 & 228\end{array}$

Best Double Couple:Mo=6.4*18*16

NP 1: Strike=350 Dip=65 S1 ip= 155

$\begin{array}{lrrr}\text { NP2: } & 91 & 67 & 27\end{array}$

Compiled by Willis S. Jacobs, Leonard E. Kerry, John H. Minsch, Russell E. Needham, Waverly J. Person,

Bruce W. Presgrave and William H. Schmieder.

ANNOUNCEMENT

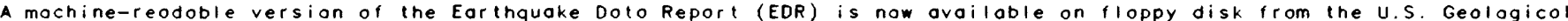
Survey's Books ond Open-File Reports Section, beginning with the EDR for Januory 1989. The disks are 1.2 megabyte,

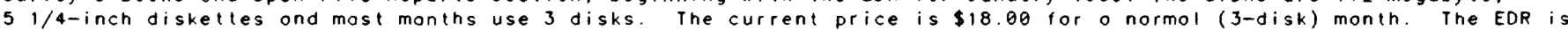

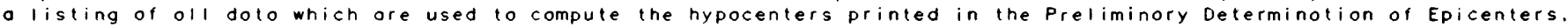
Monthly Listing

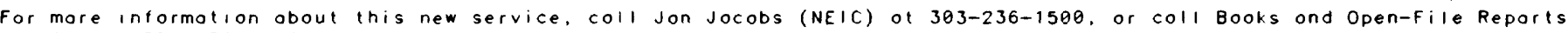
Section ot 303-236-7476 


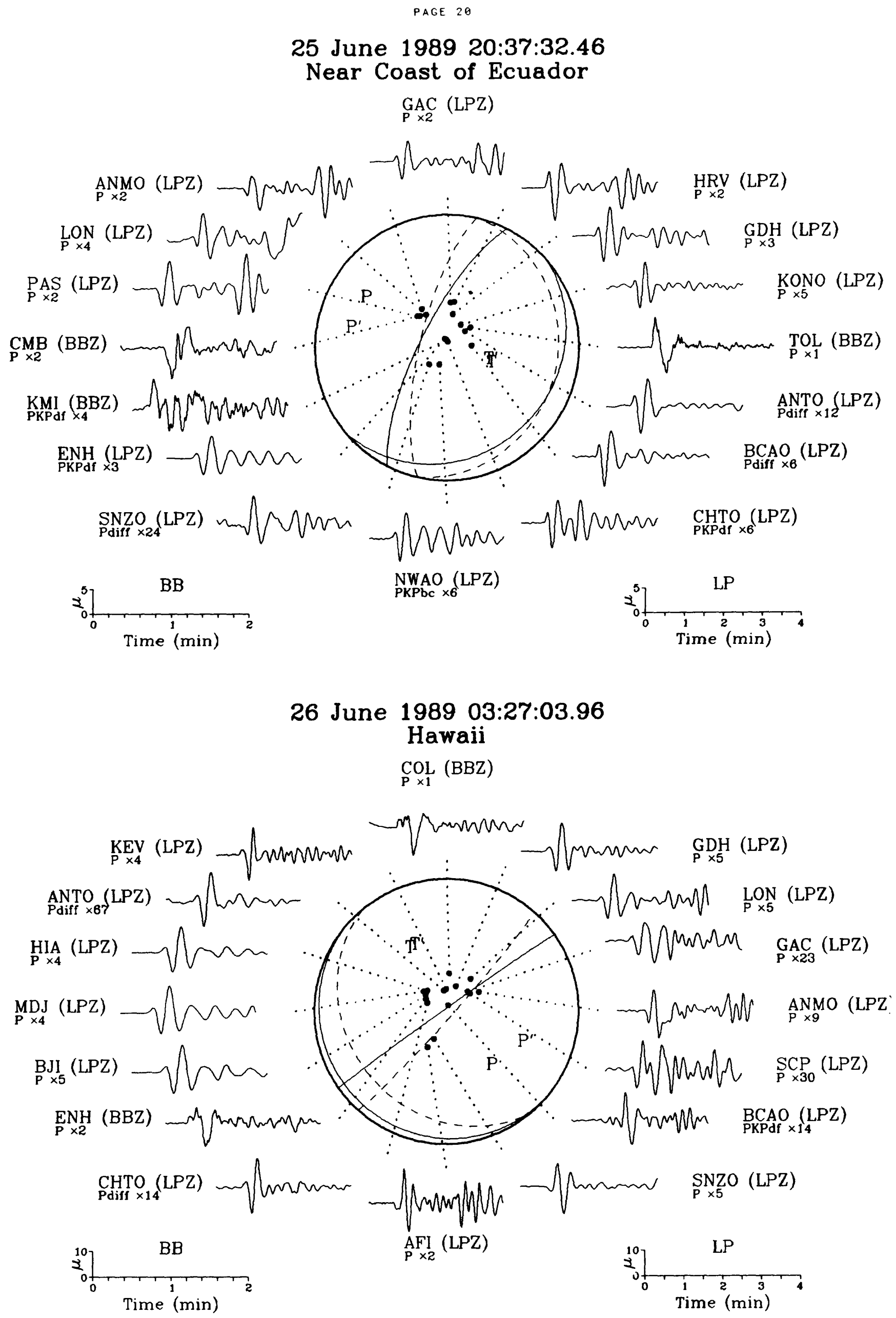


PAGE 21

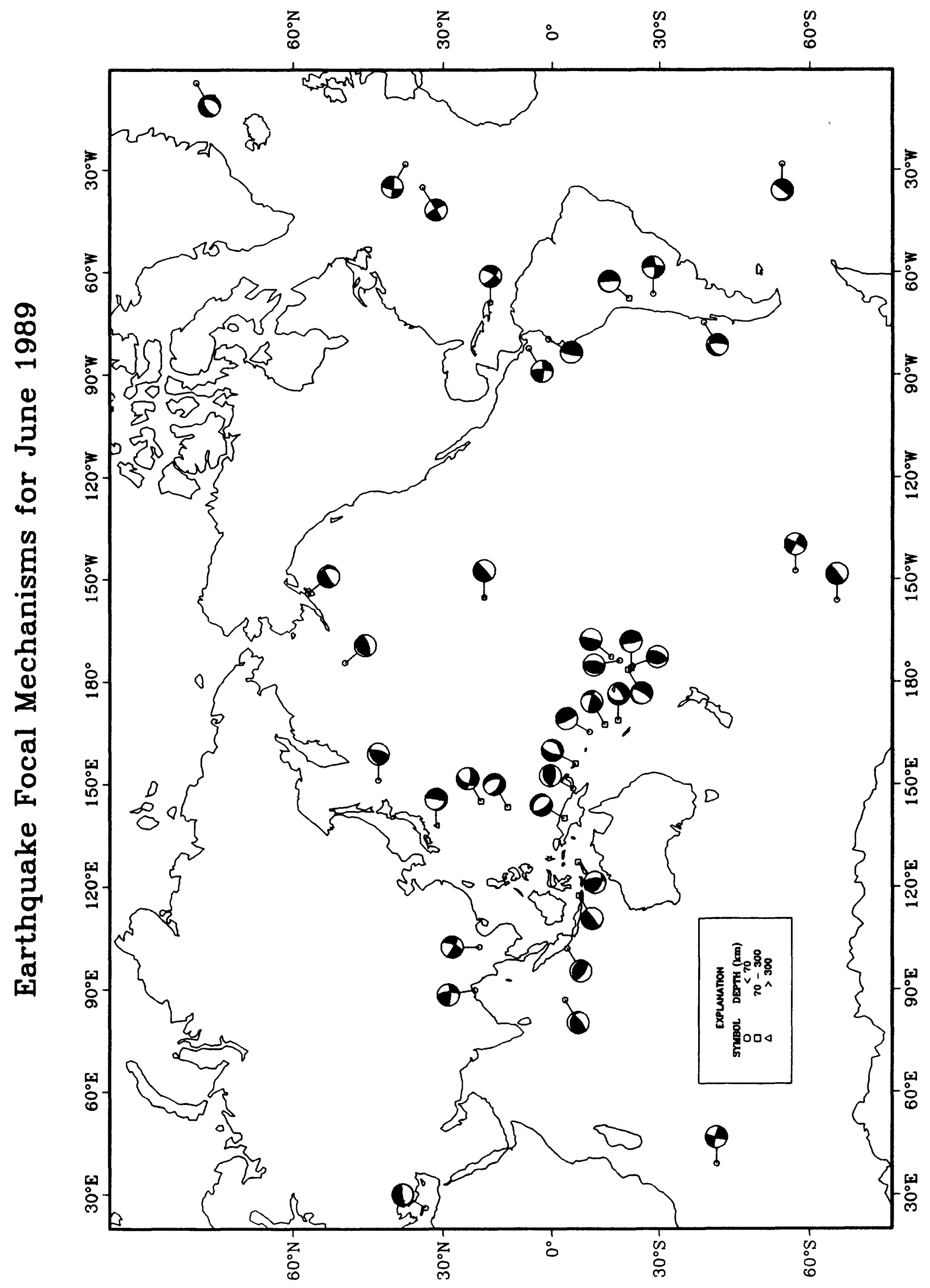




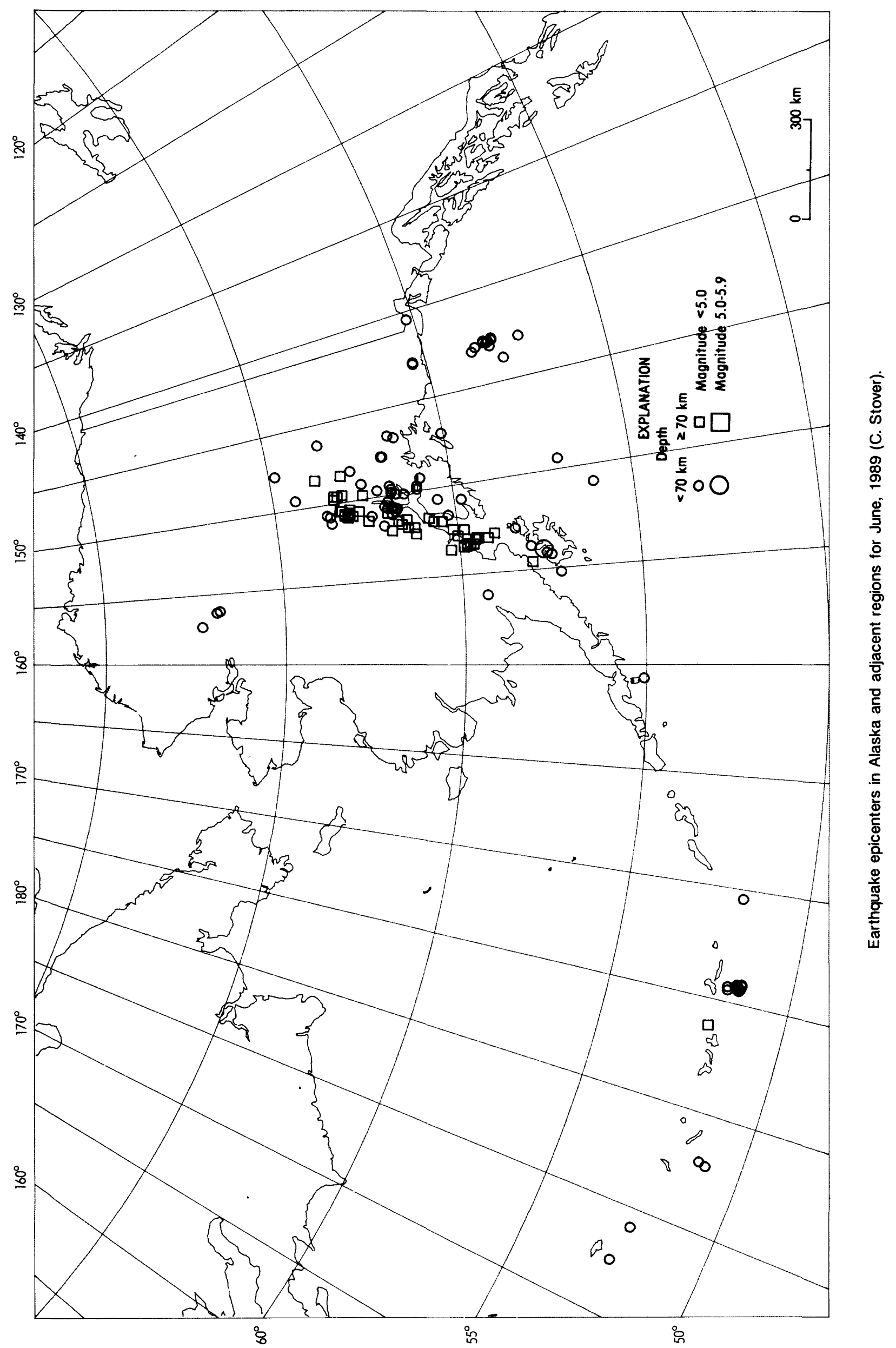




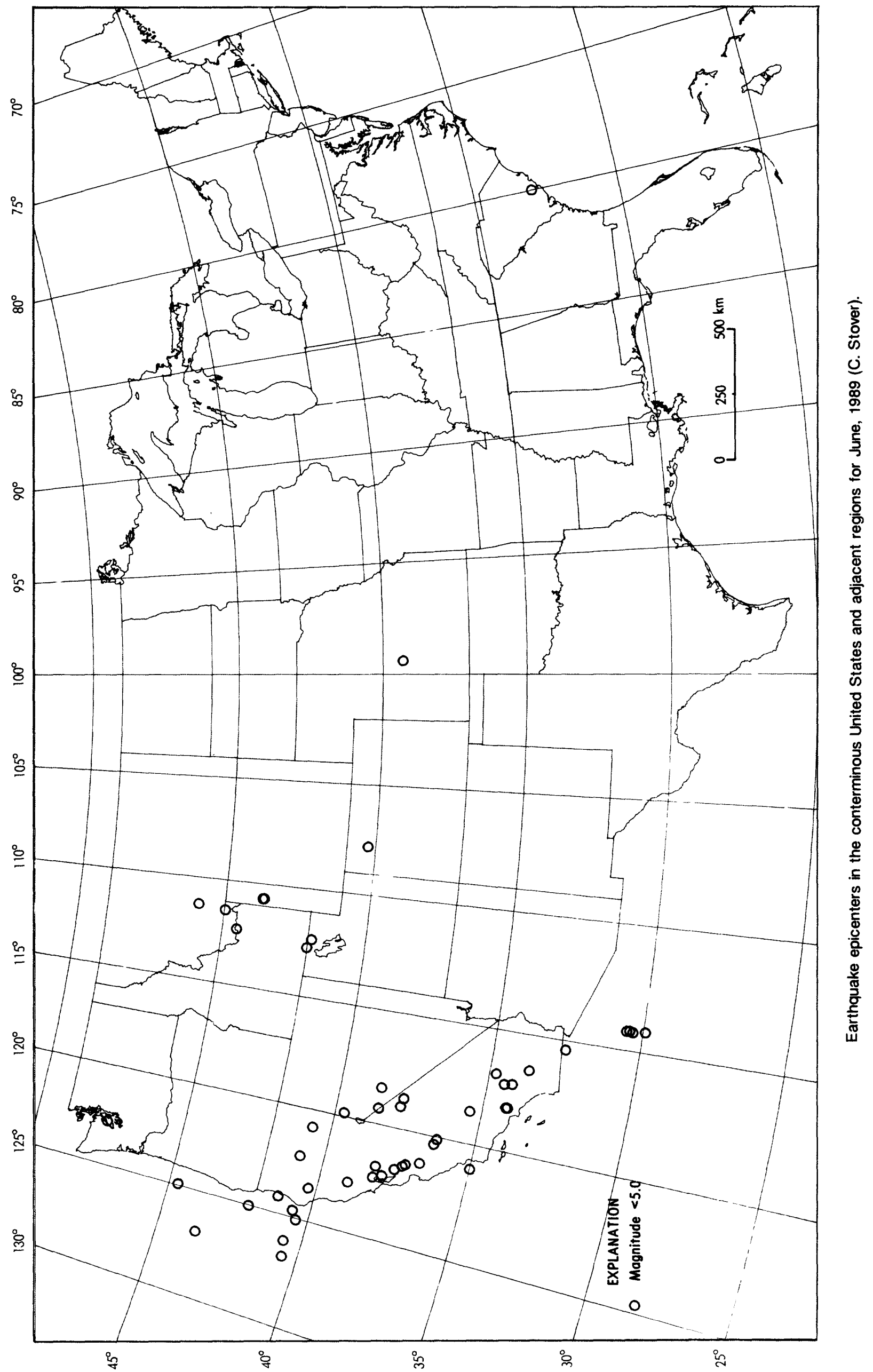




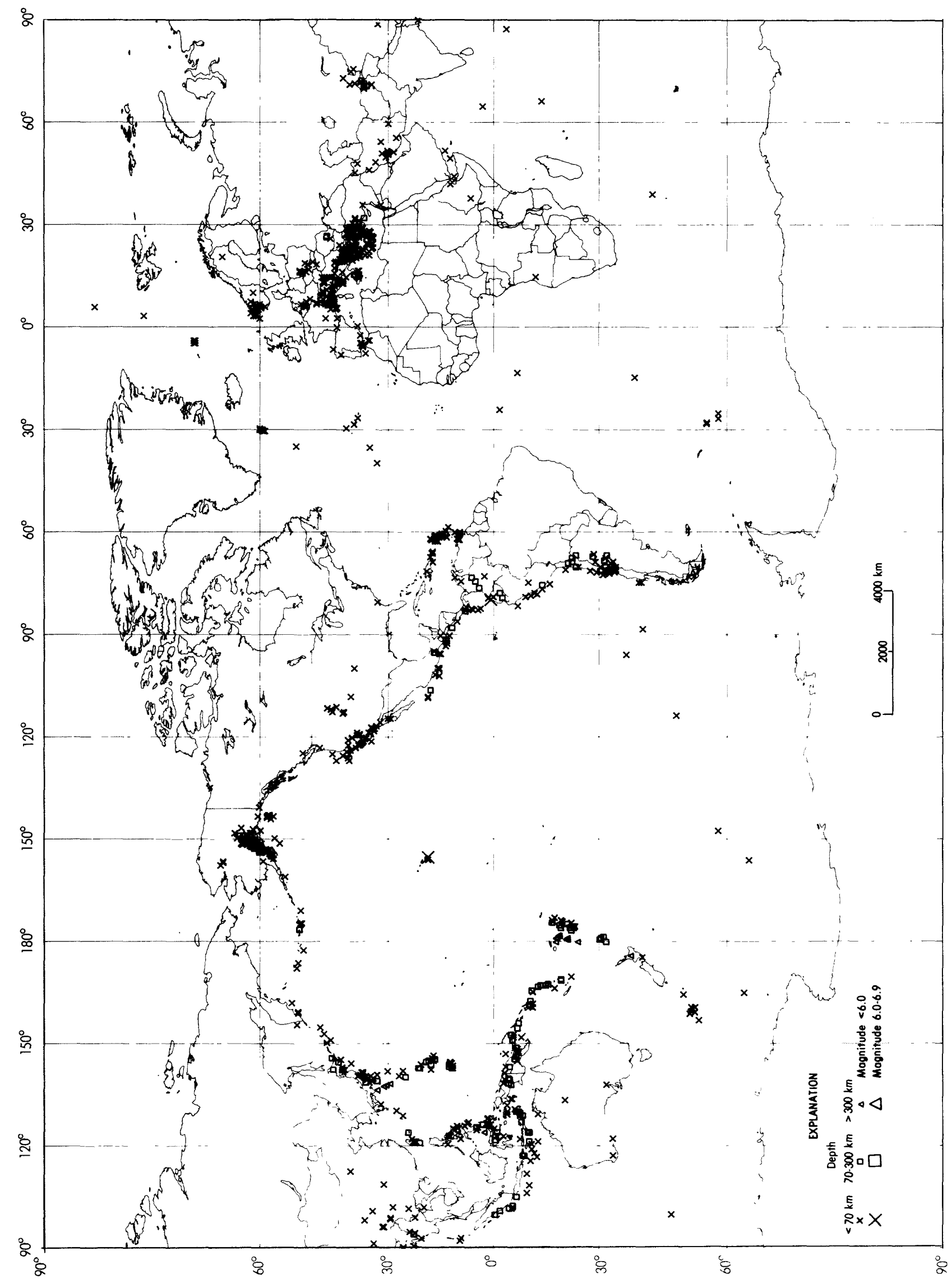

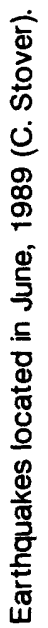

